


\section{SUSCEPTIBILITY PAYS OFF:}

Insights into the mlo-based powdery mildew resistance

Michela Appiano 


\section{Thesis committee}

\section{Promoter}

Prof. Dr R.G.F. Visser

Professor of Plant Breeding

Wageningen University \& Research

\section{Co-promoters}

\section{DrY. Bai}

Associate professor, Plant Breeding

Wageningen University \& Research

Dr A.M.A. Wolters

Senior Researcher, Plant Breeding

Wageningen University \& Research

\section{Other members}

Prof. Dr A.J.H. Bisseling, Wageningen University \& Research

Prof. Dr J.A.G.M. de Visser, Wageningen University \& Research

Dr F. Takken, University of Amsterdam

Dr C.M.P. van Dun, Rijk Zwaan Breeding B.V., Fijnaart / De Lier

This research was conducted under the auspices of the Graduate School of Experimental Plant Sciences. 


\title{
SUSCEPTIBILITY PAYS OFF: \\ Insights into the mlo-based powdery mildew resistance
}

\author{
Michela Appiano
}

Thesis

submitted in fulfillment of the requirements for the degree of doctor at Wageningen University by the authority of the Rector Magnificus

Prof. Dr A.P.J. Mol, in the presence of the

Thesis Committee appointed by the Academic Board to be defended in public on Friday 23 December 2016 at 1.30 p.m. in the Aula. 


\section{Michela Appiano}

SUSCEPTIBILITY PAYS OFF: Insights into the mlo-based powdery mildew resistance, 266 pages.

PhD thesis, Wageningen University, Wageningen, NL (2016)

With references, with summaries in English and Dutch

ISBN: 978-94-6257-948-4

DOI: $10.18174 / 391598$ 


\section{Table of contents}

$\begin{array}{lll}\text { Chapter } 1 & \text { General introduction }\end{array}$

Chapter 2 Genome-wide study of the tomato SIMLO gene family and its functional characterization in response to the powdery mildew fungus Oidium neolycopersici

Chapter 3 Functional characterization of a tomato syntaxin involved in powdery mildew resistance

Chapter 4 Identification of candidate MLO susceptibility genes in 101 cultivated Solanaceae and functional characterization of tobacco NtMLO1 and eggplant SmMLO1

Chapter 5 Monocot and dicot MLO powdery mildew susceptibility 133 factors are functionally conserved in spite of the evolution of class-specific molecular features

Chapter 6 A transposable element insertion in the susceptibility gene 159 CsaMLO8 results in hypocotyl resistance to powdery mildew in cucumber

Chapter 7 Discovery and characterization of a novel tomato mlo mutant 191 from an EMS mutagenized Micro-Tom population

Chapter 8 General Discussion

Summary

Samenvatting

Acknowledgements

About the author

List of publications 



\section{Chapter 1}

General introduction 
Plant breeding is the effort to select or modify crop plants to obtain genotypes with (more) favourable traits (Acquaah, 2012). Hereditary traits that breeders purposely want to modify are diverse. Yield and disease resistance, for example, have always been a priority. Recently, the need to select plants that are better adapted to environmental stresses has become more pressing because climate change is modifying the crop production environment. This fact implies that it is important to develop new varieties that can resist biotic (diseases and insect pests) and abiotic (e.g., salt, drought, heat, cold) stresses in their production environment.

By means of this thesis, we want to contribute to understanding and improving breeding activities aimed at obtaining resistance to one particular biotic constraint of Solanaceous and cucurbit crops, which is the powdery mildew (PM) disease.

It's in the interest of breeders to obtain durable resistance that is potentially effective against multiple pathogens. There is a class of genes that combines both features, and it is represented by the so-called susceptibility (S-) genes.

A well-known member of this class of genes is MLO (Mildew Locus $\underline{O}$ ). It is mainly studied as key factor to establish a compatible interaction between PM pathogens and plant hosts, but, at least in pepper, it is also involved in susceptibility to bacteria and oomycetes. Moreover, the resistance conferred by impaired MLO genes, called mlo-based resistance, is known to be durable, as exemplified by the long-lasting effectiveness of the barley Hvmlo gene in the field.

\section{The PM disease and the pathogens that cause it}

PM disease is one of the most important diseases of food and ornamental plants (Glawe, 2008).

\section{PM distribution and host range}

This disease is worldwide present, and sometimes more widely distributed in semiarid regions than in areas with high rainfall, where other diseases flourish. The fungal species responsible are a conspicuous number (more than 400). They can either infect a broad range of plants or prefer very specific plant species reaching in total almost 10,000 possible hosts. For example, the pathogen, Blumeria graminis f.sp. hordei (further mentioned as $B g h$ ), causing the PM disease in barley is listed among the top ten most detrimental fungal pathogens based on the scientific and economic importance (Dean et al., 2012).

New reports on PM disease between 2010 and 2016 indicate that this disease is still plaguing many countries in the world, therefore very actual. It also appears that the number of host species is enlarging, which means that climate change might be influencing powdery mildew geographic expansion and host range (Figure 1, Harvell et al., 2002; Garrett et al., 2006). 
General introduction | 9

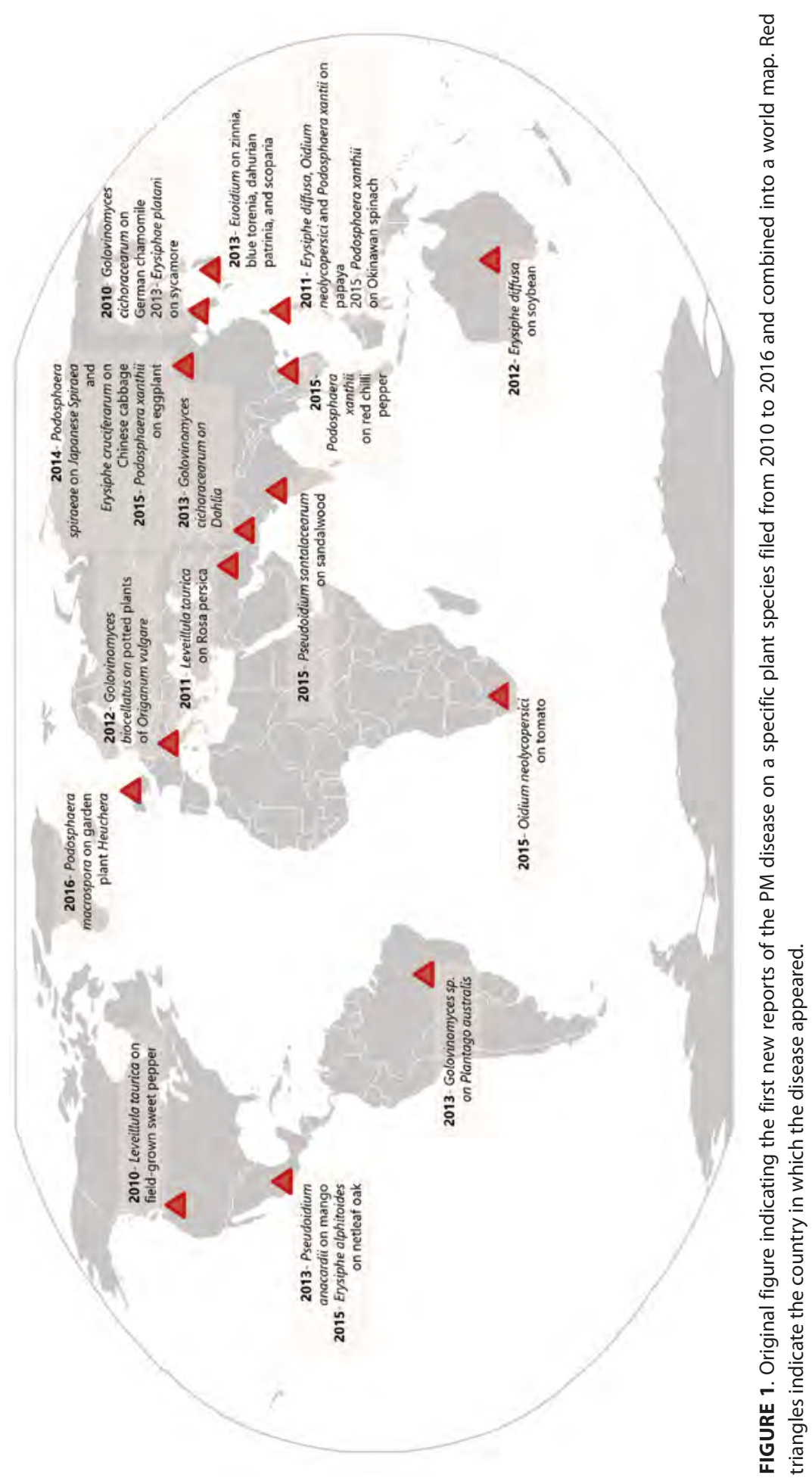




\section{PM symptoms}

PM disease is easy to recognize by the white powder covering all the aboveground plant organs, predominantly leaves and stems and occasionally flowers and fruits. It can cause various types of damage, such as deformation of stems and leaves, defoliation, reduced yields and lowered quality but also death of the attacked tissue (Figure 2).

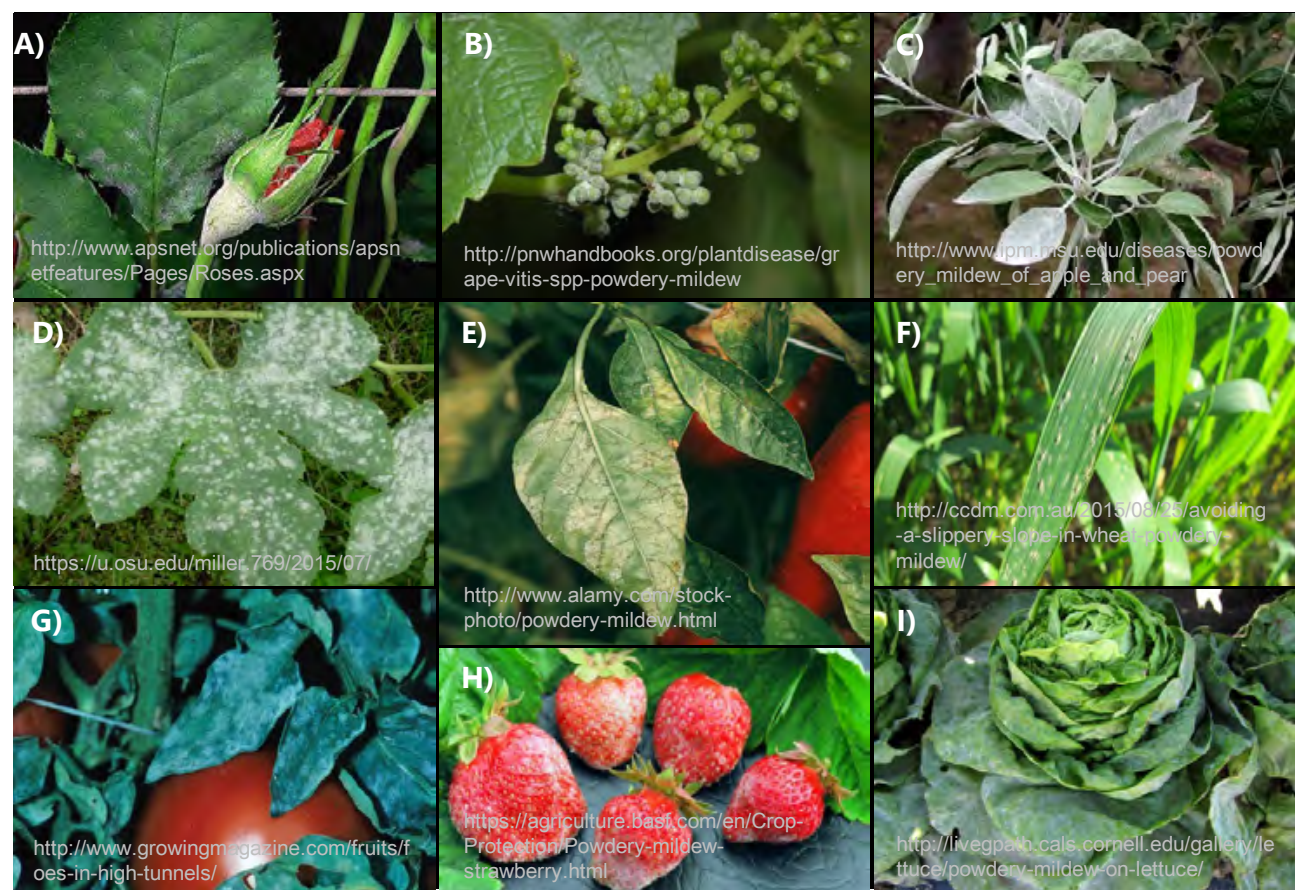

FIGURE 2. Powdery mildew symptoms on A) rose, B) grape, C) apple, D) squash, E) pepper, F) wheat, G) tomato, H) strawberry and I) lettuce. Photos are taken from Google images according to the website link given for each panel.

\section{PM lifestyle and life cycle}

PMs are obligate biotrophic pathogens as they rely upon the plant host to live, survive, reproduce, and thrive.

Each cycle begins with the landing of an airborne conidia on a host surface (Figure 3). When a conidium germinates, it forms a short hypha, called germination tube. The distal tip differentiates into a swollen, elongated appressorium. From the appressorium, a penetration peg breaks the plant epidermal cell wall and forms a haustorium within the cell surrounded by a plant-derived membrane. The haustorium represents the feeding structure of the fungus and the centre from where effector molecules are secreted. Once nutrients are available, the hyphae grow quickly, in most of the cases only epiphytically, producing secondary appressoria and haustoria, conidiophores and new airborne conidiospores. Altogether, they contribute to the powdery appearance of the disease on plant tissues. 


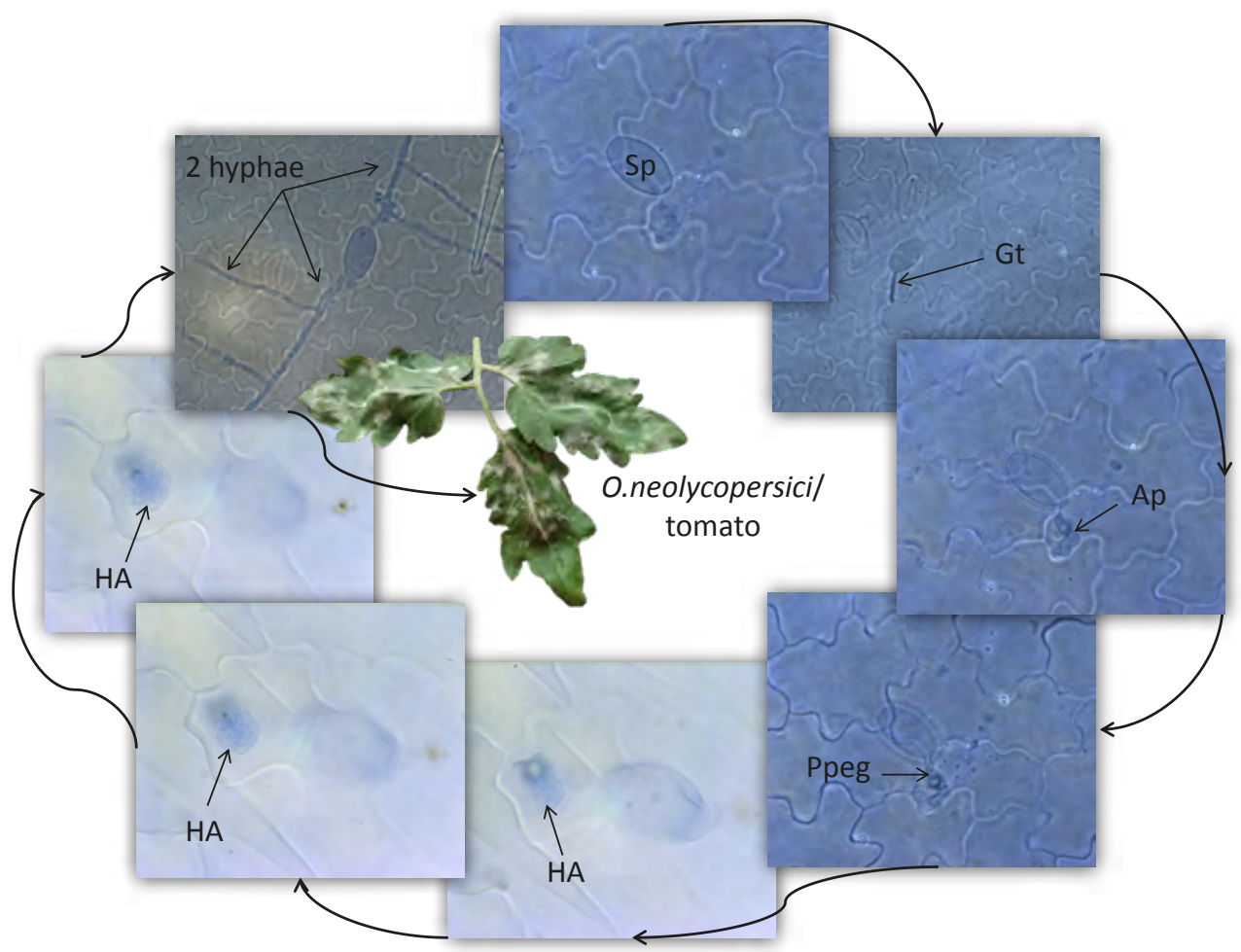

FIGURE 3. Life cycle of the PM pathogen Oidium neolycopersici on the susceptible tomato cv Moneymaker. Photos composing the figure are original and were taken during our microscopic studies. $\mathbf{S p}=$ spore; $\mathbf{G} \mathbf{t}=$ germination tube; $\mathbf{A p}=$ appressorium; $\mathbf{P p e g}=$ =penetration peg; $\mathbf{H A}=$ haustorium; $\mathbf{2}$ hyphae=secondary hyphae.

There are some PM species that, differently from the majority, penetrate through stomata. One of these PMs is an important pathogen for tomato, pepper, eggplant and other crops, namely Leveillula taurica (Braun and Cook, 2012). The haustoria of this fungus are formed in the mesophyll cells. Therefore, the mycelium is partially endophytic. Conidiophores emerge through stomata, mainly on the abaxial leaf surface, while on the adaxial surface, chlorotic spots are usually visible, indicating the development of mildew colonies underneath (Figure 2, panel E) (Zheng et al., 2013).

\section{PM classification}

All the PM fungi belong to the ascomycete order of the Erysiphales.

Mildews known only in their asexual reproductive stage (anamorph state) are called by the genus name Microidium (formerly Oidium). All PMs with Oidium anamorph can be grouped into two genera: Euodium, if they exhibit catenate type of conidial development, or Pseudoidium, when conidia mature one at the time (Braun and Cook, 2012). 
The latest classification of Braun and Cook (2012), based on holomorph (both the asexual and sexual stages), conidial germination and SEM (Scanning Electron Microscope) data, distinguishes six tribes: Erysipheae, Golovinomyceteae, Cystotheceae, Phyllactinieae, Blumeriae and one unnamed tribe to place the anamorph genus Microidium.

\section{PM-Omics}

The genome of several powdery mildew species has been recently sequenced, such as Erysiphe necator, Blumeria graminis f.sp hordei (Bgh), Erysiphe pisi, Golovinomyces orontii and Blumeria graminis f.sp tritici (Bgt) (Jones et al., 2014; Spanu et al., 2010; Wicker et al., 2013). A number of genomes of other pathogenic fungi are currently underway, like tomato PM Oidium neolycopersici, lettuce PM Golovinomyces cichoracearum, pepper PM Leveillula taurica, cucumber PM Podosphaera xanthii and strawberry PM Podosphaera aphanis (Bindschedler et al., 2016).

Data obtained from the sequenced genomes indicate that although about 4-times larger than other related Ascomycetes (average size is 36.9 Mbp; Mohanta \& Bae, 2015), PM genomes miss many coding genes otherwise present in Ascomycetes species (average number of coding genes is more than 11.000; Mohanta \& Bae, 2015).

The genome size increased because of the proliferation of transposons. The close association of transposon with effector-encoding genes most probably led to the rapid evolutionary adaptation of powdery mildews (Hacquard et al., 2013).

A first transcriptome analysis of conidia, germinated conidia, and hyphae of Bgh revealed a coordinated change in gene expression patterns between pre- and post-penetration stages (Thomas et al., 2001). Transcripts associated with lipid metabolism, for example, accumulated during fungal germination, while transcripts of genes involved in glycolysis were high in mature appressoria and infected epidermal cells. During the later phase of plant colonization, transcripts related to protein biosynthesis accumulated.

A second transcriptome analysis of epidermal peels of heavily infected Bgh leaves, provided insights into the transcripts of candidate effector proteins (Godfrey et al., 2010). This analysis resulted in the identification of a conserved sequence motif ( $\mathrm{Y} / \mathrm{F} / \mathrm{W}-\mathrm{X}-\mathrm{C}$ ) in most of the candidate effectors. However, the functional relevance is still under investigation. Another transcriptome study of $G$. orontii-infected $A$. thaliana plants revealed transcripts encoding proteins for the detoxification of reactive oxygen species (Weßling et al., 2012).

Regarding the PM proteome, several attempts have been made to study it (Bindschedler et al., 2009; Bindschedler et al., 2011; Godfrey et al., 2009; Noir et al., 2009). However, the low biomass of the pathogen in the early stages of infection and the dependence on a wellsequenced, well-assembled and well-annotated genome are challenges still to overcome to achieve good proteomic analyses. In a protein-protein interaction of secreted G. orontii 
PM effector candidates with their possible Arabidopsis targets, it was found that multiple PM effectors converged onto a limited number of host targets (hubs). These hubs are themselves interconnected with other host proteins and targeted by other pathogens, like oomycetes and bacteria (Weßling et al., 2014).

\section{PM epidemiology}

An enormous number of conidia are produced during the growing season (Bélanger et al., 2002). Under disease-conducive conditions, a cycle is usually completed within 3-7 days after infecting the host. The rapid rate of asexual reproduction can lead to exponential growth of powdery mildew populations resulting in epidemics.

In regions with mild climates, powdery mildews can survive winter in the form of dormant mycelium within buds of infected plants (Braun and Cook, 2002). Moreover, in these regions sexual fruiting bodies (also called ascocarps) do not seem to occur frequently. When ascocarps are produced, they can survive harsh conditions since they are resistant to low temperatures and drought.

\section{PM and climate change}

Disease symptoms are the product of the interaction not only between a susceptible host plant and a virulent pathogen but also of the two of them with the environment. The changes associated with global warming (i.e., increased temperatures, changes in the quantity and pattern of precipitation, increased $\mathrm{CO}_{2}$ and ozone levels, drought, etc.) may affect the incidence and severity of plant diseases. The same changes can influence the further coevolution of plants and their pathogens (Burdon et al., 2006; Chakraborty 2005; Crowl et al., 2011; Garrett et al., 2006).

For example, the effect of changes in temperature has been studied in the pathosystem tomato-PM caused by O. neolycopersici (Elad et al., 2009). They observed that disease severity increased dramatically from $18^{\circ} \mathrm{C}$ to $26^{\circ} \mathrm{C}$.

Elevated $\mathrm{CO}_{2}$ levels are expected to increase plant canopy size and density, which, if combined with increased humidity at the canopy level, will promote PM growth (Manning \& Tiedemann, 1995). On the other hand, other studies showed that wheat grown in a high level of $\mathrm{CO}_{2}$ was more resistant to the pathogen Blumeria graminis f.sp. tritici (Bgt) because plants were able to mobilize the assimilates into defense structures, like papillae (Hibberd et al., 1996a and 1996b). Nevertheless, if able to penetrate, colonies of Bgt grew quicker in $2 \mathrm{X}-\mathrm{CO}_{2}$ than in normal conditions (Hibberd et al., 1996a).

Salinity is also a major environmental stress and increased salinization of arable land is expected to have devastating global effects. Given this problem, a recent study addressed at elucidating the interaction between abiotic and biotic stress in tomato indicates that 
a mild salt stress $(50 \mathrm{mM} \mathrm{NaCl})$ in combination with PM inoculation results in enhanced susceptibility of the cultivated tomato (Kissoudis et al., 2016).

\section{PM control}

Traditionally, sulphur dust, lime sulphur, and copper sprays have been considered specific remedies for PMs. Fungicides like sterol-biosynthesis-inhibitors (DMI), quinone outside inhibitors (Qol), and quinoline are often used in PM management programs, but some powdery mildew pathogens have developed resistance to a range of these fungicides (Pirondi et al., 2014).

Pruning the plants, spacing them correctly, irrigating properly to reduce the humidity and limiting nitrogen fertilization are among the good agricultural practices to consider. Furthermore, crop rotation, removal of debris, optimal sowing conditions and timing are other good strategies to control this disease.

Recently the biological control of PMs has become possible through the use of mycoparasitic fungi or yeasts. The European project called BIOCOMES, for example, is looking for sustainable biological control agents of the pathogen Bgt causing the PM disease on wheat.

For the cucumber PM disease caused by Podosphaera xanthii, a recent study reported the efficacy of a biologically active isolate of the epiphytic yeast Pseudozyma aphidis (Gafni et al., 2015). This yeast has an antagonistic effect and an antibiosis effect on P. xanthii: the crude extract of the yeast affects the fungal spores germination, while application of $P$. aphidis suspension on cucumber leaves can reduce disease severity up to $75 \%$.

Last but not least, powdery mildew can be fought using resistant varieties. In tomato, for example, nine loci have been found to confer resistance to O. neolycopersici, five of which are dominant genes (Ol-1 to Ol-6, except ol-2), and three are QTLs (Bai et al., 2005; Seifi et al., 2014). The ol-2 gene is a recessive allele of the SIMLO1 gene (Bai et al., 2008). The Ol-4 and ol-2 genes are, currently, used in breeding programs.

This overview shows the importance of this disease regarding the economic impact and scientific interest. As mentioned above, chemical control of this disease is limited and not always effective. Genetic control, on the other hand, is a valuable and probably durable solution. 


\section{PM perception and the lines of defence}

\section{The first layer of defence}

\section{Plant barriers}

The PM infection starts with a conidium landing on the host surface. The first barrier for a PM to overcome is the constitutive layer of the plant defence like cuticle, epicuticular waxes, and cell wall. Unlike other pathogens that use cell wall degrading enzymes, PM pathogens, or at least $B g h$, presumably use hydrostatic pressure to penetrate this first layer of defence (Pryce-Jones et al., 1999). Such pressure is built up by the appressorium and might activate plant mechanosensors. It might also damage the cell wall, releasing damage-associated molecular patterns (DAMPs) or the fungus itself can produce microbeassociated molecular patterns (MAMPs). However, it is important to notice that because of their biotrophic lifestyle, PM infection strategy is to invade the plant host creating as little damage as possible. Indeed, a recent study pointed out that in the early stages of $O$. neolycopersici infection of a susceptible tomato cultivar, the detection of biotic emissions, such as biogenic volatile organic compounds (BVOC) stocked in the trichomes and released if damaged, was very low (Kasal et al., 2016).

Part of the early line of defence responses includes cell wall reinforcement below the fungal appressorium. This process, starting with cell polarization, cytoskeleton rearrangement and focal accumulation of defence-related proteins, leads to the formation of a domeshaped apposition between the epidermal wall and the plasma membrane. This structure is called papilla (Assaad et al., 2004; Collins et al., 2003). Interestingly, papillae are a front line of defence in response to both adapted and non-adapted formae speciales of powdery mildew fungi (Aghnoum \& Niks, 2010). Their effectiveness depends on timing and structural composition (Chowdhury et al., 2014).

\section{MAMP-triggered immunity (MTI)}

MAMPs and DAMPs can be detected by pattern recognition receptors (PRR) located in the plasma membrane. The recognition triggers a plethora of defence responses, such as alkalinization of the apoplast and production of reactive oxygen species (ROS), influx of $\mathrm{Ca}^{2+}$, and activation of mitogen-activated protein kinase (MAPK) pathways. Finally, biosynthesis and excretion of antimicrobial products like PR (pathogenesis-related) proteins and phytoalexins occur. These responses lead to the MAMP-triggered immunity (MTI).

Chitin is a major component of fungal cell walls. Plant cells have chitin degrading enzymes to digest fungal cell walls during an infection. Chitin fragments, considered as MAMP, are subsequently perceived by the plant triggering an immune response. It was discovered in 
Arabidopsis that chitin is perceived by the membrane-localized PRR CERK1 (Chitin Elicitor Receptor Kinase 1) and LYKs 4/5 (Lysin motif receptor-like kinases 4/5) (Cao et al., 2014; Miya et al., 2007). CERK1 is a plasma membrane protein containing extracellular LysM motif and intracellular kinase domain. Arabidopsis cerk1 mutants are more susceptible than wild type to the adapted PM Golonovinomyces orontii (Go) and do not respond anymore to chitin elicitor (Miya et al., 2007). Similarly, lyk4/lyk5 mutants show complete loss of chitin response. The results of Cao et al. (2014) indicate that AtLYK5, primary receptor for chitin, forms a chitin-inducible complex with AtCERK1 to induce plant immunity.

\section{The second layer of defence: ETI}

The recognition of MAMPs is counteracted by the action of pathogen-secreted intracellular effectors that prevent the induction of PRR-mediated signaling, initiating the EffectorTriggered Susceptibility (ETS) (Jones \& Dangl, 2006). The effectors are microbial molecules known in the past as Avr proteins. Whether recognized or not, the primary role of the Avr protein is to aid the invader in colonizing the host either by suppressing the plant immune signalling or by altering the plant's physiology to the pathogen's advantage (Chen et al., 2010). Effectors are delivered into the host cells by the haustorium. However, the delivery mechanisms of the effectors are largely unknown. Two papers provided a model for the mechanism of PM effectors' delivery into the plant cell (Voegele et al., 2009; Zhang et al., 2013). Effectors are hypothesized to be transferred from the extra-haustorial matrix to the cytosol through retrotranslocon pores located in the endoplasmatic reticulum (ER). A protein called Sec61 is hypothesized to be necessary for pore function. This trafficking is thought to take place in vesicles, dependent or independent of Golgi. The silencing in barley of the HvSec61ßa translocon reduces barley's susceptibility to Bgh. This result suggests that Sec61ßa is recruited by the PM pathogen to allow effector's delivery in the cytoplasm.

In turn, plant intracellular receptors also called NB-LRRs- or R-proteins can evolve to recognize these effectors and trigger a new immunity called Effector-Triggered Immunity (ETI). Often, the recognition of the pathogen-Avr and plant-R protein is accompanied by a form of programmed cell death called hypersensitive response (HR). Effectors can be recognized either directly or indirectly by the NB-LRR proteins. In the case of indirect recognition, NB-LRRs 'guard' host proteins called effector target. Modification of this target by the effector results in the activation of the $\mathrm{R}$ protein, which triggers the immune response (Dangl \& Jones, 2001).

There are several examples of $R$-genes taken from the plant-PM pathosystem. In wheat, the $P m 3 b$, a member of the coiled-coil nucleotide binding site leucine-rich repeat (NBSLRR), is responsible for the race-specific resistance to the PM pathogen Bgt (Yahiaoui et al., 2004). The Pm3 locus is syntenic to the Mla locus of barley which confers resistance to 


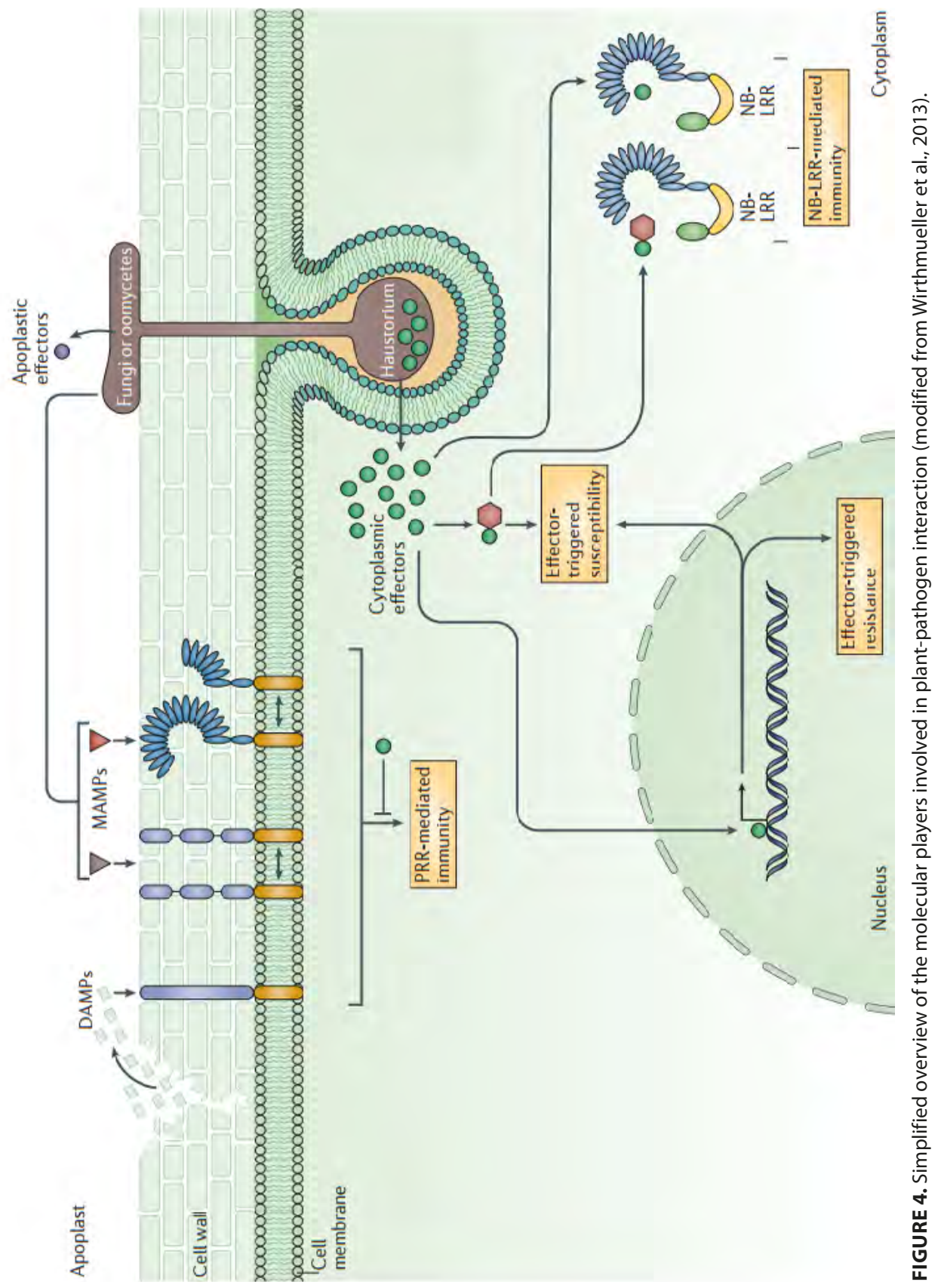


the barley PM Bgh (Hartl et al., 1993; Zhou et al., 2001). In both cases, recognition of the specific pathogen avirulence proteins by the cognate NBS-LRRs induces HR (Hückelhoven et al., 2000; Hückelhoven \& Kogel, 1998; Bourras et al., 2015). In Arabidopsis, two paralogs RPW8.1 and RPW8.2 contribute to the resistance against PM-Go (Xiao et al., 2001). The resistance mediated by RPW 8 occurs after the formation of haustoria and it is typically associated with the accumulation of hydrogen peroxide and localized host cell death (Xiao et al., 2001; Göllner et al., 2008). In tomato, the Ol-4 gene, deriving from the wild tomato species S. peruvianum LA2172, was mapped on the short arm of chromosome 6 where a cluster of $R$-genes is found (Seifi et al., 2010). It was also shown to confer PM resistance towards $O$. neolycopersici through fast hypersensitive response (Bai et al., 2005).

This model of continued co-evolution in which pathogens overcome the layers of plant immunity and plants recognize conserved and variable pathogen extracellular and intracellular elicitors is known as the 'zig-zag' model (Figure 4, Dodds \& Rathjen, 2010; Jones \& Dangl, 2006).

In this simplistic model, it may appear as if MTI and ETI are strictly separated. However, several studies show that there is no clear-cut distinction between the two signalling pathways but a continuum (Thomma et al., 2011). The difference lies mostly in the amplitude and kinetics of the response since both pathways overlap at multiple levels (Qi et al., 2011; Tsuda \& Katagiri, 2010).

\section{Genes contributing to the susceptibility to powdery mildew}

After the discovery of the gene PMR6, required for susceptibility to powdery mildew in Arabidopsis (Vogel et al., 2002), Eckardt (2002) pointed out how little research was aimed at studying the so-called plant susceptibility genes (S-genes).

S-genes are plant genes that facilitate the infection and support a compatible interaction with the pathogens (van Schie \& Takken, 2014). As described above, adapted pathogens have evolved ways to interfere with the plant immune system. Its consequent failure allows entry of the pathogens. In this process, the host is tricked to collaborate with the biotroph invader. The haustorial complex, for example, is partially fungus-derived, and partially (or fully) plant-derived. Following the haustorial complex, the host plant reroutes nutrients and hormones, like cytokinines, towards the infection sites, which become metabolically active "green islands" (Fotopoulos et al., 2003; Walters, 2015). Hence, the host contributes to the accommodation of the biotroph pathogen in many ways.

Several S-genes have been characterized for their role in PM disease. Based on the reviews of van Schie and Takken (2014) and Lapin \& Van den Ackerveken (2013), they can be grouped in three classes. Genes of class I allow a basic compatibility, attracting 
the pathogen and allowing its penetration. Genes of class II suppress the plant immune signalling. Genes in class III are involved in the sustained compatibility, providing pathogen accommodation and meeting its metabolic and structural needs.

In the context of powdery mildews, a representative of the class I S-genes is the maize (Zea mays) GLOSSY1 (GL1). Spores of Bgh do not germinate, and appressoria do not develop on leaves of this mutant because it lacks long chains of aldehydes normally present in the waxy cuticle (Hansjakob et al., 2011). This cuticle compound is, therefore, particularly important for the successful germination and differentiation of the PM pathogen Bgh.

Once the pre-penetration process is completed, the next barrier to overcome is the cell wall. One of the best examples of S-genes required for powdery mildew penetration is the $M L O$ (Mildew Locus O) gene. Like the MLO, a number of proteins encoded by other S-genes are involved in the penetration phase. Lifeguard (LFG) proteins, among which the BAXinhibitor 1 (BI1), but also RAC/ROP G-proteins and GAP, are related to vesicle trafficking and focal orientation of the cytoskeleton towards the infection site (Eichmann et al., 2010; Hückelhoven, et al., 2003; Opalski et al., 2005; Schmidt et al., 2014; Schultheiss et al., 2003; Weis et al., 2013). Probably these proteins help the invagination of the cell membrane, and the accommodation of the fungal haustorium. Interestingly, these genes confer susceptibility, not only towards adapted pathogens, among which powdery mildews, but also resistance towards non-adapted biotrophic and necrotrophic fungi because they cannot bypass the first layer of induced defences.

Once the haustorium is developed, the pathogen needs to maintain the interaction with the host. At this point, other S-genes (class II and III) are involved to ensure a long-lasting intimate relationship with the PM pathogen by suppressing the plant immune responses and providing nutrients for the pathogen's growth. One of these genes is DMR1. It encodes a homoserine kinase which is an enzyme involved in the biosynthesis of the amino acids methionine, threonine, and isoleucine. They represent presumably essential metabolites for the sustainment of the pathogen and for the plant itself (Huibers et al., 2013). PMR4 encodes a callose synthase which negatively regulates the salicylic acid (SA) pathway and may suppress MTI by preventing the accumulation of callose precursors (Ellinger et al., 2013; Nishimura et al., 2003; Huibers et al., 2013). In addition to PMR4, the CESA3 (cellulose synthases) gene may function as a suppressor of the MTI by negatively regulating jasmonic acid (JA) and ethylene (ET) (Ellis \& Turner, 2001). Finally, EDR1 encodes an MAPK kinase-kinase which is a negative regulator of multiple defence responses leading to the MTI (Frye \& Innes, 1998; Frye et al., 2001; Gao et al., 2015). 


\section{Turning susceptibility into a winning factor}

S-genes are interesting because, if mutated, they can be turned into powerful resistance factors. For all the S-genes mentioned above, there are mutants or silenced transgenic plants that are resistant to the powdery mildew disease and in some cases also to other diseases. Indeed, one of the powerful effects of the loss-of-function of S-genes is a broadspectrum resistance against multiple isolates of the same pathogen, but, in some cases, also against different pathogens.

Moreover, contrary to the use of R-genes, the resistance offered by the S-genes is expected to be durable. The introduction of R-genes in cultivated crops represents a longstanding procedure. However, the selective pressure on the pathogen, conferred by the racespecificity of the R-gene, is high. A mutation appearing in the effector is sufficient to avoid the recognition of the R-gene, making the deployed resistance ineffective. Therefore, the resistance conferred by the R-genes is rarely durable. Overcoming the S-gene resistance, on the other hand, implies that the pathogen acquires a new way to infect the host. It is highly improbable for this to happen, also considering how much powdery mildews, as biotroph, are dependent on host factors. The MLO and eukaryotic initiator factor $4 \mathrm{E}$ (elF4E) genes represent well-known examples of durable S-gene resistance. The mlobased resistance was discovered in the 1940's in barley (Lundqvist 1991). It is active against all known isolates of barley PM Bgh (Jørgensen, 1992). The pvr-based resistance, due to the loss-of-function of elF4E, was described in pepper in the 1960's to be effective against potyvirus (Cook, 1960). Since their discovery, mlo and pvr resistances have been commercially used with great success (Brown, 2015; Moury \& Verdin, 2012).

Effectiveness regarding duration and spectrum of action are features that have attracted a lot of attention from public and private sectors. Consequently, the use of impaired S-genes is considered now an alternative and, possibly more successful breeding strategy than the classical use of R-genes (Pavan et al., 2010).

So, how to apply S-genes into a breeding program? The first step consists of searching for homologs in the crop species of interest. Much research has been performed to screen Arabidopsis mutant collections and natural accessions in search for resistant phenotypes towards several pathogens. Therefore, many S-genes are described in Arabidopsis. Nevertheless, the availability of genome sequence information of many important crop species allows the identification of the corresponding homologs.

If the desired S-gene exists in the crop species of interest, the second step is to verify if it is functional towards corresponding crop pathogens. Constitutive gene silencing by RNA interference (RNAi), knock-out expression by cleavage of targeted DNA sequences using sequence-specific nucleases, or overexpression of the candidate S-gene to verify its ability to restore the susceptibility of a resistant mutant, are suitable options in crop 
species amenable to transformation. Transient gene silencing through viral vectors is an alternative for crops that are recalcitrant to transformation.

If the function is, indeed, conserved, it is important to verify that there are no pleiotropic effects associated to the loss-of-function of the S-gene of interest. Negative effects of the disruption of S-gene function consist of dwarfing, induced lesions, senescence, susceptibility to other pathogens, reduced root colonization by mycorrhizal fungi and lower fertility (Jarosch et al., 1999; Jørgensen, 1992; Kessler et al., 2010; Kumar et al., 2001; Lorrain et al., 2003; Poraty-Gavra et al., 2013; Ruiz-Lonzano et al., 1999; Zheng et al., 2013). At this point, the possible use of the $\mathrm{S}$-gene depends on a careful consideration of benefits and drawbacks.

An elegant example of the application of this strategy is given by Huibers (2013). The authors describe the identification of two Arabidopsis homologs in tomato, the callose synthase SIPMR4, and the homoserine kinase SIDMR1. Upon RNAi silencing of both genes, tomato plants became resistant to the PM disease. However, silenced SIDMR1 plants suffered from reduced growth, while silenced SIPMR4 plants did not present any pleiotropic effect.

If the use of the S-gene is promising, a stable mutant can be obtained through different strategies. The first strategy consists of finding natural mutations among wild relatives of the crop of interest, then crossing with the cultivated species, and performing extensive backcrossing programs. Alternatively, mutations can be induced through chemical or physical mutagens. Afterwards, a screening of large populations to identify mutants with desirable properties is needed. Another option involves targeted knockdown of the gene of interest via RNAi or knock-out by genome editing tools like zinc finger nucleases (ZFNs), transcription activator-like effector nucleases (TALENs) and CRISPR-Cas9 system (Pavletich \& Pabo, 1991; Joung \& Sander, 2013; Liu \& Fan, 2014; Urnov et al., 2010). The latter has been successfully and fast adopted by the scientific community because its application is simpler, less costly and more efficient compared to the former technologies (Liang et al., Zhang, 2014). Moreover, there is confidence that in the future the plants obtained with this editing tool are considered non-transgenic by the European regulatory office (Bortesi \& Fischer, 2015).

One final consideration concerns the possibility that the susceptibility factor is encoded by multiple (redundant) genes of a gene family. In this case, it is necessary to evaluate the effect of each homolog on the phenotype and eventually target multiple genes to obtain a complete loss-of-function mutant. The CRISPR/Cas 9 system can be particularly useful to knock out redundant genes (Wang et al., 2014). 


\section{Molecular components of the mlo-based resistance}

The $M L O$ gene is a well-known example of S-genes. It encodes a plant-specific protein which typically contains seven transmembrane domains embedded in the plasma membrane.

Disease resistance is characterized by early termination of fungal infection due to failed host cell invasion. The typical histological feature is the occurrence of papillae deposited at the sites of attempted penetration.

The mlo-based resistance relies on two pathways, one dependent and the other independent from the actin of the cytoskeleton (Miklis et al., 2007). The molecular components of the actin-dependent pathway are conserved among the plant species investigated, namely barley, Arabidopsis, and grapevine (Consonni et al., 2006; Feechan et al., 2013; Panstruga, 2005). They participate in two distinct secretory systems. In one, a ternary complex is formed between a plasma membrane protein AtPEN1/HvRor2 and two other SNARE proteins to allow vesicle fusion (Collins et al., 2003; Freialdenhoven et al., 1996). In the second secretory system, toxic compounds produced by the glycosyl hydrolase PEN2 are transported across the plasma membrane by a PEN3 transporter (Lipka et al., 2005).

The three PENETRATION (PEN) genes were identified by screening Arabidopsis mutants with increased penetration success of the non-adapted barley powdery mildew Bgh (Collins et al., 2003). Later, all three genes have also been found to be required for mlo-based powdery mildew resistance in Arabidopsis plants (Consonni et al., 2006). These facts support the hypothesis that non-host immunity and mlo resistance rely on functionally overlapping defence pathways (Humphry et al., 2006). Moreover, they are both independent from SA, JA, and ET and share the same histological mechanism of defence response (Ellis, 2006; Trujillo et al., 2004).

\section{The charm of the MLO genes dwells in their mystery}

With the availability of genomic information, it was found that multiple copies of the MLO gene are present in the genome of a plant species. A recent evolutionary study, which reconstructed the likely evolution of the MLO family in embryophytes, traced back MLOlike proteins not only to plants but also to unicellular eukaryotes, like green and red algae (Ellis, 2006; Kusch et al., 2016; Trujillo et al., 2004). The number of homologs depends on the plant species investigated. However, in general, the $M L O$ gene families of land plants range from small (seven members) to medium size (39 members) (Acevedo-Garcia et al., 2014; Kush et al., 2016). The phylogenetic analyses of the MLO gene families resulted in the distinction of seven clades, although not all plant species harbor representatives of all clades (Kusch et al., 2016). 
MLO genes in Clade IV and V act as powdery mildew susceptibility factors of monocot and dicot species, respectively. In Arabidopsis three of its $15 \mathrm{MLO}$ homologs are involved in PM resistance, although AtMLO2 has a larger effect compared to the other two genes, AtMLO6 and AtMLO12 (Consonni et al., 2006).

Studies on the pepper Clade V-CaMLO2 indicate that this gene is involved in modulating biotic responses not only to powdery mildew but also to bacterial and oomycete pathogens like Xanthomonas campestris pv vesicatoria, Pseudomonas syringae pv tomato and Hyaloperonospora arabidopsis (Zheng et al., 2013; Kim \& Hwang, 2012). Additionally, this gene is a negative regulator of ABA signaling, implicating CaMLO2 involvement in drought stress responses (Lim \& Lee, 2014).

Other Clades harbor MLO genes associated with two distinct developmental processes. Arabidopsis null mutants Atmlo4 and Atmlo11 show aberrant root thigmomorphogenesis, which is exemplified as unusual root curling upon a tactile stimulus under in vitro conditions (Bidzinski et al., 2014; Chen et al., 2009). Both AtMLO4 and AtMLO11 are grouped into Clade I.

Another Arabidopsis mutant called nortia, which carries a 20-bp deletion in the AtMLO7 gene, shows reduced fertility and pollen tube overgrowth in the synergid cells (Kessler et al., 2010). These cells, present in the mature embryo sac, guide pollen tubes towards the double fertilization process in angiosperms. The AtMLO7 belongs to Clade III. The involvement of the remaining AtMLO genes in any other processes is still unknown.

In conclusion, MLO proteins seem to be involved in many biological processes, although their core biochemical activity is not known yet. Based on topology and subcellular localization, the MLO proteins resemble G protein-coupled receptors (GPCRs). However, no experimental evidence supports the hypothesis that MLO proteins function as a GPCRs (Kim et al., 2002). A conserved peptide motif is represented by the intracellular calmodulin-binding domain (CaMBD) which interacts in vitro with calmodulin (CaM) in a $\mathrm{Ca}^{2+}$-dependent manner (Kim et al., 2002). Nevertheless, the precise mechanism of the modulation of CaM by MLO proteins is not clear yet. In an experiment, aimed at investigating genes co-expressed with barley HvMlo and Arabidopsis AtMLO2, a considerable number of receptor-like kinases (RLK) was found (Humphry et al., 2010). This finding might link MLO proteins to other components of plant immunity, but it requires further experimental evidence to explain how they are interconnected in a functional way. 


\section{Scope of the thesis}

This work is part of a line of studies investigating mlo-based resistance in plants, and particularly in crop species.

After the characterization in tomato of ol-2 as a loss-of-function allele of the SIMLO1 gene (Bai et al., 2008), one part of the research described in this thesis focused only on MLO genes in tomato and on components of the tomato mlo-based resistance; the other part on the investigation of $M L O$ genes from difference species.

In Chapter 2, we used tomato sequence information to complete the characterization of the SIMLO homologs. Particular attention was given to the Clade V members of the tomato MLO phylogenetic tree. The outcome showed that the simultaneous silencing of SIMLO1 and two of its closely related homologs confers a higher resistance level than the one obtained by using the ol-2 allele alone. In Chapter 3, we investigated the role of the SIPEN1-like genes as factors required for the mlo-based resistance in host and non-host interactions strengthening the assumption that "non-host and mlo-based resistance may be the two faces of the same coin" (Humphry et al., 2006).

The second line of research was inspired by the fact that $M L O$ susceptibility genes are conserved across plant species and therefore can be used in breeding programs if 1) identified and 2) natural or induced mutations are found. In Chapter 4, we identified and functionally characterized the orthologs of SIMLO1 in tobacco and eggplant, two other important Solanaceae crops. We named the newly identified genes NtMLO1 and SmMLO1, in the respective species. We also verified that a Q198R mutation in the protein sequence of NtMLO1 leads to complete loss of function, enlarging the list of point mutations with a detrimental effect on the MLO proteins.

In Chapter 5, we proved that despite phylogenetic distance and evolution of different molecular features, monocot and dicot MLO proteins involved in the interaction with powdery mildews are functionally conserved. Also, we confirmed that functional monocot and dicot MLO proteins are both recruited by adapted and non-adapted powdery mildew pathogens during the early phase of the infection, allowing them to penetrate the cell wall. Finally, we set up a suitable complementation assay to validate the function of putative susceptibility MLO genes in new species.

In Chapter 6, we characterized the resistance observed in an advanced cucumber breeding line due to the insertion of a transposable element (TE) in the CDS of the CsaMLO8 gene. We verified that this disrupted allele is not functional, contrary to the wild-type CsaMLO8 allele. Also, we showed that the TE-containing allele represents a natural mutation of the functional CsaMLO8 because it is present also in a wild cucumber accession. 
Finally, in Chapter 7, we describe the in-house development of an EMS Micro-Tom population aimed at finding new sources of resistance to different pathogens. Regarding powdery mildew, we discovered a new loss-of-function allele of the SIMLO1 gene, named $m 200$, which confers a higher resistance level than the one provided by the ol-2 mutant allele. A macroscopic and microscopic comparison between plants carrying these alleles hints at the influence of the genetic background on the resistance level observed. If this is verified, it becomes an important factor to consider in breeding programs.

Based on the results obtained, in Chapter 8, I summarize our findings and discuss their implications for a successful use of the mlo-based resistance in breeding programs. Furthermore, I discuss the importance of this study as exemplary work to apply also to other S-genes and hopefully achieve a durable broad-spectrum resistance without any drawbacks on other important plant traits. 


\section{References}

Acevedo-Garcia, J., Kusch, S., \& Panstruga, R. (2014). Magical mystery tour: MLO proteins in plant immunity and beyond. New Phytologist, 204(2), 273-281.

Acquaah, G. (2012). Plant genetic resources principles of plant genetics and breeding. John Wiley \& Sons, Ltd, 199-225.

Aeeinfar, H. (2011). First report of powdery mildew of Rosa persica caused by Leveillula taurica from Iran. Iranian Journal of Plant Pathology, 47(3), 105.

Aghnoum, R., \& Niks, R. E. (2010). Specificity and levels of non-host resistance to nonadapted Blumeria graminis forms in barley. New Phytologist, 185(1), 275-284.

Assaad, F. F., Qiu, J.-L., Youngs, H., Ehrhardt, D., Zimmerli, L., Kalde, M.,Thordal-Christensen, H. (2004). The PEN1 syntaxin defines a novel cellular compartment upon fungal attack and is required for the timely assembly of papillae. Molecular Biology of the Cell, 15(11), 5118-5129.

Bai, Y., Pavan, S., Zheng, Z., Zappel, N. F., Reinstädler, A., \& Lotti, C. (2008). Naturally occurring broad-spectrum powdery mildew resistance in a Central American tomato accession is caused by loss of Mlo function. Molecular Plant-Microbe Interaction, 21(1), 30-39.

Bai, Y., van der Hulst, R., Bonnema, G., Marcel, T. C., Meijer-Dekens, F., Niks, R. E., \& Lindhout, P. (2005). Tomato defense to Oldium neolycopersici: dominant $O I$ genes confer isolate-dependent resistance via a different mechanism than recessive ol-2. Molecular Plant-Microbe Interactions, 18(4), 354-362.

Bélanger, R.R., Bushnell, W.R., Dik, A.J., \& Carver, T.L.W. (2002). The Powdery mildews: a comprehensive treatise. APS Press. St. Paul.

Bertetti, D., Martini, P., Repetto, L., Gullino, M. L., \& Garibaldi, A. (2012). First report of powdery mildew caused by Golovinomyces biocellatus on Origanum vulgare L. "Compactum" cultivated in Italy. Protezione delle Colture, 1(23), 25.

Bidzinski, P., Noir, S., Shahi, S., Reinstadler, A., Gratkowska, D. M., \& Panstruga, R. (2014). Physiological characterization and genetic modifiers of aberrant root thigmomorphogenesis in mutants of Arabidopsis thaliana MILDEW LOCUS O genes. Plant, Cell \& Environment, 37(12), 2738-2753.

Bindschedler, L. V., Burgis, T. A., Mills, D. J. S., Ho, J. T. C., Cramer, R., \& Spanu, P. D. (2009). In planta proteomics and proteogenomics of the biotrophic barley fungal pathogen Blumeria graminis f. sp. hordei. Molecular \& Cellular Proteomics, 8(10), 2368-2381.

Bindschedler, L. V., McGuffin, L. J., Burgis, T. A., Spanu, P. D., \& Cramer, R. (2011). Proteogenomics and in silico structural and functional annotation of the barley powdery mildew Blumeria graminis $\mathrm{f}$. sp. hordei. Methods, 54(4), 432-441.

Bindschedler, L. V., Panstruga, R., \& Spanu, P. D. (2016). Mildew-omics: how global analyses aid the understanding of life and evolution of powdery mildews. Frontiers in Plant Science, 7, 123.

Bortesi, L., \& Fischer, R. (2015). The CRISPR/Cas9 system for plant genome editing and beyond. Biotechnology Advances, 33(1), 41-52.

Bourras, S., McNally, K. E., Ben-David, R., Parlange, F., Roffler, S., Praz, C. R., \& Keller, B. (2015). Multiple avirulence loci and allele-specific effector recognition control the $\mathrm{Pm} 3$ race-specific resistance of wheat to powdery mildew. The Plant Cell, 27(10), 2991-3012.

Braun, U., \& Cook, R. T. A. (2012). Taxonomic manual of the Erysiphales (Powdery Mildews). CBS Biodiversity Series No. 11. CBS, Utrecht, Netherlands.

Brown, J. K. M. (2015). Durable resistance of crops to disease: a darwinian perspective. Annual Review of Phytopathology, 53(1), 513-539.

Burdon, J. J., Thrall, P. H., Ericson, \& Lars. (2006). The current and future dynamics of disease in plant communities. Annual Review of Phytopathology, 44(1), 19-39.

Cao, Y., Liang, Y., Tanaka, K., Nguyen, C. T., Jedrzejczak, R. P., Joachimiak, A., \& Stacey, G. (2014). The kinase LYK5 is a major chitin receptor in Arabidopsis and forms a chitin-induced complex with related kinase CERK1. eLife, 3, e03766. 
Chakraborty, S. (2005). Potential impact of climate change on plant-pathogen interactions. Australasian Plant Pathology, 34(4), 443-448.

Chen, L.-Q., Hou, B.-H., Lalonde, S., Takanaga, H., Hartung, M. L., Qu, X.-Q., \& Frommer, W. B. (2010). Sugar transporters for intercellular exchange and nutrition of pathogens. Nature, 468(7323), 527-532.

Chen, Z., Noir, S., Kwaaitaal, M., Hartmann, H. A., Wu, M. J., Mudgil, Y., Jones, A. M. (2009). Two seventransmembrane domain MILDEW RESISTANCE LOCUS O proteins co-function in Arabidopsis root thigmomorphogenesis. Plant Cell, 21(7), 1972-1991.

Chowdhury, J., Henderson, M., Schweizer, P., Burton, R. A., Fincher, G. B., \& Little, A. (2014). Differential accumulation of callose, arabinoxylan and cellulose in nonpenetrated versus penetrated papillae on leaves of barley infected with Blumeria graminis f. sp. hordei. New Phytologist, 204(3), 650-660.

Collins, F. S., Green, E. D., Guttmacher, A. E., \& Guyer, M. S. (2003). A vision for the future of genomics research. Nature, 422(6934), 835-847.

Consonni, C., Humphry, M. E., Hartmann, H. A., Livaja, M., Durner, J., \& Westphal, L. (2006). Conserved requirement for a plant host cell protein in powdery mildew pathogenesis. Nature Genet., 38, 716 - 720.

Cook, A. A. (1960). A mutation for resistance to potato virus $Y$ in pepper. Phytopathology, 50, 550-552.

Crowl, T. A., Crist, T. O., Parmenter, R. R., Belovsky, G., \& Lugo, A. E. (2008). The spread of invasive species and infectious disease as drivers of ecosystem change. Frontiers in Ecology and the Environment, 6(5), 238-246.

Dallagnol, L. J., de Castro, F. R., Garcia, E. N., \& Camargo, L. E. A. (2012). First report of powdery mildew caused by Golovinomyces sp. on Plantago australis in Brazil. Plant Disease, 97(3), 421-421.

Dangl, J. L., \& Jones, J. D. G. (2001). Plant pathogens and integrated defence responses to infection. Nature, 411(6839), 826-833.

Dean, R., Van Kan, J. A. L., Pretorius, Z. A., Hammond-Kosack, K. E., Di Pietro, A., Spanu, P. D., Foster, G. D. (2012). The Top 10 fungal pathogens in molecular plant pathology. Molecular Plant Pathology, 13(4), 414430.

Dodds, P. N., \& Rathjen, J. P. (2010). Plant immunity: towards an integrated view of plant-pathogen interactions. Nature Reviews Genetics, 11(8), 539-548.

Eastburn, D. M., McElrone, A. J., \& Bilgin, D. D. (2011). Influence of atmospheric and climatic change on plantpathogen interactions. Plant Pathology, 60(1), 54-69.

Eckardt, N. A. (2002). Plant Disease Susceptibility Genes? The Plant Cell, 14(9), 1983-1986.

Eichmann, R., Bischof, M., Weis, C., Shaw, J., Lacomme, C., Schweizer, P., Hückelhoven, R. (2010). BAX INHIBITOR-1 is required for full susceptibility of barley to powdery mildew. Molecular Plant-Microbe Interactions, 23(9), 1217-1227.

Elad, Y., Jacob, D., Rav David, D., Burshtein, M., Sztjenberg, A., Yehezkel, H., Messika, Y. (2009). Development of climate control methods for integrated management of powdery mildew of tomato caused by Oidium neolycopersicum. Acta Horticolturae, 807, 727-732.

Ellinger, D., Naumann, M., Falter, C., Zwikowics, C., Jamrow, T., Manisseri, C., Voigt, C. A. (2013). Elevated early callose deposition results in complete penetration resistance to powdery mildew in Arabidopsis. Plant Physiology, 161(3), 1433-1444.

Ellingham, O., Denton, G.J., Denton, J.O., Robinson, R.J. ( 2016). First report of Podosphaera macrospora on Heuchera in the United Kingdom. New Disease Reports 33, 23.

Ellis, C., \& Turner, J. G. (2001). The Arabidopsis mutant cev 1 has constitutively active jasmonate and ethylene signal pathways and enhanced resistance to pathogens. The Plant Cell, 13(5), 1025-1034.

Ellis, J. (2006). Insights into non-host disease resistance: can they assist disease control in agriculture? The Plant Cell, 18(3), 523-528.

Feechan, A., Jermakow, A. M., Ivancevic, A., Godfrey, D., Pak, H., Panstruga, R., \& Dry, I. B. (2013). Host cell entry of powdery mildew is correlated with endosomal transport of antagonistically acting VuPEN1 and VvMLO to the papilla. Molecular Plant-Microbe Interactions, 26(10), 1138-1150. 
Félix-Gastélum, R., Herrera-Rodríguez, G., Martínez-Valenzuela, C., Longoria-Espinoza, R. M., MaldonadoMendoza, I. E., Quiroz-Figueroa, F. R., \& Espinosa-Matías, S. (2013). First report of powdery mildew (Pseudoidium anacardii) of mango trees in Sinaloa, Mexico. Plant Disease, 97(7), 994-994.

Fotopoulos, V., Gilbert, M. J., Pittman, J. K., Marvier, A. C., Buchanan, A. J., Sauer, N., Williams, L. E. (2003). The monosaccharide transporter gene, AtSTP4, and the cell-wall invertase, At $\beta$ fruct 1 , are induced in Arabidopsis during infection with the fungal biotroph Erysiphe cichoracearum. Plant Physiology, 132(2), 821-829.

Freialdenhoven, A., Peterhansel, C., Kurth, J., Kreuzaler, F., \& Schulze-Lefert, P. (1996). Identification of genes required for the function of non-race-specific mlo resistance to powdery mildew in barley. The Plant Cell, 8(1), 5-14.

Frye, C. A., \& Innes, R. W. (1998). An Arabidopsis mutant with enhanced resistance to powdery mildew. The Plant Cell, 10(6), 947-956.

Frye, C. A., Tang, D., \& Innes, R. W. (2001). Negative regulation of defense responses in plants by a conserved MAPKK kinase. Proceedings of the National Academy of Sciences of the United States of America, 98(1), 373-378.

Gafni, A., Calderon, C. E., Harris, R., Buxdorf, K., Dafa-Berger, A., Zeilinger-Reichert, E., \& Levy, M. (2015). Biological control of the cucurbit powdery mildew pathogen Podosphaera xanthii by means of the epiphytic fungus Pseudozyma aphidis and parasitism as a mode of action. Frontiers in Plant Science, 11(6), 132.

Gao, D., Appiano, M., Huibers, R. P., Loonen, A. E. H. M., Visser, R. G. F., Wolters, A.-M. A., \& Bai, Y. (2015). Natural loss-of-function mutation of EDR1 conferring resistance to tomato powdery mildew in Arabidopsis thaliana accession C24. Molecular Plant Pathology, 16(1), 71-82.

Garrett, K. A., Dendy, S. P., Frank, E. E., Rouse, M. N., \& Travers, S. E. (2006). Climate change effects on plant disease: genomes to ecosystems. Annual Review of Phytopathology, 44(1), 489-509.

Glawe, D.A., \& Barlow, T. (2010). First report of powdery mildew caused by Leveillula taurica of field-grown sweet pepper in the Pacific Northwest. Plant Health Progress, doi: 10.1094/PHP-2007-0708-01-BR.

Glawe, D. A. (2008). The powdery mildews: a review of the world's most familiar (yet poorly known) plant pathogens. Annual Review of Phytopathology, 46:27-51.

Godfrey, D., Böhlenius, H., Pedersen, C., Zhang, Z., Emmersen, J., \& Thordal-Christensen, H. (2010). Powdery mildew fungal effector candidates share N-terminal Y/F/WXC-motif. BMC Genomics, 11, 317-317.

Godfrey, D., Zhang, Z., Saalbach, G., \& Thordal-Christensen, H. (2009). A proteomics study of barley powdery mildew haustoria. PROTEOMICS, 9(12), 3222-3232.

Göllner, K., Schweizer, P., Bai, Y., \& Panstruga, R. (2008). Natural genetic resources of Arabidopsis thaliana reveal a high prevalence and unexpected phenotypic plasticity of RPW8-mediated powdery mildew resistance. New Phytologist, 177(3), 725-742.

Hacquard, S., Kracher, B., Maekawa, T., Vernaldi, S., Schulze-Lefert, P., \& Ver Loren van Themaat, E. (2013). Mosaic genome structure of the barley powdery mildew pathogen and conservation of transcriptional programs in divergent hosts. Proceedings of the National Academy of Sciences of the United States of America, 110(24), E2219-E2228.

Hansjakob, A., Riederer, M., \& Hildebrandt, U. (2011). Wax matters: absence of very-long-chain aldehydes from the leaf cuticular wax of the glossy11 mutant of maize compromises the prepenetration processes of Blumeria graminis. Plant Pathology, 60(6), 1151-1161.

Hartl, L., Weiss, H., Zeller, F. J., \& Jahoor, A. (1993). Use of RFLP markers for the identification of alleles of the Pm3 locus conferring powdery mildew resistance in wheat (Triticum aestivum L.). Theoretical and Applied Genetics, 86(8), 959-963.

Harvell, C. D., Mitchell, C. E., Ward, J. R., Altizer, S., Dobson, A. P., Ostfeld, R. S., \& Samuel, M. D. (2002). Climate warming and disease risks for terrestrial and marine biota. Science, 296(5576), 2158.

Hibberd, J. M., Whitbread, R., \& Farrar, J. F. (1996a). Effect of $700 \mu \mathrm{mol}$ mol -1CO2 and infection with powdery mildew on the growth and carbon partitioning of barley. New Phytologist, 134(2), 309-315.

Hibberd, J. M., Whitbread, R., \& Farrar, J. F. (1996b). Effect of elevated concentrations of $\mathrm{CO}_{2}$ on infection of barley by Erysiphe graminis. Physiological and Molecular Plant Pathology, 48(1), 37-53. 
Hoshi, H., Sato, Y., Kagiwada, S., \& Horie, H. (2013). First report of powdery mildew on zinnia, blue torenia, dahurian patrinia and scoparia caused by genus Euoidium in Japan. Journal of General Plant Pathology, 79(2), 89-95.

Hückelhoven, R., Dechert, C., \& Kogel, K.-H. (2003). Overexpression of barley BAX inhibitor 1 induces breakdown of mlo-mediated penetration resistance to Blumeria graminis. Proceedings of the National Academy of Sciences of the United States of America, 100(9), 5555-5560.

Hückelhoven, R., Fodor, J., Trujillo, M., \& Kogel, K.-H. (2000). Barley Mla and Rar mutants compromised in the hypersensitive cell death response against Blumeria graminis f.sp. hordei are modified in their ability to accumulate reactive oxygen intermediates at sites of fungal invasion. Planta, 212(1), 1624.

Hückelhoven, R., \& Kogel, K.-H. (1998). Tissue-specific superoxide generation at interaction sites in resistant and susceptible near-isogenic barley lines attacked by the powdery mildew fungus (Erysiphe graminis f. sp. hordei). Molecular Plant-Microbe Interactions, 11(4), 292-300.

Huibers, R. P., Loonen, A. E. H. M., Gao, D., Van den Ackerveken, G., Visser, R. G. F., \& Bai, Y. (2013). Powdery mildew resistance in tomato by impairment of SIPMR4 and SIDMR1. PLOS ONE, 8(6), e67467.

Humphry, M., Bednarek, P., Kemmerling, B., Koh, S., Stein, M., Göbel, U., \& Panstruga, R. (2010). A regulon conserved in monocot and dicot plants defines a functional module in antifungal plant immunity. Proceedings of the National Academy of Sciences of the United States of America, 107(50), 21896-21901.

Humphry, M., Consonni, C., \& Panstruga, R. (2006). mlo-based powdery mildew immunity: silver bullet or simply non-host resistance? Molecular Plant Pathology, 7(6), 605-610.

Jarosch, B., Kogel, K.-H., \& Schaffrath, U. (1999). The ambivalence of the barley Mlo Locus: mutations conferring resistance against powdery mildew (Blumeria graminis f. sp. hordei) enhance susceptibility to the rice blast fungus Magnaporthe grisea. Molecular Plant-Microbe Interactions, 12(6), 508-514.

Jones, J. D. G., \& Dangl, J. L. (2006). The plant immune system. Nature, 444, 323-329.

Jones, L., Riaz, S., Morales-Cruz, A., Amrine, K. C. H., McGuire, B., Gubler, W. D., \& Cantu, D. (2014). Adaptive genomic structural variation in the grape powdery mildew pathogen, Erysiphe necator. $B M C$ Genomics, 15(1), 1-18.

Jørgensen, I. H. (1992). Discovery, characterization and exploitation of Mlo powdery mildew resistance in barley. Euphytica, 63(1-2), 141-152.

Joung, J. K., \& Sander, J. D. (2013). TALENs: a widely applicable technology for targeted genome editing. Nature reviews molecular cell biology, 14(1), 49-55.

Kasal, T. (2016). Use of biogenic volatile organic compounds to assess the health status of tomato plants (Solanum lycopersicum) cv. "Moneymaker", PhD thesis, Bonn University

Kessler, S. A., Shimosato-Asano, H., Keinath, N. F., Wuest, S. E., Ingram, G., Panstruga, R., \& Grossniklaus, U. (2010). Conserved molecular components for pollen tube reception and fungal invasion. Science, 330(6006), 968-971.

Kim, D. S., \& Hwang, B. K. (2012). The pepper MLO gene, CaMLO2, is involved in the susceptibility cell-death response and bacterial and oomycete proliferation. The Plant Journal, 72(5), 843-855.

Kim, M. C., Lee, S. H., Kim, J. K., Chun, H. J., Choi, M. S., \& Chung, W. S. (2002). Mlo, a modulator of plant defense and cell death, is a novel calmodulin binding protein. Isolation and characterization of a rice Mlo homologue. Journal of Biological Chemistry, 277, 19304-19314.

Kim, M. C., Panstruga, R., Elliott, C., Muller, J., Devoto, A., Yoon, H. W., \& Schulze-Lefert, P. (2002). Calmodulin interacts with MLO protein to regulate defence against mildew in barley. Nature, 416(6879), 447451.

Kissoudis, C., van de Wiel, C., Visser, R. G. F., \& van der Linden, G. (2016). Future-proof crops: challenges and strategies for climate resilience improvement. Current Opinion in Plant Biology, 30, 47-56.

Kumar, J., Hückelhoven, R., Beckhove, U., Nagarajan, S., \& Kogel, K.-H. (2001). A compromised Mlo pathway affects the response of barley to the necrotrophic fungus Bipolaris sorokiniana (teleomorph: Cochliobolus sativus) and its toxins. Phytopathology, 91(2), 127-133. 
Kusch, S., Pesch, L., \& Panstruga, R. (2016). Comprehensive phylogenetic analysis sheds light on the diversity and origin of the MLO family of integral membrane proteins. Genome Biology and Evolution, 8(3): 878-895.

Lapin, D., \& Van den Ackerveken, G. (2013). Susceptibility to plant disease: more than a failure of host immunity. Trends in Plant Science, 18(10), 546-554.

Lebeda, A., Mieslerová, B., Jankovics, T., Kiss, L., \& Van der Linde, E. J. (2015). First detection of tomato powdery mildew caused by Oidium neolycopersici in South Africa. South African Journal of Botany, 99, 153157.

Lee, H. B., Lee, H. W., \& Mun, H. Y. (2013). First report of powdery mildew caused by Erysiphe platani on sycamore (Platanus occidentalis) in South Korea. Plant Disease, 97(6), 841-841.

Liang, Z., Zhang, K., Chen, K., \& Gao, C. (2014). Targeted mutagenesis in Zea mays using TALENs and the CRISPR/Cas system. Journal of Genetics and Genomics, 41(2), 63-68.

Lim, C. W., \& Lee, S. C. (2014). Functional roles of the pepper MLO protein gene, CaMLO2, in abscisic acid signaling and drought sensitivity. Plant Molecular Biology, 85(1), 1-10. doi: 10.1007/s11103-0130155-8

Lipka, V., Dittgen, J., Bednarek, P., Bhat, R., Wiermer, M., Stein, M., \& Schulze-Lefert, P. (2005). Pre- and postinvasion defenses both contribute to nonhost resistance in Arabidopsis. Science, 310(5751), 1180-1183.

Liu, L., \& Fan, X.-D. (2014). CRISPR-Cas system: a powerful tool for genome engineering. Plant Molecular Biology, 85(3), 209-218.

Lorrain, S., Vailleau, F., Balagué, C., \& Roby, D. Lesion mimic mutants: keys for deciphering cell death and defense pathways in plants? Trends in Plant Science, 8(6), 263-271.

Lundqvist, U., (1991). Swedish mutation research in barley with plant breeding aspects. A historical review. In: Plant Mutation Breeding for Crop Improvement, IAEA-SM-311/25, pp. 135-147.

Manning, W. J., \& v. Tiedemann, A. (1995). Climate change: potential effects of increased atmospheric carbon dioxide $\left(\mathrm{CO}_{2}\right)$, ozone $\left(\mathrm{O}_{3}\right)$, and ultraviolet-B (UV-B) radiation on plant diseases. Environmental Pollution, 88(2), 219-245.

McTaggart, A. R., Ryley, M. J., \& Shivas, R. G. (2012). First report of the powdery mildew Erysiphe diffusa on soybean in Australia. Australasian Plant Disease Notes, 7(1), 127-129.

Miklis, M., Consonni, C., Bhat, R. A., Lipka, V., Schulze-Lefert, P., \& Panstruga, R. (2007). Barley MLO modulates actin-dependent and actin-independent antifungal defense pathways at the cell periphery. Plant Physiology, 144(2), 1132-1143.

Miya, A., Albert, P., Shinya, T., Desaki, Y., Ichimura, K., Shirasu, K., \& Shibuya, N. (2007). CERK1, a LysM receptor kinase, is essential for chitin elicitor signaling in Arabidopsis. Proceedings of the National Academy of Sciences of the United States of America, 104(49), 19613-19618.

Mohanta, T. K., \& Bae, H. (2015). The diversity of fungal genome. Biological Procedures Online, 17, 8.

Moury, B., \& Verdin, E. (2012). Chapter 4 - Viruses of pepper crops in the mediterranean basin: a remarkable stasis. In L. Gad \& L. Hervé (Eds.), Advances in Virus Research, 84, 127-162

Mukhtar, I., Mustaq, S., \& Khokhar, I. (2013). First report of powdery mildew on Dahlia (Dahlia variabilis) caused by Golovinomyces cichoracearum in Lahore, Pakistan. Australasian Plant Disease Notes, 8(1), $1-3$

Nieto-López, E.H., Leyva-Mir, S.G., Ayala-Escobar, V., Nava-Díaz, C., \& Tovar-Pedraza, J.M. (2015). First report of powdery mildew caused by Erysiphae alphitoides on Quercus rugosa in Mexico. Journal of Plant Pathology, 97(3), 541-551

Nishimura, M. T., Stein, M., Hou, B.-H., Vogel, J. P., Edwards, H., \& Somerville, S. C. (2003). Loss of a callose synthase results in salicylic acid-dependent disease resistance. Science, 301(5635), 969.

Noir, S., Colby, T., Harzen, A., Schmidt, J., \& Panstruga, R. (2009). A proteomic analysis of powdery mildew (Blumeria graminis f.sp. hordei) conidiospores. Molecular Plant Pathology, 10(2), 223-236.

Opalski, K. S., Schultheiss, H., Kogel, K. H., \& Hückelhoven, R. (2005). The receptor-like MLO protein and the RAC/ ROP family G-protein RACB modulate actin reorganization in barley attacked by the biotrophic powdery mildew fungus Blumeria graminis f.sp. hordei. Plant Journal, 41(2), 291-303. 
Panstruga, R. (2005). Discovery of novel conserved peptide domains by ortholog comparison within plant multi-protein families. Plant Molecular Biology, 59(3), 485-500.

Park, M. J., Choi, Y. J., Han, J. G., \& Shin, H. D. (2010). First report in Korea of powdery mildew of Matricaria chamomilla caused by Golovinomyces cichoracearum. Plant Pathology, 59(4), 801-801.

Patel, R. P., Pandey, G.N., Gyanendra Tiwari, Patidar H., \& Patidar, D. K. (2013). First report of powdery mildew fungi on sandlwood in Madhya Pradesh. International Journal of Current Research, 7(6), 16705-16708

Pavan, S., Jacobsen, E., Visser, R. G. F., \& Bai, Y. (2010). Loss of susceptibility as a novel breeding strategy for durable and broad-spectrum resistance. Molecular Breeding, 25: 1-12

Pavletich, N. P., \& Pabo, C. O. (1991). Zinc finger-DNA recognition: crystal structure of a Zif268-DNA complex at 2.1 A. Science, 252(5007), 809.

Pirondi, A., Nanni, I. M., Brunelli, A., \& Collina, M. (2014). First report of resistance to Cyflufenamid in Podosphaera xanthii, causal agent of powdery mildew, from melon and zucchini fields in Italy. Plant Disease, 98(11), 1581-1581.

Poraty-Gavra, L., Zimmermann, P., Haigis, S., Bednarek, P., Hazak, O., Stelmakh, O. R., Yalovsky, S. (2013). The Arabidopsis Rho of Plants GTPase AtROP6 Functions in Developmental and Pathogen Response Pathways. Plant Physiology, 161(3), 1172-1188.

Pryce-Jones, E., Carver, T. I. M., \& Gurr, S. J. (1999). The roles of cellulase enzymes and mechanical force in host penetration by Erysiphe graminis f.sp.hordei. Physiological and Molecular Plant Pathology, 55(3), 175-182.

Qi, Y., Tsuda, K., Glazebrook, J., \& Katagiri, F. (2011). Physical association of pattern-triggered immunity (PTI) and effector-triggered immunity (ETI) immune receptors in Arabidopsis. Molecular Plant Pathology, 12(7), 702-708.

Ruiz-Lozano, J.M., Gianinazzi, S., Gianinazzi-Pearson, V. (1999). Genes involved in resistance to powdery mildew in barley differentially modulate root colonization by the mycorrhizal fungus Glomus mosseae. Mycorrhiza, 9:237-40

Schmidt, S. M., Kuhn, H., Micali, C., Liller, C., Kwaaitaal, M., \& Panstruga, R. (2014). Interaction of a Blumeria graminis f. sp. hordei effector candidate with a barley ARF-GAP suggests that host vesicle trafficking is a fungal pathogenicity target. Molecular Plant Pathology, 15(6), 535-549.

Schultheiss, H., Dechert, C., Kogel, K.-H., \& Hückelhoven, R. (2003). Functional analysis of barley RAC/ROP G-protein family members in susceptibility to the powdery mildew fungus. The Plant Journal, 36(5), 589-601.

Seifi, A., Gao, D., Zheng, Z., Pavan, S., Faino, L., Visser, R. G. F., \& Bai, Y. (2014). Genetics and molecular mechanisms of resistance to powdery mildews in tomato (Solanum lycopersicum) and its wild relatives. European Journal of Plant Pathology, 138(3), 641-665.

Seifi, A., Kaloshian, I., Vossen, J., Che, D., Bhattarai, K. K., Fan, J., \& Bai, Y. (2010). Linked, if not the same, Mi-1 homologues confer resistance to tomato powdery mildew and root-knot nematodes. Molecular Plant-Microbe Interactions, 24(4), 441-450.

Shen, Y.-M., Huang, T.-C., Liu, H.-L., \& Chao, C.-H. (2015). First report of the powdery mildew Podosphaera xanthii on Gynura bicolor. Australasian Plant Disease Notes, 10(1), 27.

Spanu, P. D., Abbott, J. C., Amselem, J., Burgis, T. A., Soanes, D. M., Stüber, K., \& Panstruga, R. (2010). Genome expansion and gene loss in powdery mildew fungi reveal tradeoffs in extreme parasitism. Science, 330(6010), 1543.

Tam, L.T.T., Dung, P.N., Liem, N.V., \& Sato, Y. (2015). First report of Podosphaera xanthii causing powdery mildew on red chilli pepper in Vietnam. New Disease Reports 32, 23

Thomas, S. W., Rasmussen, S. W., Glaring, M. A., Rouster, J. A., Christiansen, S. K., \& Oliver, R. P. (2001). Gene identification in the obligate fungal pathogen Blumeria graminis by expressed sequence tag analysis. Fungal Genetics and Biology, 33(3), 195-211.

Thomma, B. P. H. J., Nürnberger, T., \& Joosten, M. H. A. J. (2011). Of PAMPs and effectors: the blurred PTI-ETI dichotomy. The Plant Cell, 23(1), 4-15.

Trujillo, M., Troeger, M., Niks, R. E., Kogel, K.-H., \& HÜCkelhoven, R. (2004). Mechanistic and genetic overlap of barley host and non-host resistance to Blumeria graminis. Molecular Plant Pathology, 5(5), 389-396. 
Tsay, J. G., Chen, R. S., Wang, H. L., Wang, W. L., \& Weng, B. C. (2011). First report of powdery mildew caused by Erysiphe diffusa, Oidium neolycopersici, and Podosphaera xanthii on papaya in Taiwan. Plant Disease, 95(9), 1188-1188.

Tsuda, K., \& Katagiri, F. (2010). Comparing signaling mechanisms engaged in pattern-triggered and effectortriggered immunity. Current Opinion in Plant Biology, 13(4), 459-465.

Urnov, F. D., Rebar, E. J., Holmes, M. C., Zhang, H. S., \& Gregory, P. D. (2010). Genome editing with engineered zinc finger nucleases. Nat Rev Genet, 11, 636-646.

van Schie, C. C. N., \& Takken, F. L. W. (2014). Susceptibility genes 101: how to be a good host. Annual Review of Phytopathology, 52(1), 551-581.

Voegele, R.T., Hahn, M., \& Mendgen, K. (2009) .The uredinales: cytology, biochemistry, and molecular biology. In: Plant relationships, 2nd edition, The Mycota V. Springer, Berlin, pp 79-94

Vogel, J. P., Raab, T. K., Schiff, C., \& Somerville, S. C. (2002). PMR6, a pectate lyase-like gene required for powdery mildew susceptibility in Arabidopsis. The Plant Cell, 14(9), 2095-2106.

Walters, D. R. (2015). The interaction between a plant and its attacker physiological responses of plants to attack (pp. 1-23): John Wiley \& Sons, Ltd.

Wang, Y., Cheng, X., Shan, Q., Zhang, Y., Liu, J., \& Gao, C. (2014). Simultaneous editing of three homoeoalleles in hexaploid bread wheat confers heritable resistance to powdery mildew. Nature Biotechnology, 32, . 947-951.

Weis, C., Hückelhoven, R., \& Eichmann, R. (2013). LIFEGUARD proteins support plant colonization by biotrophic powdery mildew fungi. Journal of Experimental Botany, 64(12), 3855-3867.

Weßling, R., Epple, P., Altmann, S., He, Y., Yang, L., Henz, S. R., Braun, P. (2014). Convergent targeting of a common host protein-network by pathogen effectors from three kingdoms of life. Cell host \& microbe, 16(3), 364-375.

Weßling, R., Schmidt, S. M., Micali, C. O., Knaust, F., Reinhardt, R., Neumann, U., Panstruga, R. (2012). Transcriptome analysis of enriched Golovinomyces orontii haustoria by deep 454 pyrosequencing. Fungal Genetics and Biology, 49(6), 470-482.

Wicker, T., Oberhaensli, S., Parlange, F., Buchmann, J. P., Shatalina, M., Roffler, S., Keller, B. (2013). The wheat powdery mildew genome shows the unique evolution of an obligate biotroph. Nature Genetics, 45(9), 1092-1096.

Wirthmueller, L., Maqbool, A., \& Banfield, M. J. (2013). On the front line: structural insights into plantpathogen interactions. Nature Reviews Microbiology, 11(11), 761-776.

Xiao, S., Ellwood, S., Calis, O., Patrick, E., Li, T., Coleman, M., \& Turner, J. G. (2001). Broad-spectrum mildew resistance in Arabidopsis thaliana mediated by RPW8. Science, 291(5501), 118.

Xing, H. H., Liang, C., Cho, S. E., \& Shin, H. D. (2013). First report of powdery mildew caused by Podosphaera spiraeae on Japanese spiraea in China. Plant Disease, 98(4), 571-571

Yahiaoui, N., Srichumpa, P., Dudler, R., \& Keller, B. (2004). Genome analysis at different ploidy levels allows cloning of the powdery mildew resistance gene Pm $3 b$ from hexaploid wheat. The Plant Journal, 37(4), 528-538.

Zhang, W.-J., Hanisch, S., Kwaaitaal, M., Pedersen, C., \& Thordal-Christensen, H. (2013). A component of the Sec61 ER protein transporting pore is required for plant susceptibility to powdery mildew. Frontiers in Plant Science, 16(4), 127.

Zhao, H. H., Xing, H. H., Liang, C., Yang, X. Y., Cho, S. E., \& Shin, H. D. (2013). First report of powdery mildew caused by Erysiphe cruciferarum on Chinese cabbage in China. Plant Disease, 98(3), 421-421.

Zheng, Z., Nonomura, T., Appiano, M., Pavan, S., Matsuda, Y., \& Toyoda, H. (2013). Loss of function in Mlo orthologs reduces susceptibility of pepper and tomato to powdery mildew disease caused by Leveillula taurica. PLoS One., 8(7): e70723

Zheng, Z., Nonomura, T., Bóka, K., Matsuda, Y., Visser, R. G. F., Toyoda, H., \& Bai, Y. (2013). Detection and quantification of Leveillula taurica growth in pepper leaves. Phytopathology, 103(6), 623-632.

Zhou, F., Kurth, J., Wei, F., Elliott, C., Valè, G., Yahiaoui, N., Schulze-Lefert, P. (2001). Cell-autonomous expression of barley Mla1 confers race-specific resistance to the powdery mildew fungus via a Rar1independent signalling pathway. The Plant Cell, 13(2), 337-350. 


\section{Chapter 2}

Genome-wide study of the tomato SIMLO gene family and its functional characterization in response to the powdery mildew fungus Oidium neolycopersici

Michela Appiano ${ }^{\dagger}$, Zheng Zheng ${ }^{\dagger}$, Stefano Pavan, Valentina Bracuto, Luigi Ricciardi, Richard G.F. Visser, Anne-Marie Wolters, Yuling Bai* ${ }^{\dagger}$ these authors contributed equally to the work * corresponding author

Published chapter in Frontiers in Plant Science, Apr 2016 Link to published paper here: http://journal.frontiersin.org/article/10.3389/fpls.2016.00380/full 


\begin{abstract}
The MLO (Mildew Locus O) gene family encodes plant-specific proteins containing seven transmembrane domains and likely acting in signal transduction in a calcium and calmodulin dependent manner. Some members of the MLO family are susceptibility factors towards fungi causing the powdery mildew disease. In tomato, for example, the loss-of-function of the MLO gene SIMLO1 leads to a particular form of powdery mildew resistance, called ol-2, which arrests almost completely fungal penetration. This type of penetration resistance is characterized by the apposition of papillae at the sites of plant-pathogen interaction. Other $M L O$ homologs in Arabidopsis regulate root response to mechanical stimuli (AtMLO4 and AtMLO11) and pollen tube reception by the female gametophyte (AtMLO7). However, the role of most MLO genes remains unknown.

In this work, we provide a genome-wide study of the tomato SIMLO gene family. Besides SIMLO1, other fifteen SIMLO homologs were identified and characterized with respect to their structure, genomic organization, phylogenetic relationship, and expression profile. In addition, by analysis of transgenic plants, we demonstrated that simultaneous silencing of SIMLO1 and two of its closely related homologs, SIMLO5 and SIMLO8, confer higher level of resistance than the one associated with the ol-2 mutation.

The outcome of this study provides evidence for functional redundancy among tomato homolog genes involved in powdery mildew susceptibility. Moreover, we developed a series of transgenic lines silenced for individual SIMLO homologs, which lay the foundation for further investigations aimed at assigning new biological functions to the MLO gene family.
\end{abstract}

\title{
Keywords
}

MLO gene family, tomato, susceptibility, powdery mildew disease 


\section{Introduction}

Many important crop species can be affected by the powdery mildew (PM) disease, resulting in great yield losses in agricultural settings. In barley, recessive loss-of-function mutations occurring in the HvMLO (Hordeum vulgare Mildew Resistance Locus $\underline{0}$ ) gene confer resistance to all known isolates of the PM fungus Blumeria graminis f.sp hordei. Therefore, natural or induced mlo-mutant alleles are in use for about seven decades to introduce resistance in spring barley breeding programs (Jørgensen, 1992; Büschges et al., 1997; Reinstädler et al., 2010).

Biochemical analysis showed that the barley HvMLO protein contains seven transmembrane domains integral to the plasma membrane, with an extracellular aminoterminus and an intracellular carboxy-terminus. The latter harbours a calmodulin-binding domain likely involved in sensing calcium influxes into cells (Devoto et al., 1999). Although the domain structure of MLO proteins is related to that of metazoan G-protein coupled receptors (GPCRs), several studies could not confirm the role of MLO proteins as canonical GPCRs (Kim et al., 2002; Lorek et al., 2013). Despite further intensive efforts to explain the biochemical function of the HvMLO protein, its core activity remains elusive (Panstruga, 2005). However, HvMLO might be exploited by the fungus to impair vesicle-associated defence mechanism at plant-pathogen interaction sites, thus facilitating its penetration (Panstruga and Schulze-Lefert, 2003; Opalski et al., 2005; Miklis et al., 2007). This feature makes HVMLO a typical representative of susceptibility genes (S-genes) (Miklis et al., 2007; van Schie and Takken 2014).

The robustness of barley mlo-resistance, due to its non-race-specific spectrum and durability, led in the last years to an extensive quest for identification and functional characterization of the $M L O$ genes in other species affected by the PM disease. The search resulted in the identification of multiple $M L O$ gene families, ranging from 12 to 39 members in Arabidopsis, rice, grapevine, cucumber, apple, peach, woodland strawberry, tobacco and soybean (Devoto et al., 2003; Feechan et al., 2008; Liu and Zhu, 2008; Shen et al., 2012; Zhou et al., 2013; Pessina et al., 2014; Appiano et al., 2015). Moreover, specific homologs were shown to play a major role in plant-pathogen interactions (Consonni et al., 2006).

A detailed phylogenetic analysis distinguished up to eight clades in which Angiosperm MLO proteins can be found (Feechan et al., 2008; Acevedo-Garcia et al., 2014; Pessina et al., 2014). The MLO homologs involved in the interaction with PM pathogens (Arabidopsis AtMLO2, AtMLO6, AtMLO12, tomato SIMLO1, pea Er1/PsMLO1, grapevine VvMLO3 and VvMLO4, tobacco NtMLO1, pepper CaMLO2, cucumber CsaMLO8, Lotus japonicus LjMLO1 and barrel clover MtMLO1) are grouped into clade V. On the other hand, all the known monocot MLO homologs acting as susceptibility factors (barley HvMLO, rice OsMLO3, 
and wheat TaMLO_A1 and TaMLO_B1) do not cluster in clade V, but in clade IV, which is primarily but not exclusively represented by monocot MLO proteins. For example, grapevine VvMLO14, strawberry FvMLO17 and peach PpMLO12 belong also to clade IV (Elliott et al., 2002; Feechan et al., 2008; Acevedo-Garcia et al., 2014; Pessina et al., 2014).

In Arabidopsis, the PM resistance conferred by the loss-of-function of AtMLO2 is incomplete and only mutations in all the three AtMLO homologs in clade $\mathrm{V}$ can completely prevent fungal entry (Consonni et al., 2006). In addition, more recent studies in Arabidopsis indicated that other members of the $M L O$ gene family play a role in different biological processes. The homologs AtMLO4 and AtMLO11 are together involved in root thigmomorphogenesis, i.e. root responses to mechanical stimuli (Chen et al., 2009), while AtMLO7 regulates pollen tube reception from the synergid cells during fertilization (Kessler et al., 2010). The biological roles of other MLO homologs still remain elusive.

Tomato (Solanum lycopersicum) is one of the most economically important vegetables in the world. It can be host of three PM species, namely Oidium neolycopersici, Oidium lycopersici and Leveillula taurica (Seifi et al., 2014). Since 1996, when it was found that all the tomato cultivars were susceptible to 0 . neolycopersici, extensive researches were conducted by our group for sources of resistance (Seifi et al., 2014). An allele containing a 19 bp deletion in the coding region of the PM susceptibility gene SIMLO1 was found in a wild accession of S. lycopersicum var. cerasiforme. This mutant allele, named ol-2, was shown to confer recessively inherited broad-spectrum resistance to a series of isolates of O. neolycopersici (Bai et al., 2008). Through histological analysis, it was shown that its mechanism of resistance is based on the early abortion of fungal pathogenesis at the sites of attempted penetration (Bai et al., 2005). This type of penetration resistance is characterized by papillae apposition, the same as described also for the PM resistance in the Atmlo2 mutant of Arabidopsis (Consonni et al., 2006). Although papilla formation can significantly reduce fungal development at the host cell entry level, fungal penetration was not fully prevented in the ol-2 mutant (Bai et al., 2005).

In this study, we exploited tomato sequence information, derived from the tomato genome sequencing Heinz 1706 and the 150 tomato genome resequencing projects (Tomato Genome Consortium et al., 2012; The 100 Tomato Genome Sequencing Consortium et al., 2014), in order to identify tomato MLO homologs (SIMLO). These were characterized with respect to 1) their genomic organization, 2) relation with $M L O$ homologs from other species, 3) occurrence of tissue-specific differentially spliced variants, 4) expression in different tissues in axenic condition, and 5) upon inoculation with the powdery mildew pathogen O. neolycopersici. Finally, an RNAi-based reverse genetic approach was followed to investigate the possibility that SIMLO homologs other than SIMLO1 could play additional roles in the interaction with $O$. neolycopersici. 


\section{Results}

\section{In silico identification and sequencing of the tomato SIMLO gene family}

A total of 17 tomato MLO-like loci were identified through BLAST interrogation of the tomato genomic sequence database (SGN), using AtMLO protein sequences as query. Two of them (referred to as Solyc09g18830 and Solyc09g18840 in the SGN database) were noticeably shorter than other predicted $M L O$ homologs and physically close to each other, suggesting they are different parts of the same gene (Table 1). Search in the tomato EST database and gene prediction analysis in the S. pimpinellifolium genome with the FGENESH software allowed identifying a hypothetical full-length $M L O$ transcript encompassing Solyc09g18830 and Solyc09g18840. PCR from leaf of the tomato cultivar Moneymaker (MM) confirmed the presence of this transcript, which was named SIMLO7 (Supplementary Fig. 1). The other 15 predicted SIMLO genes were named from SIMLO1 to SIMLO6, and from SIMLO8 to SIMLO16, as reported in Table 1. For all of them, information is available with respect to putative amino acid length and number of introns.

With the exception of SIMLO4, information on chromosomal localization could also be inferred (Table 1). Most SIMLO homologs are scattered throughout the tomato genome, thus suggesting that segmental duplication events have been a major source for the evolution of the SIMLO gene family. Exceptions are represented by two physical gene clusters, one containing SIMLO6, SIMLO10 and SIMLO15 on chromosome 2, and the other containing SIMLO3 and SIMLO16 on chromosome 6.

Sequence and expression of all the predicted SIMLO homologs were verified by PCR amplification of CDNAs derived from four different tissues (leaf, root, flower and ripened fruit) of MM. All the SIMLO homologs could be amplified at least from one plant tissue. In total, 15 SIMLO homologs could be cloned from leaf (with the exception of SIMLO12), 10 from flower, 9 from fruit and 8 from root (Supplementary table 1).

Sequence alignment of cloned SIMLO transcripts with corresponding SGN predicted coding sequence (CDS), derived from the cultivar Heinz 1706, revealed polymorphisms for SIMLO7, SIMLO8, SIMLO10 and SIMLO15 (Supplementary Fig. 1). The 1339 bp SIMLO7 cloned transcript corresponds to a short open reading frame (ORF) due to a stop codon at 137-139 bp (Supplementary Fig. 1). The SGN predicted CDS of SIMLO8 misses part of the third, seventh, eighth and ninth exon present in the corresponding transcript cloned from MM leaf; compared to the SGN predicted CDS of SIMLO10, transcript cloned from MM fruit contains a base change at the beginning of the fifth exon, which results in a stop codon (Supplementary Fig. 1). Also the predicted ORF of SIMLO15 is shorter (375 aa) than the average ORF length of other SIMLOs (Table 1). The sequence cloned from MM leaf has a longer ORF (459 aa) compared to the predicted SGN sequence (Table 3a). 
TABLE 1. Features of the SIMLO gene family as inferred by the Sol Genomics Network Database.

\begin{tabular}{|c|c|c|c|c|c|}
\hline SGN locus name & MLO gene & Chromosome & Position & $\begin{array}{c}\text { ORF lenght } \\
\text { (aa) }\end{array}$ & Introns \\
\hline Solyc04g049090 & SIMLO1 & 4 & SL2.40ch04:38700445..38705951 & 507 & 14 \\
\hline Solyc08g015870 & SIMLO2 & 8 & SL2.40ch08:6074040..6078983 & 504 & 13 \\
\hline Solyc06g010030 & SIMLO3 & 6 & SL2.40ch06:4786764..4792828 & 591 & 14 \\
\hline Solyc00g007200 & SIMLO4 & $2 ?$ & SL2.40ch00:6816892..6823417 & 554 & 14 \\
\hline Solyc03g095650 & SIMLO5 & 3 & SL2.40ch03:50279919..50288063 & 517 & 14 \\
\hline Solyc02g082430 & SIMLO6 & 2 & SL2.40ch02:40694608..40700995 & 553 & 14 \\
\hline $\begin{array}{l}\text { Solyc09g018830 } \\
\text { Solyc09g018840 }\end{array}$ & SIMLO7 & 9 & SL2.40ch09:17564555..17568214 & 270 & 10 \\
\hline Solyc11g069220 & SIMLO8 & 11 & SL2.40ch11:50939533..50946726 & 506 & 13 \\
\hline Solyc06g082820 & SIMLO9 & 6 & SL2.40ch06:44779673..44784035 & 511 & 13 \\
\hline Solyc02g083720 & SIMLO10 & 2 & SL2.40ch02:41596474..41602413 & 533 & 14 \\
\hline Solyc01g102520 & SIMLO11 & 1 & SL2.40ch01:83071860..83075439 & 475 & 13 \\
\hline Solyc08g067760 & SIMLO12 & 8 & SL2.40ch08:53957062..53962884 & 532 & 14 \\
\hline Solyc $10 \mathrm{~g} 044510$ & SIMLO13 & 10 & SL2.40ch10:22128868..22135940 & 558 & 14 \\
\hline Solyc07g063260 & SIMLO14 & 7 & SL2.40ch07:62995345..63002900 & 563 & 14 \\
\hline Solyc02g077570 & SIMLO15 & 2 & SL2.40ch02:37045094..37050486 & 375 & 10 \\
\hline Solyc06g010010 & SIMLO16 & 6 & SL2.40ch06:4699552..4706571 & 477 & 14 \\
\hline
\end{tabular}

In other cases, sequence alignments of cloned SIMLO from the different tissues with their corresponding genomic regions showed various types of splice variants, consisting of intron retention, exon skipping and alternative $5^{\prime}$ and 3 ' splice sites, according to the types of alternative splicing described by Keren et al., 2010 (Table 2 and Supplementary Fig. 1).

TABLE 2. Types of differentially spliced events observed in cloned SIMLO homologs from different tissues of the tomato cv Moneymaker.

\begin{tabular}{|c|c|c|c|c|c|}
\hline \multirow{2}{*}{ SIMLO } & \multirow{2}{*}{ Plant tissue } & \multicolumn{4}{|c|}{ Type of alternative splicing } \\
\hline & & Intron retention & Exon skipping & Alternative 5' splice site & Alternative $3^{\prime}$ splice site \\
\hline SIMLO1 & flower & & & & $\sqrt{ }$ \\
\hline SIMLO5* & fruit & $\sqrt{ }$ & & & \\
\hline SIMLO6 & leaf & & & $\sqrt{ }$ & $\sqrt{ }$ \\
\hline SIMLO9 & leaf & & $\sqrt{ }$ & & \\
\hline SIMLO11* & root & $\sqrt{ }$ & & & \\
\hline SIMLO13 & leaf & & $\sqrt{ }$ & & $\sqrt{ }$ \\
\hline SIMLO15 & fruit & & $\sqrt{ }$ & $\sqrt{ }$ & \\
\hline SIMLO15* & root & $\sqrt{ }$ & $\sqrt{ }$ & $\sqrt{ }$ & \\
\hline SIMLO15* & flower & $\sqrt{ }$ & $\sqrt{ }$ & $\sqrt{ }$ & \\
\hline
\end{tabular}

The asterisk $\left({ }^{*}\right)$ indicates SIMLO transcripts that can be either incompletely spliced or alternatively spliced 
TABLE 3A. Features and motifs distribution occurring in SIMLO proteins obtained from in silico translation of leaf, root, flower and fruit transcripts of the tomato cv Moneymaker. When no deviating transcripts are present for one SIMLO, the one from leaf has been used for motif analysis. Cells highlighted in grey indicate the absence of the corresponding motif.

\begin{tabular}{|c|c|c|c|c|c|c|c|c|c|c|c|c|}
\hline & & 容 売 & $\frac{\text { 흔 }}{\text { 흘 }}$ & 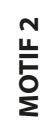 & $\begin{array}{l}m \\
\stackrel{m}{E} \\
\stackrel{0}{\Sigma}\end{array}$ & 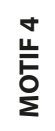 & $\begin{array}{l}\text { n } \\
\underline{E} \\
\stackrel{0}{0} \\
\Sigma\end{array}$ & $\begin{array}{l}\circ \\
\stackrel{0}{E} \\
\stackrel{0}{\Sigma}\end{array}$ & $\begin{array}{l}\text { 岂 } \\
\text { 足 }\end{array}$ & $\begin{array}{l}\infty \\
\stackrel{0}{E} \\
\sum\end{array}$ & 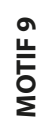 & $\begin{array}{l}\frac{0}{} \\
\frac{1}{5} \\
\frac{0}{2}\end{array}$ \\
\hline \multirow{3}{*}{ SIMLO1 } & LEAF & 507 & $\sqrt{ }$ & $\sqrt{ }$ & $\sqrt{ }$ & $\sqrt{ }$ & $\sqrt{ }$ & $\sqrt{ }$ & & & & \\
\hline & ROOT & 507 & $\sqrt{ }$ & $\sqrt{ }$ & $\sqrt{ }$ & $\sqrt{ }$ & $\sqrt{ }$ & $\sqrt{ }$ & & & & \\
\hline & FLOWER & 491 & $\sqrt{ }$ & $\sqrt{ }$ & $\sqrt{ }$ & $\sqrt{ }$ & $\sqrt{ }$ & & & & & \\
\hline SIMLO2 & LEAF & 504 & $\sqrt{ }$ & $\sqrt{ }$ & $\sqrt{ }$ & $\sqrt{ }$ & $\sqrt{ }$ & & & & & \\
\hline SIMLO3 & LEAF & 591 & $\sqrt{ }$ & & & $\sqrt{ }$ & & $\sqrt{ }$ & & & & \\
\hline SIMLO4 & LEAF & 554 & $\sqrt{ }$ & $\sqrt{ }$ & $\sqrt{ }$ & $\sqrt{ }$ & & $\sqrt{ }$ & $\sqrt{ }$ & $\sqrt{ }$ & & \\
\hline \multirow{3}{*}{ SIMLO5 } & LEAF & 517 & $\sqrt{ }$ & $\sqrt{ }$ & $\sqrt{ }$ & $\sqrt{ }$ & & $\sqrt{ }$ & & & & \\
\hline & FLOWER & 517 & $\sqrt{ }$ & $\sqrt{ }$ & $\sqrt{ }$ & $\sqrt{ }$ & & $\sqrt{ }$ & & & & \\
\hline & FRUIT & 540 & $\sqrt{ }$ & $\sqrt{ }$ & $\sqrt{ }$ & $\sqrt{ }$ & & $\sqrt{ }$ & & & & \\
\hline \multirow{4}{*}{ SIMLO6 } & LEAF & 549 & $\sqrt{ }$ & $\sqrt{ }$ & $\sqrt{ }$ & $\sqrt{ }$ & & & $\sqrt{ }$ & $\sqrt{ }$ & & \\
\hline & ROOT & 553 & $\sqrt{ }$ & $\sqrt{ }$ & $\sqrt{ }$ & $\sqrt{ }$ & & $\sqrt{ }$ & $\sqrt{ }$ & $\sqrt{ }$ & & \\
\hline & FLOWER & 553 & $\sqrt{ }$ & $\sqrt{ }$ & & $\sqrt{ }$ & & $\sqrt{ }$ & $\sqrt{ }$ & $\sqrt{ }$ & & \\
\hline & FRUIT & 553 & $\sqrt{ }$ & $\sqrt{ }$ & $\sqrt{ }$ & $\sqrt{ }$ & & $\sqrt{ }$ & $\sqrt{ }$ & $\sqrt{ }$ & & \\
\hline SIMLO7 & LEAF & 61 & & & & & & & & & & \\
\hline SIML08 & LEAF & 561 & $\sqrt{ }$ & $\sqrt{ }$ & $\sqrt{ }$ & $\sqrt{ }$ & & $\sqrt{ }$ & & & & \\
\hline \multirow{3}{*}{ SIMLO9 } & LEAF & 448 & $\sqrt{ }$ & & $\sqrt{ }$ & $\sqrt{ }$ & $\sqrt{ }$ & $\sqrt{ }$ & & $\sqrt{ }$ & & \\
\hline & FLOWER & 511 & $\sqrt{ }$ & $\sqrt{ }$ & $\sqrt{ }$ & $\sqrt{ }$ & $\sqrt{ }$ & $\sqrt{ }$ & & $\sqrt{ }$ & & \\
\hline & FRUIT & 511 & $\sqrt{ }$ & $\sqrt{ }$ & $\sqrt{ }$ & $\sqrt{ }$ & $\sqrt{ }$ & $\sqrt{ }$ & & $\sqrt{ }$ & & \\
\hline \multirow{4}{*}{ SIMLO10 } & LEAF & 533 & $\sqrt{ }$ & $\sqrt{ }$ & $\sqrt{ }$ & $\sqrt{ }$ & $\sqrt{ }$ & $\sqrt{ }$ & & & $\sqrt{ }$ & \\
\hline & ROOT & 533 & $\sqrt{ }$ & $\sqrt{ }$ & $\sqrt{ }$ & $\sqrt{ }$ & $\sqrt{ }$ & $\sqrt{ }$ & & & $\sqrt{ }$ & \\
\hline & FLOWER & 533 & $\sqrt{ }$ & $\sqrt{ }$ & $\sqrt{ }$ & $\sqrt{ }$ & $\sqrt{ }$ & $\sqrt{ }$ & & & $\sqrt{ }$ & \\
\hline & FRUIT & 178 & & $\sqrt{ }$ & & & & & & & $\sqrt{ }$ & \\
\hline \multirow{4}{*}{ SIML011 } & LEAF & 475 & $\sqrt{ }$ & $\sqrt{ }$ & $\sqrt{ }$ & $\sqrt{ }$ & $\sqrt{ }$ & $\sqrt{ }$ & & $\sqrt{ }$ & & \\
\hline & ROOT & 70 & & & & & & & & & & \\
\hline & FLOWER & 475 & $\sqrt{ }$ & $\sqrt{ }$ & $\sqrt{ }$ & $\sqrt{ }$ & $\sqrt{ }$ & $\sqrt{ }$ & & $\sqrt{ }$ & & \\
\hline & FRUIT & 475 & $\sqrt{ }$ & $\sqrt{ }$ & $\sqrt{ }$ & $\sqrt{ }$ & $\sqrt{ }$ & $\sqrt{ }$ & & $\sqrt{ }$ & & \\
\hline SIML012 & FLOWER & 532 & $\sqrt{ }$ & $\sqrt{ }$ & & $\sqrt{ }$ & & $\sqrt{ }$ & & $\sqrt{ }$ & & \\
\hline \multirow{4}{*}{ SIML013 } & LEAF & 63 & $\sqrt{ }$ & & & & & & & & & \\
\hline & ROOT & 558 & $\sqrt{ }$ & $\sqrt{ }$ & & $\sqrt{ }$ & & $\sqrt{ }$ & & & $\sqrt{ }$ & $\sqrt{ }$ \\
\hline & FLOWER & 558 & $\sqrt{ }$ & $\sqrt{ }$ & & $\sqrt{ }$ & & $\sqrt{ }$ & & & $\sqrt{ }$ & $\sqrt{ }$ \\
\hline & FRUIT & 558 & $\sqrt{ }$ & $\sqrt{ }$ & & $\sqrt{ }$ & & $\sqrt{ }$ & & & $\sqrt{ }$ & $\sqrt{ }$ \\
\hline SIMLO14 & LEAF & 563 & $\sqrt{ }$ & $\sqrt{ }$ & $\sqrt{ }$ & $\sqrt{ }$ & $\sqrt{ }$ & & & & & \\
\hline \multirow{4}{*}{ SIML015 } & LEAF & 459 & $\sqrt{ }$ & & $\sqrt{ }$ & & $\sqrt{ }$ & & & $\sqrt{ }$ & & \\
\hline & ROOT & 56 & & & & & & & & & & \\
\hline & FLOWER & 70 & & & & & & & & & & \\
\hline & FRUIT & 84 & & & & & & & & $\sqrt{ }$ & & \\
\hline SIML016 & LEAF & 477 & $\sqrt{ }$ & & $\sqrt{ }$ & $\sqrt{ }$ & $\sqrt{ }$ & & & & & \\
\hline
\end{tabular}




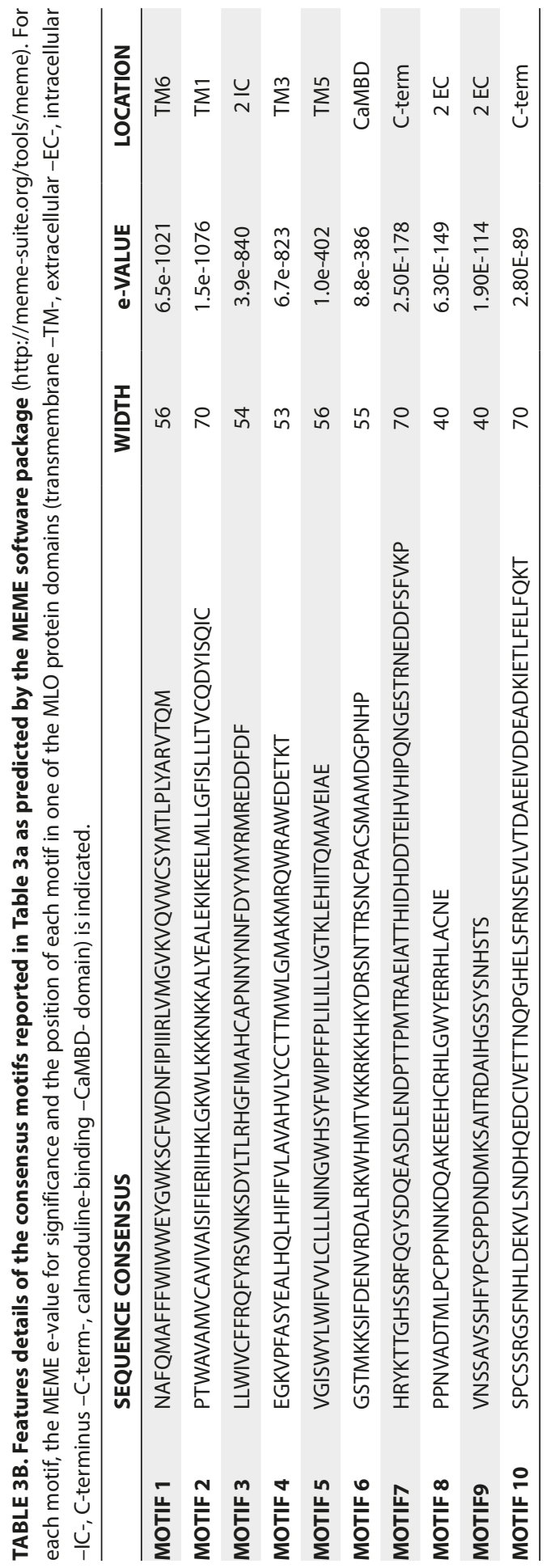




\section{Characterization of conserved amino acids and motifs of the SIMLO proteins}

To examine sequence features of the tomato SIMLO proteins, a multiple sequence alignment was performed using sequences obtained by the conceptual translation of transcripts cloned in different tissues. When no deviating transcripts were observed for a SIMLO gene, the sequence obtained from leaf was used for translation, with the exception of SIMLO12 which is the only homolog that was not cloned from leaf but from flower.

The aligned amino acid sequences of the tomato SIMLO protein family showed a high degree of conservation (92\%) of the 30 amino acid residues previously described to be invariable throughout the whole MLO protein family (Supplementary Fig. 2) (Elliott et al., 2005).

Due to aberrant transcripts, the protein sequences of SIMLO7 and SIMLO13 in leaf, SIMLO11 in root, and SIMLO15 in root, flower and fruit, were severely truncated (Table 3a). The predicted ORF of SIMLO8 in leaf was longer than the one deriving from the SGN prediction, which is missing important domains of the MLO protein. The protein sequence of SIMLO9 in leaf was shorter (448 aa length) than the ones obtained from the other two tissues (512 aa length) and it is predicted to have five transmembrane (TM) domains, instead of seven as in fruit and flower (Table 3a).

Finally, the SIMLO protein family was also used as input to search for conserved motifs. Ten patterns of consecutive amino acids, having a length ranging from 40 to 70 and shared by at least three MLO sequences (Table 3b), were found. Interestingly, four of these motifs included transmembrane domains, while the others were located in the second intracellular and extracellular domains, in the C-terminus and in the calmodulin-binding domain. The motifs seven and nine were shared only by SIMLO4/SIMLO6 and SIMLO10/ SIMLO13 respectively while the motif ten was only present in the amino acid sequences of SIMLO13 of root, flower and fruit. Those motifs might indicate regions of peculiar importance for the specific function of these homologs.

\section{Phylogenetic analysis of the tomato SIMLO protein family}

A phylogenetic analysis was carried out in order to establish the relationships between SIMLO proteins and MLO proteins of other plant species (Arabidopsis AtMLO1-15, pea PsMLO1, Lotus japonicus LjMLO1, barrel clover MtMLO1, pepper CaMLO2, tobacco NtMLO1, cucumber CsaMLO8, apple MdMLO18 and MdMLO20, strawberry FvMLO13 and FvMLO15, peach PpMLO9 and PpMLO13, barley HvMLO, rice OsMLO2 and wheat TaMLOA1b and TaMLOB1a). The resulting tree contains eight different clades (Fig. 1). These were named by Roman numerals from I to VIII, in accordance with previous studies performing phylogenetic analysis on the Arabidopsis and apple MLO protein families (Devoto et al., 2003; Pessina et al., 2014). 
Five clades, namely clade I, II, III, V and VI, contain both tomato and Arabidopsis homologs; clade IV contains only monocot MLO homologs that were selected for this study; clade VII contains only SIMLO15 together with apple, peach and strawberry MLO proteins (MdMLO18, PpMLO9 and FvMLO15, respectively). No SIMLO homologs could be assigned to clade VIII, which only contains Rosaceae MLO homologs (Fig. 1).

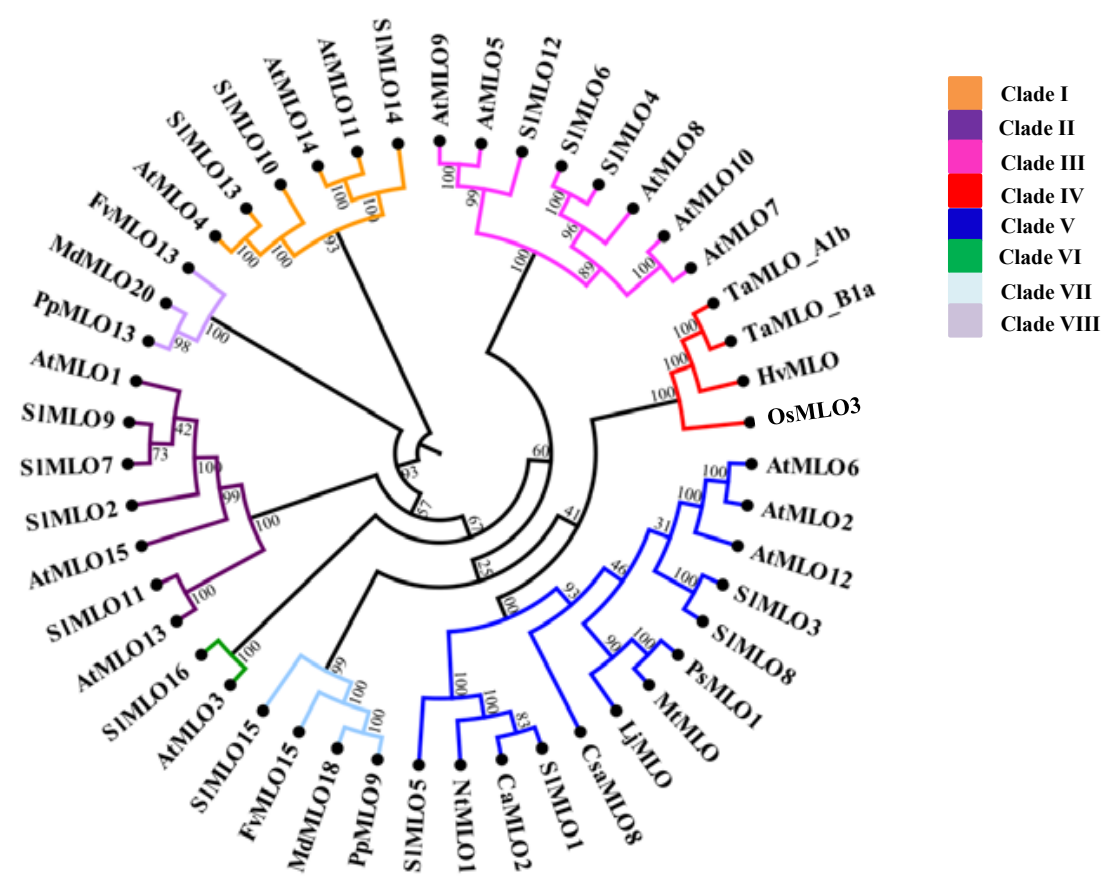

FIGURE 1. Rooted circular cladogram showing the phylogenetic relationships of the tomato SIMLO proteins. A UPGMA-based tree comprises all the sequences of the MLO protein family of Arabidopsis (At) and tomato (SI). Individual sequences of apple (Md), tobacco (Nt), cucumber (Csa), pea (Ps), Lotus japonicus (Lj), barrel clover (Mt), pepper (Ca), barley (Hv), rice (Os) and wheat (Ta) are included. Numbers on each node represent bootstrap values based on 100 replicates. Phylogenetic clades are designated with colours and Roman numbers according to the position of AtMLO homologs and apple MdMLO, as indicated by Pessina et al., 2014. The tomato SIMLO protein sequences used for this tree derived all from the translation of the transcripts cloned from leaf of the cv. Moneymaker, except for SIMLO12, which corresponds to the translated sequence of flower. Accession numbers of the sequences used, other than tomato SIMLO, are listed in Supplementary Table 2.

Three tomato MLO homologs, SIMLO3, SIMLO5 and SIMLO8, cluster together with SIMLO1 in clade $\mathrm{V}$, containing all the known eudicot MLO homologs functionally related to powdery mildew susceptibility (AtMLO2, AtMLO6, AtMLO12, PsMLO1, LjMLO1, MtMLO1, CsaMLO8, NtMLO1 and CaMLO2) (Fig. 1) (Elliott et al., 2005; Consonni et al., 2006; Bai et al., 2008; Pavan et al., 2009; Humphry et al., 2011; Várallyay et al., 2012; Zheng et al., 2013; Appiano et al., 2015; Berg et al., 2015). 
The tomato homologs SIMLO4, SIMLO6, and SIMLO12 group in clade III together with AtMLO7, which regulates Arabidopsis pollen tube reception by the synergid cells, whereas SIMLO10, SIMLO13 and SIMLO14 are the closest tomato homologs to the root thigmomorphogenesis regulating proteins AtMLO4 and AtMLO11 in clade I (Fig. 1) .

Finally, clade II includes four tomato SIMLO homologs (SIMLO2, SIMLO7, SIMLO9 and SIMLO11) together with three Arabidopsis proteins (AtMLO1, AtMLO13 and AtMLO15) and clade VI harbours only AtMLO3 and tomato SIMLO16 (Fig. 1).

\section{Expression profiles of SIMLO homologs in axenic conditions and upon powdery mildew challenge}

The expression level of SIMLO genes was determined in four different tissues (leaf, root, flower and ripened fruit). These were found to vary considerably among SIMLO genes, and it was not possible to assign clade-specific expression patterns (Fig. 2). Concerning clade V, SIMLO5 and SIMLO8 were found to be characterized by very low expression levels in all the tissues. Interestingly, SIMLO1 was found to be less expressed in leaves compared to flowers. Our results are supported by the collection of RNA-seq data, as shown by the FPKM (fragments per kilobase of exon per million fragments mapped) values for the four tissues under investigation of each homolog represented into graphs of Supplementary Fig. 3.

We investigated the expression profile of the SIMLO gene family in response to $O$. neolycopersici, using L33 as a reference gene (Fig. 3). SIMLO1 expression significantly increased at six and ten hours after pathogen challenge. No other SIMLO homolog in clade V (SIMLO3, SIMLO5, SIMLO8) showed pathogen-dependent up-regulation.

On the other hand, a significant upregulation in response to $O$. neolycopersici was observed for SIMLO homologs outside clade V, namely SIMLO2, SIMLO4, SIMLO7, SIMLO10, SIMLO13, SIMLO14 and SIMLO16. In particular, the expression of SIMLO4 and SIMLO14 at ten hours after inoculation was comparable to the one of SIMLO1, and $\sim 4$-fold and $\sim 3$-fold higher than the one of control plants, respectively.

Similar results were obtained repeating the expression analysis using Ef $1 \mathrm{a}$ as reference gene (Supplementary Fig. 4).

In order to confirm the strong up-regulation of the above mentioned genes, a second inoculation experiment was carried out, sampling leaf tissues at the same time points ( 0 hpi, $6 \mathrm{hpi}$ and $10 \mathrm{hpi}$ ). The results presented in Supplementary Fig. 5 indicate that indeed SIMLO1, SIMLO4 and SIMLO14 show a statistically significant up-regulated expression due to the $O$. neolycopersici challenge. The slight down-regulated expression of SIMLO3 observed after the first pathogen inoculation was not confirmed in the second experiment. 
SIMLO1

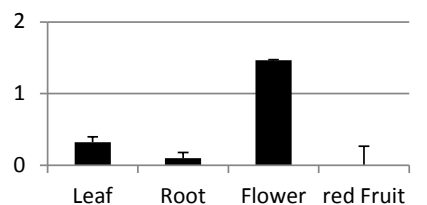

SIMLO4

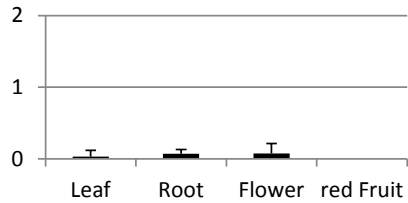

SIMLO7

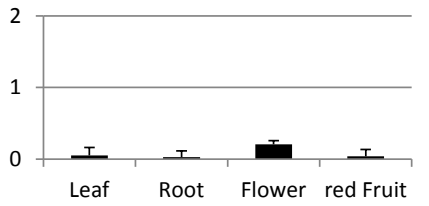

SIMLO10

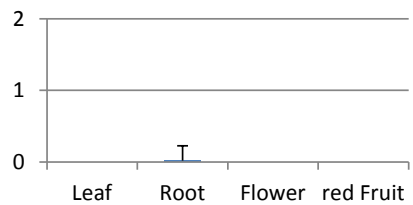

SIMLO13

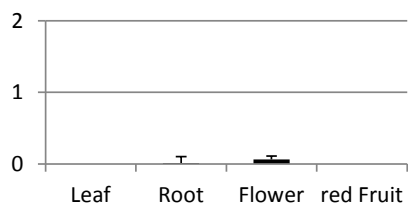

SIMLO16

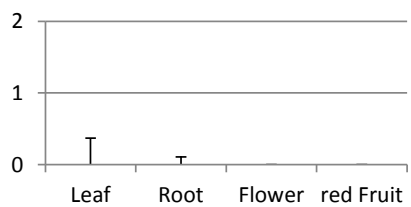

SIMLO2

SIMLO3

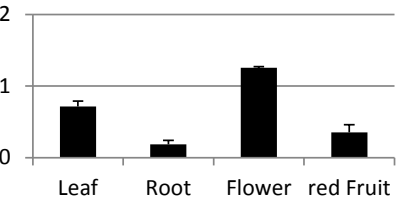

SIMLO5

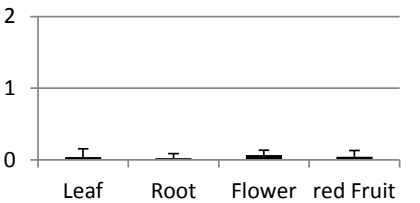

SIMLO8

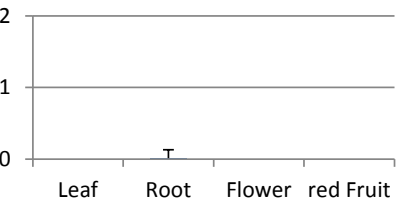

SIMLO11

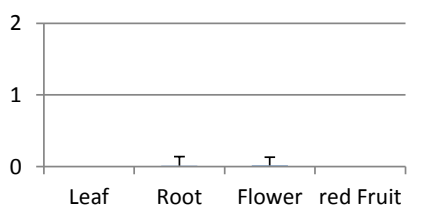

SIMLO14

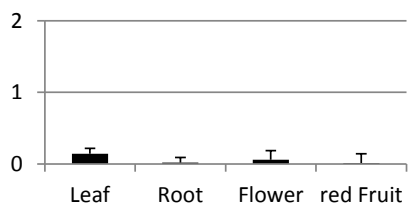

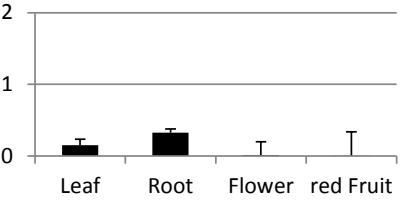

SIMLO6

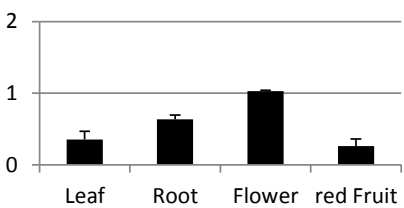

SIMLO9

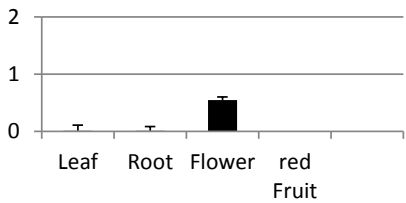

SIMLO12

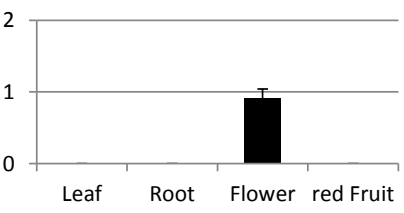

SIMLO15

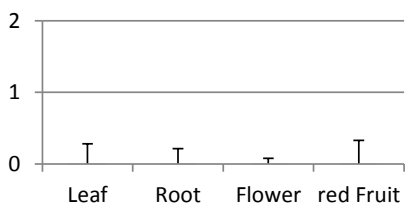

FIGURE 2. Relative expression level of SIMLO transcripts evaluated in four different tissues (leaf, root, flower and mature fruit) of the tomato cv. Moneymaker in axenic condition. The expression level of each gene is compared to the abundance of Ef1 a which was used as reference gene. Bars show standard errors based on three technical replicates. Similar trends are reported in Supplementary Fig. 3. 


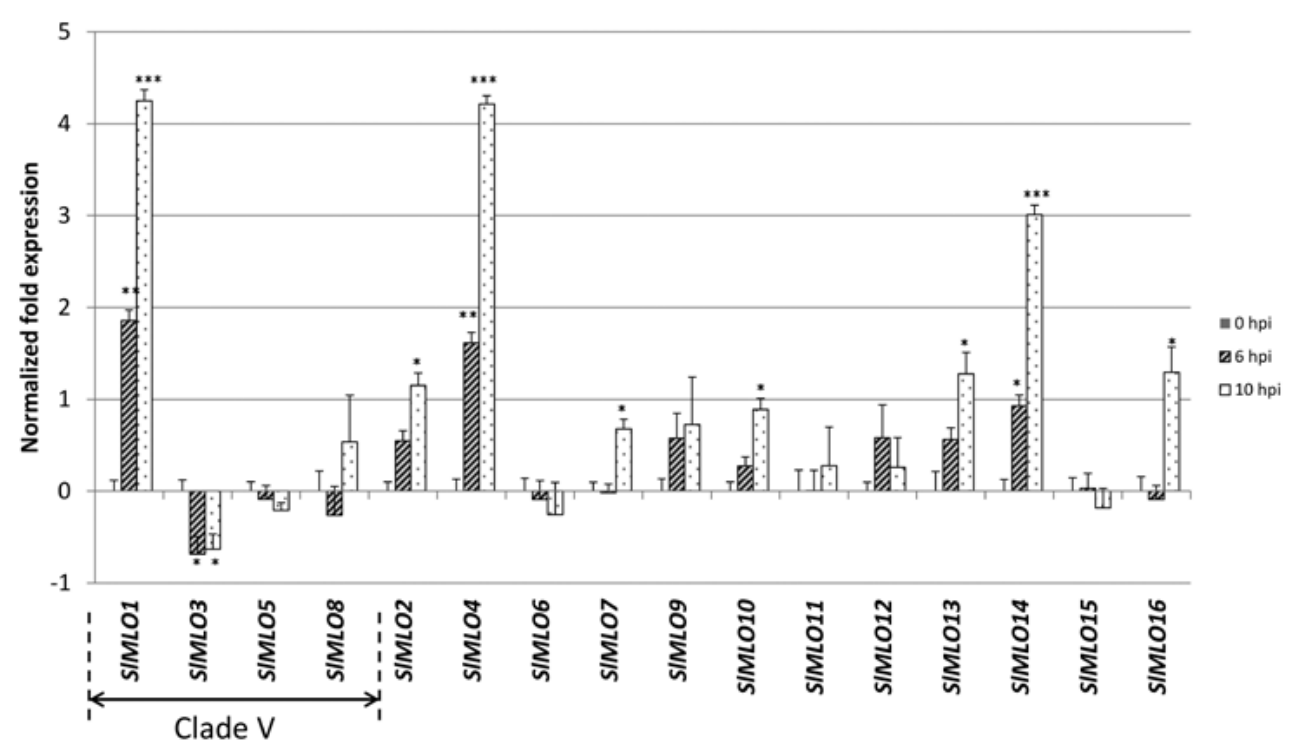

FIGURE 3. Relative expression level of the SIMLO gene family in response to 0 . neolycopersici inoculation. Samples were collected at 0, 6 and $10 \mathrm{hrs}$ after inoculation (hpi). Transcript abundance of each SIMLO homolog was normalized against the transcription level of the 60S ribosomal protein L33 used as reference gene. Bars show standard errors based on four biological replicates. Asterisks refer to significant differences with respect to non-inoculated plants ( $0 \mathrm{hpi})$, inferred by mean comparisons with a Student's t-test $\left({ }^{*} \mathrm{p}<.05,{ }^{* *} \mathrm{p}<.01,{ }^{* * *}\right.$ $\mathrm{p}<.001)$. The SIMLO genes harbored in clade $\mathrm{V}$, based on the phylogenetic tree of Fig. 1, are indicated by an arrow spanning their corresponding bars. Similar results were obtained by using the elongation factor Ef1a as housekeeping gene (Supplementary Fig. 4)

\section{Functional characterization of clade V SIMLO homologs}

Based on their relatedness with eudicot $M L O$ homologs predisposing to PM susceptibility, including SIMLO1, the newly identified SIMLO homologs in clade V (SIMLO3, SIMLO5 and $S I M L O 8$, Fig. 1) were further investigated with respect to their role in the interaction with O. neolycopersici. Therefore, specific RNAi silencing constructs for these three homologs were developed, which were used to transform the susceptible cultivar Moneymaker (MM) (Supplementary Fig. 6 and Supplementary Table 3). A silencing construct targeting SIMLO1 was included as control, which was expected to lead to a resistant phenotype.

Ten to twenty $T_{1}$ plants were obtained for each silencing construct. The expression of the target genes was assessed by means of real-time qPCR (Supplementary Fig. 7) and $T_{1}$ plants with a reduced level of expression of the target gene were allowed to self-pollinate to develop $T_{2}$ families. In total, two independent $T_{2}$ families (each segregating for the presence of the silencing construct) were developed for SIMLO1 and SIMLO8, and three were obtained for SIMLO3 and SIMLO5. Transgenic individuals of each family were further assessed for the silencing levels of target genes and other clade $V$ homologs. This revealed successful silencing of each target genes and no unwanted co-silencing in transgenic 
RNAi::SIMLO3, SIMLO5 and SIMLO8 individuals [Fig. 4, panel B), C) and D)]. Conversely, $\mathrm{T}_{2}$ transgenic plants of two $\mathrm{T}_{2}$ families carrying the RNAi::SIMLO1 silencing construct were characterized by the simultaneous silencing of SIMLO1, SIMLO5 and SIMLO8 [Fig. 4, panel A) and Supplementary Fig. 8].

As expected, $\mathrm{T}_{2}$ progenies carrying the RNAi::SIMLO1 construct segregated for PM resistance: $T_{2}$ plants carrying the silencing construct $\left[T_{2}-S I M L O 1 \_N P T(+)\right]$ were resistant, whereas non-transgenic plants [ $\left.\mathrm{T}_{2-} S I M L O 1 \_N P T(-)\right]$ were susceptible as MM (Fig. 5, panel A). In contrast, all $\mathrm{T}_{2}$ progenies segregating for SIMLO3, SIMLO5 and SIMLO8 silencing constructs visually appeared to be fully susceptible to O. neolycopersici (Fig. 5 , panel A). The quantification of disease severity on these lines using real-time qPCR supported phenotypic observations, as no significant difference was found between T 2 SIMLO3_NPT(+), $T_{2}$ SIMLO5_NPT(+), $T_{2}$ SIMLO8_NPT(+) plants and MM (Fig. 5, panel B and Supplementary Fig. 9). For each $\mathrm{T}_{2}$ family, transgenic and non-transgenic plants were phenotypically indistinguishable.

The SImlo1 line, harboring a loss-of-function mutation in the SIMLO1 gene (Bai et al. 2008), is resistant to PM, however lower leaves displayed PM symptoms (Fig. 5, panel A). Compared to the plants of the SImlo1 line, RNAi plants carrying the RNAi::SIMLO1 construct ( $\mathrm{T}_{2}$ SIMLO1_NPT(+) plants) showed no PM symptom and also a significantly lower amount of fungal biomass (Fig. 5 panel B and Supplementary Fig. 9, panel A). Therefore, further microscopic observations were carried out to study the fungal growth on the SImlo1 line and $T_{2}$ SIMLO1_NPT(+) plants.

Since the two $T_{2}$ families carrying the RNAi::SIMLO1 construct showed no difference with respect to the level of reduced expression of the SIMLO homologs and fungal biomass quantification (Supplementary Fig. 8 and 9), we used one $T_{2}$ family for microscopic study. Compared to MM, fungal growth was significantly reduced in both SImlo1 and $\mathrm{T}_{2}$ RNAi::SIMLO1_NPT(+) individuals due to the formation of a papilla beneath the appressorium (Fig.6). Interestingly, the rate of papilla formation in $\mathrm{T}_{2-}$ RNAi::SIMLO1_NPT(+) (93.3\% of the infection units) was significantly higher than in SImlo1 (64.4\% of the infection units) (Table 4). In some cases, O. neolycopersici was still able to penetrate epidermal cells and form haustoria with a rate of $48.9 \%$ in SImlo1 and 30\% in $T_{2-}$ RNAi::SIMLO1_NPT(+) (Table 4 and Fig. 6). The general development of the spores on the two genotypes was strikingly different: while on the SImlo1 line the fungus could produce mostly up to two secondary hyphae (in $36.7 \%$ of the total infection units), on $T_{2}$ RNAi::SIMLO1_NPT(+) individuals fungal growth was significantly reduced after producing a germination tube (Table 4 and Fig. 6). 

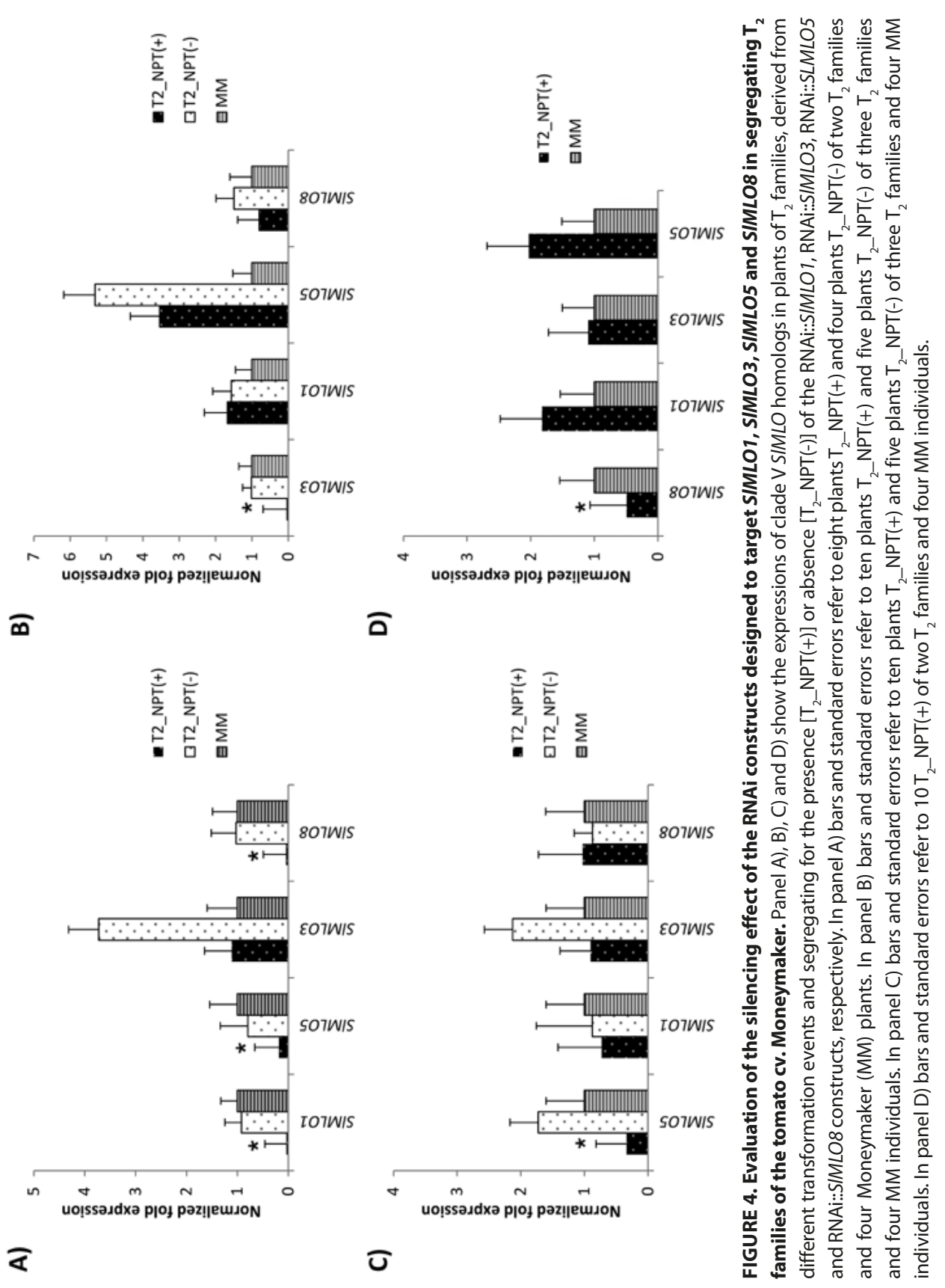


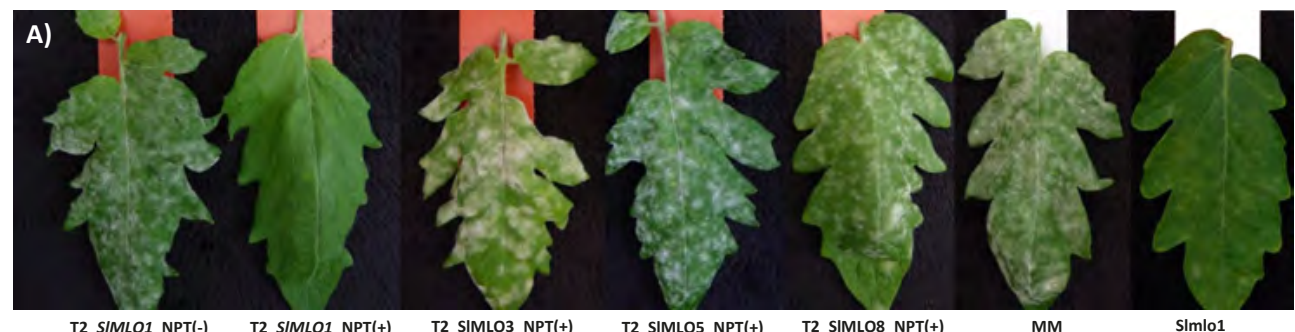

T2_SIMLO1_NPT(-)

T2_SIMLO1_NPT(+)

T2_SIMLO3_NPT(+)

T2_SIMLO5_NPT(+)

T2_SIMLO8_NPT(+)

MM

SImlo1

B)

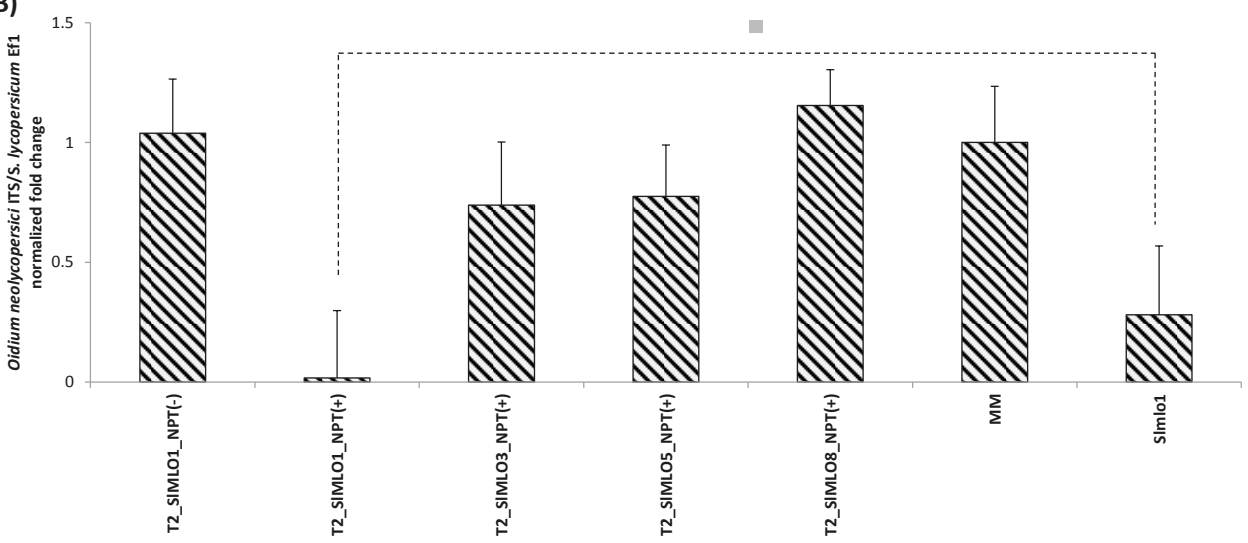

FIGURE 5. Powdery mildew evaluation on plants of segregating $T_{2}$ families obtained with silencing constructs targeting SIMLO genes to attest their involvement in O. neolycopersici susceptibility. Panel A) shows the phenotypic evaluation of the powdery mildew growth on leaves of different $T_{2}$ individuals that have been evaluated for the (from left to right) absence of the RNAi::SIMLO1, presence of the RNAi::SIMLO1, presence of the RNAi::SIMLO3, presence of the RNAi::SIMLO5 and presence of the RNAi::SIMLO8 silencing constructs, followed by one individual of the cv Moneymaker (MM) and one of the SImlo1 line carrying a loss-of-function mutation in the SIMLO1 gene. Panel B) shows the relative quantification of the ratio between Oidium neolycopersici and plant gDNAs in transgenic individuals [NPT(+)] and not transgenic individuals [NPT(-)] segregating in $\mathrm{T}_{2}$ families obtained with the silencing constructs above described. Bars and standard errors refer to (from left to right) four individuals of two independent $T_{2}$ families not carrying the RNAi::SIMLO1, eight individuals of the same two $T_{2}$ families carrying the RNAi::SIMLO1, 18 individuals of three independent $T_{2}$ segregating families carrying the RNAi::SIMLO3 construct, 18 individuals of three independent $T_{2}$ segregating families carrying the RNAi::SIMLO5 construct and 20 individuals of two $\mathrm{T}_{2}$ segregating families carrying the RNAi::SIMLO8 construct, next to $10 \mathrm{MM}$ plants and 10 plants of the SImlo1 line. The asterisk refers to the significant difference in susceptibility between individuals of the $T_{2} S_{-}$IMLO1_ $_{2}$ $\mathrm{NPT}(+)$ and SImlo1, inferred by mean comparisons with a Student's t-test $\left({ }^{*} \mathrm{p}<.05\right)$. 
TABLE 4. Development of Oidium neolycopersici growth on the susceptible genotype Moneymaker and on the two resistant genotypes, SImlo1 carrying a loss-of-function SIMLO1 gene and plants of a $T_{2}$ family selected to carry the RNAi::SIMLO1 silencing construct which can effectively silence SIMLO1, SIMLO5 and SIMLO8.

\begin{tabular}{ccccccccccc}
\hline & \multicolumn{4}{c}{ Percentage of infection units (IU) } & \multicolumn{5}{c}{ Hyphae per IU } \\
\hline \multirow{2}{*}{ Genotype } & $\begin{array}{c}\text { Primary } \\
\text { AP }\end{array}$ & $\begin{array}{c}\text { Primary } \\
\text { papilla }\end{array}$ & $\begin{array}{c}\text { Primary Secondary Secondary } \\
\text { HS }\end{array}$ & Papilla & HS & $\mathbf{1}$ & $\mathbf{2}$ & $\mathbf{3}$ & $\mathbf{4}$ & $\mathbf{5}$ \\
& 100 & 0 & 90.2 & 0 & 68.3 & 76.8 & 67.1 & 35.4 & 6.1 & 0 \\
MM & 100 & 64.4 & 48.9 & 23.3 & 14.4 & 43.3 & 36.7 & 18.9 & 3.3 & 0 \\
SImlo1 & 100 & $93.3^{*}$ & 30.0 & 2.2 & 0.0 & 11.1 & 7.8 & 3.3 & 0.0 & 0 \\
\hline
\end{tabular}

$\mathrm{AP}=$ appressorium, $\mathrm{HS}=$ haustorium

${ }^{*} \mathrm{p}<.05$ compared to SImlo1

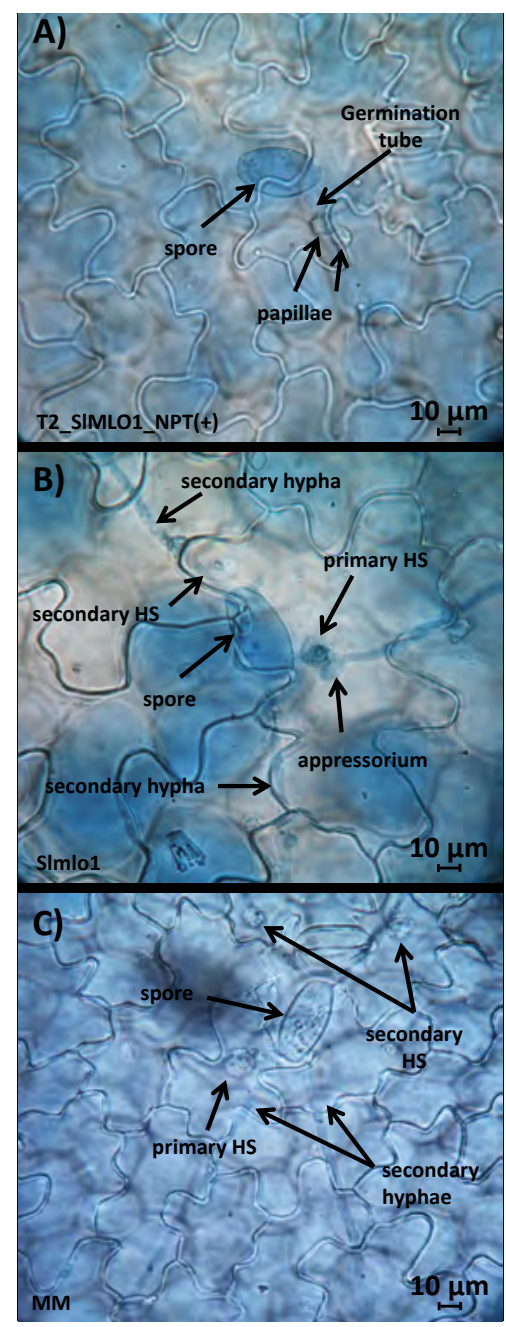

FIGURE 6. Effect of the silencing of SIMLO1, SIMLO5 and SIMLO8 in tomato cv Moneymaker background compared with the SImlo1 line harboring a loss-of-function of SIMLO1 gene. Panels $A$ ) refers to a transgenic plant carrying the RNAi::SIMLO1 construct, panels B) a plant of the SImlo1 line and panels C) a plant of the tomato (v. Moneymaker. A), B) and C) show fungal structures (spores, germination tube, appressorium, haustorium -HS- and hyphae) and the plant cellular reaction of papilla apposition at the sites of fungal penetration. 


\section{Discussion}

\section{Structure and evolution of the SIMLO gene family}

In this study, we followed an in silico approach to assign 16 homologs to the tomato MLO gene family. This is consistent with the results of previous studies reporting the MLO gene families of several diploid species made of a number of homologs variable from 13 to 21 (Devoto et al., 2003; Feechan et al., 2008; Liu and Zhu, 2008; Shen et al., 2012; Pessina et al., 2014; Schouten et al., 2014; Appiano et al., 2015). This suggests that a similar number of MLO homologs is likely to be retrieved in future genome-wide investigations involving diploid eudicot species.

Information on chromosomal localization was available for all the SIMLO homologs with the exception of SIMLO4. However, potato and tomato genomes are highly syntenic (2012) and the closest SIMLO4 homolog in potato (Sotub02g007200) is positioned on chromosome 2, thus suggesting that SIMLO4 is also located on tomato chromosome 2.

Cloning of the SIMLO gene family from different tissues of the cultivar MM revealed the occurrence of transcripts deviating from predictions available at the SGN database, indicating that, despite the efforts of the tomato resequencing project, the assembly of genomic regions and the prediction of certain loci are not correct yet. Moreover, several cases of differentially spliced variants among plant tissues were observed, mostly due to intron retention and exon skipping, as it is in the case of SIMLO5, SIMLO9, SIMLO11, SIMLO13 and SIMLO15. Due to the method used in this study to amplify the SIMLO homologs, we cannot exclude that the intron retention is the result of the amplification of non-mature mRNA. However, intron retention was previously reported to be a very common type of alternative splicing in Arabidopsis and rice (Ner-Gaon et al., 2007). There is also a well-documented evidence indicating organspecific regulation of alternative splicing in plants (Palusa et al., 2007). More studies need to be performed to unravel its complexity and functional significance. Certainly, alternative forms of splicing, such as the ones found in this study, can lead to aberrant mRNA isoforms that cause the loss-of-function of a MLO gene. An example is reported by a recent study conducted by Berg et al., 2015 in cucumber. They show that the integration of a transposable element in the genomic region of the CsaMLO8 leads to an aberrant splicing that causes the loss-of-function of this susceptibility gene in a resistant cucumber genotype.

The identification of protein motifs conserved in transmembrane domains of specific SIMLO homologs (Table 3a and 3b) corroborates previous findings in Solanaceae plant species (Appiano et al., 2015). This indicates that transmembrane domains, which are thought to provide a common scaffold invariable for the whole MLO family (Devoto et al., 1999), might also be involved in conferring specific functions to MLO homologs. Future functional studies of targeted mutagenesis of transmembrane MLO protein regions can help to unravel their actual role. 
All the SIMLO proteins were found to group in six phylogenetic clades together with other eudicot MLO homologs, including the complete Arabidopsis AtMLO family and certain members of the apple, peach and strawberry MLO family. No SIMLO homolog could be assigned to clade IV, previously shown to contain monocot MLO homologs and a few eudicot homologs (grapevine VvMLO14, strawberry FvMLO17 and peach PpMLO12) (Feechan et al., 2008; Pessina et al., 2014).

Based on their sequence relatedness with Arabidopsis AtMLO proteins of known function, it is logical to argue that one or more of the tomato SIMLO homologs in clade III and clade I could regulate the processes of root response to mechanical stimuli and pollen tube reception, respectively. The RNAi silenced lines of several SIMLO homologs generated in this study could be useful to assign new functions to MLO proteins which have gone unnoticed by the evaluation of the available panel of Arabidopsis Atmlo mutants.

\section{Possible pleiotropic effects and co-functioning of SIMLO homologs}

RNA-seq data, RT-PCR and real-time qPCR of the SIMLO gene family confirmed the expression of all the 16 SIMLO homologs. Often, it was possible to detect high level of transcript of the same SIMLO homolog in more than one of the four tissues under study (leaf, root, flower and mature fruit). This is in line with the findings of the previous study of Chen et al., 2006, investigating the expression pattern of the Arabidopsis AtMLO gene family in several tissues. Overall, this body of evidence suggest that: a) different MLO homologs may have synergistic or antagonistic roles in regulating the same biological process; b) MLO homologs may have pleiotropic effects on different biological processes. Co-functioning between MLO homologs has been demonstrated to occur in Arabidopsis, where different AtMLO genes co-participate in the same tissue to determine powdery mildew susceptibility and root response to mechanical stimuli (Consonni et al., 2006; Chen et al., 2009). A yet unidentified additional biological function could be hypothesized for the SIMLO1, previously shown to act as a susceptibility gene towards 0 . neolycopersici (Pavan et al., 2009). This gene was found to exhibit its strongest expression level in tomato flower and moderate expression in root, two tissues which are less or not attacked by the fungus, respectively. Moreover, additional biological roles for SIMLO1 would explain why this gene has not been excluded from evolution, despite promoting susceptibility to PM pathogen. Interestingly, evidence shows that the SIMLO1 orthologs in barley and Arabidopsis are involved in the interaction with pathogens other than powdery mildews, such as necrotrophs and hemibiotroph (Jarosch et al., 1999; Kumar et al., 2001; Consonni et al., 2006). Thus, it is worthwhile to test the RNAi-SIMLO1 plants with more pathogens to broaden its role in plant-pathogen interactions. 


\section{SIMLO homologs involved in powdery mildew susceptibility}

In this study, we mainly focused on the SIMLO genes grouped in the clade V containing all the $M L O$ homologs associated with PM susceptibility in eudicots. The presence of multiple tomato homologs in clade $\mathrm{V}$ is in accordance with the existence of three Arabidopsis proteins (AtMLO2, AtMLO6 and AtMLO12) associated with increased fungal penetration (Consonni et al., 2006).

We showed that tomato SIMLO3, SIMLO5 and SIMLO8, differently from SIMLO1, do not increase their expression upon $O$. neolycopersici challenge. Furthermore, strong silencing of the same homologs in a susceptible tomato background (Moneymaker) did not result in a significant reduction of disease symptoms (Fig. 3, Fig. 4 and Fig. 5).

Plants transformed with a construct meant to silence SIMLO1 showed co-silencing of SIMLO5 and SIMLO8, due to sequence relatedness between these genes (Fig. 4). Interestingly, these plants were also significantly more resistant than plants of the S/mlo1 line (Fig. 5). Since the SImlo1 line is only a BC3S2 line carrying the SImlo1 mutation (the ol-2 gene) in MM background, we cannot fully exclude background effects from the ol-2 donor, the resistant line LC-95 of S. lycopersicum var. cerasiforme, which might add to partial susceptibility phenotype of the $S / m l o 1$ line. On the other hand, our scenario is reminiscent of the one reported in Arabidopsis, where Atmlo2 single mutant displays partial PM resistance, whereas Atmlo2/Atmlo6/Atmlo12 triple mutant is fully resistant (Consonni et al., 2006). Also in grape, more than one VVMLO genes are involved in susceptibility to powdery mildew (Feechan et al. 2008; 2013). Taken together with the knowledge of functional redundancy in Arabidopsis and grape, our data suggest that in tomato SIMLO1, SIMLO5 and SIMLO8 are functionally redundant as PM susceptibility factors with SIMLO1 playing a major role. Our results showed that the contribution of SIMLO5 and SIMLO8 is too small to be observed with an RNAi approach silencing individual genes, but a complementation experiment using the SImlo1 line could be more suitable to observe the minor role of these genes.

It cannot be excluded yet that the other clade $\mathrm{V}$ tomato homolog SIMLO3 is also involved in plant-pathogen interactions. However, it is worthwhile to notice that the SIMLO3 protein is missing three of the six motifs contained in SIMLO1, two of which are also present in SIMLO5 and SIMLO8 (Table 3b). The motif three in Table 3b is located in the second intracellular domain, which is known to be involved together with the third intracellular domain in the protein functionality (Elliott et al., 2005). This would suggest that SIMLO3 might miss important features to be fully functional as susceptibility factor. Overexpressing of SIMLO3 in the SImlo1 mutant may provide a better evidence on its eventual role as functional susceptibility gene. 
Interestingly, we noticed that SIMLO4 and SIMLO14, which do not belong to clade V, are up-regulated upon $O$. neolycopersici infection (Fig. 3 and Supplementary Fig. 4 and 5). SIMLO14 is closely related to AtMLO4 and AtMLO11, which are involved in root thigmomorphogenesis (Chen et al., 2009), while SIMLO4 is related to AtMLO7, involved in pollen tube reception (Kessler et al., 2010). In Arabidopsis, mutation of AtMLO4, AtMLO7 and AtMLO11 does not result in PM resistance. Thus, we expected that silencing of SIMLO4 and SIMLO14 in tomato will not lead to PM resistance too. The up-regulated expression of SIMLO4 and SIMLO14 after challenge with O. neolycopersici might be the result of shared regulatory cis-acting elements. We used a $2 \mathrm{~kb}$ region located upstream the starting codon of SIMLO1, SIMLO4 and SIMLO14 coding sequences to search for shared regulatory elements through the online database Plant Care (http://bioinformatics.psb.ugent.be/ webtools/plantcare/html/) (Lescot et al., 2002). We found, at least, five common motifs which are associated with upregulation by multiple biotic and/or abiotic stresses: ABRE (CACGTG), involved in abscisic acid responsiveness, CGTCA- and TGACG-motifs, involved in the MeJA responsiveness, HSE (AAAAAATTC), involved in heat stress responsiveness, and TCA (CCATCTITTT/GAGAAGAATA) element, involved in salicylic acid response. It is intriguing whether SIMLO4 and SIMLO14 can act as a susceptibility gene to PM. Till now, only clade IV and clade V MLO genes have been studied for their role as a susceptibility gene. To further study these PM-induced non-clade V SIMLO genes, a complementation test using the SImlo mutant could be performed.

In conclusion, this study provides a comprehensive characterization of the MLO gene family in tomato by analyzing their genomic structure, expression profile and predicted protein motifs. In tomato, there are $17 \mathrm{MLO}$ genes which can be grouped into six clades. The expression of these MLO genes can be tissue specific and some $M L O$ genes showed alternative splicing variants in different tissues. The SIMLO1 in clade $\mathrm{V}$ is confirmed to be the major PM susceptibility factor. In addition, two clade V genes, SIMLO5, and SIMLO8 are suggested to have a partially redundant function, as described in Arabidopsis for AtMLO2, 6 and 12 genes (Consonni et al. 2006). To label an MLO gene as a PM susceptibility gene, it is recommended to combine phylogenetic analysis and expression profile to select candidates of clade IV (for monocot) and V (for dicot) that are induced by PM infection. However, the upregulation of $M L O$ genes outside clade $V$ in response to PM, as shown in this study and in Pessina et al. (2014), raises the possibility that they may act as susceptibility genes. Finally, the RNAi lines generated in this study are useful materials for further assigning new biological functions to the $M L O$ gene family members. 


\section{Materials and methods}

\section{Plant material, fungal material and inoculation}

In this study, we used the susceptible S. lycopersicum cultivar Moneymaker (MM), the SImlo1 line and transgenic $T_{2}$ families in which individual SIMLO gene was silenced via RNAi in MM background. The SImlo1 mutant (the ol-2 gene) was a natural mutation discovered in the resistant line LC-95 of S. lycopersicum var. cerasiforme. The LC-95 line was crossed with the susceptible tomato S. lycopersicum cv. Super Marmande and the F2 progeny was used for mapping in 1998 (Ciccarese et al. 1998). Later, we introgressed the ol-2 allele into S. lycopersicum cv Moneymaker (MM) by backcrossing and one BC3S2 line homozygous for the ol-2 allele (the tomato SImlo1 line) was used in the experiment.

The powdery mildew disease assay was performed by artificial inoculation in the greenhouse. For this, the Wageningen isolate of O. neolycopersici (On) was used (Bai et al., 2008). A suspension of $O$. neolycopersici conidia was prepared, by rinsing freshly sporulating leaves of infected tomato plants with tap water. This suspension was immediately sprayed on 1 month-old tomato plants. Ten plants for each of the $T_{2}$ progenies obtained from the transformation of each silencing construct, 10 Slmlo1 plants and 10 MM plants were used for disease assay. The scoring of powdery mildew symptoms was done 10 days after inoculation, inspecting and collecting the third and fourth true leaves for each plant.

For the evaluation of the expression of the SIMLO gene family, two independent inoculations were set up. In both cases, we used the cultivar MM, four and three biological replicates for each of the three time points $(0,6$ and 10 hours post inoculation -hpi- ) during the first and the second inoculation respectively.

\section{Identification and cloning of the SIMLO gene family}

Putative tomato MLO protein sequences were identified in the Sol Genomics Network (SGN) (http://solgenomics.net/) database by using the BLASTP and TBLASTN algorithms with Arabidopsis AtMLO protein sequences as a query. Chromosomal localization, sequences of the corresponding genes and introns/exons boundaries were inferred by annotations from the International Tomato Annotation Group (ITAG).

Aiming at cloning and sequencing the SIMLO gene family from the cultivar MM, total RNA from leaf, root, flower and ripened fruit was isolated (RNeasy ${ }^{\circledR}$ mini kit, Qiagen). The different tissues were collected from five MM plants and pooled together to obtain enough material for the RNA isolation. For each individual SIMLO homolog, two primer pairs specifically amplifying overlapping products of around $800 \mathrm{bp}$ of the predicted coding sequences (CDS) were designed using the Primer3 plus online software (http:// www.bioinformatics.nl/cgi-bin/primer3plus/primer3plus.cgi; Rozen and Skaletsky, 2000). 
The forward primer and the reverse primer of product $A$ and product $B$, respectively, are located in the respective UTR regions to ensure at least the cloning of the complete CDS. A one-step PCR was performed to obtain the desired product (SuperScript ${ }^{\circledast}$ III One-Step RT-PCR System, Invitrogen) (Supplementary Table 1). Indeed, a PCR performed on a cDNA obtained with oligo $d_{T}$ primers did not yield any product for many of the homologs under investigation. The use of sequence-specific primers in the one-step PCR, on the other hand, allowed the binding of only the desired mRNA sequences.

Corresponding amplicons were visualized on agarose gel and cloned into the PGEM ${ }^{\circledR}-T$ Easy vector (Promega). Recombinant plasmids were sequenced by using universal T7 and SP6 primers.

In order to reveal gene structures and polymorphisms, SIMLO sequences obtained by cloned amplicons were merged using the package Seqman of the software DNASTAR ${ }^{\circ}$ Lasergene8. The obtained consensus was aligned with the coding region of the SIMLO identified in silico and the corresponding genomic region using the CLC 7.6.1 sequence viewer software (www.clcbio.com).

Finally, for the motif analysis, the MEME (http://meme.nbcr.net/) package was used to predict consensus patterns of consecutive conserved amino acids in the SIMLO proteins deriving from the in silico translation of the cloned transcripts from leaf, root, flower and fruit of the cultivar MM (Bailey et al., 2015).

\section{Comparative analysis}

The corresponding SIMLO protein sequences of translated cloned CDS obtained from leaf and flower (in the case of SIMLO12) were used as dataset in the CLC 7.6.1 sequence viewer software (www.clcbio.com) for ClustalW alignment and the obtainment of an UPGMAbased comparative tree (bootstrap value was set equal to 100), together with those of the 15 Arabidopsis AtMLO homologs. Moreover MLO proteins experimentally shown to be required for PM susceptibility were added, namely pea PsMLO1, barley HvMLO, wheat TaMLO_A1b and TaMLO_B1a, rice OsMLO2, pepper CaMLO2, tobacco NtMLO1, cucumber CsaMLO8, Lotus japonicus LjMLO1 and barrel clover MtMLO1. Moreover MLO homologs of the Rosaceae species that cluster in clade VII (FvMLO15, MdMLO18, PpMLO9) and VIII (FvMLO13, MdMLO20 and PpMLO13) were included (Supplementary Table 2). The obtained UPGMA-comparative tree was then displayed as circular rooted cladogram with CLC software.

\section{Expression analysis of the SIMLO gene family in response to 0 . neolycopersici}

Tissue samples from the third and fourth true leaf of 1-month old tomato plants were collected immediately before fungal inoculation and at two time points after inoculation 
(6 and 10 hours). The RNA isolation was performed with MagMAX-96 Total RNA Isolation kit (Applied Biosystem), following the manufacturer's instructions. Included in the protocol is a DNase treatment using the TURBO ${ }^{\text {TM }}$ DNase. An aliquot of the RNA isolated was run on denaturing agarose gel to assess its integrity. Purity and concentration were determined by measuring its absorbance at $260 \mathrm{~nm}$ and $280 \mathrm{~nm}$ using the NanoDrop ${ }^{\circledR}$ $1000 \mathrm{~A}$ Spectrophotometer. Following this protocol for RNA isolation, intact and pure RNA was obtained and the concentration was variable between $200 \mathrm{ng} / \mu \mathrm{l}$ and $250 \mathrm{ng} / \mu \mathrm{l}$.

cDNAs were synthesized by using the SuperScript III first-strand synthesis kit (Invitrogen) using the oligo(dT) ${ }_{20}$ primer, starting from the same amount of RNA (200ng/ $\mu$ l). Specific primer pairs for each of the 16 SIMLO homologs, amplifying fragments ranging from $70 \mathrm{bp}$ to $230 \mathrm{bp}$, were designed as described above (Supplementary Table 3). The amplification of single fragments of the expected size for each homolog was verified by agarose gel electrophoresis and by the observation of the melting pick. Four tomato reference genes were tested for expression stability in order to determine which ones could be suitable for normalization of the expression of SIMLO homologs. These include the 605 ribosomal protein L33 (GeneBank number Q2MI79), the elongation factor 1a (GeneBank number X14449), actin (GeneBank XP_004236747) and ubiquitin (GeBank number XP_004248311) (Schijlen et al., 2007),(Løvdal and Lillo, 2009). Gene expression stability was assayed with the BestKeeper program (Pfaffl et al., 2004), determining as best reference genes the ribosomal protein $\mathrm{L} 33$ and the elongation factor $1 \mathrm{a}$. The cDNAs were diluted 10fold and used in real-time qPCR with a Bio-Rad CFX96TM thermal cycler. The thermal cycling conditions used were $95^{\circ} \mathrm{C}$ for $1 \mathrm{~min}$, followed by 40 cycles at $95^{\circ} \mathrm{C}$ for $15 \mathrm{~s}, 60^{\circ} \mathrm{C}$ for $1 \mathrm{~min}$ and $72^{\circ} \mathrm{C}$ for $30 \mathrm{~s}$, followed by a melt cycle of $0.5^{\circ} \mathrm{C}$ increment per min from 65 to $95^{\circ} \mathrm{C}$. Comparable amplification efficiencies between target and reference genes were determined using the LinRegPCR software (Karlen et al., 2007). Normalization was performed according to the $\Delta \Delta C_{t}$ method (Livak and Schmittgen, 2001). Four biological replicates and two technical replicates were used in this experiment. Student's t-tests were applied in order to assess significant differences between the treatments.

\section{SIMLO family expression analysis in different tissues}

To analyze $M L O$ gene expression in leaf, root, flower and ripened fruit approximately equal amount of tissues from five MM plants were pooled and used for RNA isolation and CDNA synthesis as described in the previous paragraph. Before using them as templates, cDNAs were diluted 10-fold. Real-time qPCR was performed using the set of primers reported in Supplementary Table 3 to amplify each homolog in the four tissues above mentioned. Elongation factor 1 a was used as reference gene. Data analysis was performed according to the $\Delta C_{t}$ method (Livak and Schmittgen, 2001). Three technical replicates for each sample were performed. 


\section{Generation of RNAi silencing lines}

Four primer pairs were designed to amplify and clone fragments from SIMLO1, SIMLO3, SIMLO5 and SIMLO8 into the Gateway-compatible vector PENTR D-TOPO (Invitrogen) (Supplementary Table 3). The cloned sequences of the SIMLO1, SIMLO3, SIMLO5 and SIMLO8 genes are highlighted in Supplementary Fig. 6. After cloning in E. coli (strain DH5a), the kanamycin-resistant colonies were assessed for the presence of constructs by colony PCR. Positive recombinant plasmids were further analyzed by restriction enzyme digestion and sequencing. Next, amplicons were transferred by LR recombination reaction into the pHELLSGATE12 vector for hairpin-induced RNAi (Wielopolska et al., 2005) following the instructions provided by the manufacturer (Invitrogen), and cloned again in E. coli DH5a. Bacterial colonies growing on a spectinomycin-containing medium were selected for the presence of the silencing construct by colony PCR and sequencing. Recombinant plasmids were transferred into the AGL1+virG strain of Agrobacterium tumefaciens (Lazo et al., 1991) by electroporation, and transformed bacterial cells were selected on a medium containing $100 \mathrm{mg} / \mathrm{ml}^{-1}$ spectinomycin, $50 \mathrm{mg} / \mathrm{ml}^{-1}$ carbenicillin and $50 \mathrm{mg} / \mathrm{ml}^{-1}$ chloramphenicol. Single colonies of $A$. tumefaciens were picked and the presence of the insert was confirmed by colony PCR. Ten-fold dilutions of overnight culture from single positive colonies were re-suspended in MSO medium ( $4.3 \mathrm{~g} / \mathrm{l} \mathrm{MS}$ basal salt mixture, $30 \mathrm{~g} / \mathrm{l}$ sucrose, $0.4 \mathrm{mg} / \mathrm{l}$ thiamine, $100 \mathrm{mg} / \mathrm{l}$ myoinositol, $\mathrm{pH} 5.8$ ) to a final $\mathrm{OD}_{600}$ of 0.5 and used for transformation.

The transformation procedure for tomato cotyledons was carried out similarly to the method described by (Appiano et al., 2015).

Silencing efficiency was assessed, for each of the 4 constructs, on 10 to $20 \mathrm{~T}_{1}$ plants and on selected $\mathrm{T}_{2}$ lines by real-time $\mathrm{qPCR}$, as described for the analysis of the SIMLO gene family expression in response to $O$. neolycopersici. In addition, the $\mathrm{T}_{2}$ lines were assessed for the presence of the nptll marker gene and the 355 promoter by $P C R$, using the primer pair NPTII Fw (5'ACTGGGCACAACAGACAATC3')/ NPTII_Rev (5' TCGTCCTGCAGTTCATTCAG 3') and 35S-Fw (5'-GCTCCTACAAATGCCATCA-3') / 35S-Rev (5'- GATAGTGGGATTGTGCGTCA-3'), and visualizing the product on agarose gel.

\section{Disease quantification on silenced lines}

$T_{2}$ lines originating from selfing of $T_{1}$ plants showing high level of silencing were inoculated with $O$. neolycopersici (On) by spraying four weeks old plants with a suspension of conidiospores obtained from freshly sporulating leaves of heavily infected plants and adjusted to a final concentration of $4 \times 10^{4}$ spores $/ \mathrm{ml}$. Inoculated plants were grown in a greenhouse compartment at $20 \pm 2^{\circ} \mathrm{C}$ with $70 \pm 15 \%$ relative humidity and day length of 16 hours. Two weeks later, infected tissues from the third and fourth true leaf were visually scored and sampled. Plant and fungal DNAs were extracted by using the 
DNeasy DNA extraction kit (Qiagen). In total, $15 \mathrm{ng}$ of DNA was used as template for amplification with the primer pair On-Fw (5'-CGCCAAAGACCTAACCAAAA-3') and OnRev (5'-AGCCAAGAGATCCGTTGTTG-3'), designed on On-specific internal transcribed spacer sequences (GenBank accession number EU047564). The tomato Ef1a primers (Supplementary Table 3) were used as reference to determine fungal biomass relative to host plant DNA by $\Delta \Delta C_{t}$ method.

\section{Disease tests for microscopic evaluation in histological study}

Spores of the Wageningen isolate of $O$. neolycopersici grown in a climate chamber at $20 \pm 1^{\circ} \mathrm{C}$, with $70 \pm 10 \% \mathrm{RH}$ and a 16 -h photoperiod were water-sprayed on the third leaf of 1-month old tomato plants of the susceptible tomato cv. MM, the resistant line SImlo1 and transgenic plants of one $\mathrm{T}_{2}$ family selected by PCR for the presence of the NPTII and $35 \mathrm{~S}$ marker genes of the RNAi::SIMLO1 silencing construct. The concentration of the spore suspension was $3 \times 10^{5}$ conidia $\mathrm{ml}^{-1}$. After 65 hours, a $4 \mathrm{~cm}^{2}$ segment was cut from the inoculated leaves. Three samples were taken from four plants of each genotype and from 5 plants of the $T_{2}$ family, bleached in a 1:3 (v/v) acetic acid/ethanol solution and 48 hrs later stained in $0.005 \%$ trypan blue as described by (Pavan et al., 2008). For each genotype, a total of 90 infection units (IU), defined as a germinated spore that produced, at least, a primary appressorium, were counted. Observations were performed using a Zeiss Axiophot bright field microscope and pictures were taken with an Axiocam ERc5s. For each IU, the number of hyphae, the presence/absence of a primary and secondary haustoria and presence/absence of papillae were recorded. 


\section{References}

Acevedo-Garcia, J., Kusch, S., and Panstruga, R. (2014). Magical mystery tour: MLO proteins in plant immunity and beyond. New Phytologist 204, 273-281.

Appiano, M., Pavan, S., Catalano, D., Zheng, Z., Bracuto, V., Lotti, C., Visser, R.F., Ricciardi, L., and Bai, Y. (2015). Identification of candidate MLO powdery mildew susceptibility genes in cultivated Solanaceae and functional characterization of tobacco NtMLO1. Transgenic Research, 24:847-858.

Bai, Y., Pavan, S., Zheng, Z., Zappel, N.F., Reinstädler, A., Lotti, C., De Giovanni, C., Ricciardi, L., Lindhout, P., Visser, R., Theres, K., and Panstruga, R. (2008). Naturally occurring broad-spectrum powdery mildew resistance in a Central American tomato accession is caused by loss of Mlo function. Molecular Plant-Microbe Interactions 21, 30-39.

Bai, Y., Van Der Hulst, R., Bonnema, G., Marcel, T.C., Meijer-Dekens, F., Niks, R.E., and Lindhout, P. (2005). Tomato Defense to Oidium neolycopersici: Dominant OI Genes Confer Isolate-Dependent Resistance Via a Different Mechanism Than Recessive ol-2. Molecular Plant-Microbe Interactions 18, 354-362.

Bailey, T.L., Johnson, J., Grant, C.E., and Noble, W.S. (2015). The MEME Suite. Nucleic Acids Research, 1. doi: 10.1093/nar/gkv416

Berg, J., Appiano, M., Santillan Martinez, M., Hermans, F., Vriezen, W., Visser, R., Bai, Y., and Schouten, H. (2015). A transposable element insertion in the susceptibility gene CsaMLO8 results in hypocotyl resistance to powdery mildew in cucumber. BMC Plant Biology 15, 243.

Büschges, R., Hollricher, K., Panstruga, R., Simons, G., Wolter, M., Frijters, A., Van Daelen, R., Van Der Lee, T., Diergaarde, P., Groenendijk, J., Töpsch, S., Vos, P., Salamini, F., and Schulze-Lefert, P. (1997). The barley Mlo gene: A novel control element of plant pathogen resistance. Cell 88, 695-705.

Chen, Z., Hartmann, H.A., Wu, M.J., Friedman, E.J., Chen, J.G., Pulley, M., Schulze-Lefert, P., Panstruga, R., and Jones, A.M. (2006). Expression analysis of the AtMLO gene family encoding plant-specific seventransmembrane domain proteins. Plant Molecular Biology 60, 583-597.

Chen, Z., Noir, S., Kwaaitaal, M., Hartmann, H.A., Wu, M.J., Mudgil, Y., Sukumar, P., Muday, G., Panstruga, R., and Jones, A.M. (2009). Two seven-transmembrane domain MILDEW RESISTANCE LOCUS O proteins cofunction in arabidopsis root thigmomorphogenesis. Plant Cell 21, 1972-1991.

Consonni, C., Humphry, M.E., Hartmann, H.A., Livaja, M., Durner, J., Westphal, L., Vogel, J., Lipka, V., Kemmerling, B., Schulze-Lefert, P., Somerville, S.C., and Panstruga, R. (2006). Conserved requirement for a plant host cell protein in powdery mildew pathogenesis. Nature Genetics 38, 716-720.

Devoto, A., Hartmann, H.A., Piffanelli, P., Elliott, C., Simmons, C., Taramino, G., Goh, C.S., Cohen, F.E., Emerson, B.C., Schulze-Lefert, P., and Panstruga, R. (2003). Molecular phylogeny and evolution of the plant-specific seven-transmembrane MLO family. Journal of Molecular Evolution 56, 77-88.

Devoto, A., Piffanelli, P., Nilsson, I., Wallin, E., Panstruga, R., Von Heijne, G., and Schulze-Lefert, P. (1999). Topology, subcellular localization, and sequence diversity of the Mlo family in plants. Journal of Biological Chemistry 274, 34993-35004.

Elliott, C., Müller, J., Miklis, M., Bhat, R.A., Schulze-Lefert, P., and Panstruga, R. (2005). Conserved extracellular cysteine residues and cytoplasmic loop-loop interplay are required for functionality of the heptahelical MLO protein. Biochemical Journal 385, 243-254.

Elliott, C., Zhou, F., Spielmeyer, W., Panstruga, R., and Schulze-Lefert, P. (2002). Functional conservation of wheat and rice Mlo orthologs in defense modulation to the powdery mildew fungus. Molecular Plant-Microbe Interactions 15, 1069-1077.

Feechan, A., Jermakow, A.M., Torregrosa, L., Panstruga, R., and Dry, I.B. (2008). Identification of grapevine $\mathbf{M L O}$ gene candidates involved in susceptibility to powdery mildew. Functional Plant Biology 35, 12551266.

Feechan, A., Jermakow, A. M., Ivancevic, A., Godfrey, D., Pak, H., Panstruga, R., et al. (2013) Host cell entry of powdery mildew is correlated with endosomal transport of antagonistically acting VvPEN1 and VvMLO to the papilla. Molecular Plant-Microbe Interactions, 26, 1138-1150. 
Humphry, M., Reinstädler, A., Ivanov, S., Bisseling, T., and Panstruga, R. (2011). Durable broad-spectrum powdery mildew resistance in pea er 1 plants is conferred by natural loss-of-function mutations in PsMLO1. Molecular Plant Pathology 12, 866-878.

Jarosch, B., Kogel, K,H., Schaffrath, U. (1999). The ambivalence of the barley Mlo locus: Mutations conferring resistance against powdery mildew (Blumeria graminis f. sp. hordei) enhance susceptibility to the rice blast fungus Magnaporthe grisea. Molecular Plant-Microbe Interactions 12:508-514.

Jørgensen, I.H. (1992). Discovery, characterization and exploitation of Mlo powdery mildew resistance in barley. Euphytica 63, 141-152.

Karlen, Y., Mcnair, A., Perseguers, S., Mazza, C., and Mermod, N. (2007). Statistical significance of quantitative PCR. BMC Bioinformatics 8:131.

Keren, H., Lev-Maor, G., and Ast, G. (2010). Alternative splicing and evolution: diversification, exon definition and function. Nat Rev Genet 11, 345-355.

Kessler, S.A., Shimosato-Asano, H., Keinath, N.F., Wuest, S.E., Ingram, G., Panstruga, R., and Grossniklaus, U. (2010). Conserved molecular components for pollen tube reception and fungal invasion. Science 330, 968-971.

Kim, M.C., Panstruga, R., Elliott, C., Muller, J., Devoto, A., Yoon, H.W., Park, H.C., Cho, M.J., and Schulze-Lefert, P. (2002). Calmodulin interacts with MLO protein to regulate defence against mildew in barley. Nature 416, 447-451.

Kumar, J., Hückelhoven, R., Beckhove. U, Nagarajan, S., Kogel KH. (2001) A compromised Mlo pathway affects the response of barley to the necrotrophic fungus Bipolaris sorokiniana (Teleomorph: Cochliobolus sativus) and its toxins. Phytopathology. 91:127-133.

Lazo, G.R., Stein, P.A., and Ludwig, R.A. (1991). A DNA transformation-competent Arabidopsis genomic library in Agrobacterium. Nature Biotechnology 9, 963-967.

Lescot, M., Déhais, P., Thijs, G., Marchal, K., Moreau, Y., Van De Peer, Y., Rouzé, P., and Rombauts, S. (2002). PlantCARE, a database of plant cis-acting regulatory elements and a portal to tools for in silico analysis of promoter sequences. Nucleic acids research 30, 325-327.

Liu, Q., and Zhu, H. (2008). Molecular evolution of the MLO gene family in Oryza sativa and their functional divergence. Gene 409, 1-10.

Livak, K.J., and Schmittgen, T.D. (2001). Analysis of relative gene expression data using real-time quantitative PCR and the $2-\triangle \Delta C T$ method. Methods 25, 402-408.

Lorek, J., Griebel, T., Jones, A.M., Kuhn, H., and Panstruga, R. (2013). The Role of Arabidopsis Heterotrimeric G-Protein Subunits in MLO2 Function and MAMP-Triggered Immunity. Molecular Plant-Microbe Interactions 26, 991-1003.

Løvdal, T., and Lillo, C. (2009). Reference gene selection for quantitative real-time PCR normalization in tomato subjected to nitrogen, cold, and light stress. Analytical Biochemistry 387, $238-242$.

Miklis, M., Consonni, C., Bhat, R.A., Lipka, V., Schulze-Lefert, P., and Panstruga, R. (2007). Barley MLO Modulates Actin-Dependent and Actin-Independent Antifungal Defense Pathways at the Cell Periphery. Plant Physiology 144, 1132-1143.

Ner-Gaon, H., Leviatan, N., Rubin, E., and Fluhr, R. (2007). Comparative Cross-Species Alternative Splicing in Plants. Plant Physiology 144, 1632-1641.

Opalski, K.S., Schultheiss, H., Kogel, K.H., and Hückelhoven, R. (2005). The receptor-like MLO protein and the RAC/ROP family G-protein RACB modulate actin reorganization in barley attacked by the biotrophic powdery mildew fungus Blumeria graminis f.sp. hordei. Plant Journal 41, 291-303.

Palusa, S.G., Ali, G.S., and Reddy, A.S.N. (2007). Alternative splicing of pre-mRNAs of Arabidopsis serine/ arginine-rich proteins: regulation by hormones and stresses. The Plant Journal 49, 1091-1107.

Panstruga, R. (2005). Serpentine plant MLO proteins as entry portals for powdery mildew fungi. Biochemical Society Transactions 33, 389-392.

Panstruga, R., and Schulze-Lefert, P. (2003). Corruption of host seven-transmembrane proteins by pathogenic microbes: a common theme in animals and plants? Microbes and Infection 5, 429-437. 
Pavan, S., Jacobsen, E., Visser, R.G.F., and Bai, Y. (2009). Loss of susceptibility as a novel breeding strategy for durable and broad-spectrum resistance. Molecular Breeding 25, 1-12.

Pavan, S., Zheng, Z., Borisova, M., Van Den Berg, P., Lotti, C., De Giovanni, C., Lindhout, P., De Jong, H., Ricciardi, L., Visser, R.G.F., and Bai, Y. (2008). Map- vs. homology-based cloning for the recessive gene ol-2 conferring resistance to tomato powdery mildew. Euphytica 162, 91-98.

Pessina, S., Pavan, S., Catalano, D., Gallotta, A., Visser, R., Bai, Y., Malnoy, M., and Schouten, H. (2014). Characterization of the MLO gene family in Rosaceae and gene expression analysis in Malus domestica. BMC Genomics 15, 618.

Pfaffl, M.W., Tichopad, A., Prgomet, C., and Neuvians, T.P. (2004). Determination of stable housekeeping genes, differentially regulated target genes and sample integrity: BestKeeper - Excel-based tool using pair-wise correlations. Biotechnology Letters 26, 509-515.

Reinstädler, A., Müller, J., Czembor, J.H., Piffanelli, P., and Panstruga, R. (2010). Novel induced mlo mutant alleles in combination with site-directed mutagenesis reveal functionally important domains in the heptahelical barley Mlo protein. BMC Plant Biology 10.

Rozen, S., and Skaletsky, H. (2000). Primer3 on the WWW for general users and for biologist programmers. Methods in molecular biology (Clifton, N.J.) 132, 365-386.

Schijlen, E.G.W.M., De Vos, C.H.R., Martens, S., Jonker, H.H., Rosin, F.M., Molthoff, J.W., Tikunov, Y.M., Angenent, G.C., Van Tunen, A.J., and Bovy, A.G. (2007). RNA interference silencing of chalcone synthase, the first step in the flavonoid biosynthesis pathway, leads to parthenocarpic tomato fruits. Plant Physiology 144, 1520-1530.

Schouten, H., Krauskopf, J., Visser, R.F., and Bai, Y. (2014). Identification of candidate genes required for susceptibility to powdery or downy mildew in cucumber. Euphytica 200, 475-486.

Seifi, A., Gao, D., Zheng, Z., Pavan, S., Faino, L., Visser, R.F., Wolters, A.-M., and Bai, Y. (2014). Genetics and molecular mechanisms of resistance to powdery mildews in tomato (Solanum lycopersicum) and its wild relatives. European Journal of Plant Pathology 138, 641-665.

Shen, Q., Zhao, J., Du, C., Xiang, Y., Cao, J., and Qin, X. (2012). Genome-scale identification of MLO domaincontaining genes in soybean (Glycine max L. Merr.). Genes and Genetic Systems 87, 89-98.

The 100 Tomato Genome Sequencing Consortium, C., Aflitos, S., Schijlen, E., De Jong, H., De Ridder, D., Smit, S., Finkers, R., Wang, J., Zhang, G., Li, N., Mao, L., Bakker, F., Dirks, R., Breit, T., Gravendeel, B., Huits, H., Struss, D., Swanson-Wagner, R., Van Leeuwen, H., Van Ham, R.C.H.J., Fito, L., Guignier, L., Sevilla, M., Ellul, P., Ganko, E., Kapur, A., Reclus, E., De Geus, B., Van De Geest, H., Te Lintel Hekkert, B., Van Haarst, J., Smits, L., Koops, A., Sanchez-Perez, G., Van Heusden, A.W., Visser, R., Quan, Z., Min, J., Liao, L., Wang, X., Wang, G., Yue, Z., Yang, X., Xu, N., Schranz, E., Smets, E., Vos, R., Rauwerda, J., Ursem, R., Schuit, C., Kerns, M., Van Den Berg, J., Vriezen, W., Janssen, A., Datema, E., Jahrman, T., Moquet, F., Bonnet, J., and Peters, S. (2014). Exploring genetic variation in the tomato (Solanum section Lycopersicon) clade by wholegenome sequencing. The Plant Journal 80, 136-148.

Tomato Genome Consortium. (2012). The tomato genome sequence provides insights into fleshy fruit evolution. Nature 485, 635-641.

Van Schie, C.C.N., and Takken, F.L.W. (2014). Susceptibility Genes 101: How to Be a Good Host. Annual Review of Phytopathology 52, 551-581.

Várallyay, É., Giczey, G., and Burgyán, J. (2012). Virus-induced gene silencing of Mlo genes induces powdery mildew resistance in Triticum aestivum. Archives of Virology 157, 1345-1350.

Wielopolska, A., Townley, H., Moore, I., Waterhouse, P., and Helliwell, C. (2005). A high-throughput inducible RNAi vector for plants. Plant Biotechnology Journal 3, 583-590.

Zheng, Z., Nonomura, T., Appiano, M., Pavan, S., Matsuda, Y., Toyoda, H., Wolters, A.M.A., Visser, R.G.F., and Bai, Y. (2013). Loss of Function in Mlo Orthologs Reduces Susceptibility of Pepper and Tomato to Powdery Mildew Disease Caused by Leveillula taurica. PLOS ONE 8.

Zhou, S.J., Jing, Z., and Shi, J.L. (2013). Genome-wide identification, characterization, and expression analysis of the MLO gene family in Cucumis sativus. Genetic and Molecular Research 12, 6565-6578. 


\section{Supplementary material}

SUPPLEMENTARY FIGURE 1. Sequence alignments of SIMLO homologs cloned from different tissues of the tomato $\mathrm{Cv}$. Moneymaker showing deviating transcripts when compared with the predicted CDS sequence, as inferred by the SGN database, against their respective genomic regions. Deviating transcripts are identified for SIMLO1 in flower, SIMLO5 in fruit, SIMLO6, SIMLO7, SIMLO8 and SIMLO9 in leaf, SIMLO10 in fruit, SIMLO11 in root, SIMLO13 in leaf and SIMLO15 in all the tissues investigated. Alignments were obtained with default parameters by the CLC sequence viewer software. Due to its size, this figure is only available online at http://journal.frontiersin. org/article/10.3389/fpls.2016.00380

SUPPLEMENTARY FIGURE 2. Sequence alignment of a set of $37 \mathrm{MLO}$ proteins cloned from four tissues (leaf, root, flower and fruit) of the $\mathrm{cv}$. Moneymaker. When no deviating transcripts were detected, the translated nucleotide sequence of leaf was used in the alignment, with the exception of SIMLO12 which was cloned only from flower. Shading was obtained by feeding the alignment, obtained with default parameters by the CLC sequence viewer software, to the online server Boxshade (http://sourceforge.net/projects/boxshade/) and setting as 0.7 the fraction of sequences that must agree for shading. In black shade the amino acids fully conserved, in grey the amino acids substitutions which are predicted to have similar biochemical properties and in red the amino acids identified by Elliot et al., 2005 as invariable for the whole MLO protein family. Transmembrane domains are indicated as inferred by the TMHMM online server (http://www.cbs.dtu.dk/services/TMHMM/) fed with the SIMLO1 protein sequence of leaf. Due to its size, this figure is only available online at http://journal.frontiersin. org/article/10.3389/fpls.2016.00380 
SIMLO1

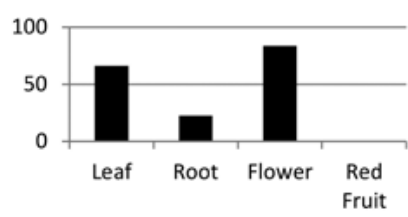

SIMLO4

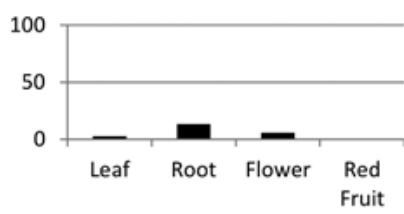

SIMLO7

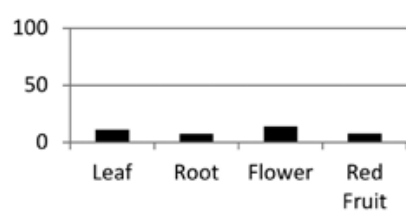

SIMLO10

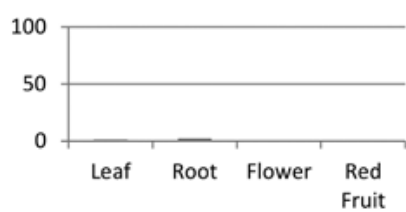

SIMLO13

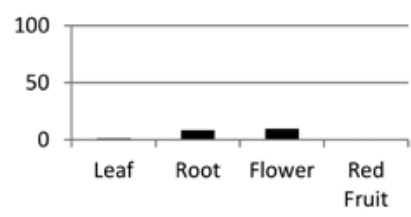

SIMLO16

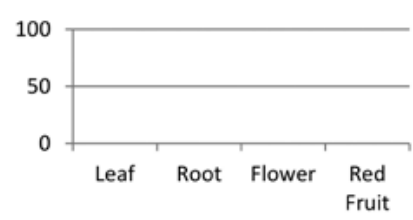

SIMLO2

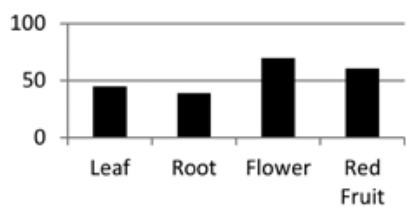

SIMLO5

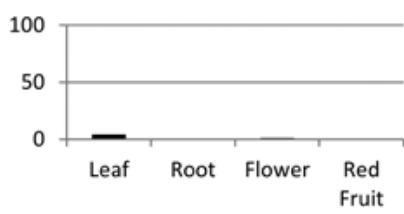

SIMLO8

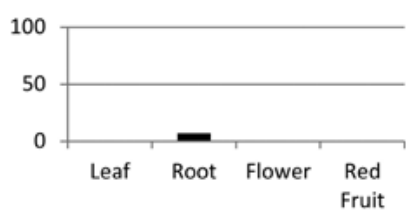

SIMLO11

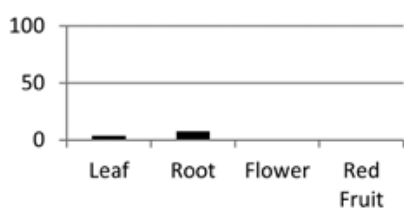

SIMLO14

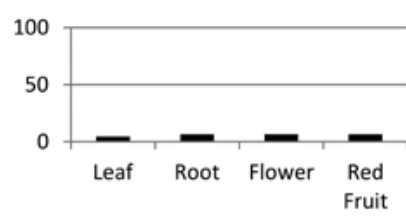

SIMLO3

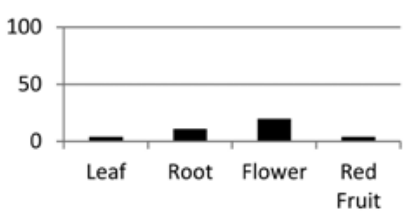

SIMLO6

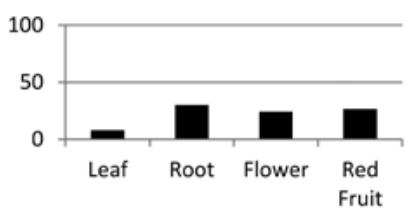

SIMLO9

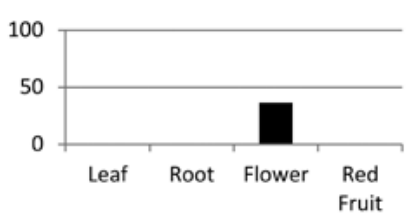

SIMLO12

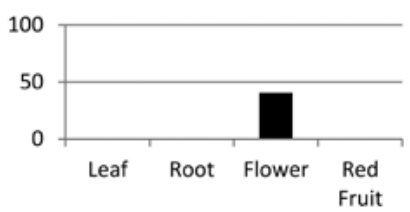

SIMLO15

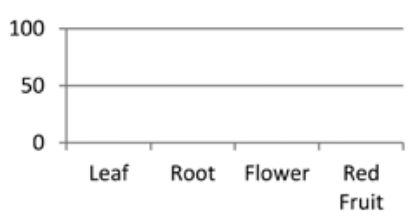

SUPPLEMENTARY FIGURE 3. FPKM values (fragments per kilobase of exon per million fragments mapped) depicted as columns for each of the four tissues investigated in this study obtained from the RNA-seq data of each of the 16 SIMLO homologs as reported by the SGN database. 


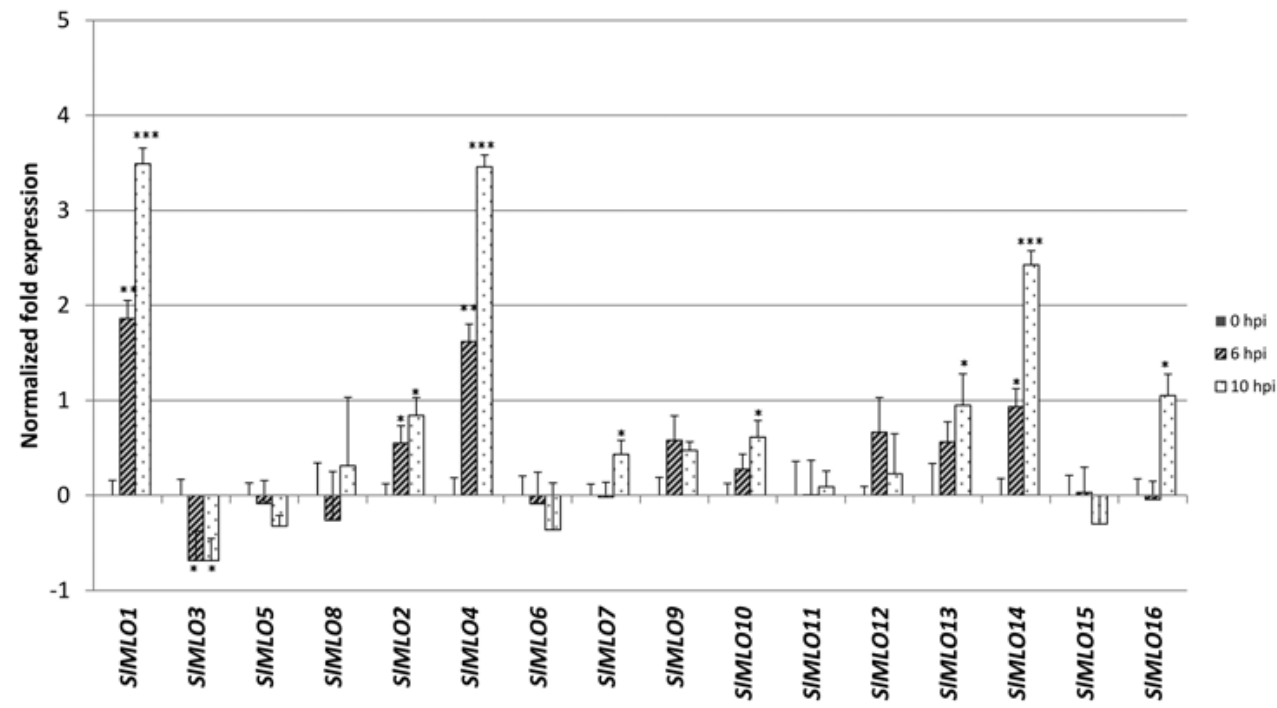

SUPPLEMENTARY FIGURE 4. Relative expression level of the SIMLO gene family members in response to $O$. neolycopersici infection. Samples were collected at 0,6 and $10 \mathrm{hrs}$ after inoculation (hpi). Transcript levels of each SIMLO homolog were normalized against the transcription level of Ef1a as reference gene. Bars show standard errors based on four biological replicates. Asterisks refer to significant differences with respect to non-inoculated plants (0 hpi), inferred by mean comparisons with a Student's t-test $\left.{ }^{*} p<.05,{ }^{* *} p<.01,{ }^{* * *} p<.001\right)$.

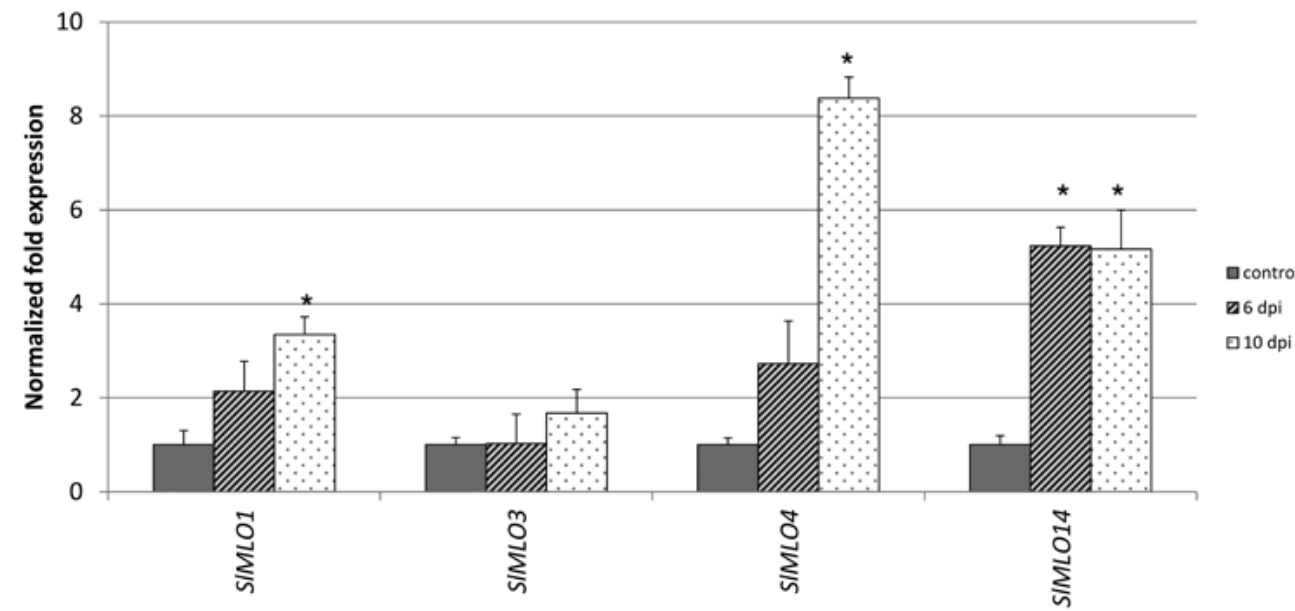

SUPPLEMENTARY FIGURE 5. Relative expression level of SIMLO1, SIMLO3, SIMLO4 and SIMLO14 compared to the reference gene Ef $1 \mathrm{a}$ upon inoculation with $O$. neolycopersici. Means are calculated from duplicate technical measurements. Bars show standard errors based on three biological replicates. Asterisks refer to significant differences with respect to non-inoculated plants used as control $(0 \mathrm{hpi})$, inferred by mean comparisons with a Student's t-test $\left({ }^{*} \mathrm{p}<.05\right)$. 


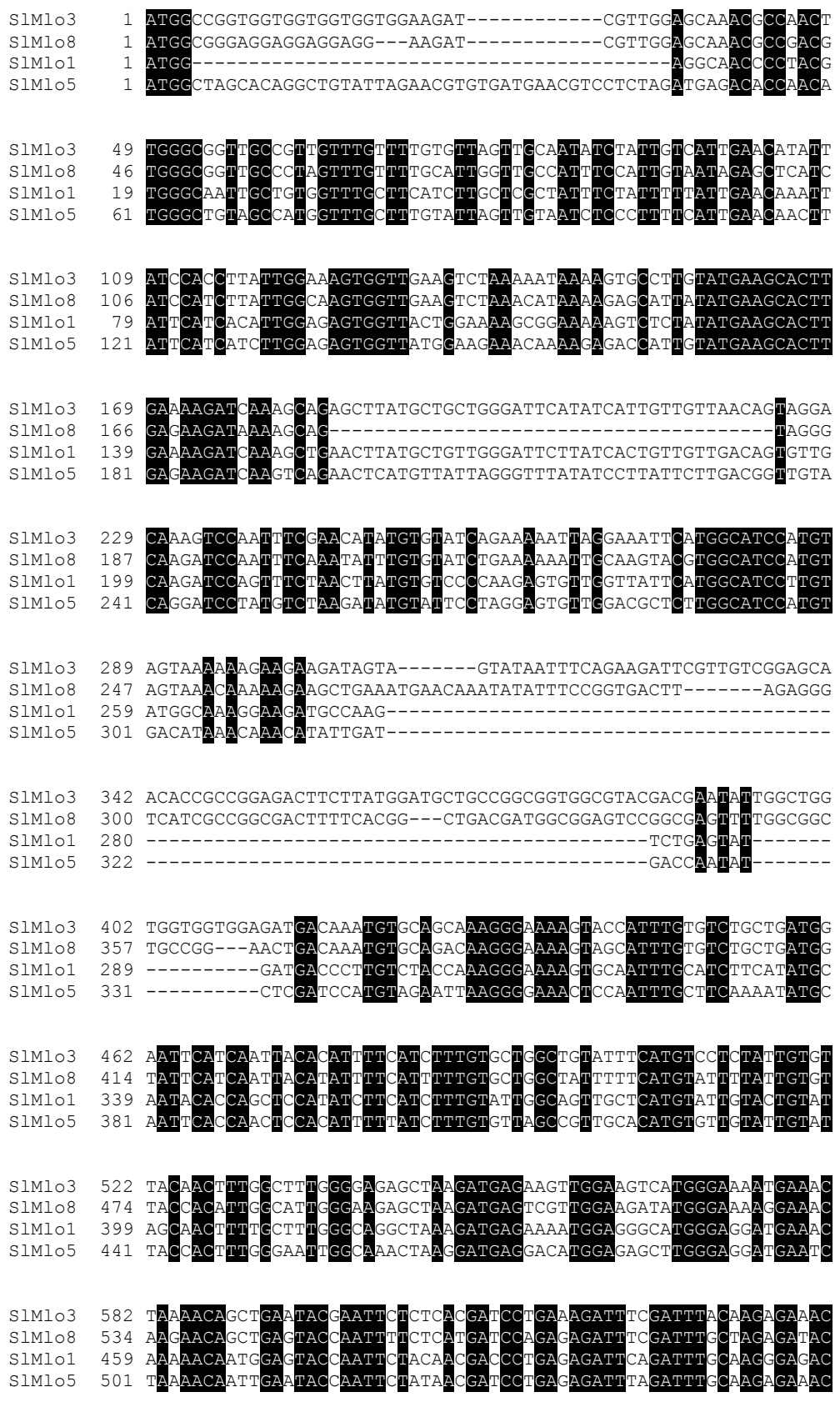




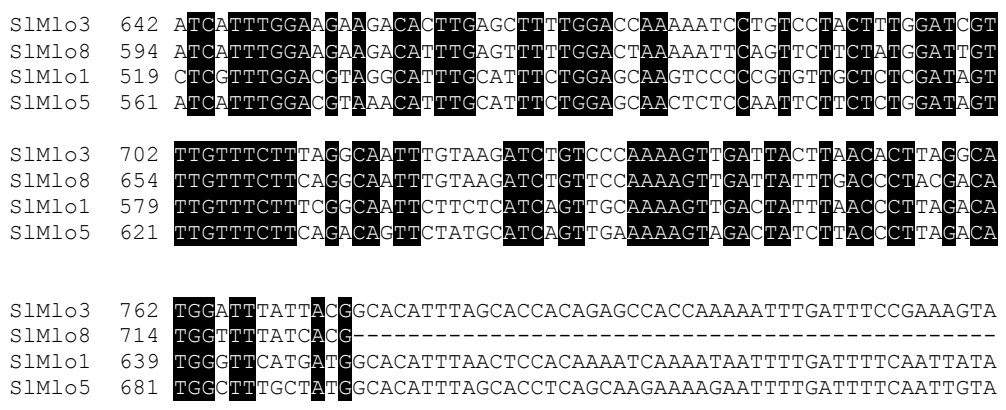

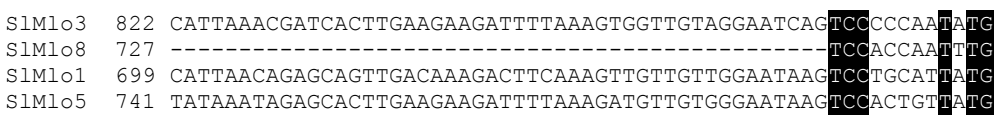

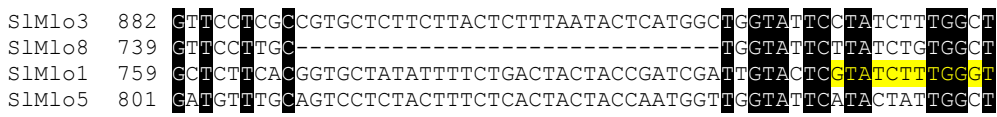

S1M103 942 ACCA TTCATCCCCT TGATTGTGATATTATTAGTAGGGACAAAGCTACAAGTGATAATAAC S1M108 768 ACCGITCATTCCGTIACTTGTGATATTATTAATA GGGACTAAACTACAAGTGATTATAAC

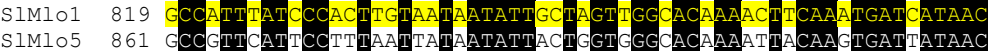

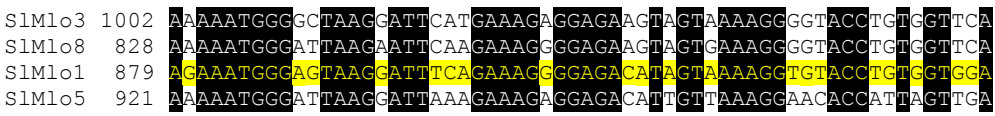

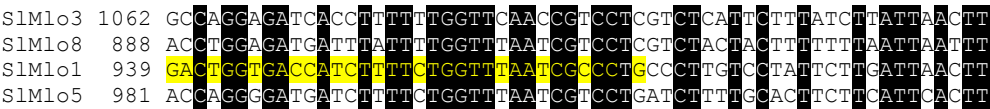

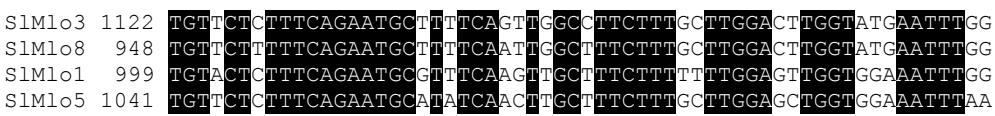

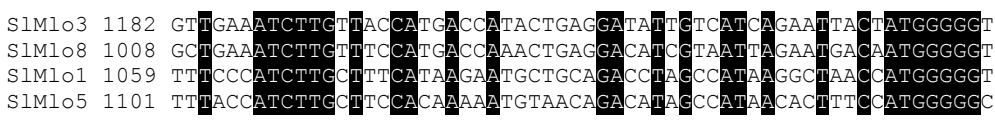

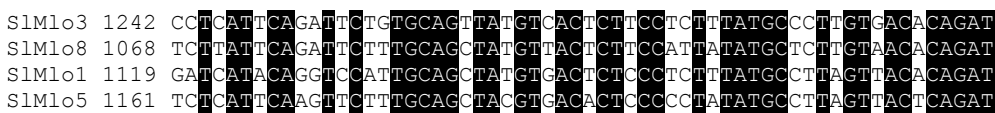



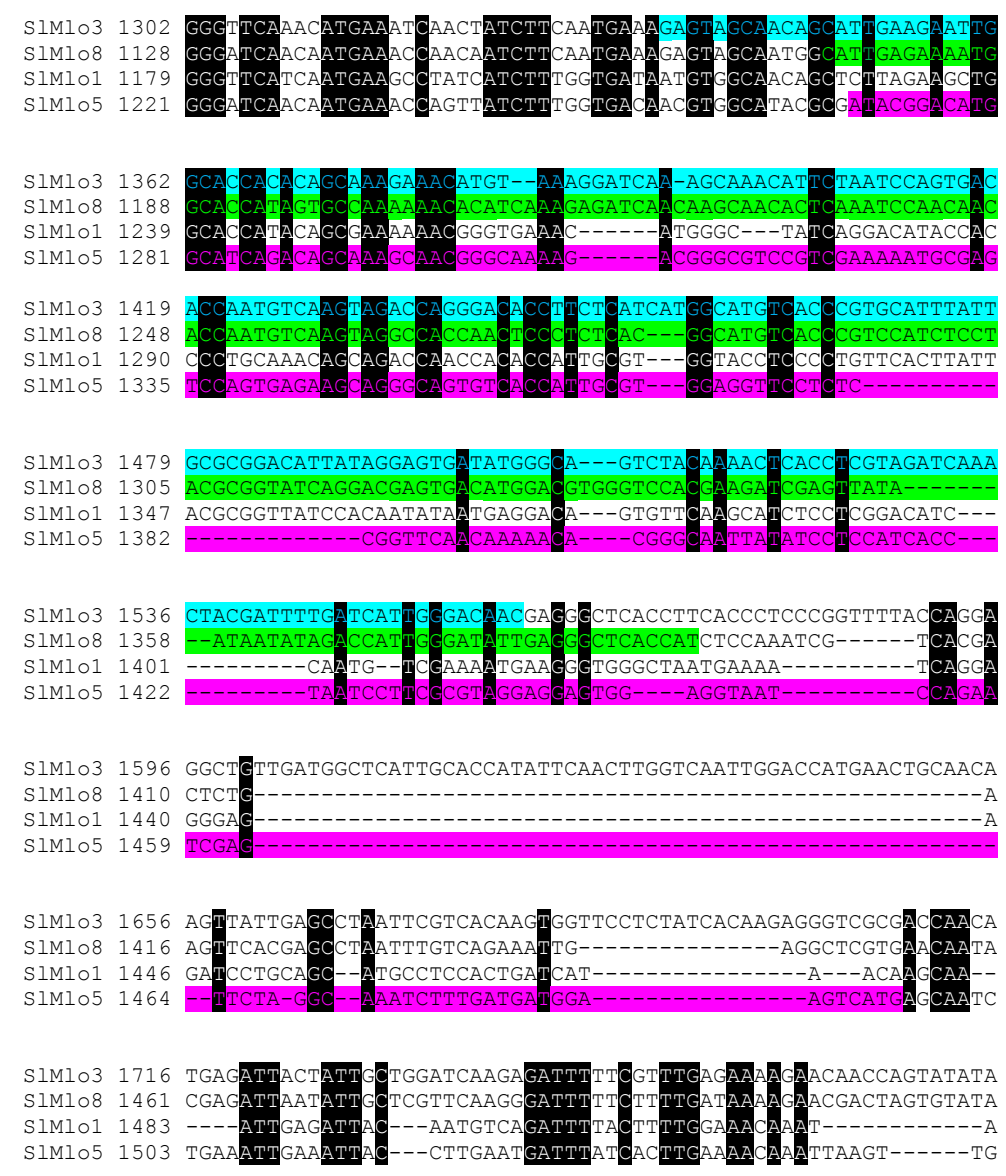

$\begin{array}{lll}\text { SlMlo3 } & 1776 & \mathrm{G} \\ \text { SlMlo8 } & 1521 & \mathrm{~A} \\ \text { SlMlo1 } & 1524 & \mathrm{~A} \\ \text { SlMlo5 } & 1554 & \mathrm{~A}\end{array}$

SUPPLEMENTARY FIGURE 6. Sequence alignment of the coding sequences of the four SIMLO homologs found in clade V, namely SIMLO1, SIMLO3, SIMLO5, and SIMLO8. The alignment was generated by CLC sequence view software using default parameter and given to BoxShade online server (http://sourceforge.net/projects/ boxshade/) to allow the shading. The fraction of sequences that must agree for shading was set as 1 . Black shade indicates nucleotides that are fully conserved among the four sequences. Regions of each homolog used for the RNAi construct are highlighted in yellow, light blue, green and pink for SIMLO1, SIMLO3, SIMLO8 and SIMLO5 respectively. Primer pairs used to prepare each RNAi construct are listed in Supplementary Table 3. 

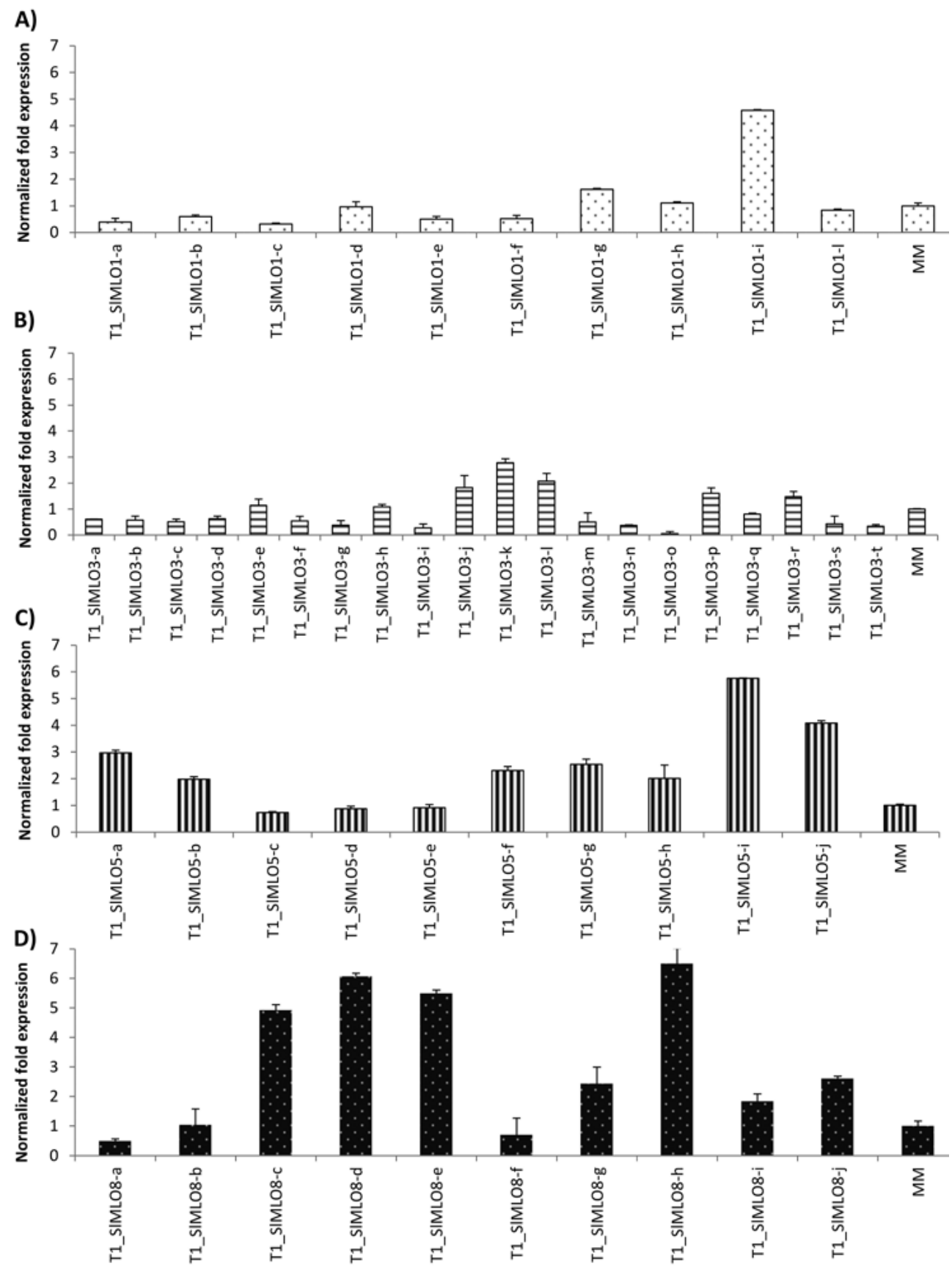

SUPPLEMENTARY FIGURE 7. Relative expression level of the target SIMLO gene in $\mathrm{T}_{1}$ plants obtained with the RNAi silencing construct for SIMLO1 (panel A), SIMLO3 (panel B), SIMLO5 (panel C) and SIMLO8 (panel D), respectively. Bars shows standard error of three technical replicates. 

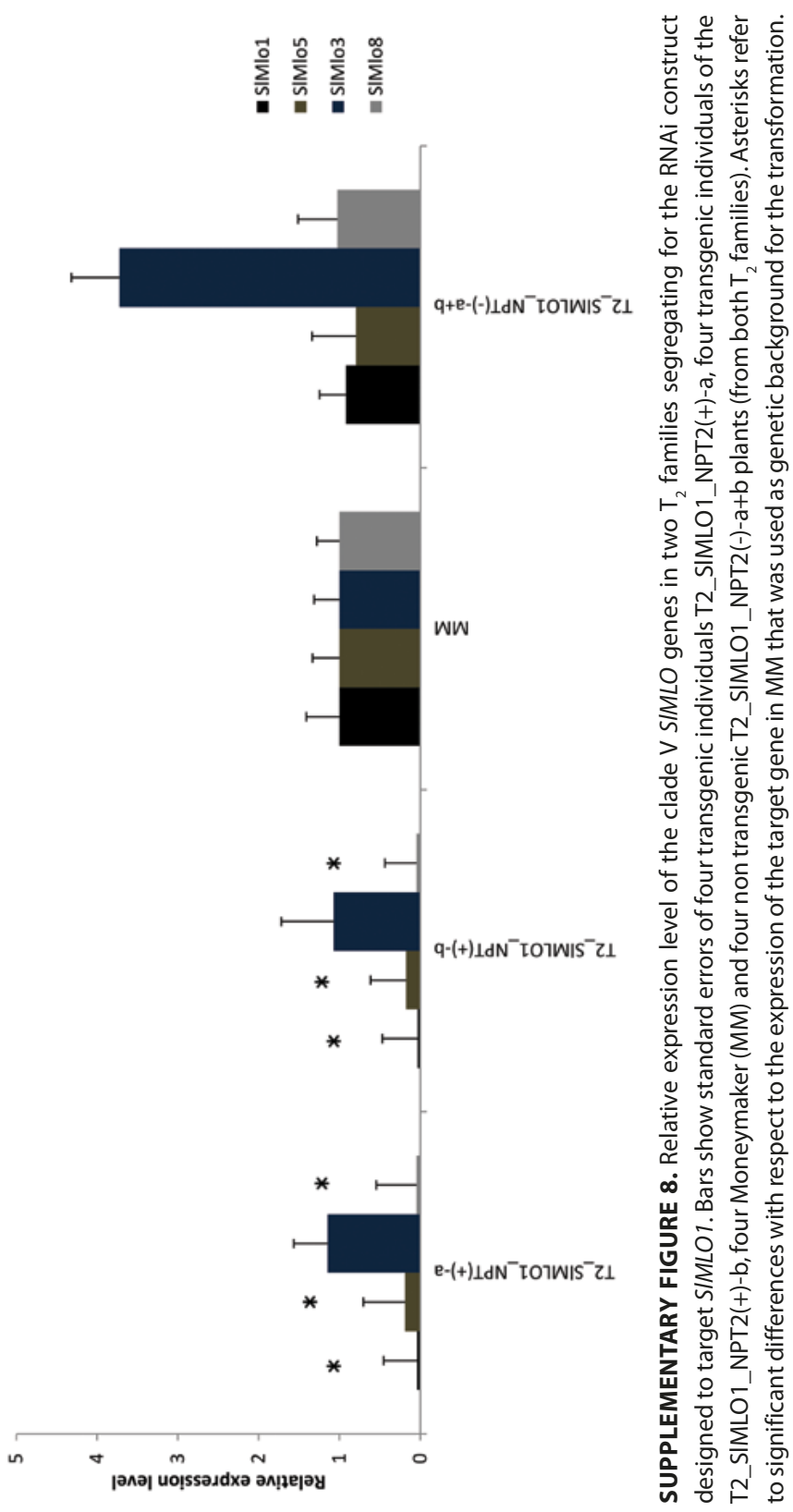
A)
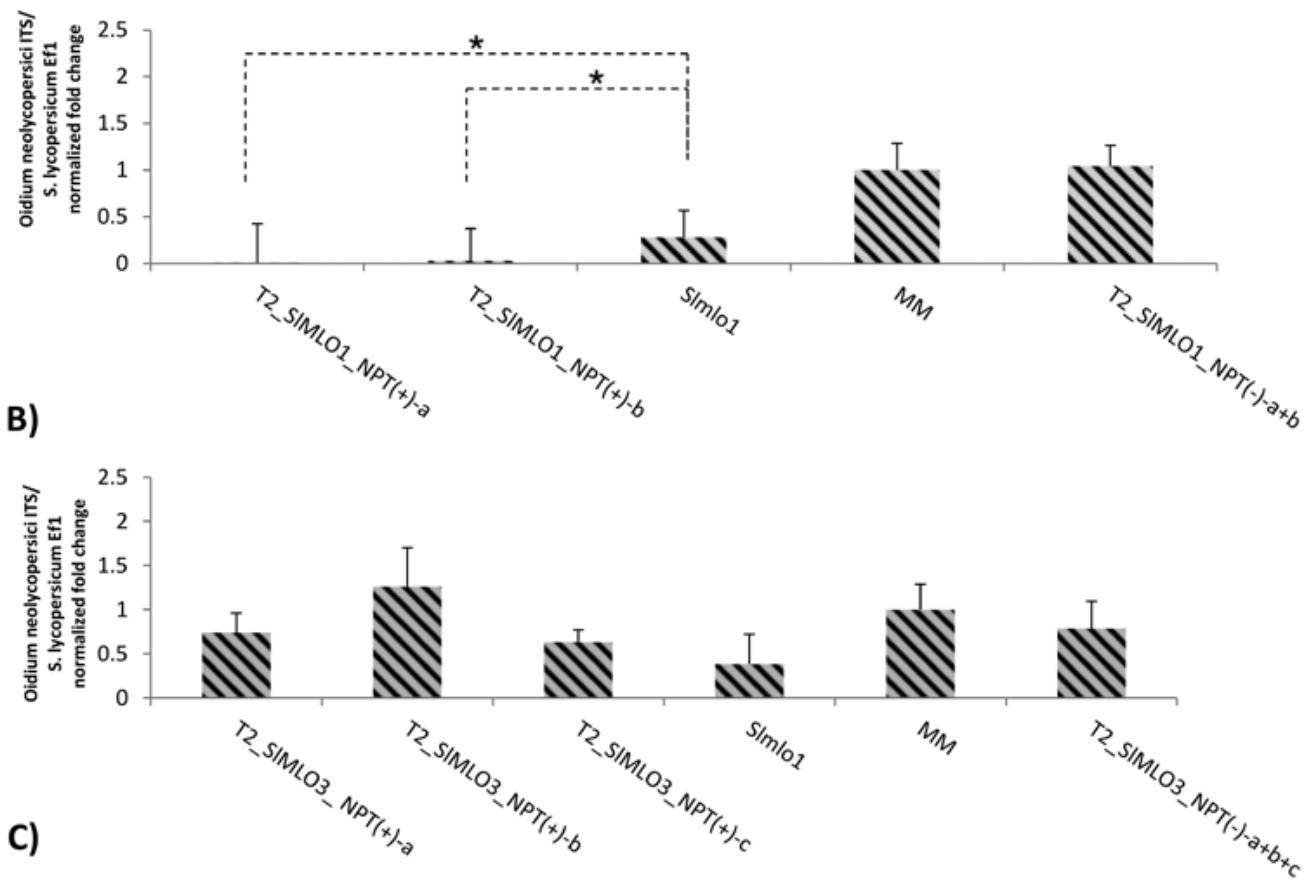

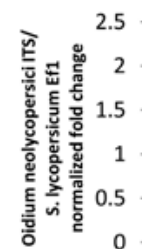
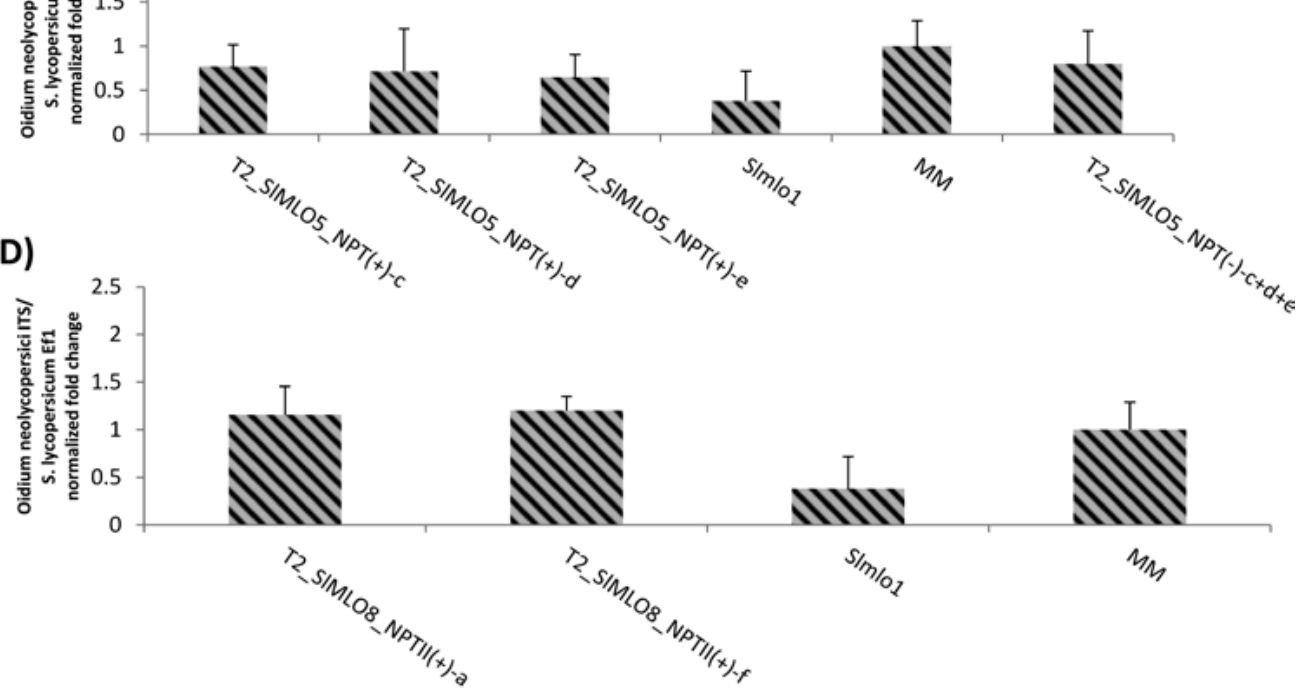
SUPPLEMENTARY FIGURE 9. Fungal biomass measured by relative quantification of the ratio between Oidium neolycopersici and plant gDNAs on independent $\mathrm{T}_{2}$ families obtained with the silencing constructs targeting SIMLO genes of clade V. Panel A and panel D refer to the two $T_{2}$ families tested in this study obtained with the constructs designed to silence SIMLO1 and SIMLO8, respectively. Panel $B$ and panel $C$ refer to the three $\mathrm{T}_{2}$ families obtained with the constructs designed to silence SIMLO3 and SIMLO5, respectively. The SIMLO gene name and extension " $a$ " to " $f$ " of the $T_{2}$ families refer to the ones in Supplementary Fig. 7.

For MM and SImlo line in each panel, bars show standard errors based on 10 plants. In panel A, bars show standard errors of four transgenic plants (NPT+) for each of the two $\mathrm{T}_{2}$ families (T2_SIMLO1-a and -b) and four plants that do not carry the silencing construct obtained from the two $\mathrm{T}_{2}$ families. Asterisks refer to significant differences compared to the SImlo1 line ( $P<0.01$; Student's t-test). In panel $B$, bars show standard errors of 10 transgenic plants of the T2_SIMLO3-a and -b families, eight plants of the T2_SIMLO3-c family and seven plants that do not carry the silencing construct obtained from the three families. In panel C, bars show standard errors of nine transgenic plants for each of the three $\mathrm{T}_{2}$ families (T2_SIMLO5-c, $-\mathrm{d}$ and $-\mathrm{e}$ ) and six plants that do not carry the silencing construct obtained from the three families. In panel D, bars show standard errors of 10 transgenic plants for each of the two $T_{2}$ families (T2_SIMLO8-a and -f). For these two $T_{2}$ families, we did not find plants without the NPTII marker. 



\section{Chapter 3}

\section{Functional characterization of a tomato syntaxin involved in powdery mildew resistance}

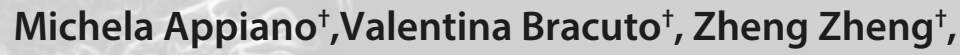
Anne-Marie A. Wolters, Zhe Yan, Luigi Ricciardi, Richard G. F. Visser, Stefano Pavan, Yuling Bai* ${ }^{t}$ these authors contributed equally to the work * corresponding author 


\begin{abstract}
The Arabidopsis syntaxin AtPEN1, as well as its barley and grapevine orthologs, contribute to the resistance conferred by loss-of-function mutations of MLO susceptibility genes (known as mlo resistance) and to non-host resistance against powdery mildew fungi. In this study, a genome-wide survey allowed the identification of 21 tomato syntaxins. Two of them, named SIPEN1 $a$ and SIPEN1b, are closely related to AtPEN1. RNAi-based silencing of SIPEN1a in a tomato mlo mutant restored its susceptibility to the tomato powdery mildew fungus Oidium neolycopersici. Moreover, it resulted in a significant increase of the penetration rate of the non-adapted powdery mildew fungus Blumeria graminis f. sp. hordei. Codon-based evolutionary analysis and multiple alignments allowed the detection of amino acids that are specifically conserved in syntaxins involved in pathogen defense. Our findings provide insights on the evolution of plant syntaxins and show that SIPEN1 $a$ is involved in tomato mlo resistance to both adapted and non-adapted powdery mildew fungi. Differently from Arabidopsis but similarly to barley, the impairment of a single syntaxin is sufficient to break mlo resistance in tomato.
\end{abstract}

\title{
Keywords
}

mlo resistance, non-host resistance, Blumeria graminis f. sp. hordei, Oidium neolycopersici, tomato syntaxins 


\section{Introduction}

In eukaryotic cells, compartmentalization through the endomembrane apparatus and exocytosis requires a highly regulated transport system. Soluble $\mathrm{N}$-ethylmaleimidesensitive-factor attachment protein receptor (SNARE) proteins are involved in such a transport, as they mediate the fusion between membranes of cargo-containing small shuttles, referred to as vesicles, with target membranes (14).

SNARE proteins were previously classified according to their localization into vesicleassociated ( $v$-SNARE) and target-membrane-associated (t-SNARE) proteins (26). An alternative classification distinguishes Q-SNARE from R-SNARE proteins by the presence of either arginine or glutamine at the centre of the SNARE domain, respectively (8). Typically, SNARE complexes which determine membrane fusion are tetrameric coiled-coil structures containing one R-SNARE protein anchored to the vesicle and one protein of each Q-SNARE family, conform to the so-called 3Q-plus-1R rule (8). Q-SNAREs are further divided into three families: Qa- (also referred to as syntaxins or SYPs), Qb- and Qc-SNAREs $(4,24)$. Based on sequence homology, syntaxins can be assigned to five subfamilies which are known as SYP1, $-2,-3,-4,-8(4,23)$.

The powdery mildew disease, affecting thousands of plant species, is caused by widespread fungal species of the Ascomycete order of Erysiphales (27). Specific members of the Mildew Locus $O(M L O)$ gene family act as susceptibility factors for powdery mildew fungi, as their loss-of-function mutations result in a particular form of resistance, referred to as mlo resistance (19). At the histological level, mlo resistance is associated with the failure of fungal penetration into host epidermal cells, as a consequence of the formation of thick cell wall appositions termed papillae (25). A number of studies suggests that MLO proteins modulate SNARE-dependent exocytosis at plant-pathogen interaction sites, which allows discharging of antimicrobial compounds (18). In barley, a syntaxin encoding gene, HVROR2, was shown to be required for mlo resistance $(5,7,10)$. Similarly, in Arabidopsis, loss of function of the HvROR2 ortholog AtPEN1 comprised the powdery mildew resistance of the Atmlo2 mutant (6).

Syntaxins have also been shown to play a major role in non-host resistance to powdery mildew fungi. In Arabidopsis, compared to the wild type, the Atpen 1 mutant allowed a significant higher penetration rate of the non-adapted barley powdery mildew pathogen Blumeria graminis f. sp. hordei (Bgh) (5). The Arabidopsis SYP122 gene encodes a syntaxin closely related to AtPEN1 and its impairment also resulted in a slight increase (10\%) of fungal penetration, which was however not reproducible (2).

In barley, the ROR2 gene is the AtPEN1 ortholog. The barley mlo ror2 double mutant showed a higher penetration rate of the wheat powdery mildew fungus Blumeria graminis f. sp. tritici (Bgt) than the single mlo mutant. The further growth of the non-adapted Bgt in 
the barley mlo ror2 double mutant was blocked since cells successfully penetrated by Bgt showed hypersensitive response $(H R)(5,15,28)$.

In this study, we exploited available genomic information to identify and characterize two tomato syntaxins. Using RNA interference technology (RNAi) functional role of the two tomato syntaxins was studied in the interactions of a tomato mlo mutant with the powdery mildew fungi Oidium neolycopersici (adapted) and Bgh (non-adapted). Evolutionary analysis and multiple alignments were performed to identify amino acids differentiate the two tomato syntaxins.

\section{Materials and methods}

\section{Plant and fungal materials}

The tomato SImlo 1 mutant, carrying a loss-of-function mutation of the SIMLO1 susceptibility gene (3), and the susceptible tomato cultivar (cv) Moneymaker (MM) were used in the disease tests as negative and positive controls, respectively. The tomato SImlo1 mutant was also used as the background of RNAi.

The Wageningen isolate of tomato powdery mildew O. neolycopersici has been maintained on cv MM plants. The Wag04 isolate of barley powdery mildew Bgh was maintained on the susceptible barley cv Manchuria. Infected tomato and barley plants were kept in a greenhouse compartment at $20 \pm 2^{\circ} \mathrm{C}$ with $70 \pm 15 \%$ relative humidity at the Unifarm of Wageningen University \& Research, The Netherlands.

Phylogenetic study of syntaxins in tomato

To retrieve the putative Qa SNARE family in tomato proteome, the Arabidopsis syntaxin AtPEN1 amino acid sequence (Genebank ID:NP_187788.1) was used as query for a tBLASTn search against the Tomato Genome CDS of Sol Genomics Network (SGN) using default settings. All the 21 putative tomato homologs retrieved, together with the complete Arabidopsis Qa SNARE family and the AtPEN1 grapevine and barley orthologs (VvPEN1 and HvROR2), for a total of 41 sequences, were used for a ClustalW alignment. The gap open cost and the gap extension cost were set equal to 10 and 4, respectively. Afterward, an Unweighted Pair Group Method with Arithmetic Mean (UPGMA) phylogenetic tree was built setting the bootstrap value equal to 100 . All the bioinformatic analyses were performed using the CLC sequence viewer software (http://www.clcbio.com/). 


\section{Generation of tomato RNAi transformants}

To generated RNAi constructs, the two tomato CDNA sequences showing the highest similarity to AtPEN1, Solyc10g081850.1.1 (named SIPEN1a) and Solyc01g006950.2.1 (named SIPEN1b), were used to design primer pairs for Gateway cloning (Invitrogen), using the Primer3 software (30). These primers, named SIPEN1a_gate_Fw/Rev (5'-caccCTGGTTGTGGACCTGGAAGT- 3'/ 5'- TGTCCTCTTCCTTGCTCCTG-3') and SIPEN1b_ gate_Fw/Rev (5'- caccACGAGCTGAAAAACCTCGAA-3'/ 5'- ACAACAGACGTCCTCGTCCT-3'), allowed the amplification of regions of 259bp and 250bp, for SIPEN1a and SIPEN1b respectively. Amplification was conducted using PfuUltrall Fusion HS DNA Polymerase (Agilent Technologies). PCR fragments were first cloned into the Gateway-compatible vector pENTR D-TOPO (Invitrogen) and then transferred to Escherichia coli competent cells (XL10-Gold Ultracompetent Cells, Agilent Technologies). Presence of the inserts was assessed by colony PCR, restriction enzyme digestion and sequencing using the universal M13 primer pair. Positive clones were used for a LR reaction, which allowed the inserts to be transferred into the pHELLSGATE12 vector, harbouring the 35S Cauliflower Mosaic Virus (CaMV) promoter for constitutive expression and the marker gene NPTII for kanamycin resistance selection. Plasmids were cloned into E. coli competent cells $\mathrm{DH} 5 \mathrm{a}$ and positive colonies were screened by colony PCR and sequencing as described before. Recombinant vectors were finally extracted and transferred to the AGL1+virG strain of Agrobacterium tumefaciens by electroporation.

The tomato SImlo1 mutant was used for transformation, as described by Appiano et al. (1). For each construct, several $T_{1}$ plants were generated and allowed to self-pollinate to obtain $\mathrm{T}_{2}$ families. To select transgenic plants of each segregating family, $\mathrm{T}_{2}$ plants were screened with primer pairs NPTII_Fw/ NPTII_Rev (5'-TCGGCTATGACTGGGCACAAC-3')/5'AAGAAGGCGATAGAAGGCGA-3'), and 35S-Fw/Rev (5'-GCTCCTACAAATGCCATCA-3')/ (5'-GATAGTGGGATTGTGCGTCA-3'). By real-time qPCR, expression of each gene in selected $T_{2}$ families was assessed using the primer pairs qPEN1a_Fw/Rev (5'CGAGATGCTTTGTGCATCAG-3'/5'- CAGTCTCCTTCAGCTCCATTTC-3') and qPEN1b_Fw/ Rev (5'- TGGTTTAGTTGTTGATGGACCTC- 3'/ 5'- ACCCCCATCCAACTTACTTACTTCTC$3^{\prime}$ ). Selected transgenic $T_{2}$ plants of each construct were crossed in order to obtain $F_{1}$ individuals in which both genes are silenced. Four-week-old $F_{1}$ plants were tested through qPCR for the expression of SIPEN1 $a$ and SIPEN1b, using the above mentioned primer pairs.

\section{Disease tests with 0 . neolycopersici and quantification of fungal biomass}

$T_{2}$ families obtained from individual $T_{1}$ plants and $F_{1}$ individuals silenced for both SIPEN1 $a$ and SIPEN1b were challenged with O. neolycopersici. Inoculation was performed as described by Pavan et al.(20), by spraying plants with a suspension of conidiospores obtained from heavily infected leaves of MM plants and adjusted to a final concentration 
of $2^{*} 10^{4}$ spores per millilitre. Inoculated plants were grown at $20 \pm 2^{\circ} \mathrm{C}$ with $70 \pm 15 \%$ relative humidity and day length of $16 \mathrm{~h}$ in a greenhouse of Unifarm of Wageningen University \& Research, The Netherlands. Disease evaluation was carried out 15 days after inoculation, powdery mildew symptom was visually scored using the scale of 0 to 3 (3) and fungal biomass was quantified by real-time qPCR as reported by Huibers et al. (11). Briefly, plant and fungal genomic DNAs were isolated from infected tomato leaves using the Qiagen DNeasy Plant Mini Kit and amplified with the primer pairs OnFw (5'-CGCCAAAGACCTAACCAAAA-3')/On-Rev (5'-AGCCAAGAGATCCGTTGTTG-3'), designed on $O$. neolycopersici internal transcribed spacer (ITS) sequences (GenBank accession number EU047564), and Ef-Fw (5'-GGAACTTGAGAAGGAGCCTAAG-3')/Ef-Rev (5'-CAACACCAACAGCAACAGTCT-3'), designed on the tomato Elongation Factor 1a (Ef1a) reference gene (17). Relative fold-change of the ratio between fungus and tomato gDNAs was inferred by the $2^{-\Delta \Delta C t}$ method $(16,21)$ and results were analyzed by the Student's t-test.

\section{Histological analysis}

Three $\mathrm{T}_{2}$ transgenic plants of each silencing construct (SIPEN1 $a$ and SIPEN $1 b$ ) were selected by PCR using 35 S and NPTI/ primer pairs. Around 18 days after sowing, these plants, together with three plants from the S/mlo1 mutant, were transferred into an infection chamber. Dry inoculum of Bgh was applied on the third and fourth leaves of four-week old tomato plants using a paintbrush to brush off the conidia from heavily infected barley leaves. At least three samples for each inoculated plant were collected 72 hours post-inoculation (hpi). These samples were stained with trypan blue as described by Freialdenhovenet al. (10) and mounted on glass slides with a 1:1 (v/v) glycerol-water solution. Observation of the slides was done using a Zeiss Axiophot bright field microscope and pictures were taken with an Axiocam ERc5s. For each sample, more than thirty infection units (one infection unit represents a germinated On spore) per slide were observed. The pathogen penetration rate was estimated as the percentage of units displaying HR (28). Statistical analysis was carried out using the Student's t-test.

\section{Detection of conserved syntaxin motifs and codons}

In order to detect conserved motifs in the syntaxin family, the whole 41 syntaxins dataset, which was used for the phylogenetic analysis above, was used as input for the BOXSHADE software (http://www.ch.embnet.org/software/BOX_form.html), using 1.0 as fraction of sequences value. Furthermore, with the aim of identifying residues specifically conserved in syntaxins acting in defense against powdery mildew fungi, another ClustalW alignment was performed, using proteins previously indicated to be involved in plant-pathogen interactions (AtSYP121, AtSYP122, HvROR12, VvPEN1), and the two tomato syntaxins SIPEN1a and SIPEN1b. This was used as input for the BOXSHADE software. Moreover, the 
same dataset was used for a codon-based evolutionary analysis, based on the difference of nonsynonymous-to-synonymous substitutions per nonsynonymous and synonymous sites (dN-dS). This was performed with the Single-likelihood Ancestor Counting (SLAC) method implemented by the Datamonkey web server (www.datamonkey.org). In order to make predictions on the kind of selection pressure (negative, neutral or positive) acting on each codon, the default $\mathrm{p}$-value of 0.1 was used as threshold for significancy.

\section{Results}

\section{In silico identification of tomato syntaxins}

In the tomato genome, 21 putative syntaxins were retrieved from a BLAST search using the Arabidopsis syntaxin AtPEN1 amino acid sequence (Genebank ID:NP_187788.1). With these 21 putative tomato syntaxins, a phylogenetic tree was constructed together with 18 syntaxins of Arabidopsis and two functional AtPEN1ortholog, barley HvROR2 and grapevine VVPEN1 (9). In the phylogenetic tree (Fig. 1), Arabidopsis syntaxins of the five syntaxin subfamilies (SYP1, $-2,-3,-4,-8)$ could be assigned to five separate clades and were named accordingly. Each clade has at least one tomato putative syntaxin. The most represented clade (SYP1), harbouring 21 homologs, was further partitioned in four subclades (indicated as SYP1a-d in Fig.1).

With strong bootstrap support, two tomato putative syntaxins, Solyc10g081850 and Solyc01g006950, were assigned to the subclade SYP1b, including all the homologs functionally shown to be involved in PM resistance, i.e. AtPEN1, HvROR2 and VvPEN1. Therefore, Solyc10g081850 and Solyc01g006950 were renamed SIPEN1a and SIPEN1b, respectively (Fig.1).

Protein sequences of SIPEN1a and SIPEN1b are highly related with each other $(76.8 \%$ nucleotide identity and $85.3 \%$ amino-acid conservation) and with AtPEN1 $(64.2 \%$ and $60.4 \%$ nucleotide identity, $72.3 \%$ and $71.7 \%$ amino-acid conservation respectively). 


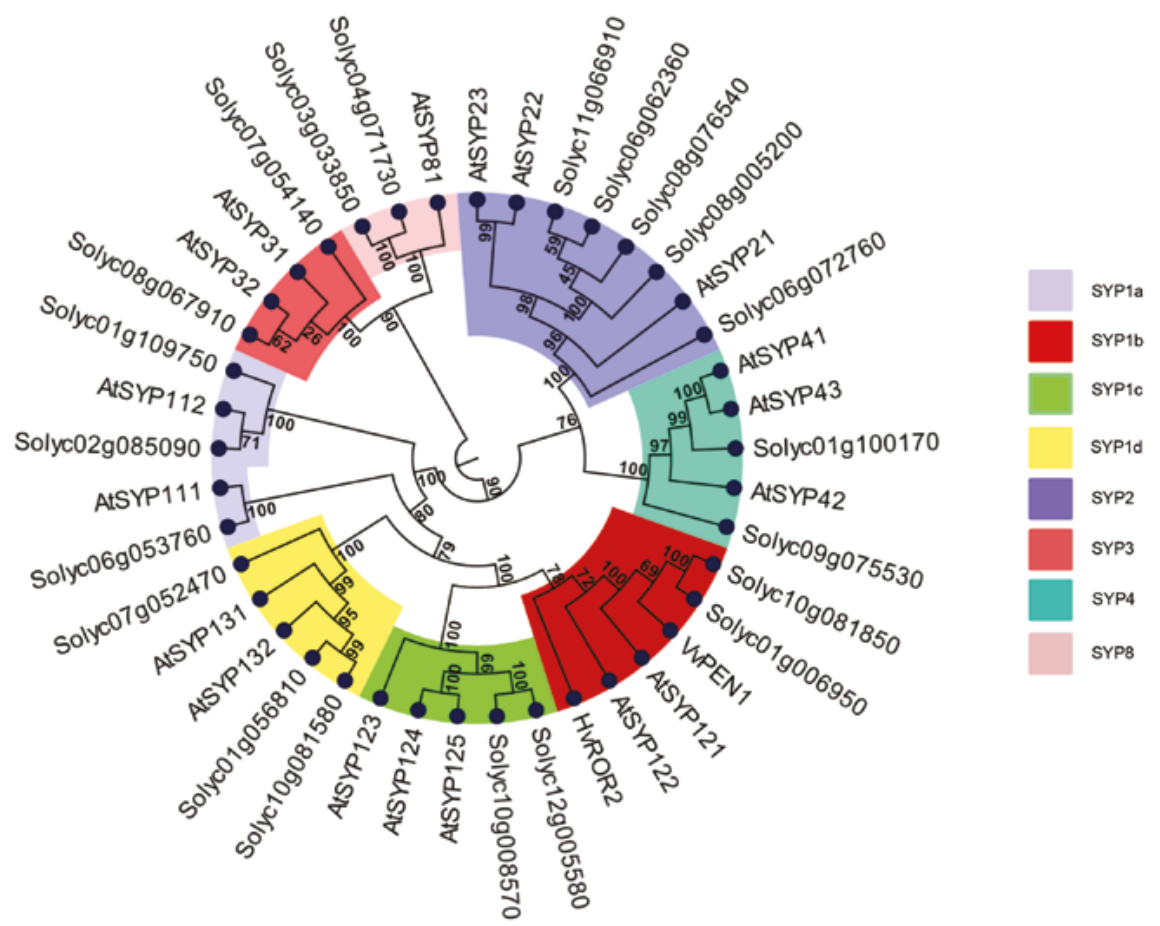

FIGURE 1. Phylogenetic relationships of 41 syntaxins. The dataset includes barley HvROR2, grapevine VvPEN1, the 18 Arabidopsis syntaxins (AtSYPs and AtPEN1) and the 21 predicted tomato syntaxins identified in this study (named with the SolGenomics Network database ID code). Numbers at nodes indicate bootstrap support values. Clades are named according to the nomenclature used for syntaxins subfamilies (SYP1, $-2,-3,-4$, -8). The clade SYP1 is further divided in four subclades (a-d). The subclade SYP1b, containing all the homologs known to be involved in plant-pathogen interactions (AtPEN1, SYSYP122, HvROR2 and VvPEN1), is highlighted in red.

\section{SIPEN1a is required for tomato PM resistance}

To gain insights on the functional role of SIPEN1 $a$ and SIPEN1b, we prepared RNAi silencing constructs for each of the two genes, which were used to transform the tomato S/mlo1 mutant. Eleven RNAi::SIPEN1a and four RNAi::SIPEN1b $\mathrm{T}_{1}$ plants were obtained and selfpollinated to produce $T_{2}$ families. Meanwhile, two cuttings per transformant were made and tested with tomato powdery mildew $O$. neolycopersici. Cuttings of all transformants showed more powdery mildew sporulation compared to the background, the tomato SImlo1 mutant (data not shown).

For each construct, two independent segregating $\mathrm{T}_{2}$ families (referred to as RNAi::SIPEN1a-I, RNAi::SIPEN1a-II, RNAi::SIPEN1b-I and RNAi::SIPEN1b-II) were selected and further characterized in a new experiment. Based on the presence of the silencing construct, transgenic $T_{2}$ individuals of each $T_{2}$ family were selected (15 plants per family), which were further referred to as RNAi::SIPEN1a(+) and RNAi::SIPEN1b(+). As control for comparison, 5 


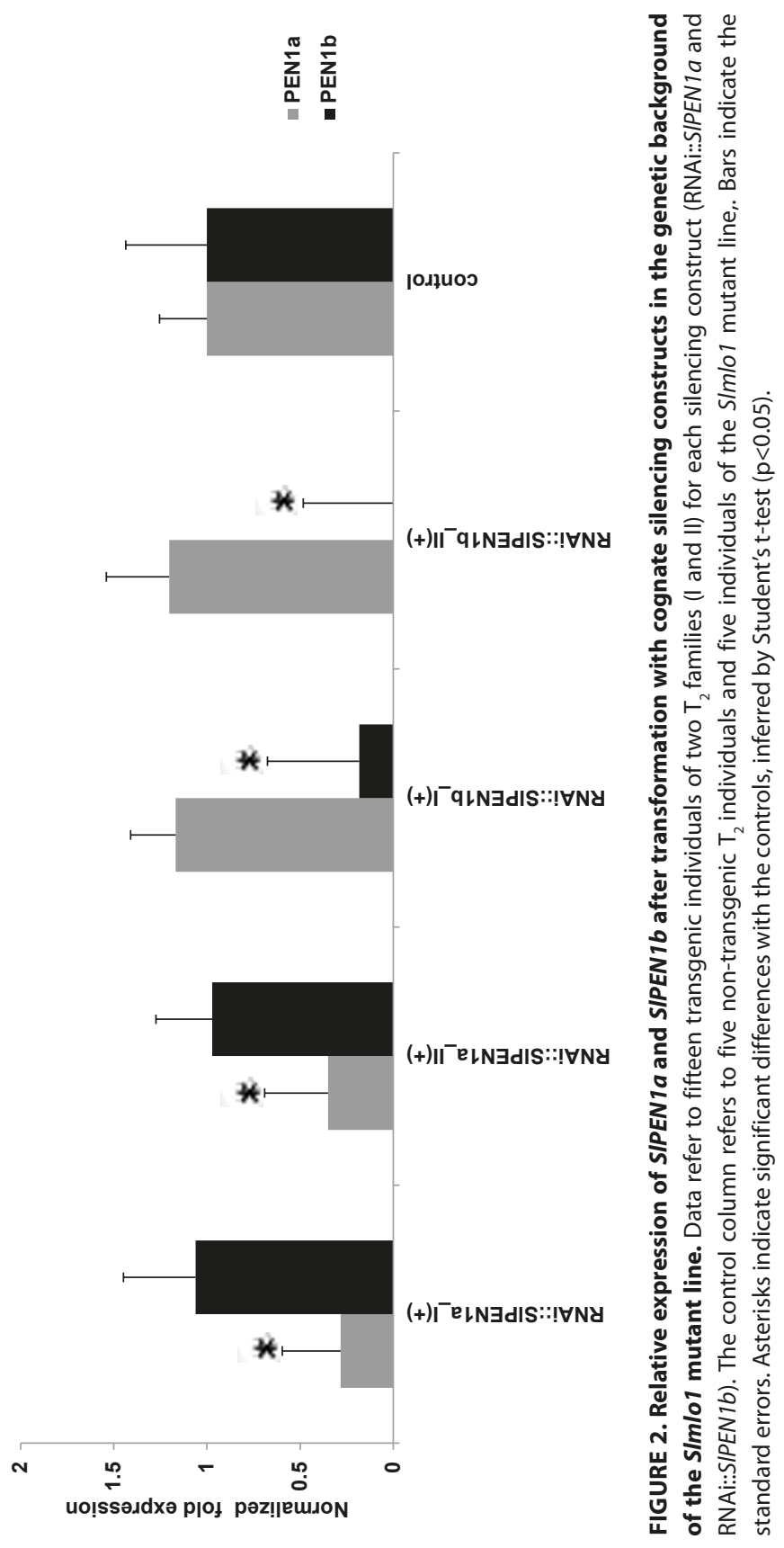


non transgenic plants per $\mathrm{T}_{2}$ family were pooled with 5 plants of the Slmlo1 mutant line since they are genetically identical. The expression level of the SIPEN1a and SIPEN1b was significantly reduced in the RNAi::SIPEN1a(+) and RNAi::SIPEN1b(+) individuals compared to the control (Fig.2). Further, no unwanted cross-silencing was found between the two target genes. RNAi::SIPEN1a(+) individuals showed clear fungal sporulation (Fig. 3A) and significantly increased fungal biomass (Fig. 3B) compared to the control plants. Although RNAi::SIPEN1b(+) individuals showed more fungal colonies than control plants, the fungal biomass was not significantly increased (Fig. 3B). Similar results were obtained by repeating the experiment.

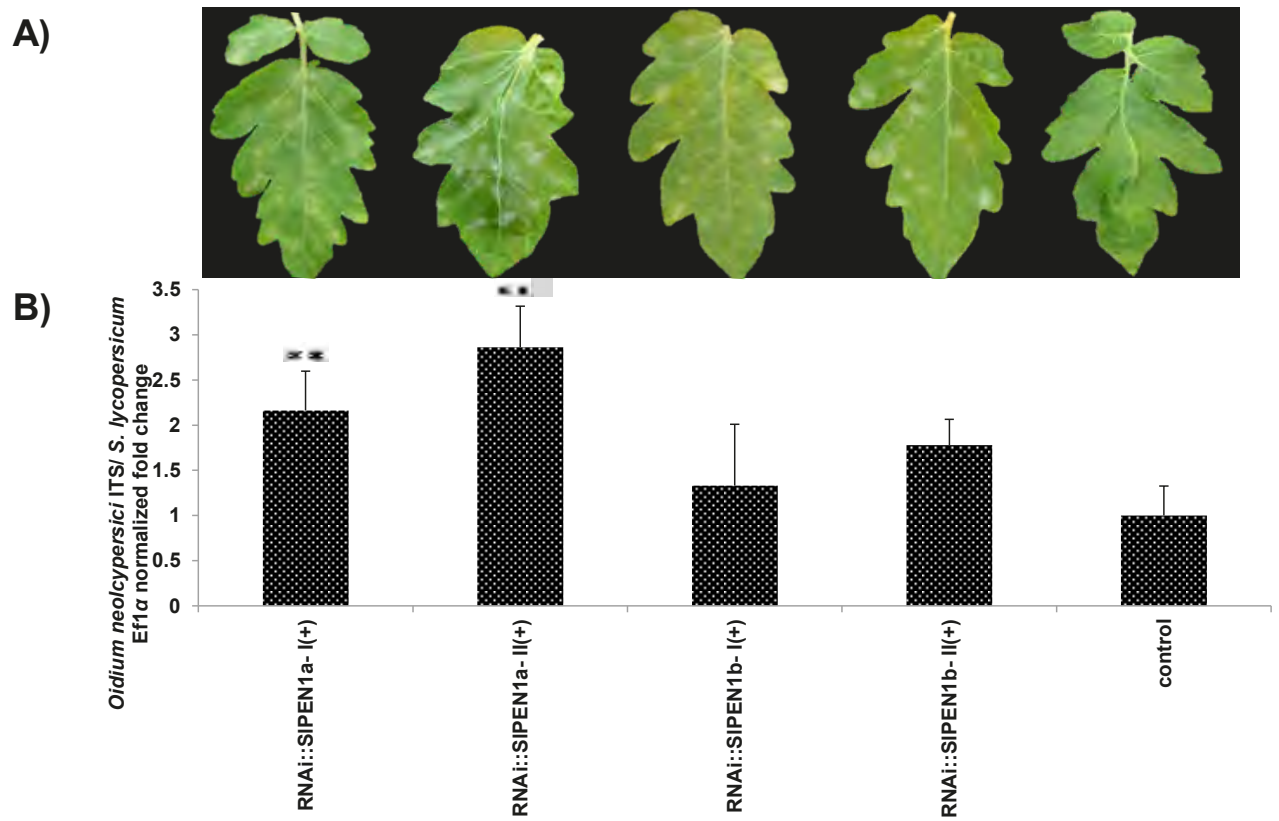

FIGURE 3. Effects of RNAi silencing of tomato SIPEN $1 a$ and SIPEN1 $b$ in the genetic background of the SImlo 1 mutant line on the interaction with the powdery mildew fungus Oidium neolycopersici. Panel a) shows the phenotype of leaves collected fifteen days after pathogen inoculation. From left to right: selected transgenic individuals of two independent $\mathrm{T}_{2}$ families carrying the SIPEN1a silencing construct [RNAi::SIPEN1a-I(+) and RNAi::SIPEN1a-II(+)], selected individuals of two independent $T_{2}$ families carrying the SIPEN1 $b$ silencing construct [RNAi::SIPEN1b-I(+) and RNAi::SIPEN1b-II(+)] and an individual of the SImlo1 resistant line. Panel b) refers to the relative quantification of the ratio between Oidium neolycopersici and tomato gDNAs in fifteen transgenic individuals of the same families above mentioned and in control plants, formed by five non-transgenic $T_{2}$ individuals and five S/mlo 1 individuals. Bars indicate the standard errors. Asterisks refer to significant differences with the control, inferred Student's t-test $\left(^{* *}=p<0.01\right.$; $\left.^{* * *}=p<0.001\right)$.

In order to investigate whether SIPEN1 $b$ has an additive effect to SIPEN1 $a$, we performed an additional experiment using the $\mathrm{F}_{1}$ progeny obtained by crossing RNAi::SIPEN1a-II(+) and RNAi::SIPEN1b-II(+) individuals. Eight $\mathrm{F}_{1}$ individuals with significantly reduced expression of both SIPEN1 $a$ and SIPEN1b were selected (Fig. 4A). Although in these individuals, higher fungal biomass (34\%) compared to RNAi::SIPEN1a-II(+) individuals was quantified, the difference was not significant (Fig. 4B). 
A)

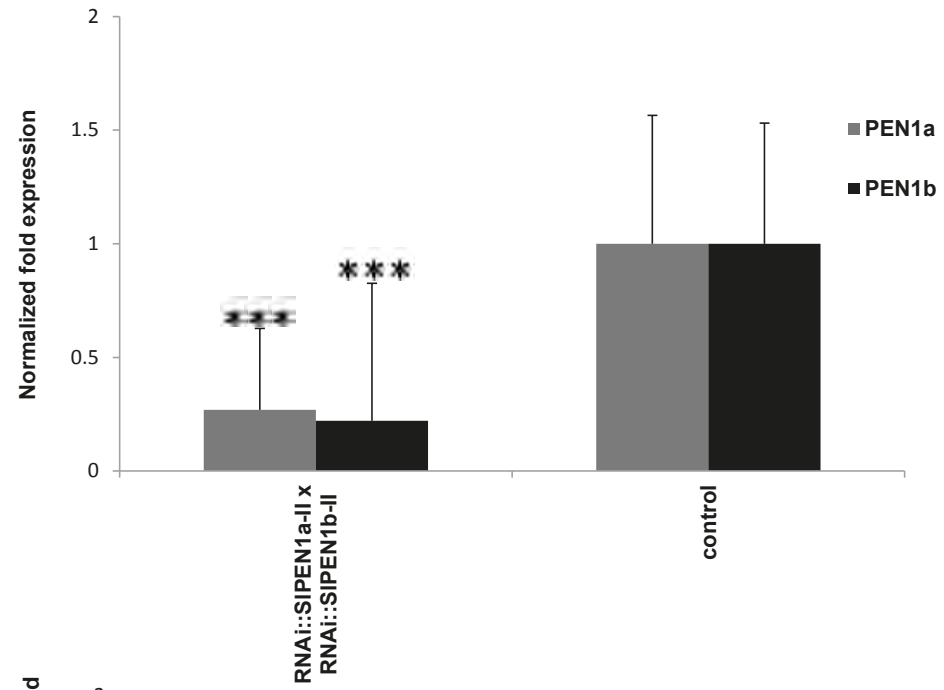

B)

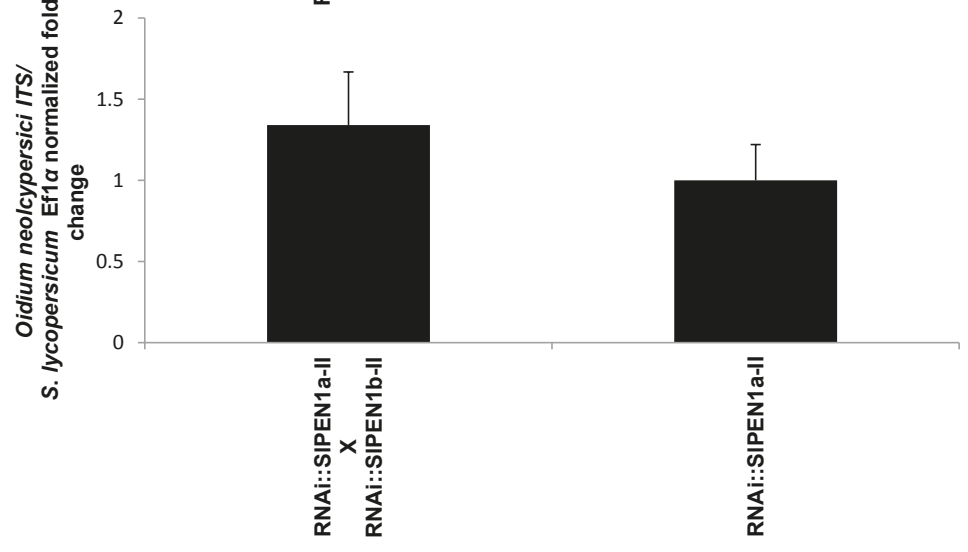

FIGURE 4. Effects of crossing of two transgenic individuals carrying silencing constructs for SIPEN1a e SIPEN1b [RNAi::SIPEN1a-II(+) and RNAi::SIPEN1b-II(+)] in the genetic background of the SImlo1 mutant line, on gene expression and growth of the powdery mildew fungus $\mathbf{O}$. neolycopersici. Panel a) shows the expression of SIPEN1a and SIPEN1b in eight individuals of the RNAi::SIPEN1a-II(+) x RNAi::SIPEN1b-II(+) hybrid progeny, compared to eleven non-transgenic control plants. Panel b) refers to the quantification of the ratio between Oidium neolycopersici biomass and plant gDNA in the same hybrids compared to fourteen RNAi::SIPEN1a-II(+) individuals. Bars indicate standard errors. Asterisks refer to significant differences, inferred by the Student's t-test $(p \leq 0.001)$.

\section{Role of SIPEN1 $a$ and SIPEN1b in non-adapted powdery mildew penetration}

Since it has been shown that PEN genes are involved in penetration resistance to both adapted and non-adapted powdery mildew, we set-up a functional assay to investigate the role of the two tomato syntaxins, SIPEN1 $a$ and SIPEN1b, in the interaction with barley powdery mildew fungus $B g h$. An artificial inoculum of this pathogen was used to inoculate RNAi::SIPEN1a-II(+) individuals, RNAi::SIPEN1b-II(+) individuals, and SImlo1 plants. The rate 
of infection units showing HR dramatically increased from $22 \%$ to $72 \%$ in RNAi::SIPEN1a$\mathrm{II}(+)$ individuals compared to the SImlo1 mutant line (Fig. 5). A slight increase (2.5\%) of HR was also found in RNAi::SIPEN1b-II(+) plants, although this value was not statistically different from the one of the SImlo1 mutant (Fig.5).
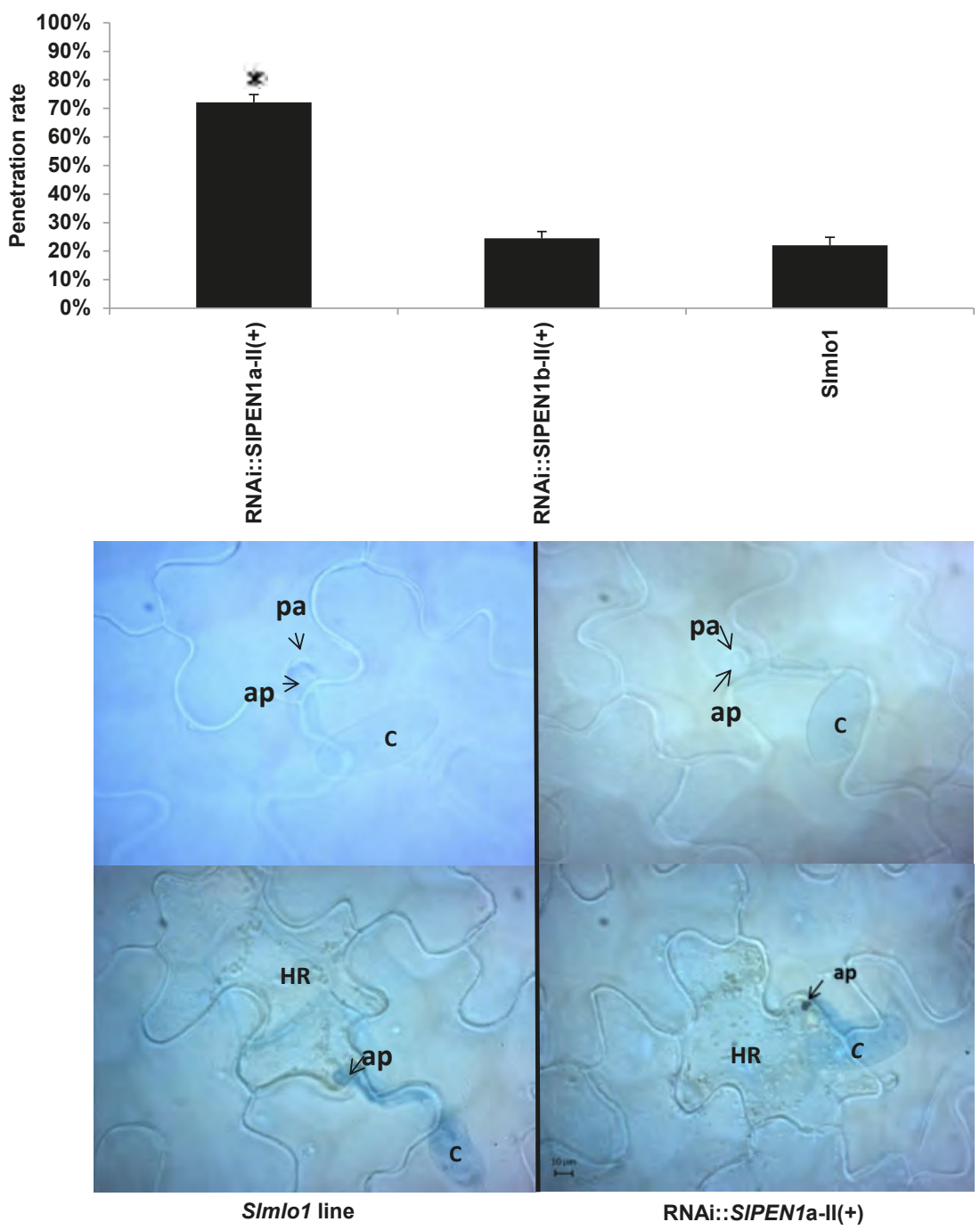

FIGURE 5. Effects of RNAi silencing of tomato SIPEN1a and SIPEN1b on the interaction with the nonadapted powdery mildew fungus Blumeria graminis $\mathbf{f}$. sp. hordei. The upper panel shows the penetration rate of Blumeria graminis $\mathrm{f}$. sp. hordei on the SImlo1 mutant line and transgenic individuals silenced for SIPEN1a and SIPEN1b [RNAi::SIPEN1a-II(+) and RNAi::SIPEN1b-II(+)], indirectly inferred as the proportion of infection units showing post-penetrative hypersensitive response. Bars refer to standard errors calculated on three biological replicates. Asterisks indicate significant difference with the SImlo1 mutant line, inferred by the Student's t-test ( $p$ $\leq 0.05$ ). The bottom panel shows the microscopic observation of papillae (pa) and hypersensitive response (HR) in epidermal cells of the SImlo1 mutant line (left) and RNAi::SIPEN1a-II(+)individuals (right) challenged with the barley powdery mildew fungus Blumeria graminis f. sp. hordei. c=conidium of; a=appressorium. 


\section{Possible molecular features required for the function of syntaxins in powdery mildew penetration}

Aiming to investigate the magnitude and direction of natural selection acting on syntaxins involved in defense against powdery mildew fungi, we used SIPEN1a, AtPEN1, HVROR2 and VVPEN1 nucleotide sequences for a codon-based single-likelihood ancestor counting (SLAC) evolutionary analysis. This is based on the dissimilarity level between non-synonymous substitution $(\mathrm{dN})$ and synonymous substitution (dS) values. The SIPEN1b and AtSYP122 were also included since they might play a minor role in tomato and Arabidopsis powdery mildew penetration. Evidence for negative selection was found on 77 codons, associated with amino acid residues scattered in syntaxin protein domains (Qa-SNARE, membrane-spanning, and the three helix domain known as $\mathrm{Ha}, \mathrm{Hb}, \mathrm{Hc}$ ) (Fig.6 and Supplementary Tab. S1). Alignment of the same subclade SYP1b proteins revealed that 65 of these residues are invariable throughout the dataset, suggesting they might play a crucial role in protein function. In order to identify syntaxin residues specifically important in pathogen defense, we performed an additional alignment with the same set of syntaxins used for phylogenetic analysis. In total, we found that 24 out of 65 residues that are invariable in the SYP1b sub-clade are not conserved in other syntaxins (Supplementary Fig. S1). In addition to these 65 invariable residues, eight amino acid differences were found between AtPEN1/SIPEN1 and AtSYP122/SIPEN1b (Supplementary Fig. S2). These eight amino acids do not belong to the 77 codons under negative selection (Fig.6 and Supplementary Tab. S1). Intriguingly, three of the eight, A24, Q31 and H72, are also biochemically different (highlighted in red in Supplementary Fig. S2). In particular, $\mathrm{H} 72$ belongs to the regulatory motif $\mathrm{Ha}$. 


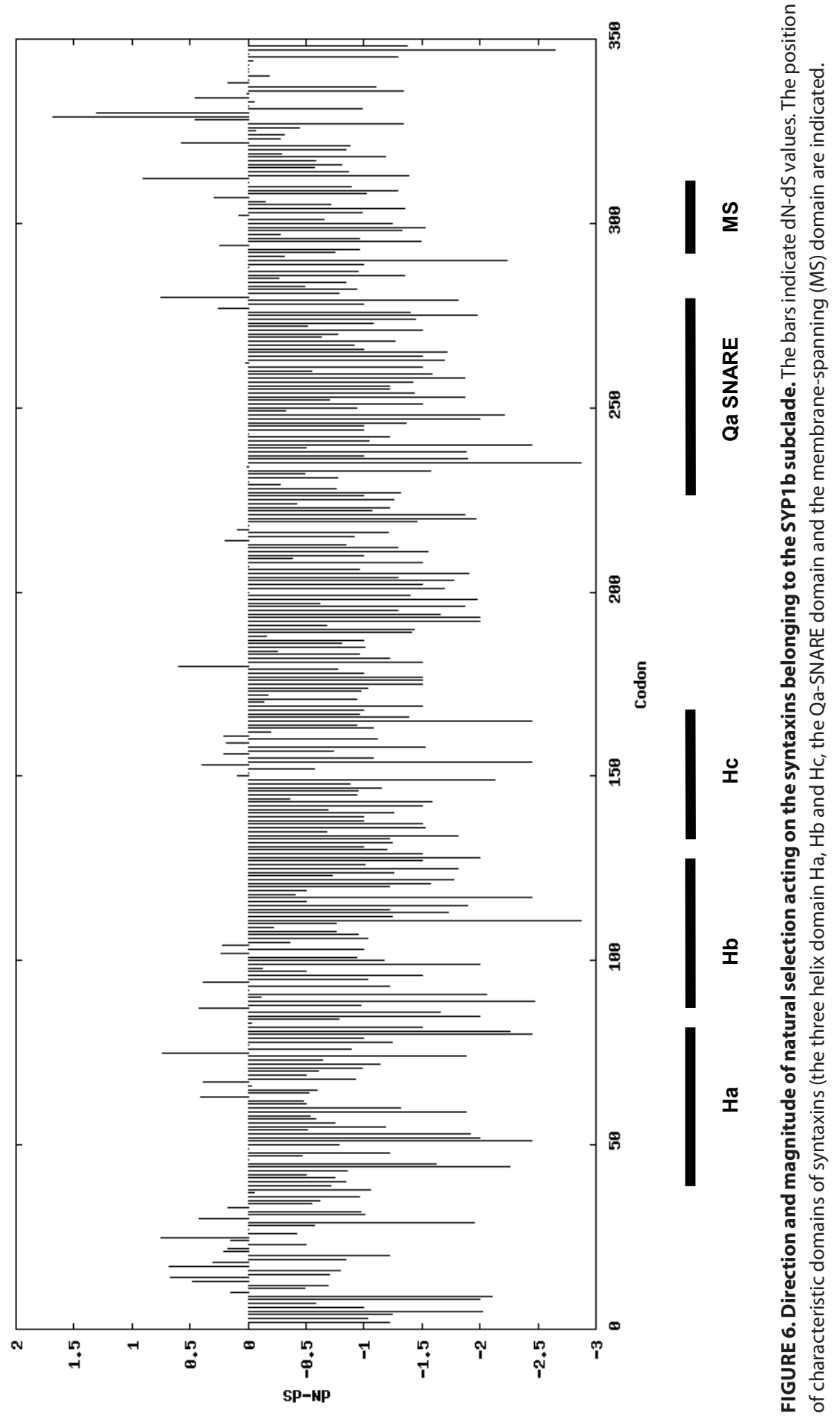




\section{Discussion}

\section{The tomato syntaxin family}

In this study, a genome-wide survey allowed the identification of 21 syntaxins in the tomato genome. The number of tomato homologs is consistent with the results of previous genome-wide studies analyzing 18 syntaxins in A. thaliana, 14 in 0 . sativa and 22 P. trichocarpa $(14,22,29)$. The identification of ten putative tomato SYP1 members corroborates previous findings indicating that this subfamily, containing homologs localized in the plasma membrane, is more represented in plants than the other subfamilies SYP2, -3, -4, and -8 (14) (Supplementary Tab. S2).

It is known that, specific SYP1 homologs in Arabidopsis are involved in other physiological processes besides defence against pathogens. Specifically, SYP111 (called KNOLLE protein) controls cytokinesis (13), whereas SYP123 and SYP132 mediate root hair tip growth (12). Phylogenetic analysis presented in this study clearly suggests that the tomato syntaxin Solyc06g053760 is an ortholog of Arabidopsis SYP111 (Fig.1). Evolutionary relatedness between SYP132 and a couple of tomato syntaxins, Solyc01g056810-Solyc10g081580 (Fig.1), might indicate that these genes are SYP132 co-orthologs due to a recent duplication event after the split of two lineages from an Arabidopsis-tomato common ancestor.

\section{Involvement of tomato syntaxins in plant-pathogen interaction}

Two tomato syntaxins, SIPEN1a and SIPEN1b, are grouped in a phylogenetic clade also containing homologs previously reported to play a major (AtPEN1, HvROR2 and VvPEN1) or minor (AtSYP122) role in penetration resistance to PM fungi (Fig.1). Silencing SIPEN1a compromised the penetration resistance to both adapted and non-adapted powdery mildew in the tomato SImlo1 mutant line (Fig. 3 and 5), showing that SIPEN1a is likely the functional ortholog of HVROR2, AtPEN1 and VVPEN1. Our data showed that SIPEN1b has a minor role in powdery mildew penetration resistance, which is in line with a previous study indicating that the Arabidopsis syntaxin AtSYP122 had a marginal influence on nonhost powdery mildew resistance (2). Thus, it is very likely that the SIPEN $1 \mathrm{~b}$ is functionally related to AtSYP122.

Similarly to barley, we found that the impairment of the tomato SIPEN1a syntaxin in the S/mlo1 mutant background macroscopically restores disease susceptibility to tomato powdery mildew. In contrast, in Arabidopsis, the Atmlo2 pen 1 double mutant, although being characterized by wild-type levels of fungal penetration, still displays a macroscopically resistant phenotype, due to enhanced post-penetration defense responses (6). AtPEN1 was shown to act as a negative regulator of the hormone salicylic acid, known to enhance post-penetration defense mechanisms against biotrophic fungi 
$(15,31)$. It might be that, differently from AtPEN1, SIPEN1 a and barley HvROR2 have minor or no role in the regulation of salicylic-acid-mediated defense pathways. In Arabidopsis, the Atpen 1 Atsyp122 double knock-out mutant displays a severely stunted and necrotic phenotype (2). In the present study, tomato individuals, silenced for both SIPEN1a and SIPEN1b homologs, were devoid of markedly visible pleiotropic effects which might be due to residual gene expression.

\section{Amino acids of syntaxins playing a potential role in penetration resistance}

In this work, a codon-based evolutionary analysis allowed us to detect 77 codons which are under significant negative selection in the SYP1b subclade containing all the six syntaxins associated with defence against powdery mildew fungi (AtPEN1, AtSYP122, HvROR2, VvPEN1, SIPEN1a and SIPEN1b). Moreover, alignment of all the 41 syntaxin sequences pointed out the occurrence of 24 residues specifically conserved in the SYP1b subclade (Supplementary Fig. S1). Three of these residues are located in the Qa-SNARE domain, whereas ten are found in the $\mathrm{Ha}, \mathrm{Hb}$ or $\mathrm{Hc}$ helices of Qa-SNARE proteins. These auto-inhibitory motifs, when folded in a closed conformation, prevent the exposure of the Qa-SNARE domain and thus the formation of SNARE complexes (5).

Here we hypothesize a regulatory function of these conserved residues since, the HvROR2 $\triangle 31$ barley mutant, harbouring a 31 amino acids deletion (S118-E148), strongly enhances its binding to HvSNAP34, a main protein of the SNARE complex(5). Interestingly, the deletion causing a constitutively open state of the protein, covers most of the Hc helix and involves four of the 24 conserved residues (namely G123, P124, T133 and G138 in HvROR2 sequence) identified in this study.

In addition to the above discussed 77 codons conserved in the SYP1b subclade, three amino acids with different biochemical functions were found between AtPEN1/SIPEN1 and AtSYP122/SIPEN1b (Supplementary Fig. S2). These three amino acids might play a role in AtPEN1/SIPEN1 for their involvement in mlo resistance to powdery mildew fungi. Future functional analyses on syntaxins, such as targeted mutagenesis, might unravel the role of these amino acids identified in this study. 


\section{References}

1. Appiano, M., Pavan, S., Catalano, D., Zheng, Z., Bracuto, V., Lotti, C., Visser, R. F., Ricciardi, L., and Bai, Y. 2015. Identification of candidate $M L O$ powdery mildew susceptibility genes in cultivated Solanaceae and functional characterization of tobacco NtMLO1. Transgenic Res. 24(5):847-858.

2. Assaad, F. F., Qiu, J. L., Youngs, H., Ehrhardt, D., Zimmerli, L., Kalde, M., Wanner, G., Peck, S. C., Edwards, H., Ramonell, K., Somerville, C. R., and Thordal-Christensen, H. 2004. The PEN1 syntaxin defines a novel cellular compartment upon fungal attack and is required for the timely assembly of papillae. Mol. Biol. Cell. 15(11):5118-5129

3. Bai, Y., Pavan, S., Zheng, Z., Zappel, N. F., Reinstädler, A., Lotti, C., De Giovanni, C., Ricciardi, L., Lindhout, P., Visser, R., Theres, K., and Panstruga, R. 2008. Naturally occurring broad-spectrum powdery mildew resistance in a Central American tomato accession is caused by loss of Mlo function. Mol. PlantMicrobe Interact. 21(1):30-39

4. Bock, J. B., Matern, H. T., Peden, A. A., and Scheller, R. H. 2001. A genomic perspective on membrane compartment organization. Nature 409(6822):839-841

5. Collins, N. C., Thordal-Christensen, H., Lipka, V., Bau, S., Kombrink, E., Qiu, J.-L., Huckelhoven, R., Stein, M., Freialdenhoven, A., Somerville, S. C., and Schulze-Lefert, P. 2003. SNARE-protein-mediated disease resistance at the plant cell wall. Nature 425(6961):973-977.

6. Consonni, C., Humphry, M. E., Hartmann, H. A., Livaja, M., Durner, J., Westphal, L., Vogel, J., Lipka, V., Kemmerling, B., and Schulze-Lefert, P. 2006. Conserved requirement for a plant host cell protein in powdery mildew pathogenesis. Nat. Genet. 38(6):716-720.

7. Enami, K., Ichikawa, M., Uemura, T., Kutsuna, N., Hasezawa, S., Nakagawa, T., Nakano, A., and Sato, M. H. 2009. Differential expression control and polarized distribution of plasma membrane-resident SYP1 SNAREs in Arabidopsis thaliana. Plant Cell Physiol. 50(2):280-289.

8. Fasshauer, D., Sutton, R. B., Brunger, A. T., and Jahn, R. 1998. Conserved structural features of the synaptic fusion complex: SNARE proteins reclassified as Q- and R-SNAREs. Proc. Natl. Acad. Sci. U. S. A 95(26):15781-15786

9. Feechan, A., Jermakow, A. M., Ivancevic, A., Godfrey, D., Pak, H., Panstruga, R., and Dry, I. B. 2013. Host Cell Entry of Powdery Mildew is correlated with endosomal transport of antagonistically acting VvPEN1 and VvMLO to the papilla. Mol. Plant-Microbe Interact 26(10):1138-1150

10. Freialdenhoven, A., Peterhansel, C., Kurth, J., Kreuzaler, F., and Schulze-Lefert, P. 1996. Identification of genes required for the function of non-race-specific $m / 0$ resistance to powdery mildew in barley. The Plant Cell 8(1):5-14.

11. Huibers, R. P., Loonen, A. E. H. M., Gao, D., Van den Ackerveken, G., Visser, R. G. F., and Bai, Y. 2013. Powdery Mildew Resistance in tomato by impairment of SIPMR4 and SIDMR1. PLoS ONE 8(6) e67467.

12. Ichikawa, M., Hirano, T., Enami, K., Fuselier, T., Kato, N., Kwon, C., Voigt, B., Schulze-Lefert, P., Baluška, F., and Sato, M. H. 2014. Syntaxin of plant proteins SYP123 and SYP132 mediate root hair tip growth in Arabidopsis thaliana. Plant Cell Physiol 55(4):790-800

13. Lauber, M. H., Waizenegger, I., Steinmann, T., Schwarz, H., Mayer, U., Hwang, I., Lukowitz, W., and Jürgens, G. 1997. The Arabidopsis KNOLLE protein is a cytokinesis-specific syntaxin. J. Cell Biol. 139(6):1485-1493

14. Lipka, V., Kwon, C., and Panstruga, R. 2007. SNARE-ware: The role of SNARE-domain proteins in plant biology. Annu. Rev. Cell Dev. Biol. 23, 147-174.

15. Lipka, V., Dittgen, J., Bednarek, P., Bhat, R., Wiermer, M., Stein, M., Landtag, J., Brandt, W., Rosahl, S., Scheel, D., Llorente, F., Molina, A., Parker, J., Somerville, S., and Schulze-Lefert, P. 2005. Plant science: Pre- and postinvasion defenses both contribute to nonhost resistance in Arabidopsis. Science 310(5751):11801183.

16. Livak, K. J., and Schmittgen, T. D. 2001. Analysis of relative gene expression data using real-time quantitative PCR and the $\mathbf{2}^{-\Delta \Delta C t}$ Method. Methods 25(4):402-8

17. Løvdal, T., and Lillo, C. 2009. Reference gene selection for quantitative real-time PCR normalization in tomato subjected to nitrogen, cold, and light stress. Anal. Biochem. 387(2):238-242 
18. Panstruga, R. 2005. Serpentine plant MLO proteins as entry portals for powdery mildew fungi. Biochem. Soc. Trans. 33(2):389-392.

19. Pavan, S., Jacobsen, E., Visser, R. G., and Bai, Y. 2010. Loss of susceptibility as a novel breeding strategy for durable and broad-spectrum resistance. Mol. Breed. 25(1):1-12.

20. Pavan, S., Zheng, Z., Borisova, M., Van Den Berg, P., Lotti, C., De Giovanni, C., Lindhout, P., De Jong, H., Ricciardi, L., Visser, R. G. F., and Bai, Y. 2008. Map- vs. homology-based cloning for the recessive gene ol-2 conferring resistance to tomato powdery mildew. Euphytica 162(1):91-98

21. Pfaffl, M. W. 2001. A new mathematical model for relative quantification in real-time RT-PCR. Nucleic Acids Res. 29(9)

22. Project, I. R. G. S. 2005. The map-based sequence of the rice genome. Nature 436(7052):793-800.

23. Sanderfoot, A. A., Assaad, F. F., and Raikhel, N. V. 2000. The Arabidopsis genome. An abundance of soluble N-ethylmaleimide-sensitive factor adaptor protein receptors. Plant Physiol. 124(4):1558-1569

24. Schilde, C., Lutter, K., Kissmehl, R., and Plattner, H. 2008. Molecular identification of a SNAP-25-like SNARE protein in Paramecium. Eukaryotic Cell 7(8):1387-1402

25. Seifi, A., Gao, D., Zheng, Z., Pavan, S., Faino, L., Visser, R. F., Wolters, A.-M., and Bai, Y. 2014. Genetics and molecular mechanisms of resistance to powdery mildews in tomato (Solanum lycopersicum) and its wild relatives. Eur. J. Plant Pathol. 138(3):641-665

26. Söllner, T., Whiteheart, S. W., Brunner, M., Erdjument-Bromage, H., Geromanos, S., Tempst, P., and Rothman, J. E. 1993. SNAP receptors implicated in vesicle targeting and fusion. Nature 362(6418):318-324.

27. Takamatsu, S. 2004. Phylogeny and evolution of the powdery mildew fungi (Erysiphales, Ascomycota) inferred from nuclear ribosomal DNA sequences. Mycoscience 45(2):147-157

28. Trujillo, M., Troeger, M., Niks, R. E., Kogel K. H. and Hückelhoven, R. 2004. Mechanistic and genetic overlap of barley host and non-host resistance to Blumeria graminis. Mol. Plant Pathol. 5(5):389-396.5.

29. Tuskan, G. A., Difazio, S., Jansson, S., Bohlmann, J., Grigoriev, I., Hellsten, U., Putnam, N., Ralph, S., Rombauts, S., and Salamov, A. 2006. The genome of black cottonwood, Populus trichocarpa (Torr. \& Gray). Science 313(5793):1596-1604.

30. Untergasser, A., Cutcutache, I., Koressaar, T., Ye, J., Faircloth, B. C., Remm, M., and Rozen, S. G. 2012. Primer3-new capabilities and interfaces. Nucleic Acids Res. 40(15):e115-e115.

31. Zhang, Z., Feechan, A., Pedersen, C., Newman, M. A., Qiu, J. I., Olesen, K. L., and Thordal-Christensen, H. 2007. A SNARE-protein has opposing functions in penetration resistance and defence signalling pathways. The Plant Journal 49(2):302-312. 


\section{Supplementary tables}

TABLE S1. Residues under significant negative selection in SYP1b syntaxins. Residue numbers refer to amino acid position in the AtPEN1 protein. DN-dS values, corresponding $\mathrm{p}$-values and residue position with respect to characteristic syntaxin domains (the three helix domain $\mathrm{Ha}-\mathrm{Hb}$ - $\mathrm{Hc}$, the Qa-SNARE domain and the membrane-spanning (MS) domain are reported.

\begin{tabular}{|c|c|c|c|}
\hline Corresponding residue in AtPEN1 & dN-dS & p-value & Domain \\
\hline F5 & -3.15883 & 0.06131 & - \\
\hline S8 & -3.1351 & 0.02213 & - \\
\hline F9 & -3.28782 & 0.09351 & - \\
\hline N43 & -3.52804 & 0.05074 & $\mathrm{Ha}$ \\
\hline L44 & -2.54257 & 0.05726 & $\mathrm{Ha}$ \\
\hline D50 & -3.83057 & 0.01861 & $\mathrm{Ha}$ \\
\hline V51 & -3.1351 & 0.01235 & $\mathrm{Ha}$ \\
\hline E52 & -3.00033 & 0.03562 & $\mathrm{Ha}$ \\
\hline L58 & -2.94697 & 0.01581 & $\mathrm{Ha}$ \\
\hline E73 & -2.94552 & 0.03696 & $\mathrm{Ha}$ \\
\hline H79 & -3.83057 & 0.01861 & $\mathrm{Ha}$ \\
\hline N80 & -3.52804 & 0.05074 & - \\
\hline A81 & -2.35132 & 0.03704 & - \\
\hline V84 & -3.1351 & 0.01235 & - \\
\hline R88 & -3.87355 & 0.01415 & $\mathrm{Hb}$ \\
\hline K90 & -3.21478 & 0.06364 & $\mathrm{Hb}$ \\
\hline V95 & -2.35132 & 0.03704 & $\mathrm{Hb}$ \\
\hline A98 & -3.1351 & 0.01235 & $\mathrm{Hb}$ \\
\hline E110 & -4.49517 & 0.00675 & $\mathrm{Hb}$ \\
\hline L112 & -2.69996 & 0.02732 & $\mathrm{Hb}$ \\
\hline R114 & -2.95551 & 0.01719 & $\mathrm{Hb}$ \\
\hline N116 & -3.83057 & 0.01861 & $\mathrm{Hb}$ \\
\hline R120 & -2.46381 & 0.03418 & $\mathrm{Hb}$ \\
\hline G124 & -2.8307 & 0.04851 & $\mathrm{Hb}$ \\
\hline G126 & -2.35132 & 0.0434 & - \\
\hline P127 & -3.1351 & 0.01235 & - \\
\hline G128 & -2.35132 & 0.03841 & - \\
\hline R133 & -2.8257 & 0.02516 & $\mathrm{Hc}$ \\
\hline R135 & -2.39318 & 0.07344 & $\mathrm{Hc}$ \\
\hline T136 & -2.35132 & 0.03704 & $\mathrm{Hc}$ \\
\hline G141 & -2.35132 & 0.03868 & $\mathrm{Hc}$ \\
\hline L142 & -2.48174 & 0.03486 & $\mathrm{Hc}$ \\
\hline L146 & -1.80166 & 0.08323 & $\mathrm{Hc}$ \\
\hline D148 & -3.33705 & 0.05724 & $\mathrm{Hc}$ \\
\hline F153 & -3.83057 & 0.01861 & $\mathrm{Hc}$ \\
\hline R157 & -2.39325 & 0.07349 & $\mathrm{Hc}$ \\
\hline Y164 & -3.83057 & 0.02885 & $\mathrm{Hc}$ \\
\hline V168 & -2.35132 & 0.03704 & - \\
\hline T174 & -2.35132 & 0.03704 & - \\
\hline V175 & -2.35132 & 0.03704 & - \\
\hline
\end{tabular}




\begin{tabular}{|c|c|c|c|}
\hline Corresponding residue in AtPEN1 & dN-dS & p-value & Domain \\
\hline T176 & -2.35132 & 0.03704 & - \\
\hline P180 & -2.35132 & 0.03704 & - \\
\hline L188 & -2.21353 & 0.04439 & - \\
\hline T191 & -3.1351 & 0.01235 & - \\
\hline G192 & -3.1351 & 0.01249 & - \\
\hline E193 & -2.58975 & 0.09884 & - \\
\hline E195 & -2.93078 & 0.03733 & - \\
\hline F197 & -3.08635 & 0.06377 & - \\
\hline L198 & -2.18715 & 0.05751 & - \\
\hline A201 & -2.35132 & 0.03704 & - \\
\hline 1202 & -2.77566 & 0.03544 & - \\
\hline E204 & -2.9842 & 0.03601 & - \\
\hline G206 & -2.35132 & 0.04295 & - \\
\hline 1217 & -2.27694 & 0.05266 & - \\
\hline E219 & -2.92783 & 0.03741 & - \\
\hline R220 & -1.6729 & 0.09756 & - \\
\hline L231 & -2.45666 & 0.06387 & Qa SNARE \\
\hline E233 & -4.49517 & 0.00675 & Qa SNARE \\
\hline L234 & -2.95785 & 0.01765 & Qa SNARE \\
\hline Q236 & -2.94552 & 0.04219 & Qa SNARE \\
\hline F238 & -3.83057 & 0.01861 & Qa SNARE \\
\hline L244 & -2.13677 & 0.06406 & Qa SNARE \\
\hline V245 & -3.1351 & 0.01235 & Qa SNARE \\
\hline E246 & -3.45719 & 0.06013 & Qa SNARE \\
\hline G249 & -2.35132 & 0.04494 & Qa SNARE \\
\hline Q251 & -2.92688 & 0.04273 & Qa SNARE \\
\hline L252 & -2.24543 & 0.04253 & Qa SNARE \\
\hline 1255 & -2.22416 & 0.05519 & Qa SNARE \\
\hline E256 & -2.93077 & 0.03733 & Qa SNARE \\
\hline V259 & -2.35132 & 0.03704 & Qa SNARE \\
\hline R261 & -2.64477 & 0.03867 & Qa SNARE \\
\hline A262 & -2.35132 & 0.03704 & Qa SNARE \\
\hline G269 & -2.35132 & 0.04325 & Qa SNARE \\
\hline L273 & -3.09493 & 0.0138 & Qa SNARE \\
\hline R277 & -2.82596 & 0.0199 & Qa SNARE \\
\hline R284 & -2.12025 & 0.06354 & - \\
\hline C288 & -3.49295 & 0.06064 & Membrane-spanning \\
\hline
\end{tabular}

TABLE S2. Distribution of Arabidopsis thaliana, Oryza sativa, Populus trichocarpa and Solanum lycopersicum syntaxins in subfamilies.

\begin{tabular}{cccccc}
\hline SNARE type & SNARE subfamily & A.thaliana & O. sativa & P.trichocarpa & S.lycopersicum \\
\hline \multirow{4}{*}{ Qa } & SYP1 & 9 & 7 & 11 & 10 \\
SNARE & SYP2 & 3 & 3 & 3 & 5 \\
& SYP3 & 2 & 1 & 3 & 2 \\
& SYP4 & 3 & 1 & 3 & 2 \\
& SYP8 & 1 & 2 & $\mathbf{2 2}$ & 2 \\
\hline
\end{tabular}




\section{Supplementary figures}

\begin{tabular}{|c|c|}
\hline AtPEN1 & DLFSSSFSRFRSGEPSPRRDVAGGGD-GVQMANPAGST \\
\hline VVPEN1 & --DLFSGSFSRFRSEEPPPS---------VEMTS---ST \\
\hline HVROR2 & ----NLFSSSWKRA---GAGGDGDLESGGG-GVEMTAPPGAA \\
\hline SIPEN1a & 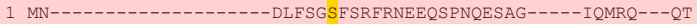 \\
\hline SIPEN1b & --DLFSGSFSRYR--ENDHDQDSHG-----IEMGD----T \\
\hline AtSYP122 & $1 \mathrm{MN}-\cdots-\cdots-\cdots$ DLLSGSEKTSVADGSSPPHS $-\cdots---\mathrm{H}-\mathrm{NIEMSKAKVSG}$ \\
\hline AtSYP124 & 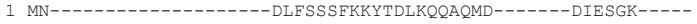 \\
\hline AtSYP123 & 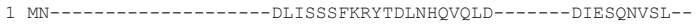 \\
\hline AtSYP111 & 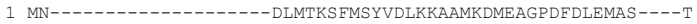 \\
\hline AtSYP131 & 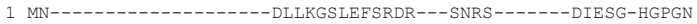 \\
\hline AtSYP125 & 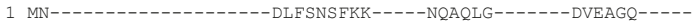 \\
\hline AtSYP132 & 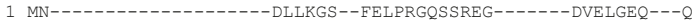 \\
\hline AtSYP112 & $1 \mathrm{MN}-\cdots--1$ \\
\hline Solyc12g005580 & 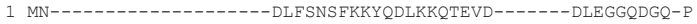 \\
\hline Solyc10g00 & $1 \mathrm{MN}----------------D L F S P S L K K Y Q D L K Q Q V Q M D------D L E L G T G G T G P$ \\
\hline Solyclog081580 & 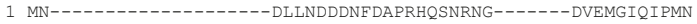 \\
\hline Solyc01g056810 & $1 \mathrm{M}-\mathrm{M}$ \\
\hline Solyc07g05 & 1 MN----------------DLLADS-SFIAGKDNASKES-------DIEMGNRFTRS \\
\hline Solyc06g05 & 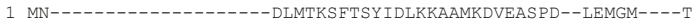 \\
\hline Solyc02g085090 & 1 MN-----------DLMTKSELSYMELKKQAHLDLET--ERDLEMGQ----- \\
\hline Solyc0lg109750 & 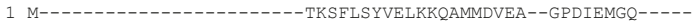 \\
\hline SOIYc0ogo & I MSEQDD \\
\hline Solyc1lg06 & ----- LDSGRS---S \\
\hline Solyc08g0d & 1 MSFQD-- - - \\
\hline Solyc08g076540 & 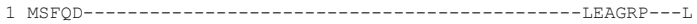 \\
\hline AtSYP22 & 1 MSFQD----------- \\
\hline AtSYP23 & ----- LEAGRGRSLA \\
\hline AtSYP21 & $1 \mathrm{MSFQ}-1-1$ \\
\hline Solyc06g072760 & I MSFED-1, \\
\hline AtSYP 41 & 1 MATRNRTLLFRKYRNSLRSVRAPLSSSS--------LTGTRSG-GVGPVIEMASTSLLN \\
\hline AtSYP 43 & 1 MATRNRTLLFRKYRNSLRSVRAPMGSSSSSTLTEHNSLTGAKSG--LGPVIEMASTSLLN \\
\hline Solyc01g100170 & 1 MASRNRTLVFRKYRDALRSVRIPAGSS---------TSTSSGHGSGPVIELATTSLLN \\
\hline AtSYP 42 & 1 MATRNRTTVYRKHRDACKSARAPLSLSA \\
\hline Solyc09g075530 & 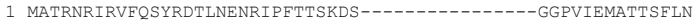 \\
\hline Solyc03g033850 & 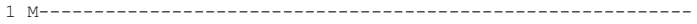 \\
\hline Solyc0 $4 \mathrm{~g} 0$ & $1 \mathrm{MS}---$ \\
\hline AtSYP81 & 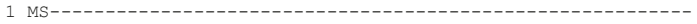 \\
\hline AtSYP31 & 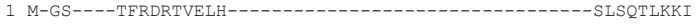 \\
\hline Solyc0 $7 \mathrm{~g}$ & 1 M-ASSGAWTYRDRTSEFA----- \\
\hline AtSYP 32 & 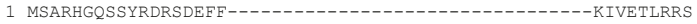 \\
\hline Solyc08g067910 & 1 MPVKVASASLRDRTQEFQ--- \\
\hline
\end{tabular}

$\mathrm{Ha}$

\begin{tabular}{|c|c|c|}
\hline AtPEN1 & 40 & -GVNLDKFFEDVESVKEELKE---- \\
\hline VVPEN1 & 28 & A----GVNLDKFFEDVESIKEELRE----- \\
\hline HVROR2 & 37 & A----GASLDRFFEDVESIKDDLRE----- \\
\hline SIPEN1a & 33 & 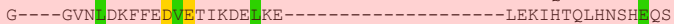 \\
\hline SIPEN1b & 30 & 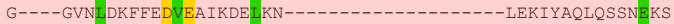 \\
\hline AtSYP122 & 35 & GSCHGGNNLDTFFLDVEVVNEDLKE-- \\
\hline AtSYP124 & 29 & 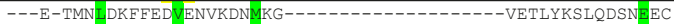 \\
\hline AtSYP123 & 32 & 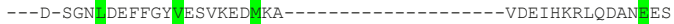 \\
\hline AtSYP111 & 37 & 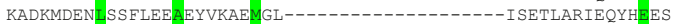 \\
\hline AtSYP131 & 30 & 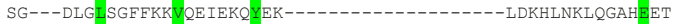 \\
\hline AtSYP125 & 24 & 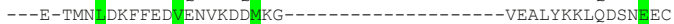 \\
\hline P132 & 29 & GG---DQGLEDFFKKVQVIDKQYDK-------------------LDKLLKKLQASHEES \\
\hline P112 & 39 & 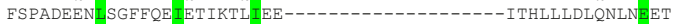 \\
\hline Solyc12g005580 & 33 & G-TE-SIDLAKFFEDVENVKEDMKD--------------VEKFHKKLQESNEES \\
\hline Solyc10g00 & 34 & 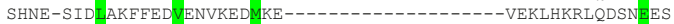 \\
\hline Solyc10 & 34 & 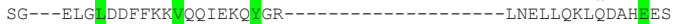 \\
\hline Solyc01go & 2 & --- LALSLIDAHEES \\
\hline Solyc07g052470 & 33 & QS---DSGIDSFNKQIQEIEKQVDR----------- \\
\hline Solyc06g053760 & 35 & 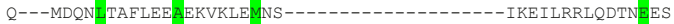 \\
\hline Solyc02 & 34 & 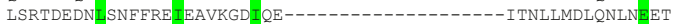 \\
\hline Solyc01g109750 & 30 & LDPTDERNLSKFFEEVAVIKSDMEE---------------------INNLLVNLQDLNRKT \\
\hline Solycuog & 13 & GPRRGFM- \\
\hline $\mathrm{s}$ & 13 & $-N G-K Q--$ \\
\hline Solyc08g005200 & 13 & $---\mathrm{NG}-\mathrm{KQ}------$ \\
\hline Solyc08g076540 & 13 & $---N G-K Q-----$ \\
\hline & 13 & 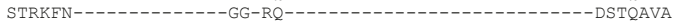 \\
\hline 23 & 16 & 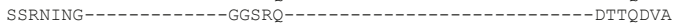 \\
\hline AtSYP21 & 12 & 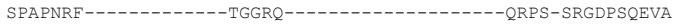 \\
\hline Solyc06g & 16 & GGSRSWE-1-1-1-1-1-1-1-RQTTQ \\
\hline P41 & 51 & 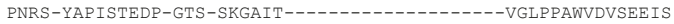 \\
\hline P43 & 59 & PNRS-YAPVSTEDP-GNS-SRGTIT-1-1-1-1 \\
\hline Solyc01g100170 & 50 & 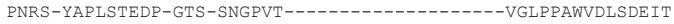 \\
\hline AtSYP 42 & 47 & SNHSSYAPLNSYDP-GPS-SSDAFT - - - - \\
\hline So & 45 & SNRS-YAPLSTEDDLGPSTSRDAFT \\
\hline Solyc03go & 2 & - \\
\hline Solyc04g071730 & 3 & --------KVRDRTEDFKDVAHRSALSLGYDESKTAALLASFIMHKPRQKSGETRAAL \\
\hline AtSYP81 & 3 & -------RERDRTEDEKDSVRNSAVS I GYNESKVASTMASFI I HKPKERSPFTKAAF \\
\hline & 23 & -GAIPSVHQ-----DEDDPASSKRS----SPGSEFNKKASRIGLGIKETSQKITRLAK-- \\
\hline Solyc07gc & 27 & AGTTGSDHE-----PQQNSASSTTKVLQI PDRSEFNKKASRI GLT I HQTFQKI DRLAK-- \\
\hline AtSYP 32 & 28 & IAPAPAANNVPYGNNRNDGARRED----LINKSEFNKRASHIGLAINQTSQKLSKLAKRI \\
\hline Solyc08g067910 & 28 & FS---SVQNGS ISTSTSSGSRSEEQRTTIAMQSEFNRRASKIGFGIHQTSQKLAKLAK-- \\
\hline
\end{tabular}

FIGURE S1. 


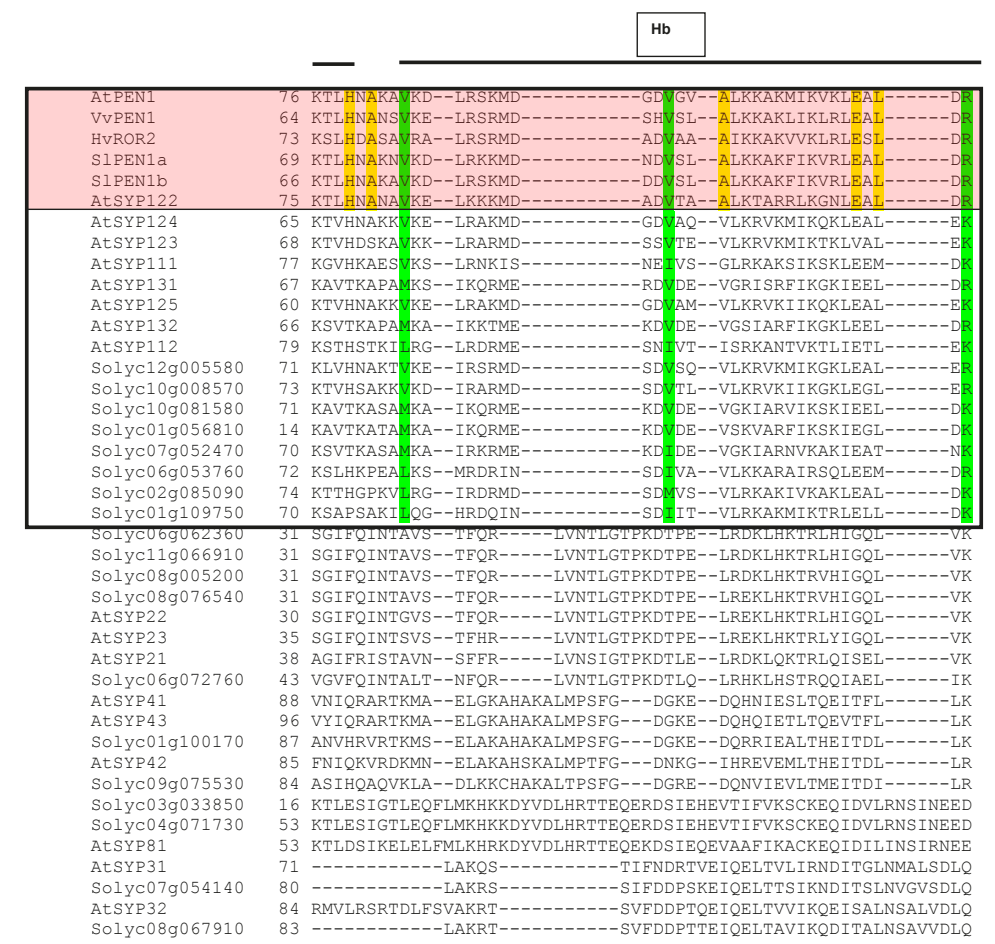

$\mathrm{Hc}$

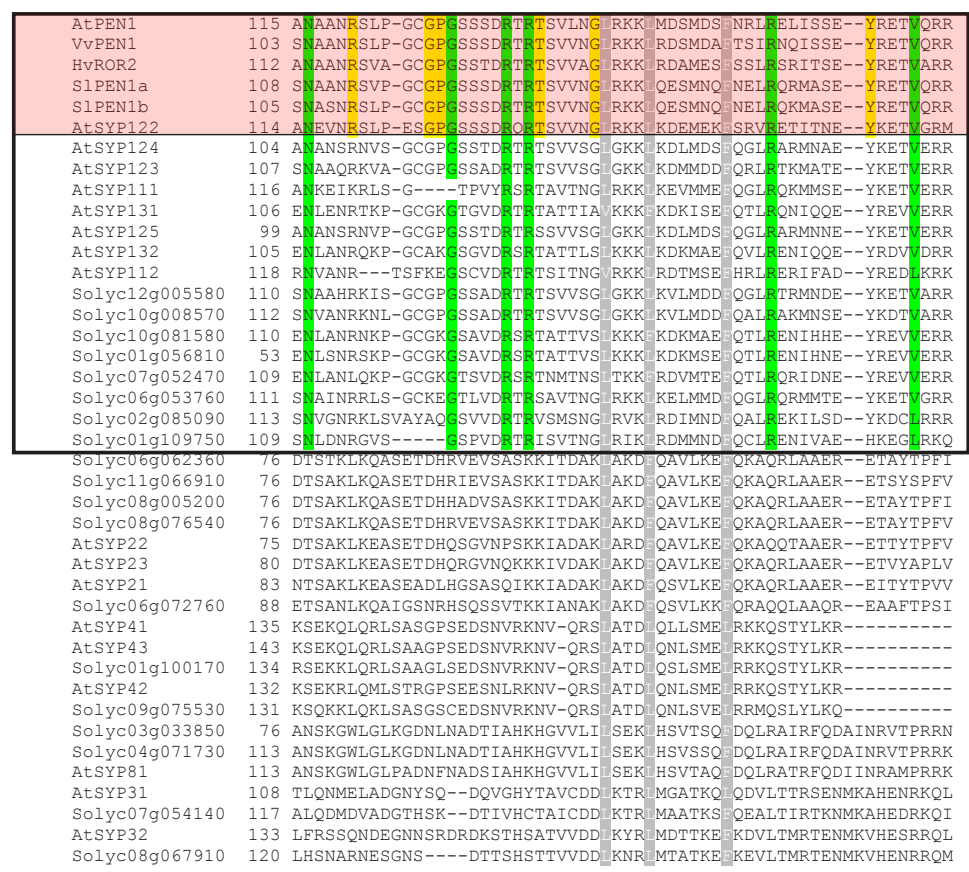




\begin{tabular}{|c|c|c|}
\hline AtPEN1 & 172 & ENPDERT \\
\hline VVPEN1 & 160 & YFTVTGENPDEKTV \\
\hline HVROR2 & 169 & YFTVTGSQPDEATL \\
\hline SIPEN1a & 165 & YFTVTGENPDEGTL \\
\hline SIPEN1b & 162 & YYTVTGENPDEAVL--------- \\
\hline AtSYP122 & 171 & CETVIGEYPDEATL $-\cdots$ \\
\hline AtSYP124 & 161 & 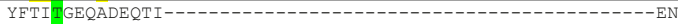 \\
\hline AtSYP123 & 164 & YFTVTGQKADEETV---------- \\
\hline AtSYP111 & 169 & YFTVTGEHANDEMI-------------------- \\
\hline AtSYP131 & 163 & 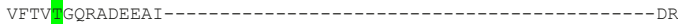 \\
\hline AtSYP125 & 156 & 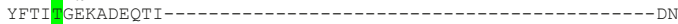 \\
\hline AtSYP132 & 162 & VYTVTGERADEDTI--- - - - - - - - \\
\hline AtSYP112 & 173 & YFLATGEEPSNEDM- - - - \\
\hline Solyc12g005580 & 167 & 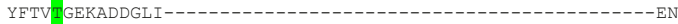 \\
\hline Solyclog008570 & 169 & 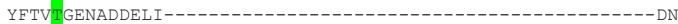 \\
\hline Solyclog0 81580 & 167 & 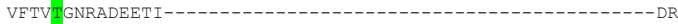 \\
\hline Solyc01g056810 & 110 & VYTVTGNRADEETI-------------------- \\
\hline Solyc07g052470 & 166 & VITVTGTRPDEETI- \\
\hline Solyc06g053760 & 168 & YFTVTGEHPDEEVI--------------------- \\
\hline Solyc02g085090 & 171 & YYNETGKEPNEEVI------ \\
\hline Solyc0lg109750 & 162 & 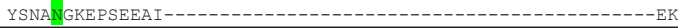 \\
\hline SOIyc06g062360 & 134 & PQAVLPSSYIDGEV - \\
\hline Solyc11g066910 & 134 & PQAVLPSSYTNSEI - - - \\
\hline Solyc08g005200 & 134 & 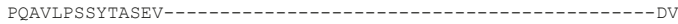 \\
\hline Solyc08g076540 & 134 & PQAVLPSSYTASEI--------------------- \\
\hline AtSYP22 & 133 & PQSALPSSYTAGEV \\
\hline AtSYP23 & 138 & HKPSLPSSYTSSEI I- \\
\hline AtSYP21 & 141 & TKE-IPTSYNAPEL----- \\
\hline Solyc06g072760 & 146 & SQEI - NSSRSIEI \\
\hline AtSYP 41 & 184 & 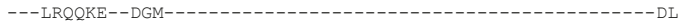 \\
\hline AtSYP 43 & 192 & $---\mathrm{LRLQKE}--\mathrm{DGA}---$ \\
\hline Solyc01g100170 & 183 & 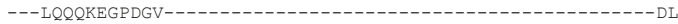 \\
\hline AtSYP 42 & 181 & ---LQQQKEGQDEV---------------------- \\
\hline Solyc09g075530 & 180 & 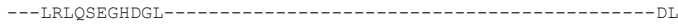 \\
\hline Solyc03g033850 & 136 & RKSTTKSNAAEASA \\
\hline Solyc04g071730 & 173 & 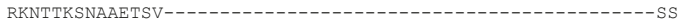 \\
\hline AtSYP81 & 173 & PKRVIKE- $\mathrm{AT}$ III I- \\
\hline AtSYP31 & 166 & FSTKNAVD--SP--------PQNNAKSVPEPPPWSSSSNPFGNLQQPLLPPLNTGAPPGS \\
\hline Solyc07g054140 & 175 & FSTNLSRE--NP--1--1--LK---QPTAEPP PWSTCQS $-1-1-1-$ LTAID - AQGSN \\
\hline AtSYP32 & 193 & 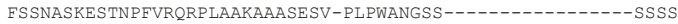 \\
\hline Solyc08g067910 & 176 & FSSSTSKEASNPFMRQRPLASRNTASTSASPPPWAN-DS-------------PSSS \\
\hline EN1 & 188 & LIS---TGES---ERFLQKAIQEQ-GRGRVLDTINEI \\
\hline VVPEN1 & 176 & LIS---TGES---ETFLQKAIQEQ-GRGRVLDTISEI \\
\hline HvROR2 & 185 & LAE---TGEG---ERLIQRAIAEQQGRGEVLGVVAEI \\
\hline SIPEN1a & 181 & LIS---TGQS---ETFLQKAIQEQ-GRGQVMDTVMEI \\
\hline SIPEN1b & 178 & LIS---TGQS---ETFLQKAIQEQ-GRGQVMDT IMEI---------QE HEAVKEIERN \\
\hline AtSYP122 & 187 & LIS---TGES---ETFLQKAIQEQ-GRGRILDTINE I---------QE HDAVKDIEKS \\
\hline AtSYP124 & 177 & LIS---SGES---ENFLQKAIQEQ-GRGQILDTISEI- \\
\hline AtSYP123 & 180 & LIS---SGES---ERFLQKAIQEQ-GRGQVMDTLSEI \\
\hline AtSYP111 & 185 & IIT-----DNAGGEEFLTRAIQEH-GKGKVLETVVEI--- \\
\hline AtSYP131 & 179 & 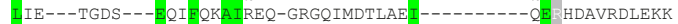 \\
\hline AtSYP125 & 172 & LIA---SGES---ENFLQKAIQEQ-GRGQILDTISEI- \\
\hline AtSYP132 & 178 & LIE---TGNS---EQIFQKAIQEQ-GRGQVMDTLAEI---------QE RHDAVRDLEKK \\
\hline AtSYP112 & 189 & MIS---GSGS--------CSDLVKTFEVKPEM- \\
\hline Solyc12g005580 & 183 & LIS---SGES---ESFLQKAIQEQ-GRGQIMDTISEI- \\
\hline Solyc10g008570 & 185 & LIS---SGES---ESFLQKAIQEQ-GRGQIMDTISEI- \\
\hline Solyclog0 81580 & 183 & LIE---TGDS---EQIFQKAIQQQ-GRGQIMGTLAEI- \\
\hline Solyc01g056810 & 126 & LIE---TGDS---EQIFQKAIREQ-GRGQIMDTLAEI- \\
\hline Solyc07g052470 & 182 & LIE---TGNS---EQIFQNAIQGM-GRGQVLSTVEEI----------QE HDAVKEIERK \\
\hline Solyc06g053760 & 184 & IIS---SGNGQGGEEFLSRAIQEH-GRGKVLETVVEI---------QD HDAREIEKS \\
\hline Solyc02g085090 & 187 & MVS --- GE ----1 SGKVQIFAAKTEM ----- NLDDKD HEAVMDIKKS \\
\hline Solyc0lg109750 & 178 & 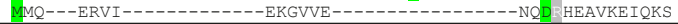 \\
\hline Solyc06g062360 & 150 & SSD---KGQE--------QRALLVESRRQEVLLLDINEISF---INEAIIEE EQGIQEVQQQ \\
\hline Solyc1lg066910 & 150 & SSD---KSQE-------QRALLVESRRQEVVLLDNE ISF---NEA I IEE DQ G IQEVQQQ \\
\hline Solyc08g005200 & 150 & ASD---KSQE-------QRALLVESRRQDVLYLDNEIAF---NEA I IEE DLGIQEVQQQ \\
\hline Solyc08g076540 & 150 & SSG---KSPE-------QRALLVESRRQEVLLLDNEIAF---NEAI IEE EQGIQEIQQQ \\
\hline AtSYP22 & 148 & ------KVPE-------QRAQLQESKRQELVLLDNEIAF---NEAVIEE EQGIQEI HQQ \\
\hline AtSYP23 & 154 & NGD---KHPE-------QRALLVESKRQELVLLDNEIAF---NEAVIEE EQGIQEIQQQ \\
\hline AtSYP21 & 156 & ESL---RISQ-------QQALLLQSRRQEVVFLDNEITF---NEA I IEE EQG REIEDQ \\
\hline Solyc06g072760 & 160 & SSS---ISPE-------SSS I LLESKRQDVVQLEHEIVF---NKAI IEE EQGMIEIQQQ \\
\hline AtSYP 41 & 195 & EMN---LSRN-------RYR-PEEDDFGDML-NEHQMSKIKKSEEVSVE EKEIQQVVES \\
\hline AtSYP43 & 203 & EMN---LNGS-------RYK-AEDDDF DDMVFSEHQMSKIKKSEE IS IE EKEIQQVVES \\
\hline Solyc01g100170 & 196 & EMN---LNGS-------HSR-RDDDDLDDLGFNEHQMAKLKKSEAFTVE EREIQQVVES \\
\hline AtSYP42 & 194 & EFN---VNGK------MSRLDEEDELGGMGFDEHQT I KLKEGQHVSAE ERE IQQVLGS \\
\hline Solyc09g075530 & 193 & EMN---EKKS-------SFL---DDDFNDVGFTELQMATGQKDEQFTAE EREIRQVLKS \\
\hline Solyc03g033850 & 152 & SLDPDMKRDSEVRDNDVSQAAPMRVQEQ-LLDDETRALQVELNSLL-----DSVQETETN \\
\hline Solyc04g071730 & 189 & NLDPNMKRDSEGLGDPDTQAAPIRVQEQ-LLDDETRALQVELNSLL-----DSVQETETK \\
\hline AtSYP 81 & 186 & TLG-----NSESIEPDEIQAQPRRLQQQQLLDDETQALQVELSNLL-----DGARQTETK \\
\hline AtSYP31 & 216 & QL--RRRSAIENAP----SQQMEMSLLQQTVPKQENYS---------QS AVALHSVESR \\
\hline Solyc07g054140 & 211 & QL--RRRLASDNPP----SNELEMSMLQDQVPRQESYS---------QS ATALQNVEST \\
\hline AtSYP32 & 235 & QLVPWKPGEGESSPLLQQSQQQQQQQQQQQQMVPLQDTYM--------QG AEALHTVEST \\
\hline Solyc08g067910 & 218 & QLFPRKQGDGDTQPLLQD---QQQQQQQQQQIVPLQDSYM---------QS AEALQNVEST \\
\hline
\end{tabular}

FIGURE S1. 
Qa- SNARE

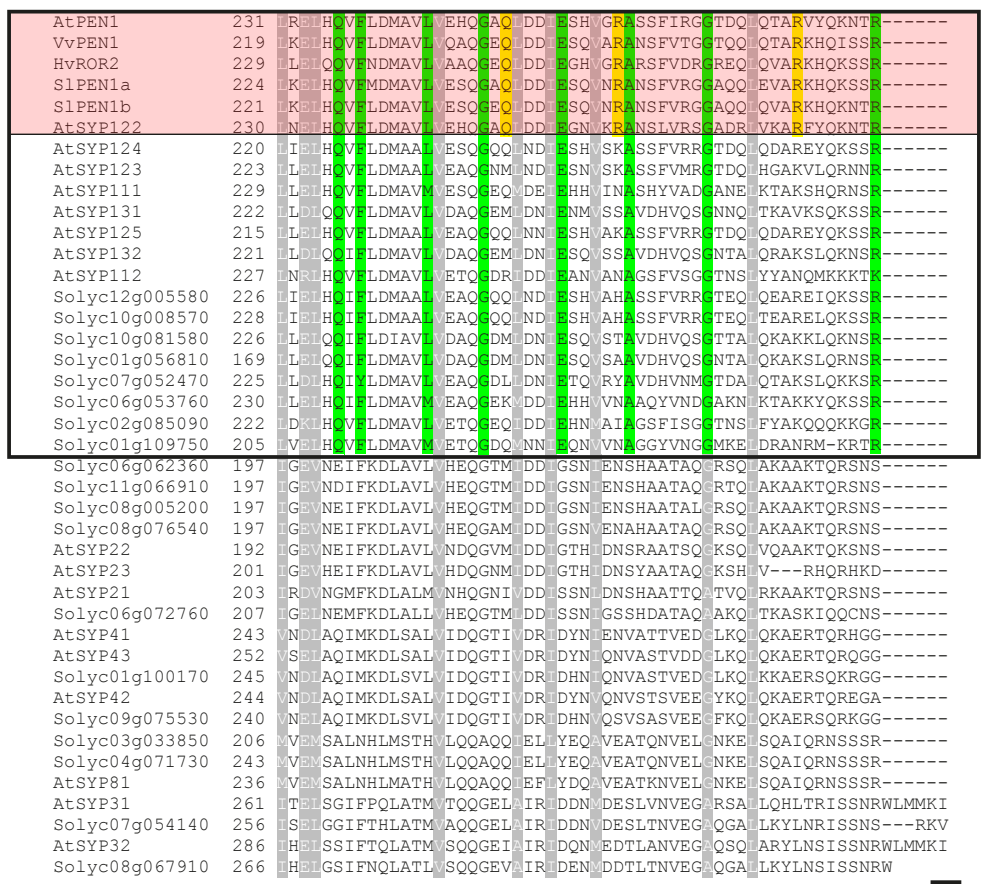

\begin{tabular}{|c|c|c|}
\hline AtPEN1 & 285 & \\
\hline VVPEN1 & 273 & $x_{1}$ \\
\hline HVROR2 & 283 & 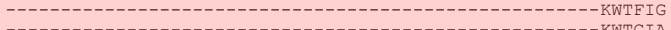 \\
\hline SIPEN1a & 278 & $---K W T C I A$ \\
\hline SIPEN1b & 275 & 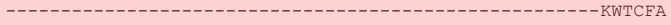 \\
\hline AtSYP122 & 284 & - \\
\hline AtSYP124 & 274 & 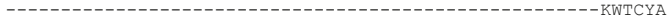 \\
\hline AtSYP123 & 277 & 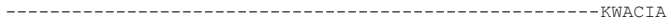 \\
\hline AtSYP111 & 283 & 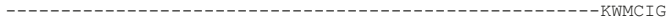 \\
\hline AtSYP131 & 276 & ---------KWMCIA \\
\hline AtSYP125 & 269 & -1--1 \\
\hline AtSYP132 & 275 & 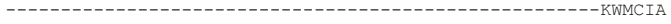 \\
\hline AtSYP112 & 281 & $-n_{n}$ \\
\hline Solyc12g005580 & 280 & -- KCACFA \\
\hline Solyclog008570 & 282 & 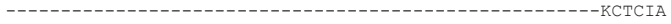 \\
\hline Solyclog0 81580 & 280 & 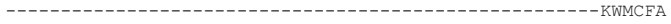 \\
\hline Solyc01g056810 & 223 & - \\
\hline Solyc07g052470 & 279 & -------KCMMIA \\
\hline Solyc06g053760 & 284 & 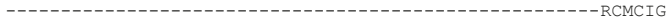 \\
\hline Solyc02g085090 & 276 & --------------- \\
\hline Solyc01g109750 & 258 & 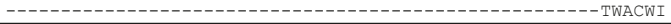 \\
\hline SOIycurgo & 251 & \\
\hline Solycllg & 251 & 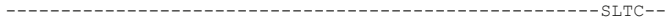 \\
\hline Solyc08g00 & 251 & 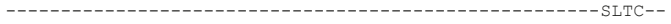 \\
\hline Solyc08g076540 & 251 & --SLTC-- \\
\hline AtSYP22 & 246 & 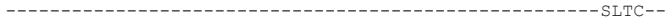 \\
\hline AtSYP23 & 252 & 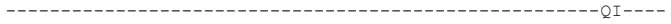 \\
\hline AtSYP21 & 257 & 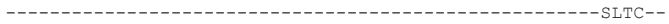 \\
\hline Solyc06g072760 & 261 & 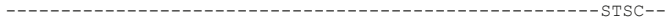 \\
\hline AtSYP41 & 297 & - \\
\hline AtSYP43 & 306 & 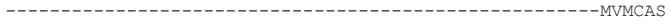 \\
\hline Solyc01g100170 & 299 & 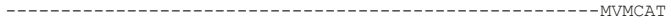 \\
\hline AtSYP42 & 298 & 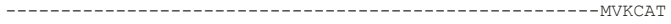 \\
\hline Solyc09go & 294 & - \\
\hline Solyc03g033850 & 260 & ------- \\
\hline Solyc04g071730 & 297 & 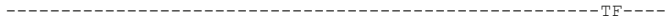 \\
\hline AtSYP81 & 290 & 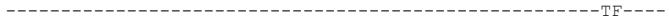 \\
\hline AtSYP31 & 321 & 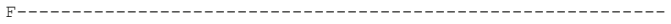 \\
\hline Solyc07g054140 & 313 & SCTENYTTAACTTCCTGCAAAGTTCATGAATAATATTCACTTTCTCACACCATACAGCGA \\
\hline AtSYP32 & 346 & \\
\hline
\end{tabular}

FIGURE S1. 


\begin{tabular}{|c|c|c|}
\hline $\begin{array}{l}\text { AtPEN1 } \\
\text { VVPEN1 }\end{array}$ & $\begin{array}{l}291 \\
279\end{array}$ & IIILIVI I LLIVLFTVRPWENN -- \\
\hline HVROR2 & 289 & IGILLVVILIIVIPIVLKNTNKSNNNNSQQ-- - \\
\hline SIPEN1a & 284 & I I I LLI IVLVVVL-SIQPWKK--------- \\
\hline SIPEN1b & 281 & I I LLLI I I LIVVL-SIQPWKK------ - \\
\hline AtSYP122 & 290 & ILLLLI IVVLIVVFTVKPWESNGGGGG \\
\hline AtSYP124 & 280 & ILLFIVVFALLLI----------- \\
\hline AtSYP123 & 283 & TILAIVVVIVILF--------- \\
\hline AtSYP111 & 289 & IIVLLLIILIVVIPIITSFSSS-- \\
\hline AtSYP131 & 282 & ILILLIIIIITVISVLKPWTQKNG-. \\
\hline AtSYP125 & 275 & I ILFIVIFILLLI - - - - - - \\
\hline AtSYP132 & 281 & 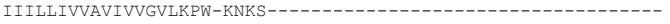 \\
\hline AtSYP112 & 287 & SILGVLILLVCVISML- - \\
\hline Solyc12g005580 & 286 & 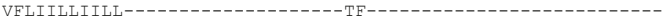 \\
\hline Solyclog008570 & 288 & ILLI I LLI IKLHLQIVSMFLKLLGHRHYSSVTFFTNPPSKESE I I HLCRSDLLSQAIKLL \\
\hline Solyclog0 81580 & 286 & IMI LLI IVAI IVVGVLKPWQSNKG \\
\hline Solyc01g056810 & 229 & I I ILLI IVAVIVVGVLKPWNSNKG- \\
\hline Solyc07g052470 & 285 & I I LLLI IAAI IVLSVIKPWKK---- \\
\hline Solyc06g053760 & 290 & AI I LLI LILVVI IPIATSFTKS--- \\
\hline Solyc02g085090 & 282 & WAVLLI I LVVCLIATL-------- \\
\hline Solyc0lg109750 & 264 & GALVLVFLLICLIAIL------ \\
\hline Solycuogur & 255 & -LLLVIFGIVLLIVII- \\
\hline Solyc1lg066910 & 255 & -LLLVIFGIVLLIVII-------- \\
\hline Solyc08g005200 & 255 & - \\
\hline Solyc08g076540 & 255 & -LLLVIFGIVLLIVIV---.---. \\
\hline AtSYP22 & 250 & -LLLVIFGIVLLIVII--------- \\
\hline AtSYP23 & 254 & --------- LLCLI ------- \\
\hline AtSYP21 & 261 & - - - - \\
\hline Solyc06g072760 & 265 & -LLLVIFGVILLIIIV- \\
\hline AtSYP41 & 303 & 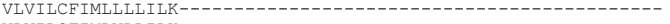 \\
\hline AtSYP4 3 & 312 & VLVILCF IMLVLLILK----- - \\
\hline Solyc01g100170 & 305 & VLVIMCF IMLVLLILK----- \\
\hline AtSYP42 & 304 & 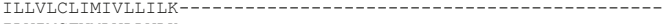 \\
\hline Solyc09g075530 & 300 & ILVIMCFVMLVLLVLK- \\
\hline Solyc03g033850 & 262 & -LLLFLVVLTFS ILFLDWYS---- \\
\hline Solyc04g071730 & 299 & 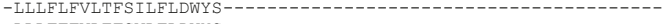 \\
\hline AtSYP81 & 292 & 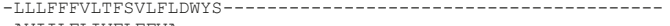 \\
\hline $\begin{array}{l}\text { AtSYP31 } \\
\text { Solyc07g }\end{array}$ & $\begin{array}{l}322 \\
373\end{array}$ & 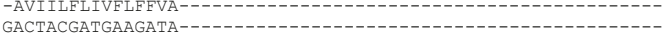 \\
\hline AtSYP32 & 347 & -FVLIAFLMIFLFFVA -- - \\
\hline
\end{tabular}

\begin{tabular}{|c|c|c|}
\hline \multicolumn{3}{|l|}{ AtPENI } \\
\hline \multicolumn{2}{|l|}{ VVPEN1 } & \\
\hline \multicolumn{2}{|l|}{ HVROR2 } & \\
\hline \multirow{2}{*}{\multicolumn{2}{|c|}{$\begin{array}{l}\text { SIPEN1a } \\
\text { SIPEN1b }\end{array}$}} & 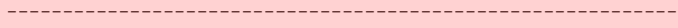 \\
\hline & & 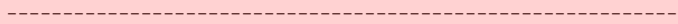 \\
\hline \multicolumn{2}{|l|}{ AtSYP122 } & $-(--------------------------------------------------------------------$ \\
\hline AtSYP124 & 293 & 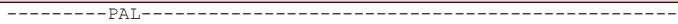 \\
\hline AtSYP123 & 296 & 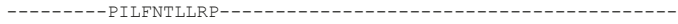 \\
\hline AtSYP111 & & - \\
\hline AtSYP131 & & - \\
\hline AtSYP125 & 288 & 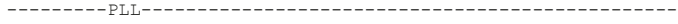 \\
\hline \multicolumn{2}{|l|}{ AtSYP132. } & - \\
\hline \multicolumn{2}{|l|}{ AtSYP112 } & 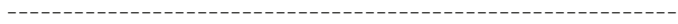 \\
\hline Solyc12g005580 & 299 & - \\
\hline Solyc10g008570 & 348 & KSTEKISSKPIVYATLIQTCTKSHSFNHGVQFHTHVIKTG IETDRFVGNSLLALYFKLGS \\
\hline \multicolumn{2}{|l|}{ Solyc10g081580 } & 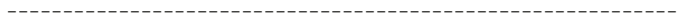 \\
\hline \multicolumn{2}{|l|}{$\begin{array}{l}\text { Solyc01g056810 } \\
\text { Solyc07g052470 }\end{array}$} & - \\
\hline \multirow{2}{*}{\multicolumn{2}{|c|}{$\begin{array}{l}\text { Solyc07g052470 } \\
\text { Solyc06g053760 }\end{array}$}} & - \\
\hline & & 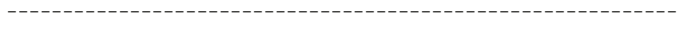 \\
\hline \multicolumn{2}{|l|}{ Solyc02g085090 } & - \\
\hline \multirow{2}{*}{\multicolumn{2}{|c|}{$\frac{\text { Solyc01g109750 }}{\text { Solycubgubzउ60 }}$}} & - - - - \\
\hline & Solyc11g066910 & 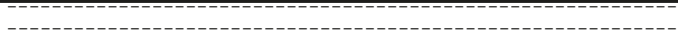 \\
\hline \multirow{2}{*}{\multicolumn{2}{|c|}{ Solyc08g005200 }} & - \\
\hline \multirow{2}{*}{\multicolumn{2}{|c|}{$\begin{array}{l}\text { Solyc08g076540 } \\
\text { AtSYP22 }\end{array}$}} & 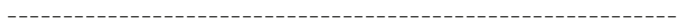 \\
\hline & & 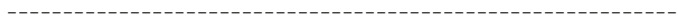 \\
\hline \multicolumn{2}{|l|}{ AtsYP23 } & - \\
\hline \multicolumn{2}{|l|}{ AtSYP21 } & 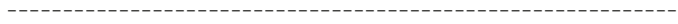 \\
\hline \multicolumn{2}{|l|}{ Solyc06g072760 } & - - \\
\hline \multicolumn{2}{|l|}{ AtSYP 41} & 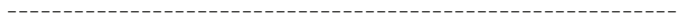 \\
\hline \multicolumn{2}{|l|}{ AtSYP 43} & 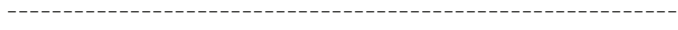 \\
\hline \multicolumn{2}{|l|}{ Solyc01g100170 } & - - - - - - - - - - - - \\
\hline \multicolumn{2}{|l|}{ AtSYP 42} & 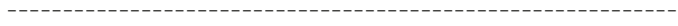 \\
\hline \multicolumn{2}{|l|}{ Solyc09g075530 } & 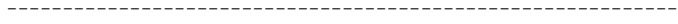 \\
\hline \multicolumn{2}{|l|}{ Solyc03g033850 } & - \\
\hline \multicolumn{2}{|l|}{ Solyc04g0 71730} & - - \\
\hline \multicolumn{2}{|l|}{$\begin{array}{l}\text { AtSYP81 } \\
\text { AtSYP31 }\end{array}$} & $\begin{array}{l}-1-1 \\
-1\end{array}$ \\
\hline AtSYP31 & & 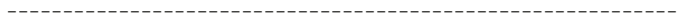 \\
\hline \multicolumn{2}{|l|}{ Solyc07g054140 } & - \\
\hline \multicolumn{2}{|l|}{ AtSYP32 } & \\
\hline
\end{tabular}

\section{FIGURE S1.}




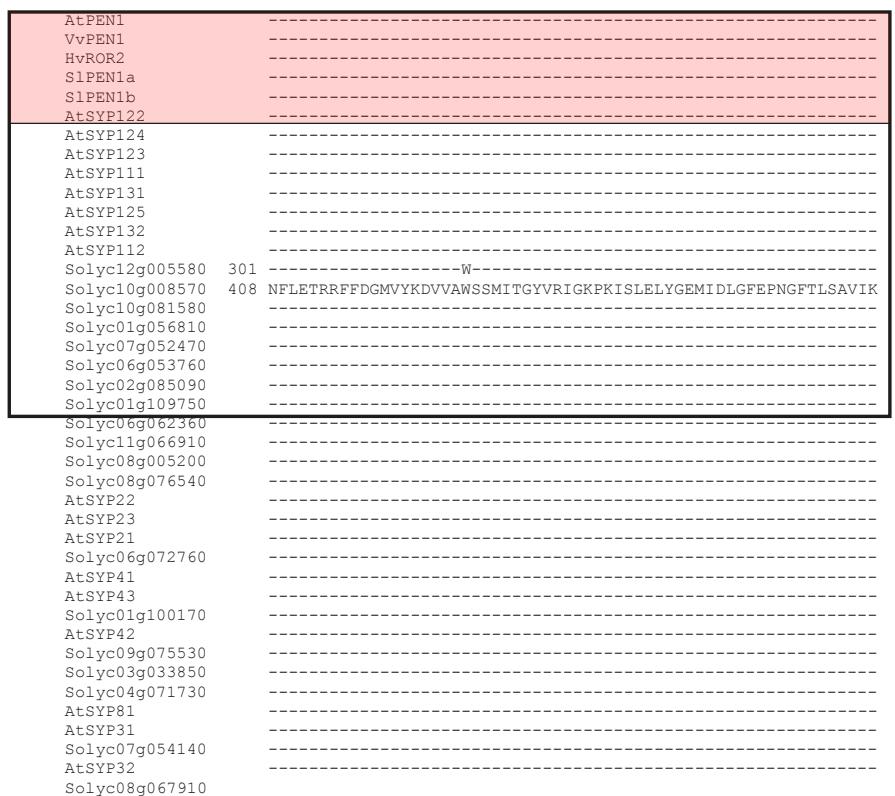

FIGURE S1. Multialignment of plant syntaxins. The dataset includes all the Arabidopsis syntaxins (AtSYPs and AtPEN1), barley HvROR2, grapevine VvPEN1 and 21 putative syntaxins retrieved from the tomato proteome in this study (indicated by the SolGenomics Network database ID code). The position of characteristic domains of syntaxins (the three helix domain $\mathrm{Ha}, \mathrm{Hb}$ and $\mathrm{Hc}$, the Qa-SNARE domain and the membrane-spanning (MS) domain are indicated. Gray color indicates residues physio-chemically conserved throughout the dataset; green color indicates residues physio-chemically conserved in SYP1 syntaxins. Yellow color highlights 24 residues specifically conserved in syntaxins of the SYP1b subclade described in this study. 


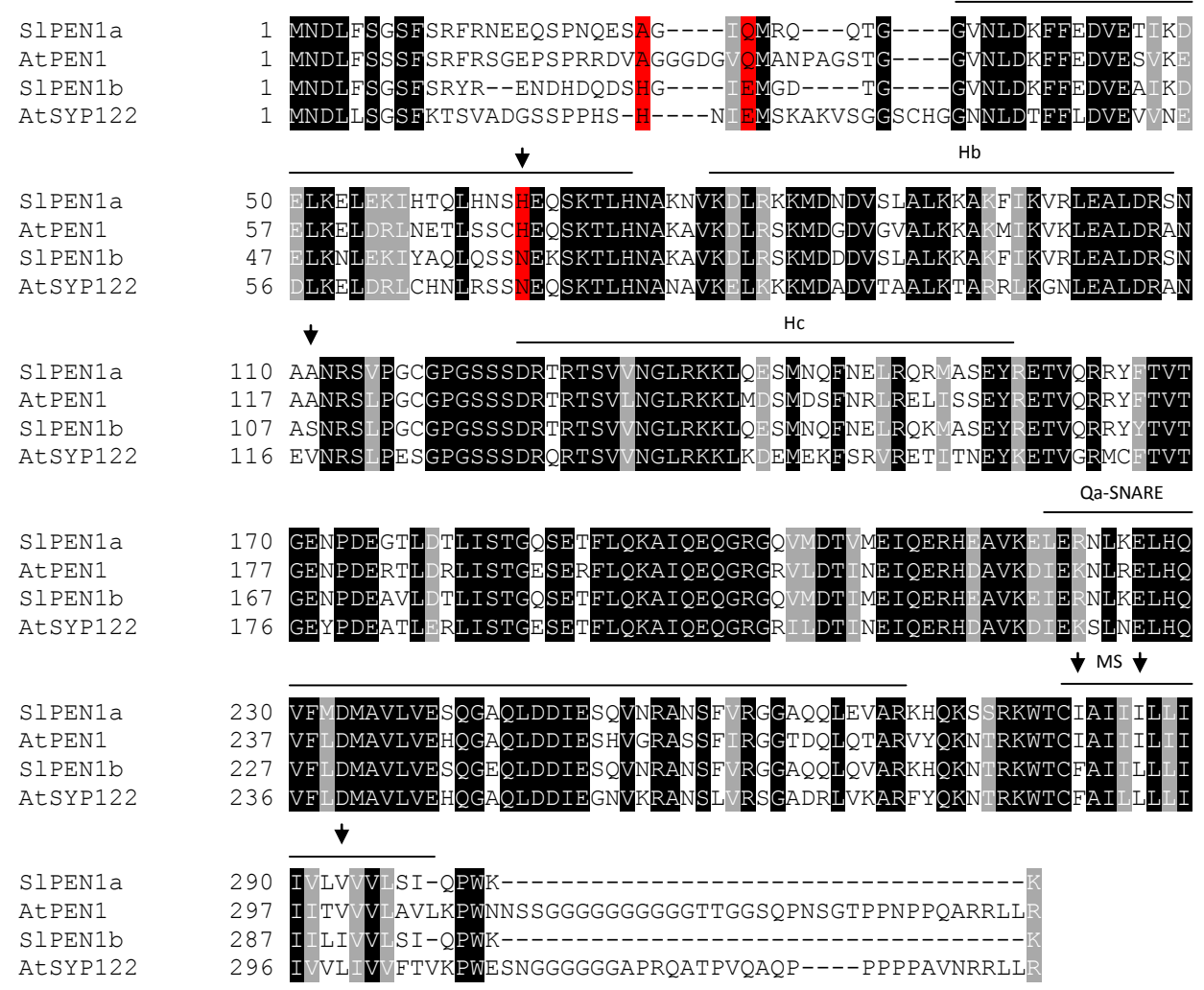

FIGURE S2. Four syntaxins multi alignment. The dataset includes Arabidopsis AtPEN1 and AtSYP122 and tomato SIPEN1a and SIPEN1b. The position of characteristic syntaxin domains (the three helix domain $\mathrm{Ha}, \mathrm{Hb}$ and Hc, the Qa-SNARE domain and the membrane-spanning (MS) domain are indicated. The arrows indicates the amino acid differences between functional syntaxins (AtPEN1 and SIPEN1a) and not functional ones (AtSYP122 and SIPEN1b). Red colour indicates substitutions in which the biochemical properties of the residues are not preserved. 



\section{Chapter 4}

Identification of candidate $M L O$ susceptibility genes in cultivated Solanaceae and functional characterization of tobacco NtMLO1 and eggplant SmMLO1

Michela Appiano ${ }^{\dagger}$, Stefano Pavan ${ }^{\dagger}$, Valentina Bracuto ${ }^{\dagger}$, Domenico Catalano, Zheng Zheng, Concetta Lotti, Richard G. F. Visser, Luigi Ricciardi, Yuling Bai* ${ }^{+}$these authors contributed equally to the work * corresponding author

Chapter composed of published data (NtMLO1) and submitted data (SmMLO1), both in Transgenic Research Link to the published paper here: http://link.springer.com/article/10.1007/s11248-015-9878-4 


\begin{abstract}
Specific homologs of the plant $\underline{M}$ ildew Locus $\underline{O}$ (MLO) gene family act as susceptibility factors towards the powdery mildew (PM) fungal disease, causing significant economic losses in agricultural settings. Thus, in order to obtain PM resistant phenotypes, a general breeding strategy has been proposed, based on the selective inactivation of MLO susceptibility genes across cultivated species. In this study, PCR-based methodologies were used in order to isolate $M L O$ genes from cultivated solanaceous crops that are hosts for PM fungi, namely eggplant, potato and tobacco, which were named SmMLO1, StMLO1 and NtMLO1, respectively.

Based on phylogenetic analysis and sequence alignment, these genes were predicted to be orthologs of tomato SIMLO1 and pepper CaMLO2, previously shown to be required for PM pathogenesis. Full-length sequences of the tobacco NtMLO1 and eggplant SmMLO1 homologs were used for two heterologous transgenic complementation assays. In both cases restoration of PM susceptibility was observed, which indicates that NtMLO1 and SmMLO1 are PM susceptibility factors in their species. The same assay showed that a single nucleotide change in a mutated NtMLO1 allele leads to complete gene loss-offunction. Results here presented, also including a complete overview of the tobacco and potato $M L O$ gene families, are valuable to study $M L O$ gene evolution in Solanaceae and for molecular breeding approaches aimed at introducing PM resistance using strategies of reverse genetics.
\end{abstract}

\title{
Keywords
}

Eggplant, tobacco, MLO, powdery mildew, resistance, plant breeding 


\section{Introduction}

Powdery mildew (PM) is a major fungal disease affecting thousands of plant species, caused by ascomycete fungi belonging to the order of Erysiphales (Glawe 2008). Chemical control of PM accounts for a large proportion of fungicides used in agricultural settings (Hewitt 1998). Therefore, the use of cultivars harbouring genetic sources of PM resistance is generally envisaged as a valuable strategy to reduce farming costs and to cope with public concerns related to environmental pollution and human health.

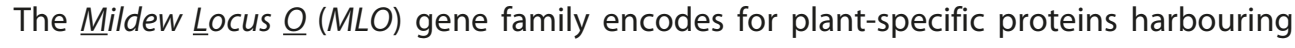
several transmembrane domains, topologically reminiscent of metazoan G-protein coupled receptors (Devoto et al. 2003). Specific homologs of the MLO family act as susceptibility genes towards PM fungi. Indeed, their inactivation, through loss-of-function mutations or silencing, has been associated with a peculiar form of PM resistance, referred to as mlo resistance (Pavan et al. 2010). This is associated with the enhancement of exocytosis defence pathways at plant-pathogen interaction sites, which are thought to contribute to the prevention of fungal penetration into host cells (Assaad et al. 2004). Initially discovered in barley, mlo resistance has been later shown to occur in other plant species as well, specifically Arabidopsis, tomato, pea, pepper and bread wheat (Bai et al. 2008; Büschges et al. 1997; Consonni et al. 2006; Humphry et al. 2011; Pavan et al. 2011; Wang et al. 2014; Zheng et al. 2013). This eventually led to the formalization of a breeding approach based on the systematic inactivation of MLO susceptibility genes across cultivated species affected by the PM disease (Dangl et al. 2013; Pavan et al. 2010; Pavan et al. 2011). Proof of concept for this strategy has been recently provided by the work of Wang et al. (2014), reporting the introduction of PM resistance in bread wheat following targeted mutagenesis of three $M L O$ homoeoalleles. In contrast with most genetic sources of PM resistance, experimental data clearly indicate that mlo immunity is not specific towards particular fungal isolates and is extremely durable. For example, loss-of-function mutations of barley HVMLO confer resistance to all known isolates of the PM fungus Blumeria graminis f. sp. hordei, and is successfully employed in barley breeding since 1979 (Lyngkjaer et al. 2000). Similarly, pea er1 PM resistance, originating from the loss of function of PSMLO1, was first reported more than sixty years ago and is the only resistance source worldwide used for breeding purposes (Harland 1948; Humphry et al. 2011; Pavan et al. 2013).

Following the completion of the respective genome sequencing projects, a number of MLO homologs variable between 12 and 19 has been identified in the diploid species Arabidopsis, rice, grapevine, peach, woodland strawberry and cucumber (Devoto et al. 2003; Feechan et al. 2008; Liu and Zhu 2008; Pessina et al. 2014; Schouten et al. 2014). Remarkably, when placed in MLO protein family phylogenetic trees, all dicot MLO 
isoforms experimentally shown to be required for PM susceptibility group in the same clade, referred to as clade $\mathrm{V}$ in scientific literature (e.g. Feechan et al. 2008; Pavan et al. 2011; Acevedo-Garcia et al. 2014). This shows that evolutionary studies on MLO proteins may predict candidates for being PM susceptibility factors.

Concerning solanaceous crops, we have functionally characterized the two MLO orthologs SIMLO1 in tomato and CaMLO2 in pepper, whose inactivation is causally associated with PM resistance (Bai et al. 2008; Zheng et al. 2013). In this work, we report the isolation, through a PCR-based approach, of three MLO genes from other cultivated Solanaceae, namely eggplant, potato and tobacco, which are likely to share a relation of orthology with SIMLO1 and CaMLO2. The tobacco NtMLO1 and the eggplant SmMLO1 homologs were chosen for a transgenic complementation assay, resulting in their functional characterization and identification of a loss-of-function mutant allele of NtMLO1. Finally, newly available tobacco and potato genome sequences (Sierro et al. 2014; The Potato Genome Consortium 2011) were exploited to provide a comprehensive overview of the $M L O$ gene families in these species.

\section{Materials and methods}

\section{PCR-based isolation and phylogenetic characterization of MLO putative orthologs}

Young leaves of eggplant (Solanum melongena cv. Half Lange Violette), potato (Solanum tuberosum cv. Desiree) and tobacco (Nicotiana tabacum cv. Petit Havana SR1) were collected for RNA extraction, which was performed using the Trizol reagent (Invitrogen). After RNA purification with the NucleoSpin RNA II kit (Macherey-Nagel), cDNA was synthesized using the SuperScript III RT first-strand cDNA synthesis kit (Invitrogen) with oligo(dT) primers.

Aiming to identify sequences of SIMLO1 putative orthologs, the primer pairs Sol-F1 (5'-CATTTGACATTCCCCTTCTTC-3') / Sol-R1 (5'-GCACCATGCATGAGTACCTCT-3') and Sol-F2 (5'-TTGGCAGTTGCTCATGTATTG-3') / Sol-R2 (5'- ATGGTGCCAGCTTCTAAGAG-3') were designed on the untranslated and coding sequences of the SIMLO1 gene (GeneBank accession number NM_001247885), respectively, (Primer3, Rozen and Skaletsky 2000) and used for PCR amplification of cDNAs. Amplicons obtained with the Sol-F2/Sol-R2 primer pair were purified using the NucleoSpin Extract II kit (Macherey-Nagel) and ligated (molar ratio 1:1) into the pGEM-T easy vector (Promega). Recombinant plasmids were cloned in $E$. coli $\mathrm{DH} 10 \beta$ chemically competent cells and recovered by using the Qiaprep spin miniprep kit (Qiagen). Sequencing reactions were performed using universal T7 and SP6 primers (Eurofins MWG Operon). 
In ordertoobtainfull-lengthcoding sequences of potatoand tobacco MLOgenes, sequences overlapping with those of the amplicons above mentioned were retrieved by BLAST search, using the tomato SIMLO1 coding sequence as query against expressed sequence tags (ESTs) and predicted coding sequence repositories, both available at the Sol Genomic Network (SGN) database (http://solgenomics.net), and then used for local alignment. The expression and sequence of candidate genes was verified by PCR amplification of CDNAs, using the primer pairs StMLO1-F (5'- ATGGCTAAAGAACGGTCG -3') / StMLO1-R (5'-TTATTTGTTTCCAAAAGT-3') and NtMLO1-F (5'-ATGGAGGCAACTCCGACTTG-3') / NtMLO1-R (5'-TCAACTCATTTTGTTGCCAAATG-3'), cloning and sequencing, which were performed as above described.

In order to amplify a full-length $M L O$ sequence in eggplant, the following primer pair was used: SmMLO1-F2 (5'-ATGGCTAAAGAACGGTCG-3') / SmMLO1-R1 (5'TTATTGTITCCAAAAGTAAAATCTGA-3'). The corresponding PCR product was cloned and sequenced as indicated above.

Full-length eggplant, potato and tobacco MLO genes (named SMMLO1, StMLO1 and NtMLO1, respectively) were translated in silico. Corresponding protein sequences were used, together with those of dicot MLO proteins experimentally associated with PM susceptibility [Arabidopsis thaliana AtMLO2 (GenBank accession code NP172598), AtMLO6 (NP176350) and AtMLO12 (NP565902), Solanum lycopersicum SIMLO1 (NP001234814), Capsicum annuum CaMLO2 (AFH68055), Pisum sativum PsMLO1 (ACO07297), Lotus japonicus LjMLO1 (AAX77015) and Medicago truncatula MtMLO1 (ADV40949) and those of the remaining twelve homologs of the Arabidopsis thaliana AtMLO protein family, for ClustalW alignment and the construction of a Unweighted Pair Group Method with Arithmetic Mean (UPGMA) phylogenetic tree. Bootstrap values were calculated from 100 replicates. All of these bioinformatic analyses were performed using the CLC sequence viewer software (http://www.clcbio.com/).

\section{Generation of transgenic plants overexpressing NtMLO1 and SmMLO1}

Two different NtMLO1 PCR products, differing for a single nucleotide polymorphism, were inserted into the Gateway-compatible vector pENTR D-TOPO (Invitrogen) and cloned in E. coli competent cells. For functional analysis of SMMLO1, the full-length sequence was amplified using primer pair Fw1aGATE (5'-CACCATGGCTAAAGAACGGTCG-3')/ RV5(5'TTATTGTTCCAAAAGTAAAATCTGA-3'), and ligated according to the manufacturer's instructions in pENTR D-TOPO (Invitrogen).

Presence of the inserts was assessed by colony PCR, restriction enzyme digestion and sequencing using the universal M13 primer pair. 
Inserts were then transferred by LR recombination into the binary plasmid vector pK7WG2, harboring the $35 \mathrm{~S}$ Cauliflower Mosaic Virus (CaMV) promoter for constitutive expression and the marker gene nptll for kanamycin resistance selection. Plasmids were inserted into $E$. coli competent cells and positive colonies were again screened by colony PCR and sequencing, as above. Recombinant vectors were finally extracted and transferred to the AGL1-virG strain of $A$. tumefaciens by electroporation.

A selected PM resistant tomato line, named SImlo1, described by Bai et al. (2008) and carrying a loss-of-function deletion in the SIMLO1 coding sequence, was used for transformation. This was performed according to the method described by McCormick et al. (1986). Briefly, seeds were surface-sterilized and sown on half-strength Murashige and Skoog (MS) agar supplemented with sucrose (10 g/l). Cotyledons were excised from 10-day-old seedlings, cut in two parts and submerged in an A. tumefaciens suspension with an $\mathrm{OD}_{600}$ value of about 0.125 . Infected cotyledonary explants were placed abaxially on the GCF10 medium (4.3 g/l MS basal salt mixture, $8 \mathrm{~g} / \mathrm{l}$ agar, $30 \mathrm{~g} / \mathrm{L}$ sucrose, 108.73 $\mathrm{mg} / \mathrm{l}$ Nitsch vitamins, $1.5 \mathrm{mg} / \mathrm{l}$ zeatin riboside, $0.2 \mathrm{mg} / \mathrm{l}$ indole-3-acetic acid, $\mathrm{pH} 5.8$ ) supplemented with $1 \mathrm{ml} / \mathrm{l}$ acetosyringone at $25^{\circ} \mathrm{C}$ for $48 \mathrm{~h}$. Then, they were transferred to the GCF10 medium to which $100 \mathrm{mg} / \mathrm{ml}$ timentin and $50 \mathrm{mg} / \mathrm{ml}$ kanamycin were added and sub-cultured onto fresh medium every 3 weeks until shoot buds were observed. These were excised from the callus and transferred to the GCF11 medium ( $4.3 \mathrm{~g} / \mathrm{l} \mathrm{MS}$ basal salt mixture, $8 \mathrm{~g} / \mathrm{l}$ agar, $30 \mathrm{~g} / \mathrm{L}$ sucrose, $108.73 \mathrm{mg} / \mathrm{l}$ Nitsch vitamins, $1.9 \mathrm{mg} / \mathrm{l}$ zeatin riboside, $\mathrm{pH}$ 5.8) with $100 \mathrm{mg} / \mathrm{ml}$ timentin and $50 \mathrm{mg} / \mathrm{ml}$ kanamycin. After meristem development, the explants were transferred to the root-inducing medium MS30B5 (4.3 g/l MS basal salt mixture, $8 \mathrm{~g} / \mathrm{l}$ agar, $30 \mathrm{~g} / \mathrm{L}$ sucrose, $112 \mathrm{mg} / \mathrm{L}$ vitamin B5, 50 mg/ml kanamycin, $\mathrm{pH}$ 5.8). Once roots were developed, plantlets were finally located on woolen rock and grown in a greenhouse compartment.

For each of the two transformations with a different NtMLO1 gene sequence, $20 T_{1}$ plants and two $T_{2}$ families (each composed by fifteen individuals derived from self-pollination of individual $T_{1}$ plants) were assayed for the presence of the construct, using the primer pair ntplIF (5'- TCGGCTATGACTGGGCACAAC-3') / ntplIR (5'-AAGAAGGCGATAGAAGGCGA-3'), designed on the ntpll gene sequence, and the primer pair 35S-F (5'-GCTCCTACAAATGCCATCA-3') / 35S-R (5'GATAGTGGGATTGTGCGTCA-3'), designed on the $35 \mathrm{~S}$ promoter sequence. DNA of individual $\mathrm{T}_{2}$ plants was isolated from using $2 \%$ CTAB in a protocol adapted for a 96 -well plate (Doyle and Doyle 1987). Expression of the transgene was assessed by qPCR using the primer pair NtMLO1_qFw (5'-GTGGAAATAAGTCCAGCATTATG-3')/ NtMLO1_qRev (5'CACCCAAAGGTACGAGTACAATC- 3').

The expression of the SmMLO1 gene in eight transformed $\mathrm{T}_{1}$ individuals was assessed by qPCR using the following primer pairs: mut-Fw (5'- TGTGCCTGTGGTTGAAACAG - $\left.3^{\prime}\right)$ /mut- 
$\operatorname{Rev}\left(5^{\prime}\right.$ - TAGCCAAATCTGCAGCGTTC - 3') and wt-Fw (5'- TCACTTATTGCGCGGTTACC - 3')/ wt-Rev (5'- TTGGTGGTTGTGAGCATGG -3'). Four T1 individuals (T1_K, T1_M, T1_P and T1_Q) were allowed to generate segregating $T_{2}$ families. DNA isolation and PCR with nptll and $35 \mathrm{~S}$ primer pair to test for the presence of the transgene in individual $\mathrm{T}_{2}$ plants were performed as above.

\section{Disease tests and Oidium neolycopersici quantification on transgenic plants}

Three cuttings per $T_{1}$ individuals and plants of the $T_{2}$ families expressing the NtMLO1 gene were challenged with an isolate of the tomato PM fungus Oidium neolycopersici maintained at the Plant Breeding Department of the University of Wageningen, The Netherlands.

Similarly, $T_{2}$ families of four $T_{1}$ individuals expressing SmMLO1 containing at least six transgenic plants were tested for their response to the same pathogen. Twelve nontransgenic individuals segregating from these families were included.

The SImlo1 mutant line and the susceptible cultivar Moneymaker (MM) were used as controls. Inoculation was performed as described by Pavan et al. (2008), by spraying plants with a suspension of conidiospores obtained from freshly sporulating leaves of heavily infected plants and adjusted to a final concentration of $4 \times 10^{4}$ spores $/ \mathrm{ml}$.

Inoculated plants were grown in a greenhouse compartment at $20 \pm 2^{\circ} \mathrm{C}$ with $70 \pm 15 \%$ relative humidity and day-length of 16 hours. Disease evaluation was carried out fifteen days after inoculation, based on a visual scoring as described by Bai et al. (2008) and/or analytically, by the relative quantification of the ratio between fungal and plant gDNAs. The latter was performed by the qPCR assay reported by Huibers et al. (2013). Specifically, plant and fungal genomic DNAs were extracted from $O$. neolycopersici infected tomato leaves (Qiagen DNeasy Plant Mini Kit) and used for amplification with the primer pairs On-F (5'-CGCCAAAGACCTAACCAAAA-3') / On-R (5'-AGCCAAGAGATCCGTTGTTG-3'), designed on $O$. neolycopersici internal transcribed spacer (ITS) sequences (GenBank accession number EU047564), and Ef-F (5'-GGAACTTGAGAAGGAGCCTAAG-3') / Ef-R (5'-CAACACCAACAGCAACAGTCT-3'), designed on the tomato Elongation Factor 1a (Ef1a) gene (Løvdal and Lillo 2009). Relative quantification was performed by the $2^{-\Delta \Delta c t}$ method (Livak and Schmittgen 2001; Pfaffl 2001).

\section{In silico characterization of the tobacco and potato $M L O$ gene families}

In order to retrieve tobacco and potato MLO homologs, nucleotide sequences of NtMLO1 and StMLO1 and corresponding translated sequences were used as query for BLAST (BLASTn and tBLASTn) search against the Sol Genomics Network (SGN) and the Potato Genomics Resource (Spud DB) databases, using default parameters. 
The number of transmembrane domains was predicted using the online software TMHMM (http://www.cbs.dtu.dk/services/TMHMM/). The putative number of introns was obtained using the online service FGENESH of Softberry (http://www.softberry.com/). Chromosomal localization and gene position of potato $M L O$ genes were inferred by the annotations of the Potato Genome Consortium. Finally, the MEME (http://meme.nbcr. net/) (Bailey et al. 2009) package was used to predict functional motifs in the NtMLO and StMLO protein families.

Predicted tobacco NtMLO and potato StMLO proteins were used to integrate the phylogenetic tree described in the previous section, according to the same methodologies above mentioned.

\section{Results}

\section{Identification of $M L O$ gene sequences from cultivated Solanaceae}

Two primer pairs, one designed on the untranslated sequence and the other on the coding sequence of tomato SIMLO1, were used to amplify homologous sequences from eggplant, potato and tobacco cDNAs. PCRs performed with the Sol-F1/R1 primer pair failed, thus suggesting the occurrence of polymorphic sequences in untranslated regions. In contrast, PCR performed with the Sol-F2/R2 primer pair, designed within the SIMLO1 coding sequence, resulted in single amplification products of $876 \mathrm{bp}$. Full-length sequences of a 1560 bp tobacco gene, named NtMLO1, and a 1557 bp potato gene, named StMLO1, were obtained by assembling partial gene sequences of PCR products with overlapping sequences retrieved by the interrogation of the SGN database. Amplification and sequencing of StMLO1 and NTMLO1 from potato and tobacco CDNAs provided evidence for their actual expression in leaves and validated their sequences. These were deposited in the GenBank database with the accession codes KM244715 (StMLO1) and KM244716 (NtMLO1).

In order to clone an eggplant MLO gene putatively involved in PM susceptibility, several primers were designed, based on the identification of conserved regions from the alignment of SIMLO1, StMLO1 and NtMLO1. These primers were then tested on eggplant CDNA. The SmMLO1-F2/SmMLO1-R1 primer pair produced a single PCR amplification product. The corresponding sequence of 1572 bp was named SmMLO1 and deposited in the GenBank database with the accession code KM244717. 


\section{Bioinformatic analyses support the identification of solanaceous MLO functional orthologs required for PM susceptibility}

StMLO1, NtMLO1 and SmMLO1 protein sequences were used to perform a phylogenetic analysis. With strong bootstrap support, they were found to group in the phylogenetic clade $\mathrm{V}$, containing all the dicot MLO homologs so far experimentally shown to be required for PM susceptibility (AtMLO2, AtMLO6, AtMLO12, SIMLO1, CaMLO2, PsMLO1, LjMLO1 and MtMLO1) (Fig. 1), thus indicating they could possibly be functionally related.

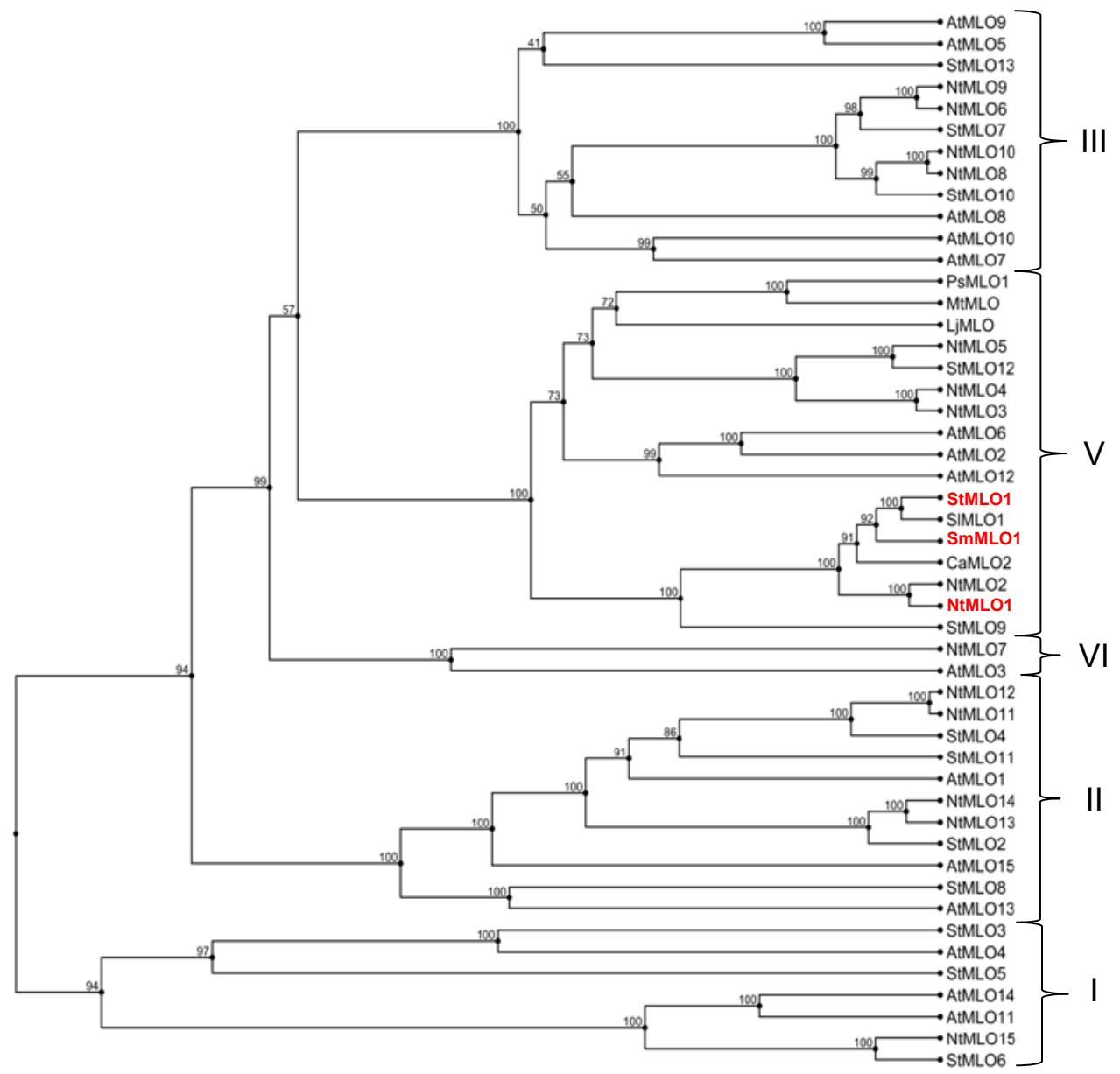

FIGURE 1. UPGMA-based tree of full-length MLO proteins. The dataset includes the tobacco NtMLO, potato StMLO and Arabidopsis AtMLO protein families, tomato SIMLO1, pepper CaMLO2, eggplant SmMLO1, pea PsMLO1, lotus LjMLO1 and barrel clover MtMLO1. Phylogenetic clades are designated with Roman numbers based on the position of AtMLO homologs, according to the nomenclature indicated by Feechan et al. (2008). Homologs identified by means of a PCR-based approach in this study (SmMLO1, StMLO1 and NtMLO1) are indicated in bold red. Numbers at each node represent bootstrap support values (out of 100 replicates). 
Previous studies highlighted the presence of amino acid residues highly conserved either in the whole MLO protein family or in MLO orthologs involved in the interaction with PM fungi, which are predicted to play a key functional role (Elliott et al. 2005; Panstruga 2005). All of these residues were found to be present in the StMLO1, NtMLO1 and SmMLO1 protein sequences (Supplementary Fig. 1), providing further evidence for the identification of $M L O$ genes required for PM susceptibility.

Finally, another strong bioinformatic indication for the identification of solanaceous MLO susceptibility genes was provided by aligning the coding sequences of STMLO1, NtMLO1 and SmMLO1 with those of the PM susceptibility genes SIMLO1 and CaMLO2, functionally characterized in tomato and pepper, respectively (Bai et al. 2008; Zheng et al. 2013) (Supplementary Fig. 2). Indeed, this revealed a very high percentage of nucleotide identity $(81,4 \%$ between tomato and tobacco, $87,5 \%$ between tomato and eggplant and $94,8 \%$ between tomato and potato), suggesting that all of these solanaceous $M L O$ genes are orthologs.

\section{Tobacco NtMLO1 complements tomato SIMLO1 in a functional complementation assay}

In order to characterize NtMLO1 at the functional level, we set up an assay based on its transgenic overexpression in the previously described tomato line SImlo1, which carries a loss-of-function mutation in the tomato SIMlo1 homolog and is thus resistant to the PM fungus O. neolycopersici (Bai et al. 2008). We hypothesised that overexpression of NtMLO1 would have restored PM susceptibility in the tomato SImlo1 mutant line, thereby demonstrating functional conservation between NtMLO1 and SIMLO1.

After transformation, cuttings of $20 \mathrm{~T}_{1}$ transgenic individuals were challenged with 0 . neolycopersici. Fifteen of the tested $T_{1}$ individuals showed restoration of PM symptoms (data not shown). In order to confirm this result, two $T_{2}$ families of the fifteen individuals ( $T_{2}$ a and $\left.T_{2}-b\right)$ derived from self-pollination of two different $T_{1}$ plants were also inoculated, together with MM (the susceptible control) and the SImlo1 mutant line (the resistant control). The presence of the overexpression construct in segregating $T_{2}$ families was assessed by PCR amplification with primer pairs designed on the $n p t / l$ gene and the $35 \mathrm{~S}$ promoter (Supplementary Fig. 3). $T_{2}$ individuals not carrying the overexpression construct $\left[\mathrm{T}_{2}(-)\right]$, as well as individuals of the SImlo1 mutant line, showed no NtMLO1 expression and an average of disease score of about 0.5 . In contrast, $T_{2}$ individuals of the two families positive for the presence of the construct $\left[\mathrm{T}_{2}(+) \_\mathrm{a}\right.$ and $\mathrm{T}_{2}(+)$ _b] showed NtMLO1 expression and an average disease score of 1.8 and 1.7, respectively (Fig. 2 and Supplementary Fig. 4). 


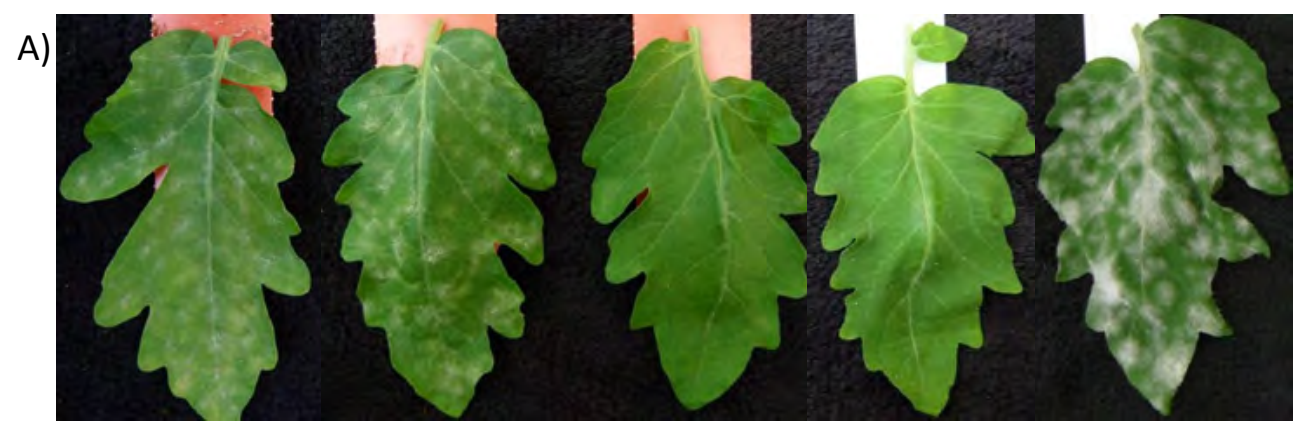

B)

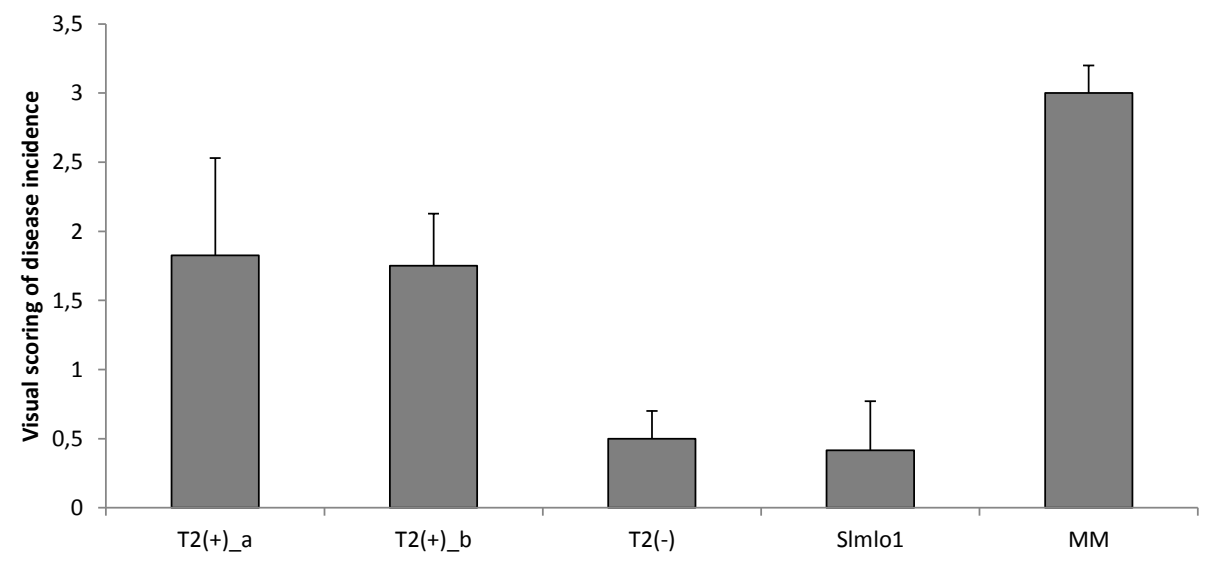

FIGURE 2. Effects of the transgenic expression of NtMLO1 in a tomato mlo loss-of-function genetic background. Panel A) refers from left to right as follows: one individual of a $\mathrm{T}_{2}$ family positive for the presence the NtMLO1 overexpression construct $\left[T_{2}-a(+)\right]$; one individual of another independent $T_{2}$ family positive for the presence of the NtMLO1 overexpression construct $\left[\mathrm{T}_{2}-\mathrm{b}(+)\right]$; one $\mathrm{T}_{2}$ individual negative for the presence of the overexpression construct $\left[\mathrm{T}_{2}(-)\right]$; one individual of the tomato SImlo1 mutant line, carrying a loss of function deletion in the SIMLO1 gene; one individual of the susceptible cultivar Moneymaker (MM). Panel B) reports the average visual scoring of disease incidence observed on the individuals of the same two $T_{2}$ families $\left[T_{2-} a(+)\right.$ and $\left.\mathrm{T}_{2-} \mathrm{b}(+)\right]$; individuals of both $\mathrm{T}_{2-}$ a and $\mathrm{T}_{2-} \mathrm{b}$ families negative for the presence of the $35 \mathrm{~S}:: N$ tMLO1 construct $\left[\mathrm{T}_{2}(-)\right]$; individuals of the SImlo1 mutant line; individuals of the cultivar MM. The scale from 0 (completely resistant) to 3 (fully susceptible) reported by Bai et al. (2008), was used for scoring. Bars and standard errors refer to $11 \mathrm{~T}_{2}(+) \_a$ plants, $10 \mathrm{~T}_{2}(+) \_$b plants, $9 \mathrm{~T}_{2}(-)$ plants, $10 \mathrm{SImlo1}$ plants and $10 \mathrm{MM}$ plants.

\section{A NtMLO1 point mutation causing the substitution of a conserved glutamine residue results in gene loss of function}

During the preparation of the 35S::NtMLO1 overexpression vector, we accidentally cloned another insert, carrying a single nucleotide polymorphism in the tobacco NtMLO1 gene. This resulted in the substitution of a glutamine residue, located in the protein second intracellular loop and previously reported to be invariable throughout the whole MLO protein family, with arginine (Q198R, Fig. 3). We could not get the same arginine-coding 
insert by repeating the cloning procedure several times from tobacco cDNA, so we assumed that this resulted from a mutation due to an error by the Taq polymerase used for amplification. Nonetheless, in order to study the effect of this substitution on protein function, we developed transgenic lines carrying an overexpression construct for this insert. Following O. neolycopersici inoculation, none of 20 individual $\mathrm{T}_{1}$ plants developed disease symptoms. Individuals of two independent $\mathrm{T}_{2}$ families positive for the presence of the construct $\left[\mathrm{T}_{2}(+) \_\mathrm{Q} 198 \mathrm{R}-\mathrm{a}\right.$ and $\left.\mathrm{b}\right]$ were found to express the transgene, as assessed by qPCR (Supplementary Fig. 4). Nevertheless, following O. neolycopersici challenge, no PM symptoms were visible on $\left[\mathrm{T}_{2}(+) \_\mathrm{Q} 198 \mathrm{R}\right]$ individuals, which were phenotypically undistinguishable from those of the SImlo1 line (Fig. 4A). In order to test whether the mutated NtMLO1 sequence maintained some residual functional activity, even so still resulting in a macroscopically resistant phenotype, we quantified, in transgenic individuals of the two $\mathrm{T}_{2}$ families, the relative fold-change of the ratio between $O$. neolycopersici and tomato gDNAs. Compared to the SImlo1 line, no significant difference was found (Fig. 4B), indicating that the point nucleotide mutation causing the substitution of glutamine with arginine in the NtMLO1 protein sequence leads to complete gene loss of function.

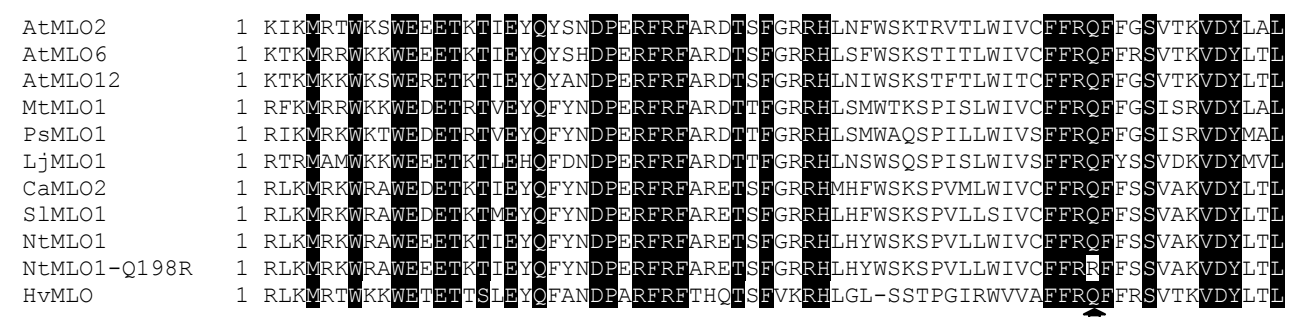

FIGURE 3. Alignment of part of the second MLO intracellular loop from several MLO proteins experimentally shown to be required for powdery mildew susceptibility (Arabidopsis AtMLO2, AtMLO6 and AtMLO12, tomato SIMLO1, pepper CaMLO2, pea PsMLO1, lotus LjMLO1, barrel clover MtMLO1 and barley HVMLO), and NtMLO1 proteins derived from the conceptual translation of the two inserts obtained during the cloning procedure (NtMLO1 and NtMLO1-Q198R). The latter is characterized by the substitution of an invariable glutamine with arginine, whose position is indicated by an arrow. 
A)

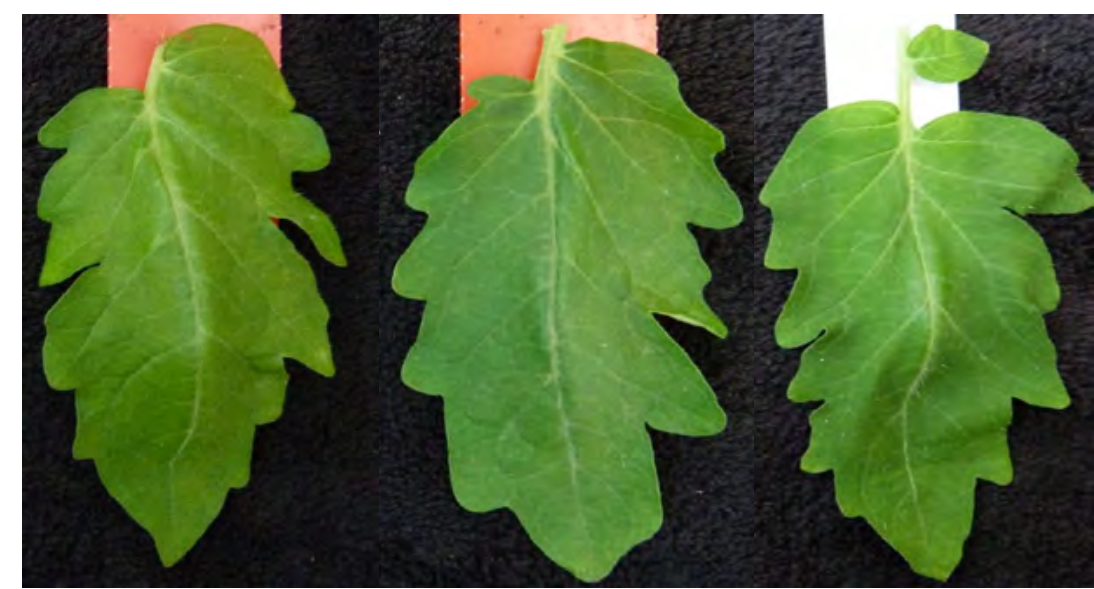

B)

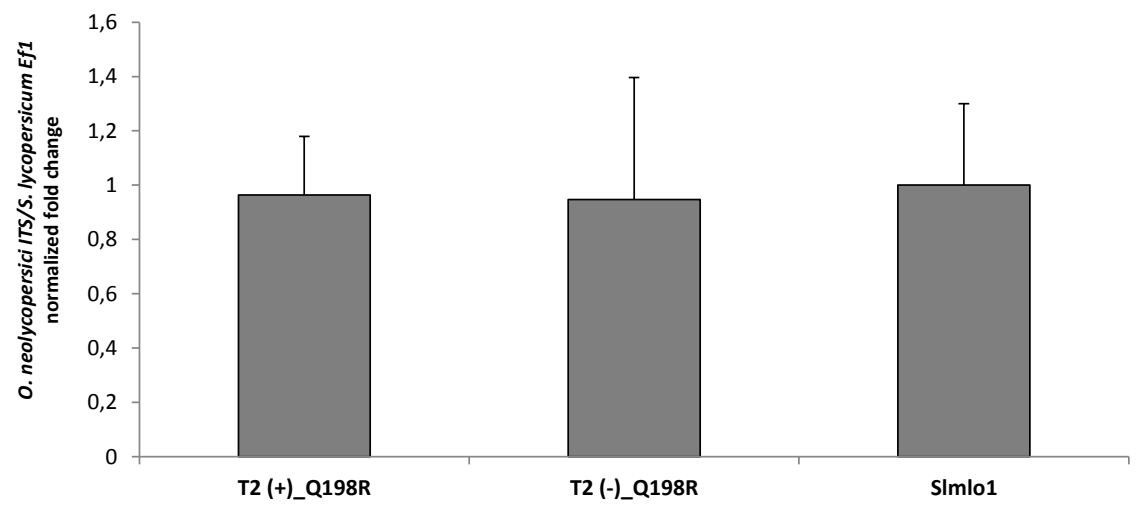

FIGURE 4. Effects of the transgenic expression of a NtMLO1 mutant sequence, resulting in the substitution of a glutamine residue with arginine in the protein second intracellular loop (Q198R). Panel A) shows the phenotype of a plant of the tomato loss-of-function SImlo1 line (right) and transgenic individuals from two different $T_{2}$ families (left and centre) assessed for transgene overexpression. Panel B) shows the relative quantification of the ratio between Oidium neolycopersici and plant gDNAs in transgenic individuals of the same $T_{2}$ families assessed for the presence or absence of the overexpression construct $\left[T_{2}(+) \_Q 198 R\right.$ and $T_{2}(-) \_Q 198 R$, respectively] and in the tomato SImlo1 mutant line. Bars and standard errors refer to 11 and 7 transgenic individuals for NtMLO1_Q198R-a and b, respectively, and 10 SImlo1 plants.

\section{Eggplant SmMLO1 complements tomato SIMLO1 in a functional complementation assay}

In order to characterize SmMLO1 at the functional level, we set up an assay based on its transgenic overexpression in the tomato line S/mlo1. This carries a loss-of-function mutation in the tomato homolog SIMLO1 and is thus resistant to the fungus O. neolycopersici, causing PM in tomato (Bai et al. 2008; Seifi et al. 2014). We assumed that restoration of PM symptoms upon SmMLO1 overexpression would have proven functional conservation with the susceptibility gene SIMLO1. 
After transformation, eight individual $\mathrm{T}_{1}$ plants were obtained. All of them expressed the transgene (Fig. 5, panel A). Four randomly selected T, plants (T1_K, T1_M, T1_P and T1_Q) were allowed to self-pollinate and give segregating $T_{2}$ families. Following inoculation with O. neolycopersici, non-transgenic $\mathrm{T}_{2}$ individuals and the line SImlo1 showed no fungal sporulation, thus displayed a resistant phenotype $(\mathrm{DI}=0)$. In contrast, all the transgenic individuals were heavily infected as the susceptible control MM, indicating that SMMLO1 is a PM susceptibility gene. The lowest and the highest average DI scores were associated with transgenic T2_K $(2,1)$ and T2_M $(2,5)$ individuals (Fig. 5, panel B and C).

\section{In silico characterization of tobacco and potato MLO families}

Recently released sequences from potato (group Phureja DM1) and tobacco (cv. Basma Xanthi) prompted us to perform a genome-wide search aiming to characterize the $M L O$ gene families in these species. This search revealed a total of 15 and 13 predicted tobacco NtMLO and potato StMLO loci, respectively, which were named according to the nomenclature specified in Tables 1 and 2. A predicted tobacco coding sequence, referred to as mRNA_127718_cds in the Sol Genomics Database, was found to be identical to $N$ TMLO1. No sequence fully matching with STMLO1 could be identified by the interrogation of the Potato Genomics Resource database, but in its place a partial gene sequence showing $100 \%$ of identity with the same gene.

For tobacco and potato MLO proteins, amino acid length and number of transmembrane domains were inferred (Supplementary Table 1 and Table 2). In addition, information on chromosomal localization and intron number was available for predicted StMLO genes (Table 2).

The tobacco NtMLO and potato StMLO protein families were used as input to search for conserved motifs, using an approach similar to the one previously reported by Deshmukh et al. (2014). We looked for motifs with length ranging from 40 to 70 residues and shared by at least three homologs. For each of the two families, seven motifs were identified. Of these, five were found to be at least partially matching with those identified in the soybean protein family (Deshmukh et al. (2014) (Supplementary Table 3).

A comparative analysis was carried out in order to establish phylogenetic relationships between the NtMLO and the StMLO protein families and MLO proteins from other dicot plant species. The analysis resulted in the distinction of five clades, designated with Roman numbers based on the position of Arabidopsis AtMLO homologs, according to the nomenclature indicated by Feechan et al. (2008) (Fig. 1). Besides NtMLO1 and StMLO1, additional NtMLO (NtMLO2, NtMLO3, NtMLO4 and NtMLO5) and StMLO (StMLO9 and StMLO12) homologs were found to group in clade $V$ together with all dicot MLO proteins previously associated with PM susceptibility. 


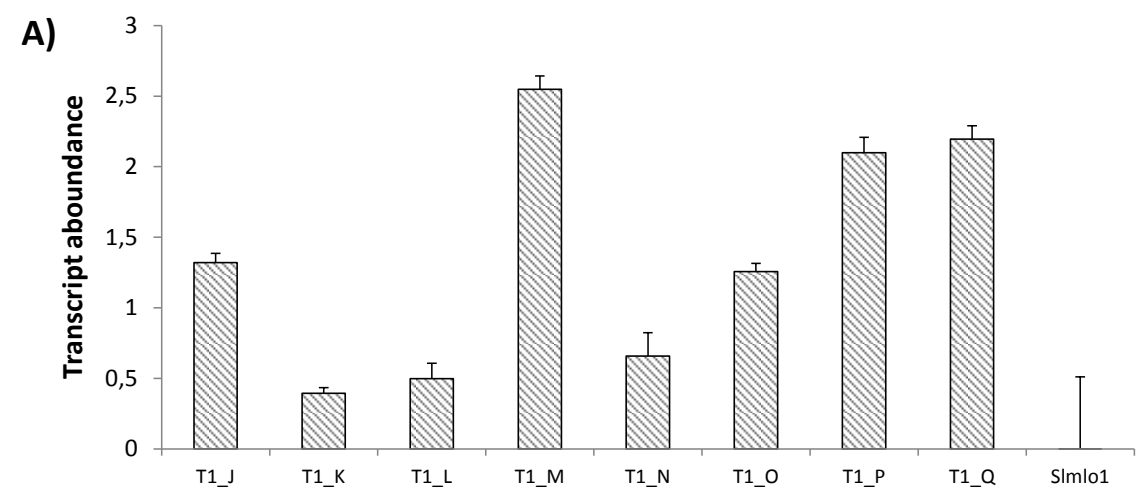

B)

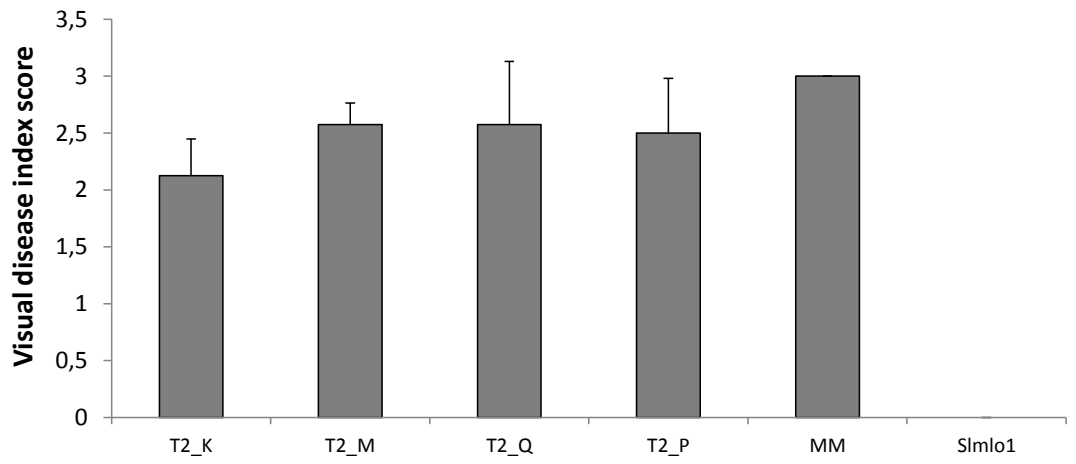

C)

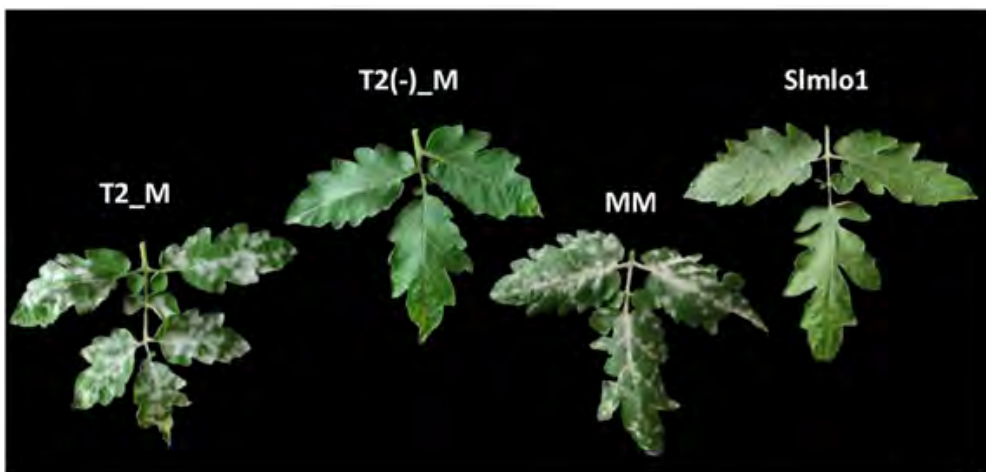

FIGURE 5. Effect of the transgenic expression of SmMLO1 in a tomato mlo loss-of-function genetic background. A) Relative quantification of $S m M L O 1$ expression levels in eight $\mathrm{T} 1$ individuals transformed with SmMLO1 (T1_J,T1_K,T1_L,T1_M,T1_N,T1_O,T1_P and T1_Q) and four individuals of the tomato line SImlo1 used as background for transformation. B) Average disease index (DI) recorded fifteen days after artificial inoculation with the powdery mildew fungus Oidium neolycopersici. The graph refers to: transgenic $\mathrm{T}_{2}$ individuals of four independent lines (T2_K, T2_M, T2_Q and T2_P) transformed with SmMLO1; the line SImlo1 (used as background for transformation). The scale from 0 (completely resistant) to 3 (fully susceptible) reported by Bai et al. (2008), was used for scoring. Bars refer to standard errors of at least six replicates $\mathbf{C}$ ) Phenotype of a selected transgenic individual of the line T2_M, a non-transgenic individual of the line T2(-)_M, a MM individual and an individual of the line SImlo1. 


\section{Discussion}

In previous studies, we functionally characterized tomato SIMLO1 and pepper CaMLO2 as two solanaceous $M L O$ susceptibility genes, as their inactivation was causally associated with PM resistance (Bai et al. 2008; Zheng et al. 2013). Starting from this information, we followed here a combined approach based on database search and PCR amplification, which resulted in the isolation of three $M L O$ genes from other widely distributed solanaceous species affected by the PM disease, namely eggplant (SmMLO1), potato (StMLO1) and tobacco (NtMLO1). PM disease represents one of the most important fungal diseases of tobacco and eggplant (Bubici and Cirulli 2008; Darvishzadeh et al. 2010) and in conducive environments may lead to important economic losses in potato cultivation (Glawe et al. 2004).

A chain of evidence, based on phylogenetic relatedness (Fig. 1) and sequence conservation with other known PM susceptibility genes and proteins (Supplementary Fig. 1 and Supplementary Fig.2) was provided, suggesting the identification of solanaceous orthologs of SIMLO1 and CAMLO2. Aiming at the functional characterization of NTMLO1 and SmMLO1, we set up an assay based on their heterologous overexpression in a tomato mlo-mutant genetic background, taking advantage from the availability of a tomato resistant line and routine protocols for tomato genetic transformation (Bai et al. 2008). Success of such an assay, as demonstrated by the restoration of symptoms in transgenic plants (Fig. 2), provides a final evidence for the role of NtMLO1 and SmMLO1 as a PM susceptibility genes.

While completing this work, newly released sequences of potato and tobacco became available. Thus, a genome-wide search was performed, which allowed to retrieve additional MLO homologs and, presumably, to characterize the complete tobacco and potato $M L O$ gene families. Phylogenetic analysis using these sequences highlighted the presence of additional NtMLO and StMLO proteins in clade V, previously shown to group dicot MLO homologs acting as PM susceptibility factors (Fig. 1). Functional redundancy of MLO homologs belonging to this clade has been shown to occur in Arabidopsis thaliana, as the simultaneous inactivation of the three homolog genes AtMLO2, AtMLO6 and AtMLO12 is required to result in complete PM resistance. Thus, functional analyses, such as the transgenic complementation test above mentioned, might lead to the identification of additional solanaceous MLO homologs playing a role in the interaction with PM fungi.

Interestingly, due to a polymerase error during the cloning procedure, we also had the opportunity to verify the crucial role of a glutamine residue localized in the second intracellular MLO domain. This amino acid has been shown to be invariable throughout the whole MLO protein family and therefore predicted to be fundamental for the role of MLO proteins as PM susceptibility factors (Elliott et al. 2005). Indeed, its replacement 
with arginine in tobacco NtMLO1 (Fig. 3) resulted in complete failure of transgenic complementation, as inferred by visual scoring and relative quantification of fungal gDNA with respect to plant gDNA (Fig. 4). This result represents a complement to earlier investigations addressed to the functional characterization of MLO proteins (Reinstädler et al. 2010; Pavan et al. 2013).

A growing body of experimental evidence supports the view that mlo-based resistance can be conveniently pursued as a strategy to cope with the PM disease in practical breeding (Pavan et al. 2010). Therefore, we predict that results here provided might be of great interest for future activities aimed at the introduction of PM resistance in Solanaceae. Targeted identification of mutations of MLO susceptibility genes can be achieved through conventional approaches of TILLING (targeted induced local lesions in genomes) or RNA interference (McCallum et al. 2000; Matthew 2006). In addition, cutting-edge technologies of genome editing are also available to the breeder, based on zinc finger nucleases (ZFNs), clustered regularly interspaced short palindromic repeat (CRISPR) and transcription activator-like effector nucleases (TALEN) (Gaj et al. 2013, Terns 2014). Noteworthy, a TALEN-based approach has been recently successfully applied to introduce PM resistance in bread wheat through simultaneous targeting of three MLO alleles, as mentioned in Wang et al. (2014). 


\section{References}

Acevedo-Garcia J, Kusch S, Panstruga R (2014) Magical mystery tour: MLO proteins in plant immunity and beyond. New Phytol doi: 10.1111/nph.12889.

Assaad FF, Qiu J-L, Youngs H, Ehrhardt D, Zimmerli L, Kalde M, Wanner G, Peck SC, Edwards H, Ramonell K, Somerville CR, Thordal-Christensen $\mathrm{H}$ (2004) The PEN1 syntaxin defines a novel cellular compartment upon fungal attack and is required for the timely assembly of papillae. Mol Biol Cell 15:5118-5129.

Bai Y, Pavan S, Zheng Z, Zappel NF, Reinstädler A, Lotti C, De Giovanni C, Ricciardi L, Lindhout P, Visser R, Theres K, Panstruga R (2008) Naturally occurring broad-spectrum powdery mildew resistance in a Central American tomato accession is caused by loss of Mlo function. Mol Plant Microbe Interact 21:30-39.

Bailey TL, Boden M, Buske FA, Frith M, Grant CE, Clementi L, Ren J, Li WW, Noble WS (2009) MEME SUITE: tools for motif discovery and searching. Nucleic Acids Res 37:W202-W208.

Bubici G, Cirulli M (2008) Screening and selection of eggplant and wild related species for resistance to Leveillula taurica. Euphytica 164:339-345.

Büschges R, Hollricher K, Panstruga R, Simons G, Wolter M, Frijters A, Van Daelen R, Van der Lee T, Diergaarde P, Groenendijk J, Töpsch S, Vos P, Salamini F, Schulze-Lefert P (1997) The barley Mlo gene: A novel control element of plant pathogen resistance. Cell 88:695-705.

Consonni C, Humphry ME, Hartmann HA, Livaja M, Durner J, Westphal L, Vogel J, Lipka V, Kemmerling B, SchulzeLefert P, Somerville SC, Panstruga R (2006) Conserved requirement for a plant host cell protein in powdery mildew pathogenesis. Nat Genet 38:716-720.

Dangl JL, Horvath DM, Staskawicz BJ (2013) Pivoting the plant immune system from dissection to deployment. Science 341:746-751.

Darvishzadeh R, Alavi R, Sarrafi A (2010) Resistance to Powdery Mildew (Erysiphe cichoracearum DC.) in oriental and semi-oriental tobacco germplasm under field conditions. J Crop Improv 24:122-130.

Deshmukh R, Singh VK, Singh BD (2014) Comparative phylogenetic analysis of genome-wide Mlo gene family members from Glycine max and Arabidopsis thaliana. Mol Genet Genomics DOI 10.1007/ s00438-014-0811-y.

Devoto A, Hartmann HA, Piffanelli P, Elliott C, Simmons C, Taramino G, Goh CS, Cohen FE, Emerson BC, SchulzeLefert P, Panstruga R (2003) Molecular phylogeny and evolution of the plant-specific seventransmembrane MLO family. J Mol Evol 56:77-88.

Elliott C, Müller J, Miklis M, Bhat RA, Schulze-Lefert P, Panstruga R (2005) Conserved extracellular cysteine residues and cytoplasmic loop-loop interplay are required for functionality of the heptahelical MLO protein. Biochem J 385:243-254.

Feechan A, Jermakow AM, Torregrosa L, Panstruga R, Dry IB (2008) Identification of grapevine MLO gene candidates involved in susceptibility to powdery mildew. Funct Plant Biol 35:1255-1266.

Gaj T, Gersbach CA, Barbas III CF (2013) ZFN, TALEN, and CRISPR/Cas-based methods for genome engineering. Trends Biotechnol 31:397-405.

Glawe DA (2008) The powdery mildews: A review of the world's most familiar (yet poorly known) plant pathogens. Annu Rev Phytopathol 46:27-51.

Glawe DA, Toit LJd, Pelter GQ (2004) First report of powdery mildew on potato caused by Leveillula taurica in North America. Plant Health Progress. doi:10.1094/PHP-2004-1214-01-HN.

Harland SC (1948) Inheritance of immunity to mildew in Peruvian forms of Pisum sativum. Heredity 2:263269.

Hewitt, H. G. (1998). Fungicides in Crop Protection, CAB International, Wallingford, UK.

Huibers RP, Loonen AEHM, Gao D, Van den Ackerveken G, Visser RGF, Bai Y (2013) Powdery Mildew Resistance in Tomato by Impairment of SIPMR4 and SIDMR1. PLOSone 8(6): e67467.

Humphry M, Reinstädler A, Ivanov S, Bisseling T, Panstruga R (2011) Durable broad-spectrum powdery mildew resistance in pea er1 plants is conferred by natural loss-of-function mutations in PsMLO1. Mol Plant Pathol 12:866-878. 
Liu Q, Zhu H (2008) Molecular evolution of the MLO gene family in Oryza sativa and their functional divergence. Gene 409:1-10.

Livak KJ, Schmittgen TD (2001) Analysis of relative gene expression data using real-time quantitative PCR and the $2^{-\triangle \Delta C T}$ method. Methods 25:402-408.

Løvdal T, Lillo C (2009) Reference gene selection for quantitative real-time PCR normalization in tomato subjected to nitrogen, cold, and light stress. Anal Biochem 387:238-242.

Lyngkjaer MF, Newton AC, Atzema JL, Baker SJ (2000) The Barley mlo-gene: An important powdery mildew resistance source. Agronomie 20:745-756.

Matthew $L$ (2004) RNAi for plant functional genomics. Comp Funct Genomics 5:240-244.

McCallum CM, Comai L, Greene EA, Henikoff S (2000) Targeting Induced Local Lesions IN Genomes (TILLING) for plant functional genomics. Plant Physiol 123:439-442.

McCormick S, Niedermeyer J, Fry J, Barnason A, Horsch R, Fraley R (1986) Leaf disc transformation of cultivated tomato (L. esculentum) using Agrobacterium tumefaciens. Plant Cell Rep 5:81-84.

Panstruga R (2005) Discovery of novel conserved peptide domains by ortholog comparison within plant multi-protein families. Plant Mol Biol 59:485-500.

Pavan S, Jacobsen E, Visser RGF, Bai Y (2010) Loss of susceptibility as a novel breeding strategy for durable and broad-spectrum resistance. Mol Breeding 25:1-12.

Pavan S, Schiavulli A, Appiano M, Marcotrigiano AR, Cillo F, Visser RGF, Bai Y, Lotti C, Ricciardi L (2011) Pea powdery mildew er 1 resistance is associated to loss-of-function mutations at a $M L O$ homologous locus. Theor Appl Genet 123:1425-1431.

Pavan S, Schiavulli A, Appiano M, Miacola C, Visser RGF, Bai Y, Lotti C, Ricciardi L (2013) Identification of a complete set of functional markers for the selection of er 1 powdery mildew resistance in Pisum sativum L. Mol Breeding 31:247-253.

Pavan S, Zheng Z, Borisova M, Van Den Berg P, Lotti C, De Giovanni C, Lindhout P, De Jong H, Ricciardi L, Visser RGF, Bai $Y$ (2008) Map- vs. homology-based cloning for the recessive gene ol-2 conferring resistance to tomato powdery mildew. Euphytica 162:91-98.

Pessina S, Pavan S, Catalano D, Gallotta A, Visser R, Bai Y, Malnoy M, Schouten H (2014) Characterization of the MLO gene family in Rosaceae and gene expression analysis in Malus domestica. BMC Genomics 15:618.

Pfaffl MW (2001) A new mathematical model for relative quantification in real-time RT-PCR. Nucleic Acids Res 29 (9): e45.

Reinstädler A, Müller J, Czembor JH, Piffanelli P, Panstruga R (2010) Novel induced mlo mutant alleles in combination with site-directed mutagenesis reveal functionally important domains in the heptahelical barley Mlo protein. BMC Plant Biol 10:31.

Rozen S, Skaletsky H (2000) Primer3 on the WWW for general users and for biologist programmers. Method Mol Biol 132:365-386.

Schouten HJ, Krauskopf J, Visser RGF, Bai Y (2014) Identification of candidate genes required for susceptibility to powdery or downy mildew in cucumber. Euphytica doi: 10.1007/s10681-014-1216-z.

Sierro N, Battey JND, Ouadi S, Bakaher N, Bovet L, Willig A, Goepfert S, Peitsch MC, Ivanov NV (2014) The tobacco genome sequence and its comparison with those of tomato and potato. Nat Commun 5:3833.

The Potato Genome Sequencing Consortium (2011) Genome sequence and analysis of the tuber crop potato. Nature 475: 189-195.

Terns RM and Terns MP (2014) CRISPR-based technologies: prokaryotic defense weapons repurposed. Trends Genet 30(3):111-8.

Wang Y, Cheng X, Shan Q, Zhang Y, Liu J, Gao C, Qiu JL (2014) Simultaneous editing of three homoeoalleles in hexaploid bread wheat confers heritable resistance to powdery mildew. Nat Biotechnol doi:10.1038/nbt.2969.

Zheng Z, Nonomura T, Appiano M, Pavan S, Matsuda Y, Toyoda H, Wolters AMA, Visser RGF, Bai Y (2013) Loss of Function in Mlo Orthologs Reduces Susceptibility of Pepper and Tomato to Powdery Mildew Disease Caused by Leveillula taurica. PLoS ONE 8(7): e70723. 


\section{Supplementary Tables}

SUPPLEMENTARY TABLE 1. Characteristics of the tobacco NtMLO gene family members identified in this study

\begin{tabular}{|c|c|c|c|c|}
\hline Gene & SGN database sequence ID & Predicted TM & Amino-acid length & Clade \\
\hline NtMLO1 & mRNA_127718_cds & 6 & 525 & V \\
\hline NtMLO2 & mRNA_127185_cds & 6 & 532 & V \\
\hline NtMLO3 & mRNA_63807_cds & 7 & 597 & V \\
\hline NtMLO4 & mRNA_106507_cds & 6 & 605 & V \\
\hline NtMLO5 & mRNA_52113_cds & 7 & 520 & V \\
\hline NtMLO6 & mRNA_44723_cds & 6 & 554 & III \\
\hline NtMLO7 & mRNA_90912_cds & 7 & 489 & VI \\
\hline NtMLO8 & mRNA_125509_cds & 7 & 555 & III \\
\hline NtMLO9 & mRNA_33476_cds & 7 & 455 & II \\
\hline NtMLO10 & mRNA_91715_cds & 7 & 410 & III \\
\hline NtMLO11 & mRNA_52133_cds & 7 & 492 & II \\
\hline NtMLO12 & mRNA_46569_cds & 7 & 490 & II \\
\hline NtMLO13 & mRNA_79933_cds & 6 & 508 & II \\
\hline NtMLO14 & mRNA_23316_cds & 7 & 505 & II \\
\hline NtMLO15 & mRNA_44406_cds & 7 & 558 & I \\
\hline
\end{tabular}

SUPPLEMENTARY TABLE 2. Characteristics of the potato StMLO gene family members identified in this study

\begin{tabular}{|c|c|c|c|c|c|c|c|}
\hline Gene & $\begin{array}{c}\text { Spud DB database } \\
\text { sequence ID }\end{array}$ & Chr. & $\begin{array}{c}\text { Position from - } \\
\text { to (bp) }\end{array}$ & $\begin{array}{l}\text { Predicted } \\
\text { introns }\end{array}$ & $\begin{array}{l}\text { Predicted } \\
\text { TM }\end{array}$ & $\begin{array}{l}\text { Amino-acid } \\
\text { length }\end{array}$ & Clade \\
\hline StMLO1* & - & - & - & - & 6 & 519 & V \\
\hline StMLO2 & PGSC0003DMG400013720 & 8 & $8,453,442-8,457,924$ & 11 & 6 & 517 & II \\
\hline StMLO3 & PGSC0003DMG400018975 & 10 & $17,809,877-17,818,901$ & 13 & 3 & 456 & 1 \\
\hline StMLO4 & PGSC0003DMG400020286 & 9 & $36,181,654-36,187,569$ & 13 & 7 & 477 & III \\
\hline StMLO5 & PGSC0003DMG400003574 & 2 & $39,242,676-39,247,920$ & 13 & 4 & 455 & I \\
\hline StMLO6 & PGSC0003DMG400012451 & 7 & $53,305,494-53,314,628$ & 14 & 7 & 565 & 1 \\
\hline StMLO7 & PGSC0003DMG400013667 & 2 & $38,189,087-38,196,233$ & 13 & 7 & 552 & III \\
\hline StMLO8 & PGSC0003DMG400018271 & 1 & $79,235,994-79,239,439$ & 12 & 5 & 414 & II \\
\hline StMLO9 & PGSC0003DMG400020605 & 3 & $36,041,611-36,048,004$ & 13 & 6 & 366 & V \\
\hline StMLO10 & PGSC0003DMG400023159 & 2 & $18,902,752-18,910,333$ & 11 & 7 & 550 & III \\
\hline StMLO11 & PGSC0003DMG400027665 & 6 & $57,855,801-57,859,958$ & 12 & 7 & 507 & II \\
\hline StMLO12 & PGSC0003DMG400030134 & 6 & $9,616,811-9,623,870$ & 13 & 7 & 589 & V \\
\hline StMLO13 & PGSC0003DMG400033623 & 8 & $42,249,518-42,256,102$ & 14 & 7 & 532 & III \\
\hline
\end{tabular}

${ }^{*}$ Features of StMLO1 refer to the homolog identified by a PCR-based approach for which no corresponding sequence is found in Spud DB 

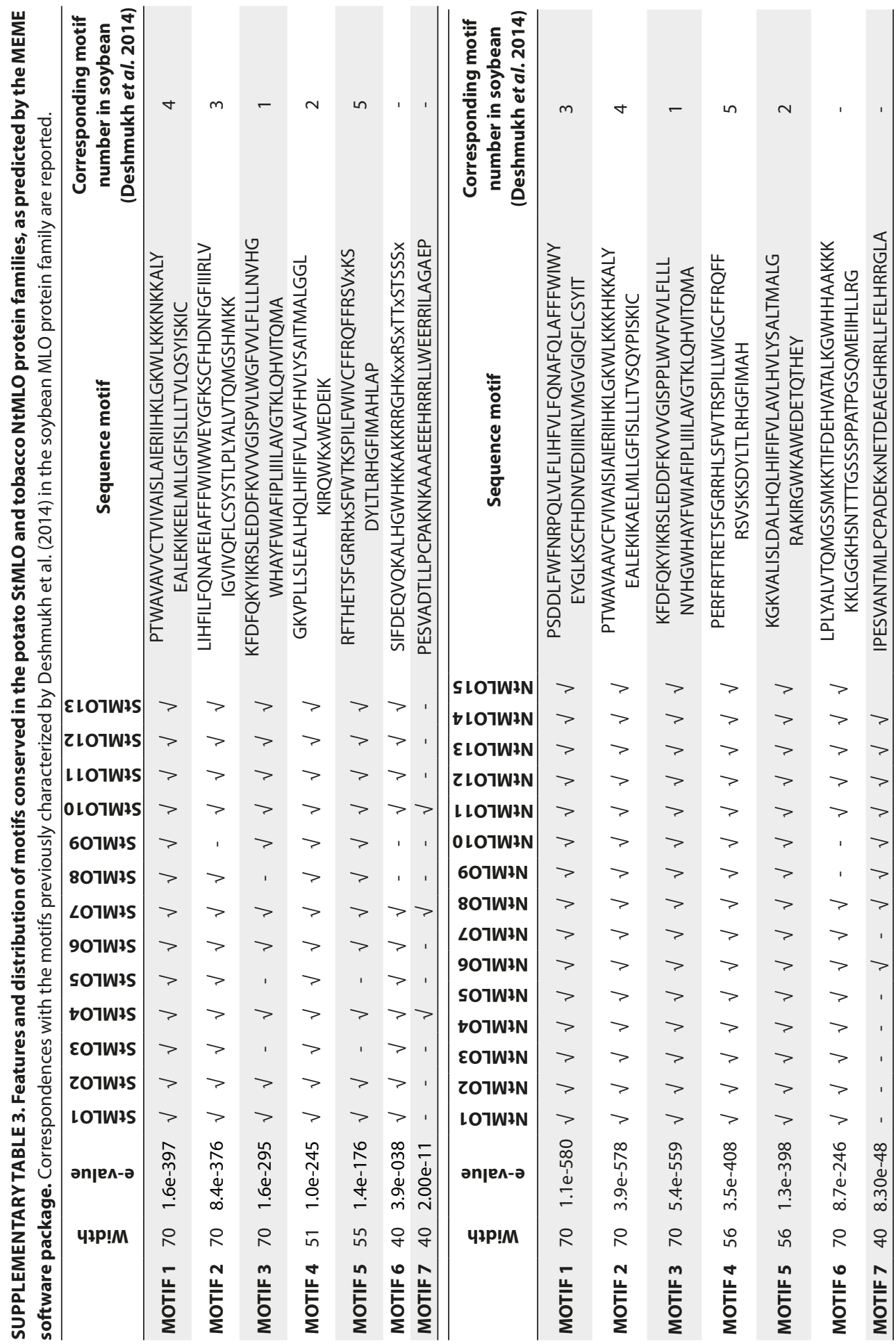

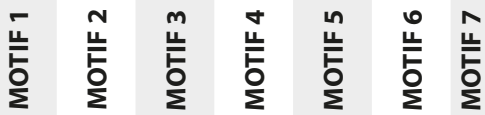




$\begin{array}{lrl}\text { AtMLO3 } & 99 \text { NRMLP-CRKTIKSHNDVSEDDDDDDGDNH-------------------DNSEFHQCSSK } \\ \text { AtMLO11 } & 98 \text { NDRFLPCTRSEIQ--------EELESG-STVKRNLLTKSLFFNIFRRRLDVIKRTTC-SE } \\ \text { AtMLO14 } & 92 \text { NDRFVPCTPSEIN--------EELESTISTVKRTQLTRSLFLHTLRRRLSGIGEDTC-SE } \\ \text { AtMLO4 } & 91 \text { NSKFYICSEEDYG--------IHKKV------LLEHTSSTNQSSLPHHGIHEASHQC-GH } \\ \text { AtMLO1 } & 90 \text { MHMLP-CSLDSRREAGASEHKNVTAKEHFQTFLPIVGTTRR----LLAEHAAVQVGYCSEK } \\ \text { AtMLO15 } & 88 \text { EKFLP-CTKPAGAEKSLKDSSHFQ---------FSETGR----HLLAGDAPAGDYCSLK } \\ \text { AtMLO13 } & 89 \text { NNMFP-CKKPLEEHHAPKSSHSI------------INNA----RHLLSTGESPDHCAAK }\end{array}$

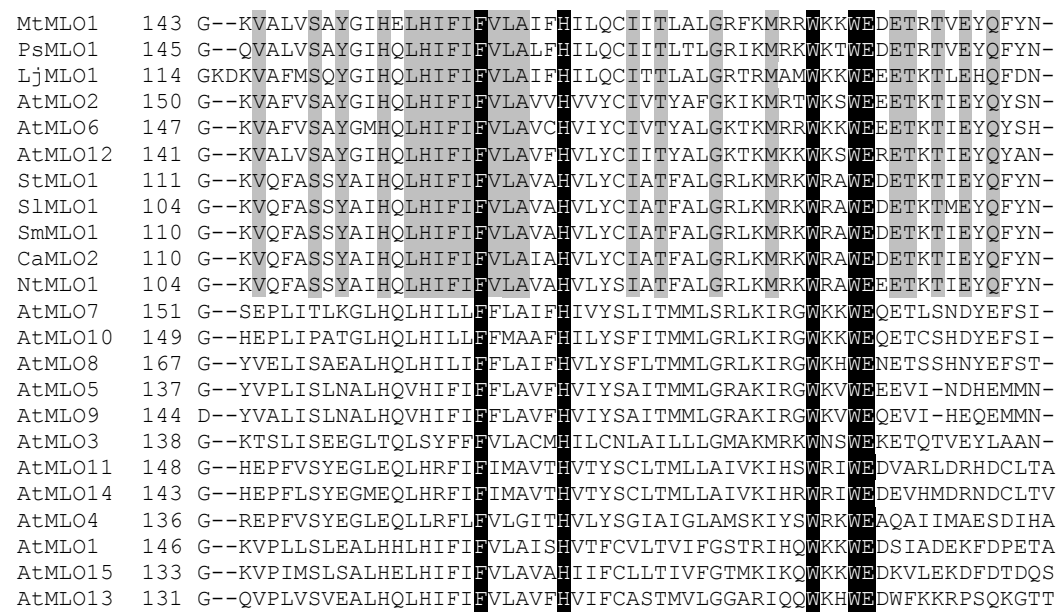

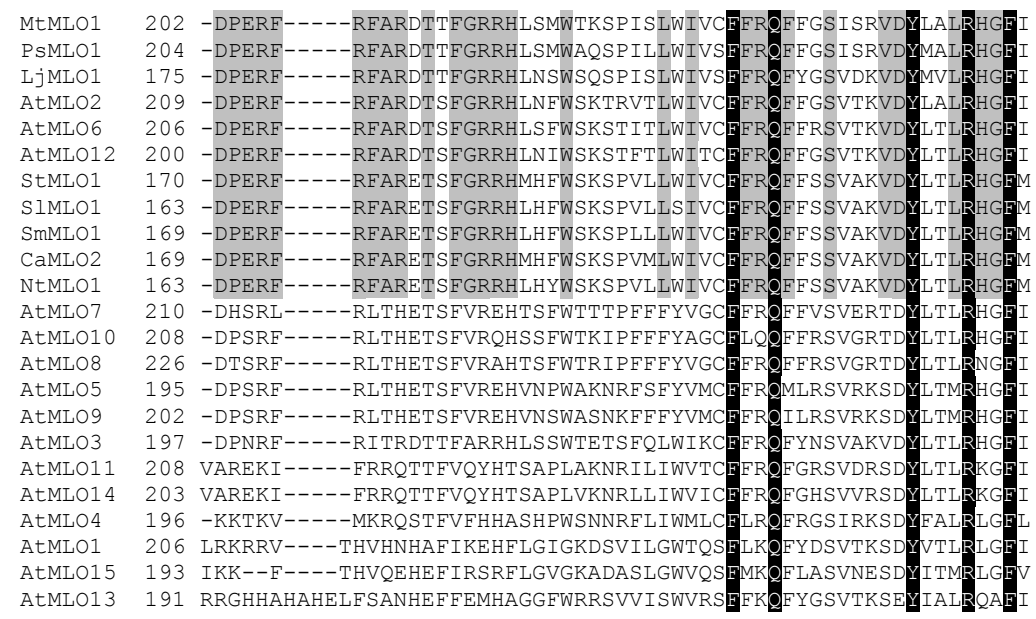

\section{SUPPLEMENTARY FIGURE 1.}



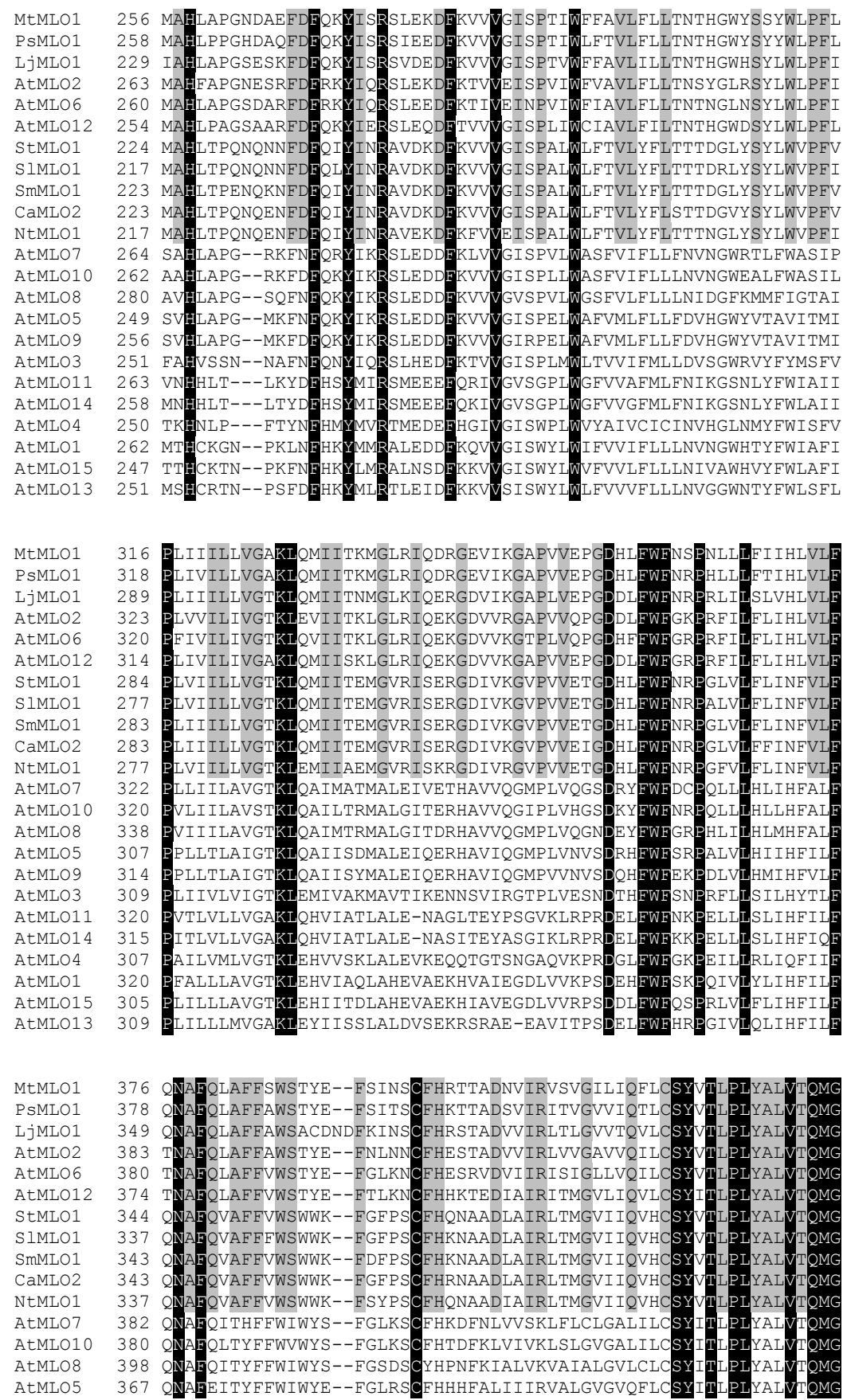

SUPPLEMENTARY FIGURE 1. 


\begin{tabular}{|c|c|c|}
\hline & & 25 \\
\hline & & \\
\hline & & \\
\hline & & \\
\hline & & IWVT- \\
\hline & & af \\
\hline & & \\
\hline & & \\
\hline ०1 & 34 & IFNERLATAL \\
\hline 01 & 36 & NERVA \\
\hline ¡MLO1 & 09 & IFHDRVA \\
\hline MLO2 & 41 & VENDRVATAL \\
\hline MLO 6 & 38 & ENERVA \\
\hline MLO12 & 432 & KQTKHG-HSGS--NTPHS \\
\hline 01 & 02 & $-\mathrm{LSGH}--\mathrm{TI}$ \\
\hline & 95 & $-\mathrm{LSGH}--\mathrm{T}$ \\
\hline MLO1 & 01 & KKRVKHGRLSGN--TTP \\
\hline MLO2 & & KKRVRHGRVSEN--TTP \\
\hline MLO1 & & KKRVKHGRLSEN--TTPVSSP \\
\hline MLO7 & 40 & IKLKKGKARKLPSKTLGVSESE \\
\hline MLO10 & 43 & KKKKGKARKPPTETLGV \\
\hline & & KKKKGVKATTKRLGGL \\
\hline & & KKSETPGQTQPPLPNLRP \\
\hline LO9 & & VFDEQTSKALE \\
\hline EMLO3 & 427 & JRDKKKTIQTPDTDNNSNNNNC \\
\hline MLO11 & 43 & RKRRHGLYGDDSTVRTETSTI \\
\hline & & KRRHGLYGDDSTIRTET \\
\hline & & ERSKHTRSVCSLDTATIL \\
\hline & & KRDLKAAASNGDEG \\
\hline 1LO15 & & RGVKKGATQVGTSHDATSPR \\
\hline MLO13 & & RGESTSEAHRIEMQPTI \\
\hline
\end{tabular}

MtMLO1 492 QTFGNSDSLQTSPRTSNYENEQWDVEGGGSTSPRNNQTVASEIEIPIVESFSTTELPVSV PSMLO1 494 HTAGNSDSLQTSPEKSDYKNEQWDIEGEGPTSLRNDQTGQHEIQIAGVESFSSTELPVRI LjMLO1 469 HHNYHNSDSPLASPRESPSNYETEQWYLEPNSPSNHTRGHDQTLQMQVLGSSATEFSPAE AtMLO2 499 FNNRSVENYPSSPSPRYSGHGHHEHQFWDPESQHQEAETSTHHSLAHESSEPVLASVELP AtMLO6 496 APHKRSRSVDESFANSFSPRNSDFDSWDPESQHETAETSNSNHRSRFGEEESEKKFVSSS AtMLO12 489 YNNRSLDQQTSFTASPSPPRFSDYSGQGHGHQHFFDPESQNHSYQREITDSEFSNSHHPQ StMLO1 459 YPQYNEDSVQASPRTSNVENEGWANEISNDNQEGEILQHASTDHNKQIEITMSDFTFGNK SIMLO1 452 YPQYNEDSVQASPRTSNVENEGWANE----NQEGEI LQHASTDHNKQIEITMSDFTFGNK SMMLO1 459 YPQYNEDSVQASPRTSNVENEGWANE IST DNKDYQEGHASTSVRPPHAHNQQIEITMSDF CaMLO2 459 YPKYNEDNVQAYPRTSNVENEGWANETSTENKDHQEEGQILQHASTSMQHPHTDQHQIEI NTMLO1 453 YPQYSNEESRTSNAENEGWANEI PTSPRRQIENI KDDDHQEGE I HASSSVHQVEIAMSEF

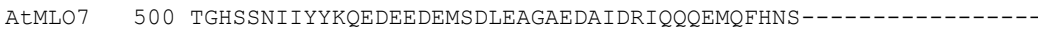
AtMLO10 498 SKTTGHSTASYMSNFEDQSMSDLEAEPLSPEPIEGHTLVRVGDQNTEIEYTGDISPGNQF AtMLO8 516 YKTTPHSMRYEGLDPETSDLDTDNEALTPPKSPPSFELVVKVEPNKTNTGETSRDTETDS

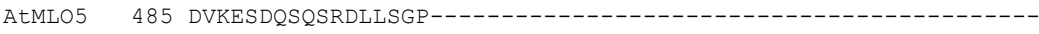
AtMLO9 - $987-10$ $\begin{array}{lll}\text { AtMLO3 } & 487 & \text { FRFSGRQSPILQEIQIQEKTER } \\ \text { AtMLO11 } & 497 & \text { TSFEQQRKQQEQGTTELELQPIQPRNDCVPNDTSSRVGTPLLRPWLSISSPTTTIELRSE }\end{array}$ $\begin{array}{lll}\text { AtMLO11 } & 497 & \text { TSFEQQRKQQEQGTTELELQPIQPRNDCVPNDTSSRVGTPLLRPWLSISSPTTTIELRSE } \\ \text { AtMLO14 } & 492 \text { TSPVQDNELELQLIRGACGNSSSVETPILRPCASISSTTFSRLQTETTDSLSRSSSLPMR }\end{array}$ AtMLO4 485 LNQITINSIDQAESIFGAAASSSSPQDGYTSRVEEYLSETYNNIGSIPPLNDEIEIEIEG

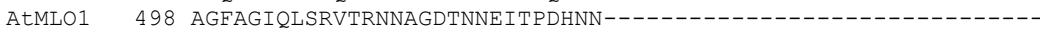

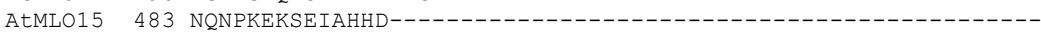
AtMLO13

\section{SUPPLEMENTARY FIGURE 1.}




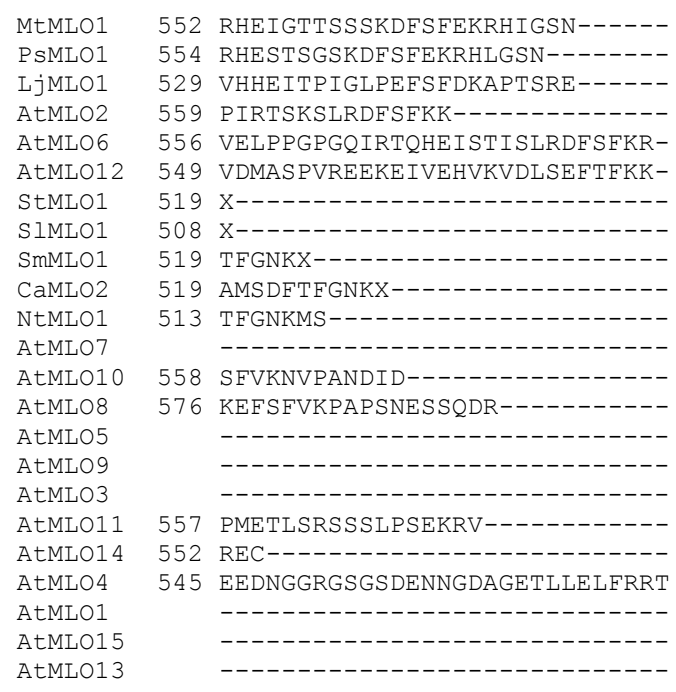

SUPPLEMENTARY FIGURE 1. Protein multiple alignment of a dataset composed of eggplant SmMLO1, potato StMLO1, tobacco NtMLO1, the fifteen Arabidopsis AtMLO homologs and the susceptibility proteins SIMLO1 (tomato), CaMLO2 (pepper), PsMLO1 (pea), LjMLO1 (lotus) and MtMLO1 (barrel clover). Black shading shows amino acid residues reported to be conserved throughout the whole MLO protein family, whereas grey shading highlights residues shared by MLO proteins experimentally shown to be required for powdery mildew susceptibility 
StMLO1

SIMLO1

SmMLO1

NtMLO1

CaMlo2

StMLO1

SIMLO1

SmMLO1

NtMLO1

CaMlo2
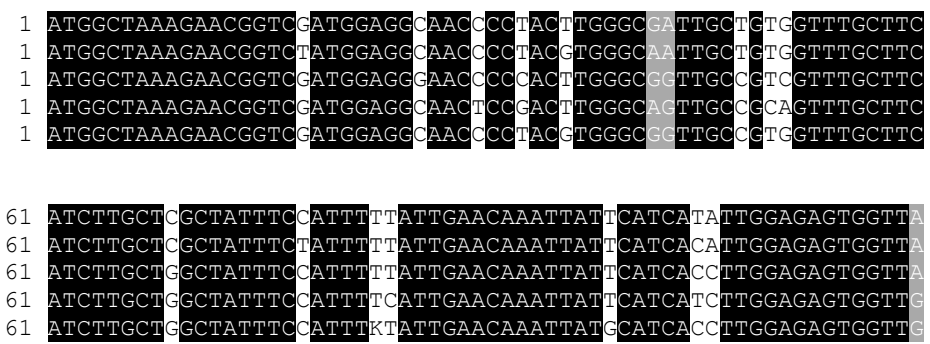

StMLO1

SIMLO1

121 CTG

121 TTG AAAA

CaMlo2 121 TTGAAAAA
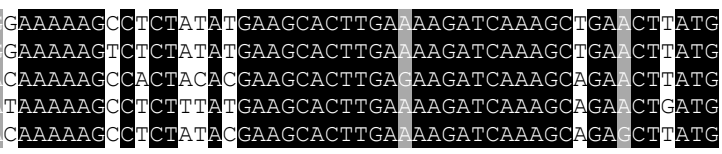

StMLO1

SIMLO1

SmMLO1

NtMLO1

181

181 CTGTTGGGATTC

181 TTGTTGGGATTC

CaMlo2

181 .
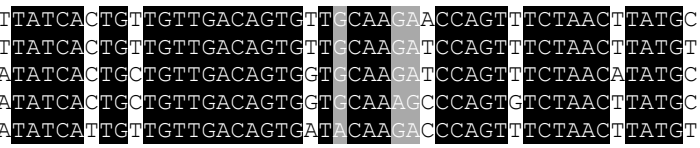

StMLO1

SIMLO1

SmMLO1

NtMLO1
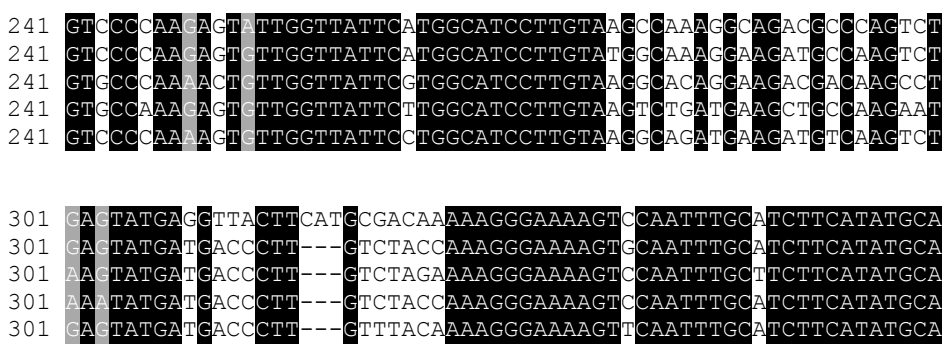

StMLO1

SIMLO1

SmMLO1

NtMLO1

CaMlo2
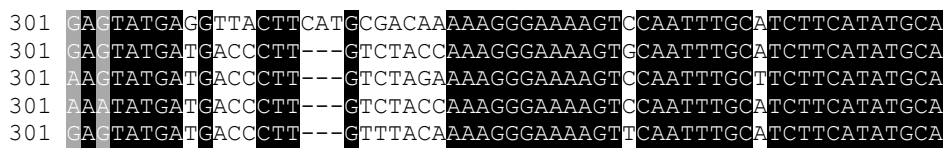

StMLO1

S1MLO1

361 ATACACCAGCTCCA

SmMLO1 358 ATACACCAGCTCCA

NtMLO1 358 ATACACCAGCTCCA

CaMlo2

358 ATACACCAGCTCCA
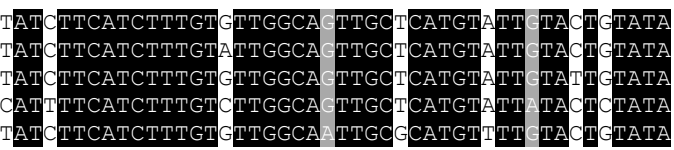

StMLO1

SIMLO1

421

418

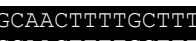

GCAACTTTTGCTTT

SmMLO1 418 GCAACTTTTGCTT

NtMLO1 418 GCAACTTTTGCTTM

CaMlo2

418 GCAACTTTTGCTTT
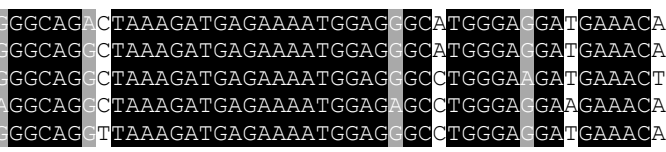

StMLO1

S IMLO1

481 AАAАCAAT

NtMLO1 478 AAAACAAT

CaMlo2

478 AAAACAAT
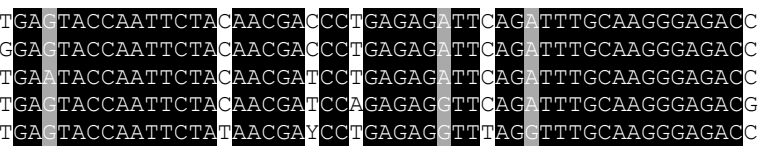

$\begin{array}{lll}\text { StMLO1 } & 541 & \text { TCG } \\ \text { SlMLO1 } & 538 & \text { TCG } \\ \text { SmMLO1 } & 538 & \text { TCC }\end{array}$
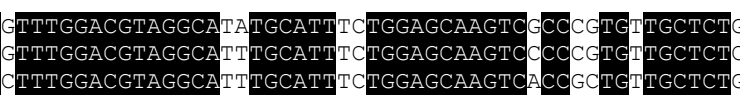

GATAGTT
GATAGTT
GATAGTT

SUPPLEMENTARY FIGURE 2. 


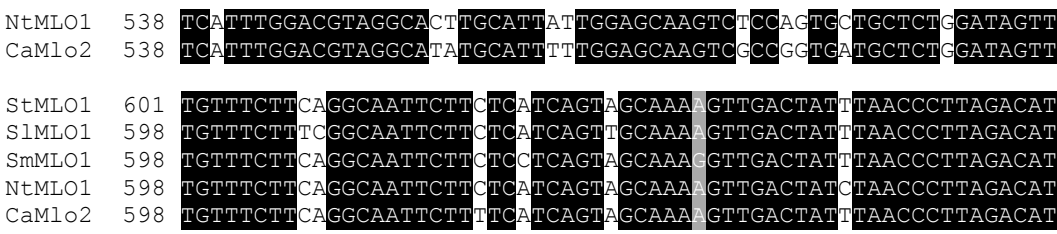

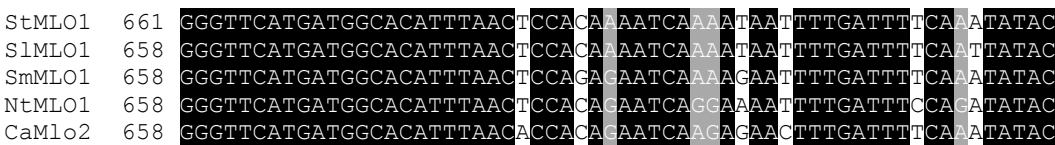

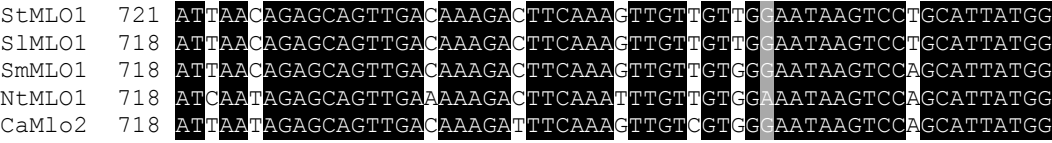

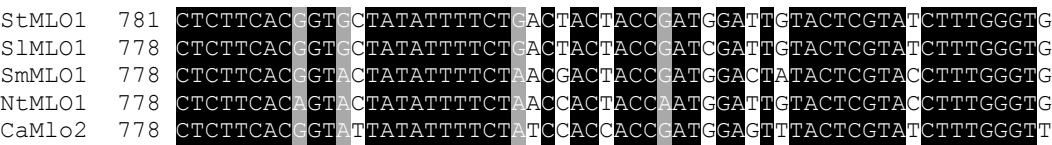
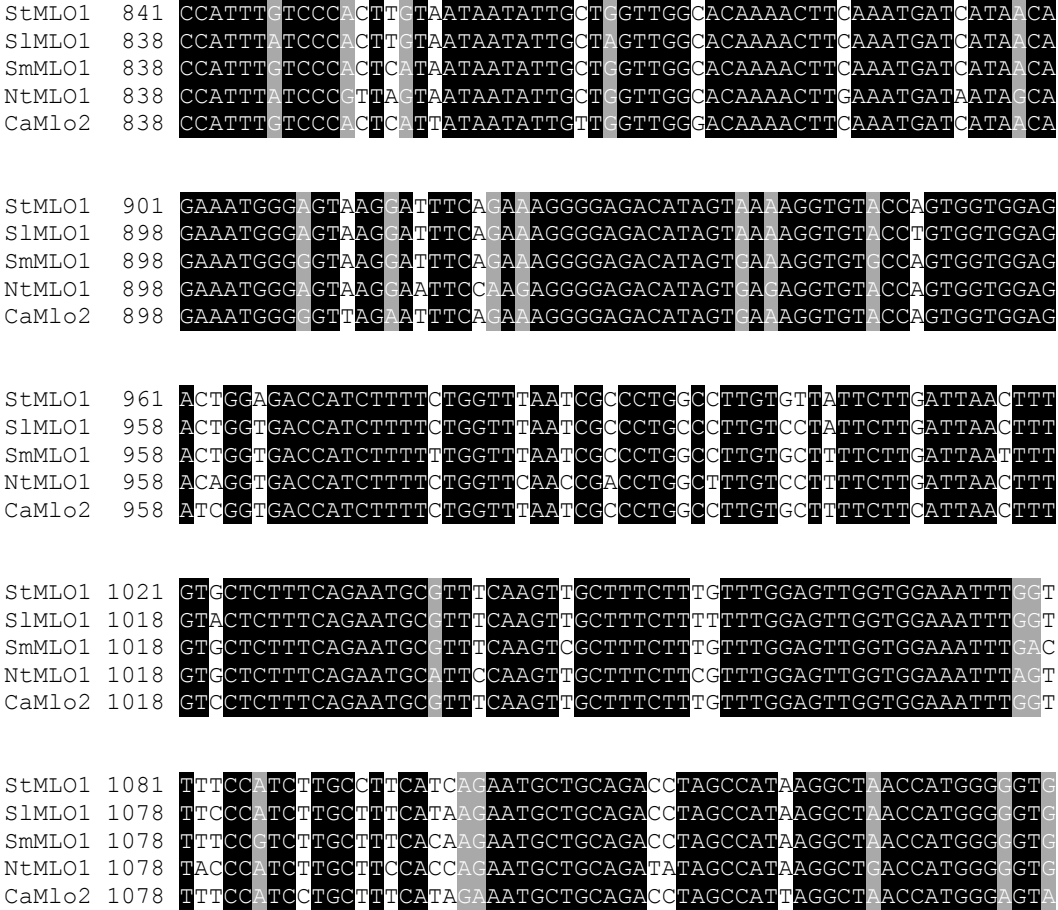

SUPPLEMENTARY FIGURE 2. 


\title{
StMLO1 1141 SIMLO1 1138 SmMLO1 1138 NtMLO1 1138 CaMlo2 1138
}
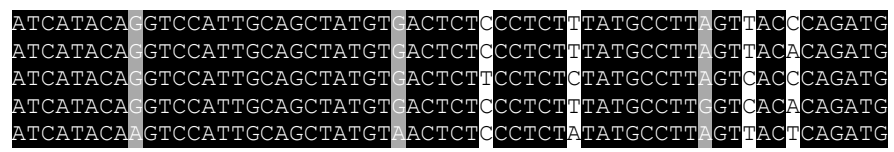

StMLO1 1201 SIMLO1 1198 SMMLO1 1198 NtMLO1 1198 CaMlo2 1198
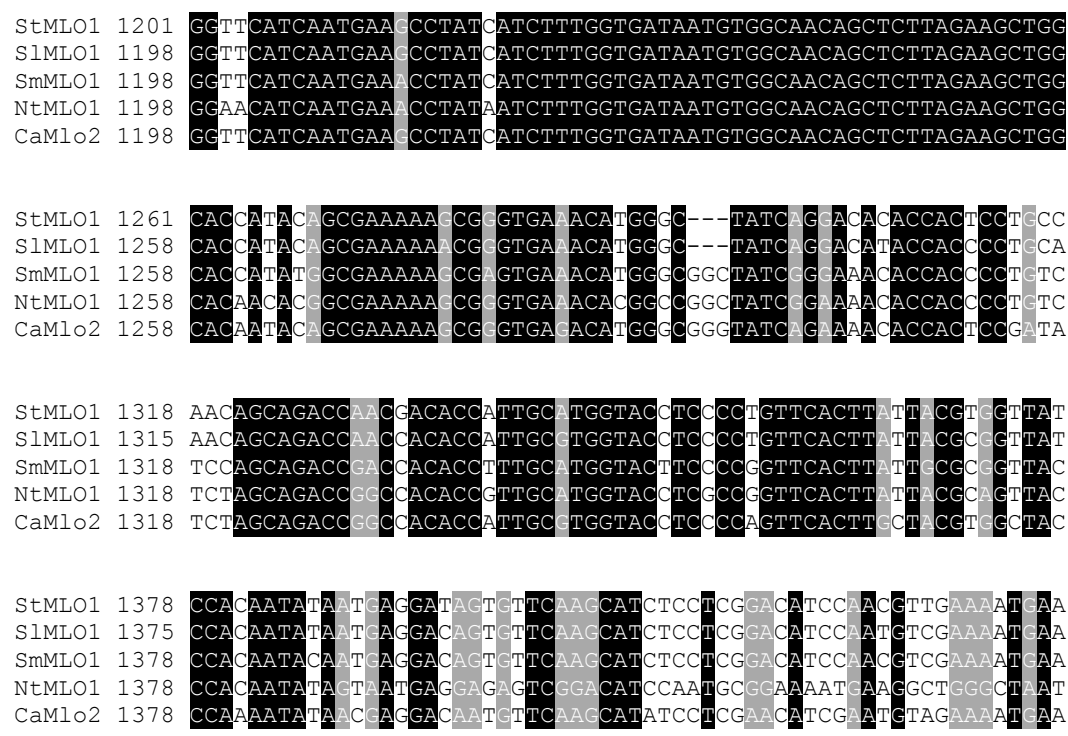

StMLO1 1438 SIMLO1 1435 SmMLO1 1438 NtMLO1 1438 CaMlo2 1438
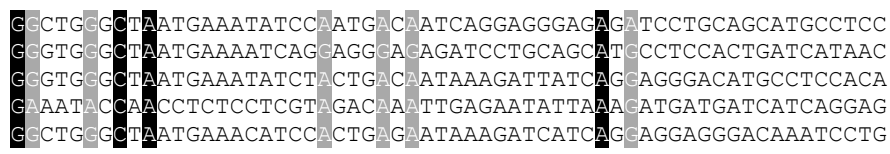

\begin{abstract}
StMLO1 1498 ACTGATCATAACAACCAAATTGAGATTACAATGTCAC SIMLO1 1495 AAGCAAATTGAGATTACAATGTCAGATTTTACTTTTC SmMLO1 1498 TCTGTGCGACCTCCCCATGCTCACAACCAGCAAATT NtMLO1 1498 GGAGAAATCCATGCCTCCAGCTCTGTGCATCAAGTTG TTTCACTTTTGGAAACAAATAA AAACAAATAAATGTAAAAACGA GATTACAATGTCAGATTTTACT CaMlo2 1498 CAGCATGCCTCCACTTCTATGCAACATCCGCATACTG TCAACATCAAATTGÄGATTGCA
\end{abstract}

StMLO1 1558 AAGATCAATGTATATGTAAAAACTAATTTCCTCTGCGTTGTTTAAGTTCATTACTGTAGA SIMLO1 1555 ATTTTCTTCTTCATTGTTTTAAGTTCATTACTGTAGTTCAAATGGCAATGATTTTGTAAA

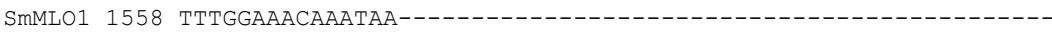

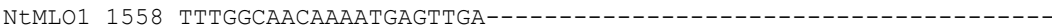
CaMlo2 1558 ATGTCAGATTTTACTTTTGGAAACAAATAG-

StMLO1 1618 GAGCATAAATGGATCCTAGATAGTTCAAGTGCCTGCTTGCTAATACACAAAGATTTTCAT SlMLO1 1615 ATTTTATACAGAGGTACTCATGCATGGTGCTCTTCATTTCAAGGTAAGAACCTTCTTATA SmMLO1

NtMLO1

$\mathrm{CaMlo2}$

StMLO1 1678 TCCAAAAAAAAAAAAAAA--------

SIMLO1 1675 TCGATTTATAGCTACTTTACATCTCA

SmMLO1

NtMLO1

CaMlo2

SUPPLEMENTARY FIGURE 2. Nucleotide multiple alignment of full-length coding sequences of eggplant STMLO1, potato StMLO1, tobacco NTMLO1, tomato SIMLO1 and pepper CaMLO2. 


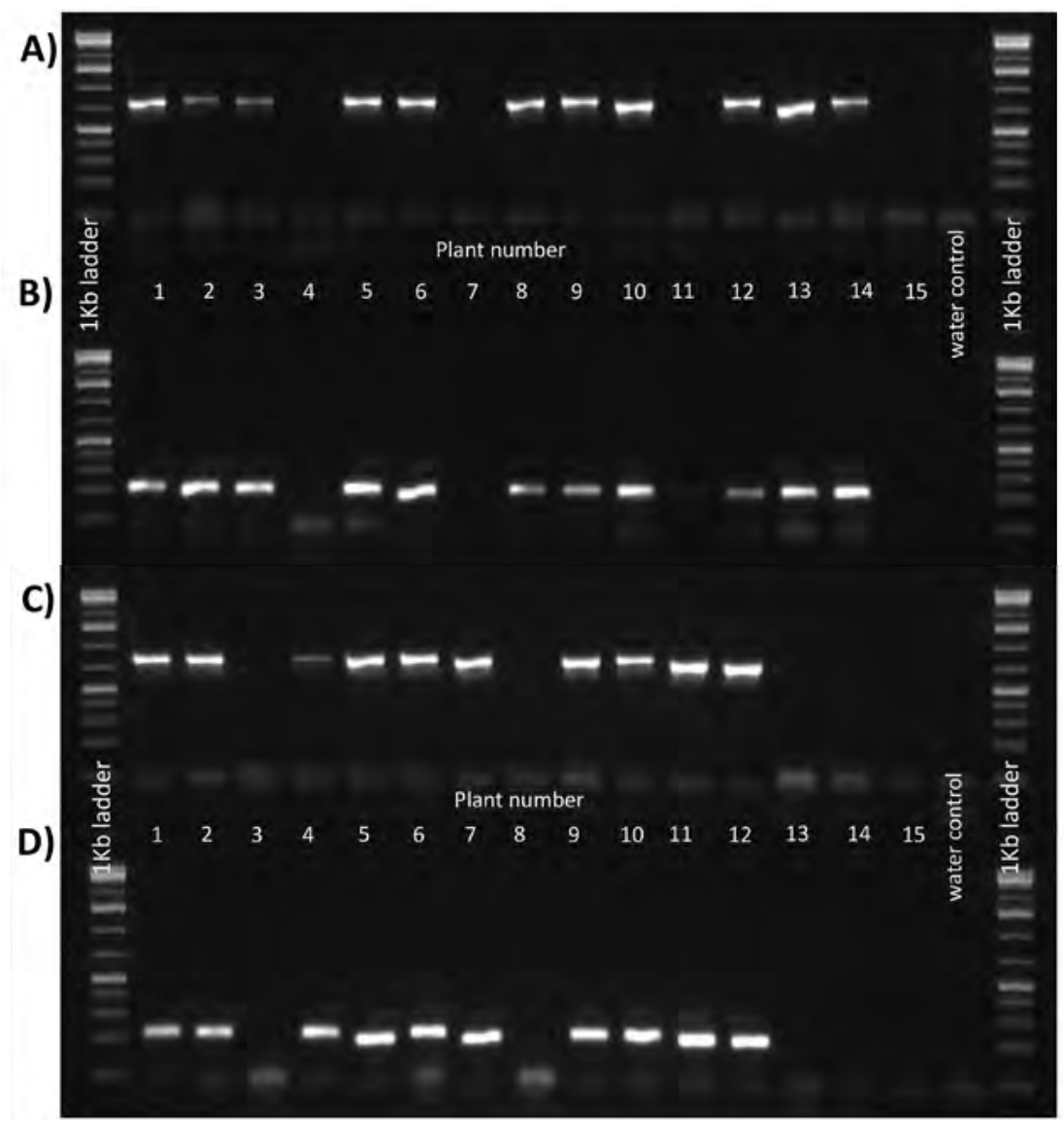

SUPPLEMENTARY FIGURE 3. Segregation of the $T_{2}-a(A$ and $B)$ and $T_{2}-b(C$ and $D)$ families for markers derived from the nptll gene (panels $A$ and $C$ ) and $35 S$ promoter (panels $B$ and $D$ ), indicating the presence of the 35S::NtMLO1 construct. 


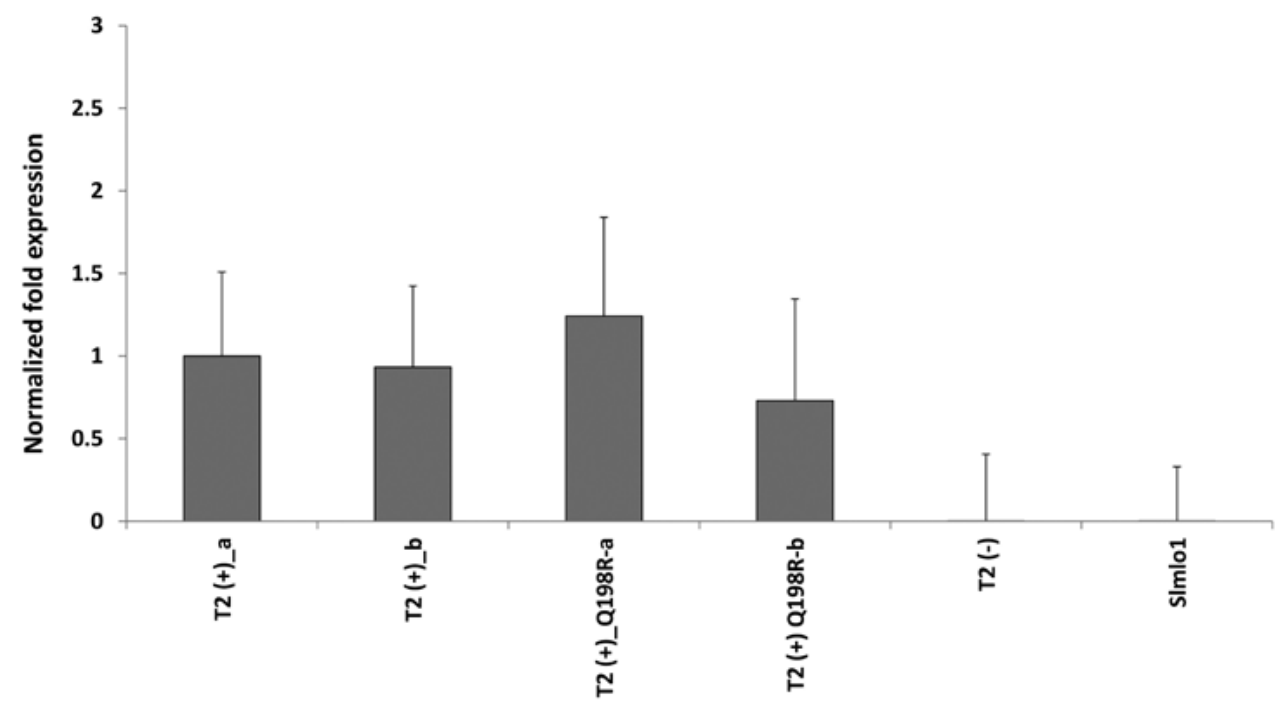

SUPPLEMENTARY FIGURE 4. Relative quantification of NtMLO1 expression levels in complementation tests, assessed by qPCR. Data refer to 11 and 10 individuals of two $T_{2}$ families $\left[T_{2}(+) \_a\right.$ and $\left.T_{2}(+) \_b\right]$ positive for the presence of the overexpression construct harboring wild-type NtMLO1; 11 and 7 individuals of two $\mathrm{T}_{2}$ families $\left[\mathrm{T}_{2}(+) \_\mathrm{Q} 198 \mathrm{R}-\mathrm{a}\right.$ and b] positive for the presence of the overexpression construct harboring a NtMLO1 mutant sequence, resulting in the substitution of a glutamine residue with arginine; 18 non-transgenic individuals from the $4 \mathrm{~T}_{2}$ families above mentioned $\left[\mathrm{T}_{2}(-)\right] ; 10$ individuals of the SImlo1 mutant line, used as background genotype for transformation. 



\section{Chapter 5}

Monocot and dicot MLO powdery mildew susceptibility factors are functionally conserved in spite of the evolution of class-specific molecular features

Michela Appiano, Domenico Catalano, Miguel I. Santillán Martínez, Concetta Lotti, Zheng Zheng, Richard G. F. Visser, Luigi Ricciardi, Yuling Bai*, and Stefano Pavan*

${ }^{*}$ corresponding author

Chapter published in BMC Plant Biology, June 2015

Link to published paper here: http://bmcplantbiol,biomedcentral.com/articles/10.1186/s12870-015-0639-6 


\begin{abstract}

\section{Background}

Specific members of the plant Mildew Locus O (MLO) protein family act as susceptibility factors towards powdery mildew (PM), a worldwide-spread fungal disease threatening many cultivated species. Previous studies indicated that monocot and dicot MLO susceptibility proteins are phylogenetically divergent.
\end{abstract}

\title{
Results
}

We show that monocot and dicot MLO susceptibility proteins evolved class-specific conservation patterns. Many of them appear to be the result of negative selection and thus are likely to provide an adaptive value. We also tested whether different molecular features between monocot and dicot MLO proteins are specifically required by PM fungal species to cause pathogenesis. To this aim, we transformed a tomato mutant impaired for the endogenous SIMLO1 gene, and therefore resistant to the tomato PM species Oidium neolycopersici, with heterologous MLO susceptibility genes from the monocot barley and the dicot pea. In both cases, we observed restoration of PM symptoms. Finally, through histological observations, we demonstrate that both monocot and dicot susceptibility alleles of the $M L O$ genes predispose to penetration of a non-adapted PM fungal species in plant epidermal cells.

\section{Conclusions}

With this study, we provide insights on the evolution and function of $M L O$ genes involved in the interaction with PM fungi. With respect to breeding research, we show that transgenic complementation assays involving phylogenetically distant plant species can be used for the characterization of novel MLO susceptibility genes. Moreover, we provide an overview of MLO protein molecular features predicted to play a major role in PM susceptibility. These represent ideal targets for future approaches of reverse genetics, addressed to the selection of loss-of-function resistant mutants in cultivated species.

\section{Keywords}

MLO, powdery mildew, Angiosperms, evolution, plant breeding 


\section{Background}

The plant Mildew Locus $\underline{O}$ (MLO) gene family codes for proteins harboring seven transmembrane domains and a calmodulin-binding site, topologically reminiscent of metazoan and fungal G-protein coupled receptors (GPCRs) [1]. Following the completion of plant genome sequencing projects, a number of homologs varying from 12 to 19 has been identified in the $M L O$ gene families of diploid species, namely Arabidopsis, rice, grapevine, cucumber, peach, woodland strawberry and sorghum [1-6].

Specific homologs of the $M L O$ gene family act as susceptibility factors towards fungi causing the powdery mildew (PM) disease, worldwide spread and causing severe losses in agricultural settings. Inactivation of these genes, through loss-of function mutations or silencing, indeed results in resistance (referred to as mlo-based resistance) in several plant species [7]. The first $M L O$ gene described as required for PM pathogenesis was barley $H v M L O[8,9]$. Since then, $M L O$ susceptibility genes have been functionally characterized in rice (OsMLO3), wheat (TaMLO_A1 and TaMLO_B1), Arabidopsis (AtMLO2, AtMLO6 and AtMLO12), tomato (SIMLO1), pepper (CaMLO2), tobacco (NtMLO1), pea (PSMLO1), lotus (LjMLO1) and barrel clover (MtMLO1) [10-17].

Defense mechanisms involved in mlo-based resistance prevent fungal penetration in epidermal cells and are associated with the formation of cell wall appositions, referred to as papillae [11]. Similar pre-penetration defense measures also take place in non-host resistance, following the interaction between PM fungal species and plant species beyond their host range. Consistent with the hypothesis of involvement of $M L O$ genes in non-host resistance, loss of function of HVMLO in the interaction between barley and the wheat PM fungus Blumeria graminis f. sp. tritici is associated with decreased rate of penetration and lower incidence of epidermal cell death, the latter being a post-penetration defense mechanism $[18,19]$.

Several studies have been addressed to the characterization of regions of relevance for the functionality of MLO proteins. Multiple alignments have pointed out the occurrence of residues highly conserved within the whole MLO family, which were therefore predicted to provide a common protein structural scaffold $[12,20]$. In addition, the occurrence of residues and motifs specifically conserved in putative orthologs of barley HVMLO has been reported [9]. Finally, functionally important residues for MLO susceptibility proteins have been inferred by the association of naturally occurring and induced mutations with partial or complete PM resistance [11, 12, 21-25].

In our previous studies, we showed that phylogenetically related dicot $M L O$ genes of the same botanic family are conserved for their function as a susceptibility gene to PM $[6,16]$. Notably, monocot and dicot MLO proteins involved in PM susceptibility group in clearly separated phylogenetic clades (e.g. [2, 9]). Here, we show that the evolution of Angiosperm 
PM susceptibility genes led to the fixation of class-specific molecular traits. Many of them appear to be the result of negative selection. By means of transgenic complementation assays, we demonstrate that, despite having different conservation patterns, monocot and dicot MLO susceptibility genes are essentially conserved with respect to functional features having a role in interactions with PM fungi. Consequences of our findings for plant breeding research are discussed.

\section{Results}

\section{Class-specific molecular features of Angiosperm MLO homologs required for PM susceptibility}

Previous studies indicated that dicot and monocot MLO proteins with a putative or ascertained role in susceptibility to PM fungi group in two different phylogenetic clades (e.g. $[2,9])$. This was confirmed by performing a new UPGMA-based phylogenetic analysis involving all the twelve MLO homologs which have been until recently functionally related to PM susceptibility (Fig. 1). Aiming to detect molecular features responsible for such phylogenetic divergence, the same MLO homologs were used as dataset for multiple

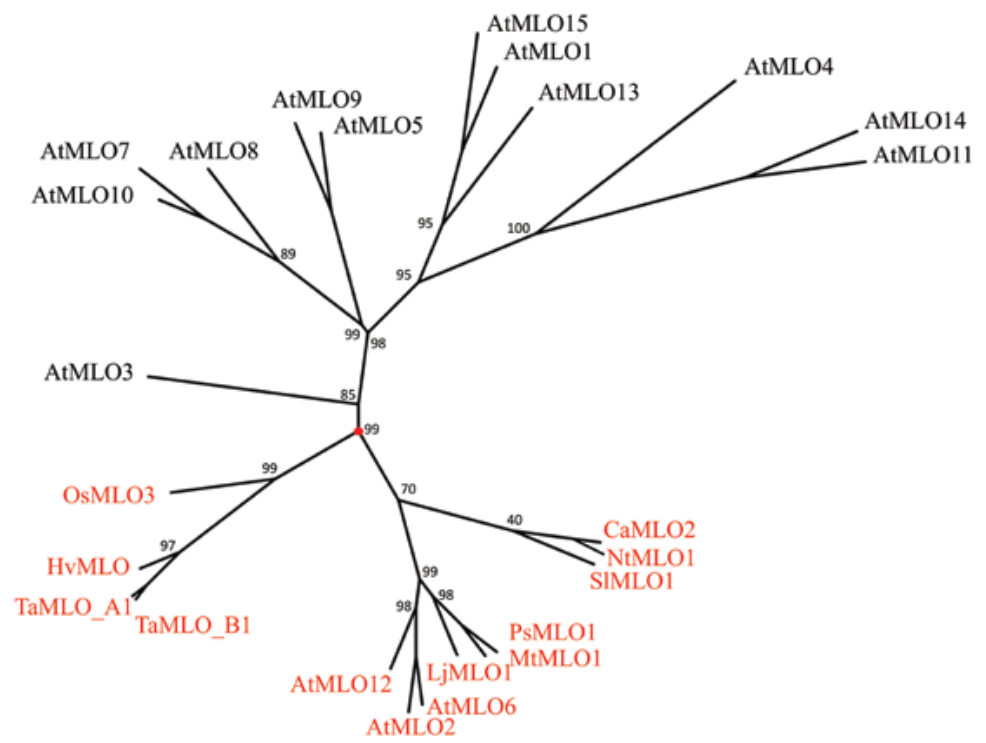

FIGURE 1. Unrooted radial phylogenetic tree of MLO powdery mildew susceptibility proteins. The tree includes, in red, all the monocot and dicot MLO homologs shown to be required for powdery mildew susceptibility (Arabidopsis AtMLO2, AtMLO6 and AtMLO12, tomato SIMLO1, pepper CaMLO2, tobacco NtMLO1, pea PsMLO1, lotus LjMLO1, barrel clover MtMLO1, barley HvMLO, wheat TaMLO_B1 and TaMLO_A1b and rice OsMLO3), and the remaining homologs of the Arabidopsis AtMLO family. Numbers at each node represent bootstrap support values (out of 100 replicates). 
protein alignment (Fig. 2). Notably, this led to the identification of 41 alignment positions in which residues invariable throughout dicots are absent in monocots, and 84 alignment positions in which residues invariable throughout monocots are absent in dicots. In 44 alignment positions, class-specific residues are replaced in the other class with residues having different properties, according to the chemical features of their side-chain group (hydrophobic, polar basic, polar acidic and polar uncharged).

\section{Adaptive relevance of class-specific molecular features supported by evolutionary analysis}

In order to make inference on the evolutionary events leading to the above mentioned class-specific molecular features, we performed a codon-based Single-Likelihood Ancestor Counting (SLAC) analysis on the difference of nonsynonymous to synonymous substitutions per nonsynonymous and synonymous sites (dN-dS). Tests were conducted to predict the evolution of each codon: neutral/dN=dS or negative (purifying) $/ \mathrm{dN}<\mathrm{dS}$. We decided to restrict the analysis to a panel of nine dicot $M L O$ susceptibility genes, as only four monocot MLO homologs have been so far associated with PM pathogenesis and the $\mathrm{dN}-\mathrm{dS}$ analysis can provide significant results only when using a sequence dataset which is not too small. We found 130 codons under significant negative selection, coding for amino acids scattered throughout MLO protein domains. Among the 130 codons, 27 are translated into class-specific residues, which are therefore predicted to provide an adaptive value (Additional file 3 ). 
AtMLO2

AtMLO 6

AtMLO12

PSMLO1

MtMLO1

LjMLO1

CaMLO2

SIMLO1

NtMLO1

TaMLO B1

TaMLO A1b

HvMLO

OsMLO3

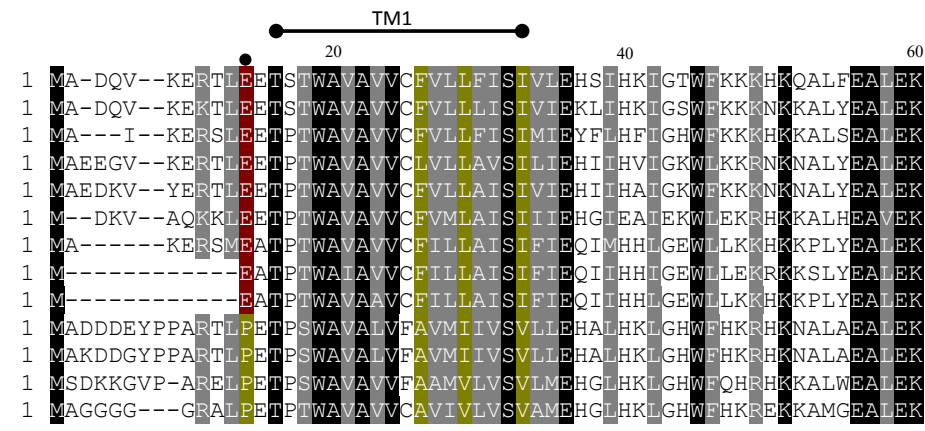

AtMLO2

AtMLO 6

AtMLO12

PsMLO1

MtMLO1

LjMLO1

CaMLO2

SIMLO1

NtMLO1

TaMLO B1

TaMLO A1b

HvMLO

OsMLO3

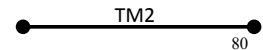

100

120

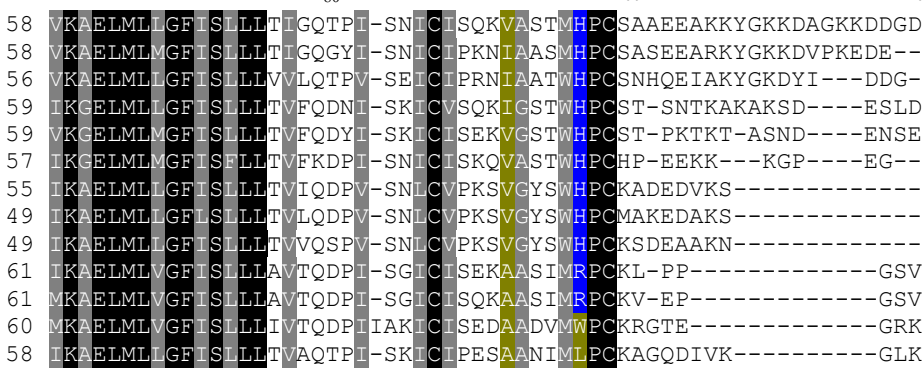

AtMLO2

140

160

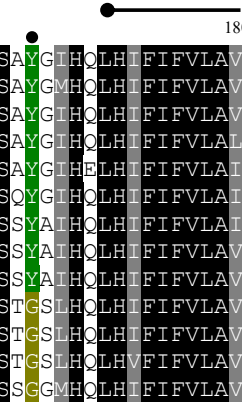

AtMLO 6

AtMLO12

PsMLO1

MtMLO1

LjMLO1

CaMLO2

SIMLO1

NtMLO1

TaMLO_B1

TaMLO_A1b

HvMLO

OSMLO3
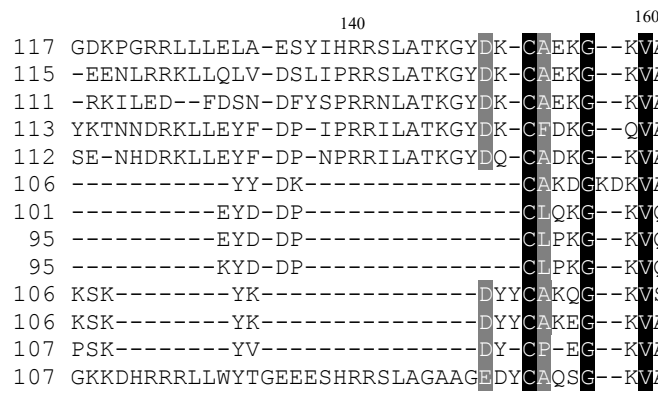

AtMLO2

AtMLO 6

AtMLO12

PsMLO1

MtMLO1

LjMLO1

CaMLO2

SIMLO1

NtMLO1

TaMLO B1

TaMLO_A1b

HvMLO

OsMLO3
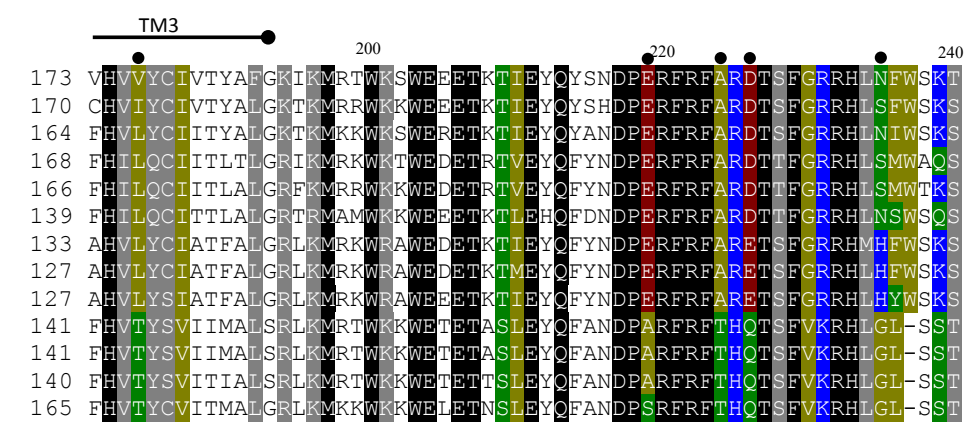

FIGURE 2. 
AtMLO2

AtMLO 6

AtMLO12

PsMLO1

MtMLO1

LjMLO1

CaMLO2

SIMLO1

NtMLO1

TAMLO B1

TaMLO_A1b

HVMLO

OsMLO3

AtMLO2

AtMLO6

AtMLO12

PSMLO1

MtMLO1

LjMLO1

CaMLO2

SIMLO1

NtMLO1

TaMLO_B1

TaMLO_A1b

HvMLO

OSMLO3

AtMLO2
AtMLO6
AtMLO12
PsMLO1
MtMLO1
LjMLO1
CaMLO2
SIMLO1
NtMLO1
TaMLO_B1
TaMLO_A1b
HvMLO
OsMLO3
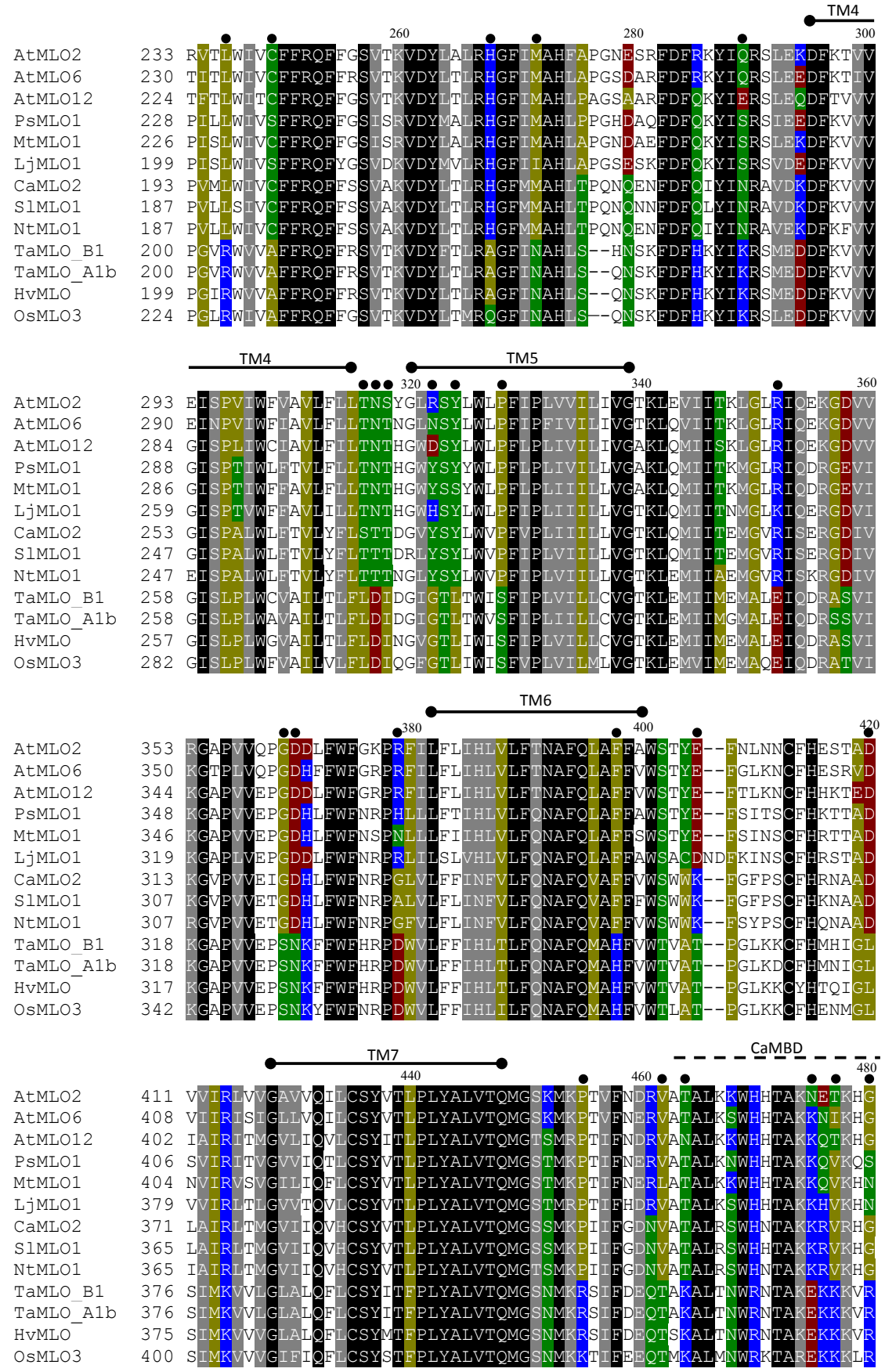

FIGURE 2. 

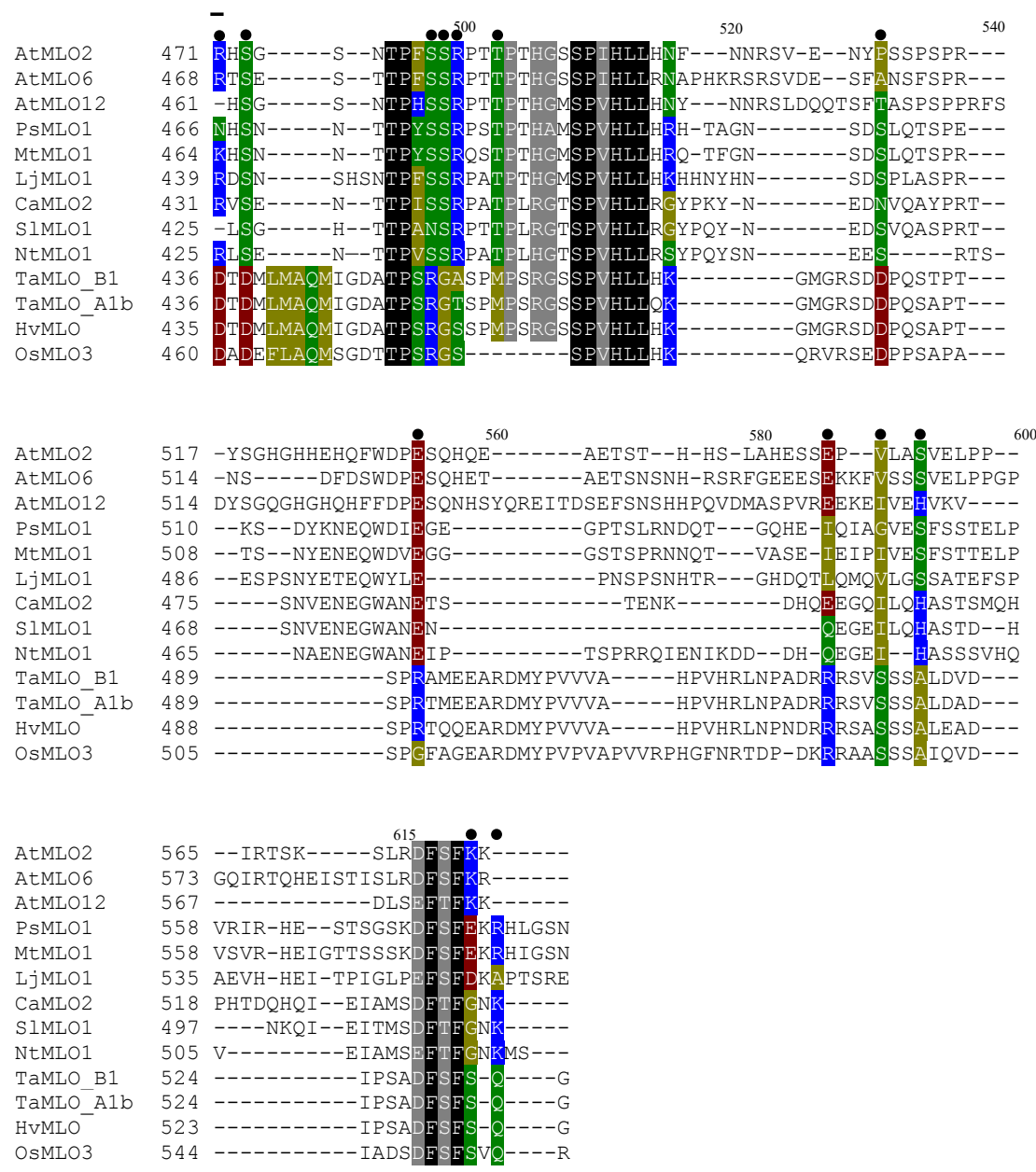

FIGURE 2. Multiple alignment of MLO powdery mildew susceptibility proteins. The dataset is composed of all the monocot (barley HvMLO, rice OsMLO3, wheat TaMLO_B1 and TaMLO_A1b), and dicot (Arabidopsis AtMLO2, AtMLO6 and AtMLO12, tomato SIMLO1, pepper CaMLO2, tobacco NtMLO1, pea PsMLO1, lotus LjMLO1 and barrel clover MtMLO1) MLO homologs shown to act as powdery mildew susceptibility factors. The positions of the seven MLO transmembrane domains (TM1-TM7) and the calmodulin binding domain (CaMBD) are identical to the ones reported by Feechan et al. (2008), Functional Plant Biology, 35: 1255-1266. Black color indicates alignment positions in which invariable residues are present. Grey color indicates alignment positions which do not contain class-specific residues and are conserved with respect to biochemical properties. Other colors indicate alignment positions in which there are class-specific residues in monocots, dicots, or both: yellow indicates hydrophobic residues $(G, A, V, L, I, F, W, M, P)$; blue indicates polar basic residues $(K, R, H)$; red indicates polar acidic residues ( $D, E)$; green indicates polar uncharged residues ( $, T, C, Y, N, Q)$. Black dots highlight 44 alignment positions in which class-specific residues are substituted in the other class by residue(s) having different biochemical properties. 


\section{Functional conservation of monocot and dicot MLO susceptibility genes}

We tested whether different molecular features between monocot and dicot MLO proteins are specifically required by PM fungal species infecting either one or the other class of Angiosperms. To this aim, we developed two constructs for the transgenic expression of a monocot (barley HvMLO) and a dicot (pea PsMLO1) MLO gene in the tomato SImlo1 line, which is homozygous for a loss-of-function mutation in the endogenous gene SIMLO1 and therefore resistant to the tomato PM fungus Oidium neolycopersici. We reasoned that complementation and restoration of PM symptoms would have occurred only in case of functional conservation between SIMLO1 and any of the two tested transgenes. In total, nineteen 35S::PsMLO1 and twenty 35S::HvMLO transformants were obtained. In both cases, eighteen individuals were obtained showing variable transgene expression levels. For each construct, three $T_{1}$ plants displaying high transgene expression (35S::PsMLO1-4,-6 and -7 and 35S::HvMLO-9,-10 and -15) were self-pollinated to generate $\mathrm{T}_{2}$ families (Additional Fig. 1). Ten individuals from each $T_{2}$ family were tested for the presence or the absence of the transgene and challenged with $O$. neolycopersici. Transgenic individuals of the three $T_{2}$ families overexpressing PsMLO1 (35S::PsMLO1_(+)) displayed PM symptoms with an average D.I. (disease index) score ranging from 2.87 to 2.92. Transgenic individuals of the three $T_{2}$ families overexpressing HvMLO (35S::HvMLO_(+)) showed an average D.l. score ranging from 1.8 to 2.4. In contrast, all non-transgenic 35S::PsMLO1_(-) and 35S::HvMLO_(-) T individuals displayed, similar to the SImlo1 plants, hardly any fungal growth (Fig. 3 and Additional file 2). For transgenic plants of the three $35 \mathrm{~S}:: \mathrm{HVMLOT}_{2}$ families, positive correlation was found between average D.I. and transgene expression level of corresponding $T_{1}$ plants (Fig. 3 and Additional file 1 and 2). Together, these results indicate that monocot and dicot MLO susceptibility genes are functionally conserved with respect to molecular features required for PM pathogenesis. 


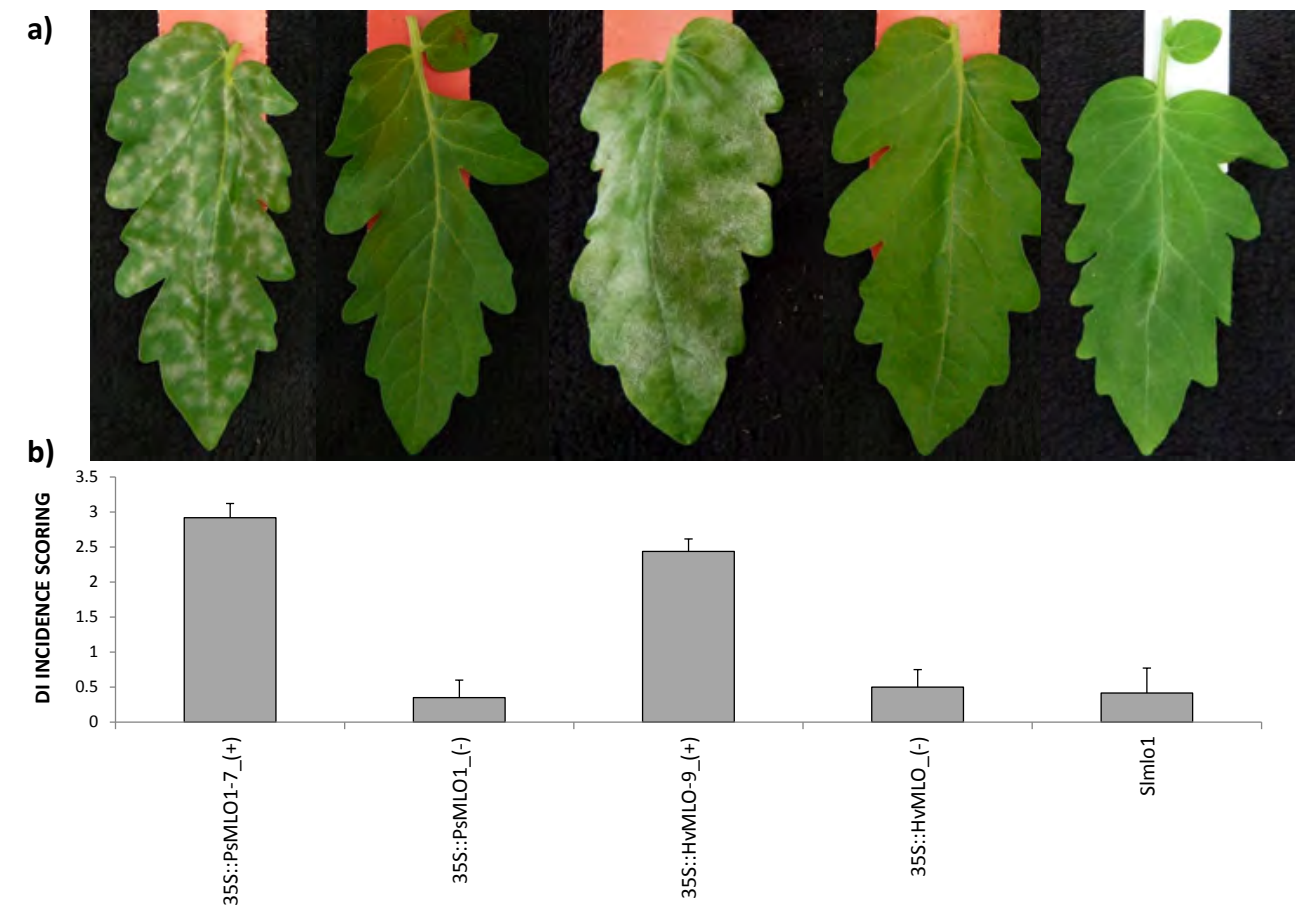

FIGURE 3. Transgenic overexpression of pea PsMLO1 and barley HvMLO in the tomato mutant line SImlo1. Panel a) shows the phenotypes of two selected individuals of the $\mathrm{T}_{2}$ family 35S::PsMLO1-7, segregating for the presence (first from the left) or the absence (second from the left) of the transgene, two selected individuals of the $\mathrm{T}_{2}$ family 35S::HVMLO-9, segregating for the presence (third from the left) or the absence (second from the right) of the transgene, and one individual of the SImlo1 line (first from the right), in response to the tomato powdery mildew fungus Oidium neolycopersici. Panel b) from left to right shows average disease index (DI) values relative to transgenic plants (+) of the 35S::PsMLO1-7 T family, non-transgenic plants (-) of three $\mathrm{T}_{2}$ families segregating for the 35S::PSMLO1 construct, transgenic plants of the 35S::HVMLO-9 $\mathrm{T}_{2}$ family, non-transgenic plants of three $\mathrm{T}_{2}$ families segregating for the 35S::HvMLO construct and the SImlo1 line. Standard deviation bars refer to six 35S::PsMLO1_(+) individuals, nine 35S::HVMLO_(+) individuals, 7 PSMLO1_(-) individuals, 7 HvMLO_(-) individuals and 10 SImlo1 individuals.

\section{Functional conservation of monocot and dicot MLO susceptibility genes in non- host interactions}

We next investigated whether functional conservation between monocot and dicot MLO homologs also holds true in non-host plant-PM interactions. To this aim, we used the PM species B. graminis f.sp. hordei ( $B g h)$ to inoculate plants of the Slmlo1 mutant line, the cultivar Moneymaker (MM), carrying wild-type SIMLO1, and two of the $35 \mathrm{~S}:: H v M L O \mathrm{~T}_{2}$ families (35S::HvMLO-9 and -10, previously described in Fig. 3, Figure S1 and S2). Bgh is an adapted PM on barley and a non-adapted PM to tomato. In the SImlo1 line, $75.4 \%$ of infection units were associated with papilla formation and $24.6 \%$ with cell death response (Fig. 4). Compared with the SImlo1 line, transgenic 35S::HvMLO-9 $\mathrm{T}_{2}$ plants displayed a 
a)

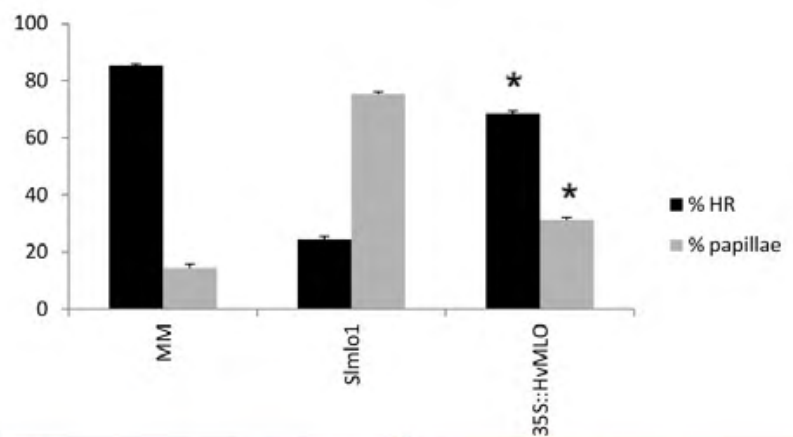

b)

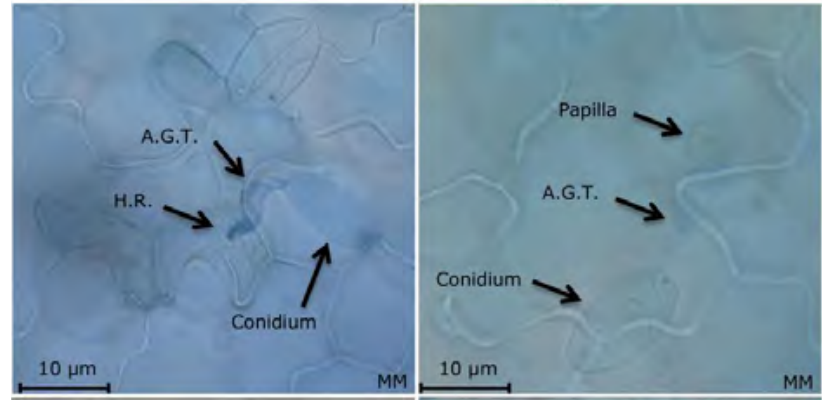

c)

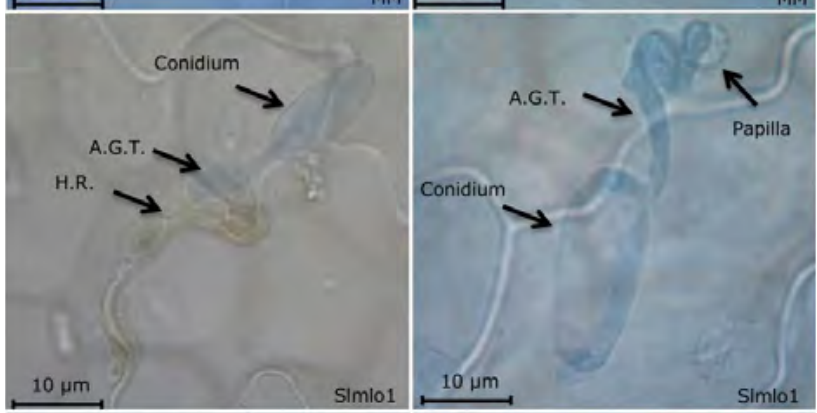

d)

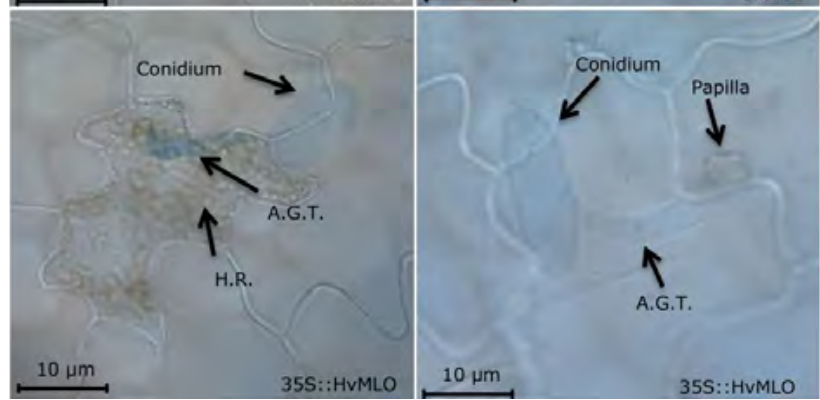

FIGURE 4. Functional conservation of SIMLO1 and HvMLO in the tomato/Blumeria graminis f.sp.hordei (Bgh) interaction. Panel a) shows the ratio of penetrated and non-penetrated epidermal cells, assessed in function of infection units showing hypersensitive response (H.R.) and papillae, respectively, in the following genotypes: the mlo mutant line SImlo1; the cultivar MM, with a similar genetic background and carrying wild-type SIMLO1; transgenic plants of a $\mathrm{T}_{2}$ family overexpressing barley HVMLO in the SImlo1 genetic background (35S::HvMLO-9). Panel b), c) and d) show, in the same genotypes, fungal structures (conidiospore and appressorium germination tube -A.G.T.-) and cellular events (the formation of papillae and H.R.) arresting fungal growth before and after penetration, respectively. 
lower level of papilla formation (31.3\%) and a higher level of cell death response (68.7\%). In MM, papilla formation and cell death occurred at a rate similar to the one in 35S::HvMLO-9 plants ( $14.6 \%$ and $85.4 \%$, respectively). Taken together, this body of evidence indicates that both HVMLO and SIMLO1 predispose to the penetration of a non-host pathogen.

\section{Discussion}

The functional characterization of $M L O$ homologs involved in PM susceptibility is of great interest for basic research on plant-microbe interactions as well as for plant breeding, as loss-of-function genotypes could be conveniently used to introduce durable and broadspectrum resistance in cultivated species [7]. Results of previous investigations indicated that mlo-based resistance in a certain plant species can be lost by the heterologous expression of $M L O$ susceptibility genes from related species of the same botanical family. Indeed, restored susceptibility has been observed in barley HVMLO mutants transformed with wheat TaMLO_B1 and rice OsMLO3, as well as in pea PsMLO1 mutants expressing lotus LjMLO1 or barrel clover MtMLO1[12,13]. Recently, similar evidence was shown on tomato SIMLO1 mutants transformed with pepper CaMLO2 or tobacco NtMLO1 [16, 17]. Here, we investigated whether complementation can also occur by transferring $M L O$ genes from more evolutionary divergent plant species. We found that, in a tomato mlo mutant background, transgenic expression of a MLO susceptibility gene from pea (a distantly related dicot species) and barley (a monocot species) is sufficient to re-establish PM susceptibility (Fig. 3 and Additional file 2). This finding indicates that, despite phylogenetic distance and the evolution of peculiar molecular traits (Fig. 1 and 2), monocot and dicot MLO proteins are essentially conserved with respect to features involved in the interaction with PM pathogens. In support of this conclusion, we show that the monocot gene HVMLO and the dicot gene SIMLO1 both enhance penetration of the non-adapted pathogen B. graminis f.sp. hordei compared to a tomato mlo-mutant (Fig. 4). Moreover, after reviewing scientific literature, we found that only one out of thirty MLO protein substitutions so far associated with PM resistance involves a class-specific residue (a monocot-specific alanine residue in position 350 of the alignment in Fig. 2) (Table 1) [22]. The same residue is replaced in dicots by a glycine (sharing similar nonpolar chemical properties of alanine, Table 1), indicating that, in this case, class-specific conservations are not associated with important changes in protein structure or function.

We cannot exclude that class-specific traits might have minor effects on interactions with PM fungi. Indeed, by comparing three independent $T_{2}$ families for each construct, we found that that overexpression of PSMLO1 results in higher D.I. index scores than the one of HVMLO (Fig. 3 and Additional file 2). Clearly, complementation tests with several other monocot and dicot transgenes could help to answer this question. 
TABLE 1. Amino acid residues in dicot AtMLO2 and monocot HvMLO whose mutation has been associated with PM resistance. For each amino-acid, localization in any of the MLO protein domains, including seven transmembrane (TM) regions, three extracellular loops (E), three intracellular (I) loops, the N-terminus and the C-terminus, is indicated.

\begin{tabular}{|c|c|c|c|c|c|c|}
\hline $\begin{array}{l}\text { Barley } \\
\text { HvMLO }\end{array}$ & $\begin{array}{l}\text { Arabidopsis } \\
\text { AtMLO2 }\end{array}$ & $\begin{array}{l}\text { Substituting } \\
\text { residue(s) in the other } \\
\text { Angiosperm class }\end{array}$ & $\begin{array}{c}\text { Conservation } \\
\text { level (\%) }\end{array}$ & Reference & $\begin{array}{c}\text { Type of } \\
\text { resistance }\end{array}$ & Domain \\
\hline R10 & & $\mathrm{R} / \mathrm{K}$ & 69,2 & [25] & full & $\mathrm{N}$-terminus \\
\hline V30 & & IN & 38,5 & [23] & full & TM1 \\
\hline \multirow[t]{2}{*}{ S31 } & & $S$ & 100 & [23] & full & TM1 \\
\hline & G66 & G & 100 & [11] & full & TM2 \\
\hline V76 & & V/I & 84,6 & [24] & partial & TM2 \\
\hline C86 & & C & 100 & [12] & full & E2 \\
\hline C98 & & C & 100 & [12] & full & E2 \\
\hline C114 & & C & 100 & [12] & full & E2 \\
\hline W159 & & W & 100 & [21] & full & 12 \\
\hline W162 & & W & 100 & [21] & full & 12 \\
\hline E163 & & $\mathrm{E}$ & 100 & [21] & full & 12 \\
\hline S187 & S220 & $\mathrm{S} / \mathrm{T}$ & 76,9 & {$[11,24]$} & full & 12 \\
\hline D219 & D253 & D & 100 & {$[11,24]$} & Partiala/full ${ }^{b}$ & 12 \\
\hline T222 & & T/V/A & 69,2 & [23] & partial & 12 \\
\hline $\mathrm{G} 226$ & & G & 100 & [23] & full & 12 \\
\hline$F 240$ & & F & 100 & [23] & partial & 12 \\
\hline D251 & D287 & D & 100 & {$[11,24]$} & Partial ${ }^{a} / f_{u} l^{b}$ & TM4 \\
\hline$\llcorner 270$ & & $F / L / I$ & 69,2 & [23] & full & TM4 \\
\hline A306 & & G & 30,7 & [22] & partial & 13 \\
\hline$\llcorner 307$ & & $\mathrm{Q} / \mathrm{L} / \mathrm{L}$ & 69,2 & [24] & partial & 13 \\
\hline $\mathrm{G} 318$ & & G & 100 & [23] & full & 13 \\
\hline P320 & & P & 100 & [24] & partial & 13 \\
\hline P324 & & $\mathrm{T} / / \mathrm{P}$ & 76,9 & [24] & partial & 13 \\
\hline$F 329$ & & $\mathrm{~F}$ & 100 & [24] & full & 13 \\
\hline W330 & & W & 100 & [24] & partial & 13 \\
\hline F331 & & F & 100 & [24] & partial & 13 \\
\hline R333 & & $R / S / K$ & 84,6 & [24] & partial & 13 \\
\hline P334 & & P & 100 & [23] & full & 13 \\
\hline C367 & & C & 100 & [12] & full & E3 \\
\hline P395 & P431 & P & 100 & {$[11,24]$} & full & TM7 \\
\hline
\end{tabular}

1) Numbers adjacent to each amino acid indicate their position in either HvMLO or AtMLO2 proteins.

2) Barley and Arabidopsis residues in the same row correspond to each other in HvMLO/AtMLO2 protein alignment.

3) Percentage of conservation is calculated based on the alignment of 13 MLO proteins functionally associated with powdery mildew susceptibility (AtMLO2, AtMLO6, AtMLO12, SIMLO1, CaMLO2, NtMLO1, PsMLO1, LjMLO1, MtMLO1, TaMLO_A1b, TaMLO_B1, OsMLO3 and HvMLO)

4) Amino acid color is according to its chemical properties: non-polar (yellow), polar, uncharged (green), polar, acidic (red), polar, basic (blue).

5) a partial resistance observed in barley, ${ }^{\text {b }}$ full resistance observed in Arabidopsis. 
Through the analysis of the $\mathrm{dN}$-dS difference, we provide evidence for negative selection acting on several class-specific residues, which are thus likely to play a major adaptive role (Additional file 3). However, as mentioned before, transgenic complementation tests indicate that these class-specific residues are not crucial for the outcome of the interaction between plants and PM pathogens. Possibly, some of the class-specific residues identified in this study might underlie roles which are not related with the interaction with PM fungi. The implication of MLO susceptibility proteins in other physiological processes would explain why, in spite of being required for pathogenesis, they have been not excluded by evolution. With this respect, it is worth to mention that PM resistance in Arabidopsis and barley mlo mutants has been associated with the induction of leaf senescence, a pleiotropic phenotype [11].

We show that $M L O$ homologs required for PM pathogenesis can complement a mlo mutant background in transgenic assays, irrespective of the phylogenetic distance between the donor and the recipient species (Fig. 3). This would be of great advantage in order to test the function of candidate $M L O$ susceptibility genes which are currently being identified by several authors across cultivated species [4,5]. Moreover, we provide an overview of MLO protein regions which are under negative selection and thus are expected to be of functional relevance. These regions represent ideal targets to select loss-of-function mutants resistant to the PM disease. With this respect, breeders may apply diverse tools, such as conventional targeted mutagenesis approaches of TILLING (targeted induced local lesions in genomes) or advanced technologies of genome editing, based on zinc finger nucleases (ZFNs), clustered regularly interspaced short palindromic repeat (CRISPR) and transcription activator-like effector nucleases (TALEN) $[26,27,28]$.

\section{Conclusion}

This work provides insights on the evolution and function of Angiosperm MLO susceptibility genes. We show that complementation assays similar to those carried out in this study are suitable for future activities aimed at the characterization of novel PM susceptibility factors across cultivated species. Moreover, we indicate a series of gene targets for the selection of loss-of-function mlo resistant mutants. 


\section{Methods}

\section{Bioinformatic analyses}

The following MLO proteins, experimentally shown to be required for PM susceptibility, were used as dataset for CLUSTAL alignment using the CLC sequence viewer software (http://clcbio.com): Arabidopsis AtMLO2 [GenBank: NP172598], AtMLO6 [GeneBank: NP176350] and AtMLO12 [GeneBank: NP565902], tomato SIMLO1 [GeneBank: NP001234814], pea PsMLO1 [GeneBank: ACO07297], pepper CaMLO2 [GeneBank: AFH68055], lotus LjMLO1 [GeneBank: AAX77015], barrel clover MtMLO1 [GeneBank: ADV40949], barley HvMLO [GeneBank: P93766], rice OsMLO3 [GeneBank: AAK94907], wheat TaMLO_B1 [GeneBank: AAK94904] and TaMLO_A1b [GeneBank: AAK94905]. The alignment was given to Geneious v8 software (http://www.geneious.com, [29] ), to highlight amino acids with different polarity, and the online web service Phylogeny.fr (http://www.phylogeny.fr/) to construct an unrooted radial phylogenetic tree.

In order to make predictions on the type of evolution (negative or neutral) of classspecific molecular features, all the above mentioned dicot $M L O$ susceptibility genes were used as dataset for a codon-based evolutionary analysis based on the difference of nonsynonymous-to-synonymous substitutions per nonsynonymous and synonymous sites (dN/dS). This was performed by using the Single-likelihood Ancestor Counting (SLAC) method implemented by the Datamonkey web server (www.datamonkey.org). The default $p$-value of 0.1 was taken as threshold to call codons under significant negative selection.

\section{Isolation and cloning of full-length PsMLO1 and HvMLO}

Total RNAs from pea (cultivar Sprinter) and barley (cultivar Maythorpe) were isolated by using the RNeasy plant mini kit (Qiagen), and corresponding cDNAs were synthesized by using the SuperScript III first-strand synthesis kit (Invitrogen) and the oligo(dT) primer. Specific primer pairs, named PsMLO1-Fw/PsMLO1-Rev and HvMLO-Fw/HvMLORev (Additional file 4: table S2) were manually designed in order to amplify the PSMLO1 and HvMLO full-length coding sequences, respectively. PCR reactions were performed by using the high-fidelity Phusion DNA polymerase (New England Biolabs) and an annealing temperature of $55^{\circ} \mathrm{C}$. Amplicons were ligated into the Gateway-compatible vector pENTR D-TOPO (Invitrogen) and cloned into the E. coli One Shot ${ }^{\circledR}$ TOP10 cells (Invitrogen), according to the manufacturer's instructions. After selecting positive colonies by colony $\mathrm{PCR}$, using the two gene-specific primer pairs above mentioned, recombinant plasmids were extracted and their inserts were sequenced. A single colony for each construct was selected, in which the inserts resulted to have sequences identical to those of HVMLO and PSMLO1 deposited in the NCBI database. 


\section{Generation and functional characterization of transgenic SIMLO1 mutant tomato plants expressing PsMLO1 and HvMLO}

Following the manufacturer instructions (Invitrogen), cloned HVMLO and PSMLO1 gene sequences were inserted by LR recombination into the binary plasmid vector pK7WG2, which harbors the $35 \mathrm{~S}$ Cauliflower Mosaic Virus (CaMV) promoter and the marker gene nptll for kanamycin resistance selection. Plasmids were then transferred to E. coli and positive colonies were screened by colony PCR and sequencing, as previously mentioned. Finally, recombinant vectors were extracted and transferred to the AGL1-virG strain of $A$. tumefaciens by electroporation.

The transformation of the tomato ol-2 mutant line, carrying a loss-of-function mutation of the PM susceptibility gene SIMLO1, was performed according to the methods described by [6] and [16]. The evaluation of the expression levels of PSMLO1 and HVMLO in $\mathrm{T}_{1}$ plants was carried out by real-time qPCR using the primer pairs qPsMLO1-Fw/qPsMLO1-Rev and qHvMLO-Fw/qHvMLO-Rev (Additional file 4). A primer pair designed on the elongation factor $1 a$ gene (qEF-Fw/qEF-Rev) was used for relative quantification (Additional file 4).

\section{Functional characterization of host and non-host interactions}

For each of the two transgenes above mentioned, three $T_{1}$ individuals showing the highest expression levels were allowed to self-pollinate, resulting in a total of six $T_{2}$ families. Individuals of each family were assayed for the presence/absence of the overexpression construct by means of PCR, using the primer pairs NPTII_FW/ NPTII_Rev and 35SFw / 35S-Rev designed on the nptll marker gene and the 35S promoter, respectively (Additional file 4). Ten resistant SImlo1 plants carrying the loss-of-function SIMLO1 allele and ten individuals of each family were challenged with an isolate of the tomato PM fungus $O$. neolycopersici maintained at the Plant Breeding Department of the University of Wageningen, The Netherlands. Inoculation was performed as described by [30], spraying 4 weeks-old plants with a suspension of conidiospores obtained from freshly sporulating leaves of heavily infected plants and adjusted to a final concentration of $4 \times 10^{4}$ spores/ $\mathrm{ml}$. Inoculated plants were grown in a greenhouse compartment at $20 \pm 2^{\circ} \mathrm{C}$ with $70 \pm 15 \%$ relative humidity. Disease evaluation was visually carried out 15 days after inoculation, based on the presence of disease signs on the third and fourth leaf, according to the scale from 0 to 3 reported by [10].

For the functional characterization of a non-host interaction, seeds from one of the three 35S::HVMLO T 2 families previously tested were surface-sterilized and sown on halfstrength Murashige and Skoog (MS) agar supplemented with $50 \mathrm{mg} / \mathrm{ml}$ kanamycin for selection of transgenic plants. Seeds were left for 2 days at $4^{\circ} \mathrm{C}$ and then transferred to a growing chamber for 10 days. Five transgenic seedlings were transplanted in pots and 
transferred to a greenhouse compartment. Three barley plants of the PM susceptible cultivar Manchuria, five SImlo1 plants and five MoneyMaker plants were used as controls. An isolate of $B$. graminis f. sp. hordei (Bgh) collected in Wageningen (Wag.04) was used for the inoculation. This was performed by rubbing Manchuria leaves heavily infected with Bgh on the third tomato leaf. After 72 hours, in which inoculated plants were kept in a climate chamber at $20^{\circ} \mathrm{C}, 16$ hours of light/day and $70 \% \mathrm{RH}$, a $4 \mathrm{~cm}^{2}$ segment was cut from the inoculated leaves (third leaf). Three samples were taken from 3 plants of each genotype.

Each leaf segment was bleached is a 1:3 $(\mathrm{v} / \mathrm{v})$ acetic-acid/ethanol solution and $48 \mathrm{hrs}$ later stained in $0.005 \%$ Trypan Blue as described by [31]. The rate of fungal penetration was estimated by the frequency of infection units showing epidermal cell death. For each genotype, three biological replicates were considered, considering at least 100 infection units. 


\section{References}

1. Devoto A, Hartmann HA, Piffanelli P, Elliott C, Simmons C, Taramino G, Goh CS, Cohen FE, Emerson BC, Schulze-Lefert $P$ et al: Molecular phylogeny and evolution of the plant-specific seven-transmembrane MLO family. Journal of Molecular Evolution 2003, 56(1):77-88.

2. Feechan A, Jermakow AM, Torregrosa L, Panstruga R, Dry IB: Identification of grapevine MLO gene candidates involved in susceptibility to powdery mildew. Functional Plant Biology 2008, 35(12):12551266.

3. Liu Q, Zhu H: Molecular evolution of the MLO gene family in Oryza sativa and their functional divergence. Gene 2008, 409(1-2):1-10.

4. Pessina S, Pavan S, Catalano D, Gallotta A, Visser R, Bai Y, Malnoy M, Schouten H: Characterization of the MLO gene family in Rosaceae and gene expression analysis in Malus domestica. BMC Genomics 2014, 15(1):618.

5. Zhou SJ, Jing Z, Shi JL: Genome-wide identification, characterization, and expression analysis of the MLO gene family in Cucumis sativus. Genet Mol Res 2013, 12(4):6565-6578.

6. Singh VK, Singh AK, Chand R, Singh BD: Genome wide analysis of disease resistance mlo gene family in sorghum [Sorghum bicolor (I.) Moench]. J Plant Genom 2012, 2(1): 18-27.

7. Pavan S, Jacobsen E, Visser RGF, Bai Y: Loss of susceptibility as a novel breeding strategy for durable and broad-spectrum resistance. Molecular Breeding 2009, 25(1):1-12.

8. Büschges R, Hollricher K, Panstruga R, Simons G, Wolter M, Frijters A, Van Daelen R, Van der Lee T, Diergaarde $\mathrm{P}$, Groenendijk J et al: The barley Mlo gene: A novel control element of plant pathogen resistance. Cell 1997, 88(5):695-705.

9. Panstruga R: Discovery of novel conserved peptide domains by ortholog comparison within plant multi-protein families. Plant Molecular Biology 2005, 59(3):485-500.

10. Bai Y, Pavan S, Zheng Z, Zappel NF, Reinstädler A, Lotti C, De Giovanni C, Ricciardi L, Lindhout P, Visser R et al: Naturally occurring broad-spectrum powdery mildew resistance in a Central American tomato accession is caused by loss of Mlo function. Molecular Plant-Microbe Interactions 2008, 21(1):30-39.

11. Consonni C, Humphry ME, Hartmann HA, Livaja M, Durner J, Westphal L, Vogel J, Lipka V, Kemmerling $\mathrm{B}$, Schulze-Lefert $\mathrm{P}$ et al: Conserved requirement for a plant host cell protein in powdery mildew pathogenesis. Nature Genetics 2006, 38(6):716-720.

12. Elliott C, Müller J, Miklis M, Bhat RA, Schulze-Lefert P, Panstruga R: Conserved extracellular cysteine residues and cytoplasmic loop-loop interplay are required for functionality of the heptahelical MLO protein. Biochemical Journal 2005, 385(1):243-254.

13. Humphry M, Reinstädler A, Ivanov S, Bisseling T, Panstruga R: Durable broad-spectrum powdery mildew resistance in pea er1 plants is conferred by natural loss-of-function mutations in PsML01. Molecular Plant Pathology 2011, 12(9):866-878.

14. Pavan S, Schiavulli A, Appiano M, Marcotrigiano AR, Cillo F, Visser RGF, Bai Y, Lotti C, Ricciardi L: Pea powdery mildew er 1 resistance is associated to loss-of-function mutations at a MLO homologous locus. Theoretical and Applied Genetics 2011, 123(8):1425-1431.

15. Várallyay É, Giczey G, Burgyán J: Virus-induced gene silencing of Mlo genes induces powdery mildew resistance in Triticum aestivum. Archives of Virology 2012, 157(7):1345-1350.

16. Zheng Z, Nonomura T, Appiano M, Pavan S, Matsuda Y, Toyoda H, Wolters AMA, Visser RGF, Bai Y: Loss of Function in Mlo Orthologs Reduces Susceptibility of Pepper and Tomato to Powdery Mildew Disease Caused by Leveillula taurica. PLOS ONE 2013, 8(7).

17. Appiano M, Pavan S, Catalano D, Zheng Z, Bracuto V, Lotti C, Visser RF, Ricciardi L, Bai Y: Identification of candidate MLO powdery mildew susceptibility genes in cultivated Solanaceae and functional characterization of tobacco NtMLO1. Transgenic Res 2015:1-12.

18. Peterhansel C, Freialdenhoven A, Kurth J, Kolsch R, Schulze-Lefert P: Interaction Analyses of Genes Required for Resistance Responses to Powdery Mildew in Barley Reveal Distinct Pathways Leading to Leaf Cell Death. The Plant Cell 1997, 9(8):1397-1409. 
19. Lipka V, Dittgen J, Bednarek P, Bhat R, Wiermer M, Stein M, Landtag J, Brandt W, Rosahl S, Scheel D et al: Pre- and Postinvasion Defenses Both Contribute to Nonhost Resistance in Arabidopsis. Science 2005, 310(5751):1180-1183.

20. Devoto A, Piffanelli P, Nilsson I, Wallin E, Panstruga R, Von Heijne G, Schulze-Lefert P: Topology, subcellular localization, and sequence diversity of the Mlo family in plants. Journal of Biological Chemistry 1999, 274(49):34993-35004.

21. Müller J, Piffanelli P, Devoto A, Miklis M, Elliott C, Ortmann B, Schulze-Lefert P, Panstruga R: Conserved ERAD-like quality control of a plant polytopic membrane protein. 2005, 17(1):149-163.

22. Panstruga R, Molina-Cano JL, Reinstädler A, Müller J: Molecular characterization of mlo mutants in North American two- and six-rowed malting barley cultivars. 2005, 6(3):315-320.

23. Piffanelli P, Zhou F, Casais C, Orme J, Jarosch B, Schaffrath U, Collins NC, Panstruga R, Schulze-Lefert P: The barley MLO modulator of defense and cell death is responsive to biotic and abiotic stress stimuli. 2002, 129(3):1076-1085.

24. Reinstädler A, Müller J, Czembor JH, Piffanelli P, Panstruga R: Novel induced mlo mutant alleles in combination with site-directed mutagenesis reveal functionally important domains in the heptahelical barley Mlo protein. BMC Plant Biology 2010, 10.

25. Wiberg A: Sources of resistance to powdery mildew in barley. 1974, 78(1):1-40.

26. McCallum CM, Comai L, Greene EA, Henikoff S: Targeting Induced Local Lesions IN Genomes (TILLING) for Plant Functional Genomics. Plant Physiology 2000, 123(2):439-442.

27. Gaj T, Gersbach CA, Barbas CF, III:ZFN, TALEN, and CRISPR/Cas-based methods for genome engineering. Trends in Biotechnology, 31(7):397-405.

28. Terns RM, Terns MP: CRISPR-based technologies: prokaryotic defense weapons repurposed. Trends in Genetics, 30(3):111-118.

29. Kearse M, Moir R, Wilson A, Stones-Havas S, Cheung M, Sturrock S, Buxton S, Cooper A, Markowitz S, Duran C et al: Geneious Basic: An integrated and extendable desktop software platform for the organization and analysis of sequence data. Bioinformatics 2012, 28(12):1647-1649.

30. Pavan S, Zheng Z, Borisova M, Van Den Berg P, Lotti C, De Giovanni C, Lindhout P, De Jong H, Ricciardi L, Visser RGF et al: Map- vs. homology-based cloning for the recessive gene ol-2 conferring resistance to tomato powdery mildew. Euphytica 2008, 162(1):91-98.

31. Anker C, Niks R: Prehaustorial resistance to the wheat leaf rust fungus, Puccinia triticina, in Triticum monococcum (s.s.). Euphytica 2001, 117(3):209-215. 


\section{Additional material}

a)

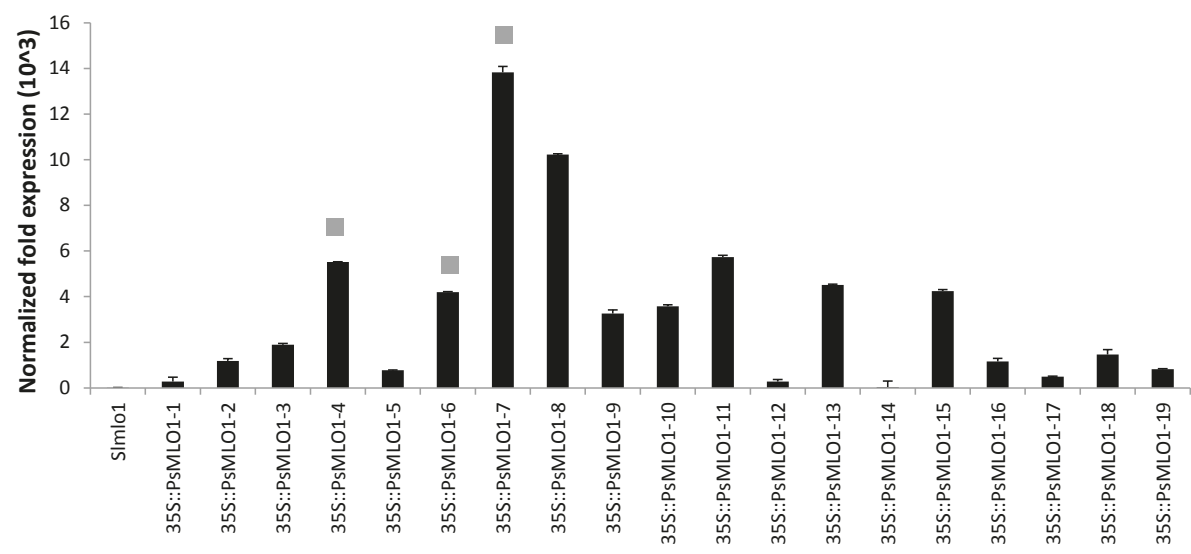

b)

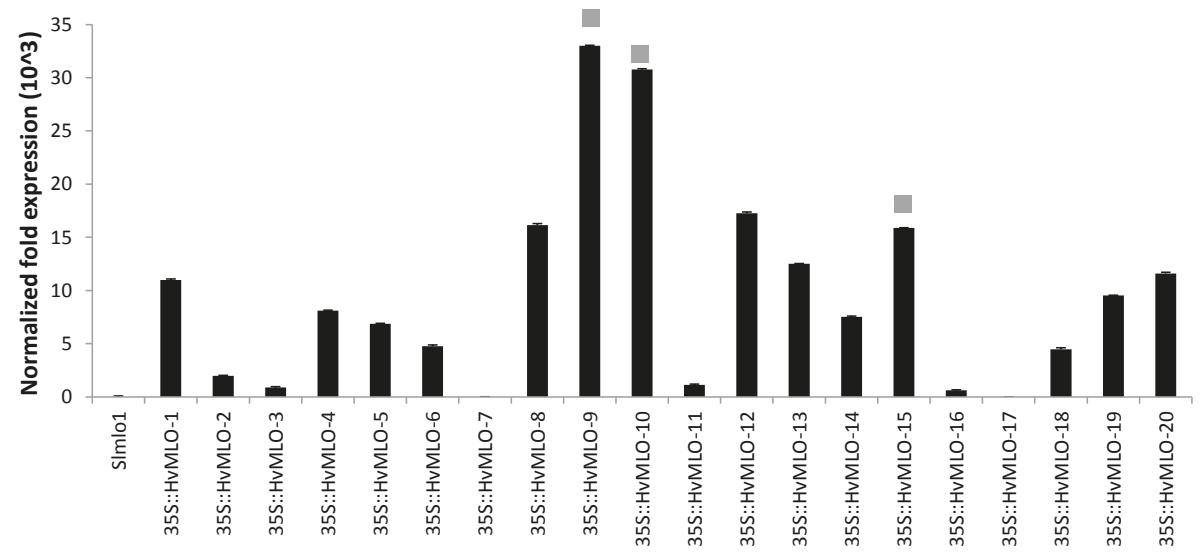

ADDITIONAL FILE 1. Figure S1. Expression levels of PSMLO1 and HvMLO after transformation. Panel A) and panel B) show the expression of PSMLO1 and HVMLO in 19 and $20 \mathrm{~T}_{1}$ individuals, respectively, which were obtained by the transformation of the tomato mutant line SImlo1, harboring a loss-of-function mutation of the endogenous SIMLO1 gene. Asterisks indicate $\mathrm{T}_{1}$ individuals selected for self-pollination and the development of $\mathrm{T}_{2}$ families. 


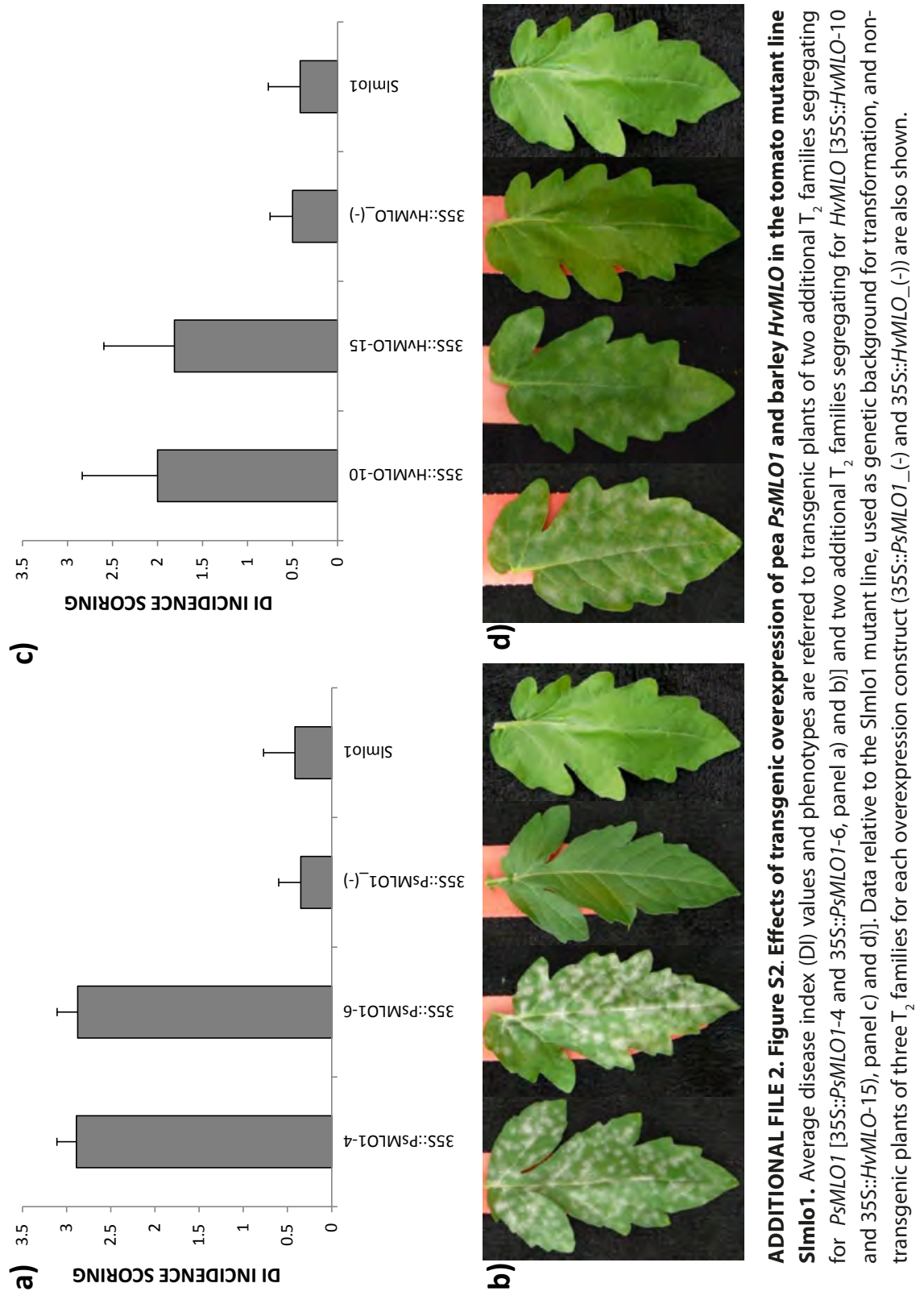


ADDITIONAL FILE 3. Table S1. Codons under significant negative selection in PM susceptibility genes. Codon numbers refer to positions in the alignment of nine dicot MLO genes (AtMLO2, AtMLO6, AtMLO12, PsMLO1, MtMLO1, LjMLO1, CaMLO2, SIMLO1, NtMLO1) experimentally shown to act as powdery mildew susceptibility genes. Amino acid residues corresponding to each codon in barley HvMLO and pea PsMLO1 are indicated. For each residue, localization in any of the MLO protein domains, including seven transmembrane (TM) regions, three extracellular loops (E), three intracellular (I) loops, the $\mathrm{N}$-terminus and the C-terminus, is indicated. Codons marked in bold are translated into class-specific residues. The threshold p-value was 0.1, representing the default value for Single-likelihood Ancestor Counting (SLAC) analysis implemented by the Datamonkey web server.

\begin{tabular}{|c|c|c|c|c|c|c|}
\hline Codon & dN-dS & $\begin{array}{l}\text { Normalized } \\
\text { dN-dS }\end{array}$ & p-value & $\begin{array}{c}\text { Residue } \\
\text { in HvMLO }\end{array}$ & $\begin{array}{c}\text { Residue } \\
\text { in PsMLO1 }\end{array}$ & Domain \\
\hline 8 & -4.82 & -2.70 & 0.021 & A9 & E8 & $\mathrm{N}$-terminus \\
\hline 14 & -3.29 & -1.85 & 0.012 & T15 & T14 & TM1 \\
\hline 16 & -4.12 & -2.31 & 0.004 & S17 & T16 & TM1 \\
\hline 18 & -3.30 & -1.85 & 0.012 & A19 & A18 & TM1 \\
\hline 20 & -3.30 & -1.85 & 0.012 & $\mathrm{~A} 21$ & A20 & TM1 \\
\hline 21 & -2.88 & -1.61 & 0.045 & V22 & V21 & TM1 \\
\hline 24 & -2.60 & -1.46 & 0.091 & A25 & L24 & TM1 \\
\hline 27 & -2.92 & -1.64 & 0.020 & V28 & L27 & TM1 \\
\hline 30 & -2.47 & -1.38 & 0.044 & S31 & S30 & TM1 \\
\hline 31 & -2.02 & -1.13 & 0.073 & V32 & 131 & TM1 \\
\hline 49 & -4.87 & -2.73 & 0.007 & K50 & K49 & 11 \\
\hline 55 & -2.47 & -1.38 & 0.037 & A56 & A55 & 11 \\
\hline 58 & -3.16 & -1.77 & 0.037 & K59 & K58 & 11 \\
\hline 62 & -4.83 & -2.71 & 0.006 & E63 & E62 & 11 \\
\hline 65 & -3.49 & -1.96 & 0.012 & L66 & L65 & TM2 \\
\hline 72 & -3.09 & -1.73 & 0.018 & L73 & L72 & TM2 \\
\hline 73 & -2.93 & -1.64 & 0.025 & L74 & L73 & TM2 \\
\hline 81 & -2.47 & -1.38 & 0.040 & A83 & S81 & E1 \\
\hline 83 & -2.88 & -1.62 & 0.057 & 185 & 183 & E1 \\
\hline 123 & -6.11 & -3.42 & 0.072 & I & D118 & E1 \\
\hline 137 & -7.01 & -3.93 & 0.023 & I & R131 & E1 \\
\hline 142 & -3.30 & -1.85 & 0.085 & I & K136 & E1 \\
\hline 145 & -3.26 & -1.83 & 0.034 & D112 & D139 & E1 \\
\hline 153 & -4.12 & -2.31 & 0.004 & V119 & V147 & E1 \\
\hline 162 & -2.98 & -1.67 & 0.034 & $\mathrm{H} 128$ & H156 & E1 \\
\hline 165 & -2.98 & -1.67 & 0.034 & H131 & H159 & TM3 \\
\hline 166 & -2.80 & -1.57 & 0.038 & V132 & 1160 & TM3 \\
\hline 170 & -2.47 & -1.38 & 0.037 & V136 & V164 & TM3 \\
\hline 171 & -2.71 & -1.52 & 0.029 & L137 & L165 & TM3 \\
\hline 172 & -2.47 & -1.38 & 0.037 & A138 & A166 & TM3 \\
\hline 176 & -3.94 & -2.21 & 0.014 & V142 & 1170 & TM3 \\
\hline 180 & -2.56 & -1.44 & 0.046 & V146 & I174 & TM3 \\
\hline 186 & -2.47 & -1.38 & 0.044 & S152 & G180 & TM3 \\
\hline 197 & -4.76 & -2.67 & 0.007 & E163 & E191 & 12 \\
\hline 199 & -3.32 & -1.86 & 0.032 & E165 & E193 & 12 \\
\hline 201 & -2.84 & -1.59 & 0.094 & T167 & R195 & 12 \\
\hline 202 & -2.47 & -1.38 & 0.037 & S168 & T196 & 12 \\
\hline 204 & -3.23 & -1.81 & 0.034 & E170 & E198 & 12 \\
\hline
\end{tabular}




\begin{tabular}{|c|c|c|c|c|c|c|}
\hline Codon & $d N-d S$ & $\begin{array}{l}\text { Normalized } \\
\text { dN-dS }\end{array}$ & p-value & $\begin{array}{c}\text { Residue } \\
\text { in HvMLO }\end{array}$ & $\begin{array}{c}\text { Residue } \\
\text { in PsML01 }\end{array}$ & Domain \\
\hline 207 & -2.62 & -1.47 & 0.099 & F173 & F201 & 12 \\
\hline 209 & -2.65 & -1.48 & 0.089 & N175 & N203 & 12 \\
\hline 211 & -2.47 & -1.38 & 0.037 & P177 & P205 & 12 \\
\hline 214 & -4.47 & -2.51 & 0.006 & F180 & F208 & 12 \\
\hline 215 & -3.49 & -1.96 & 0.016 & R181 & R209 & 12 \\
\hline 218 & -2.17 & -1.22 & 0.065 & H184 & R212 & 12 \\
\hline 219 & -2.67 & -1.50 & 0.090 & Q185 & D213 & 12 \\
\hline 222 & -2.98 & -1.67 & 0.034 & F188 & F216 & 12 \\
\hline 224 & -4.09 & -2.30 & 0.007 & K190 & R218 & 12 \\
\hline 225 & -2.28 & -1.28 & 0.063 & R191 & R219 & 12 \\
\hline 226 & -2.98 & -1.67 & 0.034 & H192 & $\mathrm{H} 22 \mathrm{O}$ & 12 \\
\hline 233 & -3.63 & -2.03 & 0.030 & T198 & S227 & 12 \\
\hline 234 & -3.55 & -1.98 & 0.052 & P199 & P228 & 12 \\
\hline 237 & -2.62 & -1.47 & 0.036 & R202 & L231 & 12 \\
\hline 239 & -2.24 & -1.25 & 0.060 & V204 & 1233 & 12 \\
\hline 243 & -2.98 & -1.67 & 0.034 & F208 & F237 & 12 \\
\hline 244 & -3.47 & -1.94 & 0.017 & R209 & R238 & 12 \\
\hline 247 & -2.64 & -1.48 & 0.090 & F212 & F241 & 12 \\
\hline 250 & -3.79 & -2.13 & 0.016 & V215 & 1244 & 12 \\
\hline 254 & -2.98 & -1.67 & 0.034 & D219 & D248 & 12 \\
\hline 258 & -2.94 & -1.65 & 0.022 & L223 & L252 & 12 \\
\hline 259 & -2.26 & -1.27 & 0.069 & R224 & R253 & 12 \\
\hline 261 & -2.47 & -1.38 & 0.040 & G226 & G255 & 12 \\
\hline 262 & -2.98 & -1.67 & 0.034 & F227 & F256 & 12 \\
\hline 265 & -3.29 & -1.85 & 0.012 & A230 & A259 & 12 \\
\hline 270 & -2.77 & -1.55 & 0.097 & I & G264 & 12 \\
\hline 280 & -2.98 & -1.67 & 0.047 & Y243 & Y274 & 12 \\
\hline 281 & -3.05 & -1.71 & 0.019 & 1244 & 1275 & 12 \\
\hline 288 & -4.47 & -2.51 & 0.006 & D251 & D282 & 12 \\
\hline 293 & -3.29 & -1.85 & 0.012 & V256 & V287 & TM4 \\
\hline 296 & -4.13 & -2.32 & 0.021 & S259 & S290 & TM4 \\
\hline 297 & -2.47 & -1.38 & 0.037 & L260 & P291 & TM4 \\
\hline 302 & -4.42 & -2.48 & 0.022 & V265 & F296 & TM4 \\
\hline 304 & -2.47 & -1.38 & 0.037 & 1267 & V298 & TM4 \\
\hline 308 & -2.83 & -1.59 & 0.027 & F271 & L302 & TM4 \\
\hline 309 & -2.88 & -1.61 & 0.045 & L272 & Т303 & E2 \\
\hline 321 & -2.47 & -1.38 & 0.037 & S284 & P315 & TM5 \\
\hline 324 & -2.47 & -1.38 & 0.037 & P287 & P318 & TM5 \\
\hline 325 & -2.78 & -1.56 & 0.099 & L288 & L319 & TM5 \\
\hline 328 & -4.16 & -2.34 & 0.008 & L291 & 1322 & TM5 \\
\hline 329 & -2.95 & -1.65 & 0.030 & L292 & L323 & TM5 \\
\hline 330 & -4.67 & -2.62 & 0.008 & C293 & L324 & TM5 \\
\hline 331 & -2.47 & -1.38 & 0.037 & V294 & V325 & TM5 \\
\hline 332 & -3.29 & -1.85 & 0.016 & G295 & G326 & TM5 \\
\hline 334 & -3.35 & -1.88 & 0.033 & K297 & K328 & 13 \\
\hline 338 & -2.36 & -1.32 & 0.054 & 1301 & 1332 & 13 \\
\hline
\end{tabular}




\begin{tabular}{|c|c|c|c|c|c|c|}
\hline Codon & $d N-d S$ & $\begin{array}{l}\text { Normalized } \\
\text { dN-dS }\end{array}$ & p-value & $\begin{array}{c}\text { Residue } \\
\text { in HvMLO }\end{array}$ & $\begin{array}{c}\text { Residue } \\
\text { in PsML01 }\end{array}$ & Domain \\
\hline 342 & -5.60 & -3.136 & 0.010 & M305 & M336 & 13 \\
\hline 343 & -2.47 & -1.38 & 0.046 & A306 & G337 & 13 \\
\hline 348 & -4.27 & -2.40 & 0.042 & D311 & D342 & 13 \\
\hline 350 & -2.47 & -1.38 & 0.045 & A313 & G344 & 13 \\
\hline 351 & -2.69 & -1.51 & 0.088 & S314 & E345 & 13 \\
\hline 357 & -3.29 & -1.85 & 0.012 & P320 & P351 & 13 \\
\hline 360 & -2.79 & -1.57 & 0.096 & E323 & E354 & 13 \\
\hline 361 & -4.13 & -2.32 & 0.019 & P324 & P355 & 13 \\
\hline 366 & -2.98 & -1.67 & 0.034 & F329 & F360 & 13 \\
\hline 368 & -4.47 & -2.51 & 0.006 & F331 & F362 & 13 \\
\hline 375 & -2.45 & -1.38 & 0.038 & L338 & L369 & TM6 \\
\hline 376 & -2.54 & -1.42 & 0.095 & F339 & F370 & TM6 \\
\hline 381 & -3.29 & -1.85 & 0.012 & T344 & V375 & TM6 \\
\hline 383 & -2.98 & -1.67 & 0.034 & F346 & F377 & TM6 \\
\hline 386 & -2.47 & -1.38 & 0.037 & A349 & A380 & TM6 \\
\hline 392 & -2.98 & -1.67 & 0.034 & F355 & F386 & TM6 \\
\hline 401 & -2.98 & -1.67 & 0.034 & P363 & F393 & E3 \\
\hline 408 & -2.98 & -1.67 & 0.033 & H370 & $\mathrm{H} 400$ & E3 \\
\hline 413 & -4.47 & -2.51 & 0.006 & L375 & D405 & E3 \\
\hline 416 & -4.37 & -2.45 & 0.004 & M377 & 1408 & E3 \\
\hline 417 & -2.33 & -1.31 & 0.065 & K378 & R409 & E3 \\
\hline 424 & -3.20 & -1.79 & 0.067 & L385 & 1416 & TM7 \\
\hline 425 & -4.86 & -2.73 & 0.009 & Q386 & Q417 & TM7 \\
\hline 430 & -2.98 & -1.67 & 0.047 & Y391 & Y422 & TM7 \\
\hline 433 & -2.91 & -1.63 & 0.021 & F394 & L425 & TM7 \\
\hline 435 & -3.19 & -1.79 & 0.014 & L396 & L427 & TM7 \\
\hline 438 & -2.01 & -1.12 & 0.076 & L399 & L430 & TM7 \\
\hline 443 & -2.47 & -1.38 & 0.038 & G404 & G435 & C-terminus \\
\hline 447 & -3.92 & -2.20 & 0.054 & K408 & K439 & C-terminus \\
\hline 448 & -2.47 & -1.38 & 0.037 & R409 & P440 & C-terminus \\
\hline 450 & -2.74 & -1.54 & 0.059 & 1411 & 1442 & C-terminus \\
\hline 451 & -2.98 & -1.67 & 0.034 & F412 & F443 & C-terminus \\
\hline 458 & -2.47 & -1.38 & 0.037 & A419 & A450 & C-terminus \\
\hline 459 & -3.49 & -1.96 & 0.012 & L420 & L451 & C-terminus \\
\hline 466 & -3.29 & -1.85 & 0.012 & A427 & A458 & C-terminus \\
\hline 468 & -2.86 & -1.61 & 0.093 & E429 & K460 & C-terminus \\
\hline 472 & -4.14 & -2.32 & 0.022 & V433 & Q464 & C-terminus \\
\hline 476 & -3.29 & -1.85 & 0.022 & D437 & S468 & C-terminus \\
\hline 483 & -2.47 & -1.38 & 0.037 & P449 & P473 & C-terminus \\
\hline 487 & -5.72 & -3.21 & 0.001 & S453 & R477 & C-terminus \\
\hline 496 & -3.29 & -1.85 & 0.013 & S462 & S486 & C-terminus \\
\hline 497 & -4.12 & -2.31 & 0.004 & P463 & P487 & C-terminus \\
\hline 499 & -2.98 & -1.67 & 0.034 & $\mathrm{H} 465$ & H489 & C-terminus \\
\hline 500 & -3.36 & -1.89 & 0.013 & L466 & L490 & C-terminus \\
\hline 501 & -3.59 & -2.01 & 0.023 & L467 & L491 & C-terminus \\
\hline 502 & -2.98 & -1.67 & 0.077 & $\mathrm{H} 468$ & H492 & C-terminus \\
\hline
\end{tabular}


ADDITIONAL FILE 4. Table S2. Primer pairs used in this study.

\begin{tabular}{ccc}
\hline Name & Fw primer sequence (5'--> $\mathbf{3}^{\mathbf{\prime})}$ & Rev primer sequence (5'--> $\mathbf{\text { ') }}$ \\
\hline PsMLO1 & CACCATGGCTGAAGAGGGAGTTAAGGAAC & CTAATTGCTCCCTAAGTGGCGCTT \\
HvMLO & CACCGACCGATGTCGGACAAAA & TCATCCCTGGCTGAAGGAAAA \\
qPsMLO1 & AGGTTTGCAAGGGACACAAC & TTGTGCATCATGTCCTGGAG \\
qHvMLO & TTTCATCCCTCTCGTGATCC & CCACTGTCCACACAAAATGC \\
NPTII & TCGGCTATGACTGGGCACAAC & AAGAAGGCGATAGAAGGCGA \\
35S & GCTCCTACAAATGCCATCA & GATAGTGGGATTGTGCGTCA \\
Oid & CGCCAAAGACCTAACCAAAA & AGCCAAGAGATCCGTTGTTG \\
qEf & ACAGGCGTTCAGGTAAGGAA & GAGGGTATTCAGCAAAGGTCTC \\
\hline
\end{tabular}





\section{Chapter 6}

A transposable element insertion in the susceptibility gene CsaMLO8 results in hypocotyl resistance to powdery mildew in cucumber

Michela Appiano ${ }^{\dagger}$, Jeroen A. Berg ${ }^{\dagger}$, Miguel Santillán Martínez, Freddy W.K. Hermans, Wim H. Vriezen, Richard G. F. Visser, Yuling Bai, Henk J. Schouten ${ }^{\ddagger}$ t these authors contributing equally to the work

${ }^{\ddagger}$ corresponding author

Chapter published in BMC Plant Biology, Oct 2015 Link to published paper here: http://bmcplantbiol.biomedcentral.com/articles/10.1186/s12870015-0635-x 


\section{Abstract}

\section{Background}

Powdery mildew (PM) is an important disease of cucumber (Cucumis sativus L.). CsaMLO8 was previously identified as a candidate susceptibility gene for PM in cucumber, for two reasons: 1 ) This gene clusters phylogenetically in clade $V$, which has previously been shown to harbour all known MLO-like susceptibility genes for PM identified in dicot species; 2) This gene co-localizes with a QTL on chromosome 5 for hypocotyl-specific resistance to $\mathrm{PM}$.

\section{Results}

We cloned CsaMLO8 alleles from susceptible and resistant cucumber genotypes, the latter carrying the QTL for hypocotyl resistance. We found that insertion of a non-autonomous Class LTR retrotransposable element in the resistant genotype leads to aberrant splicing of CsaMLO8 mRNA. Heterologous expression of the wild-type allele of CsaMLO8 in a tomato mlo-mutant restored PM susceptibility. However, heterologous expression of the CsaMLO8 allele cloned from the resistant cucumber genotype failed to restore PM susceptibility. Furthermore we showed that inoculation of susceptible cucumber with the PM pathogen Podosphaeraxanthii induced transcriptional upregulation of CsaMLO8 in hypocotyl tissue, but not in cotyledon or leaf tissue. This coincides with the observation that the QTL at the CsaMLO8-locus causes full resistance in hypocotyl tissue, but only partial resistance in cotyledons and true leafs. We studied the occurrence of the loss-of-function allele of CsaMLO8 in cucumber germplasm by an in silico approach using resequencing data of a collection of 115 cucumber accessions, and found that this allele was present in 31 out of 115 accessions.

\section{Conclusions}

CsaMLO8 was characterised as a functional susceptibility gene to PM, particularly in the hypocotyl where it was transcriptionally upregulated upon inoculation with the PM pathogen P. xanthii. A loss-of-function mutation in CsaMLO8 due to the insertion of a transposable element was found to be the cause of hypocotyl resistance to PM. This particular allele of CsaMLO8 was found to occur in $27 \%$ of the resequenced cucumber accessions.

\section{Keywords}

Powdery mildew, MLO, susceptibility gene, Cucumber (Cucumis sativus L.), hypocotyl resistance, non-autonomous transposable element 


\section{Background}

Cucumber (Cucumis sativus L.) is an economically important crop, with an annual global production of over 65 megatons [1]. Powdery mildew (PM) is one of the most widespread diseases in cucurbits, and a limiting factor for cucumber production. Two species of fungi have been reported to cause PM in cucumber, i.e. Podosphaera xanthii (synonymous with P. fusca, previously named Sphaerotheca fuliginea) and Golovinomyces cichoracearum (previously named Erysiphe cichoracearum). Of these, P. xanthii is considered to be the main causal agent of PM in cucurbits $[2,3]$.

Breeding of resistant cucumber varieties has been undertaken for several decennia (e.g. [4-6]), but underlying resistance genes have to date not been functionally characterised. As the genome of cucumber ('Chinese long' inbred line 9930) was published in 2009 [7], and several other cucumber accessions have been resequenced $[8,9]$, the time is now ripe to identify causal genes for cucumber resistance to mildew diseases.

Traditionally, breeding of disease resistant crops is performed by introgression of resistance $(R)$ genes, often from wild relatives of the crop. R proteins, most commonly of the nucleotide-binding, leucine-rich-repeat (NB-LRR) type, are able to recognise either corresponding avirulence (Avr) gene products of the pathogen, or degradation products of host factors associated with pathogen attack [10]. This triggers a defence response in the host cell, often associated with a hypersensitive response (HR), leading to cell death [10]. As $R$ genes recognise very specific products, introgression and subsequent employment of a new $R$ gene puts selective pressure on the pathogen to evolve in such a way that it is no longer recognised by the host plant. Therefore, $R$-gene based resistance is often breached by new, virulent, races of the pathogen quite soon, especially for versatile pathogens, such as powdery mildew fungi [10].

An alternative for $R$-gene mediated resistance is the identification of impaired susceptibility (S) genes [11]. Most pathogens require cooperation of their host plant to be able to successfully establish a compatible interaction [12]. This is especially true for biotrophic pathogens such as mildew species, as they greatly rely on a long-lasting interaction with (living) host cells to facilitate their propagation [12]. Therefore, the expression of several host genes is essential for the pathogen. Such genes can be regarded as $S$ genes, and can function for instance in facilitating host recognition and penetration, negative regulation of host defences or fulfilling metabolic and structural needs of the pathogen [12]. Loss-offunction mutations in a $S$ gene is thought to lead to durable, broad spectrum, recessively inherited resistance $[13,14]$.

The barley mlo gene is one of the best-known examples of an impaired $S$ gene. After it first was found in the 1940s in a mutagenized barley population [15], deployment of lossof-function mlo alleles in barley has resulted in PM resistant barley varieties. These have 
been grown in the field for several decades already without breaching of resistance by virulent new mildew races to date, providing evidence for the durability of $S$-gene based resistance [16]. After the barley MLO gene was cloned [17], it was found that MLO genes are conserved throughout the plant kingdom and occur in higher plants as a multi-copy gene family $[18,19]$. In several plant species, MLO-like genes have been found to be involved in PM susceptibility, such as Arabidopsis, tomato, pea, pepper, tobacco, bread wheat and potentially also grapevine and peach [20-27]. It has been found that in phylogenetic trees of the MLO gene family all MLO-like S-genes for PM detected in monocotyledonous species cluster in clade IV, whereas all MLO-like S-genes identified in dicotyledonous species cluster in clade V. The other clades (I, II, III and VI) harbour MLO-like genes that have not been proven to be S-genes [19].

The genome of cucumber harbours 13 putative $M L O$-like genes [28]. Of these, three (i.e. CsaMLO1, CsaMLO8 and CsaMLO11,with respective Cucurbit Genomics Database IDs [Csa1M085890.1], [Csa5M623470.1] and [Csa6M292430.1]) cluster in clade V of the MLO gene family, and can therefore be considered candidate S-genes for powdery mildew resistance [28]. CsaMLO8 is of particular interest, as its position on the genome (Chr5: $24,827,408 . .24,831,456)$ co-localizes with $p m 5.2$, a recently identified major QTL explaining $74.5 \%$ of the phenotypic variation for 'hypocotyl' resistance in F3 families derived from the resistant cucumber inbred line WI 2757 [29]. 'Hypocotyl' or intermediate resistance of cucumber to PM was previously shown to be a recessively inherited monogenic trait in crossings between several cucumber lines, and was characterised by completely resistant hypocotyl, stem and petiole tissue and partially resistant leaves and cotyledons. Hypocotyl resistance is suggested to play an important role in overall PM resistance of cucumber, as it appears that complete resistance in leaves is not possible without the recessive hypocotyl resistance gene [5]. In breeding practice loss of the hypocotyl resistance allele leads to PM susceptible seedlings. The allele is present in almost all modern pickling cucumber varieties, and most of the resistant long cucumber varieties (Freddy Hermans, personal communications), showing the agricultural significance of hypocotyl resistance in cucumber.

Here, we report the cloning of CsaMLO8 from both susceptible and (hypocotyl) resistant cucumber genotypes. We show that at the transcript level the allele obtained from the resistant genotype has deletions of 72 or $174 \mathrm{bp}$ due to alternative splicing, caused by the insertion of a LTR retrotransposable element in this gene at the genomic level. Complementation of the tomato mlo-mutant with the wild-type and $\Delta 174$ alleles of CsaMLO8 showed that wild-type CsaMLO8 is a functional susceptibility gene (S-gene), whereas the $\Delta 174$ allele has lost its function as S-gene, thus leading to PM resistance. Furthermore, qRT-PCR showed that CsaMLO8 is transcriptionally upregulated upon inoculation with $P$. xanthii in hypocotyl tissue, but not in leaves or cotyledon, explaining why loss-of-function of CsaMLO8 provides particularly resistance in the hypocotyl. 


\section{Results}

\section{Cloning and sequencing of the CsaMLO8 coding sequence from susceptible and resistant genotypes}

We performed RT-PCR using RNA derived from either a susceptible wild-type cucumber cultivar or a resistant breeding line known to be homozygous for the hypocotyl resistance QTL as a template. Whereas the product we obtained from the susceptible genotype was of the expected size (i.e. $1726 \mathrm{bp}$ ), we obtained two different products from the resistant genotype, both smaller than expected (Fig. 1A). Sequence analysis revealed that the CsaMLO8 mRNA variant obtained from the susceptible genotype was identical to the predicted coding sequence. The two mRNA products obtained from the resistant genotype however had (non-frameshift) deletions of respectively 72 and $174 \mathrm{bp}$. The 174 bp deletion variant corresponds to a loss of the complete $11^{\text {th }}$ exon of the CsaMLO8 gene, whereas the 72 bp deletion variant corresponds to the loss of a fragment of the $11^{\text {th }}$ exon with canonical splice sites (5'-GT and AG-3') (Fig. 1B). Furthermore, the coding sequence of the resistant genotype has five (synonymous) SNPs compared to the reference genome (Additional file 1).

To determine the impact of the 72 and 174 bp deletions found in the mRNA on the predicted CsaMLO8 protein sequence, the predicted CsaMLO8 protein was aligned to a dataset of MLO proteins encoded by clade V S-genes from several other species i.e. Arabidopsis, barrel clover, pea, lotus, tomato, pepper and tobacco (Additional file 2). It appeared that the region encoded by the deleted area in the 72 and 174 bp deletion variants is highly conserved among different MLO proteins (Fig. 1C). Furthermore, the transmembrane structure of the CsaMLO8 protein (wild-type allele) was predicted using HMMTOP 2.1 software [30]. The predicted transmembrane structure of the wild-type protein was largely consistent with the barley MLO structure determined by Devoto et al. $[18,19]$. The 72 and 174 bp deletions correspond to removal of a region of 24 respectively 58 amino acid residues in the (predicted) third cytoplasmic loop of CsaMLO8 (Fig. 1D). The relative transcript abundances of the two CsaMLO8 splice variants characterised by the 72 and 174 bp deletions were determined by qRT-PCR using splice junction spanning primers on different tissues (i.e. hypocotyl, cotyledon and true leaf) of PM resistant cucumber, either inoculated with PM or non-inoculated. It appeared that the $174 \mathrm{bp}$ deletion splice variant was the most abundant isoform, whereas the 72 bp deletion splice variant was less abundant in each tissue regardless whether tissues were inoculated or not (Additional file 3). 
A

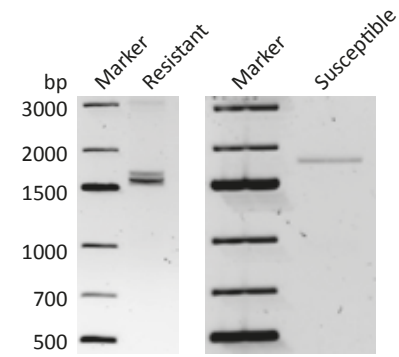

B

\begin{tabular}{|c|c|c|c|c|c|c|}
\hline & & 20 & & 40 & & $\stackrel{60}{1}$ \\
\hline CSaMLO8 WT & TTCATCTCCT & TAATTATAAT & TCTATTGGTG & GGAACAAAGC & TCCATGTTAT & TATAACTCAT \\
\hline$\Delta 72$ & $\mathrm{TCCT}$ & TATAAT & G - - - & $\ldots \ldots$ & $\ldots$ & $\ldots \ldots$ \\
\hline$\Delta 174$ & СТСС T & & $\cdots$ & & $\ldots \ldots$ & \\
\hline & ${ }_{1}^{80}$ & & $\begin{array}{c}100 \\
1\end{array}$ & & $\begin{array}{c}120 \\
1\end{array}$ & \\
\hline CSaMLO8 WT & CAATTCAAGA & AAGGGGTCAT & GTTGTGAAGG & CGT & CGTTCAGCCT & $A C C$ \\
\hline CsaMLO8 $\triangle 72$ & $\ldots \ldots$ & $\cdots$ & $\cdots \cdots-G$ & GTGTTCCGGT & CGTTCAGCCT & CGGGATGACC \\
\hline $\operatorname{saMLO8} \Delta 174$ & $\ldots \ldots$ & $\ldots$ & $\ldots \ldots$ & $\ldots \ldots$ & $\ldots \ldots$ & 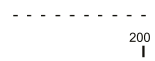 \\
\hline CsaMLO8 WT & $C A$ & TTC & & GTT & GA & TCA \\
\hline CsaMLO8 $\triangle 72$ & GGACGTCCA & АТTC & AT & GT T & GA & \\
\hline$M L 08 \triangle 1$ & 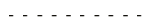 & $\ldots \ldots$ & 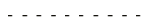 & $-2-2-2$ & - & ATGCATTTC \\
\hline
\end{tabular}

C

\begin{tabular}{|c|c|c|c|c|c|c|c|}
\hline & & & & VVVQ & NFG & LITF & \\
\hline & PEIPL & VGTKLEVIIIT & KLGLRIQEKG & DVVRGAPVVQ & PGDDLFWEG & PRFILFLIHL & VLFTN \\
\hline MLO6 & PEIPFIVILI & VGTKLQVIIT & KLGLRIQEKG & DVVKGTPLVQ & PGDHFFWFGR & PRFILFLIHL & VLFTNAFQLA \\
\hline$F$ & & VGAKLQMIIIS & KLGLRIQQEKG & DVVKGAPVVE & PGDDLFWFGE & PRFILFLIHL & \\
\hline 1L01 & & AKLQMIIIT & GLRIQDRG & GVIKGAPVVE & GDHLFWFNS & PNLLLFIIHL & 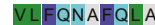 \\
\hline PsML01 & IIILL & VGAKLQMIIT & KMGLRIQDRG & EVIIKGAPVVE & $P G D$ & PHLLLFTIHL & \\
\hline LjMLO1 & PFIPLIIIILL & $\overline{\mathrm{V}}$ GTKLQMIIIT & NMGLKI QER & DVIKGAPLVE & PGDDLFWFNE & PRLILSLVHL & VLFQI \\
\hline SIMLO1 & PEIPLVIILL & VGTKLQMIIIT & EMGVRISERG & DIVKGVPVVE & TGDHLFWFNR & PALVLFLIINF & VLFC \\
\hline & & VGTKLQMIIT & EMGVRISERG & DIVKGVPVVE & IGDHLFWFNR & PGLVLFFINF & ON \\
\hline NIMLO & HiTL & VGTKLEMIIA & EMGVRIISKRG & ARGVPVVE & GDHLFWFNR & PGFVLFLINA & EQVA \\
\hline ion & & & & & & & \\
\hline
\end{tabular}

D

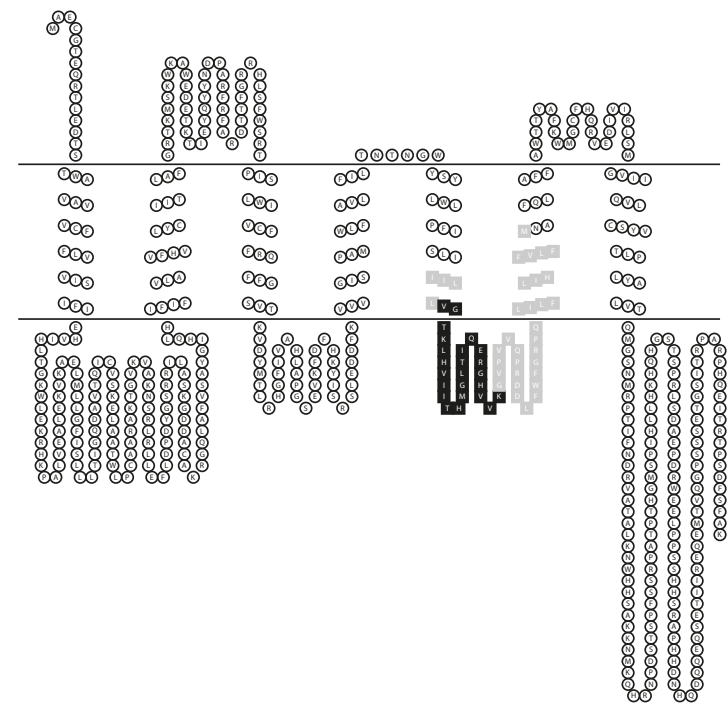

FIGURE 1. Characterization of CsaMLO8 alleles from resistant and susceptible cucumber genotypes.

(A) CDNA of resistant (left panel) and susceptible (right panel) cucumber genotypes was used as template for PCR with CsaMLO8 specific primers. Amplified products were analysed on $1.25 \%$ agarose gels. Whereas the 
product amplified from cDNA of the susceptible genotype gives a single band of the expected size, cDNA of the resistant genotype results in two separate bands, both of a smaller size than expected.

(B) Full length CsaMLO8 amplified from CDNA from susceptible and resistant cucumber genotypes was sequenced. A partial alignment is shown between the (wild-type) sequence as obtained from the susceptible genotype and the sequences from two deletion variants ( $\Delta 72$ and $\Delta 174)$ obtained from the resistant genotype. Numbers are relative to the start of the alignment.

(C) Partial alignment of the CsaMLO8 protein and other proteins encoded by clade V MLO S-genes of several species. Amino acid residues are coloured according to the RasMol colour scheme. The 24 and 58 amino acid residues deleted in the proteins encoded by the $\triangle 72$ and the $\triangle 174$ variants of CsaMLO8 are indicated by red arrows. A bar graph underneath the alignment indicates the conservedness of each amino acid position.

(D) Graphic representation of the transmembrane structure of the predicted CsaMLO8 protein, determined using HMMTOP 2.1 [30]. The plasma membrane is indicated by two horizontal lines. Amino acid residues highlighted in black are predicted to be deleted in the protein encoded by the $\triangle 72$ variant of the CsaMLO8 gene, residues highlighted in black and grey are predicted to be deleted in the protein encoded by the $\Delta 174$ variant of the CsaMLO8 gene.

\section{Complementation of SIMLO1 loss-of-function tomato mutant with CsaMLO8 WT and CsaMLO8D174}

The sequence analysis of the transcripts of CsaMLO8 from susceptible and resistant genotypes led to the hypothesis that CsaMLO8 is a functional S-gene for PM, whereas the 174 bp deletion allele (CsaMLO8D174) has lost its function as S-gene. To test these hypotheses, both alleles were overexpressed in a previously described tomato mlomutant, which carries a mutation in the tomato SIMLO1 gene and is resistant to tomato powdery mildew, Oidium neolycopersici [21].

Cuttings of ten independent transgenic individuals per construct (35S::CsaMLO8 WT and 35S::CsaMLO8D174) were challenged with the tomato PM pathogen O. neolycopersici. Powdery mildew susceptibility was evaluated qualitatively, by looking for PM symptoms on the leaves (Fig. 2A, Additional file 4). Six out of ten individual transformants expressing CsaMLO8 WT were scored as susceptible to PM, whereas none of the transformants expressing CsaMLO8D174 were scored as susceptible to PM. PM susceptibility was confirmed quantitatively, by performing qPCR on DNA isolated from inoculated leaves, using O. neolycopersici specific primers. This showed that the biomass of $O$. neolycopersici in plants scored as susceptible to PM was at least 0.20 , relative to the biomass in the susceptible control MM, whereas the biomass in plants scored as resistant was less than 0.20 (Fig. 2B). Furthermore, transcript abundances of the transgenes in each of the transgenic individuals were determined by qRT-PCR using CsaMLO8 specific primers (Fig. 2C). This confirmed that transcript levels of CsaMLO8 WT and CsaMLO8D174 were comparable. The six CsaMLO8 WT transformants scored as susceptible to PM had a higher CsaMLO8 expression than the four CsaMLO8 WT transformants scored as resistant to PM. 
A
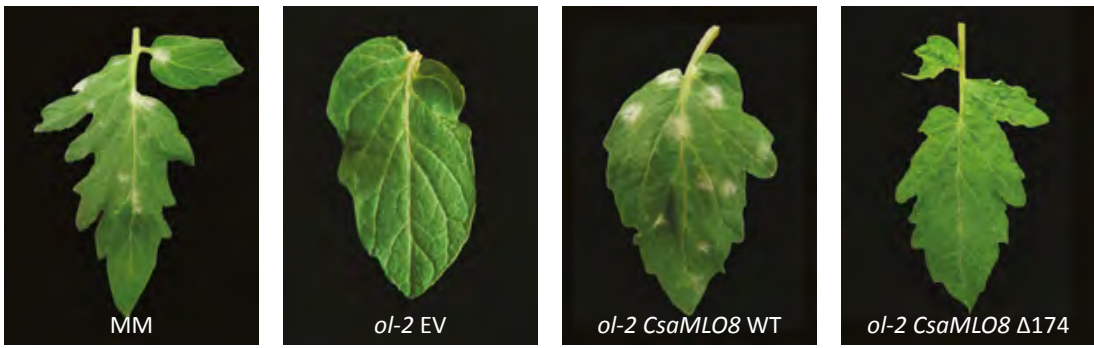

B

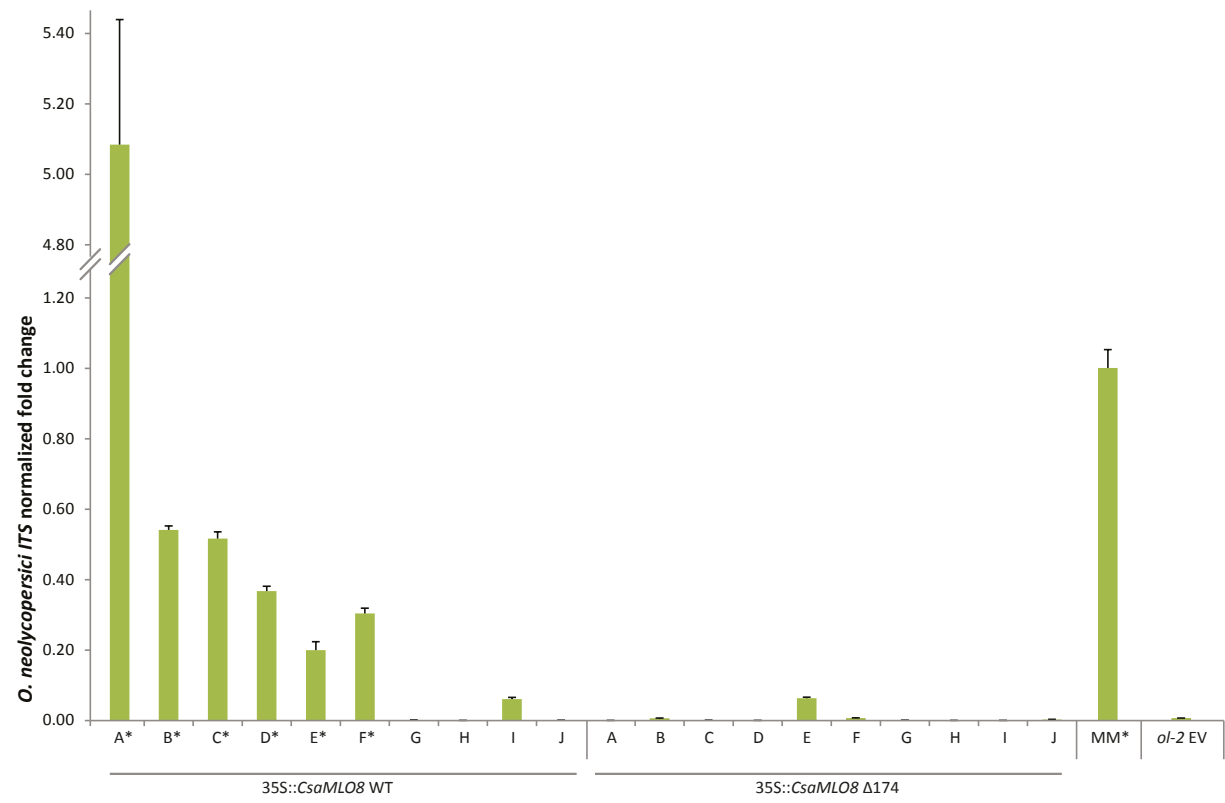

C

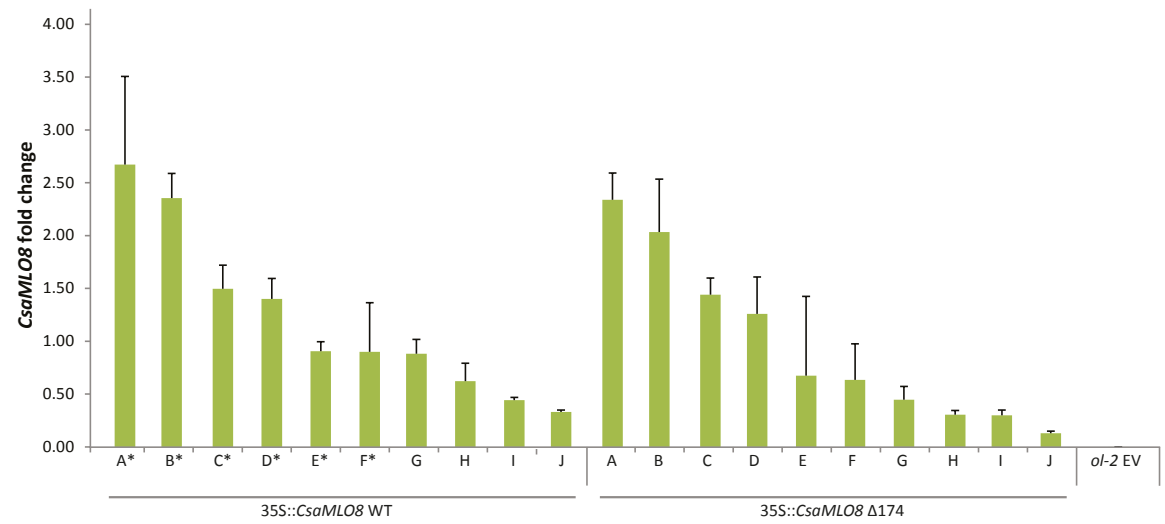

FIGURE 2. Complementation of ol-2 tomato with CsaMLO8 WT restores PM susceptibility, whereas complementation with CsaMLO8D174 does not.

The PM resistant ol-2 tomato mutant with a deletion in SIMLO1 [21] was transformed with either a 35S::CsaMLO8 WT construct, a 35S::CsaMLO8D174 construct, or an empty vector (EV) control. Cuttings from these transformants were inoculated with a Oidium neolycopersici spore suspension. As additional control we used the wild-type, susceptible cv. Moneymaker (MM). 
(A) The phenotype of susceptible control MM, resistant EV transformed ol-2, and transgenic individuals overexpressing either CsaMLO8 WT or CsaMLO8D174 in ol-2 background. Photographs were taken 16 days post inoculation.

(B) Relative quantification by qPCR of the ratio between Oidium neolycopersiciand plant gDNA in susceptible MM, resistant EV transformed ol-2, and transgenic individuals overexpressing either CsaMLO8 WT or CsaMLO8D174 in ol-2 background. Fold changes were normalised relative to the susceptible control MM. based on macroscopic evaluation. Bars represent the average fold change over 3 technical replicates. Error bars indicate standard deviation. Asterisks indicate plants scored as susceptible to powdery mildew.

(C) Relative quantification by qRT-PCR of the ratio between CsaMLO8 expression and expression of tomato housekeeping gene SIEF- $\alpha$ in EV transformed ol-2 and transgenic individuals overexpressing either CsaMLO8 WT or CsaMLO8A174 in ol-2 background. Bars represent the average fold change over 3 technical replicates. Error bars indicate standard deviation. Asterisks indicate plants scored as susceptible to powdery mildew.

\section{Sequencing and characterization of a transposable element in CsaMLO8}

To investigate the cause of the deletions in the CsaMLO8 coding sequence, we performed PCR using DNA from both the susceptible and resistant cucumber genotypes as a template, with primers designed to amplify the region that contained the deletions in CsaMLO8. The product amplified from the susceptible genotype had the expected size (i.e. $346 \mathrm{bp}$ ), whereas the product amplified from the resistant genotype was larger (ca. $1500 \mathrm{bp}$, Fig. 3A). Sequence analysis of the amplified product revealed a $1449 \mathrm{bp}$ insertion in the genomic DNA sequence of the resistant genotype compared to the susceptible genotype. This insertion in the DNA of the resistant genotype coincided with the region that contained the deletion in the CsaMLO8 mRNA of this genotype. Characterization of this genomic insertion by a dot-plot (Fig. 3B) revealed the presence of long terminal repeats (LTRs) with a length of ca. $200 \mathrm{bp}$. An alignment between the first and last $200 \mathrm{bp}$ of the insertion confirmed the presence of 184 bp long LTRs beginning with a $5^{\prime}$-TG-3' and ending with a $5^{\prime}-$ TA-3' (Fig. 3C). The LTRs share $100 \%$ sequence identity with one another. After the $3^{\prime}$ LTR, there is a duplication of the 5 bp of CsaMLO8 before the insertion (Target Site Duplication, TSD, 5'-ATTAT- $3^{\prime}$ ). No open reading frames (ORFs) could be detected in the insertion. Taken together, these findings led us to the conclusion that the insert is most likely a non-autonomous transposable element (TE) of Class I, Order LTR, according to the transposable element classification scheme proposed by Wicker et al. [31]. 


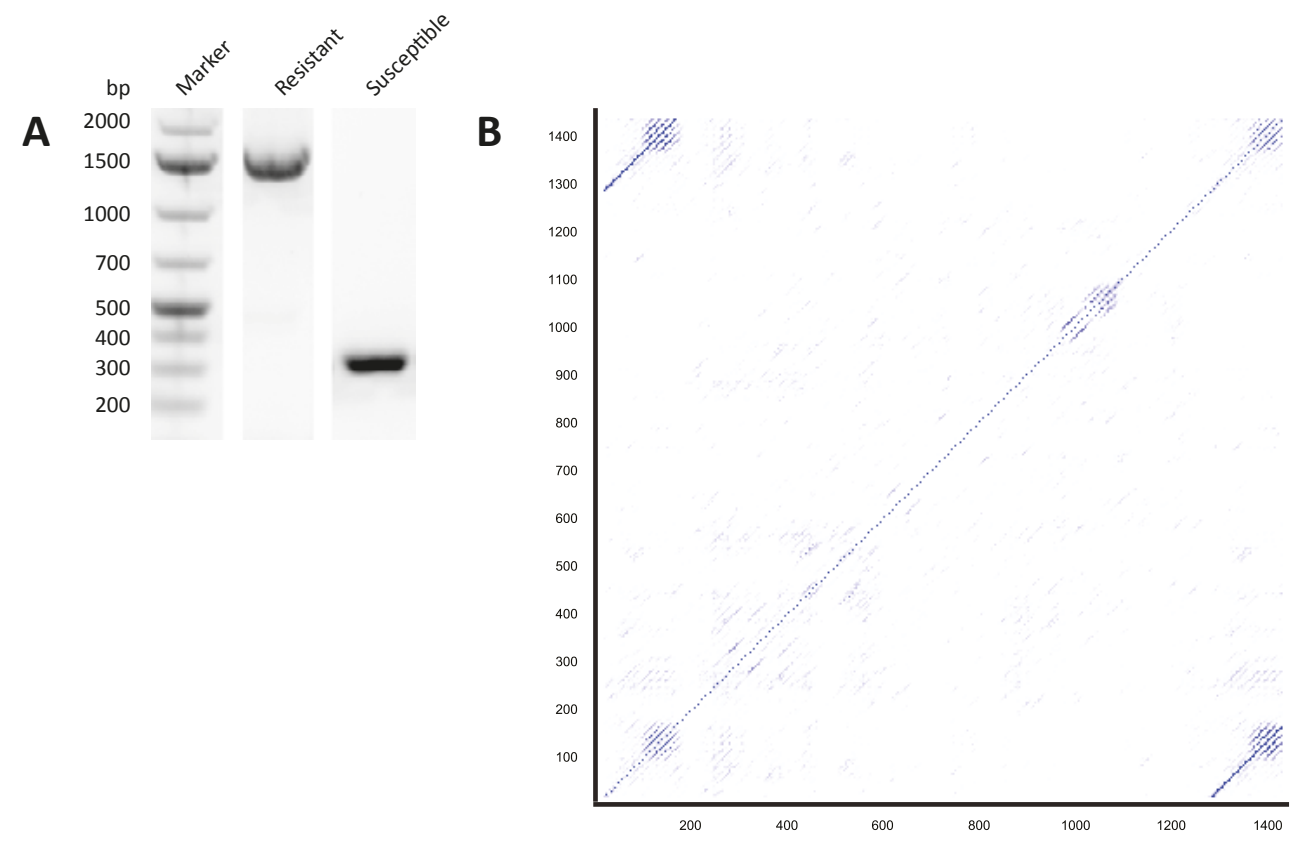

\section{C}

\begin{tabular}{|c|c|c|c|c|c|}
\hline & & 20 & & $\begin{array}{l}40 \\
1\end{array}$ & \\
\hline TE first $200 \mathrm{bp}$ & - GCTCCATGT & TATTATTGTT & GATTTTATGG & AATCAATCTA & TATTTCATTC \\
\hline \multirow[t]{2}{*}{ TE last $200 \mathrm{bp}$} & CTCTGATACC & A $\ldots$ TGTT & GATTTTATGG & AATCAATCTA & TATTTCATTC \\
\hline & $\begin{array}{c}60 \\
1\end{array}$ & & $\begin{array}{c}80 \\
1\end{array}$ & & $\begin{array}{c}100 \\
\end{array}$ \\
\hline TE first $200 \mathrm{bp}$ & ATTACTTAAT & AGAGATACAA & GCCTATATAT & AACCATAGAG & AAATACACTT \\
\hline \multirow[t]{2}{*}{ TE last $200 \mathrm{bp}$} & АT TACTTAAT & AGAGATACAA & GCCTATATAT & AACCATAGAG & AAATACACTT \\
\hline & & $\begin{array}{c}120 \\
\mathbf{1}\end{array}$ & & $\begin{array}{c}140 \\
\mathbf{I}\end{array}$ & \\
\hline TE first $200 \mathrm{bp}$ & AAGGAAATAA & TATCAAATAA & TATCTCCTAA & GAATAATATC & TCCTAAGAAT \\
\hline \multirow[t]{2}{*}{ TE last $200 \mathrm{bp}$} & AAGGAAATAA & TATCAAATAA & TATСТССТАA & GAATAATATC & TCCTAAGAAT \\
\hline & $\begin{array}{c}160 \\
1\end{array}$ & & 180 & & 200 \\
\hline TE first $200 \mathrm{bp}$ & AАTATCTCCT & AAGAATAATA & TCTCCTAAGA & ATAATCTAAT & TATATTAATA \\
\hline \multirow[t]{2}{*}{ TE last $200 \mathrm{bp}$} & ААТАТСТССТ & AAGAATAATA & TCTCCTAAGA & АТАATCTAAT & TATATTAATA \\
\hline & & & & & \\
\hline $200 \mathrm{bp}$ & $\cdots-$ СССТС & ССТСAAACTC & A & & \\
\hline TE last $200 \mathrm{bp}$ & ATTATAACTC & ATATGGGATT & - & & \\
\hline
\end{tabular}

D

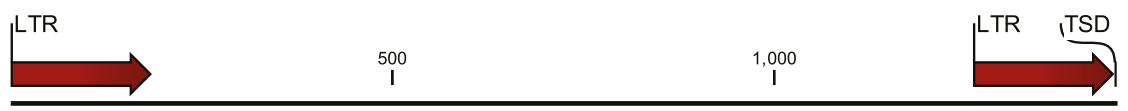

FIGURE 3. Amplification and sequencing of CsaMLO8 from genomic DNA isolated from the resistant genotype reveals the insertion of an 1449 bp long Transposable Element (TE).

(A) The genomic region of CsaMLO8 in which deletions in the coding sequence were observed in the resistant genotype was amplified from DNA isolated from both the susceptible and resistant genotypes. Amplified products were analysed on $1.25 \%$ agarose gel. Whereas the product amplified from the susceptible genotype was of the expected size, the product amplified from the resistant genotype was larger than expected.

(B) The product amplified from the resistant genotype as described in (A) was sequenced, which revealed an insertion with a length of $1449 \mathrm{bp}$. A dot-plot was made of the insertion to see whether the sequence contains repetitive elements. 
(C) The first and last $200 \mathrm{bp}$ of the insertion, plus $15 \mathrm{bp}$ of CsaMLO8 before and after the insertion were aligned to one another, to verify the presence of long terminal repeats (LTRs). Non-aligned parts of the sequence are highlighted in red. It can be seen that the first $184 \mathrm{bp}$ of the insertion are completely identical to the last 184 $\mathrm{bp}$ of the insertion. There is a duplication of $5 \mathrm{bp}$ from CsaMLO8 before and after the insertion (Target site duplication, 5'-ATTAT-3').

(D) Schematic representation of the insertion. The locations of LTRs and the 3' TSD are indicated.

\section{Similar TEs in the cucumber genome}

In an attempt to identify homologous, potentially autonomous, transposable elements in the cucumber genome, we performed a BLASTn search on the cucumber reference genome (Chinese long inbred line ' 9930 ', v2) with the LTR sequence of the TE found in CsaMLO8 as query. We identified 169 putative homologous LTRs. A previously designed tool [32] was used to screen the genome for regions bordered by two putative homologous LTR sequences. Two putative homologous LTR sequences within a window of $20 \mathrm{~kb}$ were considered to be the borders of a putative homologous TE. The $20 \mathrm{~kb}$ window was decided upon based on the observation that LTR retrotransposons are generally between 3-15 kb of size [33], the only exception to our knowledge being the very large Ogre retrotransposons found in legumes [34]named Ogre, which is over $22 \mathrm{~kb}$ long and makes up at least $5 \%$ of the pea (Pisum sativum L., which have ca. $5 \mathrm{~kb}$ LTRs and are therefore ca. $22 \mathrm{~kb}$ in size. A total of 44 putative TEs was identified, randomly distributed over all seven chromosomes of the cucumber reference genome (Fig. 4, Additional file 5). For 20 putative TEs, the complete sequence in between the LTRs was extracted from the genome, and compared to the sequence of the TE found in CsaMLO8 (Additional file 6). It was found that most of the putative TEs have a length comparable to the CsaMLO8$\mathrm{TE}$, being between 1 and $2 \mathrm{~kb}$. One putative TE was considerably larger than average, with 7,142 bp, whereas one putative TE was considerably smaller than average, i.e. 367 bp. In only one out of the 20 putative TEs (TE37), an open reading frame (ORF) could be detected. This ORF, with a length of $411 \mathrm{bp}$, does not lead to a predicted protein with any similarity to known proteins according to a BLASTp search against all non-redundant protein databases, and is therefore considered a false positive ORF. We conclude that we could not detect an autonomous TE that contained the genes that could have been responsible for the insertion of the non-autonomous TE in CsaMLO8. 


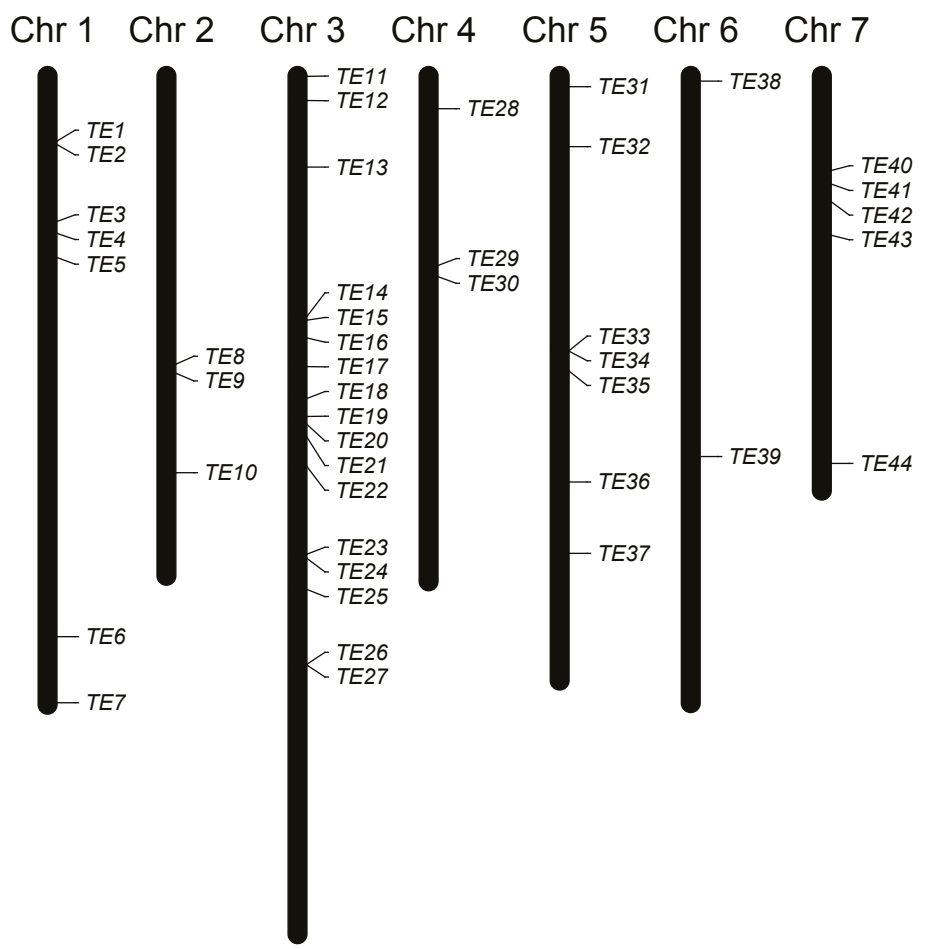

FIGURE 4. There are 44 putative homologous TEs in the cucumber reference genome.

A BLASTn search was performed on the cucumber reference genomes with the LTR sequence of the TE found to be inserted in CsaMLO8. Pairs of putative LTRs within $20 \mathrm{~kb}$ of one another were considered borders of putative TEs. 44 putative TEs were identified, chromosomal locations of which are indicated.

\section{Occurrence of the TE-allele of CsaMLO8 in cucumber germplasm}

We were interested to see how frequently the TE-allele of CsaMLO8 we have characterised in our resistant cucumber genotype occurs in the cucumber germplasm. As Qi et al. (2013) resequenced a core collection of 115 very divergent cucumber accessions [8], we decided to perform an in silico search for the presence of the mutant CsaMLO8 allele containing the TE) and/or the wild type (WT) allele among those genotypes. For 21 resequenced accessions (18\%) we could only detect reads indicating presence of the TE-allele. For 82 resequenced accessions $(71 \%)$ we could only find reads indicating presence of the WT-allele. For 10 accessions (9\%) we found reads indicating presence of both alleles. For the remaining two accessions (2\%), presence of neither of the alleles could be identified (Table 1, Additional file 7). The TE-allele of CsaMLO8 was present in three out of the four geographic groups of accessions (i.e. East Asian, Eurasian and Indian but not Xishuangbanna) as defined by Qi et al. [8]. One of the 31 accessions in which the TE-allele of CsaMLO8 was detected (i.e. PI 215589) belongs to the wild form of cucumber, Cucumis sativus var. hardwickii, whereas the other 30 accessions belong to the cultivated form of cucumber, C. sativus var. sativus. 


\section{Inoculation with $P$. xanthii induced transcription of CsaMLO8 in hypocotyl tissue, but not in leaf tissue of susceptible cucumber}

$M L O$ genes involved in PM susceptibility are upregulated in several plant species several hours after inoculation (e.g [26, 35, 36]we sequenced additional mlo resistance alleles, two of which confer only partial resistance. Wild-type MLO dampens the cell wall-restricted hydrogen peroxide burst at points of attempted fungal penetration of the epidermal cell wall, and in subtending mesophyll cells, it suppresses a second oxidative burst and cell death. Although the Bgh-induced cell death in mlo plants is spatially and temporally separated from resistance, we show that the two processes are linked. Uninoculated mutant mlo plants exhibit spontaneous mesophyll cell death that appears to be part of accelerated leaf senescence. Mlo transcript abundance increases in response to Bgh, rice (Oryza sativa). To see whether the same holds true for CsaMLO8, we performed qRT-PCR experiments to quantify CsaMLO8 transcript abundances in hypocotyl, cotyledon and leaf tissues of PM susceptible and resistant cucumber plants, prior to and at 4, 6, 8 and 24 hours after PM inoculation (Fig. 5). For PM susceptible plants, we found that in hypocotyl tissue CsaMLO8 transcript abundance was significantly higher at 4 hpi $(P=0.037)$ and $6 \mathrm{hpi}(P=$ $0.004)$ compared to the transcript abundance prior to inoculation (0 hpi). The significant difference had disappeared 8 hpi $(P=0.212)$ and 24 hpi $(P=0.281)$. Contrastingly, CsaMLO8 transcript abundances in cotyledons and true leaves were not significantly altered at any of the evaluated time points after PM inoculation $(P>0.05)$ (Fig. 5A). For PM resistant plants, we found that CsaMLO8 transcript abundance was not significantly higher in any tissue at any time point after inoculation compared to the transcript abundance prior to inoculation $(P>0.05)$. In hypocotyl tissue, transcript abundance was significantly lower at $6 \mathrm{hpi}(P=0.046), 8 \mathrm{hpi}(P=0.006)$ and $24 \mathrm{hpi}(P=0.009)$ compared to the transcript abundance prior to inoculation (0 hpi). In cotyledon tissue, transcript abundance was significantly lower at $8 \mathrm{hpi}(P=0.002)$ compared to the transcript abundance prior to inoculation (Fig. 5B). 
TABLE 1. 31 out of 115 resequenced cucumber accessions have the TE-allele of CsaMLO8.

Total reads of 115 recently resequenced cucumber accessions [8] were assayed in silico for the presence of reads indicating the presence of either the allele of CsaMLO8 characterised by the insertion of a TE, or the wild-type allele. The amount of reads indicating presence of either the TE-allele or the WT-allele of CsaMLO8 is given. Database number, accession names and geographic groups of accessions were obtained from [8].

\begin{tabular}{|c|c|c|c|c|c|c|}
\hline $\begin{array}{c}\text { Accession } \\
\text { number } \\
\text { NCBI SRA }\end{array}$ & $\begin{array}{c}\text { TE-allele } \\
\text { reads }\end{array}$ & $\begin{array}{c}\text { WT-allele } \\
\text { reads }\end{array}$ & $\begin{array}{l}\text { Putative } \\
\text { genotype }\end{array}$ & $\begin{array}{l}\text { PI or CGN } \\
\text { number }\end{array}$ & Name Accession & Group \\
\hline SRR543205 & 9 & 0 & Homozygous & PI 215589 & 13598 & Indian \\
\hline SRR543216 & 17 & 0 & Homozygous & V05A0674 & Bei Jing Xiao Ci & East Asian \\
\hline SRR543221 & 1 & 9 & Heterozygous & V05A1333 & Liao Tong Mi Ci & East Asian \\
\hline SRR543223 & 19 & 0 & Homozygous & V05A0920 & He Cha Huang Gua & East Asian \\
\hline SRR543224 & 19 & 0 & Homozygous & V05A1115 & Qian Qi Li Huang Gua & East Asian \\
\hline SRR543225 & 1 & 7 & Heterozygous & V05A0985 & Ye San Bai & East Asian \\
\hline SRR543226 & 23 & 0 & Homozygous & V05A0428 & Liao Yang Ye San & East Asian \\
\hline SRR543228 & 1 & 0 & Homozygous & - & 228 & East Asian \\
\hline SRR543230 & 18 & 0 & Homozygous & V05A0522 & Huang Gua & East Asian \\
\hline SRR543231 & 5 & 8 & Heterozygous & V05A0552 & Qing Dao Qiu Ye Er San & East Asian \\
\hline SRR543240 & 1 & 13 & Heterozygous & CGN19828 & - & East Asian \\
\hline SRR543242 & 22 & 0 & Homozygous & V05A0034 & Da Ci Huang Gua & East Asian \\
\hline SRR543243 & 12 & 1 & Heterozygous & V05A1427 & Qiu Huang Gua & East Asian \\
\hline SRR543244 & 1 & 7 & Heterozygous & V05A0291 & Leng Lu Huang Gua & East Asian \\
\hline SRR543246 & 1 & 0 & Homozygous & - & Bai Ye San & East Asian \\
\hline SRR543251 & 4 & 0 & Homozygous & - & 2004348 & East Asian \\
\hline SRR543252 & 11 & 0 & Homozygous & CGN20266 & Hok & Eurasian \\
\hline SRR543253 & 6 & 0 & Homozygous & - & $151 G$ & Eurasian \\
\hline SRR543257 & 5 & 0 & Homozygous & CGN20512 & 752 & Eurasian \\
\hline SRR543258 & 9 & 0 & Homozygous & CGN20515 & Gy 3 (S4) & Eurasian \\
\hline SRR543264 & 2 & 0 & Homozygous & - & $65 \mathrm{G}$ & Eurasian \\
\hline SRR543265 & 11 & 0 & Homozygous & - & G8 & Eurasian \\
\hline SRR543267 & 14 & 0 & Homozygous & V05A0726 & Jin Yan Er Hao & East Asian \\
\hline SRR543269 & 10 & 3 & Heterozygous & CGN19579 & 1972 B-2 & Eurasian \\
\hline SRR543271 & 15 & 0 & Homozygous & CGN19844 & 2163 & Eurasian \\
\hline SRR543272 & 2 & 5 & Heterozygous & $\begin{array}{l}\text { PI 234517/ } \\
\text { CGN20898 }\end{array}$ & SC 50 & Eurasian \\
\hline SRR543274 & 11 & 0 & Homozygous & CGN21627 & Spartan Garden MSU-C7-63 & Eurasian \\
\hline SRR543275 & 4 & 0 & Homozygous & - & Marketmore76 & Eurasian \\
\hline SRR543276 & 4 & 0 & Homozygous & - & GY14 & Eurasian \\
\hline SRR543281 & 9 & 3 & Heterozygous & PI 482412 & TGR 580 & Indian \\
\hline SRR543293 & 6 & 8 & Heterozygous & PI 605943 & USM 307 & Indian \\
\hline
\end{tabular}



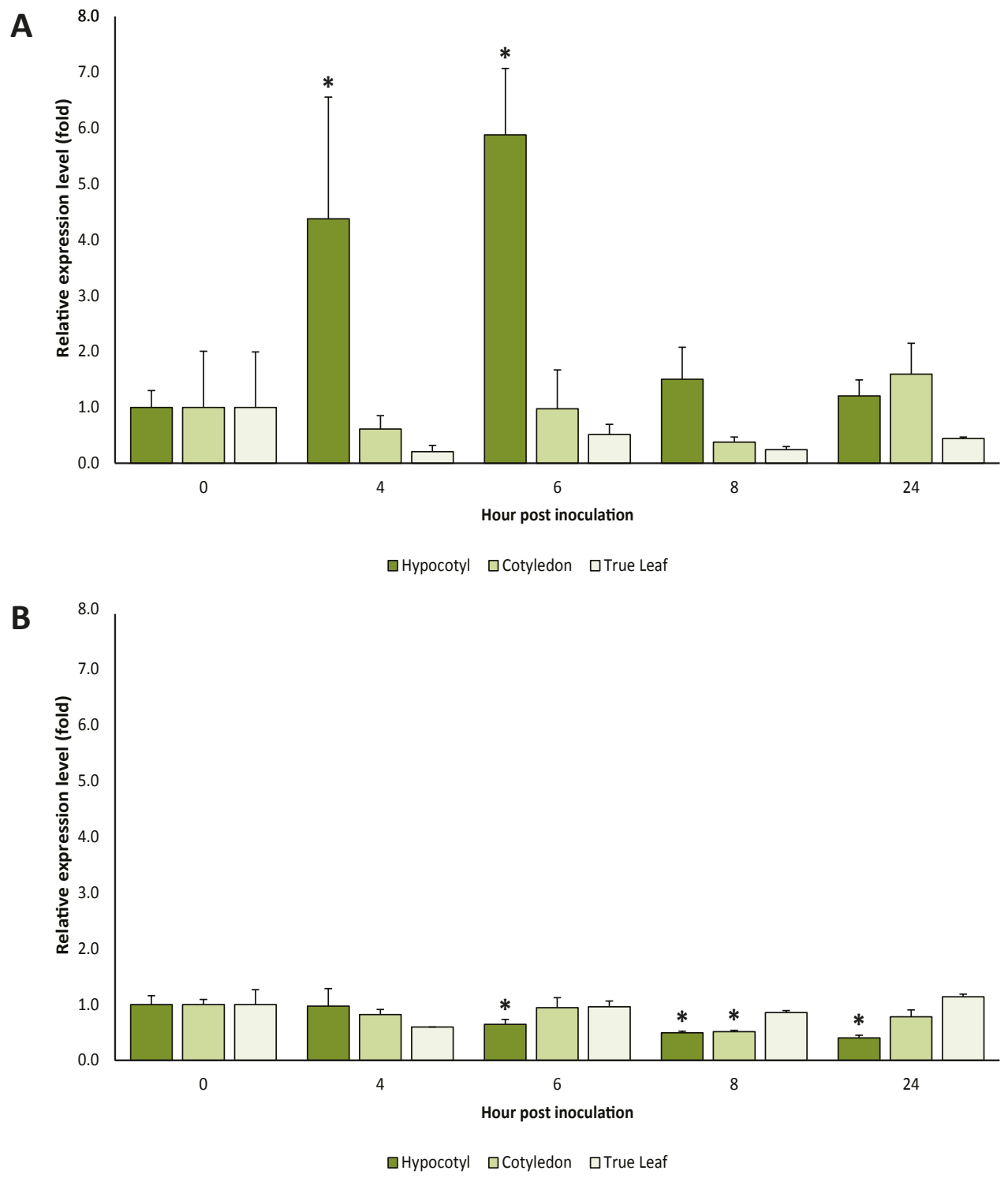

FIGURE 5. CsaMLO8 transcription is induced after inoculation with Podosphaera xanthii in hypocotyl tissue, but not in cotyledon or true leaf tissue.

Susceptible (A) and resistant (B) cucumber seedlings were inoculated with a $P$. xanthii spore suspension. Prior to and 4, 6, 8 and 24 hours post inoculation, hypocotyl, cotyledon and true leaf tissue were harvested and immediately frozen in liquid nitrogen. Relative quantification of CsaMLO8 expression was performed by qRT-PCR. Fold changes were normalised relative to CsaMLO8 expression prior to inoculation. Bars represent the average fold change over three independent biological replicates. Error bars indicate standard errors of the mean. Asterisks indicate significant differences to the expression prior to inoculation (Student's T test, $P<0.05$ ). 


\section{Discussion}

\section{CsaMLO8 is a functional susceptibility gene for PM in cucumber}

Several studies characterised some, but not all, clade V MLO genes as being required for PM susceptibility in different dicotyledonous plant species [20-23, 25-27]. Here we have shown that heterologous expression of the cucumber gene CsaMLO8 in SImlo1 mutant tomato background restored PM susceptibility, providing evidence for the role of CsaMLO8 as a susceptibility gene for PM in cucumber (Fig. 2). As the role of clade V MLO genes in susceptibility to PM seems to be evolutionary conserved between divergent dicotyledonous plant families, e.g. Brassicaceae [20]the ability to cause disease in plants and animals has been gained and lost repeatedly during phylogenesis. In monocotyledonous barley, loss-of-function mlo alleles result in effective immunity against the Ascomycete Blumeria graminis f. sp. hordei, the causal agent of powdery mildew disease. However, mlo-based disease resistance has been considered a barley-specific phenomenon to date. Here, we demonstrate a conserved requirement for MLO proteins in powdery mildew pathogenesis in the dicotyledonous plant species Arabidopsis thaliana. Epistasis analysis showed that mlo resistance in $A$. thaliana does not involve the signaling molecules ethylene, jasmonic acid or salicylic acid, but requires a syntaxin, glycosyl hydrolase and $A B C$ transporter. These findings imply that a common host cell entry mechanism of powdery mildew fungi evolved once and at least 200 million years ago, suggesting that within the Erysiphales (powdery mildews, Solanaceae [21, 23, 25], Fabaceae [22] tomato, Vitaceae [26], Rosaceae $[27,36]$ and now also Cucurbitaceae, it is probable that in other economically important species belonging to the family Cucurbitaceae, such as melon (Cucumis melo) and pumpkin (Cucurbita pepo) clade V MLO genes will also play a role in PM susceptibility. Indeed, in a patent application a functional complementation of Arabidopsis Atmlo2, Atmlo2,6 and Atmlo2,6,12 mutants by a melon MLO-like gene was claimed to partially restore PM susceptibility, based on the percentage of diseased leaf area in 4 to 9 primary transformants [37]. Alignment of this melon MLO gene with the three Clade $V$ genes of cucumber revealed that the gene from melon is most similar to CsaMLO8, and less alike to the two other Clade V genes (i.e. CsaMLO1 and CsaMLO11) [28]. This is consistent with our finding that CsaMLO8 is a S-gene for PM. In tomato we observe that complementation of SIMLO1 loss-of-function mutants with CsaMLO8 restores PM susceptibility, with individual transformants with higher CsaMLO8 expression generally being more susceptible to PM than transformants with lower CsaMLO8 expression (Fig. 2). It seems possible that in the case of complementation of Arabidopsis mutants by the melon $M L O$ gene there is also a quantitative effect due to different levels of melon $M L O$ expression in individual transformants, leading to the conclusion that the melon MLOgene only partially restores susceptibility whereas it is actually due to the fact that transgene expression was not high enough to fully complement the loss of AtMLO function. 


\section{Transposon insertion in CsaMLO8 leads to aberrant splicing and therefore to loss of the $S$-gene function}

By cloning CsaMLO8 from cDNA of a PM resistant cucumber genotype that is homozygous for the hypocotyl resistance QTL, we found evidence for aberrant splicing of CsaMLO8 in this genotype, leading to products with deletions of respectively 72 and $174 \mathrm{bp}$ in exon 11, compared to the WT gene. We showed that these deletions are predicted to lead to loss of 24 respectively 58 amino acid residues in the third cytoplasmic loop of the CsaMLO8 protein, in a highly conserved region between clade V MLO proteins from different species (Fig. 1). As it was previously shown that cytoplasmic loop-loop interplay is required for MLO function [38], we anticipated that such rather big deletions in one of the cytoplasmic loops, if the protein should properly fold at all, would lead to lossof-function of the protein. Indeed, we showed here that expression of the $\Delta 174$ variant of CsaMLO8 in SImlo mutant tomato background failed to restore PM susceptibility (Fig. 2). This makes cucumber, after barley [17], tomato [21] and pea [22], the fourth plant species in which a natural mutation in an $M L O$ gene has been found to lead to resistance. Although we did not try to complement S/mlo mutant tomato with the 72 bp deletion variant of CsaMLO8, and thus cannot rule out the possibility that it is (partially) functional as an S gene, we expect that the result will be similar to the $174 \mathrm{bp}$ deletion variant, given the conservedness of the deleted region.

To determine the reason for the aberrant splicing of CsaMLO8 in the resistant cucumber genotype, we set out to amplify and sequence the genomic region of CsaMLO8 in which the deletions were detected. In this way, we discovered a 1,449 bp insertion in exon 11 of the gene compared to the reference genome. Sequence analysis of the insertion revealed the presence of $100 \%$ identical LTRs and TSDs, but no open reading frames or any similarity to known proteins or genes (Fig. 3), leading to the conclusion that the insertion is probably a Class I, Order LTR (retro)transposable element (TE), following the TE classification scheme proposed by Wicker et al. [31]. The fact that the LTRs are completely identical to one another is an indication that the TE is relatively recently inserted. The integration of a transposable element in a MLO gene, leading to aberrant splicing of transcripts and in that way to loss of gene function, is reminiscent of the findings in the pea PSMLO1 gene, where in one of the alleles (found in PM resistant pea cultivar Jl 2302) the integration of an Ogre LTR retrotransposon lead to aberrant splicing [22].

We analysed putative TEs with similar LTRs (Fig. 4), and found no functional ORFs in these TEs, confirming that we are dealing with a family of non-autonomous TEs. Additionally, a large amount of LTR singlets (i.e. LTR sequences without a partner) were detected, as only 88 out of the 169 detected LTRs could be assigned to a putative TE (Additional file 5). LTR singlets presumably originate from the unequal recombination between two LTRs of a single element [39], or from assembling errors of the reference genome. It is known 
that plant genomes are to a great extent shaped by the integration of large amounts of transposable elements, with LTR retrotransposons being the most abundant among them (e.g. [40,41]. The cucumber genome was shown to be no exception to this, with $24 \%$ of the genome consisting of transposable elements and LTR retrotransposons comprising $10.4 \%$ of the genome [7]. To our knowledge, the TE we found to be inserted in CsaMLO8 is the first TE with a reported effect on a cucumber gene. It seems likely that more TEs with an effect on genes in cucumber will be found in the future.

\section{CsaMLO8 is upregulated upon P. xanthii inoculation in hypocotyl tissue only}

Resistance to PM in cucumber has previously been reported to be tissue specific, with an important, recessively inherited gene providing full PM resistance in hypocotyl tissue and partial resistance in leafs [5]. Recently, PM resistance of cucumber was mapped in multiple tissues separately. The strongest QTL for hypocotyl resistance, pm5.2 was mapped on chromosome 5, in a region containing CsaMLO8 [29]. In this study, we showed that CsaMLO8 was, in susceptible cucumber, transcriptionally upregulated in hypocotyl tissue at 4 and 6 hours post inoculation, but not in cotyledon or leaf samples (Fig. 5A). Apparently, the ability of the pathogen to upregulate CsaMLO8 expression is specific for hypocotyl tissue. Therefore, we postulate that it is very well possible that PM resistance caused by a loss of function allele of CsaMLO8 would also be specific for hypocotyl tissue.

Interestingly, CsaMLO8 was not found to be transcriptionally upregulated in hypocotyl tissue (or any other tissue) in the resistant cucumber line (Fig. 5B). This is in sharp contrast with the findings in barley [35] where transcription of the MLO gene seemed to be even stronger induced upon PM inoculation in mlo loss-of-function mutants compared to wild type plants. In tomato it was found that transcription of the SIMLO1 gene was slightly upregulated upon PM inoculation in s/mlo1 loss of function mutants, but to a far lesser extent than in wild type plants [21]. Although it remains a question why the pathogen is unable to upregulate CsaMLO8 expression in our resistant cucumber line several explanations might be offered, e.g. lesser transcript stability of the mutant CsaMLO8 transcripts, differences in the promotor region of the mutant allele of CsaMLO8 or differences in other genes required for CsaMLO8 expression compared to the susceptible cultivar.

Previously, RNA-seq experiments on cucumber leaf tissue revealed that of the thirteen CsaMLOgenes only CsaMLO1, another clade V MLO gene, was transcriptionally upregulated after inoculation with P. xanthii [28]. This is consistent with our finding that CsaMLO8 is not upregulated in leaf samples after PM inoculation (Fig. 5). It is possible that CsaMLO1 and CsaMLO8 are functionally redundant, but are specifically expressed in separate tissues (i.e. CsaMLO1 specific in leaf tissue and CsaMLO8 in hypocotyl tissue). To our knowledge there are no other examples of tissue specialization in $M L O$-like $S$ genes of other species. 
In Arabidopsis, which also has three clade V MLO genes, Atmlo2 mutants were found to be partially resistant, double mutants Atmlo2/Atmlo6 or Atmlo2/Atmlo12 were more resistant than Atmlo2 single mutants, and triple mutants Atmlo2/Atmlo6/Atmlo12 were completely resistant [20]the ability to cause disease in plants and animals has been gained and lost repeatedly during phylogenesis. In monocotyledonous barley, loss-of-function mlo alleles result in effective immunity against the Ascomycete Blumeria graminis $\mathrm{f}$. sp. hordei, the causal agent of powdery mildew disease. However, mlo-based disease resistance has been considered a barley-specific phenomenon to date. Here, we demonstrate a conserved requirement for MLO proteins in powdery mildew pathogenesis in the dicotyledonous plant species Arabidopsis thaliana. Epistasis analysis showed that mlo resistance in A. thaliana does not involve the signaling molecules ethylene, jasmonic acid or salicylic acid, but requires a syntaxin, glycosyl hydrolase and $A B C$ transporter. These findings imply that a common host cell entry mechanism of powdery mildew fungi evolved once and at least 200 million years ago, suggesting that within the Erysiphales (powdery mildews. It is not yet known by what mechanism MLO genes are transcriptionally upregulated upon PM infection, although it would seem intuitive to hypothesise that it is an active process caused by an effector of the fungus. Given the tissue specificity of MLO upregulation in cucumber, this might be an interesting model to investigate the mechanism of MLO upregulation by PM fungi.

\section{The transposon insertion allele of CsaMLO8 occurs frequently in cucumber germplasm}

Interestingly, during the preparation of this manuscript, another group reported the fine-mapping of a QTL for PM resistance on the long arm of chromosome 5, which they called pm5.1, to a region of $170 \mathrm{~kb}$ containing 25 predicted genes. The main candidate gene in this region was found to be a $M L O$ like gene, which appears to be the same as CsaMLO8 in our study. By cloning and sequencing of this gene from genomic DNA of their resistant parent, line S1003, as well as two additional unrelated resistant lines, S02 and S06, they found that they contained a $1449 \mathrm{bp}$ insert in the $11^{\text {th }}$ exon of the gene [42]. Sequence analysis indicates that the location and sequence of the insertion found in their study are completely identical to the LTR retrotransposon described in this study. These researchers did not report on cloning the coding sequence of CsaMLO8 in their material, nor on complementation experiments.

Additionally, a patent was filed describing an allele of CSKIP2, a gene claimed to provide PM resistance, shown to harbour a 72 bp deletion in the coding sequence [43]. Although it is not shown in the patent, the occurrence of this allele is claimed to be caused by the integration of a transposon-like element in the $11^{\text {th }}$ exon of the gene. Sequence analysis revealed that CSKIP2 is in fact the same gene as CsaMLO8, and the 72 bp deletion allele 
they describe is the same as the $72 \mathrm{bp}$ deletion we found in our material. Interestingly the patent does not describe the 174 bp deletion which we found, but an in silico prediction showed that the $174 \mathrm{bp}$ deletion variant would not be amplified by the primers they chose to amplify the partial CsaMLO8 sequence. In the patent no functional proof is given that this allele of CsaMLO8 indeed leads to resistance.

As several groups independently found the same allele of CsaMLO8 in different, to our knowledge unrelated, resistant cucumber genotypes, we were interested to know how often this allele occurs in the global cucumber germplasm. Therefore, we performed an in silico screen on a collection of 115 recently resequenced cucumber accessions [8] for the presence and/or absence of the transposable element (TE) allele of CsaMLO8. We found evidence for the presence of the TE-allele, either homozygously or heterozygously, in at least 31 out of the 115 accessions (Table 1), indicating that this particular allele of CsaMLO8 occurs quite often. For some accessions only a small number of reads indicating presence/ absence of the TE allele was found, potentially due to a low read coverage at this locus. It is therefore possible that in some accessions now identified as homozygous for either the TE-allele or the WT allele of CsaMLO8, reads indicative of the other allele were missed due to low read coverage, so there might be some heterozygous accessions misidentified as being homozygous for one of the alleles.

As we found that the TE allele of CSaMLO8 leads to PM resistance, it might have been selected for by cucumber breeders, by selecting for the most resistant plants. Interestingly one of the accessions found to have the TE-allele of CsaMLO8 was PI 215589, a wild accession of C. sativus var. hardwickii collected in India in 1954. This indicates that the TEallele of CsaMLO8 does occur in the wild, and might have been introgressed in cultivated cucumber from PI 215589 or a related hardwickii accession.

\section{Conclusions}

In this study we provide evidence for a role of CsaMLO8 as a $S$ gene for powdery mildew (PM) susceptibility. We show that complementation by CsaMLO8 overexpression in SImlo 1 mutant tomato background restores PM susceptibility. We also show that a mutant allele of CsaMLO8 cloned from resistant cucumber fails to restore PM susceptibility. As CsaMLO8 is located in the region where a QTL for hypocotyl specific resistance was detected, we determined CsaMLO8 expression in different tissues of PM inoculated plants, and found that CsaMLO8 was only transcriptionally upregulated in hypocotyl tissue. On this basis we conclude that the mutant allele of CsaMLO8 is causal to the observed hypocotyl resistance towards PM in cucumber. 


\section{Methods}

\section{Plant materials and fungal strain}

Two cucumber genotypes were used in this study: the PM susceptible cv. Sheila and an advanced breeding line, related to the resistant $c v$. Anaxo, homozygous for a recessively inherited QTL on chromosome 5 conferring hypocotyl resistance $(p m-h)$.

Two tomato genotypes were used: PM susceptible cv. Moneymaker (MM), and a PM resistant breeding line ol-2, homozygous for a 19 bp deletion mutation in the coding sequence of SIMLO1 [21].

Unless otherwise indicated, plants were grown under standard conditions in a closed greenhouse.

An isolate of $P$. xanthii (causing PM in cucumber) was obtained from infected cucumber plants in the greenhouse of a seeds company from The Netherlands and maintained on cv. Sheila in a greenhouse compartment at Wageningen University, The Netherlands. The species of the isolate was confirmed by sequencing of the ITS sequence from fungal DNA by primer pair 5'- CGTCAGAGAAGCCCCAACTC-3' (ITS $P$. xanthii Forward) and 5'-AGCCAAGAGATCCGTTGTTG-3' (ITS P. xanthii Reverse) (data not shown).

The Wageningen isolate of Oidium neolycopersici (tomato PM) was maintained on cv. MM as described [44].

\section{Cloning and sequencing of CsaMLO8 CDS}

Young leaves of cucumber cv. Sheila and the resistant breeding line were harvested and immediately frozen in liquid nitrogen. Total RNA was isolated by using the RNeasy Kit (Qiagen, Germany). Possible DNA contamination of RNA samples was removed by treatment with DNase I, Amp Grade (Invitrogen life technologies, U.S.A.). cDNA was synthesised using $2 \mu \mathrm{g}$ of RNA samples with an iScript cDNA Synthesis Kit (Bio-Rad Laboratories, U.S.A.).

For amplification of CsaMLO8 coding sequences, CDNA was amplified with primers 5'- caccCTGCCTCTCCACATGCATAA-3' (Full length CsaMLO8 Forward) and 5'-GCGCCCTGTACATGAAGAAC-3' (Full length CsaMLO8 Reverse). As template $50 \mathrm{ng}$ cDNA was used in $50 \mu \mathrm{l}$ reactions using $1 \mathrm{u}$ PfuUltra II Fusion HS DNA polymerase (Agilent Technologies, U.S.A.), $1 \mathrm{x}$ reaction buffer, $1 \mathrm{mM}$ dNTP and $200 \mathrm{nM}$ of each primer. Cycling conditions were: $1 \mathrm{~min}$. initial denaturation at $95^{\circ} \mathrm{C}$, followed by 40 cycles of $20 \mathrm{sec}$. denaturation at $95^{\circ} \mathrm{C}, 20 \mathrm{sec}$. annealing at $60^{\circ} \mathrm{C}$ and $2 \mathrm{~min}$. extension at $72^{\circ} \mathrm{C}$. Reactions were finished by $3 \mathrm{~min}$. incubation at $72^{\circ} \mathrm{C}$. $\mathrm{PCR}$ products were separated by gel electrophoresis in ethidium bromide stained agarose gels. Bands were cut out and purified using QIAquick Gel 
Extraction Kit (Qiagen, Germany). Purified products were cloned into Gateway-compatible vector pENTR D-TOPO (Invitrogen life technologies, U.S.A.) and transformed to chemically competent Escherichia coli strain One Shot TOP10. Presence of the right fragment was assessed by colony PCR using primers and conditions as above. Plasmids were recovered using the Qiaprep spin miniprep kit (Qiagen, Germany). Sequencing reactions were performed in triplicates using pUC/M13 forward and reverse sequencing primers (GATC Biotech, Germany).

\section{Complementation of tomato ol-2 mutant with CsaMLO8 WT and CsaMLO8D174}

Entry plasmids pENTR:CsaMLO8 WT and pENTR:CsaMLO8D174, obtained as described above, were transferred by Gateway LR cloning into binary vector pK7WG2, which harbours the constitutively active 35S Cauliflower Mosaic Virus promotor and the nptll marker gene for kanamycin resistance [45]. Recombinant plasmids were transformed to chemically competent $E$. coli strain dh5a. Positive recombinant bacterial colonies were screened by colony PCR using CsaMLO8 specific primers as described above, and sequenced. Recombinant plasmids were recovered using the Qiaprep spin miniprep kit (Qiagen, Germany). pK7WG2:CsaMLO8 WT and pK7WG2:CsaMLO8D174 binary vectors were transformed to electrocompetent cells of Agrobacterium tumefaciens strain AGL1virG by electroporation [46].

Cotyledon explants of ol-2 mutant tomato seedlings were transformed as previously described [25]. Obtained tomato transformants were assessed for presence of CsaMLO8, the nptll marker gene and the 35S CaMV promotor sequence by PCR with primers $5^{\prime}$ - caccCTGCCTCTCCACATGCATAA-3' (Full length CsaMLO8 forward) and 5'-GCGCCCTGTACATGAAGAAC-3' (Full length CsaMLO8 reverse), 5'-GAAGGGACTGGCTGCTATTG-3' (nptll forward) and 5'-AATATCACGGGTAGCCAACG-3' (nptll reverse), and 5'-TACAAAGGCGGCAACAAACG-3' (35S forward) and 5'-AGCAAGCCTTGAATCGTCCA-3' (35S reverse), with conditions as described above.

For each of the two transformations with a different construct, ten independent transgenic plants were selected, and were assessed for CsaMLO8 expression by qRT-PCR using primer pair sequences specific for CsaMLO8 5'-GCGACGGCATTGAAGAACTG-3' (Forward) and 5'-AGGAGACATGCCGTGAGTTG-3' (Reverse). As housekeeping gene for normalization of CsaMLO8 expression in tomato, SIEF-a was used, with primer pair 5'-ATTGGAAACGGATATGCCCCT-3' (SIEF- $a$ forward) and 5'-TCCTTACCTGAACGCCTGTCA-3' (SIEF-a reverse). qRT-PCR was performed using the CFX96 Real-Time PCR machine (BioRad Laboratories, U.S.A.). Each $10 \mu$ reaction contained $300 \mathrm{nM}$ of each primer, $1 \mu \mathrm{l}$ (50ng) cDNA template and $1 \times$ iQ SYBR Green Supermix (Bio-Rad Laboratories, U.S.A.). Cycling conditions were an initial denaturation step of $95^{\circ} \mathrm{C}$ for $3 \mathrm{~min}$., followed by 40 cycles of 10 sec. denaturation at $95^{\circ} \mathrm{C}$ and $30 \mathrm{sec}$. annealing and extension at $60^{\circ} \mathrm{C}$, finished by a melt cycle of $0.5^{\circ} \mathrm{C}$ increment per $10 \mathrm{sec}$. from $65^{\circ} \mathrm{C}$ to $95^{\circ} \mathrm{C}$. 


\section{Evaluation of PM resistance of ol-2 tomato, overexpressing CsaMLO8 WT or CsaML084174}

Cuttings originating from ten individual transgenic plants per construct (two cuttings per plant) were inoculated with 0 . neolycopersici. Cuttings of an empty vector (EV) transformed ol-2 plant and the susceptible cultivar Moneymaker (MM) were used as controls. A spore suspension was prepared by washing heavily infected leaves of cv. $\mathrm{MM}$ with water, and adjusting the spore concentration to $8 \times 10^{4}$ conidiospores $/ \mathrm{ml}$. The spore suspension was evenly sprayed on the cuttings. Sixteen days after inoculation the disease severity was assessed by eye, and scored as either susceptible (sporulating powdery mildew colonies visible on leaves) or resistant (no powdery mildew symptoms at all). Additionally, leaf samples were taken for quantification of $O$. neolycopersici biomass. Infected leaves (the 2nd or 3rd leaf) were sampled for each cutting. Total plant and fungal DNA was extracted using the DNeasy Plant Kit (Qiagen, Germany). Isolated DNA was used for qPCR with primer pair 5'-CGCCAAAGACCTAACCAAAA-3' (Oidium ITS forward) and 5'-AGCCAAGAGATCCGTTGTTG-3' (Oidium ITS reverse), specific for the internal transcribed spacer (ITS) of O. neolycopersici ribosomal DNA, to quantify O. neolycopersici biomass, and with SIEF-a primers as described above for normalization. qPCR was performed using the CFX96 Real-Time PCR machine (Bio-Rad Laboratories, U.S.A.). Each $10 \mu$ reaction contained $300 \mathrm{nM}$ of each primer, $2 \mu \mathrm{l}$ (20ng) cDNA template and 1 x iQ SYBR Green Supermix (BioRad Laboratories, U.S.A.). Cycling conditions were identical to those described above for quantification of CSAMLO8 expression in transformed tomato.

\section{Amplification, sequencing and characterization of CsaMLO8-insertion}

DNA was isolated from young leaves of cucumber cv. Sheila and the resistant breeding line, which were immediately frozen in liquid nitrogen afterharvesting, using the DNeasy Plant Kit (Qiagen, Germany). DNA was amplified with primers 5'-AGCATTTTGCCATCCATACTTCA-3' (CsaMLO8 insertion region Forward) and 5'-CTGCAAGCACAGGATGAATGTC-3' (CsaMLO8 insertion region Reverse). As template $30 \mathrm{ng}$ DNA was used in $25 \mu$ reactions using $1.25 \mathrm{u}$ DreamTaq DNA polymerase (Thermo Scientific, U.S.A.), 1x DreamTaq buffer, $0.8 \mathrm{mM}$ dNTP and $200 \mathrm{nM}$ of each primer. Cycling conditions were: $3 \mathrm{~min}$. initial denaturation at $95^{\circ} \mathrm{C}$, followed by 35 cycles of $30 \mathrm{sec}$. denaturation at $95^{\circ} \mathrm{C}, 30 \mathrm{sec}$. annealing at $57^{\circ} \mathrm{C}$ and $2 \mathrm{~min}$. extension at $72^{\circ} \mathrm{C}$. Reactions were finished by $5 \mathrm{~min}$. incubation at $72^{\circ} \mathrm{C}$. PCR products were visualised by staining with GelRed and electrophoresis on agarose gels. PCR products were purified using Qiaquick PCR purification kit (Qiagen, Germany). Sequencing reactions were performed in duplo, using primers 5'-AGCATTTTGCCATCCATACTTCA-3' (CsaMLO8 insertion region Forward), 5'-ACGAAGAGCGAAACGAAGAA-3' (CsaMLO8 insertion sequencing Forward), 5'- GCTCCTGCCCAATTCAGACC-3' (CsaMLO8 insertion sequencing Reverse) and 5'-CTGCAAGCACAGGATGAATGTC-3' (CsaMLO8 insertion region Reverse) 
(GATC Biotech, Germany). Obtained sequences were aligned using CLC Genomics Workbench 7.5 software. The consensus sequence for the amplified region was extracted from the alignment. This consensus sequence was aligned to the genomic reference sequence of CsaMLO8 to determine the exact location and sequence of the insertion.

A dot plot was constructed for the sequence of the insertion, using CLC Genomics Workbench 7.5 standard settings. The first and last $200 \mathrm{bp}$ of the insertion sequence were extracted and aligned to each other to identify the length and sequence of the LTRs. The sequence of the insertion was scanned for open reading frames using CLC Genomics Workbench 7.5 standard settings, which gave no results.

\section{In silico mining of the cucumber reference genome for homologous TEs}

The previously determined LTR sequence of the CsaMLO8-TE was used as query to perform a BLASTn search in the genome of the cucumber reference genome (Chinese long inbred line '9930', v2 [7] to identify putative homologous LTRs. The resulting output was stored as a tabular file. A python script described by Wolters et al. (2014) was used to search for LTR matches within $20 \mathrm{~kb}$ from each other [32]. Sequences with a length smaller than 20 kb flanked by two LTRs were considered as putative homologous TEs, and were extracted from the genome using the BEDtools suite [47]. The list of putative TEs was manually curated to remove sequences with two LTRs in opposite directions (two instances) and sequences with large (>100 bp) gaps (25 instances). In three instances, putative TEs were found to be nested (i.e. three LTRs were found to be within $20 \mathrm{~kb}$ of each other), in which cases the smaller putative TEs were discarded in favour of the bigger, nested model. Putative TEs were aligned to one another and to the CsaMLO8-TE using CLC Genomics Workbench 7.5 software, to determine sequence identity compared to the CsaMLO8-TE. Putative TEs were screened for open reading frames using CLC Genomics Workbench 7.5 standard settings. Putative TEs were used as query to perform tBLASTx searches to the REPbase database [48].

\section{In silico screening of resequenced lines for presence of CsaMLO8-TE allele}

Reads of the resequencing project of 115 cucumber accessions by Qi et al. [8] were downloaded from the NCBI short read archive, accession SRA056480. By a simple Bash script, total reads were screened for the presence of $30 \mathrm{bp}$ sequences comprised of:

1) The last $15 \mathrm{bp}$ of CsaMLO8 before the TE insertion and the first $15 \mathrm{bp}$ of the TE insertion, in forward (5'- GCTCCATGTTATTATTGTTGATTTATGGA-3') or reverse (5'-TCCATAAAATCAACAATAATAACATGGAGC-3') orientation;

2) The last $15 \mathrm{bp}$ of the TE insertion and the first $15 \mathrm{bp}$ of CsaMLO8 after the TE insertion, in forward (5'-TATATTAATAATTATAACTCATATGGGATT-3') or reverse $\left(5^{\prime}\right.$ AATCCCATATGAGTTATAATTATTAATATA-3') orientation; 
3) The $30 \mathrm{bp}$ of CsaMLO8 surrounding the TE insertion site, without TE sequence, in forward (5'- GCTCCATGTTATTATAACTCATATGGGATT-3') or reverse (5'-AATCCCATATGAGTTATAATAACATGGAGC-3') orientation.

The number of detected reads per accession with each of the six bait sequences was stored as a tabular file. The total number of reads indicating presence of the TE allele and the total number of reads indicating presence of the WT allele were summated, the genotype of the accessions was determined to be either homozygous TE-allele, homozygous WTallele or heterozygous.

\section{CsaML08 expression analysis PM-inoculated cucumber}

PM susceptible and resistant cucumbers were grown in a climate chamber at $20^{\circ} \mathrm{C}$ (day) and $16^{\circ} \mathrm{C}$ (night), with a $16 \mathrm{~h} / 8 \mathrm{~h}$ day/night cycle, and a relative humidity of $90 \% .18$ days post seeding, plants were inoculated with a $P$. xanthii spore suspension by spray method, using inoculum that was obtained by washing heavily infected cucumber leaves with water. The inoculum was adjusted to a final concentration of $1.0 \times 10^{4}$ conidia/ml. The spore suspension was evenly sprayed on leaves, cotyledons and hypocotyl of the seedlings. Prior to inoculation and at 4, 6, 8 and 24 hours post inoculation (hpi), from three individual plants per time point hypocotyl, cotyledon and (first) true leaf samples were harvested separately, and were immediately frozen in liquid nitrogen.

Total RNA was isolated using the MagMAX-96 Total RNA Isolation kit (Ambion, U.S.A.). cDNA was synthesised using $1 \mu \mathrm{g}$ of RNA samples with an iScript cDNA Synthesis Kit (BioRad Laboratories, U.S.A.). Before use in qRT-PCR, cDNA samples were diluted 10-fold.

Quantitative real-time PCR was performed using a CFX96 Real-Time PCR machine (Bio-Rad Laboratories, U.S.A.). Primer pair sequences specific for CsaMLO8 5'-GCGACGGCATTGAAGAACTG-3' (Forward) and 5'-AGGAGACATGCCGTGAGTTG-3' (Reverse) were used to quantify CsaMLO8 expression. Primer pairs specific for cucumber housekeeping genes TIP41, CACS and EF- $a$, as described by Warzybok et al. [49]nitrogen is the most important nutritional factor limiting the yield of cultivated crops. Since nitrogen is essential for synthesis of nucleotides, amino acids and proteins, studies on gene expression in plants cultivated under different nitrogen availability require particularly careful selection of suitable reference genes which are not affected by nitrogen limitation. Therefore, the objective of this study was to select the most reliable reference genes for qPCR analysis of target cucumber genes under varying nitrogen source and availability. Among twelve candidate cucumber genes used in this study, five are highly homologous to the commonly used internal controls, whereas seven novel candidates were previously identified through the query of the cucumber genome. The expression of putative reference genes and the target CsNRT1.1 gene was analyzed in roots, stems and leaves of cucumbers grown under nitrogen deprivation, varying nitrate availability or 
different sources of nitrogen (glutamate, glutamine or $\mathrm{NH} 3$, were used for normalization of CsaMLO8 expression. Each $10 \mu \mathrm{l}$ reaction contained $300 \mathrm{nM}$ of each primer, $1 \mu \mathrm{l}$ (50ng) cDNA template and $1 \mathrm{x}$ iQ SYBR Green Supermix (Bio-Rad Laboratories, U.S.A.). Cycling conditions were an initial denaturation step of $95^{\circ} \mathrm{C}$ for $3 \mathrm{~min}$. followed by 40 cycles of $10 \mathrm{sec}$. denaturation at $95^{\circ} \mathrm{C}$ and $30 \mathrm{sec}$. annealing and extension at $60^{\circ} \mathrm{C}$, finishing with a melt cycle of $0.5^{\circ} \mathrm{C}$ increment per $10 \mathrm{sec}$. from $65^{\circ} \mathrm{C}$ to $95^{\circ} \mathrm{C}$.

Two technical replicates for each sample were tested. CsaMLO8 expression of each sample was determined by the $\Delta \Delta C_{t}$ method [50]provides the necessary accuracy and produces reliable as well as rapid quantification results. But accurate quantification of nucleic acids requires a reproducible methodology and an adequate mathematical model for data analysis. This study enters into the particular topics of the relative quantification in real-time RT-PCR of a target gene transcript in comparison to a reference gene transcript. Therefore, a new mathematical model is presented. The relative expression ratio is calculated only from the real-time PCR efficiencies and the crossing point deviation of an unknown sample versus a control. This model needs no calibration curve. Control levels were included in the model to standardise each reaction run with respect to RNA integrity, sample loading and inter-PCR variations. High accuracy and reproducibility $(<2.5 \%$ variation, normalised by the geometric mean of the three housekeeping genes. Averages and standard errors of CsaMLO8 transcript abundance were calculated over three biological replicates per tissue/time point combination, and statistical significance of differences in $\Delta \Delta C_{t}$ value between time points 4, 6, 8 and 24 hpi and 0 hpi were determined, using Student's T-tests.

\section{Relative quantification of CsaMLO8 transcript isoforms in resistant cucumber}

cDNA samples of non-inoculated and inoculated (6 hpi) resistant cucumber tissues, obtained as described above, were used to quantify relative transcript abundance of the $\Delta 174$ and $\Delta 72$ splice isoforms. Quantitative real-time PCR was performed using a CFX96 Real-Time PCR machine (Bio-Rad Laboratories, U.S.A.). Four primer pairs were designed to specifically amplify one of the two CsaMLO8 splice isoforms: 5'-CTCCTTAATTAATGCATTTCAGC-3' (Forward) with 5'-CTTGTATGATAACCCCCATTGAG-3' (Reverse) or 5'-TTCATTGTTGCACATCTTGC-3' (Forward) with 5'-AAGCTGAAATGCATTAATTAAGG-3' (Reverse) for specific quantification of CsaMLO8D174 and 5'-ATTCTATTGGGTGTTCCCGTC-3' (Forward) with 5'-CTTGTATGATAACCCCCATTGAG-3' (Reverse) or 5'-TTCATTGTTGCACATCTTGC-3' (Forward) with 5'-GAACGACGGGAACACCCAAT-3' (Reverse) for specific quantification of CsaMLO8D72. Primer pairs specific for cucumber housekeeping genes TIP41, CACS and EF-a, as described by Warzybok et al. [49]nitrogen is the most important nutritional factor limiting the yield of cultivated crops. Since nitrogen is essential for synthesis of nucleotides, amino acids and proteins, studies on gene expression in plants cultivated under different nitrogen availability require particularly careful selection of suitable 
reference genes which are not affected by nitrogen limitation. Therefore, the objective of this study was to select the most reliable reference genes for qPCR analysis of target cucumber genes under varying nitrogen source and availability. Among twelve candidate cucumber genes used in this study, five are highly homologous to the commonly used internal controls, whereas seven novel candidates were previously identified through the query of the cucumber genome. The expression of putative reference genes and the target CsNRT1.1 gene was analyzed in roots, stems and leaves of cucumbers grown under nitrogen deprivation, varying nitrate availability or different sources of nitrogen (glutamate, glutamine or $\mathrm{NH} 3$, were used for normalization of CsaMLO8 expression. Each 10 $\mu \mathrm{l}$ reaction contained $300 \mathrm{nM}$ of each primer, $1 \mu \mathrm{l}$ (50ng) cDNA template and $1 \mathrm{x}$ iQ SYBR Green Supermix (Bio-Rad Laboratories, U.S.A.). Cycling conditions were an initial denaturation step of $95^{\circ} \mathrm{C}$ for $3 \mathrm{~min}$. followed by 40 cycles of $10 \mathrm{sec}$. denaturation at $95^{\circ} \mathrm{C}$ and 30 sec. annealing and extension at $60^{\circ} \mathrm{C}$, finishing with a melt cycle of $0.5^{\circ} \mathrm{C}$ increment per 10 sec. from $65^{\circ} \mathrm{C}$ to $95^{\circ} \mathrm{C}$.

Two technical replicates for each sample were tested. CsaMLO8 expression of each sample was determined by the $\Delta \Delta C_{t}$ method [50]provides the necessary accuracy and produces reliable as well as rapid quantification results. But accurate quantification of nucleic acids requires a reproducible methodology and an adequate mathematical model for data analysis. This study enters into the particular topics of the relative quantification in realtime RT-PCR of a target gene transcript in comparison to a reference gene transcript. Therefore, a new mathematical model is presented. The relative expression ratio is calculated only from the real-time PCR efficiencies and the crossing point deviation of an unknown sample versus a control. This model needs no calibration curve. Control levels were included in the model to standardise each reaction run with respect to RNA integrity, sample loading and inter-PCR variations. High accuracy and reproducibility $(<2.5 \%$ variation, normalised by the geometric mean of the three housekeeping genes. Averages and standard errors of CsaMLO8 splice isoform abundance were calculated over three biological replicates per tissue, per tissue the average of the relative abundances calculated with the two different primer pairs per splice isoform was calculated. 


\section{References}

1. Food and Agriculture Organization of the United Nations, Statistics Division [http://faostat3.fao.org/]

2. Pérez-García A, Romero D, Fernández-Ortuño D, López-Ruiz F, De Vicente A, Torés JA: The powdery mildew fungus Podosphaera fusca (synonym Podosphaera xanthii), a constant threat to cucurbits. Mol Plant Pathol 2009, 10:153-60.

3. Block CC, Reitsma KR: Powdery Mildew Resistance in the U. S. National Plant Germplasm System Cucumber Collection. Hortic Sci 2005, 40:416-420.

4. Jahn M, Munger HM, McCreight JD: Breeding cucurbit crops for powdery mildew resistance. In The powdery mildews: a comprehensive treatise; 2002:239-248.

5. Shanmugasundaram S, Williams PH, Peterson CE: Inheritance of Resistance to Powdery Mildew in Cucumber. Phytopathology 1971, 61:1218-1221.

6. Sitterly WR: Breeding for Disease Resistance in Cucurbits. Annu Rev Phytopathol 1972, 10:471-490.

7. Huang S, Li R, Zhang Z, Li L, Gu X, Fan W, Lucas WJ, Wang X, Xie B, Ni P, Ren Y, Zhu H, Li J, Lin K, Jin W, Fei Z, Li G, Staub J, Kilian A, van der Vossen EAG, Wu Y, Guo J, He J, Jia Z, Ren Y, Tian G, Lu Y, Ruan J, Qian W, Wang M, et al.: The genome of the cucumber, Cucumis sativus L. Nat Genet 2009, 41:1275-81.

8. Qi J, Liu X, Shen D, Miao H, Xie B, Li X, Zeng P, Wang S, Shang Y, Gu X, Du Y, Li Y, Lin T, Yuan J, Yang X, Chen J, Chen H, Xiong X, Huang K, Fei Z, Mao L, Tian L, Städler T, Renner SS, Kamoun S, Lucas WJ, Zhang Z, Huang $\mathrm{S}$ : A genomic variation map provides insights into the genetic basis of cucumber domestication and diversity. Nat Genet 2013, 45:1510-5.

9. Wóycicki R, Witkowicz J, Gawroński P, Dąbrowska J, Lomsadze A, Pawełkowicz M, Siedlecka E, Yagi K, Pląder W, Seroczyńska A, Śmiech M, Gutman W, Niemirowicz-Szczytt K, Bartoszewski G, Tagashira N, Hoshi Y, Borodovsky M, Karpiński S, Malepszy S, Przybecki Z: The genome sequence of the North-European cucumber (Cucumis sativus L.) unravels evolutionary adaptation mechanisms in plants. PLoS One 2011, 6:e22728.

10. Jones JDG, Dangl JL: The plant immune system. Nature 2006, 444:323-9.

11. De Almeida Engler J, Favery B, Engler G, Abad P: Loss of susceptibility as an alternative for nematode resistance. Curr Opin Biotechnol 2005, 16:112-117.

12. Van Schie CCN, Takken FLW: Susceptibility Genes 101: How to Be a Good Host. Annu Rev Phytopathol 2014, 52:551-581.

13. Pavan S, Jacobsen E, Visser RGF, Bai Y: Loss of susceptibility as a novel breeding strategy for durable and broad-spectrum resistance. Mol Breed 2010, 25:1-12.

14. Eckardt NA: Plant disease susceptibility genes? Plant Cell 2002, 14:1983-1986.

15. Freisleben R, Metzger I:Über die Auffindung einer mehltauresistenten Mutantenach Röntgenbestrahlung einer anfälligen reinen Linie von Sommergerste. Naturwissenschaften 1942, 24:608.

16. Jorgensen $\mathrm{JH}$ : Discovery, characterization and exploitation of Mlo powdery mildew resistance in barley. Euphytica 1992, 63(Table 1):141-152.

17. Büschges R, Hollricher K, Panstruga R, Simons G, Wolter M, Frijters A, van Daelen R, van der Lee T, Diergaarde P, Groenendijk J, Töpsch S, Vos P, Salamini F, Schulze-Lefert P: The barley Mlo gene: a novel control element of plant pathogen resistance. Cell 1997, 88:695-705.

18. Devoto A, Piffanelli P, Nilsson M, Wallin E, Panstruga R, von Heijne G, Schulze-Lefert P: Topology, Subcellular Localization, and Sequence Diversity of the Mlo Family in Plants. J Biol Chem 1999, 274:34993-35004.

19. Devoto A, Hartmann HA, Piffanelli P, Elliott C, Simmons C, Taramino G, Goh C-S, Cohen FE, Emerson BC, Schulze-Lefert P, Panstruga R: Molecular Phylogeny and Evolution of the Plant-Specific SevenTransmembrane MLO Family. J Mol Evol 2003, 56:77-88.

20. Consonni C, Humphry ME, Hartmann HA, Livaja M, Durner J, Westphal L, Vogel J, Lipka V, Kemmerling B, Schulze-Lefert $\mathrm{P}$, Somerville SC, Panstruga R: Conserved requirement for a plant host cell protein in powdery mildew pathogenesis. Nat Genet 2006, 38:716-20. 
21. Bai Y, Pavan S, Zheng Z, Zappel NF, Reinstädler A, Lotti C, De Giovanni C, Ricciardi L, Lindhout P, Visser R, Theres K, Panstruga R: Naturally Occurring Broad-Spectrum Powdery Mildew Resistance in a Central American Tomato Accession Is Caused by Loss of Mlo Function. Mol Plant-Microbe Interact 2008, 21:30 39.

22. Humphry M, Reinstädler A, Ivanov S, Bisseling T, Panstruga R: Durable broad-spectrum powdery mildew resistance in pea er 1 plants is conferred by natural loss-of-function mutations in PsMLO1. Mol Plant Pathol 2011, 12:866-78.

23. Zheng Z, Nonomura T, Appiano M, Pavan S, Matsuda Y, Toyoda H, Wolters A-MA, Visser RGF, Bai Y: Loss of function in Mlo orthologs reduces susceptibility of pepper and tomato to powdery mildew disease caused by Leveillula taurica. PLoS One 2013, 8:e70723.

24. Wang Y, Cheng X, Shan Q, Zhang Y, Liu J, Gao C, Qiu J-L: Simultaneous editing of three homoeoalleles in hexaploid bread wheat confers heritable resistance to powdery mildew. Nat Biotechnol 2014, Advance on(July):1-6.

25. Appiano M, Pavan S, Catalano D, Zheng Z, Bracuto V, Lotti C, Visser RGF, Ricciardi L, Bai Y: Identification of candidate $M L O$ powdery mildew susceptibility genes in cultivated Solanaceae and functional characterization of tobacco NtMLO1. Transgenic Res 2015.

26. Feechan A, Jermakow AM, Torregrosa L, Panstruga R, Dry IB: Identification of grapevine MLO gene candidates involved in susceptibility to powdery mildew. Funct Plant Biol 2008, 35:1255-1266.

27. Jiwan D, Roalson EH, Main D, Dhingra A: Antisense expression of peach mildew resistance locus 0 (PpMlo1) gene confers cross-species resistance to powdery mildew in Fragaria $x$ ananassa. Transgenic Res 2013, 22:1119-1131.

28. Schouten HJ, Krauskopf J, Visser RGF, Bai Y: Identification of candidate genes required for susceptibility to powdery or downy mildew in cucumber. Euphytica 2014:1-12.

29. He X, Li Y, Pandey S, Yandell BS, Pathak M, Weng Y: QTL mapping of powdery mildew resistance in WI 2757 cucumber (Cucumis sativus L .). Theor Appl Genet 2013, 126:2149-2161.

30. Tusnady GE, Simon I: The HMMTOP transmembrane topology prediction server. Bioinformatics 2001, 17:849-850.

31. Wicker T, Sabot F, Hua-Van A, Bennetzen JL, Capy P, Chalhoub B, Flavell A, Leroy P, Morgante M, Panaud $\mathrm{O}$, Paux $\mathrm{E}$, SanMiguel $\mathrm{P}$, Schulman $\mathrm{AH}$ : A unified classification system for eukaryotic transposable elements. Nat Rev Genet 2007, 8:973-82.

32. Wolters PJ, Schouten HJ, Si-Ammour A, Baldi P: Genomic characterisation of the "Wijcik" mutation. Wageningen University; 2014.

33. Kumar A, Bennetzen JL: Plant Retrotransposons. Annu Rev Genet 1999, 33:479-532.

34. Neumann P, Požárková D, Macas J: Highly abundant pea LTR retrotransposon Ogre is constitutively transcribed and partially spliced. Plant Mol Biol 2003, 53:399-410.

35. Piffanelli P, Zhou F, Casais C, Orme J, Jarosch B, Schaffrath U, Collins NC, Panstruga R, Schulze-Lefert P: The barley MLO modulator of defense and cell death is responsive to biotic and abiotic stress stimuli. Plant Physiol 2002, 129:1076-1085.

36. Pessina S, Pavan S, Catalano D, Gallotta A, Visser RGF, Bai Y, Malnoy M, Schouten HJ: Characterization of the MLO gene family in Rosaceae and gene expression analysis in Malus domestica. BMC Genomics 2014, 15:1-12.

37. Diergaarde PJ, van Enckevort LJG, Posthuma KI, Prins MW: Powdery Mildew Resistance Providing Genes in Cucumis Melo. 2012:1-50.

38. Elliott C, Uller JM, Miklis M, Bhat RA, Schulze-lefert P, Panstruga R: Conserved extracellular cysteine residues and cytoplasmic loop - loop interplay are required for functionality of the heptahelical MLO protein. J Biol Chem 2005, 385:243-254.

39. Bennetzen JL: Transposable element contributions to plant gene and genome evolution. Plant Mol Biol 2000, 42:251-269.

40. Tenaillon MI, Hollister JD, Gaut BS: A triptych of the evolution of plant transposable elements. Trends Plant Sci 2010, 15:471-478. 
41. Oliver KR, McComb JA, Greene WK: Transposable elements: Powerful contributors to angiosperm evolution and diversity. Genome Biol Evol 2013, 5:1886-1901.

42. Nie J, He H, Peng J, Yang X, Bie B, Zhao J, Wang Y, Si L, Pan J-S, Cai R: Identification and fine mapping of pm5.1: a recessive gene for powdery mildew resistance in cucumber (Cucumis sativus L.). Mol Breed 2015, 35:7.

43. Diergaarde PJ, van Enckevort LJG, Posthuma KI, Prins MW: Powdery Mildew Resistance Providing Genes in Cucumis Sativus. 2013:1-50.

44. Bai Y, van der Hulst R, Bonnema G, Marcel TC, Meijer-Dekens F, Niks RE, Lindhout P: Tomato defense to Oidium neolycopersici: dominant $O$ I genes confer isolate-dependent resistance via a different mechanism than recessive ol-2. Mol Plant-Microbe Interact 2005, 18:354-362.

45. Karimi M, Inzé D, Depicker A: GATEWAY vectors for Agrobacterium-mediated plant transformation. Trends Plant Sci 2002, 7:193-195.

46. Lazo GR, Stein PA, Ludwig RA: A DNA transformation-competent Arabidopsis genomic library in Agrobacterium. Nat Biotechnol 1991, 9:963-965.

47. Quinlan AR, Hall IM: BEDTools: A flexible suite of utilities for comparing genomic features. Bioinformatics 2010, 26:841-842.

48. Jurka J, Kapitonov V V., Pavlicek a., Klonowski P, Kohany O, Walichiewicz J: Repbase Update, a database of eukaryotic repetitive elements. Cytogenet Genome Res 2005, 110:462-467.

49. Warzybok A, Migocka M: Reliable reference genes for normalization of gene expression in cucumber grown under different nitrogen nutrition. PLoS One 2013, 8:e72887.

50. Pfaffl MW: A new mathematical model for relative quantification in real-time RT-PCR. Nucleic Acids Res 2001, 29:2002-2007. 


\section{Additional files}

Due to the size, all the additional files are available online following the links indicated.

Additional file 1: https://static-content.springer.com/esm/art\%3A10.1186\%2Fs12870-015-0635-x/

MediaObjects/12870 2015635 MOESM1 ESM.pdf

Format: PDF

Full length alignment of CsaMLO8 WT, CsaMLO8A72 and CsaMLO8A174 coding sequences

Additional file 2: $\underline{\text { https://static-content.springer.com/esm/art\%3A10.1186\%2Fs12870-015-0635-x/ }}$ MediaObjects/12870 2015635 MOESM2 ESM.pdf

Format: PDF

Multiple sequence alignment of MLO proteins encoded by clade V MLO S-genes from different species

Additional file 3: https://static-content.springer.com/esm/art\%3A10.1186\%2Fs12870-015-0635-x/ MediaObjects/12870 2015635 MOESM3 ESM.pdf

Format: PDF

Relative quantification of CsaMLO8D174 and CsaMLO8D72 transcript abundances by qRT-PCR on CDNA samples obtained from non-inoculated (A) or inoculated (B) cucumber tissue samples. Fold changes were normalised relative to CsaMLO8D174 expression. Bars represent the average fold change over three independent biological replicates. Error bars indicate standard errors of the mean.

Additional file 4: https://static-content.springer.com/esm/art\%3A10.1186\%2Fs12870-015-0635-x/ MediaObjects/12870_2015_635_MOESM4_ESM.pdf

Format: PDF

Photographs of 20 independent ol-2 tomato plants transformed with either CsaMLO8 WT or CsaMLO8A174

Additional file 5: http://bmcplantbiol.biomedcentral.com/articles/10.1186/s12870-015-0635-x

Format: XLSX

Complete overview of putative LTRs and putative TEs homologous to the TE identified in CsaMLO8

Additional file 6: https://static-content.springer.com/esm/art\%3A10.1186\%2Fs12870-015-0635-x/ MediaObjects/12870_2015_635_MOESM6_ESM.pdf

Format: PDF

Multiple sequence alignment of the TE identified in CsaMLO8 and putative homologous TEs

Additional file 7: $\underline{\text { http://bmcplantbiol.biomedcentral.com/articles/10.1186/s12870-015-0635-x }}$

Format: XLSX

Full table of 115 resequenced accessions. The amount of reads identified is given at the overlap between CsaMLO8 and the start of the insertion in forward (TE start-F) and reverse (TE start-R) direction, at the overlap between the end of the insertion and CsaMLO8 in forward (TE end-F) and reverse (TE end-R) direction, and at the site of the insertion with only CsaMLO8 sequence in forward (WT-F) and reverse (WT-R) direction. 



\section{Chapter 7}

Discovery and characterization of a novel tomato mlo mutant from an EMS mutagenized Micro-Tom population

Michela Appiano, Ageeth van Tuinen, Danny Schipper, Robin Huibers, Dongli Gao, Richard GF Visser, Anne-Marie Wolters, Yuling Bai 


\section{Abstract}

In tomato, there are at least three SIMLO genes contributing to the powdery mildew disease caused by Oidium neolycopersici, namely SIMLO1, SIMLO5 and SIMLO8. Of the three homologs, the SIMLO1 gene plays a major role since a natural mutant allele called ol-2 can almost completely prevent fungal penetration by forming a papilla. The ol-2 allele contains a 19-bp deletion in the coding sequence of the SIMLO1 gene, resulting in a premature stop codon within the second cytoplasmic loop of the predicted protein.

In this study, we describe a mutant containing a novel allele (the $m 200$ allele) of the tomato SIMLO1 gene, which was discovered from an EMS mutagenized Micro-Tom population. Compared to the sequence of the SIMLO1 gene, the m200 allele carries a point mutation at T65A. The SNP results in a premature stop codon located in the first transmembrane domain of the complete SIMLO1 protein. The length of the predicted protein is 21 amino acids, while the SIMLO1 full-length protein is 513 amino acids. We developed a HighResolution Melting (HRM) marker to distinguish the mutated m200 allele from the SIMLO1 allele in backcross populations. Tomato plants homozygous for the $m 200$ allele showed resistance to Oidium neolycopersici. The allele was recessively inherited and conferred resistance that was associated with papilla formation at fungal penetration sites of plant epidermal cells. 


\section{Introduction}

Tomato (Solanum lycopersicum L.) is a model crop species of high economic value with interesting developmental features such as compound leaves, fleshy fruits, and sympodial shoot branching. The amount of information currently available for the domesticated tomato is abundant. Its genome (Sato et al., 2012), transcriptome (Tomato Functional Genomics Database, http://ted.bti.cornell.edu/) and metabolome (Moco et al., 2006) are available, as well as functional genomic tools, like the RNA interference (RNAi, Xiong et al., 2005, Schijlen et al., 2007, De Jong et al., 2009), transcription activator-like effector nucleases (TALENs, Lor et al., 2014), and clustered regularly interspaced short palindromic repeats (CRISPR)/Cas9 (Brooks et al., 2014; Ron et al., 2014). Tomato's amenability to Agrobacterium transformation allows the use of all these technologies.

An important aspect of the domesticated tomato is its lack of genetic diversity because of years of selection for a limited set of traits, such as fruit shape and size (Rodríguez et al., 2011). However, given the upcoming challenges for agriculture regarding climate change and food safety, it has become a prominent issue to improve tomato also for resistance or tolerance to biotic and abiotic stresses (Kissoudis et al., 2016). One way to achieve this goal is to use the diversity present in wild relatives. S. lycopersicum can be crossed with its wild relatives for the introgression of resistance traits, although crossing barriers and carry-over of undesirable traits represent substantial limitations (Bai and Lindhout, 2007). It has been a general practice in tomato breeding to use wild relatives as a donor for introgression of valuable traits present in tomato varieties. For example, almost all tomato genes conferring resistance to pathogens are derived from wild species (Barham and Winstead, 1957; Parniske et al., 1999; Chunwongse et al., 2002; Seah et al., 2004; Lanfermeijer, et al., 2005; Ji et al., 2007; Menda et al., 2014). This so-called introgression breeding is nowadays facilitated by the available genome sequences of many wild species, such as S. habrochaites, S. pennellii, S. pimpinellifolium and S. arcanum (Sato et al., 2012; Bolger et al., 2014; Aflitos et al., 2014;).

Another way to increase genetic diversity is to introduce new mutations artificially. Chemical and physical mutagenesis are frequently used for this purpose in most of the economically important crop species (Shu, 2012). Of the chemical mutagens, ethyl methane sulfonate (EMS) is very popular. EMS selectively alkylates guanine bases, which, during DNA replication, are preferably coupled with a thymine over a cytosine residue, resulting in a random point mutation. Most of these mutations (70-99\%) consist of substitutions from $C$ to $T$ or from $G$ to $A$ (abbreviated as C/G to T/A) (Greene et al., 2003; Till et al., 2004; Till et al., 2007).

Five EMS tomato populations were developed during the last years (Table 1), two of which used the tomato cultivar Micro-Tom (MT). In contrast to most of the cultivated tomatoes, the MT is a miniature determinate tomato cultivar, released in 1989 by J. Scott 
and B. Harbaugh for home gardening purposes and introduced in the genetic research by Avraham Levi's group in 1997 (Scott \& Harbaugh, 1989; Meissner et al., 1997). Because of its small size (8-10 cm when grown in $14 \mathrm{~cm}$ diameter pots) and short life cycle (7090 days from sowing to fruit-ripening), MT has been compared to Arabidopsis as a model system to carry out molecular research in tomato. Recently, the Japanese mutant database, TOMATOMA became available, together with MT's genome and a wholegenome resequencing analysis of EMS-MT mutants (Saito et al., 2011; Shirasawa et al., 2016). Altogether, these features make MT a suitable cultivar for large-scale mutagenesis.

Breeders aim at finding and introducing durable resistance in cultivated crops. A known way to achieve it consists of using impaired plant susceptibility genes (S-genes) (Pavan et al., 2010, van Schie \& Takken, 2014). The MLO gene is the best-characterized example of $S$-genes in several crops. Functional MLO proteins are required by adapted powdery mildew (PM) pathogens to penetrate the cell wall and cause disease (Hückelhoven, 2005). The $M L O$ gene encodes a plant transmembrane protein which typically spans across the plasma membrane seven times and ends in the cytoplasm with a C-terminus domain. It is highly conserved in plant species and can be tracked back to green algae (Kusch et al., 2016). However, the biochemical function of the MLO protein is still unknown. The only indication lays in the conserved calmodulin-binding site in the C-terminus domain which was shown to interact in vitro with calmodulin in a $\mathrm{Ca}^{2+}$ dependent manner (Kim et al., 2002).

In 1976 a natural loss-of-function barley mlo allele was discovered, called $\mathrm{mlo}-11$, and since then used in the field for spring barley cultivation (Jørgensen, 1971; Jørgensen, 1992). Thanks to its durable effectiveness, barley PM disease has greatly declined in importance in countries where barley is widely cultivated, like the United Kingdom (Brown, 2015).

Each plant species contains a certain number of MLO paralogs. Members of clade IV and $\mathrm{V}$ are described as susceptibility factors towards pathogens causing the PM disease (Feechan et al., 2008; Kusch et al., 2016; Panstruga, 2005). In tomato, the SIMLO gene family comprises 16 homologs, of which four belong to clade V, namely SIMLO1, SIMLO3, SIMLO5 and SIMLO8 (Zheng et al., 2016). A naturally mutated allele of the SIMLO1 gene, called ol-2, was described in the past years (Ciccarese et al., 1998; De Giovanni et al., 2004; Pavan et al., 2008; Bai et al., 2008). The ol-2 variant contains a 19-bp deletion in the coding sequence resulting in a premature stop codon within the second cytoplasmic loop of the predicted protein. This mutation, inherited from S. lycopersicum var cerasiforme, when in homozygous state, mediates broad-spectrum resistance to Oidium neolycopersici. The ol-2 conferred resistance is characterized by the formation of papillae beneath the fungal appressoria which can significantly reduce pathogen penetration (Bai et al., 2005). In the following years, transgenic RNAi lines were developed to silence simultaneously multiple clade V-SIMLO homologs (Bai et al., 2005; Zheng et al., 2016). One construct, in particular, 
was described to silence SIMLO1, SIMLO5 and SIMLO8. When ol-2 plants were compared to plants of the RNAi lines, the authors noticed a higher level of resistance associated with the latter. Because of these results, it was concluded that the three SIMLO genes contribute to the tomato susceptibility towards PM, with SIMLO1 having the major role (Zheng et al., 2016).

In the present study, we describe the in-house development of an EMS mutant population of the tomato cultivar MT. With its development, we aimed at finding new sources of resistance to different pathogens, including PM. In this EMS population, we discovered a new mutant defective in the SIMLO1 gene, called $m 200$. Then, we performed a comparison of the novel allele with the ol-2 mutant as well as the RNAi line in which three clade V SIMLO homologues are silenced. Results and implications are further presented and discussed in the context of mlo mutations occurring in other plant species.

TABLE 1. Collection of EMS tomato populations described in literature.

\begin{tabular}{|c|c|c|c|}
\hline Year & Title & Tomato cultivar used & Reference \\
\hline 으 & A new model system for tomato genetics & Micro-Tom & Meissner et al., 1997 \\
\hline ঠ্ণ & $\begin{array}{l}\text { In silico screening of a saturated mutation } \\
\text { library of tomato }\end{array}$ & M82 & Menda et al., 2004 \\
\hline \multirow{2}{*}{ ஓ्̀ } & $\begin{array}{l}\text { Mutant resources for the miniature tomato } \\
\text { (Solanum lycopersicum L.) 'Micro-Tom' }\end{array}$ & Micro-Tom & Saito et al., 2009 \\
\hline & $\begin{array}{c}\text { Implementation of two high through-put technologies } \\
\text { in a novel application: detecting point mutations } \\
\text { in large EMS mutated plant population }\end{array}$ & Tpaadasu & Gady et al., 2009 \\
\hline$\frac{\circ}{\circ}$ & $\begin{array}{l}\text { A new mutant genetic resource for tomato } \\
\text { crop improvement by TILLING technology }\end{array}$ & Red Setter & Minoia et al., 2010 \\
\hline
\end{tabular}

\section{Results}

\section{A novel EMS mlo mutant (m200) shows resistance to powdery mildew}

An EMS-mutagenized population of tomato cv MT was developed and phenotypically screened for resistance to the powdery mildew pathogen $O$. neolycopersici (On).

During the EMS treatment, the $1 \% \mathrm{v} / \mathrm{v}$ EMS concentration was mostly used to maximize the genomic variation with a minimum decrease in viability.

The $M_{1}$ plants derived from the first two rounds of EMS treatment (about 1000 seeds per round) were inoculated with spores of the pathogen On by spray inoculation. In the first group of approximately $1000 \mathrm{M}_{1}$ plants, one plant (M200) showed no fungal sporulation, while all other plants were severely infected (Figure 1, panel A). 
The $M_{1}$ plants were allowed to self-pollinate and $M_{2}$ seeds were collected. All the tested M200 $\mathrm{M}_{2}$ plants were free of PM symptoms, and thus resistant. Except for the resistant phenotype, no other morphological differences were observed in $M 200 M_{1}$ and $M_{2}$ plants compared to wild-type MT (not subjected to the EMS treatment).

To find the causal mutation for the highly resistant phenotype of the M200 plant and its $\mathrm{M}_{2}$ progeny, we chose SIMLO1 as the first candidate gene. We cloned the coding sequence (cds) of the SIMLO1 gene. A SNP (T65A) was detected in the SIMLO1 cds of the M200 plant compared to the sequence in MT and tomato cultivar Heinz (Figure 1, panel B). This point mutation results in a premature stop codon. The stop codon affects the triplet, which is translated into the amino acid leucine $(\mathrm{L})$ in the full-length SIMLO1 protein of Heinz, and is located in the first transmembrane domain (Figure 1, panel C). The resulting truncated protein contains 21 aa instead of 513 aa. Using the program Protter, the truncated 21 aa protein does not contain transmembrane regions and is located in the intracellular space. This new SIMLO1 allele was named m200.

A)

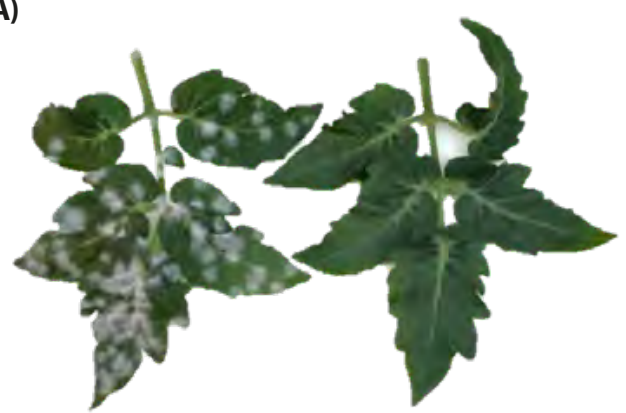

B) $200 \_$sIMLO1 Micrō-7om sinto Heinz_S1M̄ot

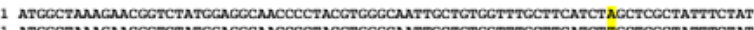
1. ATGGCTMAACANCGGTCTATGGAGGCAACCCCTACGTGGGCANTGCTGTGGTTTGCTTCATCTTOCTCOCTATTTCTA:

M200_S1MLO1 Micro-7om SINLO Heinz_sIMËO1

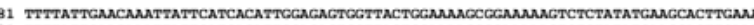

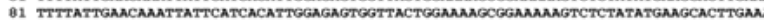
B1 TTTTATTGAACAAATTATTCATCACATTGGAGAGTGGTTACTGGAHAAGCGGAAAAGGTCTCTATATGAAGCACTTGAA

M200_S1MLO1 161 AGATCAMAGCTGAACTTATGCTGTTGGGATTCTTATCACTGTTGTTGACAGTGTTGCAAGATOCAGTTTCTAACTTATGI

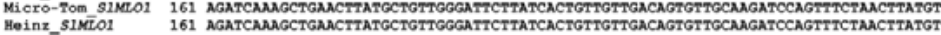

N200_S1MLO1 241 GTCCCCAMGAGTGTTGGTTATTCATGGCATCCTTGTATGGCARAGGAAGA TGCCAAGTCTGAGTATGATGACCCTTGTCT

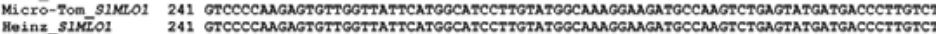
Meinz_SIMËOI

M200_S1MLO1 321 ACCAMAGGMUAAGTGCANTTGCATCTTCATATGCAMTACACCAGCTCCATATCTFCATCTTTGTATTGGCAGTTGCTC MiCrö-Tom_SINZO1 321 ACCAAAGGGAMAAGTGCAATTTGCATCTTCATATGCAATACACCAGCTCCATATCTTCATCTTTGTATTGGCAGTTGCTC

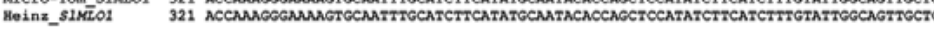

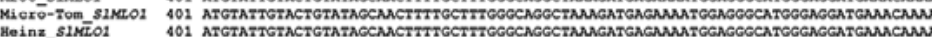

N200_S1MLO1 561 TITCTGCAGCAAGTCCCCCGTGTTECTCTCGATAGTTTGTTTCTTTCGGCAATTCTTCTCATCAGTTGCAMAAGTTGACT

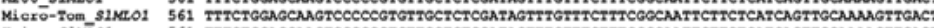

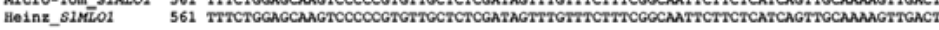


B)

200_S1MLO Miero-Zom_sime Heinz_SIML̃ol

N200_S1MLO Micro-7om_sin Heinz S1MTiol

N200_S1MLO1 Miero-7om_siM

M200_S1MLO1 Miero-rom_siN Heinz_SIML̃OI

N200_sIMLO1 Miero-rom S1

M200_S1MLOD

M200 S1MLO

Mierō-rom

Heinx_S1MIO Micrō-rom

721 AMCAGAOCAGTTGACAMAGACTTCAMATTGTTGTTGGAATAMGTCCTOCATTATCGCTCTTCACGGTOCTATATTITCT 721 AACAGAOCAGTTGACANAGACTTCANAGTIGTTGTTGGAATAAGTCCTCCATTATGGCTCTTCACGGTGCTATATTTIC:

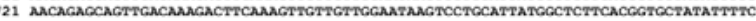

801 GACTACTACCGATCGATTGTACTCGTATCTITGGGTGCCAITTATCCCACTTGTAATAATATTGCTAGTTGGCACANAAC

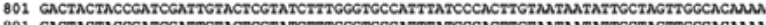

881 TTCANATGATCATAACAGAATGGGAGTAAGGATTTCAGAUAGGGGAGACATAGTANAGGTGTACCTGTGGTGGAGACT

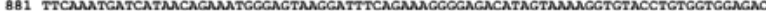

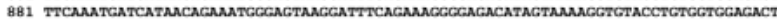

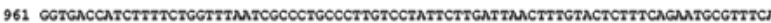

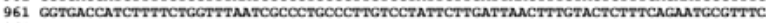
961 GGTGACCATCTTTTCTGGTTTMATCGCOCHGCCCTTGTCCTATTCTTGATTANCTTTGTACTCTITCAGA TGCGTTTCA

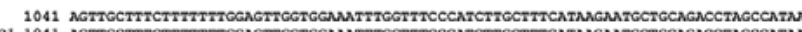

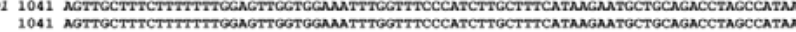

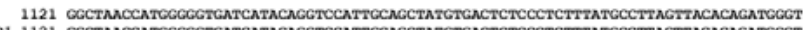

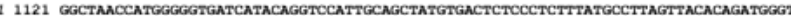
1121 GGCTAACCATGGGGTGATCATACAGGTCCATTGCAGCTATGTGACTCTCCCTCTTIATGCCTTAGTTACACAGATGGGT

N200_S1MEO1 1281 GAMACATGGGCTATCAGGACATACCACOCCTGCAMACAGCAGACCAACCACACCATRGCGTGOTACCTCCCCTGTTCACT Micro-Tom_SINLO1 1281 GAAACATGGGCTATCAGGACATACCACCCCTGCAAACAGCAGACCAACCACACCATTGCGTGGTACCTCCCCTGTTCAC:

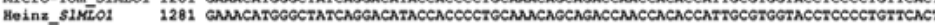

N200_S1MEO1 1361 TATTACGCGGTTATCCACMATATMATGMGACAGTGTTCAMGCATCTCCTCGGACATCCAMTGTCGMAMTGMGGGTGG Mierö-Tom_SIMEO1 1361 TATTACGCGGTTATCCACAATATAATGAGGACAGTGTTCAMGCATCTCCTCGGACATCCAATGTCGMAMTGMAGGGTGC

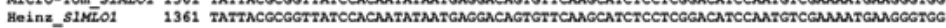

N200_S1MLO1 1441 QCTAATGAAMATCAGGAGGGAGAGATCCTGCAGCATGCCTCCACTGATCATAACAAGCAAATTGAGATTACAATGTCAG

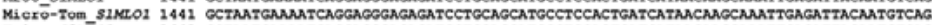

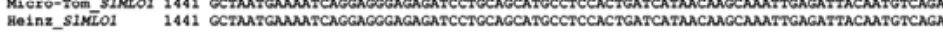

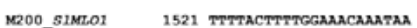
Micro-Tom_SINLO1 1521 TTTTACTTTTGGAAACAMATAA Heinz_sINİO1 1521 TITTACTTTEGAMACAMATA

C)

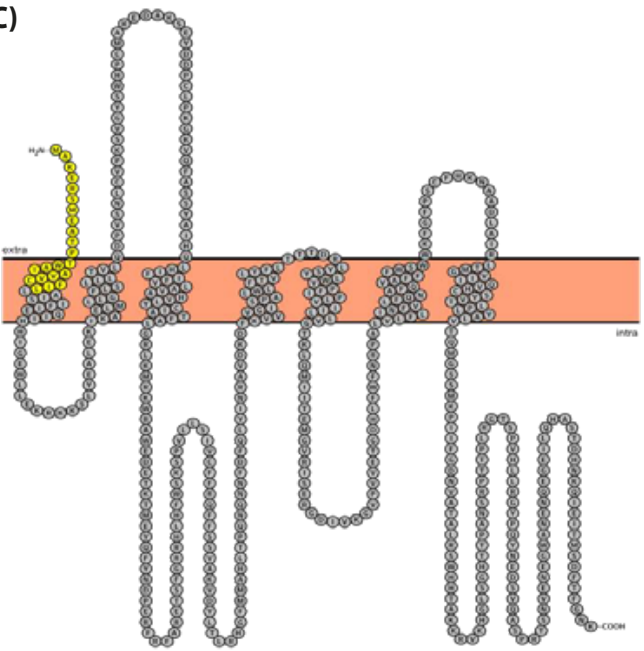

FIGURE 1. A novel EMS mlo mutant ( $m 200$ ) shows resistance to powdery mildew. A) Contrasting phenotypes of susceptible leaves of an $\mathrm{M}_{1}$ plant (on the left) and resistant leaves of the M200 plant (on the right) after Oidium neolycopersici inoculation. B) Coding sequences (cds) alignment of the SIMLO1 gene in M200, Micro-Tom (MT), and tomato $\mathrm{cv}$. Heinz. Highlighted in yellow is the base change $\mathrm{T} \rightarrow \mathrm{A}$ responsible for the premature stop codon in M200 plant. C) Schematic representation of the SIMLO1 protein of the cv. Heinz. The predicted $m 200$ protein is indicated in yellow, while the region that is absent in $m 200$ is shadowed in grey. 


\section{The resistance associated with the novel $\mathbf{m} 200$ allele is recessively inherited}

Aiming at observing the inheritance of the $m 200$ allele, the $M_{200} M_{1}$ plant was backcrossed to MT and crossed to the tomato cv. Moneymaker (MM). Initially, three BC families derived from different fruits of the cross between M200 and MT were tested with On. All the BC plants showed clear fungal sporulation, and were as susceptible as the controls, MM and MT (Supplementary Table 1).

A ㅂigh Resolution Melting (HRM) marker was developed which could clearly distinguish the SIMLO1 allele carried by the wild-type MT from the mutated m200 allele (Figure 2). All $B C_{1}$ plants were heterozygous for the $m 200$ allele (Supplementary Table 1).

Five $B C_{1} S_{1}$ families were produced and used in another disease test. The segregation ratio observed within the progenies of each backcross fitted the 3:1 (susceptible : resistant) hypothesis (Supplementary Table 2 and 3). All BC $\mathrm{S}_{1}$ resistant plants were homozygous for the $m 200$ allele, and all susceptible plants were either homozygous or heterozygous for the MT/MM allele (Figure 2). Overall, these results confirm that resistance to powdery mildew observed in plants carrying the $m 200$ allele exhibits a recessive inheritance.

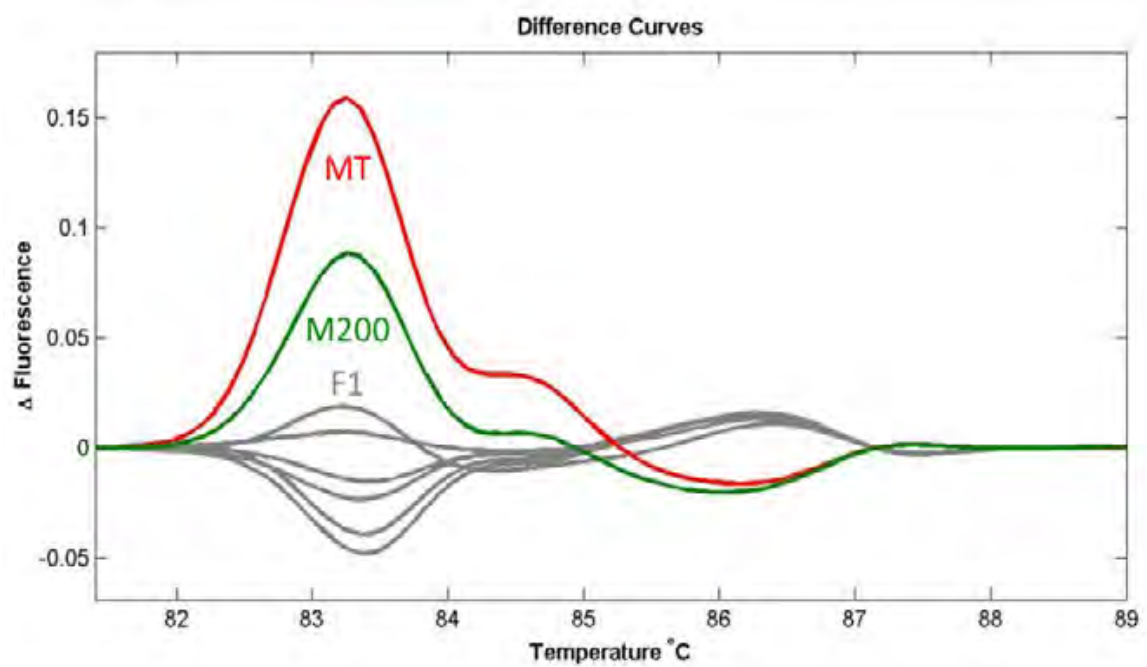

FIGURE 2. High Resolution Melting (HRM) profiles of two parental lines and their BC population. Red plot derived from the Micro-Tom plant -MT- (homozygous for the wild-type SIMLO1 gene), green plot from the M200 plant (homozygous for the mutated allele $\mathrm{m200}$ ) and grey plots from the six individuals of the $\mathrm{BC}_{1}$ population (heterozygous) obtained after crossing M200 with Micro-Tom.

\section{Papilla formation is associated with resistance in the $\mathbf{m} 200$ mutant}

An experiment was conducted to 1) study the histological mechanism of the $m 200$ resistant mutant and 2) compare the level of resistance conferred by the m200 mutant in MM background with other genotypes. The other genotypes included in this experiment 
are the ol-2 mutant in two different genetic backgrounds (MM and Super Marmande -SM) and the RNAi::SIMLO1 line in which SIMLO1, SIMLO5 and SIMLO8 are silenced.

Symptoms were visually monitored at 10, 15 and 18 days post inoculation (dpi). The control MM plants started showing PM symptoms at $10 \mathrm{dpi}$ and were heavily infected at 18 dpi (Figure 3, panel A). In contrast, no fungal sporulation was observed on all plants of the $\mathrm{m} 200$ mutant, the ol-2 mutant (in MM background) and the RNAi::SIMLO1 line, at all time-points (Figure 3, panel B, C, D and F). For the ol-2 mutant in SM background, no fungal sporulation was observed on the $3^{\text {rd }}$ and $4^{\text {th }}$ leaves at all time points (Figure 3, panel E). Occasionally, weak mycelium growth could be seen on the $1^{\text {st }}$ and $2^{\text {nd }}$ true leaves of the ol-2 mutant in SM background, at 15 and $18 \mathrm{dpi}$.

At 72 hours post-inoculation (hpi) on infected MM leaves, 91\% of the infection units (IU, a germinated spore) developed colonies having 1 to 6 secondary hyphae (Table 2 and Figure 3, panel A).

At the same time point in the $m 200 \_\mathrm{BC}_{1} \mathrm{~S}_{3}-1$ family about $10 \%$ of the 97 IUs succeed in forming colonies with 1 to 4 secondary hyphae. Seventy-six of the 97 IU observed differentiated an appressorium, while 21 IUs formed only a germination tube. Of the IU that formed an appressorium, 34 were stopped by papilla formation at the fungal penetration sites and 10 IUs further developed a haustorium and formed colonies (Table 2 and Figure 3, panel B).

Of the $101 \mathrm{IU}$ recorded on leaf samples of the family $m 200 \_B C_{1} S_{3}-2$, all formed an appressorium. In addition, $33 \mathrm{IU}$ of the 101 were stopped by papilla formation and $68 \mathrm{IU}$ did not develop further than the appressorium (Table 2 and Figure 3, panel C). None of the IUs formed a haustorium.

On samples of plants carrying the ol-2 allele in MM background, all the 90 observed IUs formed an appressorium. Of these $90 \mathrm{IU}, 55$ were stopped by a papilla, 4 developed colonies with haustorium and 2 to 4 secondary hyphae, and 31 did not develop further than the appressorium where papilla was not visible (Table 2 and Figure 3, panel D).

On samples of plants carrying the ol-2 allele in SM background, $100 \mathrm{IU}$ were counted and all produced an appressorium. Of the 100 IUs, 51 were stopped by a papilla, 11 developed into colonies where 2 to 5 secondary hyphae were visible and 38 formed only the appressorium without any visible papilla (Table 2 and Figure 3, panel E).

When comparing the IU stopped by papillae on plants carrying the $m 200$ allele with those carrying the ol-2 allele (either in MM or SM), we noticed that the papillae frequency (\% papilla per appresorium in Table 2) was significantly lower (Student t test, $p=0.043$ ) in the m200 than in the ol-2 plants.

On samples of transgenic RNAi::SIMLO1 plants, all the 109 IU observed formed an appressorium, of which 78 were blocked by a papilla (Figure 3, panel F), which represents 
a significant increase compared to the numbers recorded on both ol-2 (Student t test, $p=$ 0.016 ) and $m 200$ plants (Student $t$ test, $p=0.002$ ) (Table 2). Six IU were able to penetrate the cell wall, producing a haustorium and differentiating 1 to 3 secondary hyphae, and 25 IU did not developed further than the appressorium.

TABLE 2. O. neolycopersici development $72 \mathrm{~h}$ after the artificial inoculation.

The infection units (IU = spore producing a germination tube) were counted on each genotype along with any fungal structure and plant response.

\begin{tabular}{|c|c|c|c|c|c|c|}
\hline & \multirow[b]{2}{*}{ IU } & \multicolumn{3}{|c|}{$\begin{array}{c}\text { Number of fungal structures } \\
\text { observed }\end{array}$} & \multirow{2}{*}{ \%papilla/AP } & \multirow[t]{2}{*}{ \%HS/AP } \\
\hline & & AP & papilla & HS & & \\
\hline$m 200 \_B C_{1} S_{3}-1$ & 97 & 76 & 34 & 10 & 44.74 & 13.2 \\
\hline$m 200 \_B C_{1} S_{3}-2$ & 101 & 101 & 33 & 0 & 32.7 & 0 \\
\hline ol-2_MM & 90 & 90 & 55 & 4 & 61.1 & 4.4 \\
\hline ol-2_SM & 100 & 100 & 51 & 11 & 51 & 11 \\
\hline RNAi::SIMLO1 & 109 & 109 & 78 & 6 & 71.6 & 7.3 \\
\hline MM & 102 & 101 & 1 & 92 & 0.99 & 91.1 \\
\hline
\end{tabular}

AP, appressorium; HS, haustorium 

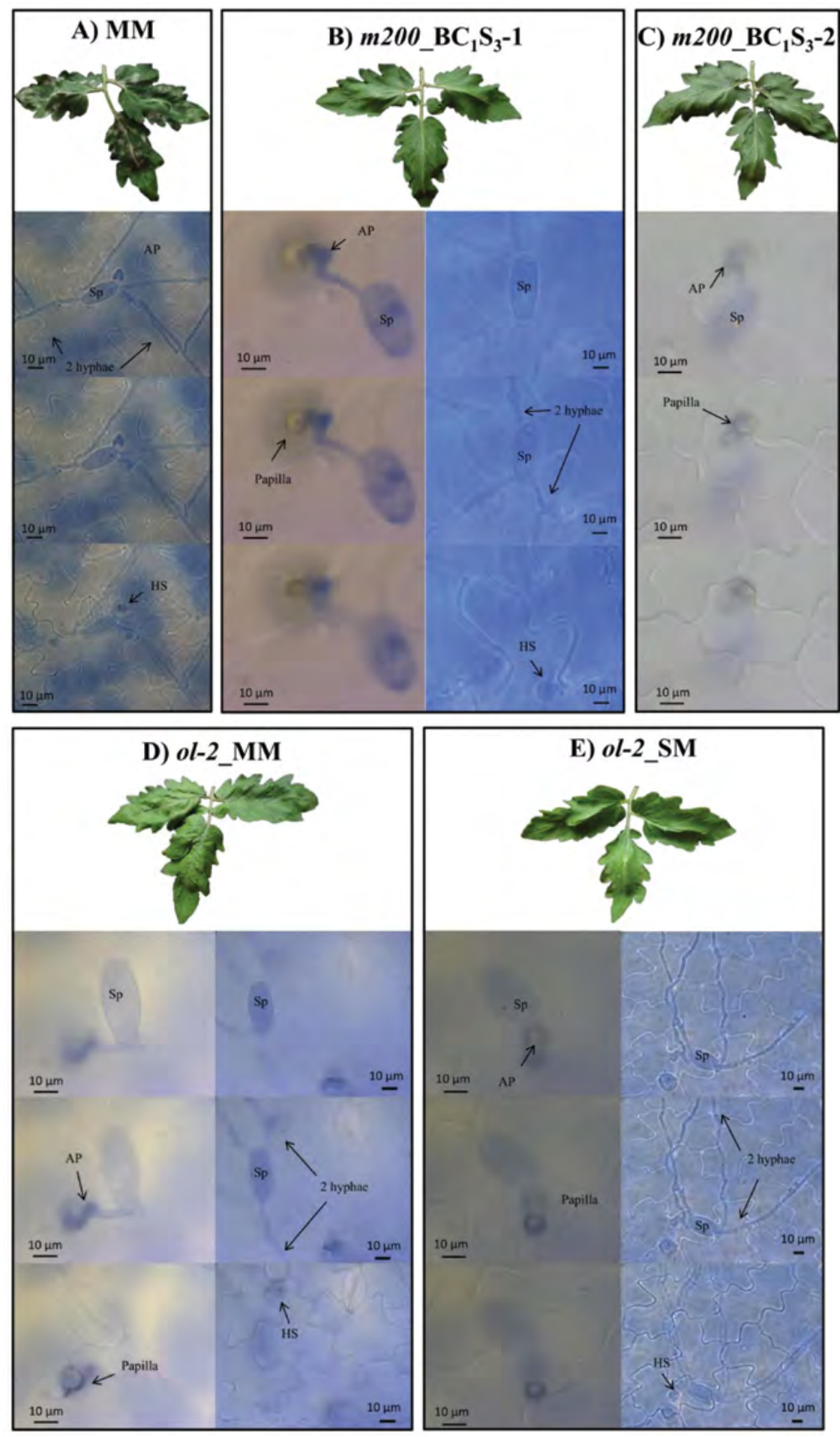


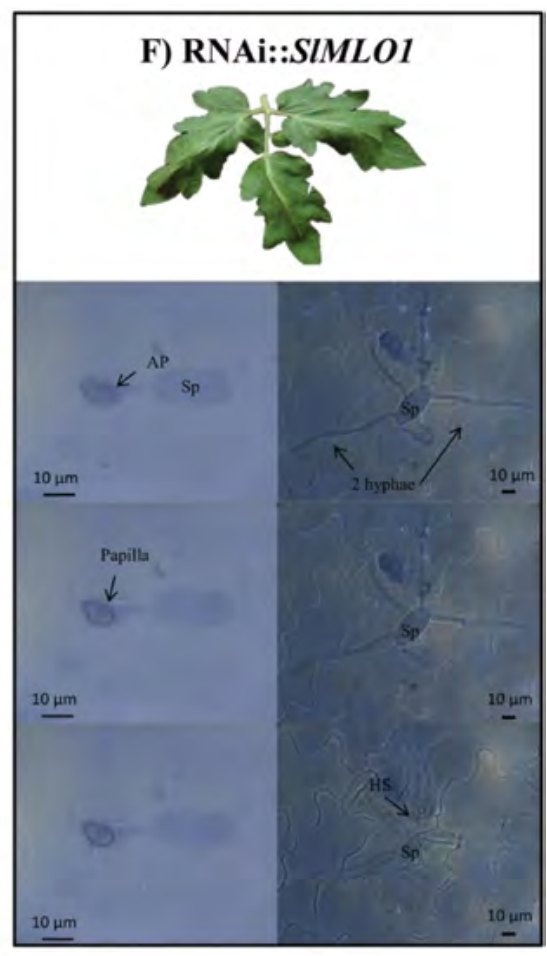

FIGURE 3. Phenotypic evaluation of the powdery mildew symptoms and development of the infection units (IU) of Oidium neolycopersici on six different genotypes.

In each panel photos are taken from A) Moneymaker (MM), B) and C) plants carrying the $m 200$ allele, D) and E) plants carrying the ol-2 allele in MM and Super Marmande (SM), respectively, and in panel F) from a plant carrying the RNAi::SIMLO1 construct.

On the top part of each panel, photos of leaves are depicted collected 18 days after the pathogen inoculation. Below that, photos of two IU/genotype are shown, except for the $\mathrm{MM}$ and $\mathrm{BC}_{1} \mathrm{~S}_{3}-2$ carrying the $m 200$ allele where only one IU is shown. Each photo is taken with different focus to observe all the fungal structures, from the most superficial to the deepest ones.

$\mathrm{Sp}=$ spore, $\mathrm{AP}=$ appressorium; $\mathrm{HS}=$ haustorium; 2 hyphae $=$ secondary hyphae.

\section{Discussion}

Powdery mildew disease can be a problem in greenhouses and field tomato cultivations. The humidity that forms at the leaf surface when cold nights change to warm days or when plants are grown in crowded locations without sufficient air circulation is enough to ignite an infection (Horst, 2013). The availability of resistant cultivars is, therefore, essential to control this disease in a sustainable way. The resistance can be achieved in several ways, each of them with pros and cons. One of the ways consists of inducing mutations artificially with chemical mutagens.

In this work we describe the set-up of an EMS mutant population of the tomato cv. MT with which we aimed at finding new sources of resistance to various diseases. Here we focused, in particular, on finding sources of resistance to the PM disease caused by $O$. neolycoperisici.

\section{Is the $\mathbf{m} \mathbf{2 0 0}$ mutation a real product of the EMS mutagenesis?}

A PM disease test was performed on the $M_{1}$ plants initially obtained with the intention of finding dominant mutations. We spotted the M200 mutant in the first disease test, and the sequence analysis showed that the resistance was due to a nonsense mutation in the coding region of the SIMLO1 gene (Figure 1, panel B). This mutation results in a stop codon at 
T65 (Figure 1, panel C). This location differs from the stop codon identified in the ol-2 allele, located in the second intracellular loop.

It is unexpected that a recessive mutation occurred homozygously in an $M_{1}$ plant since the probability of having a mutation on both alleles has been shown to be extremely low (Oladosu et al., 2016).

In order to verify whether any natural impaired SIMLO1 allele is already present in MT, we blasted the full-length nucleotide sequence of the Heinz SIMLO1 gene to the MT database MiBASE (http://www.kazusa.or.jp/jsol/microtom/indexe.html). Two transcripts with 100\% identity were found, the LEFL1037DE09 and the LEFL1063CA10, both shorter than the full-length SIMLO1 (623 bp and $610 \mathrm{bp}$, respectively), indicating that the transcript of MT SIMLO1 is not complete. However, we could further clone the full-length sequence by PCR amplification of mRNA derived from MT plants. A multi-alignment composed of the two partial EST sequences retrieved by blasting, the sequenced MT SIMLO1 transcript and the known Heinz SIMLO1 did not reveal any mutation (Supplementary Fig. 1). Thus, these findings indicate that the SIMLO1 gene in MT does not differ from the one in other cultivated tomatoes, like Heinz and MM. This is to be expected since MT originated from two cultivated tomatoes (Scott and Harbaugh, 1989).

In addition, we searched for any predicted mutations of the SIMLO1 gene among the 115 tomato accessions, recently sequenced, using the haploSmasher online server (http://www. plantbreeding.wur.nl/hs/) (Aflitos et al., 2014). The output of this analysis also revealed that there are no predicted natural mutations at the T65 where the m200 SNP occurs.

It is also important to notice that the mutation detected is not typically produced by the EMS mutagen. The latter notoriously favors transitions, e.g. purine replaced by purine $A \leftrightarrow G$, and pyrimidine replaced by pyrimidine $C \leftrightarrow T$ (indicated as $G / C \rightarrow A / T$, Sega, 1984; Griffiths et al., 1999). In Arabidopsis, almost all the EMS mutations so far described correspond to a $\mathrm{G} / \mathrm{C}$ to $A / T$ transitions (Greene et al., 2003). We have also investigated the available literature regarding EMS mlo-alleles and found 16 in wheat and 11 in barley (Table 3, Reinstädler et al., 2010; Acevedo-Garcia et al., 2016). Recently, two additional mlo-alleles have been detected in petunia EMS mutants (Jiang et al., 2016). In all cases, except three, the mutagenized treatment produced the expected base substitutions $(\mathrm{G} / \mathrm{C} \rightarrow \mathrm{A} / \mathrm{T})$. In contrast, the barley mutants mlo13, mlo-26 and mlo-30 are characterized by transversions (purine replaced by a pyrimidine, and vice-versa) $\mathrm{T} \leftrightarrow \mathrm{A}$, as observed in $\mathrm{m} 200$. In mlo-13 and mlo-26, the transversion caused two missense mutations, $\mathrm{V} 30 \mathrm{E}$ and $\mathrm{L} 270 \mathrm{H}$ respectively, which in both cases lead to the loss-of-function of the protein. In mlo-30, the mutation occurred in intron sequences which affected transcript splicing (Piffanelli et al., 2002) and resulted in one transcript containing an 18-nucleotide deletion of exon 12 and another containing the entire unspliced intron 11.

Therefore, although not common, the mutation observed in the M200 mutant is not an exception. 
The fact that the mutation occurred homozygously in an $M_{1}$ plant, can also lead to the hypothesis that it spontaneously occurred. Spontaneous mutations in Arabidopsis are known to take place at a rate of $10^{-7}$ to $10^{-8} \mathrm{bp} / \mathrm{generation}$ (Kovalchuk et al., 2000; Bashir et al., 2014). Anyway, it is reported that the large majority of spontaneous mutations are G/ $\mathrm{C} \rightarrow \mathrm{A} / \mathrm{T}$ transitions (Ossowski et al., 2009).

In tomato, the ol-2 is a natural mutation originated from the wild accession LA-1230 of S. lycopersicum var. cerasiforme (cherry tomato) caused by a 19-bp deletion in corresponding of the $7^{\text {th }}$ exon which determines a truncated protein (Ciccarese et al., 1998; Bai et al., 2008). The molecular reasons behind this deletion were not investigated, although it would be interesting to know what kind of mutation triggered the deletion and if it is also one of the most frequent spontaneous transitions.

TABLE 3. EMS mlo null alleles reported in literature.

Mutations corresponding to base transition are indicated by bold characters.

\begin{tabular}{|c|c|c|c|c|c|c|}
\hline \multirow{2}{*}{$\begin{array}{l}\text { Plant } \\
\text { species }\end{array}$} & \multirow{2}{*}{$\begin{array}{l}\text { Gene } \\
\text { name }\end{array}$} & \multirow{2}{*}{$\begin{array}{l}\text { Allele } \\
\text { name }\end{array}$} & \multicolumn{2}{|c|}{ SNP } & \multirow{2}{*}{$\begin{array}{c}\text { Effect on } \\
\text { transcript/protein }\end{array}$} & \multirow{2}{*}{ Reference } \\
\hline & & & WT & Mutant & & \\
\hline \multirow{2}{*}{ Petunia } & \multirow{2}{*}{ PhMLO1 } & n.a. & G & $A$ & S130L & \multirow{2}{*}{ Jiang et al., 2016} \\
\hline & & n.a. & $\mathrm{C}$ & $\mathrm{T}$ & G176E & \\
\hline \multirow{11}{*}{ Barley } & \multirow{11}{*}{ HvMlo1 } & mlo-5 & $A$ & G & M1I & \multirow{11}{*}{ Reinstdler et al., 2010} \\
\hline & & mlo-6 & $\mathrm{G}$ & $A$ & aberrant splicing variants & \\
\hline & & mlo-7 & G & $A$ & G226D & \\
\hline & & mlo-8 & A & G & M1V & \\
\hline & & mlo-9 & $C$ & $\mathrm{~T}$ & R10W & \\
\hline & & mlo-13 & $\mathbf{T}$ & A & V30E & \\
\hline & & mlo-16 & G & $A$ & aberrant splicing variants & \\
\hline & & mlo-17 & $\mathrm{C}$ & $\mathrm{T}$ & S31F & \\
\hline & & mlo-26 & $\mathbf{T}$ & A & $\mathrm{L} 270 \mathrm{H}$ & \\
\hline & & mlo-27 & G & $A$ & G318E & \\
\hline & & mlo-30 & A & $\mathbf{T}$ & aberrant splicing variants & \\
\hline \multirow{16}{*}{ Wheat } & \multirow{16}{*}{ TaMLO } & TaMLO-A1 & $C$ & $\mathrm{~T}$ & P325L & \multirow{16}{*}{$\begin{array}{c}\text { Acevedo-Garcia et al., } \\
2016\end{array}$} \\
\hline & & TaMLO-A1 & C & $\mathrm{T}$ & A354V & \\
\hline & & TaMLO-B1 & $\mathrm{G}$ & A & G296E & \\
\hline & & TaMLO-B1 & $C$ & $T$ & T297I & \\
\hline & & TaMLO-B1 & $C$ & $T$ & R313W & \\
\hline & & TaMLO-B1 & G & A & S315N & \\
\hline & & TaMLO-B1 & $\mathrm{G}$ & A & G319R & \\
\hline & & TaMLO-B1 & G & A & А320T & \\
\hline & & TaMLO-B1 & $C$ & $\mathrm{~T}$ & T345M & \\
\hline & & TaMLO-D1 & $\mathrm{G}$ & $A$ & V316T & \\
\hline & & TaMLO-D1 & G & $A$ & G319R & \\
\hline & & TaMLO-D1 & G & A & А320T & \\
\hline & & TaMLO-D1 & $C$ & $\mathrm{~T}$ & P321S & \\
\hline & & TaMLO-D1 & $\mathrm{G}$ & A & V323I & \\
\hline & & TaMLO-D1 & $C$ & $\mathrm{~T}$ & P335L & \\
\hline & & TaMLO-D1 & $C$ & $\mathrm{~T}$ & T345M & \\
\hline
\end{tabular}




\section{Is the level of $\mathbf{m l o}$-based resistance influenced by the position of the mutation?}

We initially assumed that the cause of the higher resistance of the $m 200$ plant is the severe truncation of this allele because $m 200$ plants are always free of any PM symptoms while ol-2 plants (at least in SM background) can occasionally show faint mycelium on $1^{\text {st }}$ and $2^{\text {nd }}$ leaves.

After reviewing the available literature on barley mlo-mutants, we found three interesting cases, namely mlo-13, mlo-17 and mlo-32 (Supplementary Fig. 2). The first two were obtained from cv Plena after EMS treatment; the last was obtained from cv Prudentia with sodium azaide $\left(\mathrm{NaN}_{3}\right.$ ) (Büschges et al., 1997; Molina-Cano et al., 2003; Panstruga et al., 2005). All three mutants carry mutations leading to a stop codon in the first transmembrane of the HvMlo protein, which corresponds to the same domain where the $m 200$ mutation is found. No difference in level of resistance was observed between mlo-13 or mlo-17 and mlo-32. They were all indicated as complete resistant mutants.

Moreover, another barley mutant, the mlo-43, was found to carry a stop codon in the second intracellular domain, the same as the nonsense mutation identified in tomato ol-2 mutant (Reinstädler et al., 2010). The mlo-43 is a mutant of the cv Bonus and it was also described as completely resistant (Lundqvist et al., 1991).

A mutant of the same cultivar, mlo-36, was described to contain a nonsense mutation at W357, in the sixth transmembrane domain (Supplementary Fig. 2, Lundqvist et al., 1991; Reinstädler et al., 2010). Both mutants were only phenotypically scored, and considered highly resistant, with mlo-36 even annotated as immune (Lundqvist et al., 1991).

We have not found other more recent evaluations of barley impaired alleles due to premature protein truncation. The reason is that it was shown that defective protein variants would probably not pass the quality test of the ERAD machinery (Endoplasmic Reticulum-Asssociated protein Degradation, Müller et al., 2005). The ER-localized quality control system monitors and validates proper folding and modification of proteins, among which the membrane proteins. Though it is currently largely unknown which signatures classify malformed membrane proteins, Muller et al. 2005 and Reinstadler et al. 2010 hypothesized that the second cytoplasmic loop and the transmembrane regions are the major quality determinant of the HvMlo protein variants. Premature truncations heavily affect protein folding, therefore, mutants containing amino acid substitutions were preferred to truncated mlo alleles in studies addressed at evaluating the biological activity of the Mlo variant.

If this holds true, the extremely truncated m200 protein, as well as the ol- 2 variant, should be subjected to a dramatic reduction in accumulation. Thus, both variants should lead to a similar level of resistance, if compared in the same background. 
A verification of this hypothesis in tomato would require more advanced crosses using as a recurrent parent the $\mathrm{cv}$ MM to observe the contribution to the resistance of each impaired mlo alleles in the same background.

Alternatively, it is possible to exploit the results described in Appiano et al., 2015 to select predicted amino acid positions that, being under negative selection, can represent targets of protein loss-of-function. Using one of the recent genome editing methods, mutants with predicted protein truncations at different level of the SIMLO1 protein could be systematically obtained. In this way, we would have a larger panel of MM mutants to understand the influence of several degrees of truncation on the resistance level.

\section{Materials and methods}

\section{Development of the Micro-Tom EMS populations}

Approximately 1000 seeds $\left(M_{0}\right)$ of the tomato cultivar Micro-Tom (MT), obtained from the Beekenkamp Plants B.V. company (The Netherlands), were pre-soaked in distilled water for 8 hours and treated overnight with two concentrations of an EMS (ethyl methane sulfonate) solution, $0.5 \%(\mathrm{v} / \mathrm{v})$ and $1 \%(\mathrm{v} / \mathrm{v})$, respectively.

In total, five rounds of EMS treatment were performed. In the first round, two different concentrations $(0.5 \% \mathrm{v} / \mathrm{v}$ and $1 \% \mathrm{v} / \mathrm{v})$ of EMS were tested. Several studies showed that the $1 \%$ concentration yielded almost 2 -fold more mutations per genome than other concentrations, like $0.5 \%$ or $0.75 \%$, without affecting too much the rate of viability (Minoia et al., 2010; Saito et al., 2011). Therefore, in the following rounds only the $1 \% \mathrm{v} / \mathrm{v}$ dilution was used.

The obtained $M_{1}$ seeds were then thoroughly washed with distilled water, dried, sown in the greenhouse and grown under standard agricultural practice. Three-weeks-old seedlings were transplanted individually to $14 \mathrm{~cm}$ pots and grown until 5 to 10 fruits per plant could be harvested. The $\mathrm{M}_{2}$ seeds, collected from these fruits, were surface sterilized in $2 \%(\mathrm{v} / \mathrm{v})$ of $\mathrm{HCl}$, following by air drying.

\section{Disease tests set-up and evaluation of the symptoms}

The approximately $1000 \mathrm{M}_{1}$ plants of the first round of EMS treatment were inoculated by spraying a fresh suspension of Oidium neolycopersici (On-Wageningen isolate) spores, after being transplanted into individual pots.

The suspension was made by rinsing heavily sporulating leaves of the cultivar MM with tap water and adjusting this suspension to a concentration of $2 \times 10^{4}$ spores per milliliter. The On isolate was maintained on the cv MM as previously described by (Bai et al., 2005). 
Ten to fifteen days after the inoculation, the $M_{1}$ plants were visually inspected. To each plant, a score was given based on a disease index (DI) varying from 0 to 3 , where 0 indicates that no fungal sporulation is visible and three that fungal colonies cover most of the surface of the inoculated leaves, as in the cv. MM.

One $M_{1}$ plant showing resistance to powdery mildew (M200) was crossed with Moneymaker (MM) and backcrossed to $M T$ to obtain $B C_{1}$ seeds which were harvested from each fruit and kept separately. Three BC deriving from the cross M200 x MT were tested with powdery mildew and all the plants of each family kept for self-pollination and seed production. Two of the three corresponding progenies $\left(B C_{1} S_{1}\right)$ were further tested with powdery mildew and selected for seed production if showing a resistant phenotype.

The $\mathrm{BC}_{1}$ plants deriving from the cross M200 x MM, selected for the loss of the dwarf and determinate growth characteristics of cultivar Micro-Tom, were allowed to self-pollinate. Their progenies $\left(\mathrm{BC}_{1} \mathrm{~S}_{1}\right)$, tested with powdery mildew, were selected if, next to being $\mathrm{MM}$ like in their appearance, were homozygous for the $m 200$ allele.

The disease test and the visual inspection of further generations were performed as for the $\mathrm{M}_{1}$ plants.

\section{Cloning and sequencing of the SIMLO1 coding sequence from the mutagenized resistant Micro-Tom plant $\mathrm{M} 200$}

Leaves of the M200 plant and two MT plants (not subjected to the EMS treatment) were collected after the powdery mildew test and total RNA was isolated with the RNeasy ${ }^{\circledR}$ plant mini kit (Qiagen) according to manufacturer's instructions. The concentration of the total RNA was measured using the Nanodrop. Approximately $1 \mu \mathrm{g}$ of RNA was treated with DNase (Invitrogen) to remove any DNA contamination. This treated RNA was used in a one-step PCR with the SuperScript ${ }^{\circledR}$ III (Invitrogen) and the specific primers for the SIMLO1 gene used in Zheng et al., 2016 (sequences in supplementary Table 4). The amplified PCR products were run on a $1 \%$ agarose gel. The bands with the desired product size (1743 bp) were excised from the gel and the products recovered using the QIAquick gel extraction kit (Qiagen). The eluted PCR products were sequenced and the obtained sequences aligned with the known SIMLO1 coding sequence (cds) of Heinz (Solyc04g049090) using the package MegAlign of the software DNASTAR ${ }^{\circ}$ Lasergene8. The predicted protein derived from the SIMLO1 sequence cloned from the M200 plant was analyzed using the TMHMM software (http://www.cbs.dtu.dk/services/TMHMM/) and the PROTTER web-tool to predicted sequence features and visualize the protein (Omasits et al., 2014). 


\section{Development of an HRM marker for detection of the mutation in the SIMLO1 gene}

In order to follow the segregation of the SNP associated with the $m 200$ allele in $\mathrm{BC}_{1}$ and $\mathrm{BC}_{1} \mathrm{~S}_{1}$ progenies of the backcrosses and crosses between the M200 plant $x$ MT and M200 plant $x$ MM, the DNA of each plant was isolated using $2 \%$ CTAB in a protocol adapted for a 96-well plate (Doyle, 1987). The quantity and integrity of genomic DNA were determined using the Nanodrop and running $1 \mu \mathrm{l}$ of the isolated DNA on an agarose gel (1\%), respectively.

Primers amplifying a DNA fragment of 225 bp containing the mutation site were designed for a high-resolution melting assay (HRM). The sequences of these primers are reported in the Supplementary Table 4.

PCR amplifications were carried out in a $10 \mu \mathrm{L}$ reaction mixture containing $10 \mathrm{ng}$ of genomic DNA, $2 \mu \mathrm{L}$ of $5 \mathrm{X}$ PCR buffer, $0.4 \mu \mathrm{L}$ of $5 \mathrm{mM}$ dNTPs, $0.5 \cup$ Phire ${ }^{\mathrm{TM}}$ Hot Start II DNA Polymerase (ThermoFisher), $0.25 \mu \mathrm{M}$ of forward and reverse primer (10 mM each) and $1 \mu \mathrm{L}$ of LC Green ${ }^{\text {Plus }}$ (Idaho technology inc., Salt Lake City, Utah, USA). The amplification included an initial denaturation at $98^{\circ} \mathrm{C}$ for $30 \mathrm{~s}$, followed by 41 cycles of $98^{\circ} \mathrm{C}$ for $5 \mathrm{~s}, 60^{\circ} \mathrm{C}$ for $5 \mathrm{~s}$ and $72{ }^{\circ} \mathrm{C}$ for $15 \mathrm{~s}$, and finishing with a final elongation at $72{ }^{\circ} \mathrm{C}$ for $30 \mathrm{~s}$.

The HRM genotyping was performed on a Light Scanner instrument (HR96 model, Idaho technology Inc., Salt Lake City, Utah, USA) with continuous melting curve acquisition (10 acquisitions per ${ }^{\circ} \mathrm{C}$ ) during a $0.1^{\circ} \mathrm{C} / \mathrm{s}$ ramp from 40 to $95^{\circ} \mathrm{C}$.

Data were retrieved and analyzed using the Light Scanner software followed by manual curation of the obtained genotype calls.

\section{Experimental set-up for histological study}

In order to study the resistance mechanism of the $m 200$ allele, an experiment for microscopic analysis was set up.

Eight $\mathrm{BC}_{1} \mathrm{~S}_{3}$ plants carrying the $\mathrm{m} 200$ allele derived from two backcrosses M200 x MM were chosen for this experiment. We also included three plants of two $\mathrm{BC}_{3} \mathrm{~S}_{2}$ lines derived from a cross between a resistant plant homozygous for the ol-2 allele and $\mathrm{MM}$ (Ciccarese et al., 1998; Zheng et al., 2016). Moreover, we added three resistant $F_{4}$ plants also carrying the ol-2 allele derived from the self-pollination of the cross between the original line LC-95 of S. lycopersicum var. cerasiforme and the cv Super Marmande (SM). For simplicity during the description of Figures and Tables, we refer to the first ol-2 genotype as ol-2_MM and the second as ol-2_SM. Furthermore, three transgenic plants of a $T_{2}$ family carrying the RNAi construct able to silence SIMLO1, SIMLO5, and SIMLO8 as described in Zheng et al., 2016 were selected. As susceptible control, three MM plants were included in this experiment. The transgenic plants carrying the RNAi construct were selected by standard 
PCR performed on DNA isolated with the $2 \%$ CTAB method (described above) from all the germinated seedlings, using two primer pairs, one targeting the NPTII gene and the other the 35 S promoter. Primer pair sequences are reported in Supplementary Table 4.

The powdery mildew disease assay was performed on four-weeks-old plants as described in the previous paragraph, but using a higher concentration of On spores equal to $3 \times 10^{5}$ conidia / $\mathrm{ml}$. From the sowing to the sampling, plants were grown in a climate-controlled compartment with $16 \mathrm{~h}$ light $/ 21^{\circ} \mathrm{C}, 8 \mathrm{~h}$ dark/ $19^{\circ} \mathrm{C}$ and $60 \%-70 \%$ relative humidity.

Four samples were collected $72 \mathrm{~h}$ post inoculation, bleached in a 1:3 (v/v) acetic acid/ ethanol solution, stained $48 \mathrm{~h}$ later by boiling in $0.005 \%$ trypan blue in lactophenol : ethanol (1:2 v/v) solution for 3-5 min and finally cleared in a nearly saturated aqueous solution of chloral hydrate $(5: 2 \mathrm{w} / \mathrm{v})$. We defined an infection unit (IU) as a spore with a germination tube. About $100 \mathrm{IU}$ per genotype were randomly counted at $65 \mathrm{X}$ magnification using a Zeiss Axiophot bright field microscope. For each IU, the presence of appressorium, haustorium, papilla, and the number of secondary hyphae was recorded. For some IU, photos were taken using the 100x magnification coupled with the differential interface contrast (DIC) technique at different focus to be able to observe all of the fungal structures eventually developed. 


\section{References}

Acevedo-Garcia, J., Spencer, D., Thieron, H., Reinstädler, A., Hammond-Kosack, K., Phillips, A. L., \& Panstruga, R. (2016). mlo-based powdery mildew resistance in hexaploid bread wheat generated by a nontransgenic TILLING approach. Plant Biotechnology Journal, n/a-n/a. doi: 10.1111/pbi.12631

Aflitos, S., Schijlen , E., de Jong, H., de Ridder, D., Smit, S., Finkers, R., Wang, J., Zhang, G., Li, N., Mao, L., Bakker, F., Dirks, R., Breit, T., Gravendeel, B., Huits, H., Strus,s D., Swanson-Wagner, R., van Leeuwen, H., van Ham, RC., Fito, L., Guignier, L., Sevilla, M., Ellul, P., Ganko, E., Kapur, A., Reclus, E., de Geus, B., van de Geest, H., Te Lintel, Hekkert, B., van Haarst, J., Smits, L., Koops, A., Sanchez-Perez, G., van Heusden, AW., Visser, R., Quan, Z., Min, J., Liao, L., Wang, X., Wang, G., Yue, Z., Yang, X., Xu N., Schranz, E., Smets, E., Vos, R., Rauwerda, J., Ursem, R., Schuit, C., Kerns, M., van den Berg, J., Vriezen, W., Janssen, A., Datema, E., Jahrman, T., Moquet, F., Bonne,t J., Peters, S. (2014). Exploring genetic variation in the tomato (Solanum section Lycopersicon) clade by whole-genome sequencing. Plant J. 80(1): 136-148

Appiano, M., Catalano, D., Santillán Martínez, M., Lotti, C., Zheng, Z., Visser, R. G. F., Ricciardi, L., Bai, Y., Pavan, S. (2015). Monocot and dicot MLO powdery mildew susceptibility factors are functionally conserved in spite of the evolution of class-specific molecular features. BMC Plant Biology, 15(1), 1-10. doi: 10.1186/s12870-015-0639-6

Bai, Y., \& Lindhout, P. (2007). Domestication and Breeding of Tomatoes: What have We Gained and What Can We Gain in the Future? Annals of Botany, 100(5), 1085-1094.

Bai, Y., Pavan, S., Zheng, Z., Zappel, N. F., Reinstädler, A., Lotti, C., De Giovanni, C., Ricciardi, L., Lindhout, P., Visser, L., Theres, K., Panstruga, R. (2008). Naturally occurring broad-spectrum powdery mildew resistance in a Central American tomato accession is caused by loss of Mlo function. Molecular Plant-Microbe Interactions, 21(1), 30-39.

Bai, Y., van der Hulst, R., Bonnema, G., Marcel, T. C., Meijer-Dekens, F., Niks, R. E., \& Lindhout, P. (2005). Tomato Defense to Oldium neolycopersici: Dominant OI Genes Confer Isolate-Dependent Resistance Via a Different Mechanism Than Recessive ol-2. Molecular Plant-Microbe Interactions, 18(4), 354-362. doi: 10.1094/MPMI-18-0354

Barham WS, Winstead NN, 1957. Inheritance of resistance to root-knot nematodes in tomatoes. Proceedings of the American Society for Horticultural Sciences 69, 372-7

Bashir, T., Sailer, C., Gerber, F., Loganathan, N., Bhoopalan, H., Eichenberger, Baskar, R. (2014). Hybridization Alters Spontaneous Mutation Rates in a Parent-of-Origin-Dependent Fashion in Arabidopsis. Plant Physiology, 165(1), 424-437.

Bolger, A., Scossa, F., Bolger, M. E., Lanz, C., Maumus, F., Tohge, T., Fernie, A. R. (2014). The genome of the stresstolerant wild tomato species Solanum pennellii. Nat Genet, 46(9), 1034-1038. doi: 10.1038/ng.3046

Brooks, C., Nekrasov, V., Lippman, Z. B., \& Van Eck, J. (2014). Efficient Gene Editing in Tomato in the First Generation Using the Clustered Regularly Interspaced Short Palindromic Repeats/CRISPRAssociated9 System. Plant Physiology, 166(3), 1292-1297.

Brown, J. K. M. (2015). Durable Resistance of Crops to Disease: A Darwinian Perspective. Annual Review of Phytopathology, 53(1), 513-539. doi: 10.1146/annurev-phyto-102313-045914

Büschges, R., Hollricher, K., Panstruga, R., Simons, G., Wolter, M., Frijters, A., Schulze-Lefert, P. (1997). The barley Mlo gene: A novel control element of plant pathogen resistance. Cell, 88(5), 695-705.

Chunwongse, J., Chunwongse, C., Black, L., \& Hanson, P. (2002). Molecular mapping of the Ph-3 gene for late blight resistance in tomato. The Journal of Horticultural Science and Biotechnology, 77(3), 281-286. doi: 10.1080/14620316.2002.11511493

Ciccarese, F., Amenduni, M., Schiavone, D., \& Cirulli, M. (1998). Occurrence and inheritance of resistance to powdery mildew (Oidium lycopersici) in Lycopersicon species. Plant Pathology, 47(4), 417-419. doi: 10.1046/j.1365-3059.1998.00254.x

De Giovanni, C., Dell'Orco, P., Bruno, A., Ciccarese, F., Lotti, C., \& Ricciardi, L. (2004). Identification of PCR-based markers (RAPD, AFLP) linked to a novel powdery mildew resistance gene (ol-2) in tomato. Plant Science, 166(1), 41-48. doi: http://dx.doi.org/10.1016/j.plantsci.2003.07.005 
De Jong, M., Wolters-Arts, M., Feron, R., Mariani, C., \& Vriezen, W. H. (2009). The Solanum lycopersicum auxin response factor $\mathbf{7}$ (SIARF7) regulates auxin signaling during tomato fruit set and development. The Plant Journal, 57(1), 160-170. doi: 10.1111/j.1365-313X.2008.03671.x

Doyle, J. J. (1987). A rapid DNA isolation procedure for small quantities of fresh leaf tissue. Phytochem Bull, 19, 11-15.

Feechan, A., Jermakow, A. M., Torregrosa, L., Panstruga, R., \& Dry, I. B. (2008). Identification of grapevine MLO gene candidates involved in susceptibility to powdery mildew. Functional Plant Biology, 35(12), 1255-1266.

Greene, E. A., Codomo, C. A., Taylor, N. E., Henikoff, J. G., Till, B. J., Reynolds, S. H., Henikoff, S. (2003). Spectrum of Chemically Induced Mutations From a Large-Scale Reverse-Genetic Screen in Arabidopsis. Genetics, 164(2), 731.

Griffiths, A.J.F., Miller, J.H., Suzuki, D.T., et al. An Introduction to Genetic Analysis. 7th edition. New York: W. H. Freeman; 2000. Induced mutations. Available from: http://www.ncbi.nlm.nih.gov/books/NBK21936/

Horst, R. K. (2013). Powdery Mildews. In K. R. Horst (Ed.), Westcott's Plant Disease Handbook (pp. 285-293). Dordrecht: Springer Netherlands.

Hückelhoven, R. (2005). Powdery mildew susceptibility and biotrophic infection strategies. FEMS Microbiology Letters, 245(1), 9-17. doi: 10.1016/j.femsle.2005.03.001

Ji, Y., Scott, J. W., Hanson, P., Graham, E., \& Maxwell, D. P. (2007). Sources of Resistance, Inheritance, and Location of Genetic Loci Conferring Resistance to Members of the Tomato-Infecting Begomoviruses. In H. Czosnek (Ed.), Tomato Yellow Leaf Curl Virus Disease: Management, Molecular Biology, Breeding for Resistance (pp. 343-362). Dordrecht: Springer Netherlands.

Jiang, P., Chen, Y., \& Wilde, H. D. (2016). Identification and mutagenesis of disease susceptibility genes of Petunia hybrida. Plant Cell, Tissue and Organ Culture (PCTOC), 126(1), 117-125. doi: 10.1007/s11240016-0982-9

Jørgensen, I. H. (1992). Discovery, characterization and exploitation of Mlo powdery mildew resistance in barley. Euphytica, 63(1-2), 141-152. doi: 10.1007/BF00023919

Jørgensen, J. H. (1971). Comparison of induced mutant genes to spontaneous genes in barley conditioning resistance to powdery mildew (pp. 117-124): International Atomic Energy Agency. IAEA, Publications, Sales and Promotion Unit.

Kim, M. C., Panstruga, R., Elliott, C., Muller, J., Devoto, A., Yoon, H. W., Schulze-Lefert, P. (2002). Calmodulin interacts with MLO protein to regulate defence against mildew in barley. Nature, 416(6879), 447451.

Kissoudis, C., van de Wiel, C., Visser, R. G. F., \& van der Linden, G. (2016). Future-proof crops: challenges and strategies for climate resilience improvement. Current Opinion in Plant Biology, 30, 47-56. doi: http:// dx.doi.org/10.1016/j.pbi.2016.01.005

Kovalchuk, I., Kovalchuk, O., \& Hohn, B. (2000). Genome-wide variation of the somatic mutation frequency in transgenic plants. The EMBO Journal, 19(17), 4431.

Kusch, S., Pesch, L., \& Panstruga, R. (2016). Comprehensive Phylogenetic Analysis Sheds Light on the Diversity and Origin of the MLO Family of Integral Membrane Proteins. Genome Biology and Evolution, 8(3), 878-895.

Lanfermeijer, F. C., Warmink, J., \& Hille, J. (2005). The products of the broken Tm-2 and the durable Tm-22 resistance genes from tomato differ in four amino acids. Journal of Experimental Botany, 56(421), 2925-2933.

Lor, V. S., Starker, C. G., Voytas, D. F., Weiss, D., \& Olszewski, N. E. (2014). Targeted Mutagenesis of the Tomato PROCERA Gene Using Transcription Activator-Like Effector Nucleases. Plant Physiology, 166(3), 1288-1291.

Lundqvist, U., 1991. Swedish mutation research in barley with plant breeding aspects. A historical review. In: Plant Mutation Breeding for Crop Improvement, IAEA-SM-311/25, pp. 135-147.

Meissner, R., Jacobson, Y., Melamed, S., Levyatuv, S., Shalev, G., Ashri, A., Levy, A. (1997). A new model system for tomato genetics. The Plant Journal, 12(6), 1465-1472. doi: 10.1046/j.1365-313x.1997.12061465.x 
Menda, N., Strickler, S. R., Edwards, J. D., Bombarely, A., Dunham, D. M., Martin, G. B., Mueller, L. A. (2014). Analysis of wild-species introgressions in tomato inbreds uncovers ancestral origins. BMC Plant Biology, 14(1), 1-16. doi: 10.1186/s12870-014-0287-2

Minoia, S., Petrozza, A., D'Onofrio, O., Piron, F., Mosca, G., Sozio, G., Carriero, F. (2010). A new mutant genetic resource for tomato crop improvement by TILLING technology. BMC Research Notes, 3, 69-69. doi: 10.1186/1756-0500-3-69

Moco, S., Bino, R. J., Vorst, O., Verhoeven, H. A., de Groot, J., van Beek, T. A., de Vos, C. H. R. (2006). A Liquid Chromatography-Mass Spectrometry-Based Metabolome Database for Tomato. Plant Physiology, 141(4), 1205-1218. doi: 10.1104/pp.106.078428

Molina-Cano, J. L., Simiand, J. P., Sopena, A., Pérez-Vendrell, A. M., Dorsch, S., Rubiales, D., Jahoor, A. (2003). Mildew-resistant mutants induced in North American two- and six-rowed malting barley cultivars. Theoretical and Applied Genetics, 107(7), 1278-1287. doi: 10.1007/s00122-003-1362-5

Müller, J., Piffanelli, P., Devoto, A., Miklis, M., Elliott, C., Ortmann, B., Panstruga, R. (2005). Conserved ERAD-like quality control of a plant polytopic membrane protein. Plant Cell. 17(1), 149-163.

Oladosu, Y., Rafii, M. Y., Abdullah, N., Hussin, G., Ramli, A., Rahim, H. A., Usman, M. (2016). Principle and application of plant mutagenesis in crop improvement: a review. Biotechnology \& Biotechnological Equipment, 30(1), 1-16. doi: 10.1080/13102818.2015.1087333

Omasits, U., Ahrens, C. H., Müller, S., \& Wollscheid, B. (2014). Protter: interactive protein feature visualization and integration with experimental proteomic data. Bioinformatics, 30(6), 884-886.

Ossowski, S., Schneeberger, K., Lucas-Lledó, J. I., Warthmann, N., Clark, R. M., Shaw, R. G., Lynch, M. (2009). The Rate and Molecular Spectrum of Spontaneous Mutations in Arabidopsis thaliana. Science, $327(5961), 92$.

Panstruga, R. (2005). Serpentine plant MLO proteins as entry portals for powdery mildew fungi. Biochemical Society Transactions, 33(2), 389-392.

Panstruga, R., Molina-Cano, J. L., Reinstädler, A., \& Müller, J. (2005). Molecular characterization of mlo mutants in North American two- and six-rowed malting barley cultivars. Molecular Plant Pathology. 6(3), 315-320.

Parniske, M., Wulff, B. B. H., Bonnema, G., Thomas, C. M., Jones, D. A., \& Jones, J. D. G. (1999). Homologues of the Cf-9 Disease Resistance Gene (Hcr9s) Are Present at Multiple Loci on the Short Arm of Tomato Chromosome 1. Molecular Plant-Microbe Interactions, 12(2), 93-102. doi: 10.1094/MPMI.1999.12.2.93

Pavan, S., Jacobsen, E., Visser, R. G. F., \& Bai, Y. (2010). Loss of susceptibility as a novel breeding strategy for durable and broad-spectrum resistance. Molecular Breeding, 25(1), 1-12.

Pavan, S., Zheng, Z., Borisova, M., Van Den Berg, P., Lotti, C., De Giovanni, C., Bai, Y. (2008). Map- vs. homologybased cloning for the recessive gene ol-2 conferring resistance to tomato powdery mildew. Euphytica, 162(1), 91-98.

Piffanelli, P., Zhou, F., Casais, C., Orme, J., Jarosch, B., Schaffrath, U., Schulze-Lefert, P. (2002). The barley MLO modulator of defense and cell death is responsive to biotic and abiotic stress stimuli. Plant Physiology. 129(3), 1076-1085.

Reinstädler, A., Müller, J., Czembor, J. H., Piffanelli, P., \& Panstruga, R. (2010). Novel induced mlo mutant alleles in combination with site-directed mutagenesis reveal functionally important domains in the heptahelical barley Mlo protein. BMC Plant Biology, 10.

Rodríguez, G. R., Muños, S., Anderson, C., Sim, S.-C., Michel, A., Causse, M., van der Knaap, E. (2011). Distribution of SUN, OVATE, LC, and FAS in the Tomato Germplasm and the Relationship to Fruit Shape Diversity. Plant Physiology, 156(1), 275-285.

Ron, M., Kajala, K., Pauluzzi, G., Wang, D., Reynoso, M. A., Zumstein, K., Brady, S. M. (2014). Hairy Root Transformation Using Agrobacterium rhizogenes as a Tool for Exploring Cell Type-Specific Gene Expression and Function Using Tomato as a Model. Plant Physiology, 166(2), 455-469.

Saito, T., Ariizumi, T., Okabe, Y., Asamizu, E., Hiwasa-Tanase, K., Fukuda, N., Ezura, H. (2011). TOMATOMA: A Novel Tomato Mutant Database Distributing Micro-Tom Mutant Collections. Plant and Cell Physiology, 52(2), 283-296. doi: 10.1093/pcp/pcr004 
Sato, S., Tabata, S., Hirakawa, H., Asamizu, E., Shirasawa, K., Isobe, S., Gianese, G., The tomato genome consortium (2012). The tomato genome sequence provides insights into fleshy fruit evolution. Nature, 485(7400), 635-641.

Schijlen, E. G. W. M., de Vos, C. H. R., Martens, S., Jonker, H. H., Rosin, F. M., Molthoff, J. W., Bovy, A. G. (2007). RNA Interference Silencing of Chalcone Synthase, the First Step in the Flavonoid Biosynthesis Pathway, Leads to Parthenocarpic Tomato Fruits. Plant Physiology, 144(3), 1520-1530.

Scott, J. W., \& Harbaugh, B. K. (1989). Micro-tom : a miniature dwarf tomato. Gainesville, Fla.: Agricultural Experiment Station, Institute of Food and Agricultural Sciences, University of Florida.

Seah, S., Yaghoobi, J., Rossi, M., Gleason, C. A., \& Williamson, V. M. (2004). The nematode-resistance gene, Mi1 , is associated with an inverted chromosomal segment in susceptible compared to resistant tomato. Theoretical and Applied Genetics, 108(8), 1635-1642. doi: 10.1007/s00122-004-1594-z

Sega, G. A. (1984). A review of the genetic effects of ethyl methanesulfonate. Mutation Research/Reviews in Genetic Toxicology, 134(2), 113-142.

Shirasawa, K., Hirakawa, H., Nunome, T., Tabata, S., \& Isobe, S. (2016). Genome-wide survey of artificial mutations induced by ethyl methanesulfonate and gamma rays in tomato. Plant Biotechnology Journal, 14(1), 51-60. doi: 10.1111/pbi.12348

The 100 Tomato Genome Sequencing, C., Aflitos, S., Schijlen, E., de Jong, H., de Ridder, D., Smit, S., Peters, S. (2014). Exploring genetic variation in the tomato (Solanum section Lycopersicon) clade by wholegenome sequencing. The Plant Journal, 80(1), 136-148. doi: 10.1111/tpj.12616

Till, B. J., Cooper, J., Tai, T. H., Colowit, P., Greene, E. A., Henikoff, S., \& Comai, L. (2007). Discovery of chemically induced mutations in rice by TILLING. BMC Plant Biology, 7(1), 1-12. doi: 10.1186/1471-2229-7-19

Till, B. J., Reynolds, S. H., Weil, C., Springer, N., Burtner, C., Young, K., Henikoff, S. (2004). Discovery of induced point mutations in maize genes by TILLING. BMC Plant Biology, 4(1), 1-8. doi: 10.1186/1471-2229-4-12

van Schie, C. C. N., \& Takken, F. L. W. (2014). Susceptibility Genes 101: How to Be a Good Host. Annual Review of Phytopathology, 52(1), 551-581. doi: 10.1146/annurev-phyto-102313-045854

Xiong, A.-S., Yao, Q.-H., Peng, R.-H., Li, X., Han, P.-L., \& Fan, H.-Q. (2005). Different effects on ACC oxidase gene silencing triggered by RNA interference in transgenic tomato. Plant Cell Reports, 23(9), 639-646. doi: 10.1007/s00299-004-0887-7

Zheng, Z., Appiano, M., Pavan, S., Bracuto, V., Ricciardi, L., Visser, R. G. F., Wolters, A.M.A., Bai, Y. (2016). GenomeWide Study of the Tomato SIMLO Gene Family and Its Functional Characterization in Response to the Powdery Mildew Fungus Oidium neolycopersici. Frontiers in Plant Science, 7, 380. 


\section{Supplementary Tables}

SUPPLEMENTARY TABLE 1. Genotyping and phenotyping of three $B C_{1}$ populations derived from the cross between the resistant M200 plant and Micro-Tom (M200 x MT). High resolution Melting (HRM) profiles correspond to the ones reported in Figure 2, panel A.

\begin{tabular}{cccccc}
\hline & & \multicolumn{2}{c}{ HRM profiles } \\
\hline M200 x MT & N susceptible plants & N resistant plants & Wild-type allele & heterozygous & m200 allele \\
\hline $\mathrm{BC}_{1-4}$ & 12 & 0 & 0 & 12 & 0 \\
$\mathrm{BC}_{1-5}$ & 3 & 0 & 0 & 3 & 0 \\
$\mathrm{BC}_{1-6}$ & 4 & 0 & 0 & 4 & 0 \\
total & $\mathbf{1 9}$ & $\mathbf{0}$ & $\mathbf{0}$ & $\mathbf{1 9}$ & $\mathbf{0}$ \\
\hline
\end{tabular}

$\mathrm{N}=$ number

SUPPLEMENTARY TABLE 2. Genotyping and phenotyping of four progenies $\left(B C_{1} S_{1}\right)$ derived from two each of the three crosses M200 x MT listed in Supplementary Table 1.

\begin{tabular}{|c|c|c|c|c|c|c|c|}
\hline \multirow[b]{2}{*}{ M200 x MT } & \multirow[b]{2}{*}{$\begin{array}{c}\text { N susceptible } \\
\text { plants }\end{array}$} & \multirow[b]{2}{*}{$\begin{array}{c}\text { N resistant } \\
\text { plants }\end{array}$} & \multicolumn{2}{|c|}{ Expected 3S:1R } & \multicolumn{3}{|c|}{ HRM profiles } \\
\hline & & & $X^{2}$ & $\mathbf{p}$ & $\begin{array}{c}\text { Wild-type } \\
\text { allele }\end{array}$ & heterozygous & $\begin{array}{l}m 200 \\
\text { allele }\end{array}$ \\
\hline $\mathrm{BC}_{1} \mathrm{~S}_{1-4-1}$ & 10 & 6 & & & 2 & 8 & 6 \\
\hline $\mathrm{BC}_{1} \mathrm{~S}_{1-4-2}$ & 13 & 4 & & & 4 & 9 & 4 \\
\hline $\mathrm{BC}_{1} \mathrm{~S}_{1-4} 4-4$ & 13 & 6 & & & 3 & 10 & 6 \\
\hline$B C_{1} S_{1-4} 4-10$ & 12 & 7 & 2.07 & 0.15 & 7 & 4 & 7 \\
\hline $\mathrm{BC}_{1} \mathrm{~S}_{1-6-1}$ & 14 & 4 & & & 4 & 10 & 4 \\
\hline $\mathrm{BC}_{1} \mathrm{~S}_{1-} 6-6$ & 11 & 7 & & & 4 & 7 & 7 \\
\hline $\mathrm{BC}_{1} \mathrm{~S}_{1-} 6-7$ & 17 & 2 & & & 4 & 13 & 2 \\
\hline $\mathrm{BC}_{1} \mathrm{~S}_{1-6} 6-9$ & 11 & 8 & 0.45 & 0.50 & 2 & 9 & 8 \\
\hline
\end{tabular}

$\mathrm{S}=$ susceptible, $\mathrm{R}$ = resistant, $\mathrm{N}=$ number

Supplementary Table 3. Genotyping and phenotyping of the progenies $\left(B C_{1} S_{1}\right)$ of three crosses between the resistant M200 plant and the tomato cv Moneymaker (M200 x MM).

\begin{tabular}{ccccccccc}
\hline & & & Expected 3S:1R & \multicolumn{3}{c}{ HRM marker profiles } \\
\hline M200 x MM & $\begin{array}{c}\text { N susceptible } \\
\text { plants }\end{array}$ & $\begin{array}{c}\text { N resistant } \\
\text { plants }\end{array}$ & $\mathrm{X}^{\mathbf{2}}$ & $\mathbf{p}$ & $\begin{array}{c}\text { Wild-type } \\
\text { allele }\end{array}$ & heterozygous & $\begin{array}{c}\text { m200 } \\
\text { allele }\end{array}$ \\
\hline $\mathrm{BC}_{1} \mathrm{~S}_{1-1}$ & 25 & 14 & 2.47 & 0.12 & 9 & 16 & 14 \\
$\mathrm{BC}_{1} \mathrm{~S}_{1-2}$ & 27 & 11 & 0.32 & 0.57 & 11 & 16 & 11 \\
$\mathrm{BC}_{1} \mathrm{~S}_{1-3}$ & 25 & 14 & 2.47 & 0.12 & 5 & 19 & 14 \\
\hline
\end{tabular}

$\mathrm{S}=$ susceptible, $\mathrm{R}=$ resistant

SUPPLEMENTARY TABLE 4. Primer pairs used in this study

\begin{tabular}{ccc}
\hline Primer name & Fw primer $\left(\mathbf{5}^{\prime}\right.$...3') & Rev primer $\mathbf{( \mathbf { 5 } ^ { \prime } \text { ....3') }}$ \\
\hline SIMLO1_full-length & TTGACATTTCCCCTTCTTCTTA & TACAAAATCATTGCCATTTGAA \\
HRM_marker & TGGCTAAAGCACGGTCTA & CTGGATCTTGCAACACTGTCA \\
35S_promoter & GCTCCTACAAATGCCATCA & GATAGTGGGATTGTGCGTCA \\
NPTII_marker & TCGGCTATGACTGGGCACAAC & AAGAAGGCGATAGAAGGCGA \\
SIEf1a & ACAGGCGTTCAGGTAAGGAA & GAGGGTATTCAGCAAAGGTCTC \\
On_ITS & CGCCAAAGACCTAACCAAAA & AGCCAAGAGATCCGTTGTTG \\
\hline
\end{tabular}




\section{Supplementary Figures}

SIMLO1 MT SIMLO1 Heinz LEFL10 63 CA10 LEFL1037DE0 9

SIMLO1 MT SlMLO1 Heinz LEFL $10 \overline{6} 3 \mathrm{CA} 10$ LEFL1037DE09

SIMLO1 MT SlMLO1-Heinz LEFL10 63 CA 10 LEFL1037DE0 9

SIMLO1 MT SlMLO1_Heinz LEFL10 63CA10 LEFL1037DE09

SIMLO1 MT SIMLO1 Heinz LEFL10 63 CA10 LEFL1037DE09

SIMLO1 MT SlMLO1_Heinz LEFL1063CA10 LEFL1037DE09

SIMLO1 MT SIMLO1 Heinz LEFL10 63 CA10 LEFL1037DE09

SIMLO1 MT SlMLO1_Heinz LEFL10 63CA10 LEFL1037DE09

SIMLO1 MT SlMLO1 Heinz LEFL $10 \overline{6} 3 \mathrm{CA} 10$ LEFL1037DE0 9

SIMLO1 MT SlMLO1 ${ }^{-}$Heinz LEFL10 63CA10 LEFL1037DE09

SIMLO1 MT SIMLO1 Heinz LEFL $10 \overline{6} 3 \mathrm{CA} 10$ LEFL1037DE0 9

SIMLO1_MT SlMLO1 ${ }^{-}$Heinz LEFL1063CA10 LEFL1037DE09

SIMLO1 MT SlMLO1 Heinz LEFL $10 \overline{6} 3 \mathrm{CA} 10$ LEFL1037DE09

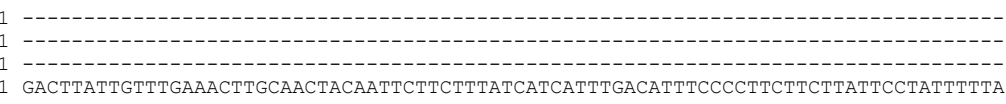

1 GACTTATTGTTTGAAACTTGCAACTACAATTCTTCTTTATCATCATTTGACATTTCCCCTTCTTCTTATTCCTATTTTTA

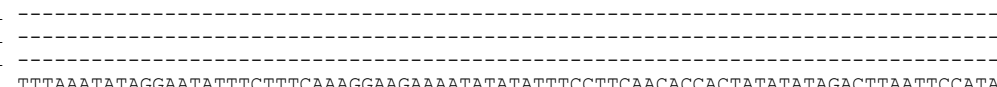

81 TTTAAATATAGGAATATTTCTTTCAAAGGAAgAAAATATATATTTCCTTCAACACCACTATATATAGACTTAATTCCATA

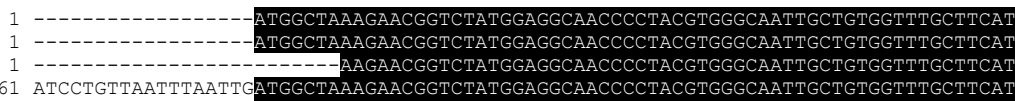

161 ATCCTGTTAATTTAATT

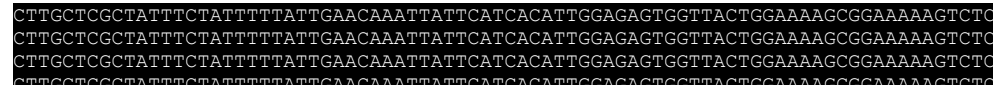

41 CTTGCTCGCTATTTCTATTTTTATTGAACAAATTATTCATCACATTGGAGAGTGGTTACTGGAAAAGCGGAAAAAGTCTC

ATATGAAGCACTTGAAAAGATCAAAGCTGAACTTATGCTGTTGGGATTCTTATCACTGTTGTTGACAGTGTTGCAAGA ATATGAAGCACTTGAAAAGATCAAAGCTGAACTTATGCTGTTGGGATTCTTATCACTGTTGTTGACAGTGTTGCAAGA ATATGAAGCACTTGAAAAGATCAAAGCTGAACTTATGCTGTTGGGATTCTTATCACTGTTGTTGACAGTGTTGCAAGA ATATGAAGCACTTGAAAAGATCAAAGCTGAACTTATGCTGTTGGGATTCTTATCACTGTTGTTGACAGTGTTGCAAGAT
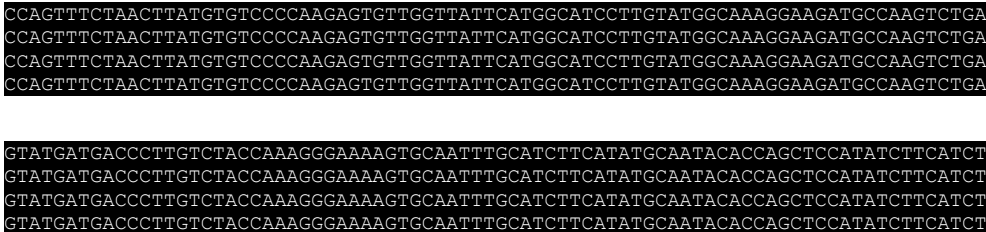
GTATGATGACCCTTGTCTACCAAAGGGAAAAGTGCAATTTGCATCTTCATATGCAATACACCAGCTCCATATCTTCATC

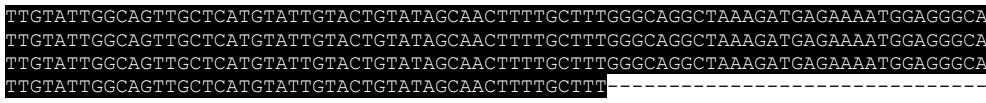

63
63
$6 \mathrm{TCCC}$
56 TGGGAGGATGAAACAAAAACAATGGAGTACCAATTCTACAACGACCCTGAGAGATTCAGATTTGCAAGGGAGACCTCGTT
TGGGAGGATGAAACAAAAACAATGGAGTACCAATTCTACAACGACCCTGAGAGATTCAGATTTGCAAGGGAGACCTCGTT
TGGGAGGATGAAACAAAAACAATGGAGTACCAATTCTACAACGACCCTGAGAGATTCAGATTTGCAAGGGAGACCTCGTT

\section{T} GGACGTAGGCATTTGCATTTCTGGAGCAAGTCCCCCGTGTTGCTCTCGATAGTTTGTTTCTTTCGGCAATTCTTCTCAT

623 CAGTTGCAAAAGTTGACTATTTAACCCTTAGACATGGGTTCATGATGGCACATTTAACTCCACAAAATCAAAATAATTTT 3 CAGTTGCAAAAGTTGACTATTTAACCCTTAGACATGGGTTCATGATGGCACATTTAACTCCACAAAATCAAAATAATTTT 616 CAGTTGC---

703 GATTTTCAATTATACATTAACAGAGCAGTTGACAAAGACTTCAAAGTTGTTGTTGGAATAAGTCCTGCATTATGGCTCTT 703 GATTTTCAATTATACATTAACAGAGCAGTTGACAAAGACTTCAAAGTTGTTGTTGGAATAAGTCCTGCATTATGGCTCTT G

783 CACGGTGCTATATTTTCTGACTACTACCGATCGATTGTACTCGTATCTTTGGGTGCCATTTATCCCACTTGTAATAATAT 783 CACGGTGCTATATTTTCTGACTACTACCGATCGATTGTACTCGTATCTTTGGGTGCCATTTATCCCACTTGTAATAATAT - -

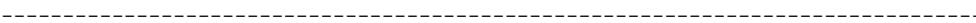

\section{SUPPLEMENTARY FIGURE 1.}


S1MLO1 MT

SlMLO1 Hein

LEFL10 $\overline{6} 3$ CA10

LEFL1037DE09
943 GTACCTGTGGTGGAGACTGGTGACCATCTTTTCTGGTTTAATCGCCCTGCCCTTGTCCTATTCTTGATTAACTTTGTACT

943 GTACCTGTGGTGGAGACTGGTGACCATCTTTTCTGGTTTAATCGCCCTGCCCTTGTCCTATTCTTGATTAACTTTGTACT

SIMLO1 MT 1023 CTTTCAGAATGCGTTTCAAGTTGCTTTCTTTTTTTGGAGTTGGTGGAAATTTGGTTTCCCATCTTGCTTTCATAAGAATG S1MLO1-Heinz 1023 CTTTCAGAATGCGTTTCAAGTTGCTTTCTTTTTTTGGAGTTGGTGGAAATTTGGTTTCCCATCTTGCTTTCATAAGAATG LEFL10 $\overline{6} 3 \mathrm{CA} 10$ LEFL1037DE09

SIMLO1 MT 1103 CTGCAGACCTAGCCATAAGGCTAACCATGGGGGTGATCATACAGGTCCATTGCAGCTATGTGACTCTCCCTCTTTATGCC SlMLO1_Heinz 1103 CTGCAGACCTAGCCATAAGGCTAACCATGGGGGTGATCATACAGGTCCATTGCAGCTATGTGACTCTCCCTCTTTATGCC LEFL10 $\overline{6} 3$ CA10 LEFL1037DE09

SIMLO1 MT 1183 TTAGTTACACAGATGGGTTCATCAATGAAGCCTATCATCTTTGGTGATAATGTGGCAACAGCTCTTAGAAGCTGGCACCA SlMLO1_Heinz 1183 TTAGTTACACAGATGGGTTCATCAATGAAGCCTATCATCTTTGGTGATAATGTGGCAACAGCTCTTAGAAGCTGGCACCA LEFL10 63 CA10 LEFL1037DE0 9

SIMLO1 MT 1263 TACAGCGAAAAAACGGGTGAAACATGGGCTATCAGGACATACCACCCCTGCAAACAGCAGACCAACCACACCATTGCGTG SlMLO1 ${ }^{-}$Heinz 1263 TACAGCGAAAAAACGGGTGAAACATGGGCTATCAGGACATACCACCCCTGCAAACAGCAGACCAACCACACCATTGCGTG LEFL1063CA10 LEFL1037DE09

SlMLO1_MT 1343 GTACCTCCCCTGTTCACTTATTACGCGGTTATCCACAATATAATGAGGACAGTGTTCAAGCATCTCCTCGGACATCCAAT SlMLO1 Heinz 1343 GTACCTCCCCTGTTCACTTATTACGCGGTTATCCACAATATAATGAGGACAGTGTTCAAGCATCTCCTCGGACATCCAAT TEFI GTACCTCCCIGT TCACTTATTACGCGGTIATCCACAATATAAGAGACAGTGTCAAGCATCTCCTCGGACATCAAT

S1MLO1 MT 1423 GTCGAAAATGAAGGGTGGGCTAATGAAAATCAGGAGGGAGAGATCCTGCAGCATGCCTCCACTGATCATAACAAGCAAAT SlMLO1-Heinz 1423 GTCGAAAATGAAGGGTGGGCTAATGAAAATCAGGAGGGAGAGATCCTGCAGCATGCCTCCACTGATCATAACAAGCAAAT LEFL10 $\overline{6} 3 \mathrm{CA} 10$ LEFL1037DE09

SIMLO1 MT 1503 TGAGATTACAATGTCAGATTTTACTTTTGGAAACAAATAA SIMLO1_Heinz 1503 TGAGATTACAATGTCAGATTTTACTTTTGGAAACAAATAA LEFL10 $\overline{6} 3 \mathrm{CA} 10$ LEFL1037DE09

SUPPLEMENTARY FIGURE 1. Nucleotide alignment of the SIMLO1 sequence experimentally obtained from the tomato cv Micro-Tom (MT), the one from the cv Heinz as in the SGN database (Solyc04g49090) and the two EST sequences obtained by blasting the Heinz SIMLO1 sequence to the MiBASE database of Micro-Tom. 


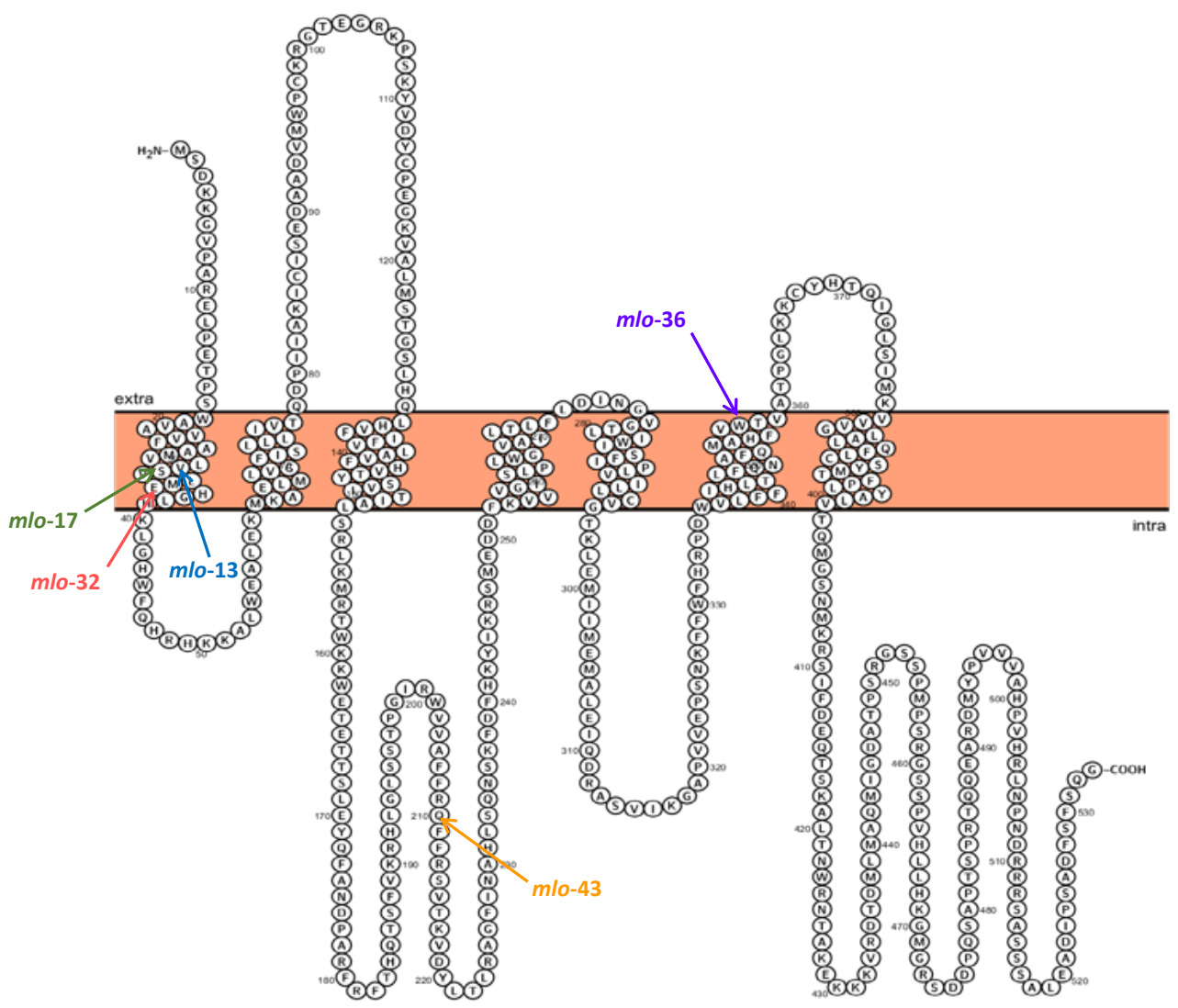

SUPPLEMENTARY FIGURE 2. Schematic representation of the complete barley HvMlo protein. The orange bar represents the plant membrane. Arrows indicate the amino acids that in their corresponding mlo-mutants are mutated into stop codons. 



\section{Chapter 8}

General discussion 
Plant diseases reduce global crop yields by $10-16 \%$ each year [1]. This constraint represents a tremendous challenge which has to be overcome if global food production should meet the demands of a growing world population by 2050.

Among plant pathogens, a small minority including powdery mildews (PM) can infect a broad range of plant species. More than 400 fungal PM species can infect thousands of plant species, causing a disease known for its whitish powdery appearance [2]. In addition, climate change is influencing the number of host species and the geographic distribution of this disease [3,4]. For example, PM disease on tomato caused by Oidium neolycopersici has been reported for the first time in 2015 in South Africa, which till 2008 was still an unexplored area of the world for PMs [2, 5]. Moreover, in 2011, the same pathogen was first identified in Taiwan on leaves of papaya (Carica papaya L.) [6]

So, how to halt its progression? There are several methods that can contribute to the control of the PM disease, from chemical and biological control to good farm management. However, my interest lies in the use of genetic resistance that ensures a durable effectiveness in crops affected by this disease.

Barley cultivars resistant to the PM pathogen Blumeria graminis f.sp. hordei (Bgh) are available since 1969 in the United Kingdom [7]. Most of the race-specific resistance to $B g h$ is conferred by the dominant resistant (R-) genes at the Mla locus. Thirty-one Mla alleles with race-specific resistances have been identified, numbered from number 1 to 31 [8]. In addition, five other resistance loci have been mapped: Mlat, MIGa, MIk, MInn and MIra [9]. The gene Mlk-1 was previously shown to be identical to Mla-4 [10]. Resistant cultivars based on the deployment of one or more allelic variants of the Mla gene, like Sultan, Wing, and Pipkin, have had a useful lifespan of just a few years (Figure 1).

On the other hand, the cultivars Atem and Alexis, introduced in the market in the 1980's, maintained a high PM-resistance level during the years (Figure 1). Both cultivars display the so-called mlo-based resistance, which, next to being durable, is recessive and broadspectrum. It is characterized at the cellular level by the timely deposition of papillae at the attempted fungal penetration sites, preventing colonization [11]. The resistance has been associated with defective alleles of the barley HvMlo gene (Mildew resistance Locus $\underline{O})$ and has for a long time been considered to be exclusive for this cereal [12]. However, more recent studies have shown that mlo-resistance is highly conserved as it occurs in many other plant species, among which Arabidopsis, pea, pepper, apple, cucumber and wheat [13-18], [19]. In tomato, a resistant cherry-type line, LC-95, harbors a natural loss-offunction allele of the tomato SIMLO1 gene, called ol-2, which is characterized by a 19-bp deletion in the coding sequence [20]. 


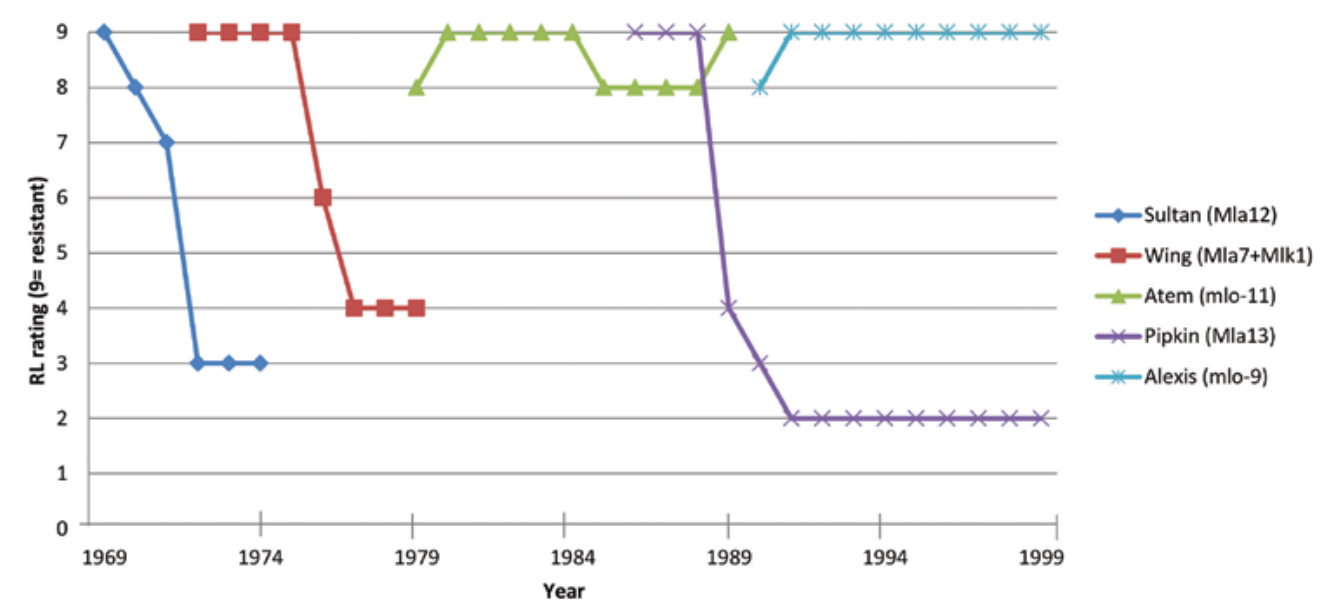

FIGURE 1. Resistance to barley powdery mildew disease in five barley cultivars deploying different

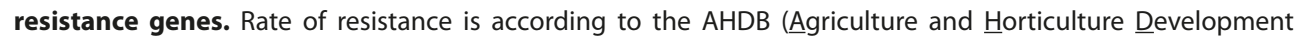
Board) Cereal and Oilseed Recommended Lists (RL) from 1968 to 1999. The cvs Sultan and Pipkin were the first ones to employ the R-genes Mla12 and Mla13, respectively. The cv Wing was the first harbouring a combination of R-genes, Mla7 and Mlk1. Atem and Alexis were the first cvs carrying loss-of-function alleles of the HvMlo gene, mlo-11 and mlo-9, respectively. This figure is a modified version of the one reported in Brown (2015).

The $M L O$ gene represents probably the best-characterized example of a class of plant genes known to facilitate the infection and provide compatibility with the pathogen. These are named susceptibility genes (S-genes) [21, 22]. In each plant species, MLO genes occur in multiple copies constituting a gene family [23]. So far, all known plant MLO genes, that act as S-genes towards the PM pathogens, cluster in two specific phylogenetic clades, clade IV for monocot and clade $V$ for dicot [24-27]. At the moment there is a body of experimental evidence showing that identification and inactivation of those genes is an effective breeding strategy to introduce resistance to PM in multiple cultivated species [21].

This thesis focused on the study of mlo-based resistance in Solanaceae and Cucurbitaceae crops. These families include several crops of agronomic importance, like tomato, eggplant, tobacco and cucumber. The results of the individual chapters represent a contribution to the current understanding of the role of MLO genes in PM susceptibility. From these findings, guidelines can be derived to aid and improve breeding activities in order to introduce the mlo-based resistance in new crop species suffering from the PM disease. These are shown in Figure 2 and discussed below. 
Introducing mlo-based resistance in a new crop species<smiles>CC</smiles>

Step 1. Identification of the main candidate $M L O$ susceptibility gene<smiles>C1CC1</smiles>

Is the genome information of the crop species available?

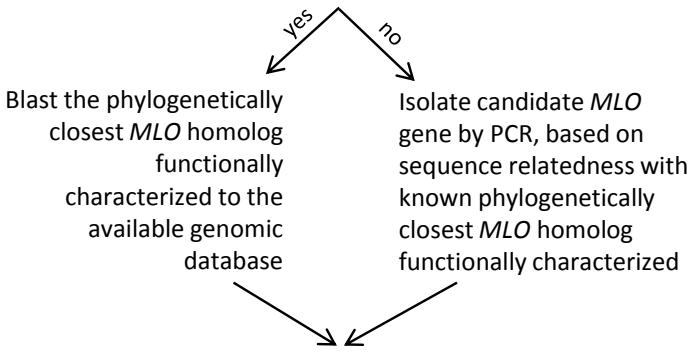

Perform a phylogenetic analysis with the newly identified $M L O$ gene(s)<smiles>C1CCC1</smiles>

Is the crop specie monocot or dicot?

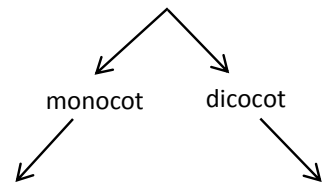

Candidate $M L O$

Candidate $M L O$

gene(s) in clade IV

gene(s) in clade $\mathrm{V}$

Presence of multiple $M L O$ genes of the crop species of interest in Clade IV or V?

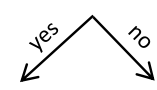

Observe which of the candidate $M L O$ genes is upregulated upon powdery mildew pathogen challenge<smiles></smiles>

Select the upregulated candidate $M L O$ gene for the functional characterization
Proceed to step 2 using the only candidate $M L O$ gene found in clade IV or V

FIGURE 2. 


\section{Step 2. Fast functional characterization of the candidate $M L O$ susceptibility gene}

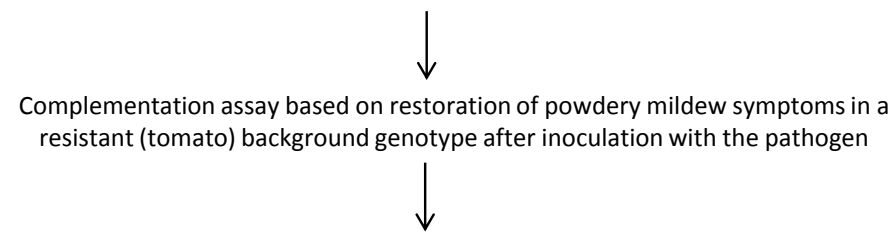

Are the symptoms visible?

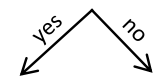

Main $M L O$ susceptibility gene in the crop species of

interest found
Test other candidates $M L O$

genes in the clade IV or $\mathrm{V}$ of

the crop species of interest<smiles>CC</smiles>

Are there pleiotropic effects visible?

Allele mining

Targeted

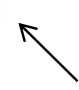

Careful consideration of

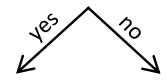

Careful consideration of

Proceed to step 3

\section{Step 3. Identification of $\mathbf{m l o}$-resistant genotypes}

genome-editing

technologies

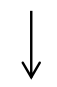

Are there natural mutations in the germoplasm of the crop species of interest?

Can the mutant allele be introgressed into the cultivated

crop species of interest?

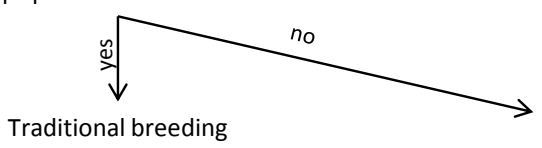

Induce variation using

mutagenesis coupled

with a high throughput $\longrightarrow$ Mutational breeding

detection method to

identify SNP in the

gene of interest

(TILLING)

๖

Obtain targeted mutations in

critical domains of the MLO

protein functionally

characterized using genome-

editing technologies

FIGURE 2. Guidelines to introduce the mlo-based resistance in new crop species affected by the PM disease. 


\section{How to find the main candidate MLO susceptibility gene(s) in the species of interest?}

\section{Identification of the main candidate $M L O$ gene}

The genome sequence of the crop of interest (or of a closely-related plant species) and the BLAST tool (http://blast.ncbi.nlm.nih.gov/Blast.cgi) are important resources to achieve this aim [28-32].

In Chapter 2, we used the available genomic information at the SOL genomic database (https://solgenomics.net/) to identify all the homologs of SIMLO1 in tomato, which was previously characterized as PM-susceptibility factor in this specie [20].

In Chapter 4, we isolated candidate MLO genes involved in PM susceptibility of three important solanaceous crops, namely tobacco, eggplant, and potato. At the time we were working on this chapter, only the genomes of potato and tobacco were published, while the one of eggplant was not available yet [33,34]. Nevertheless, due to the close phylogenetic distance of these plant species with tomato, a combined approach based on database search (of the available genomes) and PCR amplification allowed the identification of tobacco NtMLO1, eggplant SmMLO1, and potato StMLO1.

When the genome sequence of the crop species of interest is not yet available, a possible approach is to obtain first genomic or EST libraries. Feechan et al (2008) [25] prepared a grape genomic library based on a partial $\mathrm{Mbol}$ restriction digest and screened it with radiolabelled cDNA fragments of Arabidopsis MLO genes. In rose, Kaufmann et al. (2012) [35] produced two ESTs libraries of untreated and PM-inoculated leaf samples, against which they blasted Arabidopsis MLO genes.

However, since the number of available plant genome sequences is increasing, there may be genomic information available for a closely related plant species. It is feasible, then, to apply a homology-based cloning approach as shown in chapter 4 for eggplant SMMLO1, and for pepper CaMLO2 by Zheng et al. (2013) [18].

The next step is to establish which of the identified homologs cluster together with characterized MLO susceptibility genes. It is known that, following a phylogenetic analysis, dicot candidate $M L O$ susceptibility genes group in clade $\mathrm{V}$, while, monocot $M L O$ susceptibility genes are clustered in clade IV [26].

In Chapter 4, for example, we observed that five NtMLO and two StMLO genes, among which the newly identified homologs, cluster in clade $\mathrm{V}$ together with SIMLO1 and CaMLO2. Similarly, in Chapter 2, four tomato SIMLO homologs grouped in clade V.

When there are multiple $M L O$ homologs, as in the above examples, how to choose the best candidate $M L O$ susceptibility gene in the crop species of interest? 
Consonni et al. (2006) showed that Arabidopsis thaliana AtMLO2, AtMLO6 and AtMLO12 genes are functionally redundant, but AtMLO2 plays a major role in Arabidopsis susceptibility to the PM pathogen Golovinomyces orontii. Other examples followed, like the two pepper clade V-MLO homologs, CaMLO1 and CaMLO2 in Zheng et al. (2013) [18] and a more recent article published by Pessina et al. (2016) [19]. The latter showed that, of the four clade V-MLO genes in apple, MdMLO19 is the main gene responsible for the susceptibility to the PM pathogen Podosphaera leucotricha.

Because of the functional redundancy, the first criterion for selection is based on the highest level of conservation of the candidate clade $\mathrm{V}-M L O$ genes with the closest related characterized $M L O$ gene. For instance, the phylogenetic tree presented in Figure 1 of Chapter 4 showed that two of the five NtMLO genes are very homologous with each other and with SIMLO1 and CaMLO2. By PCR we isolated only NtMLO1 and our results provided evidence of its role as PM susceptibility gene. However, a recent paper showed that also a functional NtMLO2 is required for a successful PM infection of transgenic tobacco plants [36]. All together, these results show that high similarity to proven MLO-like susceptibility genes is a valuable criterion for selection.

In case none of the candidates can be excluded based on sequence relatedness, transcript abundance of each gene should be assessed upon PM infection. It was shown that the expression of MLO susceptibility genes is triggered few hours after PM inoculation, particularly between 4 and 10 hrs [18, 25, 31, 32, 37]. In Chapter 2, we showed that among the four SIMLO homologs, only the expression of SIMLO1 is significantly increased at 6 and 10 hrs. In fact, loss-of-function of this gene can almost completely prevent PM penetration [38]. In contrast, if the other three homologs are silenced individually, the susceptibility level of the background genotype in which they were tested (Moneymaker) does not change (Chapter 2).

The induction of gene expression upon powdery mildew infection should be considered as an absolute selection criterion for clade IV or clade V MLO genes. In Chapter 2 we observed that two SIMLO homologs outside clade V, SIMLO4 and SIMLO14, are up-regulated after PM inoculation. Preliminary results, which were not included in Chapter 2, indicated that SIMLO14 does not act as PM susceptibility factor in tomato. Similarly, in apple, a recent paper excluded clade VII- MdMLO18 as susceptibility gene, although its expression was induced by the PM pathogen [19].

\section{Fast approach to functionally validate the candidate $M L O$ susceptibility gene}

Once a candidate gene has been identified, it is important to verify that it acts as a PM susceptibility factor in a given plant species. This is of interest for the application of the mloresistance in breeding activities and basic research on plant-pathogen interactions. 
Commonly $M L O$ genes are validated using a resistant Arabidopsis mutant (Atmlo2/6/12) or a resistant barley mutant ( $\mathrm{mlo}-11)$ in assays involving overexpression of heterologous dicot and monocot MLO candidates, respectively [14, 19, 39-42]. If PM symptoms occur, the tested $M L O$ gene is a susceptibility factor because it is able to rescue the function of the impaired Atmlo genes or Hvmlo gene, depending on the genotype used.

The results reported in Chapter 5 indicate that the resistant tomato genotype ol-2, containing an impaired allele of the SIMLO1 gene, can be used to quickly validate the function of a candidate $M L O$ gene. We have developed a routine tomato transformation protocol in our lab which is reliable in testing both monocot and dicot $M L O$ genes. Moreover, the examples of successful complementation of closely related solanaceous homologs of SIMLO1 as well as more distant $M L O$ genes (Chapter 4, 5 and 6), indicate that a high degree of sequence conservation is not required in order to complement the loss-of-function of a susceptibility MLO gene, as was previously hypothesized by Panstruga (2005).

The implication of this finding is that the function of the MLO susceptibility genes is extremely conserved, which raises questions about its core biochemical activity. Once these questions are answered it will be possible to understand the reason for which a plant species has maintained, during evolution, a protein that is hijacked by PMs. More insights on this topic are described further in this chapter.

Another suitable option to verify the function of a candidate MLO gene consists of knockingdown its expression, in a transient or stable way. The virus-induced gene silencing (VIGS) technology offers an easy and quick alternative to the generation of stable transgenic plants, especially for species in which the latter is not achievable. This approach, for example, has been followed in barley, wheat, peach and pepper [18, 43-45]. Despite its advantages, this technology has several limitations inherent to the uneven silencing of the gene throughout an inoculated plant, the high variation between plants and experiments and the possible alteration of plant development, especially height and leaf morphology [46]. These limitations affect the observation of the phenotype upon silencing of the candidate MLO gene. For these reasons, the use of the stable silencing RNA interference (RNAi) is preferred over VIGS application.

Moreover, although the silencing approach has the advantage of using the native plantpathogen interaction, it may not be the best method to identify minor MLO genes involved in PM susceptibility. For example, in Chapter 2, the involvement of clade V-SIMLO3 gene could not be verified because no change in PM susceptibility was observed in RNAi silenced transgenic plants. Moreover, the minor effect of SIMLO5 and SIMLO8 was noticed only when comparing plants carrying the RNAi::SIMLO1 construct and ol-2 plants.

Novel genetic techniques to modify genes have been developed in recent years [47]. These include zinc finger nucleases (ZFNs), transcription activator-like effector nucleases 
(TALENs), and the CRISPR (clustered regularly interspaced short palindromic repeats)/Cas (CRISPR-associated) system [48-50]. These techniques allow genome editing by adding, removing or replacing DNA at specific locations. Therefore, they are widely used to study gene function. Recently, Wang et al. (2014) used the TALEN genome editing technology to generate transgenic winter wheat plants containing simultaneous knock-out lesions in the three TaMLO homoeologues. Their results suggest that all three genes contribute to the response of wheat to PM infection. In addition, they used the CRISPR/Cas9 technology to generate mutations (small deletions and one bp insertion) in the TaMLO-A1 allele, demonstrating for the first time the application of this novel system on $M L O$ genes. Similarly to TALENs, CRISPR/Cas9 is highly specific for the target sequence but much easier to handle. Both are particularly useful in polyploid outcrossing crops for which it is difficult to obtain homozygous mutants.

One careful consideration regarding the occurrence of pleiotropic effects is needed after verifying the involvement of the $M L O$ gene in the crop species of interest. If deleterious phenotypes are visible, it is important to consider whether or not introducing the mloresistance in a new crop.

Impaired mlo alleles produced severe pleiotropic effects in barley, consisting of spontaneous necrosis and premature leaf senescence, but also in Arabidopsis, where spontaneous callose deposition was observed [37, 51,52]. Moreover, both barley and Arabidopsis mutants were more susceptible to certain hemibiotrophic and necrotrophic pathogens which indicate that MLO proteins are exploited by a plethora of microorganisms [51, 53-55].

In Arabidopsis, it was shown that the pleiotropic effects can be uncoupled from mlo-based resistance by applying second-site mutations in the NahG gene to reduce the higher SA level observed in single and double mutants compared to wild-type plants [51]. However, since SA is involved in response to other pathogens, further analyses are needed to assess the possibilities of application in an agronomically beneficial manner.

A very recent article showed the identification of a new natural variant of the barley mlo-11 allele, called mlo-11(cnv2), found in the Ethiopian barley landrace Eth295 [56]. Developmental stage influences the resistance provided by this novel allele. The resistance obtained is partial, although effective. However, most importantly, the landrace carrying the novel variant lacks the pleiotropic effects typical of the barley mutants. The gene expression of the two alleles was compared and causally related to the phenotypic differences observed between the landrace Eth 295 and the cv Westminster, carrying the mlo- 11 allele. Therefore, they suggest the use of "temperate" MLO mutations to fine tune the balance between expression, pleiotropic effects and resistance levels.

No pleiotropic effects were observed in tomato silenced plants (Chapter 2) nor in stable silenced apple plantlets, while a reduction in plant size was shown in pepper silencing 
CaMLO2 gene $[18,19]$. Taken together, these findings indicate that pleiotropic effects vary among plant species upon knockdown or knockout of the susceptibility MLO genes. This means that there is a large space for the possible utilization of the mlo-resistance in breeding programs, without the limitations connected to the pleiotropic effects, and for a suitable compromise between level of resistance and pleiotropic effects.

\section{Identification of mlo-resistant genotypes...}

At this point of the pipeline, the correct target MLO gene has been found, functionally validated, and its utilization considered potentially beneficial for the studied crop species. In Europe, the deployment of transgenic plants (including those resulting from genome editing approaches) in plant breeding and agriculture is still socially and politically debated. Therefore, currently, non-transgenic approaches are favored in the selection of new varieties.

\section{a)...through natural variation}

The first non-transgenic strategy to identify mlo-resistant genotypes consists of searching for variation within the natural germplasm of the crop species of interest.

In Table 1, an overview of all the mlo-mutant alleles described in literature is presented, among which natural alleles are found in pea (er-1, er-2, er-3, er-4 and er-6), rose, tomato (ol-2), apple, cucumber, tobacco and barley (mlo-11 and mlo-11(cnv2)) [13, 15, 20, 35, 36, $56,57]$.

Nowadays, with the huge amount of sequence information derived from sequencing projects of cultivated vegetable and fruit crops, like tomato, cucumber and apple, allele mining is another promising approach to identify novel natural $M L O$ alleles useful for the development of new cultivars [58, 59] (http://www.fruitbreedomics.com). In Chapter 6 , we exploited the genomic information of the 115 cucumber accessions to search for the occurrence of the transposable element found in the cucumber susceptibility gene CsaMLO8. By doing so, we gathered information regarding the natural variation of the CsaMLO8 gene and found out that it probably derived from the wild cucumber species, $C$. sativus var hardwickii, from which it was introgressed in cultivated cucumbers.

Therefore, if an impaired natural allele of the MLO gene of interest is found, and the accession or wild species can be crossed, a long program aimed at inserting the desired allele in the crop under investigation starts. 


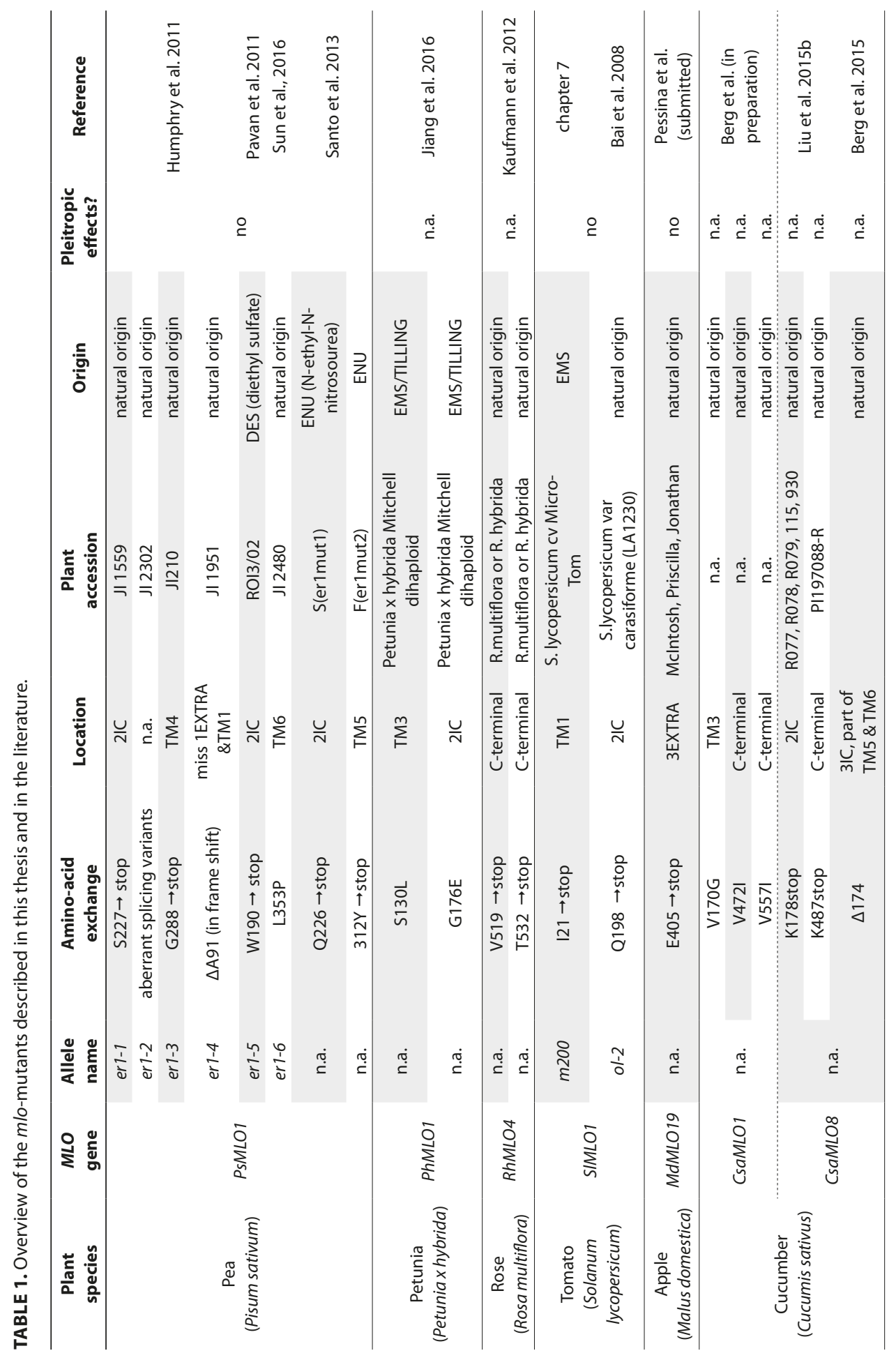




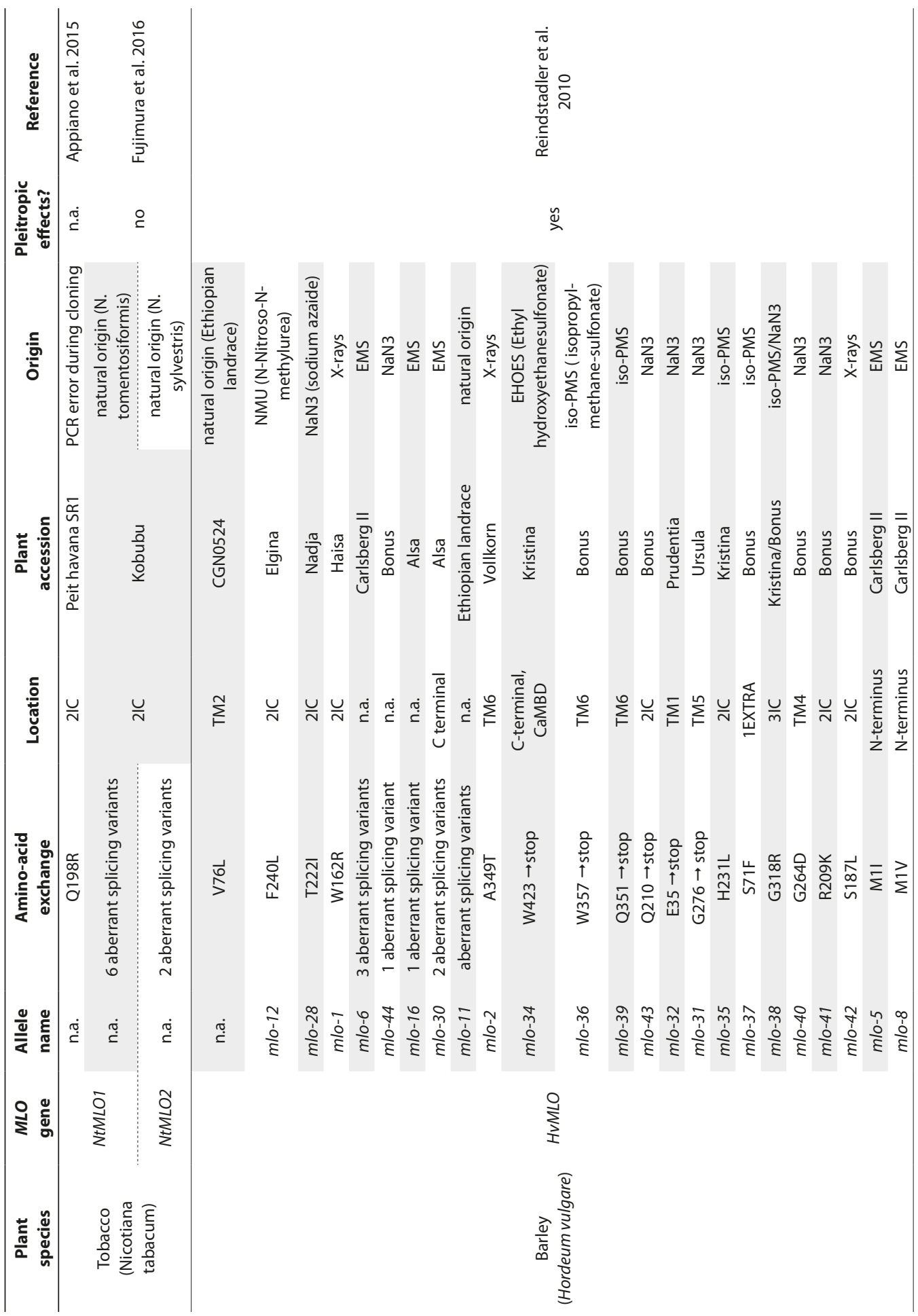




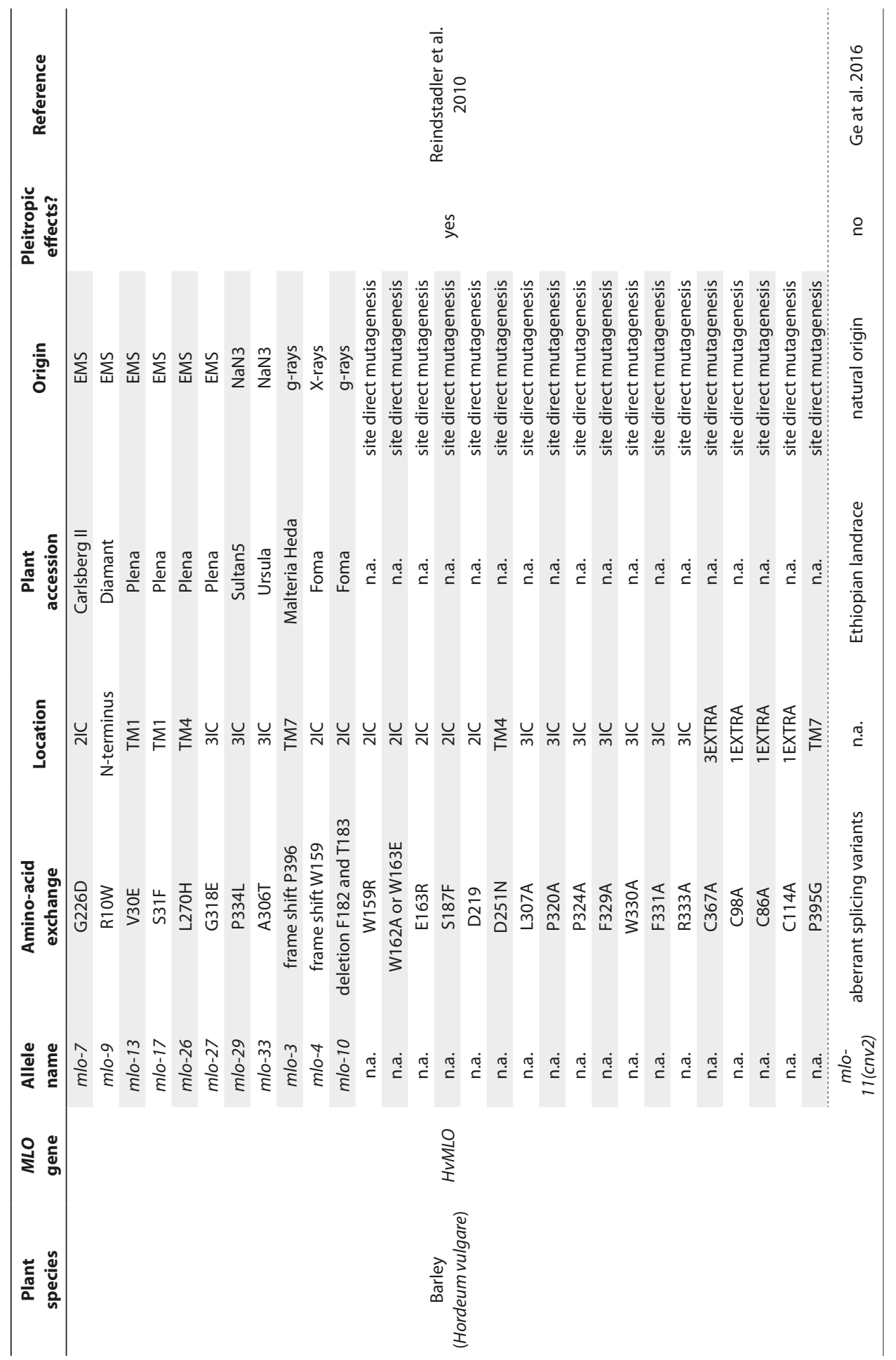




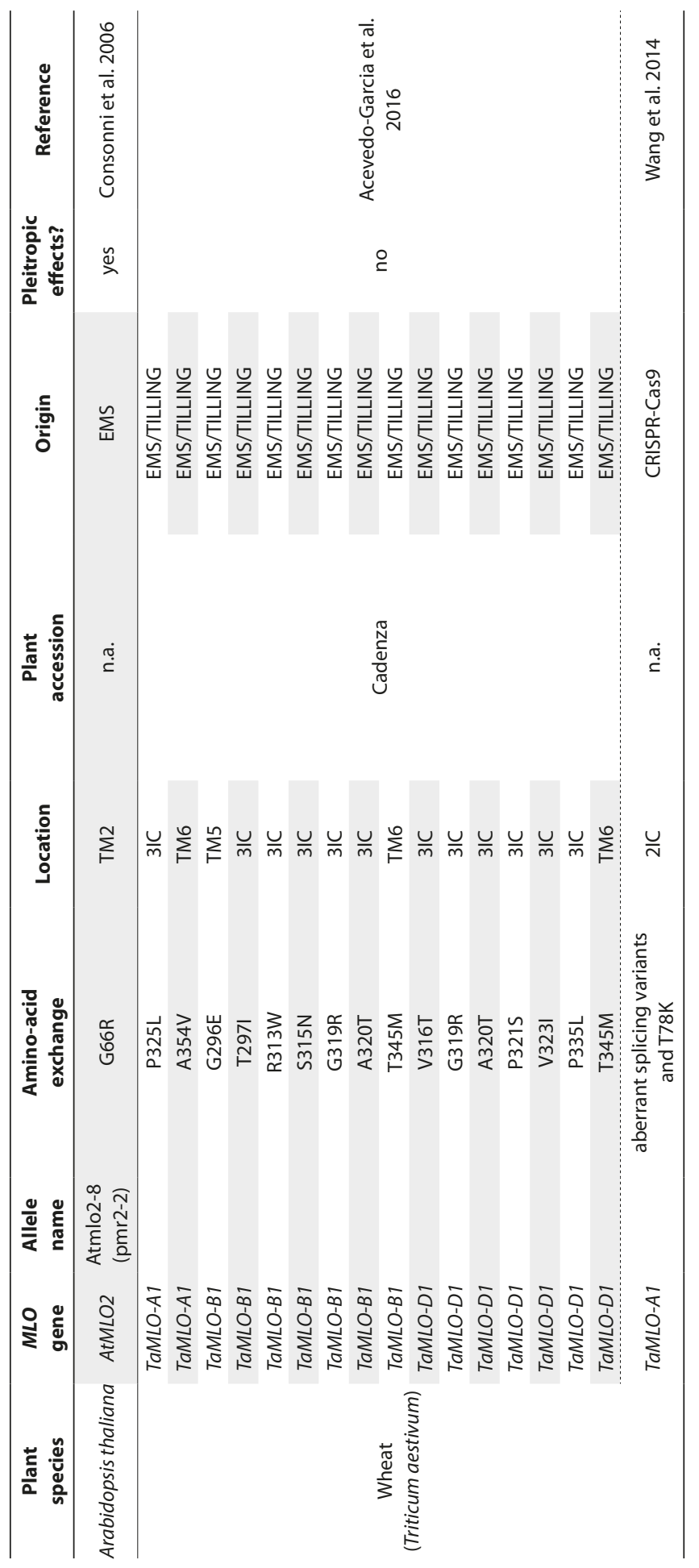




\section{b) ...through artificial mutations}

\section{i) ...obtained with mutagens}

However, the problem of many cultivated species is the lack of genetic diversity. For this reason, mutagenesis programs aimed at obtaining new mutants became very popular from the 1950's onwards. In 1995, the number of commercial mutant varieties reached 484 [60]. The mutagens used are grouped into two broad categories, namely chemical and physical mutagens [61]. Among the most commonly used physical mutagens there are X-and $\gamma$-rays, also called ionizing radiations because of the impact they have [60]. Chemical mutagens frequently used are alkylating agents, like EMS, MNU, ENU, azide, hydroxylamine, nitrous acid, acridines and base analogues. The use of chemical mutagens is preferred when point mutations are desired, while the physical mutagens usually induce larger deletion, like chromosomal aberration or rearrangements [60].

In Chapter 7, we describe the development of an EMS mutant population of tomato cv MicroTom in which a PM resistant plant was discovered. The resistance observed is of the mlo-type because associated with the loss-of-function of the SIMLO1 gene.

Normally, the identification of individuals with a target mutation takes time because it involves the screening of large populations. TILLING (Targeting Induced Local Lesions IN Genomes) is a powerful approach that integrates chemical mutagenesis with a high throughput detection method to identify single nucleotide mutations in a specific region of a gene of interest [62]. Moreover, although until recently no TILLING-derived crop variety has been released commercially, they represent a great advantage for plant breeding (especially in Europe) since these varieties will be considered non-transgenic [63]. A very recent paper showed the application of this technology to generate hexaploid bread wheat lines with enhanced resistance to the PM disease without affecting growth and development [64].

Looking at all the mlo-mutant alleles in Table 1, the highest number is found in barley (33), followed by wheat (16), petunia (2), pea (3) and tomato (1) [64-67], [68] and chapter 7].

These mutants are obtained either with ionizing radiations or with chemical agents, the latter being the predominant method. EMS (ethyl methane sulfonate) is by far the chemical mutagen most commonly used since twenty-nine mlo-mutants were obtained with it. Eight mutants were obtained with $\mathrm{NaN}_{3}$ (sodium azide), four with iso-PMS, and two with ENU (Table 1). lonizing radiation, particularly $X$ - and $Y$-rays were used only in barley to obtain four and two mutants, respectively (Table 1). The type of mutations consists of missense mutations, where a single codon is altered to encode a different amino acid, nonsense mutations, where a premature stop codon is introduced producing an incomplete protein product, and aberrant splicing variants, translated in truncated proteins or missing certain domains.

It was observed in Humphry et al. (2011) that the majority of barley mutants is characterized by single amino acid substitutions, while, in pea, mlo-mutants derive from more dramatic 
changes of the PsMLO1 protein. This observation still holds true, considering the enlarged panel of mlo-mutants, like tobacco, cucumber, rose and tomato. This might be an indication of different evolutionary strategies of these plant species.

The large majority of the mutations of Table 1 are found in the second (21) and third (23) cytoplasmic domains, which have already been identified as relevant regions for the MLO proteins acting as PM-susceptibility factors (see also Figure 3) [69]. Transmembrane (TM) regions are additional sites of loss-of-function mutations in 24 cases, with the predominant occurrence in the sixth transmembrane (7), indicating that TM domains harbor important sites for protein conformational changes.

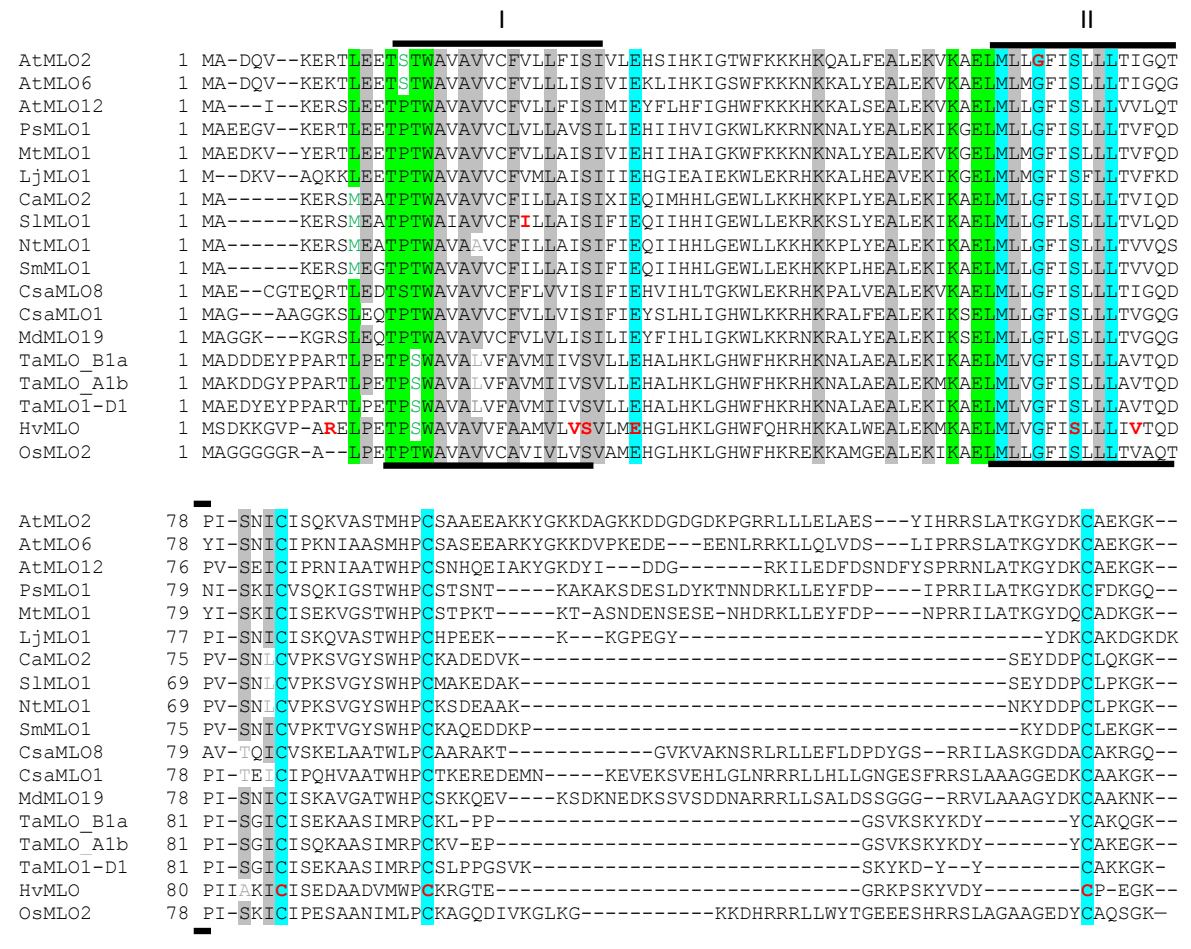

III

152 VAFVSAYGIHOLHTFIFVLAVVHVVYCIVTYAFGKIKMRTWKSWEEETKTIEYOYSNDPERFRFARDTSFGRRHLNFWSK 149 VAFVSAYGMHQLH IFIFVLAVCHVIYCIVTYALGKTKMRRWKKWEEETKTIEYQYSHDPERFRFARDTSFGRRHLSFWSK 143 VALVSAYGIHQLHIFIFVLAVFHVLYCI ITYALGKTKMKKWKSWERETKTIEYQY ANDPERERFARDTSFGRRHLNIWSK 147 VALVSAYG I HQLH IFIFVLALFH LQCI ITLTLGRIKMRKWKTWEDETRTVEYQF YNDPERFRFARDTTEGRRHLSMWAQ 145 VALVSAYGIHELH IFI FVLAIFH LQCI I TLALGRFKMRRWKKWEDETRTVEYOFYNDPERFRFARDTTFGRRHLSMWTK 118 VAFMSQYGIHQLHIFIFVLAIFH I LQCITTLALGRTRMAMWKKWEEETKTLEHQF DNDPERFRFARDTTFGRRHLNSWSQ 112 VQFASSYAIHQLHIFIFVLAIAHVLYCIATFALGRLKMRKWRAWEDETKTIEYQFYNDPERFRFARETSFGRRHMHFWSK 106 VQFASSYAIHQLHIFIFVLAVAHVLYCIATFALGRLKMRKWRAWEDETKTMEYQFYNDPERFRFARETSFGRRHLHFWSK 106 VQFASSYAIHQLHIFIFVLAVAHVLYSIATFALGRLKMRKWRAWEEETKTIEYQFYNDPERFRFARETSEGRRHLHYWSK 112 VQFASSYAIHQLHIFIFVLAVAHVLYCIATFALGRLKMRKWRAWEDETKTIEYQFYNDPERFRFARETSEGRRHLHFWSK 142 LAFVSAYGIHQLHIFIFVLAVFHVLYCI ITLAFGRTKMSKWKAWEDETKTIEYQYYNDPARFRFARDTTFGRRHLSFWSR 150 ASFISADGIHQLHIFIFVLAVFHVLYCVLTYALARAKMRSWKTWEKETKTAEYQFS DPERFRFARDTSFGRRHLSFWTK 149 VPFVSYYGIHOLHILIFVLAVFHVLYCITTLVLGRAKMRKWKTWELETKTAAYOFS DPPERFRFARDTSFGRRHLSFWSR 120 VSLMSTGSLHQLHIFIFVLAVFHVTYSVI IMALSRLKMRTWKKWETETASLEYQFANDPARFRFTHQTSFVKRHLGL-SS 120 VALMSTGSLHQLHIFIFVLAVF HVTYSVI IMALSRLKMRTWKKWETETASLEYQFANDPARFRFTHQTSFVKRHLGL-SS 120 VSLMSTGSLHQLHMF I FVLAVFHVTYSVI IMALSRLKMRTWKKWETETXSLEYQFANDPARFRFTHQTSFVKRHLGL-SS 119 VALMSTGSLHOLHVFIFVLAVFHVTYSVITIALSRLKMRTWKKWETETTSLEYOFANDPARFRFTHOTSFVKRHLGL-SS 144 VALMSSGGMHOLHIFIFVLAVEHVTYCVITMALGRLKMKKWKKWELET SLEYQFANDPSRFRFTHQTSEVKRHLGL-SS

FIGURE 3. 
IV

AtMLO2 AtMLO 6 AtMLO12 PsMLO1 MtMLO1 LjMLO1 CaMLO2 SIMLO1 NtMLO1 SmMLO1

CsaMLO8 CsaMLO1 MdMLO19 TaMLo Bla TaMLO A1b TaMLO $\overline{1}-\mathrm{D} 1$ HvMLO OSMLO2

AtMLO2 AtMLO 6 AtMLO12 PSMLO1 MtMLO1 LjMLO1 CaMLO2 SIMLO1 NtMLO1 SmMLO1 CsaMLO8 CsaMLO1 MdMLO19 TaMLO_Bla TaMLO A1b TaMLO-D1 HVMLO OsMLO2

AtMLO2 AtMLO 6 AtMLO12 PSMLO1 MtMLO1

LjMLO1

CaMLO2

SIMLO1

NtMLO1

SmMLO1

CsaMLO8

CsamLO1

MdMLO19

TaMLO_Bla

TaMLO A1b

TAMLO D1

HvMLO

OSMLO2

AtMLO2 AtMLO6 AtMLO12 PSMLO1 MtMLO1

LjMLO1 CaMLO2 SIMLO1 NtMLO1 SmMLO1 CsaMLO8 CsaMLO1 MdMLO19

TaMLO B1a TaMLO_A1b TaMLO_D1 HVMLO OSMLO2

232 TRVTLWIVCFFRQFFGSVTKVDYLALRHGFIMAHFAPGNESRFDFRKYIQRSLEKDFKTVVEISPVIWFVAVLFLLTNSY 229 STITLWIVCFFRQFFRSVTKVDYLTLRHGFIMAHLAPGSDARFDFRKYIQRSLEEDFKTIVEINPVIWFIAVLFLLTNTN 223 STFTLWITCFFROFFGSVTKVDYLTLRHGF IMAHLPAGSAARFDFOKYIERSLEODFTVVVGISPLIWCIAVLFILTNTH 227 SPILLWIVSFFRQFFGSISRVDYMALRHGF IMAHLPPGHDAQFDFQKYISRS IEEDFKVVVGISPT IWLFTVLFLLTNTH 225 SPISLWIVCFFRQFFGSISRVDYLALRHGF IMAHLAPGNDAEFDFQKYISRSLEKDFKVVVGISPTIWFFAVLFLLTNTH 198 SPISLWIVSFFRQFYGSVDKVDYMVLRHGF I IAHLAPGSESKFDFQKYISRSVDEDFKVVVGISPTVWFFAVLILLTNTH 192 SPVMLWIVCFFROFFSSVAKVDYLTLRHGFMMAHLTPONOENFDFOIYINRAVDKDFKVVVGISPALWLFTVLYFLSTTD 186 SPVLLSIVCFFRQFFSSVAKVDYLTLRHGFMMAHLTPQNQNNFDFOLYINRAVDKDFKVVVGISPALWLFTVLYFLTTTD 186 SPVLLSIVCFFRQFESSVAKVDYLTLRHGFMMAHLTPQNQNNFDFQLYINRAVDKDFKVVVGISPALWLFTVLYFLTTTD
186 SPVLLWIVCFFRQFFSSVAKVDYLTLRHGFMMAHLTPQNQENFDFQIYINRAVEKDFKFVVEISPALWLFTVLYFLTTTN 192 SPLLLWIVCFFRQFFSSVAKVDYLTLRHGFMMAHLTPENQKNFDFQIYINRAVDKDEKVVVGISPALWLFTVLYFLTTTD 222 TPISLWIVCFFROFFGSVTKVDYMTLRHGF IVAHLAPGSEVKFDFHKYIXRSLEDDFKVVVGISPAMWLFAVLFILTNTN 230 NPAL WIVCFFRQFVRSVPKVDYLTLRHGF IMAHLAPQSHTQFDFQKYINRSLEEDFKVVVGISPPIWFFAVLFLLSNTH 229 SPISLWIVCFFRQFVRSVPKVDYLTLRHGF IAAHLAPQSQTKFDFQKYINRSLEEDFKVVVGISPTIWLFAVLILMSNTH 199 TPGVRWVVAFFRQFFRSVTKVDYFTLRAGF INAHLS--HNSKFDFHKYIKRSMEDDFKVVVGISLPLWCVAILTLFLDID 199 TPGVRWVVAFFROFFRSVTKVDYLILRAGF INAHLS--ONSKFDFHKYIKRSMEDDFKVVVGISLPLWAVAILTLFLDID 199 TPGIRWVVAFFRQFFRSVTKVDYLTLRAGF INAHLS--HNSKFDFHKYIKRSMEDDFKVVVGISLPLWCVAILTLFLDID 198 TPGIRWVVAFFRQFFRSVTKVDYLTLRAGF INAHLS--QNSKFDFHKYIKRSMEDDFKVVVGISLPLWGVAILTLFLDIN 223 TPGLRWIVAFFRQFFGSVTKVDYLTMRQGF INAHLS--QNSKFDFHKYIKRSLEDDFKVVVGISLPLWFVAILVLFLDIQ

\section{$\mathrm{V}$}

312 GLRSYLWLPFIPLVVILIVGTKLEVIITKIGLRIQEKGDVVRGAPVVOPGDDLFWFGKPRFILFLIHLVLFTNAFQLAFF 309 GLNSYLWLPFIP IVILIVGTKLQVIITKLGLRIQEKGDVVKGTPLVOPGDHFFWFGRPRFILFLIHLVLFTNAFQLAFF 303 GWDSYLWLPFLPLIVILIVGAKLQMIISKLGLRIQEKGDVVKGAPVVEPGDDLFWFGRPRFILFLIHLVLFTNAFOLAFF 307 GWYSYYWLPFLPLIVILLVGAKLQMIITKMGLRIQDRGEVIKGAPVVEPGDHLFWFNRPHLLLFTIHLVLFQNAFQLAFF 305 GWYSSYWLPFLPLI I LLVGAKLQMI ITKMGLRIQDRGEVIKGAPVVEPGDHLFWFNSPNLLLF I IHLVLFQNAFQLAFF 278 GWHSYLWLPFIPLI I ILLVGTKLQMI ITNMGLKIQERGDVIKGAPLVEPGDDLFWFNRPRLILSLVHLVLFQNAFQLAFF 272 GVYSYLWVPFVPLI I I LLVGTKLQMI ITEMGVRISERGDIVKGVPVVEIGDHLFWFNRPGLVLFFINFVLFQNAFQVAFF 266 RLYSYLWVPFIPLVI ILLVGTKLOMIITEMGVRISERGDIVKGVPVVETGDHLFWFNRPALVLFLINFVLFONAFOVAFF 266 GLYSYLWVPFIPLVI ILLVGTKLEMI IAEMGVRISKRGDIVRGVPVVETGDHLFWFNRPGFVLFLINFVLFQNAFQVAFF 272 GLYSYLWVPFVPLII ILLVGTKLQMI ITEMGVRISERGDIVKGVPVVETGDHLFWENRPGLVLFLINFVLFQNAFQVAFF 302 GWYSYLWLPFISLI I I LLVGTKLHVI ITHMGLTIQERGHVVKGVPVVOPRDDLFWFGRPQLILFLIHFVLFMNAFQLAFF 310 GWRAYLWLPFIPLI ILLLIGTKLQVI ITKMALRIQERGEVVKGVPVVEPGDDLFWFNRPRL I Y LINFVLFQNAFQVAFF 309 GSRSYLWLPFVPLVMILMVGTKLQVI ITKMGLKLSERGEVVRGTPLVEPGDHLFWENNPRLLL I IHFVLFQNAFALAFF 277 GIGTLTWISFIPLVI LLCVGTKLEMI IMEMALE IQDRASVIKGAPVVE PNKFFWEHRP DWVLFF IHLTLFQNAFQMAHF 277 GIGTLTWVSFIPLIILLCVGTKLEMI IMEMALEIQDRS SVIKGAPVVEPSNKFFWEHRPDWVLFFIHLTLFQNAFQMAHF 277 GIGTLTWISFIPLVI LLCVGTKLEMI IMEMALE IQDRASVIKGAPVVEPSNKFFWFHRPDWVLFF I HLTLFQNAFQMAHF 276 GVGTLIWISFIPLVI LLCVGTKLEMI IMEMALE IQDRASVIKGAPVVEPSNKFFWFHRPDWVLFF I HLTLFQNAFQMAHF 301 GFGTLIWISFVPLVILMLVGTKLEMVIMEMAQEIQDRATVIKGAPVVEPSNKY FWENRPDWVLFFI HLILFQNAFQMAHF

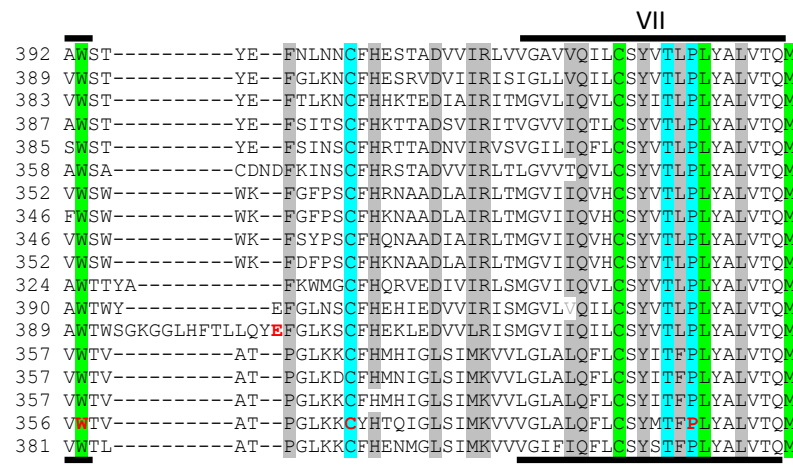

SKMKPTVFNDRVATALKKW SKMKPTVFNERVATALKSW TSMRPTIFNDRVANALKKW STMKPTIFNERVATALKNW STMKPTIFNERLATALKKW STMRPTIFHDRVATALKSW SSMKPI IFGDNVATALRSW SSMKP I IFGDNVATALRSW T SMKP I I FGDNVATALRSW SSMKPI IFGDNVATALRSW SNMRPTIFNDRVATALKNW STMKPTIFNERVAEALRNW STMKPVIFNDRVATALKKW SNMKRSIFDEQTAKALTNW SNMKRSIFDEQTAKALTNW SNMKRSIFDEQTAKALTN SNMKRS I FDEQTSKALTNW

\section{CaMBD}

460 HHTAKNETKHGRHSG-----S--NTPFSSRPTTPTHGSSPIHLLHNF---NNRSV-E--NYPSSPSPR----YSGHG--457 HHTAKKNIKHGRTSE-----S--TTPFSSRPTTPTHGSSPI HLLRNAPHKRSRSVDE--SFANSFSPR----NS----451 HHTAKKQTKHG-HSG-----S--NTPHSSRPTTPTHGMSPVHLLHNY---NNRSLDQQTSFTASPSPPRESDYSGQG--455 HHTAKKQVKQSNHSN-----N--TTPYSSRPSTPTHAMSPVHLLHRH-TAGN-------SDSLQTSPE-----KS----453 HHTAKKQVKHNKHSN-----N--TTPYSSRQSTPTHGMSPVHLLHRQ-TFGN-------SDSLQTSPR-----TS----428 HHTAKKHVKHNRDSN-----SHSNTPFSSRPATPTHGMSPVHLLHKHHNYHN-------SDSPLASPR-----ESPS-420 HNTAKKRVRHGRVSE-----N--TTPISSRPATPLRGTSPVHLLRGYPKY-N-------EDNVQAYPRT-------S--414 HHTAKKRVKHG-LSG-----H--TTPANSRPTTPLRGTSPVHLLRGYPQY-N-------EDSVQASPRT-------S--414 HNTAKKRVKHGRLSE-----N--TTPVSSRPATPLHGTSPVHLLRSYPQYSN-------EES-----RT-------S--420 HHMAKKRVKHGRLS-------GNTTPVSSRPTTPLHGTSPVHLLRGYPQYN--------EDSVQASPRT-------S---

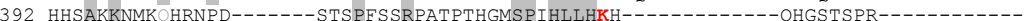
458 YHSARKHIKHNRGS--------VTPMSSRPATPTHSMSPVHLLRHY--------KSEVDSFHTSPRRSPFDTDRWDND 469 HIAAKKHVKHKNAS----_------P-ASAPGTPLHSMS PVHLLRNYKYEQDI-------DSIQTSPRMPYFDNEGSDSP

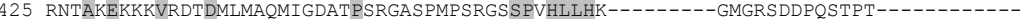

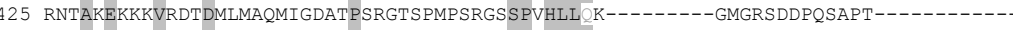
425 RNTAKEKKKVRDTDMLMAQMIGDATPSRGTSPMPSRASSPVHLLHK---------GMGRSDDPQSAPTS--------424 RNTAKEKKKVRDTDMLMAQMIGDATPSRGSSPMPSRGSSPVHLLHK---------GMGRSDDPQSAPT---------

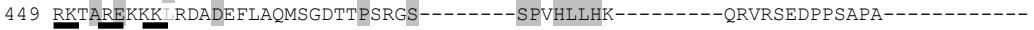

FIGURE 3. 


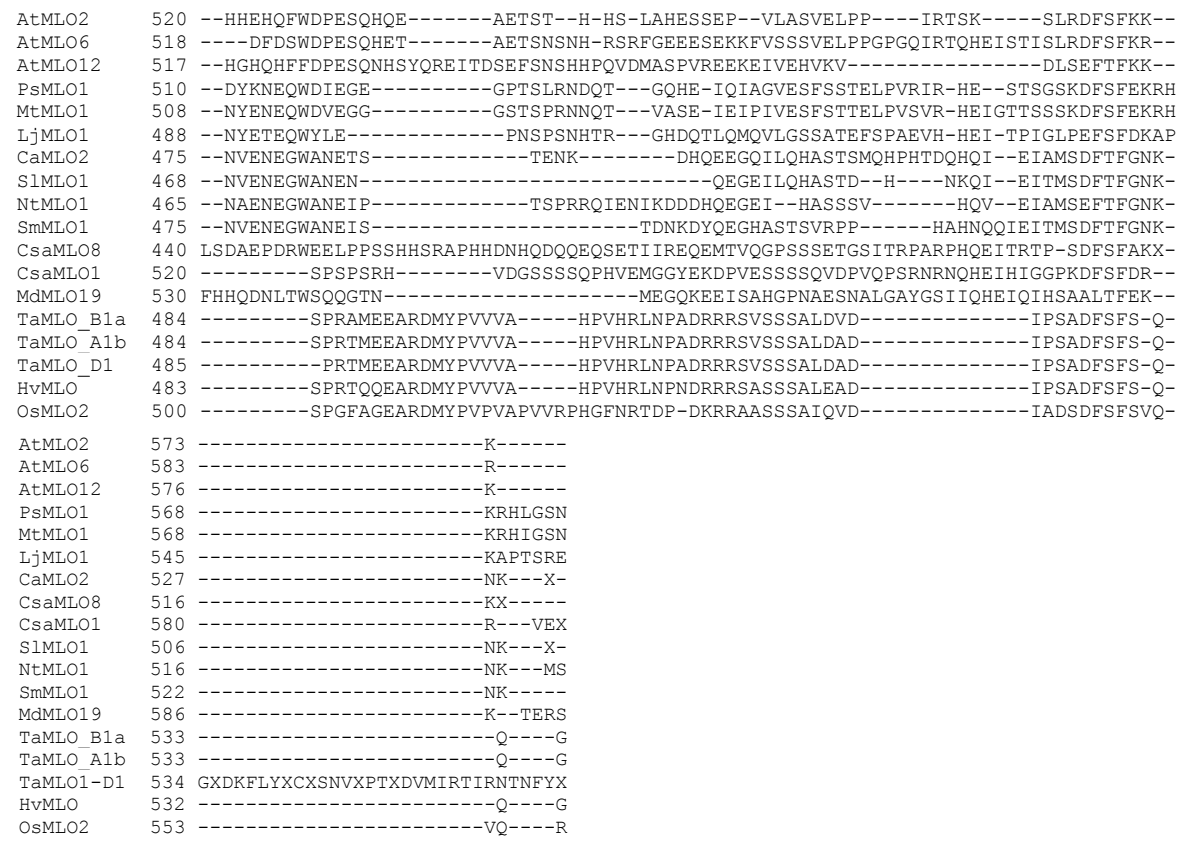

FIGURE 3. Protein alignment of functionally characterized MLO sequences of Arabidopsis (At), pea (Ps), barrel clover (Mt), Lotus japonicas (Lj), pepper (Ca), cucumber (Csa), tomato (SI), tobacco (Nt), eggplant (Sm), apple (Md), wheat (Ta), barley (Hv) and rice (Os). Highlighted in green and in light blue are the conserved amino acids among the whole MLO family indicated by Kush et al. 2016 [26] and by Elliott et al. 2005 [69], respectively. Amino acids highlighted in gray refer to the ones reported to be under negative selection by Appiano et al. 2015 [24, chapter 5). Letters displayed in green, light blue or gray indicate synonymous amino acid exchanges in each of three categories above described. Letters in red bold indicate amino acids identified in mlo-mutants for each of the plant species described above. Black lines indicate the position of the transmembrane domains which have been numbered with romans numbers.

\section{ii) ...or through targeted genome editing}

Several studies have addressed the characterization of relevant functional regions of the MLO protein $[26,40,70]$. With the increasing number of MLO sequences being identified in various plant species, multiple alignments point out the occurrence of highly conserved residues that are predicted to have an important functional/structural role (Figure 3). Also, in Chapter 5 we identified 130 codons under negative selection that are translated into amino acids predicted to be conserved during evolution (Figure 3). We also highlighted amino acids specific for monocot and dicot MLO proteins which do not seem to influence the interaction with PM pathogens. We combined the information of important predicted amino acids with the actual mutations found in natural and artificial mutants in the alignment of Figure 3.

Any novel S-MLO protein characterized in a certain crop species can be added to this alignment to pinpoint potential loss-of-function sites. If artificial or natural mutants are 
not available, the information of Table 1 and Figure 3 can be usefully coupled with the editing technologies presented above to obtain loss-of-function mutations, especially within the protein domains highlighted in the previous paragraph.

In case it was previously verified that knocked-down $M L O$ alleles are not coupled with undesired pleiotropic effect in the species under investigation, it is convenient to decide upon the artificial induction of severe mutations (i.e. nonsense) in order to ensure a severe resistant phenotype.

On the other hand, when loss of the susceptibility function needs to be adjusted in such a way that pleiotropic effects are avoided, missense mutations that do not produce drastic amino acid changes are preferable to nonsense mutations.

Missense mutations can be grouped in two categories: synonymous and non-synonymous coding changes. The first do not impact protein structure and function, while the latter might do. To evaluate the impact of non-synonymous changes, the physicochemical environment in which the amino acid exists, the structural context of the amino acid and the functional context of the amino acid within the protein should be taken into consideration [71].

The cellular location of the MLO proteins can be divided in three levels: intracellular, extracellular and transmembrane. The structural context is more difficult to determine as the crystal structure of the MLO proteins is not known because they are insoluble. The crystal structure of one GPCR (G-protein-coupled receptor), bovine rhodopsin, has been determined [72]. Since MLO proteins are structurally reminiscent of GPCRs, the rhodopsin structure could be taken into account as a model, even if with some caution because MLO function seems to be independent of G-proteins [73-75].

The final step is to evaluate the annotated features of an amino acid variant and the level of conservation of the amino acid in an alignment. Finally, protein function is the key to understanding the consequences of amino acids substitution. Since this is not known yet for MLO proteins, it becomes crucial to rely on conservation of the amino acid position and knowledge of actual loss-of-function site (through mutants).

For example, in barley three mutant alleles (V76L, T222l, and F240L) are called weak alleles because they do not confer full resistance since they still allow about $30 \%$ of PM penetration rate $[37,67]$. Nevertheless, PM progression is slowed down in these lines (penetration success on susceptible wild type MLO genotypes ranges between 50-80\%) and the use of chemicals to control the disease can be reduced. These three amino-acids are either conserved within the alignment or substituted with favorable ones (Figure 3). The latter consists of replacements with (predicted) neutral amino acids, which partially impair protein function. 
More examples of weak alleles were obtained through site direct mutagenesis in the study of Muller et al. (2004) [76] and Reinstädler et al. (2010) [67] showing how single amino acid replacement influences the functionality of the MLO protein.

The advent of the CRISPR/Cas9 technology promises the possibility to introduce any DNA modification, among which precise site mutations. Few studies have so far addressed this application, but its feasibility has been recently demonstrated in yeast (Saccharomyces cerevisiae) [77].

The CRISPR/Cas9 technique could also be used to modulate the MLO expression by targeting the promoter of the susceptibility MLO gene. It is known that its expression is induced upon PM infection and required for PM successful colonization [37, 78]. Although it is not known if MLO is directly or indirectly targeted by PM elicitors/effectors, there must be some element(s) in the promoter of the $M L O$ gene that are responsive to them, triggering the expression. Since prediction of responsive element(s) is difficult, promoter studies should be conducted first. These involve cloning both the full-length promoter and truncated promoter fragments in front of e.g. a GUS or GFP gene, transforming them into tomato or Arabidopsis, and studying which region is required for induction of expression by powdery mildew infection. Once the region is known, the effect of amino acids changes can be analyzed by CRISPR/Cas9 so that induction of MLO expression by PM pathogen is prevented, without interfering with expression levels required for normal development.

One successful application of this concept was achieved in rice using TALENs to disrupt the effector binding site of the bacterial blight, Xanthomonas oryzae pv. oryzae, located in the promoter region of the SWEET14 gene [115].

Ultimately, CRISPR/Cas9 could also be used for epigenome editing [79]. In the work of Ge et al. (2016) [56], it was shown that HVMLO expression is DNA methylation-dependent. Moreover, they associated the partial resistance level of the Ethiopian landrace carrying the mlo-11(cnv2) allele to its intermediate level of expression (and methylation) compared to wild-type barley and full resistant cv Westminster. Therefore, by modulating the methylation of the $M L O$ gene promoter, it should be possible to knockdown its expression to a degree that would not interfere with any other possible developmental function but would effectively stop PM penetration. 


\section{Towards the discovery of the function of the MLO protein: direction for future research}

Since MLO proteins are exploited by PM pathogens to cause the disease, a question still torturing MLO researchers is why plants have them.

In the recent paper of Kush et al. (2016) [26], MLO proteins were traced back to red and green algae, which finding implies a fundamental ancestral role of the MLO proteins.

As mentioned in the introduction, not all the MLO proteins act as susceptibility genes, but some have been associated with root aberrant phenotypes and others with female infertility. The MLO proteins involved in root response to mechanical stimuli group in clade I, which seems to be the most ancient embryophyte MLO clade [80]. It is tempting, then, to imagine that their ancestral role was to mediate root interaction with symbiotic organisms, like mycorrhizal fungi, important for land colonization. However, it was described that also species that do not produce true roots, like the moss Physcomitrella possess clade I MLOs [26].

It is known that HvMlo protein is able to remodel actin cytoskeleton upon PM contact with the cells [81, 82]. The cytoskeleton is a key factor in several processes including cell division and elongation, vesicle and organelle trafficking, adhesion and motility, and establishing polarity [83-85]. Cell polarity is essential for a wide range of biological processes in both unicellular and multicellular organisms [86]. Set-up of plant defense responses against pathogens, pollen tube reception, and root tip growth are processes that involve cell polarization and comprise characterized functions of MLO proteins described in Arabidopsis. Consequently, it is reasonable to imagine that the ancestral MLO proteins in the unicellular organisms were involved in cell polarity, for division, elongation, adhesion and motility. Then, when, the first embryophytes colonized the land, MLO proteins helped the emergence of roots. Later, with the development of gymnosperms, MLO proteins were involved in fertilization for seed development. One MLO-like sequence of the clade II (for pollen tube reception) is indeed present in the gymnosperm Picea abies [26].

It seems that to unravel the mystery of the core biochemical function of the MLO protein, results of researches on the cellular components of pollen tube reception, root development and PM resistance mechanisms will have to be synergistically combined to gain important insights. For example, the interaction between Receptor-like Kinases (RLK) and MLO proteins was highlighted in studies on pollen tube reception and in experiments showing genes coexpressed with $M L O$ during pathogen defense [87-89]. A functional connection between them, though, still has to be proven.

Our expertise concerns the PM resistance side. Therefore the set-up of suitable experiments could ultimately benefit this scientific discussion. Moreover, we mainly focus on using tomato, instead of Arabidopsis, as model species to carry out molecular research, because of the immediate translation of the results to other cultivated (Solanaceous) crops. 
As a starting point, it would be important to determine the expression of the tomato MLO genes in different conditions (both environmental and biotic stresses).

It is known that expression of barley HvMlo gene is induced upon inoculation with several pathogens, including its adapted PM Blumeria graminis f.sp. hordei, the non-adapted PM Blumeria graminis f.sp. tritici and the rice blast fungus, Magnaporte grisea, and upon leaf wounding and application of the herbicide paraquat [37]. Also in Arabidopsis, the expression of clade V-AtMLO genes is affected by biotic and/or abiotic stresses [78]. The tested biotic stresses comprised the adapted PM Erysiphe cichoracearum, Golovinomyces orontii, the hemibiotrophic oomycete pathogen Phytophthora infestans, the necrotrophic fungal pathogen Botrytis cinerea, and the bacterial pathogen Pseudomonas syringae. Among the abiotic stresses, Chen and collaborators tested cold, wounding, salt and osmotic stress. Barley mlo-mutants are more susceptible to the rice blast fungus M. grisea. Likewise, Arabidopsis mlo-mutants are more susceptible to $P$. infestans. Both pathogens are hemibiotroph and able to induce the expression of MLOs genes in their respective hosts [14, 53].

In tomato, we have so far verified the involvement of SIMLO1 in susceptibility towards the adapted PM pathogen Oidium neolycopersici and the other PM infecting tomato Leveillula taurica ([18]; Chapter 2). Thanks to the material we have generated, the expression and involvement of the other clade V-MLO genes could be tested with L. taurica, but also with pathogens having different infection styles. Provisory results show that expression of both wild-type SIMLO1 and impaired ol-2 allele are induced upon inoculation with adapted and non-adapted PM fungi like observed for barley HvMlo. However, contrary to the findings of Piffanelli et al. (2002) [37], in our experiment the wild-type SIMLO1 gene had a stronger induction than the ol-2 allele towards both PM fungi. It would be interesting to add in these experiments the novel EMS mutant allele, m200 (Chapter 7), to verify its expression and eventually correlate it with the resistant phenotype since it was hypothesized that the stronger the mutation, the more severe the phenotype [56].

With respect to abiotic stresses, the expression of SIMLO1 was also observed to be induced upon imposition of a mild level of salinity [90].

Together, all these studies confirm the involvement of the $M L O$ genes in various biological processes. Nevertheless, expanding the knowledge of what triggers their expressions can point towards those processes that obviously share similar molecular components. For this aim, mlo-mutants also have a central importance because they can assist the discovery of genes differentially expressed in wild-type and loss-of-function genotypes.

In barley, for example, the deployment of cDNA arrays already showed differential gene expression upon PM inoculation in mlo-mutants compared to a susceptible genotype [91, 92]. The plant membrane/signaling protein interaction network database is another tool that can serve as a resource for gene discovery (http://biodb.lumc.edu/mind/, Jones et al., 2014). 
Finally, in order to identify proteins interacting with MLOs, a complementary approach should be followed. The complication here is that the MLO protein is embedded in the plant membrane, so approaches like co-immunoprecipitation will most probably not work. Traditionally, the yeast two-hybrid approach represents the method of choice to unravel protein interaction partners. A recent paper showed its suitability also for cellwall proteins [93]. Nonetheless, since yeast two-hybrid screens are well known to produce false-positive results, verification of individual interaction partners by other approaches, preferentially in planta, is required. The fluorescence resonance energy transfer (FRET) microscopy, for example, allows monitoring protein-protein interactions in vivo and in real-time [94]. Since its introduction, the number of publications containing FRET as a keyword increased rapidly and continued to do so till now [95]. This technique was also very elegantly used in Bhat et al. (2005) [94] to show the dynamics of the interaction between barley HvMlo proteins (wild-type and impaired) with calmodulin (CaM) upon $B$. graminis f.sp. hordei inoculation.

The implementation of non-invasive imaging technologies, like FRET microscopy, with a large set of mlo-mutants, may be a step forward towards the characterization of the molecular mechanics of MLO-dependent fungal entry.

\section{Bird's-eye view of the SNARE proteins involved in plant-microbe interaction}

Eukaryotic cells are divided in several intracellular membranous compartments connected by vesicular traffic [96]. After vesicles bud off from a membrane, they must fuse with the correct target membrane. SNARE proteins (soluble $\underline{N}$-ethylmaleimide-sensitive factor attachment protein receptor) act as mediators of vesicle membrane fusion with specific organelles. Phylogenetic studies on SNAREs of animals, plants, protists, and fungi distinguished four main branches, based on specific motif profiles: Qa-, Qb-, Qc and R-SNARE $[97,98]$.

When a non-adapted pathogen is detected, pre-invasive defenses at the cell periphery are engaged as the first layer of protection. Members of the Qa- clade, also called syntaxins, are involved in this layer of plant immunity. Well-described syntaxins involved in PM resistance are found in this clade, such as Arabidopsis PEN1 (AtSYP121) and AtSYP122, barley ROR2, and grapevine VVPEN1 [99-101].

PEN1 and AtSYP122 have overlapping functions in growth and development because pen 1 syp122 double mutant plants are severely dwarfed and necrotic, but not the respective single mutants [102]. PEN1 has also a function in plant immunity because pen 1 mutants allow enhanced entry of non-adapted pathogens like Blumeria graminis f.sp. hordei (Bgh) and Erysiphe pisi into leaf epidermal cells [99]. The mutant syp122, on the other hand, shows only a slight increase (10\%) of Bgh penetration, which was not reproducible [94, 102]. 
Additionally, PEN1 is required for Atmlo2-based resistance since Atmlo2 pen 1 double mutants show elevated levels of G. cichoracearum (Go) cell entry [14]. However, following penetration, pathogen development is restricted by the increase of salicylic acid levels. This phenomenon is also observed in Arabidopsis double mutants of SYP42 and SYP43, two other Qa-SNARE proteins. The syp42 syp43 mutants exhibit pleiotropic developmental phenotypes (semi-dwarfism, short roots and a large number of lateral roots), reduced penetration resistance to non-adapted PM and extensive chlorosis upon infection with Go [103]. However, the level of susceptibility to this pathogen is macroscopically not altered. The chlorosis is dependent on the accumulation of high SA levels in response to powdery mildew challenge.

In Chapter 3 of this thesis, we identified in tomato two members of the Qa-SNARE group, designated SIPEN1 $a$ and SIPEN1b. Using the RNAi approach, both homologs were individually silenced in a tomato line harboring the impaired ol-2 allele; the transgenic plants obtained were challenged with the adapted PM O. neolycopersici (On) and nonadapted Bgh. We deduced that SIPEN1a has a major role in the mlo-based resistance in tomato because its silencing causes a significant increase of On growth. In addition, it is involved in non-host resistance because of the enhanced entry of Bgh in epidermal cells of silenced plants. The role of SIPEN1b gene could not be confirmed. Thus, we hypothesized that the difference in critical amino acids is responsible for the functional specialization. Protein alignment indicated that there are three non-synonymous substitutions in functional and non-functional tomato and Arabidopsis syntaxins that can play a role in plant defense specialization.

This explanation was also proposed for SYP122 and PEN1 [104]. To verify this hypothesis, the authors obtained a chimeric syntaxin by swapping the first 175 amino acids of the PEN1 N-terminal domain with the corresponding sequence of SYP122. The construct was then used to transform pen 1 and pen 1 syp122 mutants. They did not observe any difference in the level of Bgh entry rate, nor a rescue of the dwarfed double mutant phenotype, deducing that interplay between $\mathrm{N}$ - and $\mathrm{C}$-terminal regions is critical for the functionality of the respective proteins. If domain swap experiments are not useful in this context, a genome editing approach could target the sharp amino acid differences between SIPEN1 $a$ and SIPEN1 $b$ and verify the original hypothesis.

Interestingly, Arabidopsis PEN1 is largely accumulated in the lateral root cap [105]. The root cap is the section of tissue at the tip of a plant root, also called calyptra. It is involved in gravity perception in plants and possibly in communication with the soil microbiota [106]. Among the microbiota, root nodule-forming rhizobacteria and arbuscular mycorrhizal (AM) fungi are important beneficial microorganisms. The latter can colonize most land plants with few exceptions, such as Arabidopsis. It is, therefore, unusual that PEN1 is largely accumulated in the lateral root cap. 
There are other Qa- syntaxins expressed mostly in roots, such as the SYP123, SYP132, SYP31, and SYP32. Of these syntaxins, the SYP132 of the model legume Medicago truncatula, a homolog of AtSYP132, was recently shown to be localized not only to the plasma membrane surrounding the infection of the rhizobacteria but also abundantly to the membrane of the nitrogen-fixing nodule, called symbiosome. This finding suggests that MtSYP132 is involved in symbiosome formation $[107,108]$. Intriguingly, this contrasts with the plasma membrane syntaxin SYP132 of Nicotiana benthamiana that is thought to be involved in plant resistance against pathogenic bacteria Pseudomonas syringae [109]. In another model legume, Lotus japonicus, Qa-syntaxin SYP32-1, a homolog of AtSYP32, was shown to be required for differentiation of nodule tissues [110].

Another SNARE involved in plant-microbe interaction belongs to the Qc-clade, namely Arabidopsis SYP71. This protein was reported to be essential for successful turnip mosaic virus (TuMV) infection by mediating the fusion of the TuMV-induced vesicles with chloroplasts [111]. Its homolog in wheat, TaSYP71, is involved in resistance to the wheat stripe rust caused by P. striiformis f.sp. tritici [112]. Moreover, in Lotus japonicus, LjSYP71 is required for effective symbiotic nitrogen fixation [113].

These findings indicate that SNAREs are involved in general plant-microbes (damaging or beneficial) interactions. However, of the Qa-SNARE/syntaxin clade, only the Arabidopsis PEN1 (SYP121) and its homologs in barley, grapevine and tomato are so far shown to be involved in non-host as well as mlo-based resistance to PMs. To complete vesicle fusion, PEN1 will then form a tetrameric complex with the Qb,c-SNARE type protein, called SNAP-33, and an R-SNARE/vesicle-associated membrane protein, VAMP722 [110]. This mechanism ultimately leads to the formation of a papilla that stops PMs at the entry sites $[14,38,39,114]$.

The research in this thesis has shed light on a number of questions and has delivered new starting points for follow up research; among them, how pathogens hijack plant proteins and what are the mechanisms that allow the pathogens to use these proteins to their own advantage. 


\section{References}

1. Chakraborty S, Newton AC: Climate change, plant diseases and food security: an overview. Plant Pathology 2011, 60(1):2-14.

2. Glawe DA: The powdery mildews: a review of the world's most familiar (yet poorly known) plant pathogens. Annu Rev Phytopathol 2008, 46.

3. Garrett KA, Dendy SP, FrankEE, Rouse MN, Travers SE: Climate Change Effects on Plant Disease: Genomes to Ecosystems. Annual Review of Phytopathology 2006, 44(1):489-509.

4. Harvell CD, Mitchell CE, Ward JR, Altizer S, Dobson AP, Ostfeld RS, Samuel MD: Climate Warming and Disease Risks for Terrestrial and Marine Biota. Science 2002, 296(5576):2158.

5. Lebeda A, Mieslerová B, Jankovics T, Kiss L, Van der Linde EJ: First detection of tomato powdery mildew caused by Oidium neolycopersici in South Africa. South African Journal of Botany 2015, 99:153-157.

6. Tsay JG, Chen RS, Wang HL, Wang WL, Weng BC: First Report of Powdery Mildew Caused by Erysiphe diffusa, Oidium neolycopersici, and Podosphaera xanthii on Papaya in Taiwan. Plant Disease 2011, 95(9):1188-1188.

7. Brown JKM: Durable Resistance of Crops to Disease: A Darwinian Perspective. Annual Review of Phytopathology 2015, 53(1):513-539.

8. Jørgensen JH, Wolfe M: Genetics of Powdery Mildew Resistance in Barley. Critical Reviews in Plant Sciences 1994, 13(1):97-119.

9. Jensen HP, Christensen E, Jørgensen JH: Powdery Mildew Resistance Genes in 127 Northwest European Spring Barley Varieties. Plant Breeding 1992, 108(3):210-228.

10. Giese $\mathrm{H}$, JØRgensen $\mathrm{JH}$, Jensen $\mathrm{HP}$, Jensen J: Linkage relationships of ten powdery mildew resistance genes on barley chromosome 5. Hereditas 1981, 95(1):43-50.

11. Jørgensen $\mathrm{IH}$ : Discovery, characterization and exploitation of Mlo powdery mildew resistance in barley. Euphytica 1992, 63(1-2):141-152.

12. Büschges R, Hollricher K, Panstruga R, Simons G, Wolter M, Frijters A, Van Daelen R, Van der Lee T, Diergaarde $P$, Groenendijk J et al: The barley Mlo gene: A novel control element of plant pathogen resistance. Cell 1997, 88(5):695-705.

13. Berg J, Appiano M, Santillan Martinez M, Hermans F, Vriezen W, Visser R, Bai Y, Schouten H: A transposable element insertion in the susceptibility gene CsaMLO8 results in hypocotyl resistance to powdery mildew in cucumber. BMC Plant Biology 2015, 15(1):243.

14. Consonni C, Humphry ME, Hartmann HA, Livaja M, Durner J, Westphal L: Conserved requirement for a plant host cell protein in powdery mildew pathogenesis. Nat Genet 2006, 38.

15. Humphry M, Reinstädler A, Ivanov S, Bisseling T, Panstruga R: Durable broad-spectrum powdery mildew resistance in pea er1 plants is conferred by natural loss-of-function mutations in PsMLO1. Mol Plant Pathol 2011, 12.

16. Pavan S, Schiavulli A, Appiano M, Marcotrigiano AR, Cillo F, Visser RGF, Bai Y, Lotti C, Ricciardi L: Pea powdery mildew er 1 resistance is associated to loss-of-function mutations at a MLO homologous locus. Theoretical and Applied Genetics 2011, 123(8):1425-1431.

17. Wang Y, Cheng X, Shan Q, Zhang Y, Liu J, Gao C: Simultaneous editing of three homoeoalleles in hexaploid bread wheat confers heritable resistance to powdery mildew. Nat Biotechnol 2014, 32.

18. Zheng Z, Nonomura T, Appiano M, Pavan S, Matsuda Y, Toyoda H, Wolters AMA, Visser RGF, Bai Y: Loss of Function in Mlo Orthologs Reduces Susceptibility of Pepper and Tomato to Powdery Mildew Disease Caused by Leveillula taurica. PLOS ONE 2013, 8(7).

19. Pessina S, Angeli D, Martens S, Visser RGF, Bai Y, Salamini F, Velasco R, Schouten HJ, Malnoy M: The knockdown of the expression of MdMLO19 reduces susceptibility to powdery mildew (Podosphaera leucotricha) in apple (Malus domestica). Plant Biotechnol J 2016, 14(10):2033-2044.

20. Bai Y, Pavan S, Zheng Z, Zappel NF, Reinstädler A, Lotti C: Naturally occurring broad-spectrum powdery mildew resistance in a central American tomato accession is caused by loss of Mlo function. Mol Plant Microbe In 2008, 21. 
21. Pavan S, Jacobsen E, Visser RGF, Bai Y: Loss of susceptibility as a novel breeding strategy for durable and broad-spectrum resistance. Mol Breeding 2010, 25.

22. van Schie CCN, Takken FLW: Susceptibility Genes 101: How to Be a Good Host. Annual Review of Phytopathology 2014, 52(1):551-581.

23. Acevedo-Garcia J, Kusch S, Panstruga R: Magical mystery tour: MLO proteins in plant immunity and beyond. New Phytol 2014, 204(2):273-281.

24. Appiano M, Catalano D, Santillán Martínez M, Lotti C, Zheng Z, Visser RGF: Monocot and dicot MLO powdery mildew susceptibility factors are functionally conserved in spite of the evolution of classspecific molecular features. BMC Plant Biol 2015, 15.

25. Feechan A, Jermakow AM, Torregrosa L, Panstruga R, Dry IB: Identification of grapevine MLO gene candidates involved in susceptibility to powdery mildew. Functional Plant Biology 2008, 35(12):12551266.

26. Kusch S, Pesch L, Panstruga R: Comprehensive Phylogenetic Analysis Sheds Light on the Diversity and Origin of the MLO Family of Integral Membrane Proteins. Genome Biology and Evolution 2016, 8(3):878895.

27. Panstruga R: Discovery of novel conserved peptide domains by ortholog comparison within plant multi-protein families. Plant Molecular Biology 2005, 59(3):485-500.

28. Ablazov A, Tombuloglu H: Genome-wide identification of the mildew resistance locus $\mathbf{O}$ (MLO) gene family in novel cereal model species Brachypodium distachyon. Eur J Plant Pathol 2016, 145(2):239-253.

29. Devoto A, Hartmann HA, Piffanelli P, Elliott C, Simmons C, Taramino G: Molecular phylogeny and domainspecific co-evolution of the plant-specific seven transmembrane MLO family. J Mol Evol 2003, 56.

30. Iovieno P, Andolfo G, Schiavulli A, Catalano D, Ricciardi L, Frusciante L, Ercolano MR, Pavan S: Structure, evolution and functional inference on the Mildew Locus $O$ (MLO) gene family in three cultivated Cucurbitaceae spp. BMC Genomics 2015, 16(1):1-13.

31. Pessina S, Pavan S, Catalano D, Gallotta A, Visser R, Bai Y, Malnoy M, Schouten H: Characterization of the MLO gene family in Rosaceae and gene expression analysis in Malus domestica. BMC Genomics 2014, 15(1):618.

32. Schouten $\mathrm{H}$, Krauskopf J, Visser RF, Bai Y: Identification of candidate genes required for susceptibility to powdery or downy mildew in cucumber. Euphytica 2014, 200(3):475-486.

33. Sierro N, Battey JND, Ouadi S, Bakaher N, Bovet L, Willig A, Goepfert S, Peitsch MC, Ivanov NV: The tobacco genome sequence and its comparison with those of tomato and potato. Nat Commun 2014, 5.

34. Genome sequence and analysis of the tuber crop potato. Nature 2011, 475(7355):189-195.

35. Kaufmann H, Qiu X, Wehmeyer J, Debener T: Isolation, Molecular Characterization, and Mapping of Four Rose MLO Orthologs. Frontiers in Plant Science 2012, 3:244.

36. Fujimura T, Sato S, Tajima T, Arai M: Powdery mildew resistance in the Japanese domestic tobacco cultivar Kokubu is associated with aberrant splicing of MLO orthologues. Plant Pathology 2016, 65(8):1358-1365.

37. Piffanelli P, Zhou F, Casais C, Orme J, Jarosch B, Schaffrath U, Collins NC, Panstruga R, Schulze-Lefert P: The barley MLO modulator of defense and cell death is responsive to biotic and abiotic stress stimuli. 2002, 129(3):1076-1085.

38. Bai Y, van der Hulst R, Bonnema G, Marcel TC, Meijer-Dekens F, Niks RE, Lindhout P: Tomato Defense to Oldium neolycopersici: Dominant OI Genes Confer Isolate-Dependent Resistance Via a Different Mechanism Than Recessive ol-2. Molecular Plant-Microbe Interactions 2005, 18(4):354-362.

39. Buschges R, Hollricher K, Panstruga R, Simons G, Wolter M, Frijters A: The barley mlo gene: a novel control element of plant pathogen resistance. Cell 1997, 88.

40. Elliott C, Müller J, Miklis M, Bhat RA, Schulze-Lefert P, Panstruga R: Conserved extracellular cysteine residues and cytoplasmic loop-loop interplay are required for functionality of the heptahelical MLO protein. Biochem J 2005, 385.

41. Elliott C, Zhou F, Spielmeyer W, Panstruga R, Schulze-Lefert P: Functional conservation of wheat and rice Mlo orthologs in defense modulation to the powdery mildew fungus. Molecular Plant-Microbe Interactions 2002, 15(10):1069-1077. 
42. Nie J, Wang Y, He H, Guo C, Zhu W, Pan J, Li D, Lian H, Pan J, Cai R: Loss-of-Function Mutations in CsMLO1 Confer Durable Powdery Mildew Resistance in Cucumber (Cucumis sativus L.). Frontiers in Plant Science 2015, 6:1155.

43. Delventhal $\mathrm{R}$, Zellerhoff N, Schaffrath U: Barley stripe mosaic virus-induced gene silencing (BSMV-IGS) as a tool for functional analysis of barley genes potentially involved in nonhost resistance. Plant Signaling \& Behavior 2011, 6(6):867-869.

44. Schijlen EGWM, de Vos CHR, Martens S, Jonker HH, Rosin FM, Molthoff JW, Tikunov YM, Angenent GC, van Tunen AJ, Bovy AG: RNA Interference Silencing of Chalcone Synthase, the First Step in the Flavonoid Biosynthesis Pathway, Leads to Parthenocarpic Tomato Fruits. Plant Physiology 2007, 144(3):15201530.

45. Várallyay É, Giczey G, Burgyán J: Virus-induced gene silencing of Mlo genes induces powdery mildew resistance in Triticum aestivum. Archives of Virology 2012, 157(7):1345-1350.

46. Burch-Smith TM, Anderson JC, Martin GB, Dinesh-Kumar SP: Applications and advantages of virusinduced gene silencing for gene function studies in plants. The Plant Journal 2004, 39(5):734-746.

47. Xiong J-S, Ding J, Li Y: Genome-editing technologies and their potential application in horticultural crop breeding. Horticulture Research 2015, 2:15019.

48. Boch J, Scholze H, Schornack S, Landgraf A, Hahn S, Kay S, Lahaye T, Nickstadt A, Bonas U: Breaking the code of DNA binding specificity of TAL-type III effectors. Science 2009, 326.

49. Jinek M, Chylinski K, Fonfara I, Hauer M, Doudna JA, Charpentier E: A Programmable Dual-RNA-Guided DNA Endonuclease in Adaptive Bacterial Immunity. Science 2012, 337(6096):816.

50. Kim YG, Cha J, Chandrasegaran S: Hybrid restriction enzymes: zinc finger fusions to Fok I cleavage domain. Proc Natl Acad Sci USA 1996, 93.

51. Consonni C, Humphry ME, Hartmann HA, Livaja M, Durner J, Westphal L, Vogel J, Lipka V, Kemmerling $\mathrm{B}$, Schulze-Lefert $\mathrm{P}$ et al: Conserved requirement for a plant host cell protein in powdery mildew pathogenesis. Nature Genetics 2006, 38(6):716-720.

52. Peterhansel C, Freialdenhoven A, Kurth J, Kolsch R, Schulze-Lefert P: Interaction Analyses of Genes Required for Resistance Responses to Powdery Mildew in Barley Reveal Distinct Pathways Leading to Leaf Cell Death. The Plant Cell 1997, 9(8):1397-1409.

53. Jarosch B, Kogel K-H, Schaffrath U: The Ambivalence of the Barley Mlo Locus: Mutations Conferring Resistance Against Powdery Mildew (Blumeria graminis f. sp. hordei) Enhance Susceptibility to the Rice Blast Fungus Magnaporthe grisea. Molecular Plant-Microbe Interactions 1999, 12(6):508-514.

54. Kumar J, Hückelhoven R, Beckhove U, Nagarajan S, Kogel K-H: A Compromised Mlo Pathway Affects the Response of Barley to the Necrotrophic Fungus Bipolaris sorokiniana (Teleomorph: Cochliobolus sativus) and Its Toxins. Phytopathology 2001, 91(2):127-133.

55. McGrann GRD, Stavrinides A, Russell J, Corbitt MM, Booth A, Chartrain L, Thomas WTB, Brown JKM: A trade off between mlo resistance to powdery mildew and increased susceptibility of barley to a newly important disease, Ramularia leaf spot. Journal of Experimental Botany 2014, 65(4):1025-1037.

56. Ge X, Deng W, Lee ZZ, Lopez-Ruiz FJ, Schweizer P, Ellwood SR: Tempered mlo broad-spectrum resistance to barley powdery mildew in an Ethiopian landrace. Scientific Reports 2016, 6:29558.

57. Sun S, Deng D, Wang Z, Duan C, Wu X, Wang X, Zong X, Zhu Z: A novel er1 allele and the development and validation of its functional marker for breeding pea (Pisum sativum L.) resistance to powdery mildew. Theoretical and Applied Genetics 2016, 129(5):909-919.

58. Qi J, Liu X, Shen D, Miao H, Xie B, Li X, Zeng P, Wang S, Shang Y, Gu X et al: A genomic variation map provides insights into the genetic basis of cucumber domestication and diversity. Nat Genet 2013, 45(12):1510-1515.

59. The 100 Tomato Genome Sequencing C, Aflitos S, Schijlen E, de Jong H, de Ridder D, Smit S, Finkers R, Wang J, Zhang G, Li N et al: Exploring genetic variation in the tomato (Solanum section Lycopersicon) clade by whole-genome sequencing. The Plant Journal 2014, 80(1):136-148.

60. Oladosu Y, Rafii MY, Abdullah N, Hussin G, Ramli A, Rahim HA, Miah G, Usman M: Principle and application of plant mutagenesis in crop improvement: a review. Biotechnology \& Biotechnological Equipment 2016, 30(1):1-16. 
61. Acquaah G: Plant Genetic Resources. In: Principles of Plant Genetics and Breeding. John Wiley \& Sons, Ltd; 2012: 199-225.

62. McCallum CM, Comai L, Greene EA, Henikoff S: Targeting Induced Local Lesions IN Genomes (TILLING) for Plant Functional Genomics. Plant Physiology 2000, 123(2):439-442.

63. Chen L, Hao L, Parry MAJ, Phillips AL, Hu Y-G: Progress in TILLING as a tool for functional genomics and improvement of crops. Journal of Integrative Plant Biology 2014, 56(5):425-443.

64. Acevedo-Garcia J, Spencer D, Thieron H, Reinstädler A, Hammond-Kosack K, Phillips AL, Panstruga R: mlobased powdery mildew resistance in hexaploid bread wheat generated by a non-transgenic TILLING approach. Plant Biotechnol J 2016:n/a-n/a.

65. Jiang $P$, Chen $Y$, Wilde HD: Identification and mutagenesis of disease susceptibility genes of Petunia hybrida. Plant Cell, Tissue and Organ Culture (PCTOC) 2016, 126(1):117-125.

66. Pavan S, Schiavulli A, Appiano M, Marcotrigiano AR, Cillo F, Visser RG: Pea powdery mildew er1 resistance is associated to loss-of-function mutations at a MLO homologous locus. Theor and Appl Genet 2011, 123.

67. Reinstädler A, Muller J, Czembor JH, Piffanelli P, Panstruga R: Novel induced mlo mutant alleles in combination with site directed mutagenesis reveal functionally important domains in the heptahelical barley Mlo protein. BMC Plant Biol 2010, 10.

68. Santo T, Rashkova M, Alabaça $C$, Leitão J: The ENU-induced powdery mildew resistant mutant pea (Pisum sativum L.) lines S(er1mut1) and F(er1mut2) harbour early stop codons in the PsMLO1 gene. Molecular Breeding 2013, 32(3):723-727.

69. Elliott C, Müller J, Miklis M, Bhat RA, Schulze-Lefert P, Panstruga R: Conserved extracellular cysteine residues and cytoplasmic loop-loop interplay are required for functionality of the heptahelical MLO protein. Biochemical Journal 2005, 385(1):243-254.

70. Devoto A, Piffanelli P, Nilsson I, Wallin E, Panstruga R, Von Heijne G, Schulze-Lefert P: Topology, subcellular localization, and sequence diversity of the Mlo family in plants. Journal of Biological Chemistry 1999, 274(49):34993-35004.

71. Plumpton M, Barnes MR: Predictive Functional Analysis of Polymorphisms: An Overview. In: Bioinformatics for Geneticists. John Wiley \& Sons, Ltd; 2007: 247-280.

72. Palczewski K, Kumasaka T, Hori T, Behnke CA, Motoshima H, Fox BA, Trong IL, Teller DC, Okada T, Stenkamp RE et al: Crystal Structure of Rhodopsin: A G Protein-Coupled Receptor. Science 2000, 289(5480):739.

73. Kim MC, Panstruga R, Elliott C, Muller J, Devoto A, Yoon HW, Park HC, Cho MJ, Schulze-Lefert P: Calmodulin interacts with MLO protein to regulate defence against mildew in barley. Nature 2002, 416(6879):447451.

74. Lorek J, Griebel T, Jones AM, Kuhn H, Panstruga R: The Role of Arabidopsis Heterotrimeric G-Protein Subunits in MLO2 Function and MAMP-Triggered Immunity. Molecular Plant-Microbe Interactions 2013, 26(9):991-1003.

75. Urano $D$, Chen J-G, Botella JR, Jones AM: Heterotrimeric G protein signalling in the plant kingdom. Open Biology 2013, 3(3).

76. Müller J, Piffanelli P, Devoto A, Miklis M, Elliott C, Ortmann B, Schulze-Lefert P, Panstruga R: Conserved ERAD-like quality control of a plant polytopic membrane protein. 2005, 17(1):149-163.

77. Biot-Pelletier D, Martin VJJ: Seamless site-directed mutagenesis of the Saccharomyces cerevisiae genome using CRISPR-Cas9. Journal of Biological Engineering 2016, 10:6.

78. Chen Z, Hartmann HA, Wu MJ, Friedman EJ, Chen JG, Pulley M, Schulze-Lefert P, Panstruga R, Jones AM: Expression analysis of the AtMLO gene family encoding plant-specific seven-transmembrane domain proteins. Plant Molecular Biology 2006, 60(4):583-597.

79. Laufer $\mathrm{BI}$, Singh SM: Strategies for precision modulation of gene expression by epigenome editing: an overview. Epigenetics \& Chromatin 2015, 8(1):1-12.

80. Jiwan $D$, Roalson $E H$, Main $D$, Dhingra A: Antisense expression of peach mildew resistance locus 0 (PpMlo1) gene confers cross-species resistance to powdery mildew in Fragaria $x$ ananassa. Transgenic Res 2013, 22(6):1119-1131. 
81. Miklis M, Consonni C, Bhat RA, Lipka V, Schulze-Lefert P, Panstruga R: Barley MLO Modulates ActinDependent and Actin-Independent Antifungal Defense Pathways at the Cell Periphery. Plant Physiology 2007, 144(2):1132-1143.

82. Opalski KS, Schultheiss H, Kogel KH, Hückelhoven R: The receptor-like MLO protein and the RAC/ROP family G-protein RACB modulate actin reorganization in barley attacked by the biotrophic powdery mildew fungus Blumeria graminis f.sp. hordei. Plant Journal 2005, 41(2):291-303.

83. Mathur J, Hülskamp M: Microtubules and Microfilaments in Cell Morphogenesis in Higher Plants. Current Biology, 12(19):R669-R676.

84. Staiger CJ: SIGNALING TO THE ACTIN CYTOSKELETON IN PLANTS. Annual Review of Plant Physiology and Plant Molecular Biology 2000, 51(1):257-288.

85. Wasteneys GO, Galway ME: REMODELING THE CYTOSKELETON FOR GROWTH AND FORM: An Overview with Some New Views. Annual Review of Plant Biology 2003, 54(1):691-722.

86. Dettmer J, Friml J: Cell polarity in plants: when two do the same, it is not the same. Current Opinion in Cell Biology 2011, 23(6):686-696.

87. Humphry M, Bednarek P, Kemmerling B, Koh S, Stein M, Göbel U, Stüber K, Piślewska-Bednarek M, Loraine A, Schulze-Lefert $P$ et al: A regulon conserved in monocot and dicot plants defines a functional module in antifungal plant immunity. Proceedings of the National Academy of Sciences of the United States of America 2010, 107(50):21896-21901.

88. Kessler SA, Shimosato-Asano H, Keinath NF, Wuest SE, Ingram G, Panstruga R, Grossniklaus U: Conserved molecular components for pollen tube reception and fungal invasion. Science 2010, 330(6006):968971.

89. Lalonde S, Sero A, Pratelli R, Pilot G, Chen J, Sardi MI, Parsa SA, Kim D-Y, Acharya BR, Stein EV et al: A Membrane Protein/Signaling Protein Interaction Network for Arabidopsis Version AMPv2. Frontiers in Physiology 2010, 1:24.

90. Kissoudis C, van de Wiel C, Visser RGF, van der Linden G: Future-proof crops: challenges and strategies for climate resilience improvement. Current Opinion in Plant Biology 2016, 30:47-56.

91. Gjetting T, Hagedorn PH, Schweizer P, Thordal-Christensen H, Carver TLW, Lyngkjær MF: Single-Cell Transcript Profiling of Barley Attacked by the Powdery Mildew Fungus. Molecular Plant-Microbe Interactions 2007, 20(3):235-246.

92. Zierold UWE, Scholz UWE, Schweizer P: Transcriptome analysis of mlo-mediated resistance in the epidermis of barley. Molecular Plant Pathology 2005, 6(2):139-151.

93. Trujillo M, Troeger M, Niks RE, Kogel K-H, HÜCkelhoven R: Mechanistic and genetic overlap of barley host and non-host resistance to Blumeria graminis. Molecular Plant Pathology 2004, 5(5):389-396.

94. Bhat RA, Miklis M, Schmelzer E, Schulze-Lefert P, Panstruga R: Recruitment and interaction dynamics of plant penetration resistance components in a plasma membrane microdomain. Proceedings of the National Academy of Sciences of the United States of America 2005, 102(8):3135-3140.

95. Xing S, Wallmeroth N, Berendzen KW, Grefen C: Techniques for the Analysis of Protein-Protein Interactions in Vivo. Plant Physiology 2016, 171(2):727-758.

96. Beest MBAt, Chapin SJ, Avrahami D, Mostov KE: The Role of Syntaxins in the Specificity of Vesicle Targeting in Polarized Epithelial Cells. Molecular Biology of the Cell 2005, 16(12):5784-5792.

97. Bock JB, Matern HT, Peden AA, Scheller RH: A genomic perspective on membrane compartment organization. Nature 2001, 409(6822):839-841.

98. Kloepper TH, Kienle CN, Fasshauer D: An Elaborate Classification of SNARE Proteins Sheds Light on the Conservation of the Eukaryotic Endomembrane System. Molecular Biology of the Cell 2007, 18(9):34633471.

99. Collins NC, Thordal-Christensen H, Lipka V, Bau S, Kombrink E, Qiu J-L, Huckelhoven R, Stein M, Freialdenhoven A, Somerville SC et al: SNARE-protein-mediated disease resistance at the plant cell wall. Nature 2003, 425(6961):973-977.

100. Feechan A, Jermakow AM, Ivancevic A, Godfrey D, Pak H, Panstruga R, Dry IB: Host Cell Entry of Powdery Mildew Is Correlated with Endosomal Transport of Antagonistically Acting VvPEN1 and VvMLO to the Papilla. Molecular Plant-Microbe Interactions 2013, 26(10):1138-1150. 
101. Hückelhoven R, Fodor J, Trujillo M, Kogel K-H: Barley Mla and Rar mutants compromised in the hypersensitive cell death response against Blumeria graminis f.sp. hordei are modified in their ability to accumulate reactive oxygen intermediates at sites of fungal invasion. Planta 2000, 212(1):16-24.

102. Assaad FF, Qiu J-L, Youngs H, Ehrhardt D, Zimmerli L, Kalde M, Wanner G, Peck SC, Edwards H, Ramonell K et al: The PEN1 Syntaxin Defines a Novel Cellular Compartment upon Fungal Attack and Is Required for the Timely Assembly of Papillae. Molecular Biology of the Cell 2004, 15(11):5118-5129.

103. Uemura T, Kim H, Saito C, Ebine K, Ueda T, Schulze-Lefert P, Nakano A: Qa-SNAREs localized to the transGolgi network regulate multiple transport pathways and extracellular disease resistance in plants. Proceedings of the National Academy of Sciences of the United States of America 2012, 109(5):1784-1789.

104. Pajonk S, Kwon C, Clemens N, Panstruga R, Schulze-Lefert P: Activity Determinants and Functional Specialization of Arabidopsis PEN1 Syntaxin in Innate Immunity. Journal of Biological Chemistry 2008, 283(40):26974-26984.

105. Lipka V, Kwon C, Panstruga R: SNARE-Ware: The Role of SNARE-Domain Proteins in Plant Biology. Annual Review of Cell and Developmental Biology 2007, 23(1):147-174.

106. Raven JA, Edwards D: Roots: evolutionary origins and biogeochemical significance. Journal of Experimental Botany 2001, 52(suppl 1):381-401.

107. Catalano CM, Czymmek KJ, Gann JG, Sherrier DJ: Medicago truncatula syntaxin SYP132 defines the symbiosome membrane and infection droplet membrane in root nodules. Planta 2007, 225(3):541550.

108. Limpens E, Ivanov S, van Esse W, Voets G, Fedorova E, Bisseling T: Medicago N(2)-Fixing Symbiosomes Acquire the Endocytic Identity Marker Rab7 but Delay the Acquisition of Vacuolar Identity. The Plant Cell 2009, 21(9):2811-2828.

109. Kalde M, Nühse TS, Findlay K, Peck SC: The syntaxin SYP132 contributes to plant resistance against bacteria and secretion of pathogenesis-related protein 1. Proceedings of the National Academy of Sciences of the United States of America 2007, 104(28):11850-11855.

110. Mai HT, Nomura M, Takegawa K, Asamizu E, Sato S, Kato T, Tabata S, Tajima S: Identification of a Sed5-like SNARE Gene LjSYP32-1 that Contributes to Nodule Tissue Formation of Lotus japonicus. Plant and Cell Physiology 2006, 47(7):829-838.

111. Wei T, Zhang C, Hou X, Sanfaçon H, Wang A: The SNARE Protein Syp71 Is Essential for Turnip Mosaic Virus Infection by Mediating Fusion of Virus-Induced Vesicles with Chloroplasts. PLoS Pathog 2013, 9(5):e1003378.

112. Liu M, Peng Y, Li H, Deng L, Wang X, Kang Z: TaSYP71, a Qc-SNARE, Contributes to Wheat Resistance against Puccinia striiformis f. sp. tritici. Frontiers in Plant Science 2016, 7:544.

113. Hakoyama T, Oi R, Hazuma K, Suga E, Adachi Y, Kobayashi M, Akai R, Sato S, Fukai E, Tabata S et al: The SNARE Protein SYP71 Expressed in Vascular Tissues Is Involved in Symbiotic Nitrogen Fixation in Lotus japonicus Nodules. Plant Physiology 2012, 160(2):897-905.

114. Freialdenhoven A, Peterhansel C, Kurth J, Kreuzaler F, Schulze-Lefert P: Identification of Genes Required for the Function of Non-Race-Specific mlo Resistance to Powdery Mildew in Barley. The Plant Cell 1996, 8(1):5-14.

115. Blanvillain-Baufumé S, Reschke M, Solé M, Auguy F, Doucoure H, Szurek B, Meynard D, Portefaix M, Cunnac S, Guiderdoni E, Boch J, Koebnik R: Targeted promoter editing for rice resistance to Xanthomonas oryzae pv. oryzae reveals differential activities for SWEET14-inducing TAL effectors. Plant Biotechnology Journal 2016, doi: 10.1111/pbi.12613 



\section{Summary}

Samenvatting Acknowledgements About the author List of publications 



\section{Summary}

Powdery mildew (PM) is a worldwide-occurring plant disease caused by ascomycete fungi of the order Erysiphales. A conspicuous number of plant species are susceptible to this disease, the occurrence of which is increasing due to the influence of climate change. Symptoms are easy to recognize by the powdery whitish fungal structures growing on the surface of plant organs. Severe infections cause significant losses in crops, such as tomato, cucumber and wheat, as well as in ornamentals, like rose and petunia. Accordingly, breeding crops with a robust immunity to this disease is of great economic importance.

A significant step in this direction was the discovery of mlo (mildew locus o) mutant alleles of the barley HvMlo gene, which are responsible for the non-race specific resistance to the barley PM pathogen, Blumeria graminis f.sp. hordei (Bgh). During the years, this recessively inherited resistance was observed to be durable, contrary to the short life-span of resistances conferred by dominant resistance ( $R$-) genes used in barley breeding programs. Studies on the histological mechanisms of the mlo-based resistance showed that the PM pathogen was stopped during penetration of the cell wall by the formation of a papilla. This structure prevents the formation of the feeding structure of the pathogen, called a haustorium.

After sequencing many plant genomes, we are discovering that $M L O$ genes are not only typical of this cereal, but are ubiquitously present in higher plant species in multiple copies per species, forming a gene family. The impairment of some members of a number of ever increasing plant species lead to broad-spectrum resistance towards their adapted PM pathogens. For example, in tomato the ol-2 gene, naturally harbored by the cherry tomato Solanum lycopersicum var. cerasiforme, represents the loss-of-function allele of the SIMLO1 gene, conferring resistance to the PM pathogen Oidium neolycopersici (On). Consequently, the use of mlo mutants represents a suitable alternative to the classical use of R-genes in breeding programs.

In Chapter 2, we describe the in silico identification of the complete tomato SIMLO gene family using the available information in the SOL genomic network database. In total, 16 tomato SIMLO members were cloned from leaf, root, flower and fruit of the susceptible tomato cv. Moneymaker to confirm the sequences retrieved from the database and to verify their actual expression in these tissues. We observed the presence of various types of splicing variants, although their possible functional meaning has not been investigated. Motif analyses of each of the translated protein sequences and phylogenetic studies highlighted, on one hand, amino acid stretches that characterize the whole MLO family, and, on the other hand, stretches conserved in MLO homologs that are phylogenetically related. Following a gene expression study upon On inoculation, we identified members of the SIMLO family that are upregulated few hours after pathogen challenge. Except SIMLO1, none of the three newly identified homologs in clade V, thus phylogenetically close to SIMLO1, are induced. Interestingly, two homologs, each found in different clades, are upregulated similarly to 
SIMLO1. Using an RNAi approach, we silenced the additional clade V-SIMLO homologs, namely SIMLO3, SIMLO5 and SIMLO8, to investigate their possible role in PM resistance. We observed that none of these homologs if individually silenced, leads to PM resistance. However, if SIMLO5 and SIMLO8 are silenced together with SIMLO1, a significantly higher level of resistance is achieved compared to plants carrying the ol-2 allele. The role of SIMLO3 could not be verified. We, therefore, concluded that there are three SIMLO genes in tomato unevenly contributing to the PM disease, of which SIMLO1 has a major role.

Chapter $\mathbf{3}$ focuses on the components of the tomato mlo-based resistance. In Arabidopsis, it is known that four members of the SNARE protein family, involved in membrane fusion, are involved in mlo-based resistance. In this chapter, we focused on the identification of tomato homologs of the Arabidopsis syntaxin PEN1 (AtSYP121). Among the group of syntaxins identified in tomato, two were closely related to each other and also to AtPEN1, denominated SIPEN1a and SIPEN1b. Another Arabidopsis syntaxin that shows a high level of homology with PEN1, called SYP122, was also found to group together with the newly identified SIPEN1 genes. However, the role of SYP122 in plant immunity was not shown in literature. After obtaining individual silencing RNAi constructs, we transformed the resistant ol-2 line, and we challenged the obtained transformants with the adapted PM On, and the non-adapted Bgh. Interestingly, we observed a significant On growth and an enhanced Bgh cell entry only in SIPEN1 a silenced plants but not in SIPEN1b silenced ones. We performed a protein alignment of tomato and Arabidopsis functional and non-functional PEN sequences. The presence of three differently conserved non-synonymous amino-acid substitutions is hypothesised to be responsible for the specialization in plant immune function.

In Chapter 4 and Chapter 5, we build up a body of evidence pointing to the fact that the function of the MLO susceptibility genes is highly conserved between monocot and dicot plant species.

In Chapter 4 we started by identifying and functionally characterizing two new MLO genes of Solanaceous crops affected by the PM disease, tobacco (Nicotiana tabacum) and eggplant (Solanum melongena). We named them NtMLO1 and SmMLO1 in the respective species, as they are the closest homologs to tomato SIMLO1. By overexpressing these genes in the resistant ol-2 line, we obtained transgenic plants that were susceptible to the PM pathogen On. This finding demonstrates that both heterologous MLO proteins can rescue the function of the impaired ol-2 allele in tomato. In addition, we found in tobacco NtMLO1 an amino acid (Q198) of critical importance for the susceptibility function of this protein.

In Chapter 5, we used the same approach adopted in Chapter 4 to show that other MLO proteins of more distant dicot species, like pea PsMLO1, can rescue the loss-of-function of the tomato ol-2 allele. And finally, we stretched this concept also to monocot MLO proteins, using barley HvMlo. While performing these experiments, we could verify that the function of the monocot and dicot susceptibility MLO proteins does not rely on the presence of class- 
specific conservation. The latter can be the reason for the phylogenetic divergence, placing monocot MLO proteins in clade IV and dicot MLO proteins in clade V of the phylogenetic MLO tree. However, functional conservation might depend on crucial shared amino acids of clade IV and V MLO proteins. Therefore, we also conducted a codon-based evolutionary analysis that resulted in the identification of 130 codons under negative selection, thus strongly maintained during evolution.

In Chapter $\mathbf{6}$ we introduce the PM disease in cucumber caused by Podosphaera xanthii (Px). We cloned the candidate susceptibility gene for PM in cucumber, CsaMLO8, from susceptible and resistant genotypes. The latter was described as an advanced cucumber breeding line characterized by hypocotyl resistance. In this line, we found the presence of aberrant splicing variants of the CsaMLO8 mRNA due to the insertion in its corresponding genomic region of a Class LTR retrotransposon. Heterologous expression of the wild-type cucumber allele in the tomato ol-2 line restored its PM susceptibility, while the heterologous expression of the aberrant protein variant failed to do so. This finding confirms that the resistance of the advanced cucumber breeding line is due to the disruption of the coding region of this gene. We also showed that the expression of CsaMLO8 in the susceptible genotype is induced by $P x$ in hypocotyl tissue, but not in cotyledon or leaf. Finally, by examination of the resequencing data of a collection of 115 cucumber accessions, we found the presence of the TE-containing allele in 31 of them among which a wild cucumber accession that might have been used in breeding programs to obtain resistance to the PM disease in cucumber.

In Chapter 7 a novel loss-of-function allele of the SIMLO1 gene is described, designated $m 200$. This allele was found in a resistant plant (M200) from a mutagenized tomato MicroTom (MT) population obtained with the chemical mutagen ethyl methanesulfonate (EMS). The $m 200$ mutation corresponds to a nucleotide transversion $(T \rightarrow A$ ) which results in a premature stop codon. The length of the predicted SIMLO1 protein in the M200 plant is only 21 amino acids, thus much shorter than the predicted protein of the previously described ol-2 allele, consisting of 200 amino acids. Thanks to the development of a High-Resolution Melting (HRM) marker designed to detect the $m 200$ mutation, we observed that this allele confers recessively inherited resistance in backcross populations of the resistant M200 plant with MT and Moneymaker. Histological study showed that the resistance of the $m 200$ mutant is associated with papilla formation. Finally, we compared the rate of On penetration in epidermal cells of $m 200$ plants with the one of plants carrying the ol-2 allele and the transgenic plants in which multiple SIMLO homologs were silenced, generated in Chapter 2. Ultimately, in Chapter $\mathbf{8}$ the results of the previous chapters are discussed in the context of 1) practical applications in breeding programs aimed at introducing the mlo-based resistance in new crops, 2) possible research aimed at unraveling the function of the MLO protein and 3) the role of other SNARE proteins. 



\section{Samenvatting}

Echte meeldauw is een wereldwijd voorkomende plantziekte, veroorzaakt door schimmels uit de stam Ascomycota, de orde Erysiphales. Een opvallend aantal plantsoorten is vatbaar voor deze ziekte, en het aantal uitbraken van deze ziekte neemt verder toe door klimaatverandering. Symptomen van de ziekte zijn makkelijk te herkennen door de witte, poederachtige schimmelstructuren die op de buitenkant van de organen van de geïnfecteerde plant groeien. Heftige infecties leiden tot aanzienlijke verliezen in zowel voedingsgewassen zoals tomaat, komkommer en tarwe, maar ook in siergewassen als roos en petunia. Daarom is het van groot economisch belang om gewassen zo te veredelen dat ze een robuuste immuniteit tegen deze ziekte verwerven.

Een belangrijke stap in deze richting was de ontdekking van mlo (meeldauw locus o) mutant allelen van het gen HvMlo in gerst, die leiden tot niet ras-specifieke weerstand tegen Blumeria graminis f.sp. hordei (Bgh), de veroorzaker van meeldauw in gerst. In de loop der jaren werd ontdekt dat deze recessief overervende resistentie duurzaam bleek, in tegenstelling tot de korte levensspanne van meeldauwresistentie veroorzaakt door dominant overervende resistentie (R-) genen die in veredelingsprogramma's van gerst gebruikt werden. Studies naar de histologische mechanismen van op mlo gebaseerde resistentie wezen uit dat de infectie werd gestopt tijdens de penetratie van de celwand van de plant, door het vormen van een papilla. Deze structuur voorkomt het vormen van een haustorium, het orgaan van de ziekteverwekker waarmee de schimmel voedingsstoffen op kan nemen.

Nu het genoom van veel plantsoorten in kaart is gebracht, ontdekken we dat MLO genen niet iets typisch van dit graangewas zijn, maar alomtegenwoordig aanwezig zijn in hogere planten, met meerdere kopieën per plantsoort die samen een genfamilie vormen. Beschadigde versies van sommige genen in deze familie leiden in een toenemend aantal plantsoorten tot weerstand tegen hun respectievelijke meeldauwpathogenen. Het ol-2 gen in tomaat, dat van nature voorkomt in kerstomaat (Solanum lycopersicum var. cerasiforme), vertegenwoordigt bijvoorbeeld een verlies-van-functie allel van het gen SIMLO1, en geeft hierdoor weerstand tegen de meeldauwpathogeen Oidium neolycopersici $(\mathrm{On})$. Derhalve is het gebruik van mlo mutanten een geschikt alternatief voor de klassieke R-genen in veredelingsprogramma's.

In Hoofdstuk 2 beschrijven we de in silico identificatie van de complete tomaat SIMLO genfamilie, waarbij we gebruik maken van de beschikbare informatie in de "SOL genomic network database". We kloneerden in totaal 16 tomaat SIMLO genen uit bladeren, wortels, bloemen en vruchten van de vatbare tomaat cultivar "Moneymaker", om de sequenties uit de databank te bevestigen, en te controleren of deze genen inderdaad tot expressie komen in deze weefsels. We observeerden de aanwezigheid van verscheidene typen van alternatieve splicing, mogelijke functionele gevolgen hiervan hebben we echter niet 
bestudeerd. Analyses van motieven in de getranslateerde eiwitsequenties en fylogenetisch onderzoek markeerden enerzijds groepen van aminozuren die karakteristiek zijn voor de gehele MLO familie, maar anderzijds ook groepen van aminozuren die specifiek geconserveerd zijn tussen MLO homologen die fylogenetisch nauw aan elkaar verwant zijn. Naar aanleiding van onderzoek naar genexpressie in weefsels geïnoculeerd met On identificeerden we leden van de SIMLO familie waarvan de genexpressie enkele uren na de inoculatie opgereguleerd werd. Behalve SIMLO1 werd geen van de drie nieuwe homologen in klade V, die dus fylogenetisch nauw aan SIMLO1 verwant zijn, geïnduceerd. Interessant genoeg werden wel twee andere homologen, in verschillende klades, geïnduceerd op vergelijkbaar niveau met SIMLO1. Met een RNAi aanpak hebben we de transcriptie van de andere klade V-SIMLO homologen (SIMLO3, SIMLO5 en SIMLO8) stilgelegd, om hun mogelijke rol in meeldauwresistentie te onderzoeken. We observeerden dat bij geen van deze homologen het stilleggen van de transcriptie tot meeldauwresistentie leid, mits één homoloog tegelijk stilgelegd werd. Als daarentegen de transcriptie van zowel SIMLO5, SIMLO8 als SIMLO1 tegelijkertijd stilgelegd wordt kan een merkbaar hoger niveau van resistentie bereikt worden dan dat in planten met het ol-2 allel. Een rol van SIMLO3 kon niet worden geverifieerd. We concludeerden daarom dat er drie SIMLO genen, op ongelijke wijze, bijdragen aan de meeldauwziekte, waarvan SIMLO1 de belangrijkste rol heeft.

De focus van Hoofdstuk 3 ligt op de componenten van op mlo gebaseerde resistentie in tomaat. Het is bekend dat in Arabidopsis vier leden van de SNARE eiwitfamilie, die betrokken zijn bij fusie van membranen, betrokken zijn bij op mlo gebaseerde resistentie. In dit hoofdstuk hebben we de focus gelegd op de identificatie van tomaat-homologen van de Arabidopsis syntaxin PEN1 (AtSYP121). In de groep van geïdentificeerde tomaat syntaxins waren er twee nauw verwant aan elkaar en aan AtPEN1, die we daarom SIPEN1a en SIPEN1b noemden. Een andere Arabidopsis syntaxin die een hoge mate van homologie liet zien met PEN1, SYP122 genaamd, bleek ook samen te vallen met de groep van nieuw geïdentificeerde SIPEN1 genen. Een rol van SYP122 in immuniteit was echter niet bekend uit de literatuur. Nadat we individuele RNAi constructen verkregen hadden transformeerden we de resistente ol-2 lijn en testen de verkregen transformanten met de aangepaste meeldauwpathogeen On en de niet-aangepaste Bgh. Interessant genoeg observeerden we een significante groei van On en een verhoogde celwandpenetratie van Bgh in planten waarin expressie van SIPEN1a verzwakt was, maar niet in planten waarin de expressie van SIPEN1b verzwakt was. We voerden een eiwit-alignering uit op zowel functionele als niet-functionele PEN eiwitten in Arabidopsis en tomaat. De aanwezigheid van drie verschillende geconserveerde niet-synonieme aminozuur substituties is volgens onze hypothese verantwoordelijk voor de specialisatie in immuunsysteem functie.

In Hoofdstuk 4 en Hoofdstuk 5 bouwen we aan bewijsmateriaal dat er op duidt dat de functie van MLO vatbaarheidsgenen in grote mate geconserveerd is tussen eenzaadlobbige en tweezaadlobbige plantsoorten. 
In Hoofdstuk 4 beginnen we met het identificeren en functioneel karakteriseren van twee nieuwe MLO genen in gewassen in de familie Solanaceae die vatbaar zijn voor meeldauwziekten, tabak (Nicotiana tabacum) en aubergine (Solanum melongena). We noemen deze genen NtMLO1 en SmMLO1 in de respectievelijke plantensoorten, omdat ze de nauwste verwanten zijn van het tomaat gen SIMLO1. Door deze genen tot over expressie te brengen in de resistente ol-2 lijn verkregen we transgene planten die vatbaar waren voor de meeldauwpathogeen On. Deze bevinding laat zien dat beide heterologe MLO eiwitten de functie van het beschadigde ol-2 allel in tomaat kunnen herstellen. In aanvulling hierop ontdekten we dat in het tabak eiwit NtMLO1 een aminozuur (Q198) van vitaal belang was voor de vatbaarheidsfunctie van dit eiwit.

In Hoofdstuk 5 gebruikten we dezelfde aanpak als in Hoofdstuk 4 om te laten zien dat andere MLO eiwitten van minder aan tomaat verwante tweezaadlobbige plantsoorten, zoals erwt (Pisum sativum) PsMLO1, het verlies van functie van het tomaat ol-2 allel kunnen herstellen. Tenslotte strekken we dit concept ook uit naar eenzaadlobbige MLO eiwitten, gebruik makend van gerst HvMlo. Door middel van deze experimenten konden we nagaan dat de functie van eenzaadlobbige en tweezaadlobbige MLO eiwitten betrokken bij vatbaarheid niet berust op de aanwezigheid van klasse-specifieke conservatie. Dit laatste kan de reden zijn voor de fylogenetische divergentie, die eenzaadlobbige MLO eiwitten in klade IV plaatst en tweezaadlobbige MLO eiwitten in klade $V$ van de fylogenetische MLO boom. Echter, functionele conservatie zou af kunnen hangen van cruciale gedeelde aminozuren in klade IV en V MLO eiwitten. Daarom voerden we ook een codon-gebaseerde evolutionaire analyse uit die resulteerde in de identificatie van 130 codons onder negatieve selectie, die dus sterk bewaard bleven tijdens de evolutie.

In Hoofdstuk 6 introduceren we de meeldauwziekte in komkommer, veroorzaakt door Podosphaera xanthii (Px). We kloonden het kandidaat vatbaarheidsgen voor meeldauw in komkommer, CsaMLO8, uit vatbare en resistente genotypes. De tweede werd beschreven als een geavanceerde veredelingslijn gekarakteriseerd door hypocotyl-specifieke resistentie. In deze lijn vonden we de aanwezigheid van afwijkende splicingvarianten van het CsaMLO8 mRNA, veroorzaakt door de insertie van een klasse LTR retrotransposon in de corresponderende genomische regio. Heterologe expressie van het wild-type komkommer allel in de tomaat ol-2 lijn herstelde de meeldauwvatbaarheid, terwijl heterologe expressie van de gewijzigde eiwitvariant dat niet deed. Deze bevinding bevestigd dat de resistentie van de geavanceerde komkommer veredelingslijn komt door de ontwrichting van de coderende regio van dit gen. We lieten ook zien dat de expressie van CsaMLO8 in het vatbare genotype wordt geïnduceerd door $P_{x}$ in hypocotylweefsel, maar niet in cotyl of blad. Tenslotte ontdekten we, door resequencingdata van een collectie van 115 komkommeraccessies te bestuderen, dat het TE-bevattende allel in 31 van deze lijnen voorkomt, onder welke een wilde komkommerlijn die wellicht in het verleden gebruikt is in veredelingsprogramma's om resistentie tegen meeldauw in komkommer te verwerven. 
In Hoofdstuk 7 beschrijven we een nieuw verlies-van-functie allel van het SIMLO1 gen, die we $m 200$ noemen. Dit allel is gevonden in een resistente plant (M200) uit een gemutageniseerde tomaat Micro-Tom (MT) populatie, verkregen met de chemische mutagen ethyl methaansulfonaat (EMS). De m200 mutatie is een nucleotide transversie $(\mathrm{T} \rightarrow \mathrm{A}$ ) die leidt tot een premature stop codon. De lengte van het verwachte SIMLO1 eiwit in de M200 plant is slechts 21 aminozuren, dus veel korter dan het verwachte eiwit gecodeerd door het eerder beschreven ol-2 allel, dat uit 200 aminozuren bestaat. Dankzij de ontwikkeling van een Hoge-Resolutie Smeltcurve Analyse (HRM) merker, ontworpen om de $m 200$ te detecteren, observeerden we dat dit allel recessief overervende resistentie geeft in terugkruisingspopulaties van de resistente M200 plant met MT en Moneymaker. Histologisch onderzoek laat zien dat de resistentie van de m200 mutant geassocieerd is met papillaformatie. Tenslotte vergeleken we de verhouding tussen de On penetratie in epidermale cellen van $m 200$ planten met die in planten met het ol-2 allel, en de transgene planten waarin de expressie van meerdere SIMLO homologen stilgelegd is, gemaakt in Hoofdstuk 2.

Uiteindelijk bediscussiëren we de resultaten van de voorgaande hoofdstukken in Hoofdstuk 8 in de context van 1) praktische toepassingen in veredelingsprogramma's met als doel op mlo-gebaseerde resistentie in nieuwe gewassen te introduceren, 2) mogelijk toekomstig onderzoek met als doel de functie van het MLO eiwit te ontrafelen, en 3) de rol van andere SNARE eiwitten. 


\section{Acknowledgements}

If I can hold this thesis in my hands, it is thanks to a number of people.

Thanks Richard! You welcomed me in Plant Breeding as a guest PhD, allowed me to stay and encouraged me to write a second thesis. I also deeply appreciated all the comments and suggestions on the manuscripts, on this thesis and its title.

Thanks Yuling! It is difficult to name all the things I learned with you. I have especially come to greatly appreciate our weekly discussions, which were at the beginning very new and challenging for me. You're not easy to convince, but when I do I feel my argument is so clear that I could convince the whole world. You are the voice in my mind that makes me expand my boundaries.

Thanks Anne-Marie! I believe that in these years, we became the best team of investigators. You are my Sherlock! Invaluable are all the tips you gave me, for the experiments and the writing, and the personal encouragements. You are the voice in my mind that reassures me.

Thanks to my wonderful paranymphs, Mas Muniroh and Valentina Bracuto! You both are always there for me! Mas, you are not a'voice in my mind' (luckily :D, otherwise I would not stop laughing!) but a warm, sunny hug! How fortunate I was that day that you popped into my office! Vale, you helped me with all kinds of work: lab-work, nail-work, gym-work and no-work talks, all in Italian! Thank you both for being in my life.

Thanks to Christos Kissoudis! You are my veteran colleague, my almost-paranymph, my kind, good-hearted friend, and my scientist idol!

Thanks to Myluska Caro! We met over a cup of tea in a moment of breaking nerves. Since then I've always known I could count on you. I miss you dearly in the office.

Thanks to all my officemates, the ones that were and the ones that are: Dalia Carvalho, Deniz Gol, Peter Bourke, Charlie Chen and Ehsan Motazedi. You are the reason that makes me step into the office every day with a smile!

Thanks to all my Chinese friends and colleagues, some of which are not in Wageningen anymore: Kaile Sun, Yan Zhe, Xuan Xu, Lina Lou, and Shu Zhang. We share some funny memories and some frightening ones, long days in the lab and flower trips, delicious hotpots and lots of laughs. Thank you!

Thanks to my former daily supervisors Stefano Pavan and Zheng Zheng: how would I have done without you?! Stefano, without you I wouldn't even know where Wageningen was on the map! Zheng, you were prophetic by making my first entrance card to Radix for one year instead of six months as we initially agreed. 
Thanks to all the technicians in our lab and especially Marjon Arens, Elly Janssen, Marian Oortwijn, Gert van Arkel, Gerard Bijsterbosch, Annelies Loonen, Danny Schipper, Fien Meijer, Isolde Pereira, Marjan Bergervoet, and Iris Capel. Thanks from the bottom of my heart for all the times you help me during these years! You are an invaluable source of knowledge and friendship.

Thanks to all my students, some of which are now PhDs themselves: Margaret Kirika, Antoine Maroteaux, Lisette van der Kuip, Anindita Chakraborty, Miguel Santillán Martínez, Katharina Hanika, Xulan Wang and Däniel van den Bosch. I have learned and I am learning a lot from each of you. I have appreciated knowing some of you also outside the lab! I can never forget Antoine singing in the African choir, Miguel coming to the lab after partying, Katharina bringing caring notes... Thank you, guys!

Thanks to all my PhD, Post-Doc and staff colleagues, especially Jeroen Berg, Ageeth van Tuinen, Anne Giesbers, Jordi Petit, Cynara Romero, Xiao Lin, Ernest Aliche, Abe Vila, and Herma Koehorst-vanPutten. Special thanks to Jeroen who is co-author of one of my chapters and Dutch translator of my summary!

Thanks a lot also to Henk Schouten and Rients Niks. I deeply appreciated our talks and all your suggestions for my chapters and papers.

Thanks to the greenhouse-team which made possible the experiments: Andre Maassen,

Bertus van der Laan, Casper Pillen, Henk Smid, Teus vanden Brink, Maarten Peters, Bert Essenstam, Gerrit Stunnenberg, and Taede Stoker.

Thanks to all the friends I met in my Dutch classes, especially Alena Prusova and Arwa Shahin, who never make me feel guilty for not speaking well yet!

Thanks to all my dearest friends from the ICC choir! You all helped me to take my mind off my worries and to lift up my heart in difficult moments.

Thanks to my Italian flatmates at the Hinkeloordseweg, Mattia Pia Arena, Gisella Schiavulli, and Valentina Materia. I will never forget how nice it was to live there (almost) altogether. For this, I also want to thank our lovely adoptive parents Dominique Don and Ton Tijdink.

And finally thanks to my Italian and Dutch families: my wonderful parents, my little brother, my amazing parents in-law, and my lovely husband (who became an expert user of Endnote).

Michela 


\section{About the author}

Michela Appiano was born in Foggia (Italy) on October $2^{\text {nd }}, 1984$. She obtained her Bachelor and Master studies in Agricultural Science in 2006 and 2009, respectively, in Foggia.

During her Bachelor study she was fascinated by the course of Molecular Biology and chose to do an experimental thesis on the characterization of different varieties of artichoke through SSR marker.

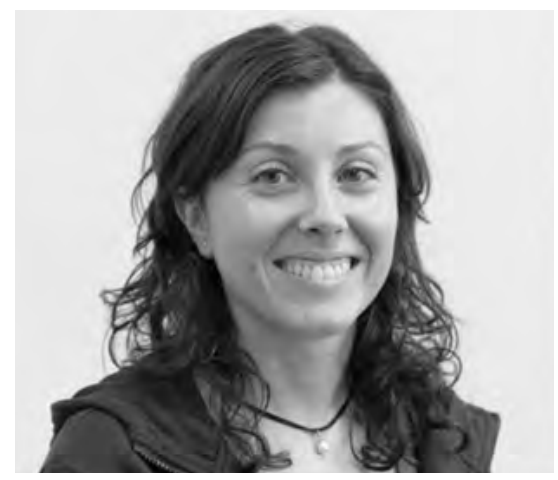

During her Master she won a fellowship granted by the International Program on Postharvest Technology, which arose thanks to the collaboration of several research groups in Europe and United States. In California, she was welcomed by the group of now emeritus - Prof. Michael Reid of UC Davis. Here she investigated the changes in gene expression during senescence and aging of a number of cut flower species.

Doing her Master thesis in this group inspired her to pursue an academic career, starting by doing a PhD. In order to decide where to do a PhD she accepted a fellowship immediately after her MSc degree at the School of Advanced Study Sant'Anna, in Pisa (Italy). Here she performed biochemical and molecular analyses on lignin biosynthesis in Gerbera cut stem. However, in January 2010 she moved back to south Italy and accepted the PhD position in Bari (Italy) within the group of prof. Luigi Ricciardi. The researcher of the group, Dr Stefano Pavan, was working on the topic of plant-powdery mildew interaction, which she found very interesting to investigate. Thus Michela started exploring the world of the $M L O$ susceptibility genes involved in this disease.

After the first year of her PhD she went to the Laboratory of Plant Breeding in Wageningen (The Netherlands) thanks to an ongoing collaboration between her Italian group and the chair of this department, Prof. Richard Visser. She spent the two following years as guest $\mathrm{PhD}$ under the supervision of Dr. Yuling Bai, leader of the group breeding for resistance in Solanaceae. In April 2013 she obtained her Italian Doctorate title.

It became clear to her that working in this multicultural, inspiring environment was a challenge she wanted to accept to deepen her scientific skills. Therefore, she continued working in the same group as PhD student of Wageningen University. The results of her project aimed at understanding the resistance based on impaired $\mathrm{mlo}$ genes are described in this thesis. 



\section{List of publications}

Zhengt Z., Appianot M., Pavan S., Bracuto V., Ricciardi L., Visser R.G.F., Wolters A.M., Bai Y. (2016) Genome-wide study of the tomato SIMLO gene family and its functional characterization in response to the powdery mildew fungus Oidium neolycopersici. Frontiers in Plant Science, 7:380.

Appiano M., Catalano D., Santillan-Martinez M., Lotti C., Zheng Z., Visser R.G.F., Ricciardi, Bai Y., Pavan S. (2015) Monocot and dicot MLO powdery mildew susceptibility factors are functionally conserved in spite of the evolution of classspecific molecular features. BMC Plant Biology, 15:257.

Berg† J., Appianot M., Santillan-Martinez M., Hermans F., Vriezen W., Visser R.G.F., Bai Y., Schouten H. (2015) A transposable element insertion in the susceptibility gene CsaMLO8 results in hypocotyl resistance to powdery mildew in cucumber. BMC Plant Biology, 15:243.

Appianot M., Pavant S., Catalano D., Zheng Z., Bracuto B., Lotti C., Visser R.G.F., Ricciardi L., Bai Y. (2015) Identification of candidate MLO powdery mildew susceptibility genes in cultivated Solanaceae and functional characterization of tobacco NtMLO1. Transgenic Research, 24: 847.

Gao D., Appiano M., Huibers R., Chen X., Loonen A., Visser R.G.F., Wolters A.M., Bai Y. (2014) Activation tagging of ATHB13 in Arabidopsis thaliana confers broadspectrum disease resistance. Plant Molecular Biology, 86: 641.

Gao D., Appiano M., Huibers R., Loonen A., Visser R., Wolters A.M., Bai Y. (2014) Natural loss-of-function mutation of EDR1 conferring resistance to tomato powdery mildew in Arabidopsis thaliana accession C24. Molecular Plant Pathology, 16, 1:71-82.

Zheng Z., Appiano M., Nonomura T., Pavan S., Wolters A.M., Matsuda Y., Toyoda H., Visser R.G.F., Bai Y. (2013) Loss-of-function in Mlo orthologs reduces susceptibility of pepper and tomato to powdery mildew disease caused by Leveillula taurica. PLOS ONE, 8(7): e70723.

Pavan S., Schiavulli A., Appiano M., Miacola C., Visser R.G.F., Bai Y., Lotti C., Ricciardi L. (2012) Identification of a complete set of functional markers for the selection of er1 powdery mildew resistance in Pisum sativum L. Molecular Breeding, 31: 247.

Pavan S., Schiavulli A., Appiano M., Marcotrigiano A.R., Cillo F., Visser R.G.F., Bai Y., Lotti C., Ricciardi L. (2011) Pea powdery mildew er1 resistance is associated to loss-of-function mutations at MLO homologous locus. Theoretical Applied, 123: 1425.

tshared first authorship 
Financial support from Wageningen University for printing this thesis is greatly appreciated.

Source material for cover picture: Prof. Pietro Spanu, Imperial College London Cover \& layout design: Iliana Boshoven-Gkini | AgileColor. com

Printed by: GVO drukkers \& vormgevers, Ede (NL) | gvo.nl 


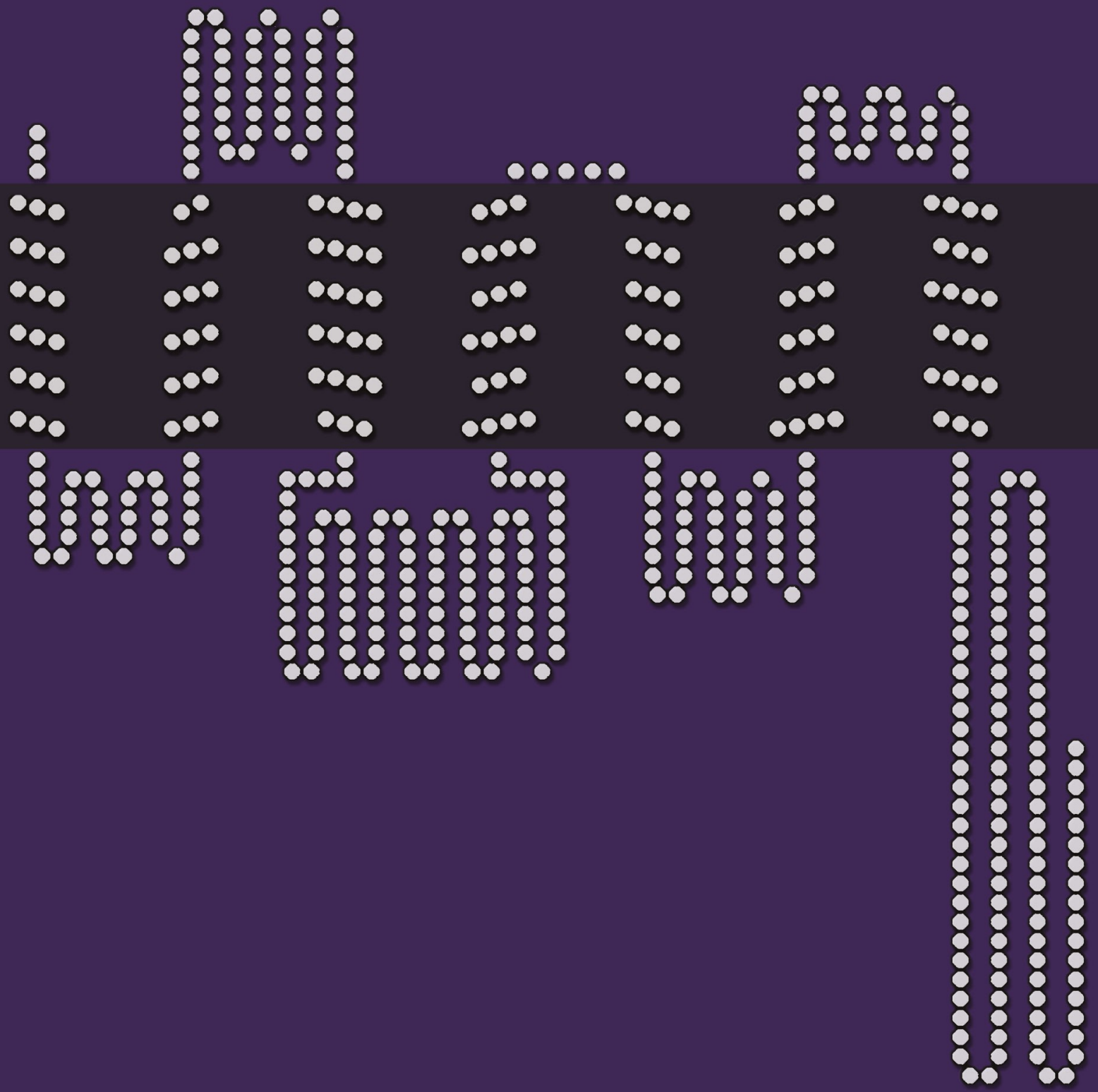

